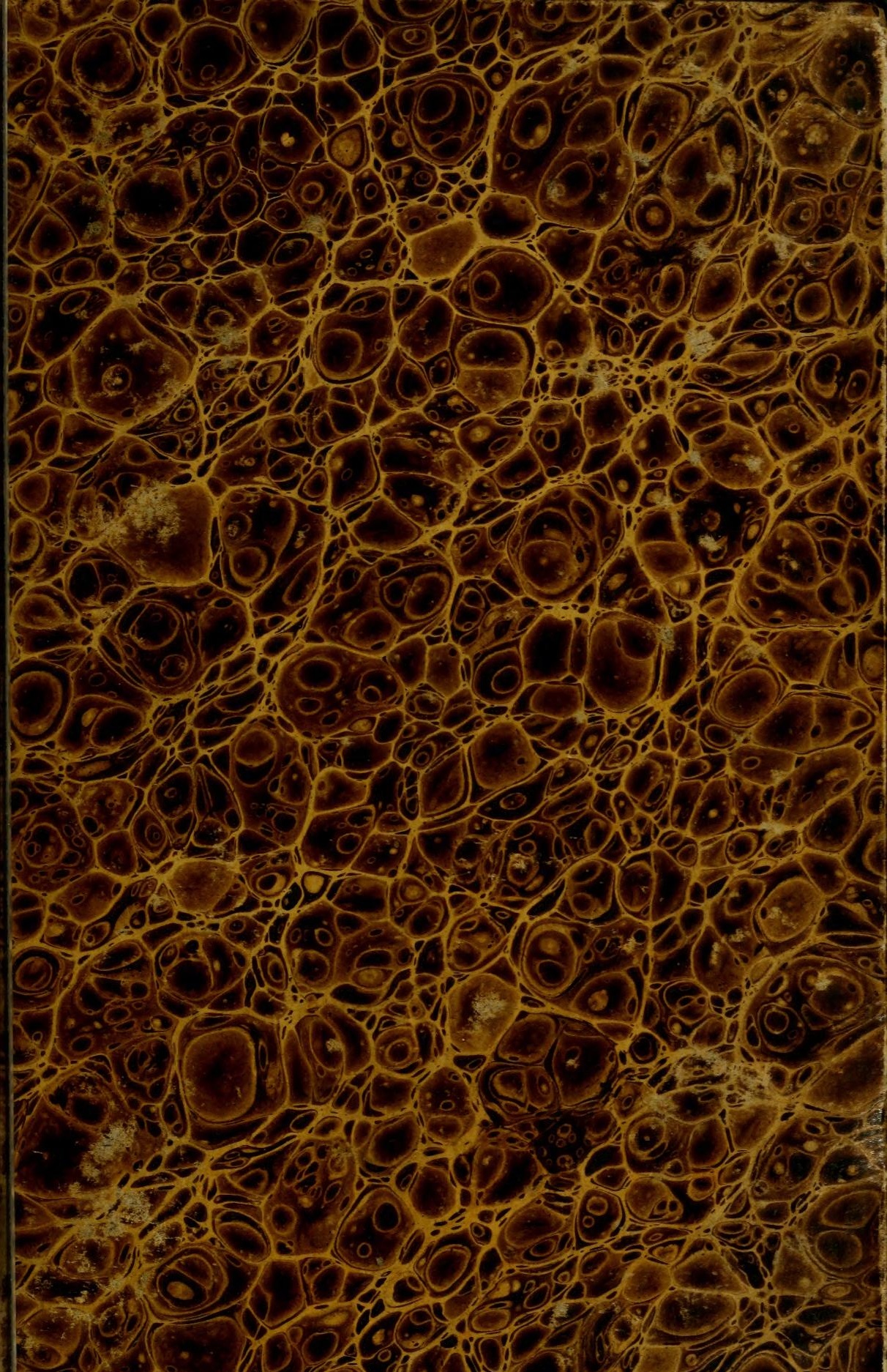




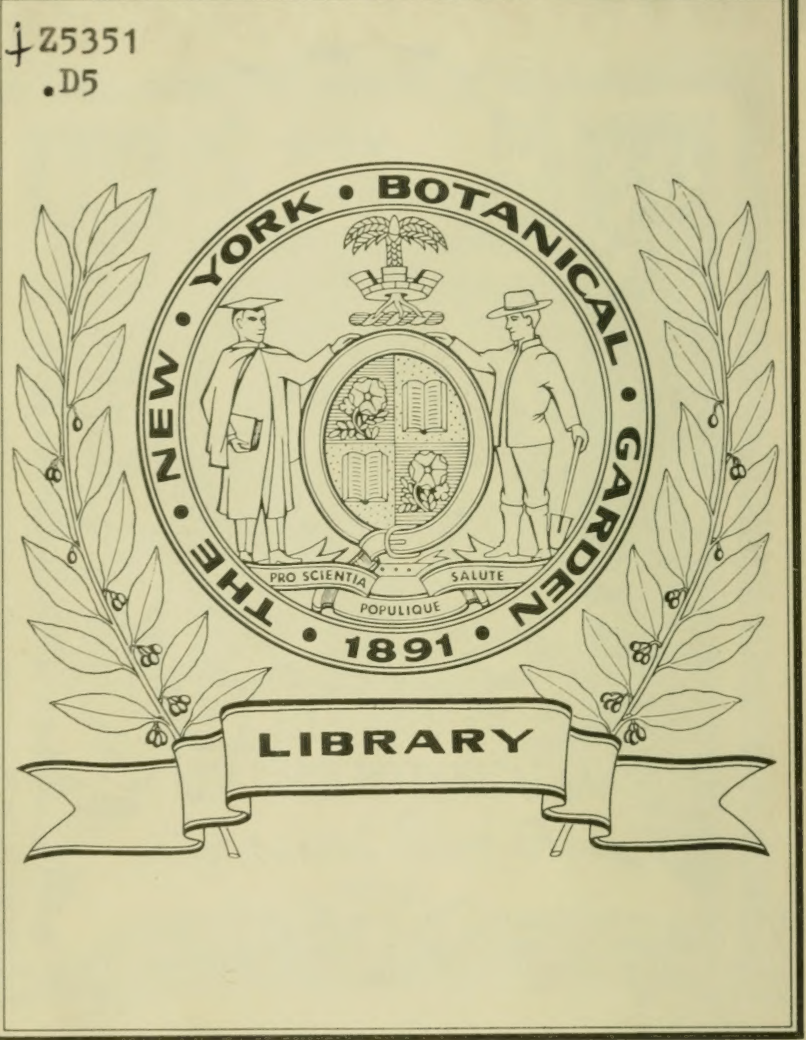






\section{Repertorium botanicum}

o d e r

\section{V e r s u c h}

e in er

\section{systematischen Darstellung der neuesten Leistun- gen im ganzen Umfange der Pflanzenkuinde}

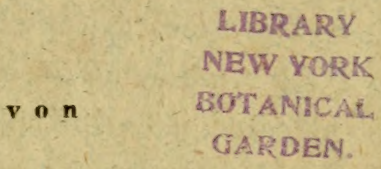

Dr. Joh. Heinrich Dierbach,

aufserordentlichem Professor der Medicin in Heidelberg, der naturforschenden Gesellschaft in Altenburg, der medicinisch-chirurgischen in Berlin, der Schlesischen Gesellschaft für vaterländische Cultur in Breslan, der physikalisch - medicinischen in Erlangen, der Senkenbergischen naturforschenden in Frarkfurt a. M., jener für Naturwissenschaften und Heilkunde in Heidelberg, der naturforschenden in Leipzig, der medicinisch - botanischen in London, der Linncischen Societät in Lyon, der K. Bair. botan. Gesellschaft in Regensburg, der Wetterauischen Gesellschaft für die gesammte Naturkunde und des Apotheker-Vereins im nördlichen Deutschlande Mitglied.

L e m g o, 1831.

Me yersehe Hof - B uch a nd lung. 
musumintod PFrimols qoH

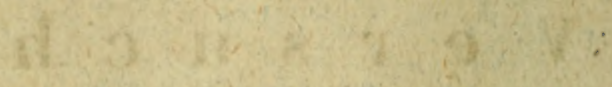

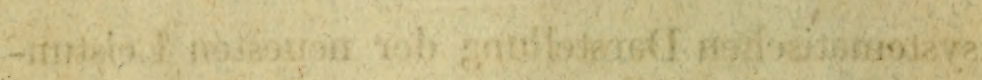

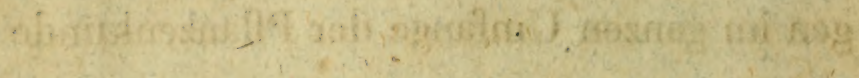

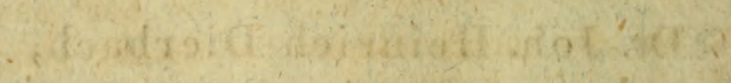

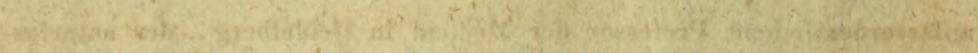

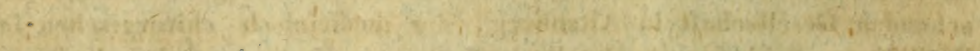
Q

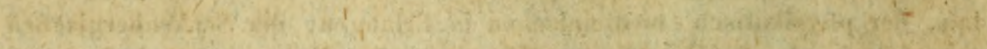

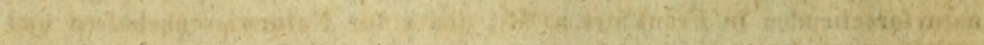

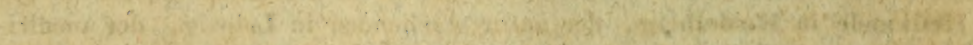

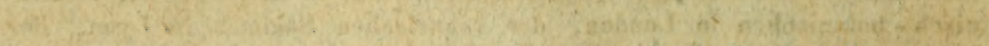

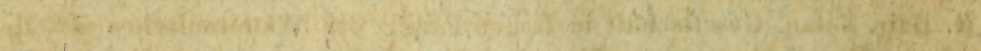

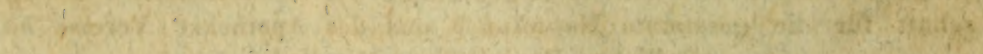

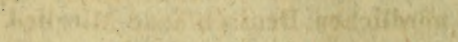

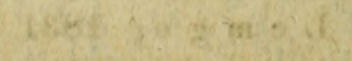

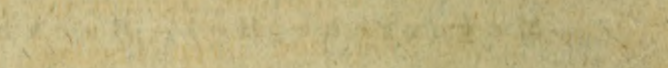




\section{$V$ o $\quad r$ w}

\section{LIBRARX \\ NEW YORK \\ BOTANICAL \\ GARDEN.}

Schüchtern nur übergebe ich diese mehrere Jahre lang schon vorbereitete Arbeit dem Publikum, das meine bisherigen Leistungen mit so vieler Nachsicht aufnahm. Wer würde aber auch nicht gleichsam zagend eine Laufbahn betreten wollen, auf der nur die Heroen der Botanik glänzen konnten? Haller's Bibliotheca botanica steht vereinzelt da, und wird so leicht nicht einen ihr würdigen Nachfolger finden. Sprengel's Geschichte der Botanik ist ein unvergänglicher Zeuge des umfassenden und seltenen Talentes ihres Verfassers, und auch die literarische Arbeit, die der verdiente Schulte's lieferte, hat die gerechtesten Ansprüche auf den wärmsten Dank der Mit - und Nachwelt.

Niemals konnte und wollte ich es wagen, die vollständige Literatur der Botanik zu bearbeiten, was meine Kräfte weit überschritten haben würde, ich beschränke mich daher nur auf die jüngste Zeit, die so Vieles und darunter so viel Schönes und Vortreffliches aufzuweisen hat.

Dieses erste Bändchen umfafst die Leistungen in der allgemeinen Botanik und ihren Theilen, das zweite wird der 
medicinisch - pharmaceutischen und chemischen Section der Pflanzenkunde gewidmet sein, und das dritte wird die zahlreichen neuen Entdeckungen in dem Gebiete der ökonomischen, technischen und Forst-Botanik darstellen, und besonders die mannichfaltigen Vortheile auf das augenscheinlichste zeigen, welche Ackerbau, Künste und Gewerbe aus der genaueren Kenntnifs des Pflanzenreichs ziehen können. Absichtlich liefs ich daher Manches in dem ersten Theile weg, das schicklicher in den übrigen eine Stelle finden wird. Alle drei machen zusammen ein Ganzes aus, doch so, dafs auch jeder Theil für sich eine eigene von den andern unabhängige Schrift bildet. - 


\section{Uebersicht des Inhalts.}

Erster Abschnitt. Allgemeine Quellen zur Kenntnifs des jetzlgen Zustandes der Pflanzenkunde und Hülfsmittel zum Studium derselben. \$. 1.

Der Botanik ausschliefslich gewidmete Zeitschriften. §. 2.

Zeit- und Gesellschafts-Schriften, welche öfters die Botanik betreffende Aufsätze enthalten. §. 3 .

a. Deutsche

b. Französische .

c. In Rufsland erscheinende Zeitschriften

d. Englisehe

e. Italienische

f. Holländische, Dinische und Schwedische:

Der Botanik allein gewidmete alphabetische Werke. §. 4.

Wörterbücher oder alphabetische Werke, die auch Gegenstände der Pflanzenkunde abhandeln. §. 5.

Propädeutische Schriften, Anleitungen zum Studium der Botanik, und dicsen Gegenstand betreffende Abhandlungen. §. 6. 14

a. Deutsche

b. Französische und Belgische

c. Englische .

d. Italienische

e. Schwedische

Specielle Hülfømittel zum Studium der Botanik. §. 7. . 18

Anstellung botanischer Excursionen . . . . . 19

Anlegung von Herbarien . . . 19

Käufliche Sammlungen getrockneter Pflanzen 
Ans Deutschland, der Schweiz und Ungarn

Aus Italien

Aus England, Rufstand und Griechenland : : 25

Aus Asien . . . . . . $\quad 25$

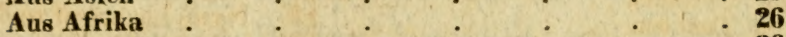

Aus Amerika : . . . . . . . 26

Aus Neu-Holland : $\quad$. $\quad$ \& $\quad 27$

Sammlungen von Cryptogamen _. . . .

Tausch-Anstalten und Reise-Vereine . . . 29

Botanische Instrumente . . . . . . 30

Nenere Phytographie und Iconographie. §. 7. . . . 32

a. Deutsche . $\quad . \quad \ldots \quad$. . 32

b. Französische $\quad: 35$

c. Englische . $\quad . \quad$. . $\quad 36$

d. Italienische $\quad \vdots \quad \vdots \quad \vdots \quad 36$

Zweiter Abschnitt. Bearbeitung des Systems. \$. 8. . . 37

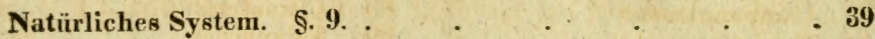

a. Schriften der Deutschen . $\quad$. . $\quad$. 39

b. Schriften der Franzosen und Italiener . $\quad . \quad 41$

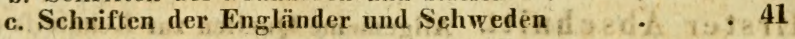

Specielle systematische Arbeiten. \$. 10.

Acotyledonen oder Cryptogamen. §. 11 . . . 42

Erste Section. Zellenpflanzen. \$. 12, .

Algen. . $\quad 0.93$ Lebermoose ... . . 57

Schwämme oder Pilze : 48 Laubmoose $: 58$

Flechten - . . . Nuls. 55

Zweite Section. Cryptogamische Gefäfspflanzen. \&.13. . . 61

Marsileaceen 61 Characeae brof . . 64

Farrenkräuter . . 62 Equisetaceae 65

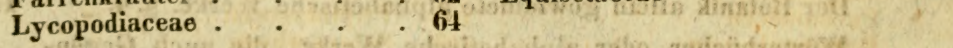

Dritte Section. Phanerogamen, Endogenen: Monokotyledonische Gefäfspflanzen. §. 14.

Gramincen $\quad 66$ Smilaceen.... 64

Cyperaceen $\quad$ 70 Dioscoreen $: 75$

Kestiaceen $\quad 71$ Orchideen.$\quad 75$

Junceen $\quad 71$ Scitamineen . . 76

Commelinaceen $\quad$. $\boldsymbol{\gamma 1}_{1}$ Palmen . . . 76

Haemadoraceen . . 71 Aroideen . . 78

Irideen . . 71 Najadeen . . . 78

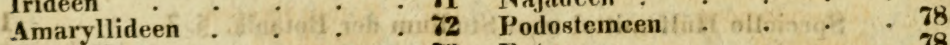

Bromeliaceen . ...mi. 72 Butomeen .78

Asphodeleen : . . , 73 Hydrocharidecm, . . 78 
Vierte Section. Exogenen oder Dicotyledonen, Chlanydoblasta. Exogenen mit verhülltem Embryo. \$. 15.

pag.

pag.

Cytineen . . . . $\quad 79$

Tacceen . . . . 80

Piperaceen $\therefore \quad 80$

Nymphaeaceen und Nelumbo-

Saurureen . . . . 80 neen....

Fünfte Section. Gymnoblasta apetala. Exogenen mit nacktem Embryo und ohne Corolle. §. 16.

Cycadeen . . . . . 81

81 Laurinren . $\quad$. 84

Abietineen _. . . 81 Thymelaeen . . $\quad .84$

Amentaceen - . . . 82 Proteaceen . $\quad .84$

Artocarpeen . . . 83 Salicineen $88^{\circ} \cdot 85$

Polygoneen . . . 83

Sechste Section. Gymnoblasta monopetala. Exogenen mit nacktem Embryo und einblättriger Corolle. §. 17. . . . 85

Plantagineen * . $\quad 86$ Gesnerieen . . $\quad . \quad 98$

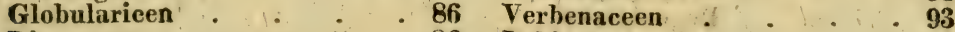

Dipsaceen * . . . 86 Labiaten . . . . . 93

Valeriancen . $\quad . \quad .86$ Acanthaceen $. \quad . \quad 95$

Synanthereen * * $\quad 86$ Bignoniaceen : * . . 95

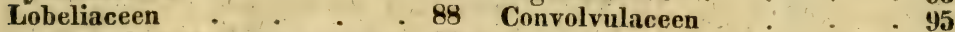

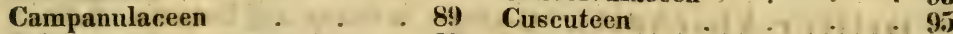

Ericineen : . * . 89 Solanaceen $\quad$ - 96

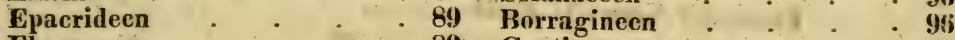

Ebenaceen $\quad \cdots \quad 89$ Gentianeen $\quad . \quad 08$

Ardisiaceen : _ _ . 90 Asclepiadeen $\quad . \quad 0 \%$

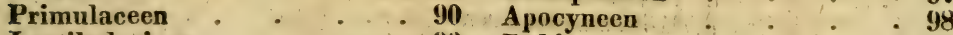

Lentibularicen . $\quad \therefore \quad 90$ Rubiaceen $\quad \cdots \quad .98$

Scrophularineen . . . 90 Caprifoliaceen . ., 99

Orobancheen - * . 92 Jasmincen — ? 99

Siebente Section. Gymnoblasta polypetala. Exogenen mit nacktem Embryo und vielblättriger Corolle. §. 18.

Lorantheen - 100

: 109

Umbelliferen $\quad \cdots \quad 101$ Frankeniaceen $\quad . \quad 109$

Araliaceen $\quad \cdot \quad 102$ Hypericineen $\quad \div \quad 109$

Menispermeen $\quad 102$ Garcinieen $\quad . \quad 110$

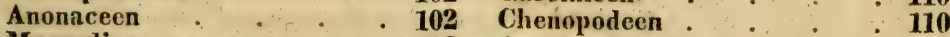

Magnoliaceen - * 102 Amaranthaceen $: \quad \div 110$

Paeoniaceen * $\quad$. 103 | Phytolacceen . $\quad \because 110$

Ranunculaceen - $\quad$ - 103 Sclerantheen : $: \quad$ : 111

Polygaleen $\div \quad \therefore \quad 114$ Paronychieen $: \div \quad \div 111$

Resedaceen - . • 104 Portulaceen : : $: 111$

Fumariaceen : $: 105$ Alsineen $\quad \div \quad \div \quad 112$

Papaveraceen $\quad 105$ Sileneen $: \div \quad 112$

Cruciferen - . 105 Crassulaceen $\quad 112$

Capparideen . . . 106 Saxifrageen . . . 113

Passifloreen - . $: 106$ Halorageen : $: 0_{13}$

Loaseen $\quad \div \quad 107$ Lythrarieen $: \div \quad \vdots 114$

Cucurbitaceen $: \div 107$ Onagrarien $: \div 11$

Grossularieen . $: \therefore 107$ Combretaceen $: \vdots 114$

Nopaleen $: \div \quad 108$ Granateen $: \div 114$

Cistineen $: \quad: \quad 108$ Calycantheen : $: \quad: 115$

Violaceen

108 Melastomaceen

Droseraceen

109 Myrtaceen 


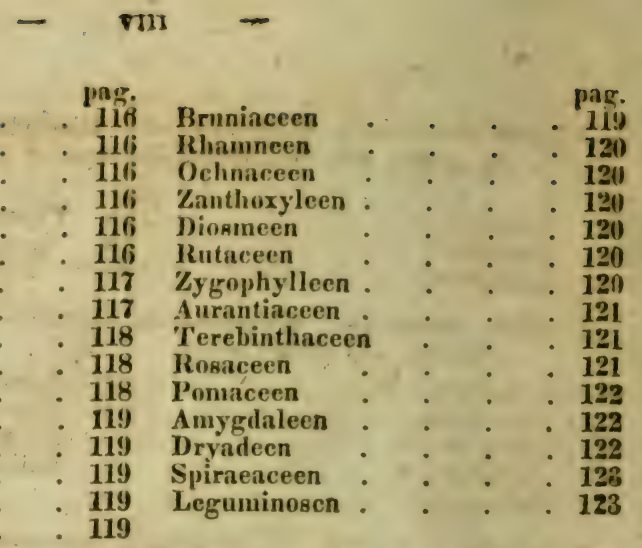

Cannelliaceen. . Pî̉ Bruniaceen ..

Ternstroemiaceen . . . 116 Rhamneen • • • $: 120$

Stereuliaceen . . . . 116 Ochnaceen . . . . 1211

Buittneriaccen - . . . 116 Zanthoxyleen : . . . 120

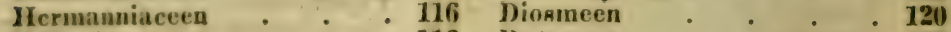

Malvaceen * . . . 116 Rutaceen . • . . 120

Geraniaceen - . . . 117 Zygophylleen . . . . 120

Lineen * . . . 117 Aurantiaceen . * . . 121

Oxalideen . . . . 118 Terebinthaceen * . . 121

Balsamineen . • - . 118 Rosaceen ? . . . 121

Ampelideen . . . . 118 Pomaceen . . . . 122

Malpighiaceen * • . 119 Amygdaleen . • . . 122

Acerineen * * . . 119 Dryadecn * * * . 122

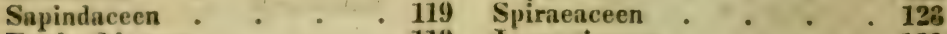

Euphorbiaceen • . . 119 Leguminosen . . . . 123

Empetreen ... . . 119

Familien, deren Stelle im System ungewifs ist. §. 19. . 125

Ceratophylleen . . . . . 125

Begoniaceen $\quad$. $\quad$. $\quad$. 125

Dritter Abschnitt. Geologie in Bezug auf Botanik. §. 20. . 125

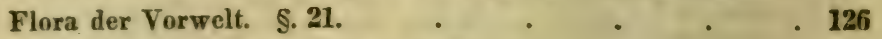

Statistik des Gewächsreiches. §. 22. . . . . 130

a. Geognostische Schriften : - . . . 131

b. Höhenmessungen $\quad . \quad$ • . . . . 131

c. Temperatur - Verhältnisse : • . 132

Schriften und einzelne Aufsätze über Pffanzen-Geographie.

§. 23. • • • • • • • . 133

a. Allgemeine $\quad \cdot \quad 133$

b. Zur Pflanzengeographic einzelner Länder : . 135

Dentrchland . . . . . . 135

Schweir $\quad . \quad$ • $\quad . \quad$. 139

Frankreich und Spanien $\quad: \quad \therefore \quad: \quad 139$

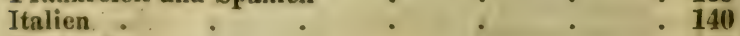

England $\quad . \quad$. $\quad . \quad$. $\quad .141$

Schweden $\quad$. $\quad . \quad$. $\quad 142$

Rufsland . . . . . . 142

Asien . . . . . . . 143

Afrika : $\quad . \quad$. $\quad . \quad$. 143

Amerika $\quad$. $\quad$ • . . 144

Nen-Holland : . . . . 145

Reiwebeschreibungen, Notizen enthaltend, die für die Geogra-

phie der Botanik benutzt werden können. §. 24. . . 145

Zeitschriften für Reisebeschrcibungen . . . 146

Reisen um die Welt . . . 146

- in Deutachland, Ungarn und lioliand : 147

- in der Schwriz und den benachbarten Alpen . 148

- in Frankreieh, Italien und Griechenland _ 149

- in England und dem übrigen nördlichen Europa 149

- in Arien und Afrika . . 150

- in Amerika und Australien : $\quad 152$ 
Literatur der Floren. \$. $25 . \quad \therefore \quad \quad \quad \quad \quad \quad 153$

A. Nord-Alpen-Länder . . . . . 154

a. Gebiet der Donau und ihrer Nebenflüsso 155

b. Gebiet des Rheins und seiner Nebenflïsse , . 159

c. Gebiet der Weser und Ems . 165

d. Gebiet der Elbe $\quad \ldots \quad \cdots \quad \ldots$

c. Gebiet der Oder $\quad 168$

f. Unteres Gebiet dor Weichsel und der nahen Kïstenflüsse.

g. Halbinsel Jütland, dänische Inseln und Helgoland . . . . . 171

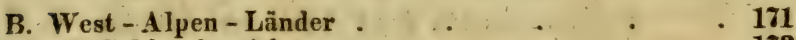

a. Gebiet der Adour . . 172

b. Gebiet der Garonne mit den Neben - und Küsten-

flüssen

173

c. Gebiet der Loire : $\quad$. 174

d. Gebiet der Seine $\quad \ldots \quad \ldots 174$

c. Gebiet der Schelde . $\quad$. $\quad$. 175

f. Gebiet der Rhone : $\quad 176$

C. Süd - Alpen - Lằnder • $\quad 177$

a. Gebiet der kleineren Alpenflïsse Etsch, Brenta,

Piave, Tagliamento, Isonzo u. 8. w. . . 177

b. Gebiet der Küstenflüsse der Gebirgakette Poglicza (Dalmatien, Croatien) . . 179)

c. Gebiet des $P_{0}$ und seiner Nebenflüsse . $\quad$ 179

d. Gebiet der Apenninenflüsse $\quad$. $\quad . \quad 180$

Italienische Inseln, Corsica . . . . 181

Sardinien, Sicilien . $\quad . \quad \quad \quad . \quad 182$

D. Pyrenäische Halbinsel nebst den balearischen Inseln 182

E. Brittische Inseln $\quad \ldots \quad \ldots \ldots$

F. Scandinavien . . . . . . 185

G. Rufsland . . . . . 185

H. Griechenland . . . . . 186

I. Asien : $\quad$ - $\quad$ - $\quad 187$

K. Afrika $\quad$. $\quad$. $\quad$. 189

1. Amerika . $\quad$. $\quad$. 191

M. Australien _ . $\quad$. $\quad$. $\quad$. 196

Vierter Abschnitt. Anatomie und Physiologie des Gewächsreiches. §. 26.

Sehriften und einzelne Aufsätze über die Structur und die Function der Pflanzentheile. §. 27.

Primitive Entwicklung der niedersten vegetabilischen Organismen und Ansichten über die Metamorphosen derselben. \$. 28.

Forschungen über die El.ementartheile des Gewächsreiches. ฐ. 29. 
Untersuchungen über dio Organe der Nutrition, Wurzel, Stengel, Blätter und ihre mecessorischen Theile. §. 30. . 203

Ernährung und Wachsthum der Pflanzen. §. 31. . . 206

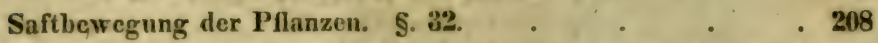

Untersuchungen über die Vermehrung der Gewiakse durch Theilung, oder die individuelle Reproduction. \$. $33 . \quad 210$

Untersuchungen über dic Eigensehaften der Blumen und ihrer Theile. \$. 34 . . . . . . . 212

Untersuchungen der Früchte und Samen. §. 35. • . 21.t

Sexualität der Pflanzen. §. 36. . . - - . $21 \%$

Secretionen vegetabilischer Theile. \$. 37. . . . 221

Wirkung der Elemente anf die Pflanzen und dieser anf ihre

Umgebung. \$. $38 . \quad$.

Untersuchungen über die Wirkung melirerer Gasarten und Gifte auf die Pflanzen. §. 39. . . . . . 2213

Untersuchungen über die Bewegungen niederer Organismen and Molecülen. §. 40. ‥ . . . 228

Krankheiten und Mifslildungen der Gewächse. §. $41 . \quad$. 22!)

Fünfter Abschnitt. Geschichte der Botanik . . . 232

Untersuchungen über die Pflanzenkenntnifs früherer Zciten. ฐ. 41.

Beiträge zur Geschichte der Botanik, besonders der neueren Zeit. \$. $42 . \quad$ • . . . . . . 235

Nachrichten von Anstalten für Naturwissenschaften, besonders von botanischen Gärten. §. 40. ․ . . . . 236

a. In Deutschland !

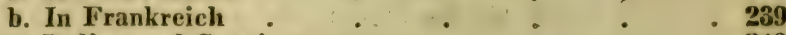

c. Italien und Spanien $\quad . \quad 240$

d. England, Dänemark, Schweden und Rufsland : 241

e. Aufserhalb Europa . . . . . 243

Nachrichten von mehreren Botanikern und Freunden der Gewrehskunde. \$. 44. … . . 243

Litcratur der Botanik §. 45 . . . . . . 250 
Seite 169 ist Endlicher's Flora Posoniensis irriger Weise unter den Floren des Odergebietes aufgeführt worden; sie ist dort zu streichen und dagegen Seite 158 nach Sadler's Flora Comitatus Pcstiensis einzuriicken.

Auf Seite 162 ist das dort angeführte Sirona-Bad bei Nierstein zwar an sciner rechten Stelle, da es in das Gebiet des Mittelrheins gehört; nufs aber von den vorhergehenden Schriften, die die Flora des Neckargebictes beleuchten, getrennt werden. -

Die Seite 161 berührte Schrift, welche eine Aufzählung der Pflanzen der Rheinpfalz enthält, ist seitdem wirklich im Druck erschienen und führt folgenden Titel:

Enumeratio plantarum in ditione Florae Palatinatus sponte crescentium. Post Pollichium, Kochium et Zizium denuo recensuit auxitque C. Petif, Med. Dr. Pars phanerogamica. Biponti typis Georgii Ritteri 1880. 8. 



\section{Erster Abschnitt.}

Allgemeine Quellen zur Kenntnifs des jetzigen Zustandes der Pllanzenkunde, und Hülfsmittel zum Studium derselben.

Nullibi suppellex literaria amplissima adeo necessaria est, ac in Botanice.

Linnaei Philos. botan. Ed. Sprengel pag. 4.

\section{§. 1.}

Jedes Jahrhundert, ja noch weit weniger von einander entfernte Zeiträume zeichnen sich jetzt durch den eignen Geist und Sinn ans, in dem die Wissenschaften bearbeitet werden. Trotz den mannichfaltigen Richtungen, die das Studium der Pflanzenkunde in verschiedenen Ländern nahm, trotz den widerstrebenden Ansichten, die noch im Schwunge sind und sich schwer werden vereinigen lassen, schreitet doch die Wissenschaft selbst mit ruhigem und sicherem Gange unaufhaltsam weiter, und aus dem Gewirre der Meinungen blickt da und dort ein Funke der Wabrheit hervor, hell leuchtend und nimmer erlüschend, oft schimmert sie an einem Orte heraus, wo man sie nicht vermuthet hälte: ja 80 manche Ansicht, die unsere Vorfahren kannten, aber verlachten, haben wir jetzt als richtig und bewährt gefunden; und Anderes, das ihnen hoch und theuer war, ist bei uns nicht ohne Grund in Vergessenheit gesunken. Dies ist der ewige und unveränderliche Gang aller Wissenschaft, wie ilın die Geschichte aller Zeiten und aller Völker lehrt; und sollten wir auch keinen andern Vortheil aus diesen Betrachtungen ziehen, so werden sie doch an eine grosse Tugend mahnen, die mehr und mehr bei unserer studierenden Jugend zu verschwinden scheint, und sie doch vor allen andern zieren wïrde, ich meine die - Bescheidenheit, die Duldsamkeit gegen Andersdenkende, die ruhige Prüfung verschiedener Ansichten, deren jede, wenn sie auch vielfältig irren sollte, doch manches Gute, manches Brauchbare in sich schliessen kanu, das der Beachtung und der Benutzung werth ist. 


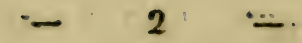

Wenn ich es wagte, eine Uebersicht der Leistungen in einem so weiten Gebiete zu liefern wie die Botanik ist, so verhehlle ich mir keineswegs die damit verbundenen grossen Schwierigkeiten, ja ich sah vollkommen gut ein, dass es fast unmöglich ist, das vorgesteckte Ziel so zu erreichen, dass daran nichts auszusetzenwäre. Leicht kann ich vermuthen, dass mir Manches entging, dass sich, trotz aller Aufmerksamkeit, Irrthümer haben einschleichen können, zumal bei den Titeln von Werken, die ich nicht selbst sah, und deren Inhalt mir blos durch fremden Bericht bekannt wurde; doch ich gab, was und wie ich es vermochte, und wern auch weiter nichts, so kann ich doch das versichern, dass es mir an redlichem Willen nicht fehlte, alles, was in meinen Kräften stand, aufzuwenden, um diese Schrift den Freunden dex Gewächskunde brauchbar zu machen, und ich hoffe, diesen Endzweck nicht ganz verfehlt zu haben.

Der Botanik ausschliefslich gewidmete Zeitschriften.

§. 2.

Nur Deutschland und England besitzen schon längere Zeit dergleichen, aber blos die des ersten Landes befassen sich mit allen Theilen der Botanik als Wissenschaft, während die englischen grofsentheils blos die Absicht haben, neue oder seltne Geu'üchse zu beschreiben und Abbildungen von ihnen zu liefern. Nur erst in den jüngsten Zeiten findet man bisueilen in ihnen vortreffliche monographische Arbeiten: Erörterungen einzelner Familien, Gruppen, Gattungen u.s. w. Doch ist auch bei diesen Arbeiten die Vorliebe für das Neuere und Seltnere nicht $\approx u$ verkennen.

Diese Zeitschriften sind aber folgende:

Flora oder botanische Zeitung, welche Recensionen, Abhandlungen, Aufsätze, Neuigkeiten und Nachrichten, die Botanik betreffend, enthält. Herausgegeben von der königl. botan. Gesellschaft in Regensburg. Erster Jahrgang, Regenshurg 1828, zwei Bände.

Diese Zeitschirift ist seitdem regclmäfsig fortgesetzt und ihr UTmfang durch verschiedene Beiträge, Beilagen und Zusätze ausgedehnt worden. Maupt-Redacteur ist der um Deutschlands Botanilk hochverdiente Professor und Sanitätsrath Dr. Hoppe.

Literaturbläter für reine und angewandte Botanik. Herausgegeben von der königl. botanischen Gesellschaft in Regensburg. Erster Band. Jahrgang 1828. Nürnberg bei Riegel und Wielsner 1828. Fine bis jetzt noch fortdauernde ungemein reichhaltige Zeitschrift, die besonders Auszüge aus französischen und englischen W'erken enthält, und überhaupt mit eben so grofser Umsicht als Thïtigkeit bearbeitet wird. Haupt-Redacteur ist, so viel mir bekunnt, Herr Dr. Eschweiler in Regensburg.

Linnaea. Ein Journal für die Botanik in ihrem ganzen Umfange. Herausgegelsen von D. F. I. von Schlechtenda1, Med. Chir. et Phil. Dr. Erster Band. Jahrgang 1826. Mit sieben Kupfertafeln. Berlin 1826. 
Diese Zeitschrift, welche noch fortwaihend besteht, erscheint in vierteljährigen Heften, und wird besonders interessant durch die Beschreibung der zahlreichen neuen Gewächse, dic in den könighlichen Sammlungen in Berlin enthalten sind. - Seit 1828 liefert dieses Journal abgesondert einen Literatur-Bericht zur Linnaea, der zuar in der That reichhaltig ist, aber wohl eben deswegen nur äufserst kurze Anzeigen liefert, wovon jedoch die Auszüge aus ausländischen Zeitschriften eine Ausnahme machen.

The botanical Register: consisting of coloured figures of exotic plants, cultivated in british gardens, with their history and mode of treatment. The designe by Sydenham Edwards, Fellow of the Linnean society. London 1815 .

Der erste Herausgeber dieser Zeitschrift ist Joh. Bellerden Gawler, genannt Ker; jetzt enthält sie bcsonders schätzbare Aufsätze von Lindley und Andern.

The botanical Magazine or flower garden displayed; in which the most ornamental foreign plants cultivated in the open ground, the greenhouse and the stove are accurately represented in their natural colours etc. By Wm. Curtis. Continued by J. Sims ete. 1787.

Enthält schon über fünfzig Bünde und wird noch immer fortgesetzt und zwar neuerdings von Hooker, dem diese Zeitschrift ihre jetzige vortreffliche Finrichtung verdankt. Schätzenswerthe Beiträge liefern $D$ on und Andre.

The botanical Cabinet, consisting of coloured delineations of plants, from all countries, with a short account of cach, direction for management etc. By Conr. Loddiges et Sons. London, seit 1817.

Exotic Flora, containing figures and descriptions of new, rare, or otherwise interesting exotic plints, especially of such as are descrving of being cultivated in our gardens etc. By W. J. Hooker etc. Edinburgh, seit 1823.

Man sehe bot. Zeit. 1823. p. 33. 1825. 2. p. 24 Beil.

W. J. Hooker Botanical Miscellany; containing Figures and Descriptions of such Plants as recommend themselves by their Novelty, Rarity, or History, or by the uses to which they are applied. Part. I- III. gr. 8. London. Treuttel and Würtz.

Siehe Bot. Literaturbl. II. p. 226.

Bulletin botanique ou Collection de notices originales et d'extraits des ouvrages botaniques, souvent accompagné de gravures représentants les analyses d'organes importantes de la fleur et du fruit, par Sèringe. Genève 1830.

Ist mir noch nicht zu Gesicht gekommen, eine kurze Nachricht davon steht in den bot. Literaturbl. IV. p. 123.

Zeit - und Gesellschafts - Schriften, welche öfters die Botanik betreffende Aufsätze enthalten.

\section{§. 3.}

Fs sind ihrer sehr viele, von denen bercits Schultes in seinem höchst schätzbaren Grundrisse einer Geschichte und Literatur der Botanik p. 6 u. d. $f$. mehrere zusammenstellte; viele derselben werden noch in den beiden folgenden Bänden dieser Schrift ihre Stelle finden; hier begnüge ich mich, die folgenden namhaft zu machen: 


\section{- 4 - \\ n. $D$ e uts $\mathrm{c}$ he.}

Notizen aus dem Gebiete der Natur- und Ireilkunde, gesammelt und migetheilt von hudwig Friedrich r. Froriep, des $\boldsymbol{K}$. $\boldsymbol{W}$. Civil-Verdienst-Ordens Ritter etc. Gedruckt in Erfurt bei Lossius, in Commisaion bei dem G. II. S. pr. Landes-Industrie-Comptoir zu Weimar. 4.

Jetzt sind zuischen zu'anzig und dreifsig Bänden dieser Zeitschrift erschienen; sie zeichnet sich besonders dadurch aus, dufs sie die den Naturforscher und Arzt interessirenden Leistungen des Auslandes sehr frühzeitig und gewöhnlich ganz gleich nach ihrem Bekanntuerden in Auszügen und deutschen Uebersetzungen mittheilt; auch die Botanik betreffende Gegenstünde mangehn nicht; doch werden zoologische Abhandlungen weit öfter mitgetheilt, als botanische. Interessunt ist noch, daf's unter der Rubrik, "Bibliographisehe Neuigkeiten" auch die Titel der neu im Auslande erschienenen botanischen Werke geu:öhnlich vollständig und richtig ungegeben uerden.

$I_{s}$ is oder encyclopiadische Zeitung, von $0 \mathrm{ken}$. Leipzig bei Brockhaus; grofs 4 ; , seit 1817.

Jetzt sind 23 Bünde vorhunden: monatlich erscheint ein Heft, in deren jedem zwar, wie die jedesmalige Inhalts-Anzeige nachueist, der Botanik eine eigenthümliche Abtheilung geuidmet ist, doch wird man leicht bemerken, dafs sie im Vergleiche mit der Zoologie etwas stiefmütterlich behandelt wirl. Uebrigens enthält die Isis nicht nur manche schitzbare dem Botaniker wichtige Original-Abhandlungen,

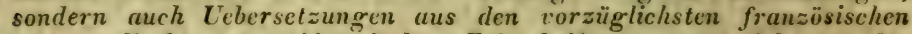
und englischen naturhistorischen Zeitschriften, namentlich aus den Mémoires du Muséum d'histoire naturelle de Paris und aus den Londner Transactions of the Linnean Society, unter andern die Commentarien Hamilton's über Theede's Hortus malabaricus, wobei ich den W'unsch nicht unterdrïclien kann, dafs auch die Comimentaricn Hamilton's über Rumph's Hortus amboinensis mitgetheilt werden möchten. Fndlich werden auch Kritiken über neuere und ältere botanische Werke aufgenommen.

Abend-Zeitung, herausgegeben von Th. Hell und Fr. Ki ind. Grofs 4. Dresden bei Arnold.

Finthält in einem Beiblatte Mittheilungen aus dem Gebicte der Flora und Pomona, woran besonders Hofrath Reichenbach thätigen Antheil nuhm. Nit Bedauern höre ich, dafs dieses so interessante Beiblatt, das der Botaniker der ganzen Abendzeitung vorzieht, eingehen soll, oder wohl schon eingegangen ist.

Nova Acta physico-medica Academiae Caesar. Leopold.-Carolinae naturae curiosorum. Auch unter dem Titel: Verhandlungen der kais. Leopold. Carolin. Akad. der Naturforscher. Bonn bei Weber, grofs 4, mit Kupfern. Seit 1818.

Herr Prof. Nees v. Esenbeck in Breslau ist jetzt Prüsident dieser sehr geschïtzten Akademie und Haupt-Redacteur der Verhandlungen. Diese einfache Bemerkung ist zureichend, um zu beweisen, dafs sie den Botaniker ganz besonders interessant sind.

1827. 8.

A b h a nd l ungen der naturforschenden Gesellschaft zu Görlitz. Seit

Verbandlungen der Schlesischen Gesellschaft für vaterländische Cultur.

Verhand lungen der Gesellschaft naturforschender Freunde in Berlin. Berlin, 4. 
Zeitschrift für Natur-und Heilkunde. Herausgegeben von Carus, Choulant u. s. w. Dresden. 8. Seit 1819.

Schriften der Gesellsehaft zur Beförderung der gesammten Naturwissenschaften in Marburg.

Naturwissenschaftliche Abhandlungen von einer Gesellschaft in Wirtemberg. Tübingen. 8 . Seit 1826.

Denkschriften der königl. Akademie der Wissenschaften zu München. 4.

Den k schriften der allgemeinen Schwcizer Gesellschaft für die gesamunten Naturwissenschaften. Zürich bei Orell und Füssli. 4.

Magazin, Nürnbergisches, zum Nutzen und Vergnügen; herausgegeben von einer Gesellschaft von Gelehrten. Nürnberg 1816, bei Schrag. gr. 8.

So viel mir bekannt, ist von dieser Zeitschrift nur ein Heft erschienen, es enthëlt, hierher gehörig, Nachrichten von dem Weinbau im südlichen Tyrol, Beiträge zur Flora von Kronach, Anzeige einiger merkwürdigen Pflanzen, welche in den nähern und fernern Umgebungen von Rosenheim wachsen; einen Aufsatz zum Andenken an J. C. D. von Schreber u. s. w.

Abhandlungen der physikalischen Klasse der königl. Akademie der Wissenselaften zu Berlin. Aus dem Jahre 182t. Berlin 1825, bei Dümmler. 4.

Ueber ihren Inhalt findet man Nachricht in der Isis 1829. $p .19$.

Monatschrift der Gesellschaft des vaterländischen Museums in Böhmen. Prag bei Calve, seit 1827. 8.

Enthalt auch botanisehe Abhandlungen, namentlich von K rombhol E.

B ïlletin der naturwissenschaftlichen und botanisehen Section der schles. Gesellschaft für vaterländische Cultur. Breslaı. 4.

Man sehe hierüber Isis 1829. p. 593.

Leopold Leo's Magazin für Heilkunde und Naturwissenschaften in Polen; in Verbindung mit einem Vereine von Aerzten herausgegeben. Warschau 1828.

Die Zeitschriften, welche vorzugsweise der Physik und Chemie gewidmet sind, können ihrer Tendenz nach die Botanik nur als Nebensache betrachten, allein sie enthalten dennoch bisweilen Aufsätze, die in das Gebiet der Pflanzen-Physiologie gehören; auch um der Pflanzen-Chemie willen dürfen sie nicht übergangen werden. Die vorzüglichsten jetzt erscheinenden dürften folgende sein:

Baumgärtner und v. Ettinghansen. Zeitschrift für Physik und Mathematik. Wien. 8. bei Carl Gerold.

Annalen der Physik und physikalischen Chemic., Fortsetzung von Gilbert's Annalen; heransgegeben zu Berlin von J. G. Poggendorf. Seit 1899. Leipzig bei Barth.

Zeitschrift für die organische Physik von Heusinger. Eisenach bei Johann Friedrich Bărecke. 8.

Eirscheint seit dem Juli 182\%.

Archiv für die gesammte Naturlehre, in Verbindung mit mehreren Gelehrten herausgegeben von $\mathbf{D}$. K. W. G. K a stner. Nürnberg. 8 . Seit 1824 .

Soll künftig unter dem Titel ,Archiv für Chemie und Meteorologie" in Nürnberg bei J. A. Stein herauskommen. 
Dio neueston Entdeckngen in der Physik, Hellktide und Chemie ete. von Dr. Fr. Sertüruer. Güttingen bei Vandenhoek und Ruprecht.

Bis jetzt sind 3 Bände erschienen.

Jahrbuch der Chemie und Physik ron Dr. Schweigger und Schweigger-Seidel. 8. Halle bei Anton und Gellhe.

Früher kam diese geschützte Zeitschrift in Nürnberg heraus, unter den Titel: Journal für Chemic und Physik.

Otto Linne Erdmann's Journal für ökonomische und technische Chemie. Scit 1828. Leiprig bei Barth.

Bis jetzt sind sieben Bände davon vorhanden.

Nächst der Chemie ist Botanik des Pharmaceuten IIauptwissenschaft; man findet darum auch in den pharmaceutischen 'Zeitschriften manche branchbare und uichtige Beiträge zur Beförderung der Pflanzenliunde, and es ist ihnen deshalb hier eine Stelle einzurüumen.

Trommsdorf's neues Journal für Pharmacic. Erfurt. 8. Jetzt 19 Bünde. - Finthült oft schr schätzenswerthe Aufsätze,

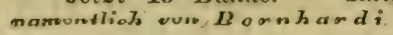

J. A. Buchner's Repertorium für die Pharmacic, unter Mitwirkung des Apothekervereins in Bayern. Nürnberg bei Schrag. 12. Jetzt sind 33 Bände vorhanden. Sie enthalten lehrreiche $\mathrm{Nach}_{\text {- }}$ richten von Martius, Kittel und Anderin.

Berlinisches Jahrbuch für die Pharmacie. Herauggegeben von Dr. W. Meissner. Berlin bei F. Oehmigke. 12.

Bereits sind ül Jahrgänge vorhanden. Sie enthalten eine Menge zumal für die medicinische Pflanzenkunde höchst vichtige Abhandhungen und Abbildungen von Sprengel, Schlechtend al u. s. w.

Archiv des Apotheker-Vereins im nördlichen Teutschlande für die Pharmacie und ihre Hülfswissenschaften, nnter Mitwirkung der Vereinsmitglieder und in Verbindung mit mehreren Gelehrten herausgegeben von Dr. Rudolph Brandes. Lemgo in Verlage der Meyerschen Hofbuchhandlung.

Erscheint seit 1822, jetzt sind 37 Bündle vorhanden. Fnthält viele interessante Abhandlungen über Botanik und einzelne Beiträge zur Flora.

Pharmaceutische Zeitung des Apothekervereins im nördlichen Teutschland, herausgegeben von Dr. Rudolph. Brandes. Lemgo, seit 1827.

Enthält öfters Recensionen botanischer Werke.

Magazin für Pharmacie und die dahin einschlagenden Wissenschaften; von Dr. Ph. I. Gei grer. Heidelberg bei Winter. 8.

Früher gab es IL̈̈nle in Karlsruhe heraus. Jetzt sind 31 Bände davon vorhanden.

Noch sind, zumul für den physiologischen Theil der Pflanzenkunde, die nuchstehenden Zeitschriften interessant:

Zeitschrift für Physiologie; in Verbindung mit mehreren Gelehrten heransgegeben von Fr. Tiedemann, G. R. und L. Ch. Treviranus. Grofs 4. Darmstadt, seit 1824:

Forstliche Beriehte und Miscellen. Eine Zeitschrift in zwanglosen Heften, von J. C. II undes hagen. Tübingen, seit 1830, bei Laupp. 
Hartig, Forst - und Jagd - Archiv. Stuttgardt und Tübingen. \&. Jetat sieben Bände.

Behlen, Zeitschrift für das Forst - und Jagdwesen, unit besonderer Rücksicht auf Baiern. Frankfurt. 8. Bereits 4 Bände.

Allgemeine Forst- und Jagdzeitung, herausgegeben von Steph an Behlen. Frankfurt, seit 1825.

Sylvanejon. Ein Conversationsblatt für unbefangene gebildete Forstmänner. Von Dr. J. L. K l auprecht.

Der a ufmerksame Forstmann; oder das Neueste und Bemerkenswertheste aus dem Forst - und Jagdfache; herausgegeben von Ch rist. Li cbich. Prag, seit 1825.

Kritische Blätter für Forst - und Jagdwissenschaft, in Verbindung mit mehreren Gelehrten herausgegeben von Dr. W. Pfeil. Leipzig, seit 1825 .

Jahrbücher der gesammten Forst - und Jagdwissenschaftlichen Literatur von C. P. Lau rop. Heidelberg, seit 1824.

A b h a ndlungen des Vereins für forstwissenschaftliche Ausbildung. Tübingen, seit 1825.8 .

Beiträge zur gesammten Forstwissenschaft. Herausgegeben von J. L. Hundeshagen. 'Tübingen, seit 1824.

Sylvan. Jahrbuch für Forstmänner, Jäger und Jagdfreunde, auf

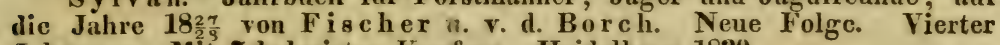
Jihrgang. Mit $\boldsymbol{\gamma}$ kolorirten Kupfern. Heidelberg 1829.

Allgemeine Jahrbücher der Forst - und Jagdkunde. Herausgegeben von G. W. Freiherrn von Wedekind und S. Behlen. Erstes Heft. Der neuen Jahrbücher der Forstkunde, herausgegeben ron G. W. Freih. v. We dek ind, siebentes Heft. Mainz 1830.

Einige dieser Zeitschriften scheinen bereits wieder eingegangen zu sein; die speciellen Werke über Forstkunde werden übrigens in dcm dritten Theile bei der ökonomischen und technischen Botanik ihre Stelle finden.

\section{b. Zeitschriften in französischer Sprache.}

Annales de la Société Linneenne de Paris. Paris. 8. avec le Bulletin Linnéen.

Thiebaut de Berneaud ist beständiger Secretär; von ihm rühren auch mehrere interessante zumal historische Aufsätze her.

Mémoires de la Société Linneenne de Calvados.

Bulletin d'histoire naturelle de la Société Linneenne de Bordeaux. Seit 1826.

Mémoires du Muséum d'histoire naturelle. Paris 4. avec pl. Chez A. Belin, Imprimeur libraire, rue des Mathurins Saint-Jacques, Hòtel Cluny.

Zwei Bände dieses klassischen Werkes bilden einen Jahrgang und jeder Band besteht aus 6 Heften.

Mémoires de la Société Linnenne de Normandie. Caen.Paris.8. chez Lance.

Enthält manche interessante Aufsätze, z. B. der 4te Band vom Jahre 1829 eine geognostische Topographie von Calvados, eine Uebersicht der Vegetation von Nieder-Normandie $u$. s. $w$. 
Mémoires de la Sociéte d'llistoire naturelle de Parls. Paris chez les éditeurs du Dictionaire classique d'histuire naturelle, Baudonin frères, Libraires-Editeurs, rue de Vaugirard Aro. 17, Rey et (iravicr, Quai des Augustins Nro. 55. 4.

Für Botanik uerden folgende Mitglieder genannt: die Herren Adolph Brongniart, Adrien de Jnssicu, Guillemin, Kunth, Kichard, Gay, Gaudichaud, Raspail, Caunbeswiedes. Den Druck der Abhandlungen besorgt Guillemin. Alle Zusendungen für die (iesellschaft müssen portofrei an das Secretariat Rue d'Anjou-Datuphine Nro. 6 addressirt werden.

Mémoires de la Société d'histoire naturelle de Strasbourg. Paris et Strashourg ehez F. G. Levrault. 4.

Einthält Einiges hierher gehörige von Herrn Prof. Nestler; namentlich einen Aufsatz über Sedum repens Schleicher mit ciner Abbildung.

Mémoires de la Société de Physique et d'histoire naturelle de Genève. Genève 4.

Enthält vortreffliche Abhandlungen, namentlich von Decandolle, Vaucher und Andorn.

Anmales scientifiques littéraires et industrielles de l'A nvergne, publicée par l'Academic des Sciences, helles lettres et arts de Clermont-Ferrand, sous la direction de M. II. Le eoq, rédacteur en chef. Clermont-Ferrand, chez Thibaud-Landriot, Libraire-Editeur. 8. Monatlich erscheint ein $H$ cft. Fs sind jetzt 3 Bünde vorhanden.

Bulletin des Scicnces de la Société philomatique. Paris 4.

Annales des Sciences naturelles, par MM. Audouin, Brogniart et Dumas: Journal complémentaire des Annales de Chimie et de Physique: Comprenant la Physiologic animale et végétale, l'Anatomie comparée des deux règnes, la Zoologie, la Botanique, la Minéralogie et la Géologie. Paris, Crochard, Libraire-étitcur, rue de Sorbonne Nro. 3 et Cloitre Saint Benoit Nro. 16. 8. avec Atlas in 4.

Bis Finde 1829) enthält es 18 Bünde. - Den letzten Bünden ist angehöngt cine Revue bibliographique, pour servir de complément aux Annales des Sc. naturelles.

Bulletin universel par de Férussac. Paris. 8.

Ist nach den $W$ issenschaften in nechrere Sectionen getheilt. Hier interessirt uns vor:ugsweise das:

Bulletin des Sciences naturelles. Paris 8. au bureau ecntral, rue do l'Abbaye. Nro. 9. chez Levrault.

Für Botanik sind Vitarbeiter die Herren Bory de Saint Vincent, A. Brongniart, Buschinger, Cambessèdes, Bupetit-Thouars, Duran, Gaudichaud, Gay, A. de Jussieu, Kunth, Mèrat, Raspail, Richard, A. de Saint-Ililaire. Ilaupt-Redacteur ist Herr Guillemin.

Bibliothèque universelle des sciences, lettres et arta, redigée à Geneve. Genere imprimerie de la bibliothèque universelle. Paris, Bossange père, libraire de S. A. R. Mons le Duc d'Orleans, rue de Richelicu. Nro. 60.

Jeden Monat erscheint ein in zwei Sectionen mit besonderer Paginirung getheiltes IIeft. Die erste ist übersclericben Littérature, die andere Sciences et Arts. Diese letstere enthält auch die botanischen Artikel und Recensionen interessanter, die Pflanzenkunde betreffender, W'erke; sie sind jetzt grafsentheils von Decandolle abgefajst. 
Annales des Sclences d'Obserration, comprenant l'Astronomie, la Physique, la Chinie, la Minéralogre, la Géologio, la Physiologie et l'Anatomie des deux règnes, la Botanique, la Zoologre, les Théories mathématiques, et les principales applications de toutes ces sciences à la Météorologie, à l'Agriculture, aux Arts et ì la Médecine, par MI. Saigay et Raspail. Paris chez Bandsuin, Rue de Vaugirard Nro. 17. 8.

Erscheint monatlich vom Monat Januar 1829 an.

Revue. encyclopédique, ou Analyse des productions les plus remarquables dans lit littérature, les sciences et les arts. in 8 . Au bureau central de la Revue, rue d'enfer St. Michel Nro. 18 et chez Treuttel et Würtz.

Annales de Chimie et de Physique, par M.M. Gay-Lussa c et Arrago. Paris. 8. Seit 1816.

Fortsetzung der Annales de Chimie.

Ami de champs. Bordeaux.

Annales de la Société d'horticulture de Paris. Paris.

Annales de l'Institut royal horticale de Fromont, dirigées par le Chevalier Soulange-Budin. Paris. Seit 1829. (April.) Chez Madame IIuzard, libraire, rue de l'Eperon Nro. 7 et au jardin de Fromont à Ris (Seine et Oise).

\section{c. In Russland erscheinende Zeitschriften.}

Mémoires de l'Academie impériale des Sciences de Petersbourg. VIte Série. Sciences mathématiques, physiques ct, naturelles. Tom. I. Livr. 1. 2. 3. gr. 4. Petersbutrg (Leipsic, Cnobloch en Comm.)

Mémoires de la Socićté impériale des Naturalistes de Moscou. Quarto.

Acta Mosquensia.

\section{d. Zeitschriften in englischer Sprache.}

Quarterly Journal of Science, Literature and Arts. London. 8. by Henry Colburn and Richard Bentley, New Burlington Strcet.

Journal of morbid anatomy, ophthalmie, medicine and pharmaceutical analysis with medico-botanical Transactions, communicated by the Mcdico-botanical Society of London. London 18:8. 8. Fiditor de Farre.

Diese Zeitschrift ist besonders interessant wegen der darin enthaltenen Nachrichten von den Arbeiten der medicinisch-botanischen Gesellschaft in London, von denen im zueiten Theile umstündlicher Bericht erstattet werden wird. Präsident der Gesellschaft ist Sir James M. Grigor, und ihr Plan ist, die officinellen Pflanzen jedes Welttheils zu sammeln und zwar geographisch geordnet mit ihren wissenschaftlichen und gewöhnlichen Namen, Angabe ihrer Eigenheiten u. s. $w_{.}$; darauf den Schiffs - und Feldärzten den Zugang zu diesen Sammlungen zu erlauben, damit sie sich vor ihrer Abreise mit den officinellen Pflanzen im Allgemeinen bekannt machen können. Auch ist schon den Officieren der verschiedenen brittischen Kolonien aufgetragen worden, die von den Fingebornen als Heilmittel gebrauchten Pflanzen an die Gesellschaft zu überschicken. Das englische Ministerium hat dic Societät, deren Director Dr. Frost ist, unter seinen besondern Schutz genommen, und die ostindische Compagnie hat die 
indischen Rgierungen beauftragt, alles für die Gesellschaft bestimnte frei passiren zu lassen. - So viel Rülımliches auch diese medicinischbotanische (iesellschaft bereits leistete, und wohl noch bei weitem mehr leisten uird, so hat sie sich doch durch Ausstofsung des bekunnten Botanikers Robert Brown befleckt; letzterer soll, wie sich eine Zeitschrift ausolrückt, den Director Dr. Frost nicht für voll passiren lassen, und so legte dieser es darauf an, Brown gar nicht mehr passiren zu lassen.

Magazine of natural History and Journal of Zoology, Botany, Mineralogy etc: by London 1828.

Transactions of the Botanical and Horticultural Society of the Countries of Durham, Northumberland and Newcastle. upon 'T y ne. London 1828.

Philosophical Magazine and Journal. London 8.

Transactions of the Linncan Society of London. 4.

Memoirs of the Wernerian natural llistory Society Edinburgh.

Journal of Science, Literature and Arta. London. 8.

Transactions of the IIorticultural Society of London. London.

The gardencrs Magaine and register of rural and domestic improvement, conducted by J. C. Loudon. London. 8.

The Edinburgh New Philorophical Journal, cxhibiting a View of the progressive Discoveries and improvements in the Sciences and tlic árts, conducted by $R \circ$ bert $J$ aneson. Edinburgh. 8.

Annals of Philosophy. London. 8.

Transactions of the royal Society of Edinburgh. Edinburgh, 4.

Edinburgh Philosophical Journal. 8.

Edinhurgh Journal of Science: conducted by David Brewster. Thomas Clark, Edinburgh. 'I'. Cadell, London. Millikin et Son, Dublin. 8.

Transactions of the literary Society of Manchester.

Ameriean Journal of Sciences and the arts, conducted by Silliman. NewYork (seit 1819).

Journal of the Acadeiny of natural Seicnces of Philadelplia. 8.

Boston Journal of Phylosophy and Art8.

Newjork medical and physical Journal.

Annals of the Lyceum of the natural listory of $\mathrm{New}$ York. 8.

As iatic Journal.

Asiatic Researehes, or Transactions of the Socicty instituted in Bengal. Calcutta. 4.

Memoirs of the Royal Asiatic Society.

e. Zeitschriften in italienischer Sprache.

Biblioteca italiana: ossia Giornale di Litteratura, Scienze ed Arti. Compilate de vari Letterati. Milano 1830. 8. presso la Direzione del Giornale.

Jetzt sind 58 Bände vorhanden. 
Configllachi e L. Brugnatelli Giornalo di Fisiı sa, Storia naturafi medicina ed àrti, Pavia. 4 .

M cmoria della reale academia della Scienze di Ten ino. 4.

Giornale arcadico di scienze, lettere ed arti. Ro ma. 8.

Giornale di Scienze, lettere et arti per la Sicilia. Palermo. 8.

Giornale di Letterati. Pisa.' 8. (bei Nistri).

Giornale Ligustico di scienze, lettere ed arti. Gcrıva. 8.

f. Zeitschriften in Holländischer, Dänischer un! Schwedischer Sprache.

Bijdragen tot de naturkundige Wetenschapen, wersammelt door van Hall, Vrolik en Mulder. Änsterdan. 8. Seit 1S26.

Det kongelige danske Videnskabernes Selskabs u laturwidenskabelige og mathematiske Afhandlingar. Kiöbenharen. 4.

Tidskrift for Naturvidenskaherne Udyivet of H. C· Dersted, J. W. Hornemann, og J. Reinhardt. Kiöbenh. 8.

Konglige Vetenskabs Academiena Handlingar. \$stockholm. 4 .

\section{Der Botanik allein gewidmete alphabetischt: Werke.}

$$
\text { S. } 4 .
$$

Der Inhalt derselben, die Att der Bearbeitung, dep dabei vorzugsweise berücksichtigte Endzuveck dieser Werke ist sehr vers chierlen, eben so ihr innerer.Werth, worauf hier nicht weiter eingegang en werden kann. Der Titel der meisten zeigt übrigens schon in Allgemeinen, was darin zu suchen ist.

Neu entdeckte Pflanzen, ihre Charakteristik, Benutzung und Behandlung, hinsichtlich der Standörter, Fortpflanzung und Vermelirung, von Dr. F. G. Dietrich. Berlin 1825. Ertser Hand Abelicea bis Bomarea. Zweiter Band Bonamia bis Citharexylum. -

Kommt auch unter dem Titel vor: Neuer Nathtrag zum vollständigen Lexicon der Gürtnerei und Botanik. Da's Werk scheint übrigens nicht fortgesetzt zu werden, w'as wirklich zu bedauern wäre. An seine Stelle scheint übrigens folgendes ges etzt zu werden.

Handlexicon der Gärtnerei und Botanik, odor alphabetische Beschreibung voun Baue, Wartung und Nutzen der vor: rigglichsten inund ausländischen, ökonomischen officinellen und zur $\mathbf{Z}$ ierde dienenden Gewächse nach dem neuesten Stande der Wissenschaft bearbeitet von Dr. Friedr. Gottlieb Dietrich, Prof. und Director diss Grofsherzogl. botanischen Gartens zu Eisenach. Erster Band Abama 1 is Chrysanthemum. Berlin bei Bethge. 1829. 2 fl. $24 \mathrm{kr}$.

Encyclopädisches Pflanzen-Wörterbuch aller einheimischen und fremden Vegetabilien, welche sich durch Nutzen, Schönheit oder sonstige Eigenthümlichkeiten besonders auszeichnen etc. Nebst einem angehăngten Polyglotten-Lexicon, von Johann Kachler. Wien 1829. 2 Bände.

Pomnozenie Dykeyonarza. Auct. J. Dzearkowski et K. Scennioki. Tom. I. et II. Varsov. 1824.

Dictionair der Botanik von Christoph Kluk, vermehrt von

D. und S. Jn polnischer Sprache. 
Targloni Tozzettl. D. Ottavio. Dizionario hotanico italiano, che comprende $\mathrm{i}$ nomi vulgari italiani, specialmente toscani e vernacoli della piante, racolti da diversi antori e della gente di campagna, col corrispondente latino e botanico. Fiorenze 1825. 8. Zureite ganz umgearbeitete und vermehrte Ausgabe.

Wörterbuch der Gattungsnamen der Pflanzen von Joh. Martinaf. Petershurg 1825.

In russischer Sprache.

Nomenclator Botanicus; enumerans ordine alphabetico nomina atque synonyma tum gencrica tum specifica et a Linnaeo ct recentioribus de re hotanica seriptoribus plantis phaneroganis imposita. Auctore Ernesto Steudel. Med. Dr. Stuttgardtiae et Tubingae. Sumptibus J. G. Cottae. MiDCCCXXI.

Nomenclatior Botanicus, enumerans ordine alphabetico nomina atque synonyma tum generica tum specifica et a Linnaco Recentioribus de re botanica socriptoribus, plantis cryptogamis imposita. Auetore Er nesto Steude I, Med. Dr. Stuttgardtiae et Tubingae. Sumptibus J. G. Cottac. MDCCCXXiv.

Voigt, Wörterbuch der botanischen Kunstsprache, zweite Auflage. Jena 1824 .

Dierbach, die botanische Terminologie älterer Zeiten im Auszuge. Karlsi'uhe 1821.

Aus G'eigers Magazin für Pharmacie besonders abgedruckt.

Botanical Terminology, or Dictionary, explaining the terms, most generally employed in Systematic Botany. By G. N. Lloyd. Member of the Plinian Society. 12. Edinburgh 1826.

Schun rmann stekhoven Kruidiundige Kunstwoordenbock. Leyden. 1526. 8.

Botanische Kunstgprache, herauggegeben von Dr. J. Leo.

Ist die: erste Abticilung der Anleitung zur allgemeinen systematischen vind pharmaceutischen Kenntnifs der Pflanzen. Die Schrift kum heraus unter dem Haupttitel: Anhang zum Taschenbuch der Arzneipflanzen. Berlin 18\%i. 8. bei Fr. Laue.

Terminologie der phanerogamischen Pflanzen, durch mehr als 690 Figuren erläntert, und besonders zum Unterricht für Seminarien und lRealgymnasien bestimmt; nebst einer Anleitung für den Lehrer, wie er in der Botanik mit Nutzen zu unterrichten hat, von Albert Dietrieh, Dr. der Philosophie, Lehrer der Botanik und Nuturgesshichte bei de:r Gärtner-Lehr-Anstalt in Neu Schöneberg etc. Berlin 1829, bei Enslin. Queer Folio. 1 fl. 21 kr.

Grundrifs der Pflanzenkunde in Gestalt eines Wörterbuchen der botanisehen Sprache. Beigefingt: Tabellarische Ueliersicht des Linncischen Systems nach Thunbergs Abänderungen. Deutsches Wörterbuch für dic botanischen Kunstausdrücke: Anhang: enthaltend das neueste System: Uebersicht des Gewächsreiches in seinen natürlichen Entwickelungsstufen. Gedrängte Darstellung dieses Systems in Tabellenform. Für Freunde der Gewächakunde cine unfassende Anlcitung zum griandlichen Sidlssktudium. Für Botaniker ein bequemes Nachschlagebuch. Von Johann li achler, corropondir. Mitgliede der Gartencultur - Gosellwchaft zu London. Lexicon - Format. Wien bei J. P. Sullinger. 1829. 4 fl. $30 \mathrm{kr}$. 
Dictionnaire raikonné des termes de botanique, contenant la definition et la description de tous les organes et de tous les termes usités en botanique; la deseription des familles naturelles, l'étymologie de tous les noms techniques, suivi d'une Table des noms latirus et des racines grecques, par H. Lecoq et J. Juillet. Paris 1828. 83. chez Maire Nyon. 8'Fr.

Wörterbücher oder alphabetische Werke, die auch Gegenstände der PHlanzenkunde abhandeln.

\section{\$. 5.}

Es gibt deren eine ungremein grofse Zahl, deren Inhali, wie man leicht denken kann, von gar verschiedenem $W$ erthe ist. Ich beschränke mich nicht ohne Grund auf die bekanntesten und brauchbarsten.

Allgemeine Encyclopãdie der Wissenschaften und Künste, in alphabetischer Folge, von genannten Schriftstellern bearbeitet, und herauggegeben von J. S. Ersch und J. G. Gruber, Professoren in Halle. Leipzig. 4.

Das höchst schützbare Werk ist jetzt in 19 Bänden bis zum Artikel Corythus vorgeschritten. Die botanischen Abschnitte rühren von Kurt Sprengel, und sind mit jener umfassenden Gelehrsamkeit bearbeitet, die diesem ber $\ddot{h}_{2} m$ ten Lehrer eigenthümlich ist. -

Wörterbuch der Naturgeschichte; dem gegenwärtigen Zustande der Botanik, Mineralogie und Zoologic angemessen. Weimar. $1824-1829$. Grofs 8 .

Bis jetzt sind 6 Bünde vorhanden, die bis zu dem Worte Graphypterus reichen. Die botanischen Artikel sollen, wie man sagt, Herrn Professor Bernhardi in Erfurt zum Verfasser haben, und sind jedenfalls mit grofser Umsicht geschrieben.

Repertorium für die Chemie als Wissenschaft und Kunst; eine möglichst vollständige alphabetisch-systematisch geordnete Darstellung des Wichtigsten über die bekannten Stoffe der Chemie, über die Bestandtheile der Mineralien, Pflanzen und Thierkörper, mit besonderer Rücksicht auf die praktische Anwendung für die Pharmacie, Medicin, Agricultur, Fabriken - und Gewerbskunde, so wie auf die Entwickelung der Grundzüge der Wissenschaft u. s. w. Von Dr. Rudolph Brande *. Hannover 1828. Hahn'sche Hofbuchhandlung. Gr. 4.

Bis jetzt sind drei Bände vorhanden, die noch nicht die zuei ersten Buchstaben des Alphabets enthalten. Von dem Buchstahen $B$. an ist mir die Bearbeitung der botanischen Artikel aufgetragen worden; mehrere derselben lieferte auch Herr Wilhelm Brandes.

Dictionnaire des Sciences naturelles, dans lequel on traite méthodiquement des différens êtres de la nature, considérés soit en eux mèmes, d'après l'état actuel de nos connoissances, soit relativement à l'utilité qu'en peuvent retirer la Médecine, l'Agriculture, le Commerce et les Arts; suiri d'une Biographic des plus célèbres naturalistes: ouvrage destiné aux Medicins, aux $\Lambda$ griculteurs, aux Manufacturiers, Artisstes, aux Commerçañs, et à tous ceux qui ont interèt à connoitre les productions de la Nature, leurs caractères génériques et specifiques, leur licu natal, leurs proprietés et lenrs usages; par plusieurs Professeurs du Jardin du Roi et des principales écoles de Paris. Paris chez Levrault.

Dieses höchst schützbare Werk besteht aus 60 Bänden Text in Octav; einem Bande Supplementen und Tabellen, 60 Heften Abbildungen, vier Bänden Biographie und 25 Heften Portraits: Die 
Botanischen Aufsaitze rühren von den Herren Brongniart, Decandolle, Cas ssini, Desfontaines, Geoffroy St. Hilaire, de Humboldt, de Jussieix, Lesson, Loiseleur Deslongchamps, Mirbel, Poiret u. s. w. her.

Dictionna ire classique d'histoire naturelle par Audonin, Bunrdon et Bury de St. Vincent. Ourrage dirigé par ee dernier collahorateur, et clans lequel on a ajouté, pour le porter au niveau de la science, un gra nd nombre de mots, qui n'avoient pu faire partie de la plupart des dictionnaires anterieur. Paris 8. Rey et Gravier, Bandonin. Dor neueste Jand dieses Trerkes, von dem mir eine Nachricht bekannt geu'orden ist, ist der zwëlfte, reichend von Pan - Pis.

Dictionnaire des Drogues simples et composés, ou Dietionnaire dhistoire naturelle médicale, de Pharmarologie et de Chimie pharmacent ique. Par A. Chevallier et A. Richard. Paris 1827 1829. Gr. 8. 5 Volumes.

Die botanischen Artikel sind theils vom IIerrn Achille Richard, theils besonclers in den beiden letzten Bünden von Herrn Guillemin abgefafst.

Propädeutische Schriften, Anleitungen zum Studinm der Botanik, und diesen Gegenstand betreffende Abhandlungen.

\section{6.}

Das Studium dieser Schriften ist eincs der: zuverläsigsten Mittel, sich mit dem jetzigen Zustunde der Botanil als Wissenschaft bekannt zu muchen. Wenn gleich sich manche nur auf Terminologie und Systemliunde beschränken, so findel man doch dagegen in andern auch mit vielein Fleifse bcurbeitete Vebersichten der übrigen Doctrinen der Pflanzenkunde, insbesondere sind gewöhnlich gerade die neuesten Lintdeckungen und Ansichten am ausführlichsten erörtert. Fs vird ferner dem Beobachter nicht entgehen, daj's die propädeutischen Schriften der einzelnen europäischen Nationen, jede für sich eine eigne Bearbeitungsart zeigt; bei den Deutschen wird man solche finden, die sich durch Vielseitiglieit und Belesenheit vor allen andern rühmlich auszeichnen, aber. zugleich wieder andere die zu den klügrlichsten Machurerken gehören. Nach den Nationen geordnet, sind folgende Anleitungsschriften zu nennen:

\section{a. Deutsche.}

Elementa Philosophiae botanicac. Auctore H. F. Link, Phil. et Med. Doct. hujus Yrof. p. o. etc. cum tabulis aeneis IV. Berolin. sumptibus Haude et Spener. 1824.

Anleitung zur Naturgeschichte des Pflanzenreichs für die ersten Anfünger. Mlit 3 Kuffertafeln. Leipzig 1821. Auch unter dem Titel: Katechismus der Botanik.

Diese Schrift ist eigentlich der dritte Theil des ganzen W'erkes; der erste kam bereits 1820 heraus und enthält die Gestaltlehre mit mehr als 6010 erläuternden Figuren. Der zueite Theil ist der Physiologie gevidmet mit 3 Kupfern, und der letzte bereits. angeführte gehört eigentlich nur der Systematik an.

Iinch, J. F. W. Botanisches IIanduch zum Selbatunterrichte für dentsehe Liebhaber der Pflanzenkunde überhaupt, und für Gartenfreunde, Apotheker, Oekonemen und Forstmäner inshesondere. Der crste Theil zählt die Pflanzengattungen, der zweite die Arten auf. Der dritte enthiilt dic Vorkenptnisse, Terminolegic und Anleitung 
zum Untersuchen und Sammeln der Pflanzen. Mit 2 Kupfertafeln. Magdeburg. 1824.

W. Tettelbach. Hülfsblätter zum Studium der Botanik. Herausgegeben von Seidel. Dresden 1825.

Fs sind blos Abbildungen von Zierpflanzen ohne allen Text, und der Titel scheint daher nicht passend geuählt zu seyn.

Lehrbuch der Naturgeschichte des Pflanzenreichs ron Karl Julius Perleb, Doctor der Philosophie u. Medicin, ordentl. öffentl. Professor der Naturgeschichte an der Grofsherzogl. Badischen Universität: zu Freiburg. Freiburg im Breisgau. Druck und Verlag von Friedrich Wagner 1826.

Kurze Anleitung zur Bötanik und rorzüglich zur Kenntnifs der wild wachsenden phancrogamischen Pflanzen Deutschlands, mit besonderer Bezeichnung der Arznei -, Gift - und Forstgewächse. Ein Lehrbuch für Gymnasien, Seminarien und höhere Bürgerschulen, so wie auch zum Sellıststudium, bearbeitet von A. Herr, Gymnasial-Lehrer in Wetzlar. Giefsen $1827.1 \mathrm{fl} .40 \mathrm{kr}$.

Uebersicht der Naturgeschichte für den mündlichon Vortrag. Düsseldorf 1827, bei J. E. Schaub. 36 kr. (Dürftig!)

Lehrbuch der Botanik. Herausgegeben von Dr. F. S. Voigt, G. H. S. W. Hofrath, ordentl. Professor der Heilkunde und Botanik, und Director des botanischen Gartens zu Jena. Zweite umgearbeitete Ausgabe. Jena 1827, bei August Schmid. 4 fl. $30 \mathrm{kr}$.

Elementarbuch der Pflanzenkenntnifs zum Unterricht und Vergnïgen für die lernbegierige Jugend. Nürnberg 1827 mit 12 nach der Natur color. Kupfern. $\mathbf{3 6} \mathbf{k r}$.

Botanik für Damen, Künstler und Freunde der Pflanzenwelt überhaupt; enthaltend eine Darstellung des Pflanzenreichs in seiner Metamorphose, eine Anleitung zum Studium der Wissenschaft, und zum Anlegen von Herbarien. Ein Verstch von H. G. Ludwig Reichenbach, Königl. Süchs. Hofrath. Leipzig bei Cnobloch, 1828. $4 \mathrm{fl} .48 \mathrm{kr}$

Taschenbuch der Botanik als Leitfaden für Schüler entworfen von C. R. Batanophilos. Leipzig 1829. 2te Auflage von K. P. A. Richter. Magdebirg 1830. 8.

Auf welchem Wege wäre das höchste Ziel der reinen Botanik zu erreichen? Ein Wort zur Beherzigung eines jeden Botanikers. Von P. H. Opiz. Prag 1829.

Handbuch der botanischen Terminologie und Systemkunde von Dr. Gottlieb Wilhelm Bischoff ete. Als zweite nach cinem völlig verānderten und erweiterten Plane umgearbeitete Ausgabe der botanischen Kunstsprache in Unrissen. Nürnberg bei Sehrag. 1830. 4. XII. u. 2fio S. nebst 21 Steindruclitafeln und $8 \mathrm{~S}$. dazu gehörigen Erklärungen. -

Kurze Anweisung fürjunge Pharmaceuten, das Studium der Botanik zweckentsprechend und selbstständig zu betreiben. Von Dr. Wilhelm Ludwig Ewald Schmidt, practischem Arzte, Wundarzte und Geburtshelfer zu Stettin. Stettin 1830. Verlag von Moritz Böhme. Klein. 8.

Die Pflanzen und ihr wisserischaftliches Studium überhaupt. Ein botanischer Grundrifs zum Gebrauche academischer Vorträge und zum Selbststudium von Jonathan Carl Zenker. Dr. der Philos., Med. $u$. 
Chinurgie, aufserordentl. Prof. der Medicin su Jena etc. Fisenach bei Joh. Friedrich Bärecke. 1830. 8. $2 \mathrm{fl} .24 \mathrm{kr}$.

Gicistreich und vortrefflich!

Grundzüge der Phytologie, zum Gebranche seines offentlichen Vortrages entworfen von Dr. Ferd. Jos. v. Zimmerinann, Sr. Oest. k. k. Apost. Majestät Rathe, bestündigem Secretür u. ordentl. Mrof. der Chemie n. Botanik an der k. k. mediciniseh-chirurg. Josephs-Academie ete. Wien 1831, in Verlage vin J. G. Henbner, $6 \mathrm{fl}$.

Ein hüchst schätzbares Handbuch!

\section{b. Französische und Bèlgische.}

Iseçons de Flore. Comrs de Botanique, explication des principanx systèmes, introduction à l'étude des plantes, par J. I. M. Poiret, exprof. dhistoire naturelle. ridition classique. Paris 18:t.

Iinna ei Philosophia botanica. Edidit van Breda. Tournai 1824.

M. I. Demerson. La Botanique enseignée en XXII. Leçons. Paris 1825, avec XI. pl. color.

Fsai sur une nourelle langue botanique par M. de Rivière.

Annales de la Sociét. Linn. de Paris. Juillet 1525.

Mémoire sur l'Etude de la Botanique par M. Victor. Au ger juge de paix:

Ibidem. Sept. 1825.

Manuel complet de Botanique élémentaire par M. Boitard 1ère. partic. Principes de Botanique élémentaire, renfermant la glossologie, l'organographie et la physiologie végétale; la phythothesie, l'histoire analytique de botanique depuis Aristole jusqu'it ce jour, et le développement du système des familles naturelles. Paris 1826.' 2de. édition, corrigrée et augmentée d'un atlas alphabetique des termes de hotanique, formant un dictionnaire tris complet. Paris 1928. 1 Vol. 18. orné de planche:: 2e. partie, Manuel d'histoire naturelle, comprenant les trois règnes de lat nature ou genre complet des animanx, des végétaux. et des minéraux. Paris. $18 \% 8$.

Dicses Mundbuch gehört in die lange Reihe von Schriften aller Art, die in Paris unter den allgemeinen Titel: Encyclopédie des sciences et arts herauskommen.

Botanographie élémentaire, ou Principes de Botanique, d'Anatomie et de Plyyiolngie végétale, contenant: la deseription des organes des plantes, la définition des termes usites en botanique; une théorie nouvelle sur l'anatomie et la physiologie végétale; lart de décrire les plantes; l'exposition des méthorles les plus employées en botanique et la description des familles naturelles, par Them. Lestiboudo is, Prof. de Botanique it Lille etc. Paris chez Roret, Lille chez Vanackere père et fils. 18\%6:

M. J. P. Lamouroux. Resumé complet de Botanique. T. I. Organographie et T'axonomie avé figures. T. II. Physique végétale, contenant la physiologie et la pathologie végétale, oú l'étude de l'organisation, des fonctions et des maladies des plantes, et la gengraphic botanique, terminé par la liographie des botanistes, une biblingraphie et un vocabulaire. Paris 12. 1826.

Manuel de P'hysique, de Chimie et de Botanique, à l'usage des aspirans itu grade des bacheliers ì-sciences, par N. Anguetín. Paris chez Croutlebais, 1826. 
Lefebure: Cours de promenades champêtres aux environs de Paris, en forme de Bulletin, dit de Flore, au moyen duquel on apprend soi - même à signaler, à reconnoitre et à classer les plantes, d'après la théorie facile, et la pratique sur les plantes vivantes du nouveau système floréal. Paris 18\%6.

Noureau Manuel de Botanique, ou Principes élémentaires de physique régétale etc., par M.M. J. Girardin et Jules Juillet; pharmaciens internes des hôpitaux de Paris. Un Vol. in 18. p. 610. avec 12 planches lithogr. et plusieurs tableaux synoptiques. Paris 1827. Compère jeune. Prix 5 Fr. 50 Cent.

Précis élémentaire de Botanique, par H. Lecoq. Paris. 1828. 4. Chez Maire-Nyon. 6 Fr.

Nourcaux Eléments de Botanique, appliquée à la Médecine, à l'usage des élèves qui suivent les cours de la faculté de Médecine et du jardin du Roi, par A chille Richard. Paris 1819. chez Béchet jeune. Quatrième édition. Paris 1828.

Von der vierten Auflage dieser Schrift ist eine deutsche Uebersetzung erschienén, betitelt:

Achilles Richard's Neuer Grundrifs der Botanik und der Pflanzenphysiologie, nach der vierten mit den Characteren der natiirlichen Familien des Gewächsreichs vermehrten und verbesscrten Original - Ausgabe ühersetzt, und mit einigen Zusätzen, Anmerkungen, einem Sachund Wort-Register rersehen von Mart. Balduin Kittel. Mit 8 Steindrucktafeln. Nürnberg 1828. bei Schrag.

Auch unter dem Titel: Vollstîndiger Inbegriff der Pharmacie, in ihren Grundlehren und practischen Theilen. Ein Handlusch für Aerzte und Apotheker von J. Andreas Buchner. Vierten Theils, zweiter Band. Nürnberg 1828.

Botanique, ou Description familière du règne végétale, d'après la classification de Linné. Paris 1828. H. Bossange.

Nur zwei Bogen stark!

Botanique des Dames, ou Méthede facile, pour connoitre les végétaux, sans maitre, par un Professeur de Botanique. Paris, 3 Vol. 18. 1828. avec figures.

Resumé du Cours de Minéralogie et de Botanique, donné au Musée des sciences et lettres à Bruxelles, par J. Kickx. Bruxelles 1828.

Botanique de F. J. Rousseau, contenant tout ce quil a écrit sur cette science, augmentée de.l'exposition de la méthode de Tournefort, de celle du système de Linné, d'un nouveau Dictionnaire de Botanique, de notes historiques etc. par M. Deville. 2de édition. Paris 1829. 12. avec 8 tableaux.

Lettres à Nanine sur la botanique, par H. Letourneur. in 18. arec pl. au palais royal Nr. 263. Paris 1827. 3 Fr. 50 C.

Lettres à Nanine sur la botanique, par Dargassies. 2 Vol. in 12. Toulouse 1828.

Principes élémentaires de botanique et de phrsique, mélangés de fables récréactives et morales, par L. de Moucheron. Paris 1828. 12. $4 \mathrm{Fr}$.

Cours de Phytologie ou de botanique générale, appliquée à l'art de cultiver les plantes, en un mot à l'horticulture, par Aubert du Petit Thouars. Paris 1828. 8. 
 \\ c. En $\mathrm{gl}$ is c he.}

J. L. Drumond: First steps in botany intended as popular ilIustrations of the science tending to its study as a branche of general education. London 1825. 12. with 100 Wood-cuts comprising 200 fig.

Botano-Theology. an arranged compendium chiefly from Smith, Keith and Thomson. Oxford and London. 1525.

R. J. Thornton. An Essay Introduction to the science of Botany. Lond. 1825.

Outlines of Botany: hy John Locke. Boston 1525.

Lectures on the Elements of Botany. Containing the descriptive Anatomy of those Organs on which the Growth and Prenervation of the Vegretable depend. In 8. with Plates and numerous Woodcuts. Iy Anthony 'Todd Thompson. Iondon 18\%6.

Notice of an Introduction to systcmatic and physiological Botany: hy Thomas Nuttall. Cambridge 1827. 8. (Nord-Aucrika.)

Some Acconnt of the Science of Botany, being the Subatance of an introductory Lecture to a Course of Botany, delivered in the Theatre of the Royal Institution of Great Britain. By John Frost F. L. and A. S. S. ete. of Emanuel College, Cambridge. 4. Pag. 17. London 1827. Dedicated by Permission to the King.

J. M. Butt: The botanical primer; heing an introduction to english botany; adapted to the Linnean system and language. London 1827. 12.

Th. Castle. An Introduction to Systematical and Physiological Botany. London 1529. 284 S. with plates.

John Lindley: An outline of the first principles of botany. London 1830. 12. VIIII. u. $106 \mathrm{~S}$. (Preis 3 Schill.)

$$
\text { d. It a } 1 \text { ie } n \text { is } \mathrm{c} \mathrm{h} \text { e. }
$$

Clavis rem herbariam addiscendi absque praceptore. Pavin 1823.

Introduzione allo studio dei Vegetabili di Nicolo Ginseppe de Jacquin, tradatta, illustrata ed accresciata di Roberto de Visiacci. Padova 1824. 8.

Ferrario P. Ottavio. La Botanica insegnata in venti due lezioni da M. J. Demerson. Versione dal francese. Milano 1826. 8.

Antonii Bertoloni. Praelectiones Rei herbariae, quae et prolegomena ad Floram Italicam. Bononiae 1827.

$$
\text { e. S ch we dis c h e. }
$$

Kalma et Widenius Sciagraphia Studii botanici. Abo 1821.

Lärabock i Botanik, af C. A. Aghard, Prof. i Botanik etc. Malmò 1829.

S. bot. Literaturbl. IV. p. 48.

\section{Specielle Hülfsmittel zum Studium der Boțanik.}

\section{§. 7.}

Man kann dahin die botanischen Excursionen, das Anlegen der Herbarien und den Besitz passender Instrumente zum Untersuchen der Ge- 
ichse zühlen. Ueber und für alle diese Gegenstånde ist in neueren Zeiten Manches gethan und geschrieben worden, wovon hier eine kurze Notiz an ihrer Stelle sein dürfte.

\section{Anstellung botanischer Excursionen.}

Die dabei zu beobachtenden Regeln findet man in mehreren der angeführten propädeutischen Schriften erörtert. Besondere Erwähnung verdient aber folgender Aufsaitz:

Des Herborisations par Lecoq.

Annales scientifiques de $l$ Auvergne T. 1. p. 545. und f.

Wras Herr Lecoq darüber sagt ist recht schön und belehrend, wenn es auch gleich für Deutsche nicht viel Neues enthalten mag; nur erinnere ich mich nicht den Rath gelesen $z u$ haben, bei botanischen Fxcursionen auch Schiefsgewehr mit sich zu führen, und zwar aus dem Grunde, weil sich bisweilen an hohen Felsen Pflanzen fänden, wo der Zugang sehr schwer oder unmöglich sei, dann könne man sich nicht selten blühende Zweige herab schiefsen!

\section{Anlegung von Herbarien.}

$\mathrm{Zu}$ den neuesten Schriften und einzelnen Aufsätzen hierüber gehören:

Gründliche Anleitung zum Einlegen der Pflanzen und wie man schöne und dauerhafte Herbarien anlegt. Ein Handbuch für Apothekerlehrlinge, angchende Botaniker und Dilettanten von L. Bauha rdt, Cand. Pharm. I. - XXIII. Klasse des Linneischen Specialsystems. Weimar. Albrechtsche Hofbuchdruckerei 1823. 356 Seiten. 8. 2 fl. $40 \mathrm{kr}$. (Nicht $\approx u$ empfehlen!)

Das Auftrocknen der Pflanzen für's Herbarium und die Aufbewahrung der Pilze nach einer Methode, wodurch jenen ihre Farbe, diesen aufserdem auch ihre Gestalt erhalten wird, von F. Luedersdorf. Berlin in der Haude und Spenerschen Buchhandlung (S. J. Joseephy.) 1827.

Thon, Handbuch für Naturaliensammler, oder Anweisung alle Naturkörper in Naturalien - Kabinetten aufzustellen und aufzubewahren, - Pflanzen zu trocknen, Herbarien, Fruchtkabinette, Holzbibliotheken u. s. w. anzulegen und zu crhalten. Mit 38 Figuren. Ilmenau 1829. 3 fl. $36 \mathrm{kr}$.

Rathschläge zur Anlegung von Herbarien.

Froriep's Notizen Bd. 22 . p. 343.

Ueber Präparation der Semperviva und Ericae für Herbarien von Beinert.

Bot. Zeit. 1822. 2 p. 574.

De la préparation des herbiers pour l'étude de la botanique par M. Lecoq. Annales scientif. de l'Auvergne. T. I.

Um Pflanzen in Herbarien vor Insekten zu bewahren empfiehlt Olislagers (Meylink, Schey-Artsenymeng en Natuurkundige Bibliotheek, Deel III. pag. 203. 1827.) ein Gemische von Quecksilber mit Gummischleim, womit man Fliefspapier bestreicht, dasselbe trocknet, in Streifen schneidet, und zu jeder Pflanze ein Stückchen legt.

Dr. Kittel rühmt $\approx u$ demselben Zwecke Opiumtinctur, womit man die Ränder des Papieres, worin die Pflanzen aufbewahrt werden, bestreicht.

Literaturblätter für Botanik. I. p. 559.

Dr. Koch schlägt Ol. Therebinthin. vor.

Bot. Zeit. 1824. 2. p. 447. 
Ćeber das Sammeln und Zubereiten der Pilze für IIerbarien und die Abhaltung der Insecten mit ilıren Larven von denselben, ist in den jüngsten Zeiten gar vicl gesagt uorden. Besonders hat der als Mykologe längst berühmte C. II. Persoon darüber zu beherzigende II inke mitgetheilt. - Man sehe Froriep's Jolizen Band 18. pag. 1i).

Das Auflewahren der Pilze in alaunhalligem Ocle, und das Anfüllen derselhen mit einer talgurligen Masse lehrte Lüdersdorff in der oben angeführten Schrift auf selir ausführliche I'eise.

Herr Moretti in Pavia hat, wie or sagrt eine sehr gute Methode crtdeckt, Sclıu:ümme natürlich zu conserviren, durch Behandlung mit gesättigter Bleizucker-Auflósung.

p. 145.

Bronn, Ergebnisse naturhistorisch-ökonomischer Reiscn. B3d. 1.

Auch in der $H_{0}$ l zsäure scheint man Schwömme erha'ten zu können. Herr Guillery schenkte dergleichen an die Linneische Gesellschaft in Paris. Man sehe:

Procédé nouveau pour la conservation de quelques espèces de champignons fugaces; par J. G. Guillery. Annales de la Sớ. Linn. de Paris Juillet 1825.

Ceber Aufbewahrung der Píze in Salzwasser stellte der. IVundarzt Cooke Fersuche an: er zeigte der Linneischen Societät in Lundon insbesondere ein Individuum von Clavaria muscoides vor, das über drei. Jature so aufbewahrt worden war. Das Fxemplar uar unversehrt und nur die Farbe etwas dunkler.

Jameson New Edinb. philosophical Journal. Jul. - Oct. 182!). p. 375.

Die gröfseste Aufmerksankeit verdient aber die Methode des Herrn Lasch in Driesen. Man sehe dessen Aufsutz:

Ueber das Auftrocknen und Aufbewahren der Fleisehpilze, vorzüglich der Blätterschwämme, zum wisenschaftichen Gebrauche.

Linnae a. 1830. Heft 3. p. 478,u. d. f.

Herr Lasch stellte die Pilze in al!en nüthigen Durchschnitten geprefst dar, so dafs durch die Segmente alle Dimensionen auf das bestimmteste $z u$ sehen sind, er trocknete sie ferner in allen Perioden der Entwickelung, so dafs man gleichsam die Lebensgeschichte dicser Gewächse deutlicher vor sich sieht, als sie je durch Abbildungen dargrestellt werden kann. Oelige Substanzen erhalten dem Ganzen die Farbe des Lebens, und die Pilze selbst liegen in leinen Bögen von apfelgrünem Oelpapier, sind mit schmalen Papierstreifchen befestigt und aufsen befindet sich die Etikette mit Citat, Diagnose und Fundort.

Reichenbach in der bot. Zeitung 1830. 1. p. 265. u. d. f.

Selbst die Blumen aus W'achs dürfen wir nicht ganz übergchen; wer darüber Einiges zu erfahren wünscht, findet es in: Dingler's polytechnischem Journal Bd. 31. p. 442.

In dem Bulletin des Sciences naturelles Mars 1829. p. 451). wird von den künstlichen Wachs-Blumen der Madame Louis (Rue du Paon Saint André No. 2. à Paris) Bericht erstattet.

Ausführliche Anweisung, künstliche Blumen zu verfertigen gibt nachstehende Schrift:

Flore artificielle par Forlier. Paris 1828. chez. Canteur, rue

St. Denis No. 326.

Fine der neuesten Methoden, Pfanzen bildlich darzustellen, ist die sogenannte IIomographie; sie besteht in ciner Art von Superposition 
auf die Pflanzenzeichnung, so dafs die Pflanze selbst als Prototype und Matrize dient. - Näheres sugt folgende Schrift:

Homographic ou Choix de 20 plantes indigènes et coloniales, par brevet d'invention, de perfectionnement et d'importation. Dédiée à S. A. R. Madame Duchesse de Berry, par Ch. d'Aignebelle. Gr. in Fol. sur papier Jesus d'Annonay. prix 10 Fr. et 15 Fr. sur papier de Chine. Paris 1825. l'auteur rue de Fauxbourg Saint-Honoré No. 111.

(Bulletin des Sc. nat. Avril 1829. p. 103.)

Pflanzen-Abdrücke sind übrigens in Deutschland längst bekannt. Man sehe unter andern:

lar $\mathbf{M a r t}$

Auch vergleiche man Schultes Grundrifs einer Geschichte und Literatur der Botanik Scite 207. \$. 109.

In F. W. L. Suckow's Vademecum für Naturaliensammler. Stuttgart 1831\%. gr. 12. (1 Fl. $30 \mathrm{kr}$.) kommt auch eine Anweisung, Pflanzen zu summeln, zu conserviren und zu versenden vor.

Zur Conservation der Herbarien hat Herr Dr. Ko ch in Kaiserslautern sehr zweckmäjsige Vorschriften bekannt gemacht.

Bot. Zeit. 1824. 2. p. 488.

M a nuel du naturaliste préparateur, ou l'art d'empailler les animaux et de conserver les végétaux et les minéraux, par Roitard 2de édition. Paris 1828. Chez Roret.

Traité de botanique élémentaire et de peinture à l'aquarelle, ou l'art de peindre les fleurs d'après nature, par deux élères des M.M. Desfontaines et Rédouté. Paris 1828. 4. Chez Lequien fils. Livr. 1. avec 3 pl. Prix de Souscription 5 Fr.

Das Werk soll aus 12 Lieferungen bestehen.

Kurze Anleitung zur Blumenzeichnung nach der Natur. München in F. G. Zeller's Kunstmagazin. Queer Fol. Vier Blätter Text und 12 Tafeln in Steindruck.

Man sehe hierüber botan. Zeilung 1821. 2. p. 736.

\section{Käufliche Sammlungen getrockneter Pflanzen.}

Sie sind ohne Zweifel ein wesentliches Hülfsmittel, sich in kurzer Zeit praktische Pflanzenkenntnifs su verschaffen; deshalb durfen dieselben, wenn sie gut zubereitet und richtig bestimmt sind, Anfängern vorzugsweise zur Beachtung empfohlen werden. - $\mathrm{Z} u$ den neuesten und bekanntesten dürften folgende gehören.

Sammlungen aus Deutschland, der Schweiz und Ungarn, besonders aus der Klasse der Phanerogamie.

Sammlung von hundert getrockneten Pflanzen aus allen Klassen des Linneischen Systems. Folio. Coburg, 1824. 3 fi.

J. F. Ruthe, Sammlung getrockneter Pflanzen aus der Gegend von Berlin, mit vollstândigen deutschen Beschreibungen begleitet, für Jugendlelrer, Forstmänner, Landwirthe und angehende Botaniker. Berlin seit 1820.

Das ganze Werk besteht aus 9 Lieferungen, deren jede 3 Rthl. p. C. kostet. - Eine nähere Anzeige steht in der botan. Zeitung 1820. 1. pag: 76. 
F. Thede beim Apotheker Bilz in Erfurt bietet Sammlungen der in dortiger Gegend wachsenden I'flanzen an; worunter allerdings manche seltene sind; fïr die Centurie verlangt er 4 Rthl.

Man sehe botan. Zeitung 1829. 1. Beilage p. 13.

T. Ph. Ekart. Gramina et plantas sul) aqua plane natintes quae in ducatu Coburgensi sponte proveniunt, sec. clarissimos betanicos cxamin. et singulis decadilus siccat. Dec. 1. 2. fol. maj. Colsurg et Lips. ap. Hinrichs. 1826. $5 \mathrm{fl} .42 \mathrm{kr}$.

A. Weihe. Dentsche Grâser für Botaniker und Oekonomen getrocknet herausgegeben. Lemgo, Meyersche Hofbuchhandlung. Seit 18:3.

Fs sind 15 Hefte vorhanden, wovon jedes $1 \mathrm{Rthl}$. kostet und 25 Arten enthält. In den Beilagen zur botan. Zeitung 1825. 2. p. 38. hat Herr $\mathrm{Dr}$. $W$. einige Berichtigungen der ausgegebenen Gräser und 1828. 1. p. 115. den Inhalt des 13. und 14. Heftes angezeigt. Die Sammlung enthält übrigrens nicht blos Gräser, sondern auch Cyperaceen und Junceen.

C. Günther et A. Schummel. Herbarium vivum, plantas in Silesia indigenas exhibens. Wratislav. sumpt. Auct. - Seit 1811.

Es sind 9 Centurien herausgekommen, deren jede 3 Rthl. kostet. -

A potheler Hornung in Aschersleben. Sammlung, getrockneter Pllanzen. Die Centuric zu 3 Rthl.

Die meisten der von ihm angebotenen Pflanzen sind in Thuringen, Mannsfeld, auf dem Harze oder im nördlichen Deutschland gesammelt; einige aber auch in der Schweiz, in dem Gebiete der Flora von Spa, im südlichen Deutschland und Frankreich. - Man sehe bot. Zeit. 1830. 1. p. 8. der Beiluge.

F. Th. Kützing, Pharmaceut, zur Zeit (1830) in Schleusingen, hietet getrocknete Pflanzen an (aus der Gegend von Magdeburg?) die Centur:e für 3 Rthl. $\frac{1}{2}$ Cent. 2 Rthl. $\frac{1}{4}$ Cent. 1 Rthl. Bot. Zeit. 1830. p. 13. Beil.

A. Sauter, Förster in Zirl bei Inspruck, ist gesonnen die Alpenpflanzen der Gebirge in den Umgebungen des Oberinthais, getrocknet, die Centuric à $7 \mathrm{fl}$. $12 \mathrm{kr}$. herauszugeben.

Bot. Zeit. 1829. 2. pag. 131. Beilage; - ferner 1830. 2. pag. 30. Beilage.

Karl Stein, Apotheker in Franenfeld, Canton Thurgau in der Schweiz, licfert getrocknete Alpenpflanzen die Centurie zu 2 Rthl, sïchs. Bot. Zeit. 1830. 2. p. 29. Beilage.

Legationsrath Wilhelm Gerhard in Leipzig biefet getrocknete Pflanzen, theils ron ihren natiurlichen Standorten genommen, theils cultivirte an, die Centurie zu 2: Rthl. Drei Centurien zu 7 Rthl. и. в. w. Je mehr man nimmt, desto wohlfeiler sind sie, aher man muls dem Herrn Legationsrath die Auswahl überlassen; wählt man selhst, so kostet dic Centurie 4 Rthl.

Man sehe bot. Zeit. 1828. 2. p. 2. der Beilage.

Joseph Klug, Pharmaceut in Reichenhall, ist erbötig, Herbarien der Flora von Bayern und Oestreich zu liefern. Die Centurio zu $10 \mathrm{fl}$.

Bot. Zeit. 1829. 2. p. 1. Beilage.

Professor und A potheker G. Hinterhuber in Salzhurg gibt getrocknete Sammlungen der Gewächse um Salzburg, so wie solche 
von den Tyroler, Kärnthner und andern Alpen; die Centurie praenumerando für 6 fl. $40 \mathrm{kr}$. C. M. W. W. oder 8 fl. R. W.

Daselbst $p .3$.

Professor Dr. Hoppe in Regensburg, längst berühmt durch die ausgezeichnete Schönheit der von ihm getrocknet ausgegebenen Gewächse um Regenshurg und der Alpen, dureh seine Gramineae selectae u. в. w., erbietet sich auf's Neuc, getrocknete Sammlungen zu liefern, wovon die Centurie nie höher als 2 Dukaten kommen soll.

Man sehe das. Specielle in der botan. Zeitung 183v. 1. p. 24. 2. 25. der Beilage.

Ferdinand Elfsmann, Provisor der Gladbachischen Apotheke in Regensburg, verkauft getrocknete Pflanzen aus den Gebirgen von Salzburg, Oberkärnthen, Süd-Tyrol u. s. w. die Centurio für $8 \mathrm{fl}$.

Bot. Zeit. 1829. 1. p. 34. der Beilage.

Flora germanica exsiccata, sive Herbarium normale plantarum selectarum criticarumque in Germania propria vel in adjacente Borussia, Austria et Hungaria, Tyroli, Helvetia, Belgiaque nascentium, concinnatum editumque a societate Florae Germanicae curante Ludovica Reichenbach. Lipsiae apud Fr. Hofmeister. Centuria prima fol. 1830. 5 Rthl.

Ueber die Finrichtung dieses für sich einzigen Unternehmens, so wie über den Inhalt dieser ersten Centurie des Normalherbarii sehe man Bot. Zeit. 1830. 2. $p$. 418. und Beilage p. 1. u. d. f.

Herbarium florae alpina helveticae, curante Oswald Heer (im Pfarrhause zu Matt, Canton Glarus in der Schweiz). Die Sammlung soll mit Ausschlul's der Pflanzen der Ebene 250 Alpengewächse enthalten und in 3 Abtheilungen ausgegeloen werden; jede Abtheilung kostet 1 Dueaten, also das Ganze 3 Ducaten oder 6 Laubthaler.

Man sehe bot. Zeit. 1828. 1. Beilage p. 9.

Die Alpenpflanzen Oestreichs sammclte früher Herr Si e ber unter dem Titel: Herbarium Florae Austriacae seu collectio exquisitissimarum stirpium in imperii Austriaci provinciis sponte crescentium Sectio 1. 300 specierum suprinios alpinarum continens. Pragae 1821. Altstadt Nr. 618. 30 fl. C. M.

Die Pflanzen dieser ersten Centurie sind im Tyrol gesammelt, ob die beiden übrigen, welche versprochen wurden, die die Gewächse von Istrien, Kroatien, Dalmatien u. s. w. enthalten sollten, herausgekommen sind, ist mir unbekannt.

Jetzt bietet derselbe an: Flora austriaca I. 300 Arten 20 Rthl. II. 260 Arten 20 Rthl. Wien, Josephsstadt Nr. 45.

Collection des Plantes de Chamouni et des montagnes, aroisinants le Mont-Blanc: par N. C. Séringe. Un Vol. 4. contenant 100 échantillons de plantes desséchées. Genève, chez l'auteur, rue du Puits. Saint Pierre No. 116. 30 Fr.

Herr Séring e lieferte früher noch ein Herbier portatif des Alpes, ein Herbarium Cereale; mehrere Hefte Saules de la Suisse; Hicracia exsiccata, Rosac exsiccatae u. s. w.

M. J. Löhr, Pharmaceut aus Coblenz, jetzt in Berlin, bietet Schweizer-Pflanzen, die Centurie zu $8 \mathrm{fl} .24 \mathrm{kr}$. an.

Bot. Zeit. 1830. 1. Beil. p. 5.

D. Trachsel in Rüggisberg bei Bern machte ein Verzeichnifs von Pflanzen bekannt, die er zum Tausche anbietet.

Daselbst. p. 5. 
A. F. Lang, Ehumeratio plantarum in Iungaria sponte nascentium, quas in usum botanicorum legit. P'ert.

Die Centurie kostet $8 \mathrm{fl}$. rheinisch.

Aro Jos. Sadler, Med. Doet, et Leop. Paner, Pharm. Mag., aammelten Pflanzen in Ungarn. Crmatien nut Siebenbuirgen, die sie in Fakcikeln horamggelien, deren jeder (ill enthilt und 4 fl. 30 kr. Angsb. Cour. kistet. Man wendet sich an die Herauscreher selbst; oder in Wien an Panl v. Partseh, in Regrensibure an Prof. Hoppe, in Leripzig an Prof. IRadius, in Dresten an IIofr. Reichenbach, in Breslau an Asscssor Günther, in Minchen an Prof. v. Martius.

- Sammlungen aus Frankreich, den Pyrenäen und Belgien.

Giv. Salznänn, Enumeratio plantar. rarior. in Gallia anstrali sponte nascentium, quas in usum botanicorum legit et exsiccavit. Montpellier 1818.

Unten dem Titel: Plantac selectae Europae australis verkaufte derselbe auch getrocknete Pflanzen aus Corsiya, der Provence und den Pyren:ëen. Man zahlte für den Fascikel 45 francs, Jeden 1... $\approx u$ 23 kr, rhein gerechnet.

P. Thomas. Zwei Centurien Pyrenäen-Pflanzen. Bern bei Schäfer it 25 francs oder $11 \mathrm{fl}$. $27 \mathrm{kr}$.

Sieber IIerbier général de France.

a. Flore des Alpes, de Dauphiné et de la Provence.

b. Flore des Pyrenées.

c. Flore de la Corse et de la région méditerranée.

d. Flore des Départemens de 1 List, de l'ouest et de l'interieur.

e. Flore des environs de Paris et des Départemens du Nord.
Faxemplare aus den Alpen der Dauphiné kosten präparirt 25 fr. die Centurie; nicht definitiv zubereitet 20 francs. Man erhult sie in Puris rue de Harlay No. 27. - Nach einer Anzeige in der bot. 6. Zeit. (1ऽâ1) 1. p. 27. der Beil.) erhält man die Pflanzen bei। $\boldsymbol{J}_{0-}$ seph Dufort, Buchhündler in Paris, Quai Voltaire No. 19. Die it Summlung enthält 172 Species in 2 Abtheil. anf weifsem Papier 40 francs, auf. grauem $34 \mathrm{fr}$.

Choix du plantes de la Belgique, par A. L. S. Lejeune et R. Courtois, 11. D. 1. et 2 . fascicle in fol. Liegre. Jeder Fascikel fostet $10 \mathrm{fr}$.

P. Michel, Agrostologie Belgique ou herbier des Graminćes, des Cyperacées et des Juncées qui croissent spontanément dans la Belgique, ou qui y sont cultivées, recucillées et publiées par Centuries, revues par A. L. S. Lejeune. Liège 18\%'s et 18\%.). Cent. II.

\section{Sammlungen aus Italien.}

G. J a n, öfentlicher Lehrer der Pflanzenliunde an der Universität zu Parma, gab heraus.

a. Flora Italiae superioris, enthaltend Gewäche ans dem venetianisch-lombardischen hönigreiche, den piemontesischen Stanten und den Herzogthümern Parma und Piacenza. Es sind 8 Centurien angekündigt worlen, jede zu 15 fr.

b. Herbier portatif des Dames. In elegant eingebundenen Büchern, deren jedes 100 Pflanzen enthalten soll und $20 \mathrm{fr}$. kostet.

c. II erbarium medico-toxicum. Zwei Centurien, jede für $\mathbf{1 6} \mathbf{f r}$.

d. Oekonomiseh-technologische Pflanzensammlung.

Man sehe bot. Zeit. 1820. 1. p. 172. u. d. f. 
Tranz Petter, Professor am Gymnasium zu Spalato in Dalmatien, bietet getrocknete Pflanzen der dortigen Gegend an, zehn Stück für $50 \mathrm{kr}$, weniger als 50 Stück werden aber nicht abgegeben. Man sehe bot. Zeit., 1829. 1. p. 24. Beilage.

K. B. Pres 1. Flora Sicula fasciculi quinque. Jeder Fascikel kostet 6 fl. Prag, Schwefelgasse Nr. 470.

Der korsikanischen Pflanzen, welche Salzmann und Sieber liefern, ist. schon oben gedacht worden, des letztern Flora Corsica enthält $\mathbf{3 7 0}$ Arten und kostet 30 Rthl.

Sammlungen aus England, Russland und Griechenland.

Dr. Marian o Lagasca, ehemals Prof. u. Director des botan. Gartens in Madrid, hat in London läufliche Herbarien von der Umgegend der' Stadt in einem 'Umkireise von 20 Mcilen angelegt, und die Pilanzen nach den Original-Exemplarien in den Banksischen und andern Herbarien bestimmt. "Sie sind bei Treuttel, W Wïrz et Comp. in London u. Strasburg für den Preis von 1 Pf. Sterling zu haben. Bötan: Litératurblätter 2. p. 220\%?

Herbariunt Florae Ruthenicae, sistens plantas rariores in Gubernio Chersonensi sponte aloviarum, Cent. I. II. Curante A. F. Lang et A. J. Szovits. 12 fl. bei Lang in Pest, Waizengasse No. 32. (Siehe Isis Bd. 20. Heft 6. u. 7. Umschlag.)

Nach einer andern Nachricht sol! man sich addressiren an Ad olph Franz Lang, Mag. d. Pharmacie, Salvators-Apotheke, Kerepeser Strasse No. 7: in Pest:

\section{Literaturblätter für Botanik 1. p. 222.}

F. W. Sicber. Herbarium creticum. Das grofse kretische Herbar von 450 Arten (die Centurie ì $16 \mathrm{fl}$. C. M.) kostet $72 \mathrm{fl}$. C. M. Das kleinere kretische von 225 der ausgesuchtesten Arten im Verzeichnifs mit * bezeichnet 28 fi. C. M.

Nack einer spitern Nachricht (bot. Zeit. 1828. 2. p. 1. d. Beil.) enthült das Herbarium Florae creticae 220 Arten und kostet 20 Rthl. Wient Josephstalt No. 45.

Ueber die Sieberschen Herbarien vergleiche man übrigens die Bemerkungen von Presl in der 1sis, Jahrgang 1828. p. 26i7.

Sieber Filices Collectio prima 80 Species. 16 Rthl. - Collectio secunda 80 Species 16i Rthl. Wien Josephstadt No. 45.

Diese Samnilungen enthalten wohl, eben so wie die folgenden, Arten aus verschiedenen Weltgegenden.

Sicber Agrostotheca. 120 Species 16 Rthi.

Daselbst. Mañ vergleiche über sie dle Bemerkungen von Nees v. Esenbeck. Bot. Zeit. 1828. 1. p. 289.

Collectio Haenkeana. Von den Reliquiis Hacnkeanis ist jetzt der erste Band, mit den Grami neen schliessend, heraussekommen. Von den darin beschriebenen Pflanzen sind getrocknete Exemplare à 20 kr. das Stück ziI erhalten. Man wendet sich an Dr. Karl Pres1, Custos an dem böhmischen Museum in Prag.

Bot. Zeit. 183\%. 1. p. 41. der Beilage.

Sammlungen aus Asien.

Sieber Flor von Palästina. 48 Arten $12 \mathrm{fl}$.

Dessen Flore de Pondichery et de Chanderagor soll einen Theil 
der Sammlung ansunachen, die Herr S. unter dem Titel: Flore franpaias coloniale, divisée en Flores partielles angekündigt hat.

\section{Sammlungen aus Afrika.}

No. 45.

Sieber Flora aegyptiaca. 150 Arten $18 \mathrm{kthl}$. Wien Josephsstadt

Salzmann Plantae lectae in itinere Mispanico-Tingitano. Der Fasciculıs zu $22 \mathrm{fr}$. Man erhält sic in Montpellier bei Ph. S alzmann, Adresse Ilern Lichtenstein in Vialars; fur Deutschland besorgte den Absatz. Herr Dr. Ziz in Mainz; wer aber nach dessen jetzt erfolgtem Ableben sich damit befafint, ist mir unbehannt.

Man sche über diese Samml. bot. Zeit. 1827. 2. p. 95. der Beilage.

Plantae capenges Zeyherianae. Es sind 6 verschiedene Sammlungen zu verschiedencn Preisen, die, so wie die Aufzählung der Pllanzen selbst nachgesehen werden kann in der botan. Zeitung 1829. 1. p. 2. der Bscilage. Die Bestimmungen sind von Kurt Sprengel und man addressirt sich an den Garten - Director Zeyher in Schwetzingen.

Die Anzeige der neuesten Sammlung steht in der bot. Zeit. 1830. 1. p. $15 \mathrm{der}$ Beil. Dort ist der Preis der Centurie auf $15 \mathrm{fl}$. rhein. bestimmt. Auch werden dort kapsche Samen à $15 \mathrm{kr}$. für die Art angeboten. - Ueber diese Sammlung vergleichie man auch die Bemerkungen des Herrn v. Voith in Regensburg. - Bot. Zeit. 1830. 1. p. 237.

Plantace capens collectae a D. Ecklon. Herr Ecklon rammelte für den naturhistorischen Verein in Würtemberg. Auf Bestellungen kïnnen aber noch kapsche Herbarien von 500 Arten zu $40 \mathrm{fl}$. abgegeben werden.

Man kann sich an Herrn Dr. Steudel in Efslingen wenden. Bot. Zeit. 1829. 1. p. 132.

Sieber Flora Capensis. Sectio prima, 150 Species, 24 Rthl. Sectio secunda, 112 Arten, 16 Rthl. Sectio tertia, 100 Arten, 16 Rthl.

Wien, Josephstadt No. 45.

$\mathrm{Zu}$ den französischen Colonial-Floren, welche Herr Sieber ankündigte, gehören :

Flore du Senegal, de Gorre et de la cote de Guinée, oder Flora Senegalensis, 50 Arten, 8 Rthl.

Flore des iles Maurice, Bourbon et Madagascar. Flore mauritiane Sect. 1. 194 Species, 32 Rthl. Sect. 2. 100 Species, 16 Rthl.

Die von Herrn Krebs auf dem Kap gesammelten Pflanzen sind, so viel mir behannt, nicht cinzeln verkauft, sondern blos an die Abnehmer der Actien vertheilt worden.

IIcrr James Bowie erbietet sich, getrocknete Pflanzen in Afrika zu bestimmten Preisen zu liefern (Kew Green Comté de Surrey) auch Saamen, $Z$ wiebeln und lebende Gewächse.

Man sehe Edinb. Journ. of Science Avril 1827. p. 366. - Bulletin des Sc. natur. Mars 1829. p. 448.

\section{Sammlungen aus Amerika.}

Sie ber Flora martinicensis 150 Species, 24 Rthl.

I d em Florae S. Trinidad. Sectio prima 100 Species 12 Rthl. Sectio necunda 120 Species 16 Rthl. 
Von der französischen Colonial-Flora kündigt Herr Sieber an 1. Samml. aus Cajenne und Gujana. 2. Samml, aus den Antillen (Martinique, Guadeloupe und St. Domingue). B. Samml. aus Terre neuve und Canada.

Schiede Collectiones e Veracruzet Jalapa.

$W$ er von diesen Sammlungen aus getrockneten Pflanzen, Saamen, Holzarten etc. etwas zu haben wünscht, wendet sich an Herrı Prof. v. Schlechtendal in Berlin. - Linnaea 1829. p. 236.

Herbarium mexicanum. Fasciculus primus, 58 Arten $16 \mathrm{fl}$.

Man wendet sich in München an die Herrn v. Martius oder Zuccarini oder auch an die Redaction der Regensburger Flora. Bot. Zeit. 1829. 1. p. 21. Beilage.

Herbarium brasiliense, gesammelt von Salzmann in Montpellier. Die Centurie kostet 40 francs.

Bull. des Sc. natur. Juillet 1830. p. 93.

Herbarium collectum in insula St. Thomas a Roberto Schomburgh Lipsiensi.

Man wendet sich an Herrn Hofrath Reichenbach in Dresden. - Bot. Zeit. 1831. 2. p. 424.

F. Volz, Pharmaceut, unternimmt eine Reise nach Georgien und Carolina, und erbietet sich, für einen Beitrag von $20 \mathrm{fl}$. wenigstens 200 - 250 Arten getrockneter Pflanzen der dortigen Gegend zu liefern. Man wendet sich an die Herren Kurroder Fleischer bei Herrn Prof. Schübler in Tübingen.

Bot. Zeit. İß̋v. 1. p. 1. Beilage.

Dr. Joh. Lhotsky zu Wien (Leopoldsstadt No. 132.) unternimmt eine botan. Reise nach Bahia in Brasilien, und erbietet sich, getrocknete Pflanzen jener Gegend zu liefern.

Man sehe das Nähere in der bot. Zeit. 1830. 1. p. 37. Beil.

\section{Sammlungen aus Neu-Holland.}

Sieber Flora novae Hollandiae, 480 Species 100 Rthl. Supplementum 100 Species 16 Rthl. Wien, Josephstadt No. 45.

Die Agrostotheca enthält auch meistens neuholländ. Arten. Ueber die vorhabende botaniscke Reise des Herrn Johann Lhotsky sehe man botan. Zeit. 1829. 2. p. 637 und 767.

\section{Sammlungen von Cryptogamen.} gezeigt.

Die Siebersche Collection von Farrenkräutern ist bereits oben an-

Sieber Cryptogama exotica 60 Arten. 7 Rthl. Wien Josephstadt No. 45.

Sammlung cryptogamischer Gewächse für Freunde der Natur. Leipzig 1824 .

C. H. Funk. Kryptogamische Gewächse, besonders des Fichtelgebirges. Leipzig seit 18u1. In Quartheften. Jedes kostet $18 \mathrm{gr}$. Jetzi sind deren 35 vorhanden. Leipzig bei Barth. Die Anzeige des letzten sehe man in der bot. Zeit. 1829. 2. p. 743.

Dietrich Herbarium Florae Germanicae, oder Deutschlands Flora in getrockneten Exemplaren. Erstes Hundert. Auch unter dem Titel : 
Dentschlands kryptegamische Gewãche. Erstes IIeft No. 1 - 50. gr. 4. Jenae 1826. ช. in Mappe $1 \mathrm{fl} .40 \mathrm{kr}$.

Plantae cryptogamicae, quas in magno ducatu Badensi collegerunt F. G. Kneiff et E. F. H a rtmann. Strashurg 1828. Fol. 10 Fr.

Fünfzig Arten aus allen Ordnungen, worunter Selerotium roseum líneifr.

Plantes cryptogames du nerd de la Françe par J. B. II. J. Desmazières. 1 - 4. fakcic. Paris et Lille 18²6. 4.

Sieke Literaturblätter 2. p. 69.

Centuria prima plantarum eryptogamicarnm Norvegicarum, yuas collegit et edidit S. Chr. Som merfelt. Christiania I826. Gröndahl. 4. Preis 4 Spec. Thaler. $11 \mathrm{fl.} 21 \mathrm{kr}$.

\section{Daselost p. 70.}

Musei thuringici vivis exemplarib. exhib. et illustr. J. G. Zenker et F. D. Dietrich. Fase. I. - IV. 8. maj. Jenae 1821 - 1825. Mit 100 getrockneten Exemplaren, 5 fl. 24 kr.

Musei frondosi, quas in Alsatia variisque IIelvetiae et Germaniac partibus collegerunt F. G. K neiff et C. P. W. Marker. Strasburg 1825 - 1ะ27. 8. Sichen Lieferungen 150 Aiten a 5 francs.

Mousses de la Normandie, recueilliés et publiées par L. Ad. de Brebisson. Caen 1826-1828. Maenel. Paris Meilhac. Cah. 1. et 2. Jedes Hleft kostet 3 francs. - Literaturblätter II. p. 70.

T. Ph. Ekart. Die Flechten, Laub- und Lebermoose, wclche im Herzogthum Coinrg auf dem Lande und im Wasser gefunden werden, nach den verdientesten Cryptogamenforsehern bestimmt, und in Fascikeln herausregeben. Fasc. I. No. 1 - s. Klein Fol. Coburg 1526. $2 \mathrm{fl} .51 \mathrm{kr}$.

Reichenbach et Schubert. Lichenes exsiccati. Die Flechten in getrockneten Exemplaren. 1 - 6. Heft, jedes mit 25 Exemplaren und mit erläutcrndem Texte, 4. Leipzig $1823-1826.14 \mathrm{fl} .24 \mathrm{kr}$.

Lichenes II elvetici exsiceati et Lichen. helveticor. Spicilegium Auct. Lud. Eman. Schacrer, ecelesiae Laupervillensis pastore. Sectio I. lichen. exsiccat. fascicul. 4 primes. Sect. II. fascicul. 5 et 6. Sect. III. fascicul. 7 et 8 illustrans. Prostant in Lauperswyl pago Helveto - Bernensi apud editorem, in Commiss. Bernae apud. C. A. Jenni et Lipsiae apod B. H. J. Hartmann. 1828.

Jeder Fascikel enthält 50 Nummern und kostet 4 .Schweizerfranken (16 auf eine Karolin). - Man sehe bot. Zeit. 1827. 1. p. 164. Literaturblätter 1. p. 222.

Lichens de France, publićes par Delise, chef de hataillon etc. Fasc. I. 4. Vire 1828.

Das Heft enthält 25 Arten oder V́arietäten. - Siehe Literalurblätter 3. p. 625.

Lichenes exsiccati Succiae, curante Flia Magno Fries. Fascicul. XIII. Cum schedulis criticis Autoris. 1825 - 1828.

Einige Fascikeln dieser Sammlung besorgte Christ. Stenhammer zu Linköping. - Man sehe bot. Zeit. 1824. p. 583. Literaturblätter ङี. $p$. 620.4. $p$. 267.

J. v. Flotow Lichenen, rorzïglich in Schlesien, der Mark und Pommern gesammelt. Cent. I. Fol. Leipzig bei Barth, 1830. In Mappen selır sorgfältig bewahrt. 16 fl. $12 \mathrm{kr}$.

Bot. Literaturbl. 4. p. 271. 
Fries. Selermoyceti Succiae exsiccati Decad. I. - XXX. apud Cl. Mauritium Bỉliopolam Gryphiswaldensem.

Pretium apud anctorem 8. Thal. pec. Saxon. Collectio hace, pro Syst. Nycol., ut pate Sphaerias et Phacidia interrupte continuatur.

Mycetologie. Sammlung ron Schwämmen en relief in natürlicher Gröfse in Wachs nachgebildet, herausgegeben von $\mathbf{J g n a z i o} \mathbf{P i -}$ zacolli in Mailand. Corso di porta orientale No. 65:3.

Nähere Nachricht über diese interessante Sammlung, ihren Preis u. s. f. findet man in dem Bulletin de's sciences natur. Janvier 1825. p. 76.

Mycologie en cire etc. Mykologie in Wachs, oder vollständige Sammlung der einheimischen Schwämme in Wachs nachgebildet, so dafs sowohl die Verschiedenheiten der Organisation, als die Geschichte ihrer Entwickelung dargestellt wird, von $k$ inspail und Ialrieh, Wachs - Bossirer der med. Facultït in Paris. 1829.

Man sehe hierüber Literaturblätter. 2. p. 129.

Mertens Prof. in Bremen, Pastor Fröhlich u. N. v. Suhr in Schleswig erbieten sich getrocknete Sammlungen von Hydrophyten oder cryptogamischen Wassergewächsen zu liefern. Die Decade soll noch nicht auf 2 Rthl. in Gold zu stehen liommen.

Bot. Zeit 1828. 1. p. 14. der Beilage.

G. H. B. Jürgens. Algae aquaticae. Wasser-Algen, anf der Nordwestküste Deutschlands gesammelt, besonders bei Jever und Ostfrieslands, so wie in den dortigen Gewissern gesammelt. Heft 1 - 19, jedes mit 12 Arten und Erklïrungen. Fol. Jercr, 1825. Jedes Heft 1 fl. $48 \mathrm{kr}$.

Man sehe bot. Zeit. 1830. 2. p. 411.

Algues de la Normandie, recueilliés et publiées, la partie des articulées, par M. Roberge, et la partie des inarticulécs, par M. Chavin:. Caen 1827. Fol.

Die. Lieferung von 25 Arten kostet 10 francs. - Man sehe Bulletin des Sc. natur. Sept. 1828. p. 122.

$\mathrm{Zu}$ den Anstalten der neueren Zeit, deren Zueck es auch ist, die Pfanzenkunde durch Verbreitung seltener getrockneter Gewächse zu befördern, kann man zühlen :

1. Die Naturalien Taush-Anstalt des Herrn Opiz in Prag.

Man sehe hierüber die neueste Nachricht in der bot. Zeit. 1829. 2. p. 109. der Ergänzungsb̉ätter. Ich führe daraus nur an, dafs die. Centurie getrockneter Pflanzon um 2 fl. C. M. abgelassen wird. Zu vergleichen noch bot. Zeit. 1828. 2. p. 494.

Ph. M. Opiz. Naturalientausch No. 1-12. gr. 8 Prag, 1823 bis 182S. Enders. 3 fl. $36 \mathrm{kr}$. No. 12. Auch unter dem Titel: Beiträgc zur Naturgeschichte, als Fortsetzung des Naturalientauschs

Herr Wiest in Tübingen errichtete eine besondere Pfianzen-Tauschanstalt für das südliche Deutschland, es ist mir aber unbekannt, ob sic noch in Thätigkeit ist.

Man sehe bot. Zeit. 1825. 2. p. 67. der Beil.

2. Wirtembergischer Reise-Verein. An der Spitze des Unternchmens stehen die Herren Prof. Hochstetter und Dr. Steudel in Efslingen. Auf Kosten des Vercins bereiste Herr Flcischer das südliche Tyrol, Müller die kärnthischen Alpen und Sardinien, ersterer 
ging spiter nach Klcinasien, Herr Hühener nach Norwegen, andere Rammelten auf dem liap u. ค. w. - Der jährliche Beitrag ist 15 fl. rhein. Man sehe bot. Zeit. 1826. 1. p. 87. 1828. 2. p. 10. der Beilage und an mehrern andern Stellen.

3. Göttinger Zoologisch-botanischer Reise-Verein. Er hat sich erst kürzlieh gelildet und wird von den Ilerren Dr. Bartling und Berthold geleitet. Der Preis der Actien ist anf 1 Lonisd'or festgesetat. Auf Rechnung dieses Vereins ist bereits Herr T $\mathbf{T}$ umann nach Morea abgereist.

Brandes Pharmaceut. Zeit. 1830. No. 11. p. 173.

4. Ueher die Mitheilung der II erbarien der englischen ostindischen Gesellschaft an verschiedene Museen und berihme Botiniker ohne Unterschied der Nation, was zumal durch Wallichs Betrieb geschieht, sehe man den Aufsatz des IIerrn A ug. J) va u im

Bulletin des Sciences naturelles. Juillet 1829. p. 88.

\section{I n s t r u m e n t e.}

Wenzel Batka in Prag hat hotanische Bestecke nach Sprengel, enthaltend in ciner Brieftasche nelost beiden Systemen, 1 zweischneidige feine Lancette, 1 Zange mit Schieher, 1 detto mit Platinspitze, 1 Nadel von Platin, 1 detto von Stahl, 1 Lupe. Kostet 8 fl.

Ferdinand $O$ echsle in Pforzheim hat botanische Bestecke mit zwei Lupen, im Preise zu $7 \mathrm{fl}$.

Mechanikus Baumann in Stuttgardt verfertigte ein botanisches Taschen-Mikroskop mit vier einfachen Linsen, durch deren Combination 8 verschiedene Vergrölserungen hervorgebracht werden können. Der Apparat geht in das ihm zum Fufsgestell dienende Kästchen. Preis 28 fl.

Ein ähnliches mit einem zusammengesetzten Mikroskop verbundones. $33 \mathrm{fl}$.

Beide sind sehr bequem eingerichtet, wenn sie auch nicht bedeutend vergröfsern. - Correspondenzblatt des wirtemb. landwirthschaftl. Vereins. Bd. 12. p. 320.

Die Mechaniker Apel und Lüders in Göttingen haben botanischc Bestecke der gröfsern Art für 3 Rthl.

Dasselbe cinfacher mit Lupe, Messer, Zange und Nadel 2 Rthlr.

Loupe mit 3 Gläsern in Horn gefafst 2 Rthl.

Diевеlbé mit 2 Gläsern. 1 Rthl. 8 ggr.

Dieselbe mit einem Glase $16 \mathrm{ggr}$.

Diese l be mit 2 Gläsern, mit ciner Fassung von Ebenholz und einer Büchse 1 Rthl.

Dieselbe mit einem Glase in Buchsbaum oder Ehenholz 12 ggr.

Microseop zu botanischen Untersuchungen mit Stativ, Spiegeln, 8 cinfachen Linsen, Messern, Zange und sonstigem Apparat, alles compendiös in einem Mahagoni-Kästehen. 5 Louisd'or.

Auch verfertigen sie zusammengesctzte Mikroskope für 16 Louisd'or und einfache für 8 Louisd'or.

G. S. PlösI, Optiker und Mechaniker in Wien, neue Wieden, Salvatorgasse No. 321. führt unter andern folgende optische Apparate.

Loupe nach Wilson, mit einer Linse von messingener Fassung 1 fl. $24 \mathrm{kr}$.

Derlei mit zwei Linsen mit Deckeln 2 fl. 48 kr. 
Einfache Loupe in Büffelhorn gefafst $1 \mathrm{fl} .12 \mathrm{kr}$. - Derlei doppelte $2 \mathrm{fl}$. - Derlei dreifache $2 \mathrm{fl}$. $48 \mathrm{kr}$.

Lo upe in Büffelhorn gefafst, mit glïsernem Lieberkühnschen Spiegel 2 fl.

Botanisches Handmikroskop mit Liebcrkühnschem Spiegel, anf messingenem Griffe, Objectivnadel mit Pincette, Messerchen und Nadel mit elfenbeinernen Heften und Pincette, in Futteral von Maroquin $7 \mathrm{fl}$.

De rlei mit zwei Linsen 9 fl.

Derlei auf büffelhornenem Griffe, eine Linse mit Lieberkühnschem Spiegel, einer Loupe und Objectivnadel mit Pincette; in Futteral ron Maroquin $4 \mathrm{fl}$. $30 \mathrm{kr}$.

Dasselbe mit schildkrïtenem Griffe. $6 \mathrm{fl}$. Pincette, Messerchen und Nadel dazu $1 \mathrm{fl}$ :

Auch hat derselbe Mikroskope für 185 fl. $279 \mathrm{fl}$. für $90 \mathrm{fl} . \mathrm{u} .60 \mathrm{fl}$.

Das optische Institut der Herrn Utrsehneider und Frauenhofer zu Mïnchen liefert verbesserte optomatische Mikroskope, worüber man nähere Nachricht findet in : p. 54.

Poggendorf's Annalen der Physik und Chemie 1829. No.9.

Bemerkungen über das neneste Mikroskop des Herrn Prof. Amici in Modena: rom Freiherrn von Jacquin findet man in:

Baumgärtner und Ettinghausen's Zeitschrift für Physik und Mathematik. Bd. 7. Heft 3. p. 257. u. d. f. Auch sehe man, uas Marx in Braunshueig darüber sagt in SchweiggerSeidels Jahrb. 1830. Heft 10. p. 180. u. d. $f$.

Ueber ein treffliches tragbares botanisches Mikroskop von Herrn Varley ist nähere Bclehrung zu finden aus Gill's Technical Repository, in :

Dingler's polytechnischem Journal. Bd. 22. p. 97. $u$. d. $f$.

G. S. PIöfsl in Wien verfertigt Linsen von Beryll, Topas und Bergkrystall von $200-300$ maliger Vergröfserung für $\mathbf{1 0} \mathrm{fl}$.

Demantlinsen für $150 \mathrm{fl}$., Saphirlinsen für $20 \mathrm{fl}$. u. в. w. worüber das Nähere nachgesehen werden kann in der

Zeitschrift für Physik und Mathematik von Baumgärtner und Ettinghau sen. Bd. 7. Heft 1. p. 119. u. d. f.

Jones Holborn in London verfertigt Diamant- und Saphirlinsen als Mikroskop brauchbar.

Darkin lobt sie im Quaterly Journal of Science, Jan. 1528,

p. 459. Auch sehe man darüber Froriep's Notizen Bd. 20. p. 218.

Ueber die von Herrn A. Pritchard, Opticus zu London, angefertigten Mikroskope mit einfachen Linsen von Saphir oder Diamant finden sich Nachrichten entlehnt aus dem No. 520.

Edinburgh Journal of. Science in Froriep's Notizen 1824. p. 209

Mechanikus Baumann in Stuttgardt verfertigt Instrumente zum Zerschneiden verschiedener Holzgattungen in Scheiben bis zur Dicke von ${ }^{3}$ है Par. Linie für mikroskopische Untersuchungen. Preis 25 fl. Sehr sinnreich eingerichtet, und mit aufserordentlicher Genauigkeit gearbeitet, für den Pflanzen-Anatom von Werth. (Siehe a. o. a. 0.)

G. S. Plö fsl in Wien liefert Sammlungen von 43 Queer - und Långs-Durchschnitten von Pflanzenstämmen und Stengeln, mit systemati- 
seher Benennung, zum Gelorauch bei dem Unterricht über den innern Bau der Pflawzen in 12 Oljectivsehiebern von Buchsbaumholz in Futteral von Maroquin fiur $12 \mathrm{fl}$. Dieselben in Ehenhols fiur $15 \mathrm{fl}$.

Prof. Schilling in Breslau erfand ein Instrument zum Nachzeichuen pflanzen-anatomischer Gegenstiande, mit der kinrichtung einer Laterua magica, doch so, dafs durch einen antebrachten Spiegel das Bild des vererrofserten Gegenstandes nicht auf die senkrechte, sondern auf die horizontale Piapierfliche geworfen wird, und so unmittelbar nachgezeichnet werden kann.

Sulzb. med. Zeit. 1827. 3. p. 272.

Mémoire sur un instrument, nommé Mierosater, ou conservateur de petites choses, par M. Morren ì Gand.

Annales des Sc. nuturelles Aout 1831. p. 404.

Das. Instrument ist bestimmt, mikroskopisclie regetabilien auf cine geeigncte Weise aufzubewahren.

\section{Neuere Phytographie und Iconographie.}

\section{§. 7.}

Unter dieser Rubrik gluube ich mehrere Schriften zusammen fassen zu müssen, dic theils heftweise erscheinend sich den Zeitschriften anschliessen, theils aber auch nur einzeln herausgregeben wurden, und Beschreibungen odler meistens auch Abbildungen von neu bekannt geurordenen, kritischen, oder zuerst in die Gï̈rten eingeführten, oder seltenen Pflanzen enthalten, und dabei auch noch manche andere Nebenzuecke zu erreichen suchen. Dergleichen Schriften gelangen nicht selten auch in die Hünde solcher Personen, denen Botanik nicht eigentlich Hauptstudium ist; sie haben aber ihrer oft gröfseren I erbreitung wegen, einen nicht zu verkennenden Einflufs auf die Bearbeitung der Pflanzenkunde, und sind noch besonders darum wichtig, da sie gleichsam die phytographische Gieschichte des 'Tages enthalten.

\section{a. D e u t s c he.}

C. L. Reichenbach. Iconographia botanica seu plantae criticac, Icones plantarum rariorum et minus rite cognitarum, indigenarum exoticarumque; sive Iconographia et Supplementum, inprimis ad opera Willdenowii, Schluhrii, Perseonii, Roemeri et Schultesii delineatae et cum commentario succincto editae Lipsiae 1823. 4. Führt uuch den Titel: Icones plantarum rariorum et minus rite eognitarum Florae Europaeae ete. Auch wie die beidlen folgenden mit deutschem Texte. Dus sehr nützliche Werk wird noch immer fortgesetst, und ist, wenn ich nicht irre, bereits auf acht Centurien Abbildungen angewachsen,

C. L. Reichenbach. Hortus botanicus, hortorum vivorum siccorumque novitates illustrans. Lipsiae 1824. 4. Decad.' 8.

C. L. Reichenbach. Iconographia botanica exotica, sive IIortus botanicus, imagrines plantarmu inprimis extra Luropam inventarum colligens; cum commentario succincto editus. - Kupfersammlung der nenesten auslïndischen Gewichse, nebst Angabe ilirer Cultur, für Gartenfrcunde. Leipzig 1827. 4.

C. L. Reichenbach. Magazin der ästretischen Botanik, oder Abbildung und Beschreibung der für die Gartencultur empfehlnngswerthen Gewächse, nebst Angabe ihrer Erzichung. Leipzig, 18\%2- Auch unter dem 'I ilel: Icones et descriptiones plantarum cultarum et colendarum, 
nddita colendiratione. Auch unter dem Titels Magaxin dex GartenBotanik etc. sind einige Hefte ausgegeben worden.

Flora exotica. Die Prachtpflanzen des Auslandes in naturgetrenen Abbildungen, herausgegeben von einer Gesellschaft von Gartenfreunden in Brüssel, mit erläuterndem Texte und Anleitung zur Cultur von Dr. H. G. L. Reichenbach. 1. Lief. Fol. 6 color. 'Tafeln, Leipzig 1830. Hofmeister. 3 fl. 36 kr.

C. L. Reichenbach. Taschenbuch für Gartenfreunde. Eine Erläuterung von 1960 Zierpflanzen, nach natürlichen Familien geordnet, und mit Nachweisungen zu ihrer Cultur begleitet. Dresten 1827. 3 fl. $36 \mathrm{kr}$.

J. G. C. Leh mann. Icones et descriptiones novarum et minus eognitarum stirpium. Fasc. V. Fol. Hamburg 1824.

Genera nova plantarum. Iconibus observationibusque illustrata. Auctore Leopoldo Trattinnick. Viennae 1825. 4.

Der erste Fascikel enthält folgende Abbildungen:

Sehrebera swictenoides Roxl. Bonplandia trifoliata, Persoonia ferruginea, Schradera capitata, Alepidea ciliaris, Thounia decandra, Cephalotus follicularis, Cyamus Nelumbo Smith, Gymnostyles anthemifolia, Brownetera aspleniifolia.

Der zueite Fascikel enthält:

Erisma floribundum, Cypselea humifusa, Sowerbeja juncea, Lightsaatia oxycoccoides, Kochia eriophora, Narthecium ossifragum, Anigosanthus flaridus, Waldsteinia gloides, Solenandria cordifolia, Joannia microphylla.

Das W'erk scheint nicht fortgesctzt worden zu sein, was man nur bedauern mufs.

Descriptiones plantarum novarum, rel minus cognitarum; auctore II. L. W endland.

In dessen und Bartlings Beitrïgen zur Botanik. 2. Heft. Göttingen 1825.

Hier sind beschrioben: Myrica ovata Wendl, Budileja glomerata W. Manulea scabra W. Mentha capensis Thunb. Cineraria geniculata Vv. Aster lanuginosus W. Baccharis cuneifolia W. Galium uncinatum Lichtenst. Bupleurum trifoliatum W. Ribes odoratum W. Ribes aureum Pursh. Eugenia australis W. Leptospermum emarginatum W. Epilobium villosum. Thunb. Diporidium atropurpureum W. D. arboreum W. Mahernia tomentosa W. Niebuhria triphylla W. Capparis capensis Thunb. Caleoides Burchell. -

Norae plantarum species: deseriptae et iconibus illustratae: anct. D. C. A. Meyer (Acta Mosquensia 'T. VII). Beschrieben und abgebildet sind: Claytonia sarmentosa, stolonifera; Ribes tubifiorum.

Literciturblätter. 2. p. 112.

Nees v. Esenbeck u. W. Sinning. Sammlung schön blühender Gewäch s e für Blumen- und Gartenfreunde, 1. Centurie Heft, 1. Fol. Düsseldorf 1826. 4 fl. $30 \mathrm{kr}$. - Ist fortgesetzt worden.

Bestimmung einiger Zierpflanzen von Bernhardi. 156 - 164. besonders über Datura.

Weimarisches Garten-Magazin. Band 2. Heft 4. 1828. pag.

Trinius plantarum novarum aut minus cognitarum, Pentas prima.

Mém. de l'Acad de Petersb. 6. p. 485. 
Plantarnm rariorum et minus cognitarum fasciculus, auctoro $x_{n-}$ dovico Griesselich, Med. Doct.

Linnaed 1830. 3. p. 411.

Sylloge plantarum novarum itemque minus cognitarum, a praestantissimis Botanicis adhuc viventibus collecta, et a Societat e regia botanica Ratishonensi edita. Cum tabula aenea. Ratisbonae, typis Viduae C. E. Brenck. 182t. Tomus secundus cum tabula lithographica 1828.

Genera und Species, deren Recht als solche unvollkommen begründet ist. Dargestellt von Dr. Griesselieh in Karlsruhe.

Geiger's Magaz. für Pharnacie. Aprilheft 1829 und durch mehrere folgende. -

C. P. Presl. Symbolae botanicae sive icones et descriptiones plantarum novarum vel minus cognitarum, Fasc. 1. Fol. Prag 1830.

IOortus Canalius seu plantarum rariorum, quae in horto botanico J. M. Com. de Canal coluntur Icones et descriptiones. Auctore J. F. Ta usch. Tom. I. Pragae 1823. Fol.

Nees r. Fsenbeck, D. C. G. et F. L. Plantarum in horto medico Bonnensi nutritarum Icones selectae. Cum 6 tab. pict. 4. maj. Etiam sub tit. Amoenitat. : botan. Bonn. Fasc. II. Bonn 1824.

Abbildungen neuer und seltener Gewächse des königl. botan. Gartens zu Berlin, nebst Beschreibungen und Anleitungen, sic zu ziehen, von H. F. Link und F. Otto etc. Berlin 1828. 8. Auch unter dem Titel:

Icones plantarum rariorum horti regii botanici Berolinensis cum dc6criptionibus et colendi ratione, auct. H. F. Link et F. Otto.

Fis sind nun 6 Hefte vorhanden. - Dieses Werk ist übrigens eine Fortsetzung der mit dem zehnten Hefte geschlossenen Abbil dungen auserlesener Gewächse, in einem verschicdenen Formut.

Descriptiones plantarum novarum, vel minus cognitarum horti botanici academici T'übingensis. Praeside Schübler. Dissert. inauguralis botanica. Auct. Schüz, Med. Dr. 'Tübing. 1825.

Index scholarum in Hamburgensium Gymnasio academico a $\mathrm{Pa}$ s:he 1828 usque ad Pascham 1829 habendarum, editus a J. G. C. Lehmanno.

Continetur his plagulis pugillus novarun quarundam plantarum in botanico Hamburgensium horto occurrentium. Hamburgi 1829. 4.

Plantas quasdam novas in horto IIamburgensi botanico cultas descripsit Lehmann, horti Director.

Linnaea 1830. 3. p. 371. Alstroemeria psittacina, Chironia serpyllifolia etc.

Amoenitates botanicae Monacenscs. Auswahl merkwïrliger Pflanzen des k. botanischen Gartens zu München mit Abbildungen und Beschreibungen, nebst Anleitung rücksichtlich ihrer Cultur von Dr. C. F. Ph. von Martius. 1. und 2. Lieferung. gr. 4. Frankfurt 1830, bei Brönner à 2 fl. $42 \mathrm{kr}$.

Horti botanici Vratislaviensis plantarum vel novarum vel minus cognitarum Manipulus. Descripsit et observationibus nec nen tabulis tribus illustravit L. C. Treviranus.

Verhandl. der k. Leopold. Akudem. der Naturforscher. Bd. 12. Abth. 1. p. 163. et 410 . 
 \\ b. F r a nz 8 s is che.}

Rapport sur les plantes rares ou nouvelles qui out fleuris dans le jardin botanique de Genèvo pendant les années 1822 et 1823 par M. De Candolle.

Mém. de las Société de phys. et d'hist. nat. de Gerève. Tom. 2 part. 2. p. 125.

Plantes rares du jardin de Genève par M. Aug. Pyr. De Candolle, avec fig. color. Genève Barbezat et Delarue. Paris Treuttel et Würz 1925.

In der zweiten Lieferung findet man unter andern Cineraria praecox, Mentha blanda, Cerastium Bicbersteinii u. s. w. Die 3 . Liefer. kam 1826 heraus.

Note sur quelques plantes observées en fleurs au mois de Janvier 1828, dans la serre de M. Saladin à Pregny, par M. De Candoll e, Prof.

Es sind: Pittosporum revolutum, Kennedia macrophylla, Astropaea Wallichii u. A. penduliflora. - Mérnoires de la Soc. do Physique de Genève T. IV. p. $85-90$.

Flore pittoresque, ou Recueil de fleurs et de fruits, peintes d'après nature, par A. Chazal, élève de M. Vanspaendonek. 2 edit. 1 livr. gr. in IV. avec 19 pl. et un frontispice gravé. Paris l'aut. rue St. Dominique d'Enter. Nro. 4 .

Sertum botanicum, ou Collection choisie des plantes les plus remarquables, par leur élégance, leur éclat ou leur utilité, par M. van Geel 1. et 2. livr. Petit in Fol. pap. velin. Prix par livr. 5 Fr. 28 6. Bruxelles 1827. De Vroom.

Collection des planches composant la botanique de J. J. Rousseau, gravées et color. d'après les desseins de J. P. R. Rédouté. Par. 8. I- XIII Livr. chacun de 5 pl. et deux feuilles de texte à 4 Fr. 50 Cent. Paris 1824.

Mlélanges botaniques ou Recueil d'observations, Mémoires et Notices sur la Botanique, par Nicolas Charles Séringe, Instituteur à l'Academic de Berne. Volumie I. 1818. Vol. II. 1826. chez l'auteur. Der Verf. lebt jetzt in Genf, als Conservator der Herbarien Decandolle's.

Choix des plus belles fleurs, prises dans différentes familles du règne végẹ́tal, de quelques branches des plus beaux fruits, groupées quelquefois et souvent animées par des insectes et des papillons; gravées et inprimées en couleurs et retouchées an pincean avec un soin, qui doit repondre de leur perfection. Par P. J. Rédouté. Paris 1928.

Es sollen 25 Lieferungen erscheinen in 4 . für 12 Francs, in fol.

24 Francs jele.

Le Guide de l'Amateur botaniste, ou Choix, description et culture des plantes étrangères, de serre et naturalisées, les plus interessantes par leur feuillage, leurs fleurs et leur odeur, par J. F. O lagnier. Paris 1827. chez Raynal. 3 France.

Loiseleur Deslongchamps. Nouvel herbier de l'Amateur, contenant la description, la culture, l'histoire et les propriétés des plantes rares et nouvelles, cultivées dans les jardina de Paris. Strash. 1830 4. et 8. Livr. 1-4. Levrault. 
B. Maund. The botanic garden, with exquiaitely coloured platen. Lond. 1825. 8. Balwin.

Ist eine Sammlung von in Grofs - Britnnnien cultivirten Pflunzen, mit illuminirten Abbildungen. Jedes Heft, das 1 Schilling kostet, enthält 4 Blumenfiguren, mit dem englischen Numen, dem systematischen nach Jussieu und Linné, Angabe des I'aterlandes, Blüthezeit, Gebrauch in der Pharmacie u. s. w.

Liste of rare plants, which hawe flowered in the Royal Botanic Garden, Edinburgh, during the last threc months. Communicated by Prof. Graham. March. 1826.

Edinburgh Philosophical Journal Nro. XXIJII. p. 353. - Eine solche Uebersicht steht noch in dem folgenden Hefte derselben Zeitschrift $p$. 171-175, wo unter andern sehr genun beschrieben sind: Conospermum acinacifolium, Epidendrum ellipticum, Fucrosia bicolor, Grevillea pubescens, Lobclia crispa, Maxillaria aromatica, Pyrethrum diversifolium, Viola hederacea.

Desoription of several new or rare plants, which have lately flowered in the neighbourhnod of Edinburgh, and chiefly in the Royal botanic Garden 10. Sept. 1829.

Alstroemeria pallida, Arabis retrofracta, Draba muricella, Eryngium comarum, Mitella pentandra, Monarda menthacfolia, Pentotemon glaucum, P. procerum, Saxifraga ferruginea, Tiarella colorans, Turritis patula, Turritis stricta. - Jameson New Edinb. philos. Journal July-Octbr. 1829. p. 34t-351. - Dergleichen oft höchst interessante Nachrichten und Beschreibungen kommen in englischen Zeitschriften öfters vor; sie können hier nicht alle namhaft gemacht werden, und es genügt, darauf aufmerksam gemacht zu haben.

Flora conspicua, by Richard Morris. Lond. 1825. Whittaker with coloured plates.

Jerlen Monat erscheint ein Heft, welches eine Auswahl von Zierpflanzen, so wie Abbildungen in--und ausländischer Bäume enthält, die zur Verschönerung europäischer Gärten dienen.

\section{d. I t a li e n is che.}

Hortus Ripulensis, seu Enumeratio plantarum, quae Ripulis coluntur, ab Aloysio Colla; additis stirpium rariorum vel nondum satis cognitarum aut forte novarum natis, descriptionibus et iconibus. Augustae Taurinorum 1824. 4. $163 \mathrm{~S}$. mit 40 Steindrücken.

Aloysii Collae illustrationes et icones rariorum stirpium, quae in ejus horto Ripulis florebant, anno 1821 , addita ad hortum ripulensem appendice $I$.

Besonders abgedruckt aus den Memorie delle reale Academie delle

Scienze di Torino. T. XXI. S.111-138. mit 7 Steindrücken.

Aloysii Colla illustrationes et icones rariorum stirpium, quac in ejus horto Ripulis florebant, anno 1825, addita ad hortum Ripulensem appendice II.

Besonders abgedruckt aus dem angeführten Memorie etc. S. 319358. mit 9 Steindrücken.

Aloys ii Colla; illustrationes et iccnes rariorum stirpium, quae in cjus horto Ripulis florebant, anno 1826, addita ad hortum ripulensem Appendice III. 
Abgedruckt aus obigen Memoric etc. T. XXXIII. S. 1-54. mit 12 Kupferstichen. - Eebor den Inhalt aller vier Schriften sehe man Linnaea 1829. $p$. 50 der Literatur.

Antonii Bartoloni Lucubrationes de Re herbaria. Bonon. 1823. 4. c. tab. aen.

Derselbe berühmte italische Botaniker giebt Nachricht von einigen neuen Pflanzen-Arten, namentlich Acacia platyacantha, Croton adanophyllum, Ipomoea fulva, I. papillosa, Myosotis strigillosa, Lysimachia decipiens. - Giorn. ligustico di Scienze, lettere ed arti Jan. 1827. p. 9 .

\section{Zweiter Abschnit.}

Be arbeitung des s y t e m s.

§. 8.

Wenn heut zu Tage von einem Pflanzensysteme die Rede ist, so unterscheidet man suar noch immer das künstliche von dem sogenannten natürlichen; allein von allen den zahlreichen künstlichen Systemen, die einst geschaffen wurden, hat sich nur lerliglich das Linnéi'sche erhalten, das bald rein, bald nach verschiedenen Ansichten abgeëndert, das bequemste Hiilfsmittel zum Auffinden der Pflanzen-Namen darbietet. Nur wenige $\boldsymbol{W}$ erke sind übrigens in der neuesten Zeit eischienen, die cine vollständige Uebersicht aller bis jetzt entdeckten Gewächse, oder eine Auswahl der bekanntesten und nutzbaren Pflanzen, nach diesem Systeme geordnet, enthielten. Fis gehören hierher aber die nachstehenden:

Martini Vahli i Enumeratio plantarum, vel ab aliis, vel ab ipжo observatorum, cum earum differentiis specificis, synonymis selectis, ef descriptionibus succinctis. Vol. I. II. Editio minoris pretii. IGottingae, apud Vandenhoeck et Ruprechit. MDCCCXXVII. 3 fi. 26 kr.

Ist nur cin neuer Titel zu dem älteren $W^{\top}$ erke, das, wie bekannt, nur einige Klassen des Systems enthält.

Caroli à Linné Equitis Systema Vegetabilium secundurn Classes, Ordines, Genera, Species. Cum characteribus, differentiis et synonymis. Editio nova, speciebus inde ad editione XV detectis aucta et locupletata. Curantibus S. J. Römer, M. D., et J. A.Sehul tes, Bajo Vol. I. Suttgardtiac sumptibus J. G. Cottae 18i'. Vol. II. 1817. Vol. III. 1818. Vol. IV. 1819. Vol. V. 1819. Vol. VI. 1820. Vol. septimi pars prima 1829; pars secunda 1830 .

Mantis s a in Vol. I et II systematis Vegetabilium, ex editione $\mathbf{J}$. $\mathbf{J}$. Römer et J. A. Schultes, cur. Jos. A. Schultes. Stuttg. 1824, 2 Vol. gr. 8. 5 thlr. Mantissa in Vol. II. Stuttg. 1826.

Ca roli Lin nei, Equitis Stellae polaris, Archiatri regii cte. Systema Vegetabilium. Editio decima scxta, curante Curtio Sprengel, equite stellae polaris et aquilae rubrae Prof. Med. et rei herb. in universit. Hal. Volumen I. Classis 1-5. Gottingae sumptibus libraiae Dieete- 
richianae. 1825. Vol. II. Cl. 1-15. 1825. Vol. III. Cl, 16-23. 1826. Voluminis IV, pars 1. CI. 21. 1827. Voluminis IV. pars 2. Curae postoriorc8. 1527.

Tentamen Supplementi nd systematis vegetabilium Linnaeani editionem decimam sextam. Auctore Antonio Sprengel, Ph. D., Gottingac 1828.

Caroli Linnaei Genera Plantarum. Editio nona, Curante $\mathbf{C u}$ tio Sprengel, Equite Stellae polaris etc. Tom I. Classis 1-13. Gottingau sumptibus Dietrichianis MDCCCXXX, 8. 4 f. $3 \mathrm{kr}$.

Carl v. Isinne's Pflanzensystem, in Aarzuge neu bearbeitet und mit den Fortachritten dieser Wisscnschaft bereichert, von B. M errem. 2 Theile, zweite Auflage. Marburg 1823.

Gemeinnutziges Handbuch der Gewüchskunde, welches mit Ausnahme der 2tsten Klasse des Lin n. Systems, die einheimischen Gewächse Deutschlands und von den ausländischen diejenigen, welche dem Arzt und Apotheker, dem Färber, Gärtner und Landwirth Nutzen bringen, enthält. Fon Dr. J. C. Mössler. Zweite Auflage, nach den neuesten Ansichten der Wissenschaft umgearbeitet, vermehrt und mit ciner Anordnung der bekannten in - und ausländischen Pflanzengattungen nach den natürlichon Verwandtschaften versehen von Dr. H. G. Ludw. Reich en bach. Altona, bei Hammerich, 1827. Erster Band. KL. 1-10. $9 \mathrm{fl}: 36 \mathrm{kr}$.

An Encyclopaedia of Plant8, comprising the Description, specific Characters, Culture, History, Application in the Arts, and every other desirable particular respecting all the Plants indigenous, cultivated in, or introduced to Britain, combining all the advantages of a Linnaean and Jussieuan Species Plantarum and Historia Plantarum, a Grammar of Botany, and a Dictionary of Botany and Vegetable Culture. The whole in English; with the Synonyms of the commoner plants in the different European and ather Languages; the scientific. Names accentuated their Etymologies explained, the Classes, Orders, and Botanical Terms illustrated by Engraving s, and with Figures of nearly Then Thouaand Species, exemplifying several individuals belonging to every Genus included in the Work. Edited by C. Loudon. IV Vol. 8. London 18\%9.

Die zahlreichen Abbildungen sind ganz kleine zwischen den Text eingedruckte Holzschnitte.

Tabellarische Uebersicht des Linneischen Pfanzen-Systems, nach Schulte bearbeitet und durch beigefügte Abbildungen der Befruchtungs-Werkzeuge möglichst versinnlicht, von Carl August L chmann. Weimar im Verlage des L. I. Comptoirs 1824. Folio.

Uebersicht des Linneischen Sexual aystema. Ein lithographirtes Blatt von Dr. Th. G. Bi s ch off. Heidelberg 1829.

Examen Classis Monneciae. Dissert. praeside Thunberg resp. J. Liden. Pars 2. resp. Bagenholm. Upsal. 1825.

Ex a men Classis Dioeciae Dissert. resp. Kiellenberg. Upsaliae 1325.

Examen Classis Polygamiae Diss. resp. E. Hedren. P، 2. resp. J. Berggren. Upenl. 18\%. 4.

Botanique, ou description familière du règne végétal, d'après la claesification de Linné. Paris 1828 in 18. 
 \\ Natürliches System.}

5. 9.

Wenn es cines Beweises bedürfte, dafs im Grunde ein natilrliches Pfanzen-System noch gar nicht bekannt ist, so würde dazu schon die einfache Bemerkung hinreichen, dafs eine Menge Schriftsteller die Pflanzen anordneten, alle angeblich nach der natürlichen Methode, obgleich sie in Hinsicht der Folgereihe der Pflanzen selbst, und der Grundsütze, die dieser zur Richtschnur dienten, auffallend von einander abueichen; indessen wird doch gerade dieses vielseitige und rege Streben nach einem idealen Ziele der Wissenschaft seljst vortheilhaft werden, so wie es nicht minder unter solchen Umständen klar wird, dafs man alle Versuche, ein natürliches System zu begründen, ohne Unterschied studiren müsse, indem ein einseitiges Beharren bei irgend einem Systeme, von wem es auch immerhin herrühren mag, dlas man für unverbesserlich ausgeben und alle übrigen verwerfen wollte, nur geeignet ist, das Fortschreiten der W'issenschaft aufzuhalten, nicht sie zu befördern.

Es gehören aber folgende neuere V'ersuche hierher.

\section{a. Schriften der Deutschen.}

G. Schübler. Uebersicht des Pflanzenreichs nach dem natürlichen Systen von Jus sieu. Tübingen 1820. grofs Folio.

Conspectus methodi plantarum naturalis. In usum auditorum scripsit D. C. J. Perleb. Friburgi Brisgoviae 1822. 4.

Auch dessen oben ang fülirtes Lehrbuch enthält zugleich eine

Uebersicht der bekanntesten Pflanzen nach sogenannten natürlichen Klassen.

Oken's Lehrbuch der Naturgeschichte. Zweiter Theil, Botanik. Zweite Abtheilung, erste Hälfte, Mark - und Stamm-Pflanzen. Jena 1825 bei Schmid. Zweite Hälfte, Blüthen - und Frucht-Pflanzen. Jena 1826.

Diese beiden Abtheilungen enthalten das System des Herrn Verf. Die erste scheint noch nicht erschienen $\approx u$ sein.

Gemailde der organischen Welt, von Jahann Gottfried Sommer, Professor am Conservatorium der Tonkunst zu Prag. Mit 1 Kupfertafel. Prag 1826. $3 \mathrm{nf} .36 \mathrm{kr}$.

Enthält eine Uebersicht des Gewächsreiches nach Oken's System.

Andeutungenzu einer natürlichen Gruppirung der Pflanzenwelt, vom Prof. Dr. Ritgen.

Schriften der Gesellschaft zur Beförderung der ges. Naturwissenschaften zu Marburg. Band II. Heft 3. p. 39-98.

Hortus regius botanicus Berolinensis, descriptus ab $H$. F. Link. Tom. primus. Berolin. 1827. 8. $384 \mathrm{~S}$.

Dieser Schrift glaubte ich hier eine Stelle einräumen $\approx u$ müssen, sie gehört keineswegs zu den Garten-Catalogen, sondern enthäl ganz vollständige Beschreibungen der Gewächse nach Familien geordnet. Dieser erste Band enthält deren nur wenige, namentlich die Gramineen und Cyperaceen.

Conspectus regni vegetabilis per gradus naturales evoluti. Tentainen auctore H. Th. L Reichenbach, Reg. Saxon. a Consil. aul. ete. Pars prima. In est Clavis herbariorum hortorumpue seu dispositio regni vegetabilis secundum Classes, Ordines, Formationes, Familias, Tribus, Gonera et Subgenera, adjecto indice lueupletissimo generuı, subgene- 
rum, synonymorum et nominum francogallicorum. Lipsiae apud Carolum Cnobloch MiDCCCXXVIII. 8.

Fin von denselben $r$ erf. angekünligtes Werk unter dem Titel: Die natürlichen Verwandschaften des Pflanzenreichs. Leipzig bei Cnobloch 1827; scheint nicht herausgekommen zu sein.

Das Pflanzenreich nach natürlichen Familien. Ein Iseitfaden beim pflanzenkundlichen Unterricht auf Schullchrer-Seminarien, höheren und niederen Bürperschulen, von Servatius Muhl, Lehrer am $k$. Preufs. Schullchrer-Siminar in Trier. Trier 1828. 54 kr.

Jus sieu's und De Candolle's natiurliche Pflanzen-Systeme, nach ihren Grundsïtzen entwickelt und mit den Pflanzen - Familien von Aghard, Batseh und Linné, so wie mit dem Linnékehen Sexualsystem verglichen. Für Forlesungen und zum Selbstunterricht, von Carl Fuhlroth, Mitglied des naturhistorischen Seminars zu Bonn. Mit einer Forrede von Dr. C. G. Nees von Esenbeck. Bonn bei Eduard Weher -1829. 8.

Uebersicht der Pflanzen-Familien, nach verschiedenen Autoren, mit Angabe der bekannten Gattungen, nebst einer kurzen Darstellung des Lin néschen Systems. Berlin 18\%9. 4. In Commission bei W. Logier.

Handbuch zur Erkennung der nutzbarsten und am häufigsten rorkommenden Gewïchse, ron Dr. II. F. Link. Firster Theil. Berlin 1829. In der Haude und Spenerschen Buchhandlung (S. J. Joseephii).

Auch unter dem Titel: Grundrifs der Kräuterkunde, zu Vorlesungen entworfen von Dr. C. L. Willdenow. Nach dessen Tode neu herausgegeben mit Zusätzen ron Dr. II. F. Link. Zweiter (jraktischer) Theil. - Enthält die Endogenen und einen Theil der Exogenen.

Systema Orbis Vegetabilium (Dissertat. inaug.) auctore Frid. Carol. Lud. Rudolphi Ratzeburgensi, Phil. Dr. A. A. L. L. M. Soc. bot. Ratisl). Sadal. Gryphiae 1930. 8. 7o s.

Der Verf. scheint sich zunächst an Oken's System zu halten, oder hatte doch dieses vorzugsweise im Auge.

Ordines naturales plantarum eorumque characteres et affinitates adjecta gencrum enumeratione, auctore Fr.'Th. Bartling, Ph. Dr., Gottingae sumptibus Dietrichianis MDCCCXXX.

Ueber das Studium der natïlichen Familien des Gewächsreiches. Von Dierbach.

G'iger's Magazin Septemberheft 1827. Januarheft 1828. Februarheft 1829. tanik.

Einige Betraehtungen über die Systemkunde in der Bo-

Botan. Zeit. 1824. II. p. 497.

Wer mag es läugnen, dafs schon aufserordentlich viel zur nüheren Frkennung des natürlichen Systems geschah? Dennoch scheint mir, läge viel W'ahres in dem Ausspruche eines bekannten deutschen Schriftstellers, der eben nicht Botaniker war:
,Statt dich, Natur! ins Herz zu fassen,
anbetungsvoll und warm, theilen sie dein Reich in Klassen, Schulgerecht, dafs Gott erbarm:"“ 
b. Schriften der Franzosen und Ilaliener.

Regni vegetabilis Systema naturale, sive Ordines, Genera et Species plantarum secundum methodi naturalis normas digestarum et descriptarum. Auctore A ug. Pyramo De Candolle. Volum. prinum, sistens Prolegomena et $O$ rdines quinque, nempe Ramunculaceas, Dilieniaceas, Magnoliaceas, Anonaceas et Menispermaceas. Parisiis sumptibus sociorum Treuttel et Würtz. 1818. Vol. II. sistens ordines sex, nempe Berberideas, Podophylleas, Nymphaeaceas, Papaveracens, Fumariaceas et Cruciferas. Parisiis 1821.

Prodromus Systematis naturalis regni vegetabilis, sive Enumeratio contracta ordinim, generum, specicrumque plantarum huc usque cognitarum, juxta methodi naturalis normas digesta; anctore $\mathbf{A}$ ug. P y ramo De Candolle. Pars prima sistens Thalamiforarum Ordines LIV. Parisiis 1524. Pars secunda sistens Calyciflorarum Ordines X. Parisiis 1825. Pars tertia sistens Calyciflorarum Ordines XXVI. Parisiis 1828. Pars quarta sistens Calyciflorarum Ordines X. Parisiis 1530.

Principes de la méthode naturelle des végétaux par M. A. F. de Jussieu. Paris et Strasbourg 1824.

Manuel d'histoire naturelle, comprenant les trois règnes de la nature, on Genera complet des animaux, des régétaux ct des ininéraux. par M. Boitard. Paris 1828.

Storia naturale de Vegetabili classificati per Familie Auct. Prof. D. A. Farin i Tom. IV. 1826. Majno.

I c on ographie des familles végétales, ou Collection de figures représentant le port, les formes et les caractères des plantes, qui penrent servir de type ponr chaque famille, avec des détails anatomiques, dessinées sur pierre par MM. S. Lamouroux, arec texte. Paris 1828. 1 Vol. in 32. avec $54 \mathrm{pl}$. Chez Bachelier. 3 Fr. $50 \mathrm{C}$.

Resumé de phytographie, ou Histoire naturelle des plantes etc. accompagnée d'une iconographie de 168 planches par J. P. Lamouroux. Paris 1828. 'Tom. I. (Acotyledonie et Monocotyledonie. Tom. II. (Dicotyledonie).

\section{c. Schriften der Engländer und Schweden.}

Robert Brown's vermischte botanische Schriften. In Verbindung mit einigen Freunden ins Dentsche übersetzt und mit Anmerkungen versehen von Dr. C. G. Ne es von Es en beck. Erster Band. Mit einer Steindrucktafel. Schmalkalden 1825. Zweiter Band. Leipzig, bei Friedrich Fleischer 1826. Dritten Bandes erste Abtheilung. Nürnberg 1827, bei Leonhard Schrag. Vierter Band 1830.

Obgleich Robert Brown's Schriften, streng genommen, nicht hierher gehören, und auch die einzelnen Abhandlungen an ihrem Orte citirt werden, so verdienen sie doch hier eine Stelle, da sie so wesentlich zur Kenntnifs der sogenannten natürlichen Methode beitrugen.

Aphorismi botanici, quas praeside C. A. Aghard pp. etc. Pars I-XVI. Lundae 1817-1826:' 8 .

Classes plantarum, quas praeside C. A. Aghard pp. etc. Pars I. Lundae 1825.

Man sehe bot. Literaturblätter I. p. 224.

A. Aghard. Conspectus regni vegetabilis, per familias et classes distributi, praemissa systematis theoria generali. 8 maj. Lipsiae V0s8 1828. 
Systema Orbis vegetabilis. Primas lineas novae constructionis, periclitatur Filia frics. Pars I. Plantao homonemenc. Lundae 1825.

\section{Specielle systematische Arbeiten.}

§. 10.

Uncrmefslich und mannichfaltig sind die Beiträge, welche die Absicht huben, cinzelne Glieder oder Abtheilungen des Geuvöhsreiches nüher zu beleuchten, sie sind es, womit bei weitem die grōste Zahl der Botaniker sich zu beschüfligen plegt, und die Nü̈zlichleit solcher Arbeiten läfst sich nicht verkennen. Alles einzeln, was geleistet wurde, hier aufzuzählen, ist auf der cinen Seite unmöglich, da mir clazu manche Mittel fehlen, und auf der andern würde dazu mehr als ein Foliant erfordert verden, und ich hutle mur die Absicht, eine kurze, Jedem zugängliche Vebersicht zu geben. Ich muste mich daher begnügen, bei jeder einzelnen Familie wenigstens cinige Nachrichten anzuführen. Selbst dieses Wenige hatte seine grofse Scluvierigkeit, $j a$ es ist, wie ich versichern darf, keine geringe Mühe, eine solche Sammlung, bei dem gänzlichen Mangel ähnlicher Torarbeiten zusammen $\approx u$ bringen.

Die Erörterungen solcher Gewächse, die einer bestimmten Gegend angehören, habe ich öfters bei den Floren derselben angeführt, und man darf daher nicht übersehen, dafs diese beiden Abschnitte sich vechıselseitig ergänzen. Bei der Anordnung der Familien folgte ich unfangs der Disposition von Richard, späterhin zog ich Bartling's kürzlich herausgekommene Methode vor; und ordnete die Familien im Ganzen nach der von diesem angenommenen Folgereihe, daher ich auch das Schema derselben mittheile.

For der Aufführung der einzelnen Gegenstände ist noch darauf aufmerksam zu machen, dafs über den Begriff von Art - Species - die Botaniker noch nichts weniger als einig sind, ja jetzt die Verschiedenheit der Meinungen auffallender wird, als dies zu Linne's Zeiten der Fall war. Man vergleiche aufser den bereits oben angeführten Aufsätzen des Regimentsurztes Griesselich, besonders Hegetschveiler's Abhandlung betitelt: Versuch ciner kritischen Aufzählung der Schweizerpflanzen, unter Anwendung der Untersuchungen über die Vielförmigkeit der Gewä̈she und zum Behuf der Reduction ihrer Arten *) (bot. Literaturbl. III. p. 502), und dessen I'ersuch über die helvetischen Arten von Rubus, nebst Bemerkungen über Species - Bildung im Allgemeinen (Denkschriften der allgem. Schweizer Gesellschaft für die gesammten Naturwissenschaften Bd. I. Abtheil. 1. p. 1-47).

Ein anderer die Systemkunde zunächst angehender Gegenstand ist dic Auswahl der Pflanzen-Namen, zumal die Benennung der Gattungen, die heut zu Tage gar sehr von Linnés so richtigen Grundsätzen abweicht. Man sehe hierüber Eв ваi historique et critique sur la Phytonomie ou nomenclature végétale, par M. F éc. Paris 1827.

\section{A c o t y l e don e n o d e r C r y tog a m e n.}

\section{§. 11 .}

Hier mögen zuvörderst einige Schriften, die sich im Allgemeinen über kryptogamische Gcwächse verbreiten, eine Stelle finden.

Ueber die Metamorphosen ciniger Cryptogamen, und über ihre animalische Organisation.

Giornale di Fisica, chimice T. VII. Bim. 4. 1821.

*) Den Aufsatz sabst sah ich bis jetzt nicht. 
Ueber mikroskopischo Vegetabilien von W. Edwards. Froriep's Notizen Bd. XIV. p. 211 in Nr. 302.

De plantarum praesertim cryptogamicarum transitu et nnalogia, Commentatio. Quam ad facultatem legendi rite adipiscendam ordini illustri Philosophorun Academiac Ruperto - Carolinae lícidelbergensis offert Theophilus G uilielmus Bisch off, Dr. Philos. Reg. Botanicor. Societ. Ratishon. Sodalis. Hcidelbergae 1825. Ex libraria J. C. B. Mohrii acadcmica.

G. W. Bischoff. Diekryptogamischen Gewächse, mit besonderer Beriicksichtigung der Flora Deutschlands und der Schweiz, organographisch, phytonomisch und systematisch bearbeitet. In 10 Licferungen mit 60 Kunfertafeln. Grol's 4. Nürnberg, bei Schrag.

I. Lief. Chareen und Equiseteen 1828. $2 \mathrm{kthl}$. $9 \mathrm{gr}$. oder $4 \mathrm{fl}$.

$12 \mathrm{kr}$. II. Rhizokarpeen und Lykopodeen 1828. $4 \mathrm{fl}$. $12 \mathrm{kr}$. III. Ophioglosseen und Farne 1829. IV. Lebermoose. $V$. Moose. IVI. Flechten. VII. Algen. VIII. IX. Pilze. X. Allgemeine Uebersicht der kryptogam. Gewächse, zugleich als Einleitung zum Ganzen, und ein genaues Register.

Considérations sur quelques végétanx du dernicr ordre ou Additions aux fragmens do Philosophie botunique, par A. L. Marquis. Rouend 1826.

\section{Erst e $S$ e c $t$ ion. Zellenpflanzen.}

§. 12.

Nach Bartling, dessen Eintheilungsart zunächst an die von Fries sich schliefst, werden diese niedern Gewuichse folgendermajsen angeordnet.

A. Homonemea.

Classis I. Fungi. Ordo 1. Coniomycetes. 2. Gasteromycetes. 3. Pyrenomyeetes. 4. Hymenomycetes.

Classis II. Lichenes. 0. 5. Coniothalami. 6. Hymenothalami. 7. Pyrenothalami.

Classis IIl. Algae. 0. 8. Nostochinae. 9. Confervaceae. 10. Florideae. 11. Fucaceae.

B. Heteronemea.

Classi is IV. Musei. 0. 12. Hepaticae. 13. Bryaceae.

Da aber die Gattungen dieser Ordnungen nicht speeiell angezeigt wurden, so zog ich es vor, die ältere Abtheilungsart dieser Gewüchse zu befolgen.

\section{Algen.}

Algae. Hydrophyta.

Um die Kenntnifs dieser niederen vegetabilischen Gebilde haben sich, nebst Andern, die Herren Bory de Saint Vincent, Bonnemaison, Aghard, Mertexs, Lyngbye, Jürgens, Lamourouxu. s. w. verdient gemacht. - 
$Z_{u}$ den neueren Arbeiten über diese Familie und ihre einzelnen Theile gehören unter andern die:

Observationes in structuram Algarum desverlebten Eysenh ardt, einst Prof. der Botanik in Königsberg; sie bezichen sich auf Fueus fastigiatus, Chordaria Filum, Conferva mutabilis und Nostochium muscorum.

Linnaea 1828. p. $147 u$. $d . f$.

Ueber krystallinische Bildungen in Algen theilte Herr Prof. $A g h$ ard in Lund mehrere Beobachtungen mit, die besonders durch Se hüb Ie r's Entdeckung des Iydrurus crystallophorus veranlafst wurden. Bot. Zeit. 18\%8. II. p. 577 u. d. f.

Examen de la question de savoir, si les crystattelles ou éponges d'eau douce sont des végétaux, par M. B. de la Pylace.

Annales de la Soc. Linn. de Paris. Sept. 1826. p. 402.

Wird affirmativ beantwortet, wie denn schon Linné und Vaucher diese Geschöpfe zu den Algen zählten. Man vergleiche bot. Literaturblätter I. p. 235.

Senator Cassebecr zu Gelnhausen lehrte die leichteste Methode, unmittelbar aus der reifen Moosbüchse die Conferve darzustellèn.

Isis März 1826. p. 289.

Bemerkenswerth ist folgende Schrift:

Experiences microscopiques et physiologiques stru une éspèce de conferve marine, par Benj. Gaillon. Rouen 1823.

Damit vergleiche man: Kritik der mikroskopischen und physiologischen Untersuchungen des Herm Gaillon über Conferya comoides Dillw rom Pastor Lyngbye. - Tidskrift for Naturvidenskab 1821. Nro. 10. p. 36.

Sehr interessant sind die Algalogischen Bemerkungen des Herrn Prosector Dr. Leible in in Wïrzburg.

Bot. Zeit. 1830. I. p. 305 u. d. $f$.

Ueber den Nutzen der Conferven in der Haushaltung der Natur theilte $H$ off mann $B$ ang seine Ansichten mit.

Abhandlungen der Societät der Wissenschaften von Kopenhagen

Bd. II. 1826. p. 209.

Ueber Conferva Aegagropila theilte F. Rudolphi in Triest einige interessante Bemerkungen mit.

Bot. Zeit. 1827. 1. p. 183.

Ueber das Genus Spirogyra Link, und über die Bewegungen und Metamorphose der S. princeps insbesondere, schrieb ausführlich Dr. Meyen.

Linnaea 1827. p. 410 .

Die Metamorphose der Ectosperma clavata Vaucher lehrte Franz Unger (Nova acta Acad. Caes. Leopold. Tom. X. p. 2). Man kann da lernen, wie die Pflanze zum Thiere wird und vice versa! Man vergleiche damit folgenden Aufsatz:

Sur les Metamorphoses et le mouvement des corps reproducteurs de direrses Conferves, et particulièrement de l'Eetosperma clavata de Vaucher.

Annales des Sc. nat. Avril 1828. p. 428 - 444. - Froriep's Notizen

22. p. 129. Nro. 471. - Siehe auch botan. Zeit. 1830. II. p. 569.

Ueber die Priestleysche grüne Materie, so wie über dio Metamorphose des Protococcus, schrieb Dr. J. Me yen.

Linnaea 1827. p. 388. 
Einige Bemerkungen über den jetzigen $\mathrm{Z}$ ustand der Algologie, liest man in der botan. Zeit. 1826. I. p. 1 อెอ.

Kritische Beitringe zum Studium der Süls - Wasser - Algen theilte Herr Dr. M e yer in Bonn mit (Bot. Zeit. 1827. II. p. 705 u.d.f.), wogegen die Herren $\mathrm{Aghard}$ (Ibid. 1829. I. p. 193-209) und $\mathrm{R}$ ud ol ph i (Ibid. 3533) mehrere Einwendungen machten.

Die Verwandschaft der Algen mit den Pilzen leuclitet auch aus nachstehendem Aufsatze ein:

Illustration du genre Inoconia dans la famille des Algues; par Mademoiselle Lil, ert. Annales de la Soc. Linn. de Paris. Sept. 1826. p. 402.

Die Inoconia soll der Trentepohlia am nächsten stehen, uelche von Martius, der letztere Gattung aufstellte, zu den Pilzen gezühlt wurde. Sehr nerkwürdig ist der Hydrurus crystall ophorus, eine neue Süfswasser-Alge Deutschlands, von Hrn. Prof. Schübler in Tübingen.

Bot. Zeit. 1828. I. p. 65 u. d. f.

Bemerkungen üher Cddium tomentosum findet man in dem Edinburgh Journal of Science. Jul. $18 \% 5$.

Vorzüglich interessant ist aber der:

Essai monographique sur les Oecillaires, par M. Bory de Saint - Vincent.

Bulletin des Sc. natur. Octobre 1827. p. 241.

Auszug aus des verstorbenen Lamouroux Abhandlung über die Seepflanzen, von Mirbel. Der Akademie der Wisscnschaften vorgelesen am 21. Febr. 1825.

Froriep's Notizen Nro. 254 oder Nro. 12 des 12. Bandes.

Rapport sur le Mémoire de M. Lamonroux intitulé: De la Geographie Botanique marine par M. Mirbel.

Annales des Sc. naturelles Juin 1825. p. 191.

Mémoire sur la Géographie des plantes marines, par M. Lamouroux.

Annales des Sc. naturelles. Janvier 1826. p. $60-82$.

Théophile Bonnemaison. Essai sur les Hydrophytes loculées (non articulées) de la famille des Epitermées et des Ceramiées.

Mémoires du Museum. T. XVI. p. 49 etc.

Seinaja. Alyarum marinarum novum genus auctore Barone Ant. Bivona. Palermo 1S22.

Resumé methodique des classifications des Thalassiophytes, par B. Gaillon. Strasbourg 1825.

Fixtr. du Dict. des Sc. natur. Vol. 53.

Rapport de M.M. Mirbel et Desfontaines sur le travail de M. Despréaux, intitulé Essai sur les Laminaires des cótes de la Normandie, lue à l'Academic des Sciences, le 29. Oct. 1827.

le Globe I. Novemb. 1827.

Joannis Targioni Tozzetti Catalogus vegretabilium marinorum musei sui, opus posthumum ad secundam partem novorum generum plantarum celcberrimi Petri Antonii Micheli inserviens, cum notis Octaviani Targioni 'Tozzetti, Joannis filii, botanices et agriculturae Professoris. Florentiae 1826. Fol.

Siehe bot. Literaturblätter II. p. 323. 
Ueber Valonin intricata Aghard, von Iferrn v. Martono in Stuttgart mit Ablild.

Bot. Zeit. 1830. II. p. 681.

Beobachtungen über Fucus vesiculosus L. Aus den hinterlassenen Papieren des C. W. Fys enhardt, weiland der Botanik Prof. zu Königsberg, sind aufgezeichnet in der Linnaea 1828. p. 279) u. $d . f$.

Einen Bericht über versehiedene Fucus-Arten, welcheDr. Hein rieh M erten a auf ciner Entdeckungreise am Bord des russ. Sehiffen Siniavin fand, theilte dessen Vater Prof. Mertens in Bremen mit. Linnaea 182!). $p .43$ u. $d . f$.

Ueber einen Fueus, den man vegetirend in dem Magen eines Cabliau fand, steht ein Bericht in dem Messager des Sciences et arts 1827. 1828. p. 386.

Die Aufzählung einiger in den östreichischen Ländern gefundenen neuen Gattungen und Arten vom Algen, nebst ihrer Diagnostik und beigefügten Bemerkungen, lieferte IIr. Prof. Aghard in Lund. Bot. Zeit. 1827. II. p. 625 u. d. f.

F. L. Nace a ri Algologia adriatica. Bologna 1830. 4. (Viennac Volko in Comm.)

Algae Britannicae, or Description of the marine and other inarticulated Plants of the British Islands belonging to the Order Algae; with Plates illustrative of the Genera. By Robert Hayne Greville. Edinburg 1830. 8.

I cones Al garum europa carum. Représentation d'Algues européennes suivi de celle des éspèces exotiques les plus remarquables recemment découvertes, publiée par C. A. Aghard Prof. ete. Livraison 1 - 3. Nro. 1-30. Leipsic. 1828 11. 18\%9. 8.

Siehe Linnaea 1830. III. p. 86 der Literatur.

C. A. Aghard, Prof. Lundensi s ete. Species Algarum rite cognitae, cum synonymis, differentiis specificis et descriptionibus succinctis. Vol. prinum. Gryphiswaldiae 18ะ3.

Jenes eigenthümliche Gebilde, das Linné Tremella Nostoc nannte, und das man bald für ein animalisches Product, bald, wie der verewigte Märklin, für eine meteorische, aus der Luft herabfallende Materie hielt, ist auch mehrfach besprochen worden. Man sehe deshalb:

Cassini. Zweifel über den Ursprung und die Natur des Nostoc. Im zweiten Band der Opuscules phytologiques.

$$
\text { Isis Band II. p. } 657 .
$$

Ein anonymer Schriftsteller (bot. Zeit. 1825. II. p. 493) scheint Märklin's Ansicht zu theilen; v. Voith in Amberg belcuchtete diese Sache nüher, und stimmt mehr für ihre vegetabilische Natur (daselbst p. 682. 1828. II. p. 672. 1830. I. p. 239).

Tremella meteorica Persoon ist nach Meyen synonym mit Actinomice Horkelii, eine neue Pilz-Art, deren unten gedacht wird, auch gehört dahin die sogenannte Sternschnuppen-Materie, die Herr Prof. Zenueck aus Hohenheim für ein animalisches von Schnecken herrührendes Erzeugnifs hielt:

Isis. Jahrgang 1830. p. 188. - Vergl. auch die früheren Untersuchungen von Brandes und Schwabe in Schweigger's Journal; und von Buchner in dessen Repertorium. 
Einige Nostochinac beschrieb Herr Aghard; namentlich Nostoch molle (daselbst 1827. II. p. 629).

Der rothe Schnee der Polurlünder, und einige andere auf glciche Weise von organischen Kügelchen gefäibte Gegenstünde, sind in den letzten Jahren vielfältig untersucht uorden. Einige literarische Nachweisungen dürften deshalb angenehm sein. Finer der schätzbarsten Aufsätze ist der des Herrn Prof. Dr. Kunze in Leipzig:

Einige Bemerkungen über den rothen Schnee. Bot. Zeit. 1825. II. p. 449 u. d. f.

Ueber die Natur des rothen Schnees von $\mathbf{A}$ ghard. Monthly Mag. Dec. 1824. Hier wirl nachgeuiesen, dafs Wrangel die rothe Matcrie als eine Flechte, Sowerly als einen Pilz (Uredo nivalis) beschrieb. Aghard aber, der die von Capit. Parry auf seiner Polar-Reise gefundene Materie untersuchen konnte, bringt sie zu den Algen, und nennt sie Protococeus nivalis.

Trommsd. Journal der Phurm. Bd.IX. Nio. 2 p. 146. - Geiger's Magazin Mai 1825. p. 150.

Ueber den in der Polarzone gefundenen rothen Schnee von Dr. C. A. Aghaird.

Verhanill. der kais. Leopold. Carol. Akadem. der Naturforsclier Band XII. 2. Abtheil. p. 735 .

Ueber den rothen Schnec, oder über die Sphaerellanivalis von $S$. C. Sommerfelt.

Magazin for Naturuilenskab. Ann. 1824. Cah. 4. p. 249.

Des differentes causes, qui colorent la neige en rouge. Bibl. univers. Octob. 1829. p. 172.

Ein besonders lehrreicher Aufsatz! Sa ussure fand rothen Schnee bereits auf den Alpen, "Vrangel im nördlichen Schweden u. s. $w$. Die rothen Kügelchen erhielten verschiedene Namen, und wurden in ganz verschiedene Abtheilungen gebracht, sic heifsen Protococcus chermesinus, Palmella nivalis, Lepraria chermesina u. s. w. Aber auch thierische Gebilde können zur rothen Fürbung. Ferculassung werden.

Analyse de la neige rouge du Pole, par M.M. Macaire Prinsep et Marcet.

Mémoires de la Soc. de Phys. et d'hist. nat. de Gencive. T. Ir. P. II. p. 185. - Bibl. univers. Dec. 1828. p. 290 - 293.

Nachrichten des Kapitains Parry rondem rothen Schnee.

Nouvelles Annales du Voyages. Avril 1828. p. 87.

Man vergleiche besonders noch Nees $v$. Fs enbeck in Robert Brown's vermischten botanischen Schriften Bd.1. p. 571 u. d. f.

Notices sur les diverses causes de la coloration de la neige et de la glace.

Annales des Sc. natur. Juin 1829. p. 212. - Froriep's Notizen Bd. XXVI. Nro. 551. p. 5. - Siehe auch botan. Literaturbl. 4. p. 135. - Nan sehe auch Dr. Unger in der Flora 1830. II. p. 782.

Beobachtungen über die Natur des sogenannten Blutregens; von A. F. Wi g mann, privatis. Apotheker in Braunschweig.

Kastner's Archiv für die gesammte Naturlehre Bd. VI. Heft 3. p. $375-381$.

Notiz über eine Materie, welche den Morat-See im Frühjahr 1825 roth fărbte, von Prof. Decandolle. 
Mínoires de la Soc. de Phys. et dhist. nat. de Genève. Tom. III. P. II. - Bibl. univers. Tom. III. p. 315. - Heusinger Zeilschrift für die orgmische Physik. Bd. I. Heft 1. Juli 1827. p. 70-79.

Die Ersache der rothen Fürbung sucht Decandolle in einer Alge, die er Oscillatoria rubescens, nennt. Heusinger redet von einer Ulva oder Tremel!a. - Man sehe hierüber auch Dr. Trechsel in dem Journal de Chimie medicale. Dec. 1827 und in Froriep's Notizen Bd.XIX.p. 282.

Ueber das $R$ oth werden der Speisen, nach Beobachtungen rom Ilerrn Dr. Sette, mitgretheilt rom Dr. Pauls, Regierungsrath in Coblenz, mit Nachtrag von Nees r. Fisenbeek und Zusammenstellung analoger Thatsachen von Dr. Seh weigerer-Seidel.

Schweiger's Jahrb. der Chemie und Physil 1827. Heft 8. p. 3 ध $u$. $d . f$.

i) Materie, wovon hier die Rede ist, wird Zongalact in a imetros a grenannt. - Man sieht, dafs gar verschiedene Gegenstände bisueilen cine rothe Furbe annehmen, deren Ursprung ungevifs und rïthselhaft ist; viellicht ist es deshalb gut, hier noch hinzuzufügen, dafs nach einer, in der Bibliothek der neuesten W' eltkunde von Malten (Aarau 1830. 4. Th. S. 238) enthaltene Mittheilung, der rothe Schnee von Fxcrementen kleiner I ögel (Alca minor) herkommen soll, die myriadenweise herum fliegen!

Mémoire sur un régétal microscopique d'un nouveau genre, proposé sous le nom de Crucigenia, par Mr. Morren à Gand.

Annales des Sc. natur. Aout. 1830 p. 404.

- Die cigentliche Stelle dieser neuen Gattung ist noch nicht gehörig ausgemittelt, doch dürfte sie $w o h$ zu den Algen gehörcn.

Confervae mucoroides varietas, ein dem Zucker sehr schädliches Gebilde, untersucht ron van Dyk und ran Beck in Uetrecht.

Siehe Linnaea 5. p. 188 Lit. - Brande 8 Archiv XXXVII.

\section{Schwämme oder Pilze.}

Fungi. Myceles.

$\boldsymbol{U} m$ diese zalelreiche und höchst vielgestaltige Pflanzenfamilie haben sich Persoon, Fries, Link, Nees von Esenbeck und viele.Indere grofse Verdienste erworben, überhaupt ist sehr viel für sie in neueren Zeiten gethan worden, wie schon die nachstehenden kurzen literarischen Nachweisungen bezeugen.

Systema mycologicum, sistens Fungorum ordines, genera et species hue usque cognitas, quas ad normam methodi naturalis determinavit, disposuit atque descripsit Elias Fries. Volumen I. Gryphiswaldiae sumptibus Ernesti Mauritii 1821. Volum. secundi Sectio prima Lundae 1822. ex officina Berlingiana. Sectio altera. Gryphiswald. 18³. Volum. III. (Sect. prior) 1829.

El. Fries. Élenchus Fungorum, sistens Commentarium in systema mycologicum. Vol. I. 1828. Pr. 1 thl. 4 gr. Vol. II. 151. S. Gryphiswaldiae. logicum.

Finthilt Nachtrüge und Verbesserungen zu dem Systema myen-

C. H. Persoon. Mycologia europaea, seu completa omnium fungorum in variis Europae regionibus detectorum enumeratio, methodo naturali dispos. ete. Erlang. Seet. 1. c. tab. XX. color. 1822. Sect. II. c. tab. X. color. 1825.5 thl. $8 \mathrm{gr}$. 
Agaricos synonymas in Personii Mycologia europaea et systcmate suo mycologico reconciliat Elias Fries.

Linnaea. T. $V$. p. 689 et seq.

Turquier de Longehamp. Concordances de Persoon (Synopsis methodica fungorum) avec De Candolle (Flore française) et des figures des champignons de Bulliard, arec la nomenclature de Fries (Systema mycologicum) I. et II. Vol. Paris 1826. 6 Bogen. 8.

Adolphe Brongniart. Fssai d'une classification naturelle des champignons, on tableau methodique des genres rapportés jusqu'a présent à cette famille. Paris chez F. G. Levrault, rue de la Harpe Nro. 81. et rue des Juifs Nro. 33 à Strasbourg 1825. 99 Seiten 8. und acht vortreffliche Kupfertufeln. Preis $3 \mathrm{fl} .18 \mathrm{kr}$.

NB. Ist auch im 23. Bande des Dictionnaire des Sciences naturelles enthalten.

P. S. Cordier Guide de l'amateur des Champignons, ou Précis de l'histoire des Champignons alimentaires, vénéneux et employés dans les arts, qui croissent sur le sol de la France. Paris 1826. avec planches color.

J. B. C. Letellier. Histoire et description des Champignons alimentaires et vénéneux, qui croissent aux environs de Paris, précédes des principes de Botanique indispensables à leur étude et suivis de planches réprésentant plus de 100 éspèces. Paris 1826. 6 Francs.

M. E. Descourtils. Des Champignons comestibles, suspects et rénéneux, avec l'indication des moyens à employer pour neutraliser les effets des éspèces nuisibles. Paris 1827. 8. avec planches.

Beschreibung der efobaren $\mathrm{Sch}$ wämme von Grofshrittannien, ron R. Kaye Greville.

Mem. of the Wern. Soc. Edinburgh. T. IV. p. 339.

Diese efsbaren Schwämme der brittischen Inseln sind: 1. 'Iuber cibarium Bull. 2. Tuber moschatum Bull. 3. Tuber album Bull. 4. Amanita cae6area Pers. 5. Agaricus procerus Scopoli. 6. A. campestris L. 7. A. edulis Bull. 8. A. orcades oder A. tortilis Decand. 9. A. odorus Bull. 10. A. eburnens Bull. 11. A. ulmarius Bull. 12. A. ostreatus Jacquin Flor. Austr. 13. A. violaceus L. 14. A. piperatus Scopoli. 15. A. acris Bolton. 16. A. deliciosus L. 17. Cantharellus cibarius Fries. 18. Boletus edulis Bull. 19. B. scaber Bull. 20. Fistulina hepatica With. Bot. Arr. ed. 6. V. 4. p. 321 oder F. buglossoides Bull. 21. Hydnum repandum L. 22. Clavaria coralloides. 23. C. cinerea Bull. 24. Morchella esculenta Pers. 25. Helvella Mitra L.

Beschreibung der Milchblätter-Schwämme (Agarici lactescentes scriptorum) im Grofsherzogthum Baden und dessen nächsten Umgebungen, als Beitrag zur Toxicologie. Von C. C. Gmelin.

Geiger's Magazin für Pharmacie. Bd. IX. p. 1-29. Ist auch

in besonderem Abdrucke zu haben. Karlsruhe 1825, bei Mïller.

Eclogac fungorum, praecipue ex herbariis Germanorum descriptorum ab Elia Fries cum 2 tabul. Linnaea 1830. 4. $p .498$. $d$. $f$.

Ignazio Pizzogalli. Saggio sui Funghi e descrizione delle spezie comprese nella Mucheta logia o raccolta di figure in cera. Milano 1825. Man sehe auch Biblioteca italiana. Juli 1825.

Carol. Vittodini Tentamen mycologicum seu Amanitarum illustratio. Mediolani 1826. 4. Mit einer Abbildung der Amanita Vittadina Moretti. Es ist eine medicinische Inaugural-Dissertation, die in Pavia vertheidigt wurde. 
D. Giuseppe Bergamaschi Osacrazioni mycologiehe ed enumerazione storica di tutti i funghi della provincia pavede.

Biblioteca italiana. Tomi XXIII. XXX. XXXI. XXXII. XXXII. und $\mathrm{XXXVII.}$

Giovanni Zantedesehi Descrizione dei funghi della provincia bresciana.

Giornale di fisica etc. Pavia 1824. Bimestre 2a-5to.

V. Briganti. De fungis rarioribus regni Neapolitani historia, picturis ad naturam ductis illustrata. Neapol. 18\%4.

Description de deux champignons nouveaux dćcourerts et dessinées par M. Louis de Blondeau; suivie d'observations et de la création d'un nouveau genre; Gyrocephalus. Paris 1824. 8. avec une planche.

(Prospectus) Fungorum Species novis iconilus norisque descriptionibus illustratae. Pars I. Fungi carnosi. Cum pluribus mygologis editum est Eduardus Sehmalz, D. Philos., Med. etc. Accedunt tabulate II coloratae. (Fistulina hepatica Fr. Agaricus volemus Fr.) Lipsiac 1827. 4 .

Es ist schade, lafs das Werk, welches wirklich sehr viel versprach, wie es scheint, bis jetzt nicht zur Ausführung kam, und wahrscheinlich nicht gehörig unterstüt zt wurde.

Fungorum javanicorum Prodromus C. G. Nees ab Esenbeck et T. F. L. Nees ab Esenbeck fratrum, ad Christ. Godofr. Ehrenberg Litterae, quas manuseriptarum loco inprimendas curaverunt sumptibus propriis. Bonnae 1821. Fol. max. cum talsula aenea.

Man sehe auch: Verhandl. der $k$. Leopold. Carolin. Akademie Bd. XIII. 1. Abtheil. p. 9, wo

Fungi Javanici editi conjunctis studiis et opera C. Blumii et Dr. T. F. L. Nees ab Esenbeck.

De Polyporo Pisachapaxi, singulorum fungorum Javanicorum specie, Nees ab Escnbeck fratrum ad C. G. Ehrenberg literae. Daselbst Bd. XIII. 1. Abtheil. p. 1.

Fungorum novorum et descriptorum illustrationes publicat $D$. F. L. de Schlechtendal.

Linnaea 1826. p. 235 und 604.

Plantarum vel novarum vel minus cognitarum descriptiones. Auctore Rudolphi. Decas prima. Fungi. Linnaea 1829. I. p. 115. Decas secunda. Heft 3. p. 387. tertia 4. p. 509.

Observationes mycologicae in species fungorum tam novas quam male cognitas, auctore Francisco Junghahnio, Med. Stud.

Linnaea 1830. III. p. 388 u. $d . f$.

A c count of a new plant of the Gastromycous order of Fungi (Encrthenema) by J. E. Boumann.

Aus den Transactions of the Linnaean Society of Lond. Yol. XII. in der Linnaea 5. p. 167 Lit.

Note relative à l'action des Champignons sur l'air et l'eau, par M. F. Marcet.

Annales de Chim. et de Physique Mars 1829. p. 318. - Der $V$ Vrf. hält dafür, dafs die Pilze Wasser einsaugen, das Oxygen desselben durch die Vegetation sich aneignen, das Hydrogen aber absondern. 
Mémoiresur les Bolets bleuissans par M. S. Macaire. Mém. de la Soc. d’hist. nat. de Genève. Tom.I. p. 2. p. 289.

Enumeratio Hymenomycetum pileaforum Marchiae Brandenburgicae nondum in Floris nostratibus nominatorum, cum observationibus incognitas et novorum descriptionibus. Auctore $\mathbf{L}$ a s ch.

Linnaea 1829. Heft 4. p. 518 u. d. f. - Enthült Beschreibung einiger Cantharelli und Merulii besonders aber Arten von Agaricus, die in der Gegend von Driessen gesammelt wurden.

Note sur l'Agaricus tubaeformis de Schaefer, par Alphorise Decandolle.

Isis 1826. Heft 2. p. 182-185.

Observations sur l'Agaricus pilosus de Hudson; par M. L. de Brondeau.

Annales de la Soc. Linn. de Paris. Sept. 1826. p. 413.

Sur l'A garicus radians, éspèce nourelle, et sur le Lycoperdum radiatum, par B. H. Desmaziè res.

Annales des Sc. natur. Fevr. 1828. p. 206-217. - Agaricus radiatus Sowerby ist Agaricus coprinus im jugendlichen Zustande, wo ihn Des ma z. A. radians nennt.

Abhandlung über den Hausschwamm, nebst Beschreibung eines ron dem Herrn Oberamtmann Castner zu' Nepenitz erfundenen zuverliissigen Mittels gegen denselben, verfafst und herausgegeben von E. W. Bourwieg, Königl. Hofrathe etc. Mit 2 lithographirten Abbildungen. Stettin 1827. 54 kr. 2. Aufl. 1828. 45 kr.

Observations sur une varieté remarquable de Merulius tremellus de Persoon; par N. Louis de Brondeau.

Annules de la Soc. Linn. de Paris. Sept.1825.

Description d'une nourelle éspèce d'Agaric, le Pleuropus eleuterophyllus ; par S. H. Leveillé.

Annal. de la Soc. Linn. de Paris. Sept. 1825.

Note sur le genre Dictyophora, dans la famille des champignons, et description d'une nouvelle éspèce provenant de l'ile de Java; par: M. Liv eillé.

Annales de la Soc. Linn. de Paris. Nov. 1826. p. 499. - Diese neue Art ist Dictyophora campanulata, man sehe bot. Literaturbl. II. $p$. 126.

Ueber den Ṕhallus impudicus sehe man Cassini im 2. Bande seiner Opuscules phytologiques. Fine Beschreibung dieses sonderbaren Schuammes lieferte auch Herr G. J. Roubieu in den Annales de la Soc. Linn. de Paris. Vol. . . p. 495. Siehe bot. Literaturbl. I. p. 239.

Eine neue Morchel-Art beschricb Herr Prof. Kromblolz in Prag, unter dem Namen Morchella bohemica.

Monatsschrift der Gesellschaft des vaterländischen Museums in Böhmen. Zweiter Jahrgang Juni 1828. p. 478 u. d.f. mit Abbildungen.

Notice sur deux Cryptogames peu connues et nouvelles pour la Flore française, par M. Lé on-Dufour.

Helotium hirsutum und Triblidium hysterinum. - Annales des

Sc. natur. Mars 1828. p. 319 - 322. 
Description d'un nouveau genre de Champignons nomué Desmaxiarella, par Marie Anne Libert.

Annales des Sc. natur. Mai 1829. p. 82. - Dic neue Gattung steht der Peziza nahe.

Monographie du genre Naemaspora des anteurs modernes, et du genre Libertella, Desmaz; par M. J. 13. Desmazières.

Annales des Sc. nat. Mars 1830. p. 269.

Notice sur les Lycoperdons de Linné et sur une éspèce de Carpobolus (Micheli) genre à ajouter à la Flore française; par M. D e smazières.

Annales de la Soc. Linn. de Paris. Mars 1825. zières.

Ueber das Lycoperdon radiatum Sowerby's, von Desma-

Siehe botan. Literaturbl. II. $p$. 334.

Note sur le Lycoperdon fulvum.

L'Ami des champs. Janv. 1829. p. 28.

Notice by M. R. Spittal of a specimen of Bovista giganten, remarkable for its great size.

Jameson New Edinb. philos. Journal. Jul. - Oct. 1829. p. 378.

Mémoiresur le genre Pilobolus; par M. J. II. Leveillé. Annales de la Soc. Linn. de Paris. Junv. 1826. p. 622.

Notice sur le Pilobolus crystallinus; par M. Durieu de Maissonueune.

Bull. de la Soc. Linn. de Bordeaux. II. livr. Juillet 1826. Annales des Sc. natur. Oct. 1826. p. 221. - Bot. Literaturbl. II. p. 335 .

Note sur le Pilobolus crystallinus; par M. Gachet.

Bullet. d'hist. nat. de la Soc. Linn. de Bordeaux. T. II. Aoñt 1828. p. 159. - Der Verf. spricht besonders von der eigenen Krümmung des Stiels.

Note sur le genre Pilobole, et sur une nouvelle éspèce découlverte par C. Montagne, D. M. Lyon 1829.

Description d'une nouvelle éspèce de Carpobolus (C. impatiens); par M. B oudier.

Annales de la Soc. Linn. de Paris. Nov. 1825. p. 556.

Phisiology of the Gyropodinm coccineum; by the Rev. Edward Hitelicoek.

Benjamin Silliman American Journal of Science and arts. Vol. IX.

Nro. 1. February 1825. p. 56-60. - Nebst einer illuminirten Abbildung des G. coccineum, woran man die Entwickelungsstufen sieht. Nach Sprengel ist es übrigens nichts anderes, als Mitremyces lutescens Schweinitz.

Observations sur le genre Asteroma et description de deux éspèces appartenant à ce genre; par Mlle. Lib ert.

Annales de la Soc. Linn. de Paris. Sept. 1826. p. 404. - Siche

bot. Literaturbl. I. p. 233 . zières.

Note sur le Sclerotinm stercorarium; par M. DesmaAnnales des Sc. natur. Févr. 1827. p. 145. 
Hall.

Beschryving ran Rhizomorpha cingens; door H. C. van

Bydragen tot de Naiuurkundige Wetensschappen T. II. p. 222. Derselbe beschrieb überhaupt die niederländischen Arten von Rhizomorpha, in derselben Zeitschrift 1826. Bd. I. p. 66. Nro. 1.

Rhizomorphen in den zartesten Klüften des Gesteins und der Steinkohle; mitgetheilt von $\mathrm{Nocg}$ gerath und $\mathrm{Necs}$ v. Esenbeck.

p. 875.

Nova Acta physico-med. Acad. Caesar. Leopold. Tom. XII. P. 2.

Turpin. Organographie végétale. Observations microscopiques sur l'organisation tissulaire, l'accroissement et le mode de reproduction de la Truffe comestible, comparée aux tisans, à la production de la Globuline, et de tous les corps reproducteurs des autres végétau.

Mémoires du Museum. Tom. $\boldsymbol{X} \boldsymbol{V} . \boldsymbol{p}$. $333-376$.

Rapport sur un Mémoire de M. 'Turpin, ayant pour objet l'organisation et la reproduction de la Truffe comestible, par MM. M irbel et Cassini

Annales des Sc. naturelles. T. XII. Oct. 182\%. p. $209-215$.

Histoire générale des Hy poxylons, au Description des genres et des éspèces, qui forment cette grande famille des végétaux, par F. Chevallier. Paris 182.

Das Ganze soll 20 Lieferungen, und jede derselben fünf Kupfer, nebst zwei Bogen Text enthalten.

Description of a $\mathrm{number}$ of new American species of Sphaeriae. By Lewis D. de Schweinitz.

Journal of the Academy of nat. sc. of Philadelphia. Vol. V. 1825.

Part. 1. p. 3. - Siche Linnaea 1829. 1. p. 44. der Literatur.

Beschreibung einer neuen Gattung (Erponema) aus der Ordnung der Hypoxyla.

Cassini im zweiten Bande der Opuscules phytologiques.

Die Gattung Phragmidium und Puccinia Potentillae, in Beaug auf Bildungsgesetz erläutert von $K$. W. Eysenhardt.

Linncea 1828. p. $84 u$. $d$. $f$.

Zur Entwickelungs-Geschichte von Puccinia Rosae und Rubi. Auszug eines Schreibens des Herrn Apotheker Schwabe in Dessau.

Daselbst p. 277.

Note sur le Pucinia Graminis par M. Gachet.

Bullet. d'hist. nat. de la Soc. Linn. de Bordeaux T. II. Dec. 1828. p. 211. - Herr G. zeigt den Unterschied zwischen Puccinia arundinacea und P. graminis, die Decandolle als Varietäten zusammen gebracht hatte.

Somme rfelt beschreibt drei neue Cryptogamen im Magazin for Naturvidenskaberne 1827. Heft 1. p. 171.

Auf Salix phylicaefolia $=$ Caenangium difforme.

Auf trocknen Blättern und Stengeln $=$ Physarum vornum.

Auf Aconitum septentrionale etc. = Circinatrichum rufum.

Trachsel über Blatt- und andere Schmarotzer - Pilze.

Botan. Zeit. 1830, I. p. 145.

Neue Art von Rost an dem Getreide, vom Prof. Fries. Es ist Accidium corruscans pscudo-peridiis laxis membranaceis elongatis congestis albidis, sporidiis globosis aureis. 
Dieser Schmarotzer ist sehr schüllich, grcift die jungen IIalme an und hindert den Wachsthum. - Physiograph. Suclskup. aursberaettelse. Lund. 1825. p. 92.

Description de deux nouvelles éspèces dUredinces par M. Baucher. (Uredo Portulacae und Uredo Tetragoniae).

Annales de la Soc. Linn. de Paris. Nov. 18\%5. p. 555.

A bhandlung über das Mutterkorn, oder neue Untersuchungen üher das Entstehen und aie Wirkungen des Mutterkorns, in hotanischer, land wirthschaftlicher und medicinischer Hinsicht betrachtet ron M. J. I. Leveillé, Ï̈é-P'äsident der Linneischen. Gesellschaft in P'uris.

Annales de la Soc. Linn. de Paris 17. Livraison. Janv. 1827. p. 56.5-589. - Aus dem Französischen mit einigen Anmerkungen von J. 11. Dierbach. - Geiger's Magazin August 1827. p. 110 u. d. $f$. - Sithe uuch ovt. Litteraturbl. X. p. 0 .

Note sur le Conoplea cylindrica de Personn par M. I. de Brondeau.

Annales de la Soc. Linn. de Paris Juillet $\mathbf{1 8 2 5}$.

Erinea sex nora descripsit D. T. L. de Schlechtendal. Linnaeq 1826. 1. p. 7t.

Recherches microscopiques et physiologiques surle genre Mycoderma; par M. Desmazières.

Annales de la Soc. Linn. de Paris. Scptbr. 1826. p. 372. + Lnnales des Sc. natur. - Janv. 18\%7. p. 42 -67.

Actinomyce. Strahlenpil\%. Fine neue Pilz-Gattung, aufgestellt von J. Meyen.

Linnaea 1827. p. $433-444$.

Bemerkungen über Sporendonema Casei, eine nene Schimmel-Gattung; ron Desmazièrcs.

Annales des Sc. nat. XI. p. 216. Juillet 1827. - Bot. Litcraturbl. I. p.. $23 \%$.

Erzengung einer Art Penicillium in der Aqua destillata Alixiae aromaticae'von Nees v. Esenlieck.

Bot. Zeit. 1824. I. p. 161 u. d. f.

Observations microscopiques sur le Blanc du Rosier. Oidium lenconitu; par M. J. B. H. J. Desmazières.

Annales des Sc. natur. Mai 1529. p. 38. - Ist keine Kranliheit, sondern Varietät von Monilia hyalina Achar.

Ueber zwei neue Gattungen ron Byssoideen, und cine neue Art Eratiom von Robert Kaye Greville. Mit einer hupfertafel.

Edinburgh Philosophical Journal. N. XXV. Juli 1823. p. 633.

Bemerkungen zur Naturgeschichte des Byssus flos Aquate L. von F. W. Wrangel.

Kongl. Fetensk. Academ. Handlingar. 1826. P. I. p.96. - Bullet. des Sc. natur. Janv. 1828. p. 100.

Ueber die Bildung einer Schimmel-Art aus Samen; von Joseph Schillinger aus Löfingen auf dem Schwarzwalde.

Kastner's Archiv Bd. 10. Heft 4. p. 428.

Iennographie de deux plantes cryptogames a ajouter a la Flore française; par M. Desmazières. (Stilbum aeruginosum, Fusisporium Betae).

Annales des Sc: nat. Avril 1830: p. 434. 
Abraham Halsey Bemerkungen über gewisse Fungi entozoici. Abhandlung, vorgelesen am 19. April 1824 an dem Lyceum zu Neu - York. Bulletin des Sc. natur. Janv. 1825. p. 66.

Neae Beobachtung der Schimmelbildung in lebenden Körper. Von Dr. Thiele in Jena.

Heusinger Zeitschrift für die organische Physik Bd. I. Heft 3. 'p. 333. - Man beobachtete sie an der Lunge eines Raben.

Ueber das Wachsen von Vegetabilien am Körper lebender Thiere, nebst Eemerliungen eines Correspondenten. Von Dr. Mitchill aus Neu - York.

Edinburgh. Journ. of Science. July 1827. - Froriep's Notizen. Bd. XVIII. p. 161 .

Views of the Process in Nature, by which under particular circumstances, regetables grow on the bodies of Living Animals. By Dr. Samuel L. Mitchill of New York. With remarks by a Correspondent.

Dies ist die Original-Aufschrift in dem angezeigten Journal von Brewster Nro. XIII. p. 310-36. - Man sche über denselben Gegenstand auch Silliman American. Journ. of Science and arts. Mars 1827. $p, 21$.

Ueber die mit Vegetation besetzte Wespe ron Guadeloupe von Ricord-Madianna.

Journal de Ph:trmacie Mars 1829. p. 158. - Froriep's Notizen Bd. XXVI. p. 18!. - Es ist da die Rede von Sphaeria entomorhiza der englischen Botaniker. Ausführliches über diesen Gegenstand findet man in F. Notizen Nro. 195 oder Nro. 19 des 9ten Bandes.

\section{Flechten.}

\section{Lichenes.}

Wie alle Gewachse aus den niedersten Abtheilungen, sind auch die Flechten erst in neueren Zeiten fleifsiger bearbeitet und untersucht worden. Es scheint jedoch, man habe die Arten-Zahl etwas über die Gebühr vergröfsert, und vielleicht dïfte bald eine grofse Reform in der Anordnung dieser schönen vegetabilischen Gebilde zu eruarten sein. Vieles Verdienst um sie haben Hoffmann, Acharius, Flörke, Meyer, Wallroth und viele andere.

Man sehe:

Auszug aus einem Berichte des Mrn. Hofraths, Ritters v. Martius, über den dermaligen Stand unserer Kienntnisse von den Flechten; erstattet in der offentlichen Sitzung der mathem.-physikal. Klasse der K. B. Akademie d. W. am 18. Febr. 1826.

$$
\text { Bot. Zeit. 1826. I. p. } 193 \text { u. d. f: }
$$

Systema Lichenum: genera exhibens rite distincta, pluribus novis adaucta. Auctore T. G. Es chweiler. Norimbergae 1824. cum tabul. lithogr.

Nebenstunden meiner Beschäftigungen im Gebiete der Pflanzenkunde. Erster Theil: Die Entwickelung, Metamorphose und Fortpflanzung der Flechten, in Anwendung auf ihre systematische Anordnung und zur Nachweisung des allgemeinen Ganges der Formbildung in den untern Ordnungen cryptogamischer Gewächse. Nach eigenen Beobachtungen und Versuchen von G. F. W. M ey e r, Dr., Königl. Grofsbrittannisch - Hannöverschem Oekonomierathe und Physiographen des Königreichs Hannover 
u. $s . w$. Mit einer doppelten illuminirten Kupfertafel und ciner Vignette. Göttingren, bei Vandenhoeck und Ruprecht 1825. 8.

Naturgeschichte der Flechten. Nach neuern Normen und in ihrem Umfange bearbeitet von Fr. Wilh. Wall roth, der Merl. und Chir. Dr., Königl. Prealfs. Plhysikus zu Heringen. Ein fafilieher Unterricht zum Selhststudium der Flechtenkunde. Erster Theil, von dem Flechtenlager im Allgemeinen. Frankfutrt 1825. $7 \mathrm{fl} .12 \mathrm{kr}$. Zweiter 'Theil, Physiologic und Pathologie des Flechtenlagers. Frankfurt 1827. 8.

A. L. A. Fée. Kssai sur les Cryptogames des écorces exotiques officinales, précédé d'une methode lichenographique, et d'un Genera, avec des considerations sur la reproduction des Agames. Paris 18\%5, Sept. Livraisons. 42 Francs:

A. L. A. Fée. Methode lichenographique et genera, ornée de 4 planches dont trois colorées, donnant les charaetères des genres qui comparent la famille des Lichens avec leurs détails grossés. Paris 1825. 4.

Glyphis et Chiodecton, genera duo nova Lichenum, auctore E. Acharius.

Linn. Transactions XII. p. 1. - Isis 1826. Heft 1. p. 30-37.

II istoire des Lichens. Genre Sticta par D. Delise. Chef de Bataillon, arec Atlas colorié, Caën 1825.

Auch in den Mémoires de la Soc. Linn. de Calvados. Tom. I.

p. 1-16\%. Tom. II. p. 13 et 598. - Dernière addition au genre

Sticta. Ibid. p. 598.

Monographie du genre Chiodecton, par A. L. A. F'ée. Lille 1829. avec une planche.

Man sehe auch Annales des Sc. naturelles. Mai 1829. p. 5-31.

Mémoire concernant les plantes cryptogames, qui peurent être réunies sous le nom de Calceioides par. Acharius.

Mém. de la Soc. Linn. de Normandie. Annal. 1826-27.

De Cladoniis difficillimo Lichenum genere Commentatio prima. Auct. H. G. Flocrke, dies festas Jesu Christi nati anniversarias pie sancteque agendas ciribus academicis indicens. Rostochii 1827. 8. Comm. secunda. Rostochii 1828. 8.

Naturgeschichte der Säulchen-Flechten, oder monogra. phischer Alischlufs über die Flechten-Gattung Cenomyce Acharius, von F. W. Wall roth. Naumburg. 1829. 2 11. $42 \mathrm{kr}$.

Schedulae criticae de Lichenihus suecanis, praeside E. M. Fries. Pars I. Lund. 18\%t. Novae schedulae criticae de Lichenihus suecanis, Auctore Elia Magno Fries, Prof. etc. Lumdae 1826. 4. 34 S.

Diese Schriften beziehen sich auf die von dem Herrn Ferf. herausgegebencn F.echten - Sammlungen, die bereits oben angezeigt worden sind.

Synoptical view of the Lichens, growing in the vicinity of the City of New York. By Alraham Halscy.

Annals of the Lyceum of Natural History of New York. Vol. I. 1823-1826. - Siche botan. Zeilung 1827. I. p. 1 u. d. $f$.

Lichenen-Flora von Würzburg, oder Anfählung und Beschreibung der $\mathrm{nm}$ Würalurg wachsenden Flechten, mit einer nenen Zusammenstellung der Gattungen und einer falslichen Erlätterung der Gattungrmerkmale begleitet; fïr Anfïnger der Flechtenkunde locarbeitet, von Philipp II epp, Ir. der Medicin etc., Arzt zu Frankenthal. Mit lithographirten Abbildungen der zu erklärenden Gattungen. Mainz 18\%4. 8. 
Wencesl. Mann. Lichenum in Bohemia observatorum dispositio succinctaque descriptio. Pragae 1826. 8.

Lichenologische Bemerkungen; von dem Herrn Rittmeister ron Flotow in Hirschberg.

Botan. Leitung 1828. 11. p. 593 u. d. f.

Siebersche Lichenen, beschrieben von $F$. Laurer, mit ciner ausgemalten Kupfertafel.

Linnaea 1827. I. 'p. 38 u. d. $f$.

Kryptogamische Parasiten auf officincllen Rinden, dargestellt vom Prof. Dr. Ze nker, mit vortrefflichen colorirten Abbildungen.

Göbel pharmaceutische I' aarenkunde Bd. I. p. 109 u. d. f. Fine sehr schätzbare Arbeit, in we?cher auch von den Flechten im Allgemeinen, ihrer Vegretationsart u. s. w. gehandelt wird, selbst die Literatur der Flechten ist angegeben, und dabei das oben angeführte Werk von Fée überall benutzt, so dafs es für Deutsche durch den Besitz dieser Warenkunde entbehrlich wird.

Ueber eine Reproductions-Art bei Borrera tenella, schrieb Cas in i im 2. Bande seiner Opuscules phytologiques.

Bemerkungen über einige in Frankreich, besonders um St. Mihiel im Departement de la Meuse gesammelten Lichenen. Vom IJern Rittmeister v. Flothow im 6. Neumärk. Dragoner-Reginent. Mit Anmerkungen von Sprengel.

Jahrb. der Gewächsk. Heft 3. p. 91 u. d. f.

\section{Lebermoose.}

Hepaticae.

Auch diese kleine Familie hat ihre Bearbeiter gefunden, und einige Gattungen derselben sind mit besonderem Fleifse untersucht worden. Sch midel, Hooker, Weber und mehrere Neueve uidmeten ihre Aufmerksamkeit diesen Gewächsen, die gleichsam in der Mitte zwischen den Flechten und Laubmoosen stehen.

Synopsis Hepaticarum Europaearum, adnexis observationibus et adnotationibus criticis illustrata. Auctore Dr. J. B. G. Lindenberg, cum tabulis duabus. Bonnae 1829, apud Ed. Weber.

Monographia Rhizospermarum et Hepaticarum. Die Wurzelfarren und Lebermoose nach ihren Gattungen und Arten, organographisch-phytotomisch bearheitet von Ag. J. Corda. Heft 1. Prag 1829. 4. VI und $16 \mathrm{~S}$. und 6 Steindrucktafeln.

Man sehe bot. Zeit. 1829. II. p. 671.

Genera Hepaticarum. Die Gattungen der Lebermoose von $A$. J. C. Corda. (Besonders alogedruckt aus Ph. M. $\mathrm{Opiz}$ Beiträgen zur Naturgeschichte. S. $613-655$.

Man sehe Linnaea 1830. 111. p. 73 der Literatur.

Specimen florae Americae septentrionalis cryptogamicae; sistens muscos hepaticos huc usque in Amer. septentrionali observatos. Auctore Lew is $\mathbf{1 0}$. de Schweinitz. Ralaigh 1821.

Literatur.

Man sehe bot. Zeit. 1824. 1. p. 363. - Linnaea 1829. I. p. 41

Hepaticae javanicae, editae conjunctis studis et opera Reinwritii, Dr. Blumii et Neesii ab Esenbeck.

Nova Acta physico medica. Tom. XII. p. 181-238. 
Ifepaticarum capensinm a C. F. Feklon collectarum brevem recensionem cum Sehlechtendalio suo communicat J. G. C. Lehmann.

Limnaea 1829. 3. p. 357 u. d. f. ,

Beschreibung einiger neuen Pfanzen aus der Ordnung Musci und Hepatici. Von Robert Kaye Greville. Bot. Zeit. 1827. I. p. 203 .

Jungermanniarum species, a Funckio, Viro cl. tum in Sudetis montibus, tum in Germaniae australis alpilus collectas, revidit $\mathrm{N}$ ecsius ab Esenbeck.

Sylloge plantar. novar. Ratisbonae 1824. p. 127. Ohlau.

Ueber Jungermannia Blasia Hooker von Beilsehmied in Botan. Zeit. 182.t. II. p. 611. - Zusutz von Nees von Esenbeck. Daselbst p. 6 ff5.

Bemerkungen über Targionia hypophylla.

Edinburgh Journal of Science. Juli 1825.

Bemerkungen über Sphaerocarpus terrestris Micheli von Dr. G. W. Bisch off.

Nova_Act. Nat. Curios. Bonn Vol. XIII. P. 2. p. 831 -838. mit einer Tafel.

L unularia alpina und Corsinia lamellosa, zwei neue europäische Lebermoose, aufgestellt von Nees von Esenbeck und Bisch off.

Bot. Zeit. 1839. II. p.' 393.

Brissocarpus und Oxymitra Bischof zwei neue Gattungen. Daselbsi 1830. I. p. i1.

Guentheria; eine nene Gattung von Lebermoosen, beschrieben von C. L. Treviranus, Prof. zu Breslau.

Jahrb. der Gewë̈hskunde Heft 3. p. 1 u. d. f.; ferner 157, wo auf Corsinia marchantioides Raddi aufmerksam gemacht wird, die mit der Guntheria synonym zu sein scheint, und bereits von Micheli gekant war.

Riccia natans nach Hooker.

Botan. Miscellamy etc. by Hooker. Pars I. April 1829. - Bot. Literaturbl. 1I. p. 125.

Sur un genre nouveau d'Ilépati ques, Lejeunia, par Mademoiselle Marie Anne Libert. Aus den Annales générales des Sc. physiques Tom. 6. in Sprengel's neuen Fintdeckungen III. p. 291.

\section{Laubmoose.}

Musci frondosi.

Die Laubmoose sind, zumal in Deulschland, mil besonderer Iorliebe untersucht worden, auch haben sich mehrere Botaniker vorzugsueise mit dem Studium derselben beschäftigt. Die gröfsten Verdienste um diese Gewächse erwarben sich II edwig, Schwägrichen, Bridel, Funk, IIornschuch und viele Andere.

Ueber die Entwickelung der Moose schrieb Prof. Nees von Esenbeck.

Isis $B d . X V \cdot p .762$. 
Ueber das Keimen der Moose theilte Drummond mehrere Bemerkungen mit.

Daselbst; literarischer Anzeiger p. 347.

Ueber die Bildung der Monse trug Prof. Dietrich von Eisenach bei der Versammlung $\mathbf{d e r}$ Naturforscher in Heidelberg seine Ansichten vor.

$$
\text { Isis 183!, p. } 519 .
$$

Einige Betrachtungen über die Befuchtungstheile der Moose, nebst den Charakteren und Beschreibungen zweier neuer Gattungen dieser Familie von Robert B rown.

Transactions of the Linn. Soc. Fol. X. - Fermischte botanische Schriften II. $p .683$ u. d. f.

J. Hedwig Species muscorum frondosorum descriptac, icon. illustrat. Opus posthumum edit. C. F. Schwägrichen. Lipsiac 1801. cum tal. Supplementi primi pars anterior 1811, posterior 1816. Supplement. secundum 1827.

Joan. Hedwig Species muscorum frondosorum descriptae et tabulis aeneis coloratis illustratae. Opus posthumum. Supplementum tertium scriptum a Friderico Schwägrichen, Prof. Lipsiensi. Lipsiae apud Joan. Ambros. Barth. 4. Sect. I. Tab. CCI-CCXXV. 1827. Sect. II. Tab. CCXXVI - CCL. $18: 8$.

Siehe bot. Zeit. 1830. Il., Ergënzungsbl. p. 1 u. d. f.

Sam. El. a Bridel-Brideri etc. Bryologia universa, seu systematica ad norum methodum dispositio, historia et descriptio omnium muscorum frondosorum hucusque cognitorum, cum synonymia et auctoribus probatissimis. Accedunt tabulae aeneae tredecim. Volumen primum. Lipsiae 1826. 8. 745 S. Volumen secundum 1827.

Neue Anordnung der Moos-Gattungen mit ihren Charakteren und Beobachtungen über ihre Vertheilung, ihre Geschichte und ihre Structur von den Herren Greville und G. A. Walker-Arnott.

Transactions of the W'

Nourelle disposition methodique des éspèces de Mousses exactement connues; par M. G. A. Walk cr-Arnott. Membre des Sociétés royales et Wernerienne d'Edinbourgh et correspondent de celle d'histoire naturelle de Paris.

Lue à la Société d'histoire naturelle, le 18 Mars 1825. - Mémoires de la Société d'histoire naturelle de Paris. Tome second. II Partie. Avril 1826. p. 249-320. - Ist auch besonders abgedruckt zu haben unter dem-Titel:

Description methodique des éspèces de Mousses, par G. A. Walker-Arnott. Paris 1825. 4.

Mémoires d'histoire naturelle, par M. le docteur B. M. Kittel. Paris 182(i.

Enthält Bemerkungen über $\boldsymbol{W}$. Arnott's Muscologic.

Versuch einer: nähern Beleuchtung der Disposition methodique des éspèces de mousses par G. A. Walker-Arnott, von Herrn Fürnrohr.

Bot. Zeit. 1827. II. p. 22-86 der. Beilage.

Bemerkungen über diese letzte Abhandlung schricb Herr Dr. Schultz in Neu-Brandenburg.

Bot. Zeit. 1828. I. p. 252. 
Caroli a Linné Species plantarum. Editio Willdenow, continuata ad Muscas a F. Sehwägriehen, Prof. Lips. 'Tomus V'. P. 2. Sect. prima. Berolini 1830.

Exposition methodique des genres de la famille des Mousses, pour servir de complément au travail de feu P'alisot-Beauvois, par M. Desveaux.

-Innales de la Soc. Linn. de Paris. T. I. p. 211. Juillet 1824.

Beitrag zur Geschichte der Fortschritte in der Kienntnifo der Moose, nach den Schriften der älteren Botanilier bis auf unsere Zeit; von Herrn Prof. II orn sehueh.

Bot. Zeit. 1825. I. p. 209 u. d.f.

Bryologia germanica; oder Beschreibung der in Deutschland und in der Schweiz wachsenden Laubmoose, von C. G. Ne es v. Esenbeck, J.Sturm et F. II ornschuch. Nürnberg, 1. Theil 1823. Zweiter Theil erste Abtheilung Leipzig bei Fleischer 1827. 8. Mit Kupfern.

Man sehe bot. Zeit. 1830. II. Ergänzungsbl. p. 18.

Will. Jackson Hooker's Muscologia britannica, containing the Moses of Great - Britain and Ireland, systematically arranged and described. London, Longmann et Comp. 1827. 8. with plates.

Erstes Verzeichnifs Sardinischer Lauboose, wie auch derjenigen, welche ron Herm Fleis cher bei Smyrua aufgefunden worden sind, nebst Beschreibungen und Abbildungen einiger neuen Arten, von Herrn Apotheker F. A. Mïller.

Botan. Zeitung 1829. II. p. 385 u. d. f.

Beschreibung einiger neuen Pflanzen, die zur Familie der Laub - und Lebermoose gehören von R. Kaye Greville. Annals of the lyceum of nat. hist. of New York. Juni 1825. p.

271. - Botan. Zeit. 1827. I. p. 33.

Beschreibung wweier neuer Arten Moose der Gattung Neckera (americana) und Hypnum (remotifolium) von R. Kaye Greville. Transactions of the Wernerian Society Vol. V. P.2. 1826. p. 481.

W. J. Hooker. Ueber die Moosgattungen Calymperes Swartz und Syrrhopodon Schwaegrichen.

Edinburgh Journ. of Science. Oct. 1825. p. 218.

Beschreibung einer neucn Moos-Gattung Grevilleanum; von Lewis Beek, M. Dr., und Ebenezer Emmons, M. D.

Sillim. American. Journ. of Science. Vol YI. Nro. 1. p. 183. tab. 1. - Botan Literaturbl. I. p. 84.

Charakter und B ese hre ibung der neuen Moos - Gattung Lyellia, mit Bemerkungen über die Abtheilung der Familie, zu welcher sie gehört, und mit einigen Zusätzen über Leptostomum und Buxbaumia, von Robert. Brown.

Transactions of the Linnean Society of London. Vol. XII. P.II. p. 560-583. - Iermischte botan. Schriften II. p. 701. brücken.

Bryologische Bemerkungen vom Apotheker Bruch in ZweiBotan. Zeit. 1826. I. p. 161 und 1827. I. p. 177.

Beitrag zu der Moos-Gattung Sphagnum vom Herrn Inspector Breutel zu Nenwied.

Bot. Zeit. 1824. II. p. 433. 
Ueber Sphagna, nebst Bemerkungen zu den in Nro. 88 der botan. Zeitung für 1824 durch Herrn Inspector B reutel mitgetheilten Beobachtungen; von Hrn. Apotheker Bruch in Zweibrücken.

Bot. Zeit. 1825. II. p. 625.

Bemerkungen über die Vegetation der Moose und Rerision des Genus Sphagnum von Dr. Jakob Hegetschweiler in Rifferschweil.

Denkschriften der allgem. Schweizer Gesellschaft für die gesammten Naturwissenschaften. Frster Band, erste Abtheilung p. 130-143.

Trichostomum La ureri; eine neue Lanbmoos-Art, iufgestellt vom Herrn Hofrath Dr. Schultz in Neubrandenburg.

Bot. Zeit. 1827. I. p. 161.

Noch ein Wort über Barbula obtusifolia und Bryum erythrocarpon; nebst einigen andern bryologischen Bemerkungen; vom Herru Apotheker Fürn rohr in Zweibrücken.

Bot. Zeit. 1829. II. p. 577.

Eremodon Rudolphianus; eine neue bestimmte Laubmoos-Art, aufgestellt vom Hrn. Prof. Hornschuch in Greifswalde.

Bot. Zeit. 1830. I. p. 209.

Scouleria, eine neue Moos - Gattung. Aus Hooker Botanical miscellany in den bot. Literaturbl. Ir. p. 4 7.

\section{$\mathrm{Z}$ weite Section.}

Cryptogamische Gefäfspflanzen.

§. 13.

Classis V. Rhizocarpac. 0. 14. Salviniaceac. 15. Marsileaceac. 16. Isoeteae.

Classis VI. Filices. O. 17. Polypodiaceae. 18. Osmundaceac. 19. Ophioglosseae.

Classis VII. Lycopodineae. O. 20. Lycopodineae.

Classis VIII. Gonyopterides 0. 21. Characcae. 22. Equisetaceac.

\section{Marsileaceen.}

Marsileaceae (Rhizocarpeàe).

Fine kleine Gruppe von Wassergewächsen, die zuerst Robert Brown als eigene Familie betrachtete, und ihre Charakteristik in seinem Prodromus Florae Novae Hollandiae mittheilte, wobei noch das zu vergleichen ist, was er insbesondere über die Gattung Azolla sagte.

Vermischte botan. Schriften 1. p. 162.

Monographia Rhizospermarum et Hepaticarum. Die Wurzelfarren und Lebermoose nach ihren Gattungen und Arten organographischphytotomisch bearbeitet von Aug. J. Corda. Prag 1829. 4. Mit lithographirten Abbildungen: Erstes Heft $48 \mathrm{kr}$.

Siehe bot. Zeit. 1829. II. p. 671.

Examen de la Vegetation de l'Isoetes setacea, ex exposition de scs caractères, par M. Roffeneau Delile, Prof. de Botanique à Montpellier.

Mémoires du Museum d'Hist. naturelle. Sept. Année. Huit. Cahier p. 100. 
Dissertatio inauguralis botanica de Salvinia natante. Praeside Schubler. Auct. ('. L. 1) uvernoy. Tübingae 1825. 4.

Bisc hoff über die Naturgeschichte der Salvinia natans mit 3 Tafeln. Nova Act. Natur. curiosor. Fol. XIV. P.1. p. 45.

Sulla Salvinia natans. Memoria de Dr. Paolo Sari Ajuto del Prof. di botanica di Pisa. Biblinteca italiana 1820. t. 20. p. $313-350$.

Siehe sprengel Neue Entdeckungen Bil. III. p. $2 u ̈ 6$.

\section{Farrenkräuter.}

\section{Filices.}

Unser deutsches raterland besitzt aus dieser schönen Familie nur vorhuillnifsmüfsig wenige und allezeit krautartige Formen, während dem zahlreiche baumartige Specics die warmen Frdstriche bewohnen; auch isl von den Botanikern, die die Gieü̈̈chse heifser Giegenden zu sehen Gelegrenheit hatten, das Meiste zur Kenntnifs der Arten dieser Famulie geschehen, während dem der physiologische Theil, zumal von deutschen Botanikern, auf das fleifsigste bearbeitet wurde.

Einen Beitrag zur Geschichte der Fortschritte in der Kenntnifs der Farrenliäuter, von Brunfels bis auf unsere Zeit, lieferte Herr Prof. Nees v. Esen beck. d. J.

Botan. Zeit. 1825. I. p. 1 u. d. f.

Das Wesen der Farrenkräuter, besonders ihrer Fruchttheile, zugleich mit Rücksicht auf systematische Anordnung und mit einer Darstellung der Entwicklung der Pteris serrulata aus dem Saamen begleitet, ron Dr. Geor $g$ Friedrich Ka ulfuls, aufserordentlichem Professor zu Hal'e. Lrste Iälfte. Mit ciner Kiupfertafel. Leipzig bei Carl Cnobloch 182\%. $3 \mathrm{ff}$.

Ueber die Art und Weise der Entwickelung der Farrenkräuter findet man die Ansichten des Herrn Prof. Nees von Esenbeck in der Isis Band XV. p. 762, und jene des Herrn Prof. Kaulfufs. Duselbst p. 360 .

Finige Bemerkungen über den innern Bau der holzigen Farrenkräuter von $H$. F. Link.

Linnaca 1826. Heft 3. p. $414-417$.

Ueber die Bedeutung der Organe der Farrenkräuter, rom Prof. L. M e yer in Königsberg.

Isis 1829. p. 390.

Ueber die Anzueht der Farnkräuter aus Samen, deren Vermchrung und Bchandlung, von dem K. bot. Gärtner Scitz in München.

Terhandl. des Vereins zur Beförderung des Gurtenbaues in den K. Preufs. Staulen. Bd. 4. - Siche Linnaeu 1829. I. p. 13 der Literat. und Botan. Literaturblätter I. p. 530.

De la distribution des fougères sur la surface du globe terrestre par M. d'Urville.

Annules des Sc. nuturelles Sept. 1825. p.51. - Froriep's Notizen Nro. 251 oder Nro. 9 des 12. Bandes.

I cones Filicum, ad eas potissimum species illustrandas destinatae, quae hactenus vel in herbariis delituerunt prorsus incognitae, vol saltem nondum per Icones botanicis innotuerunt, auctor. J. G. Hook er 
et Roberto Kaye Greville. Strasbourg Trenttel et Würz 1826. Fasc. III. 1827.

Preis jeder Lieferung mit schwarzen Kupfern 9 Rthl.

Prodrome de la famille des Fougères, par M. Desveaux, Directeur du Jardin des plantes d'Angers.

Annales de la Soc. Linn. de Paris Mai 1827. p. 181. - Juillet

p. 213 et suiv. - Siehe botanische Literaturbl. 1. p. 239.

G. T. Ka ulfus. Enumeratio Filicum, quas in itinere circa terram legit C. A. de Chamisso, adjectis in omnia harum plantarum genera permultae species cum tab. duab. aeneis. Lips. 1824.

Nya cllar mindre kända arter of Ormbunkar (Filices) beskrifne af Jol. Em.'Wickström.

Kongl. Ietenskaps Academiens Handlingar, för är 1825. Stockbolm 1826. 8. p. 43.t - Siehe Linnaea 153. Hieft 3. p. $112 \mathrm{der} \mathrm{Li-}$ terctur. - Fis scheint von dieser. Abhandlung auch ein besonderer Abdiuck vorhanden zu sein.

Reliquiae Haenkeanae seu descriptiones et icones plantarum, quas in America meridiona'i et boreali, in insulis Philippinis et Marianis collegit Thaddaeus Haenke, philosophiae Doctor, phytographus regis Hispaniac. Cura Musci bohemici Fasc. I. cum tab. XII acri incis. Pragae 1825. Fol. XV. und $83 \mathrm{~S}$.

Dieses erste Heft enthält grofsentheils Farrenkrüuter, von L. B. Presl beschrieben, der auch schon in den Deliciis Prugensibus 39 neue Arten dieser Familie bezeichet hatte. Man sehe auch Geiger's Magazin für Pharmacie April 1826. p. 103.

Raddi Plantarum brasiliensium nova genera et Species norae vel minus cognitae. Collegit et descripsit. Pars I. (Filices). Florentiae ex typographia Aloisii Fezzati 18\%5. Fol.

Alumbrationes plantarum. Edidit D. F. L. de Schlechtendal. Fascicul. prim. Berolini apud Ferd. Dümmler. MDCCCXXV.

Fnthält die Beschreibung kapischer Farrenkräuter, worunter auch Arten von Equisetum, Ophioglossum und Lycopodium. Man vergleiche die Literatur der afrikanischen Floren.

Essai sur les fougères de Calvados, par M. Chaurin.

Mémoires de la Soc. Linn. de Calvados. 1825. p. 381.

Ueber Woodsia. Eine neue Gattung Farrenkraut, von Robert Brown.

Transactions of the Linnean Society of London. Vol. XI. 1816.

p. 180 - 174. - Vermischte botan. Schriften II. p. 675.

Sur un Sousgenre à former parmi les Polypodes, sous le nom de Drynaire, par M. Bory de Saint Vincent.

Annales des Sc. natur. Aoút 1825. p. 462-473.

Botrychium Lunaria. D. F. L. de Schlechtendal Racpero suo S. P. D.

Linnaea 1829. I. p. 128.

S. W. Conrad. Bemerkungen über Osmunda Claytoniana L. mit einer Abbildung.

Journ. of the Acad. of nat. Sc. of Philadelphia Jun. 1827. p. 39. Ist nicht synonym mit Osmunda interrupta Michaux, wie Mühlenberg will, noch Varietät von 0. cinnamomea nach Pursh, sondern eigene, wiewohl seltene Art. 
Bemerkungen über die Farrenkrãuter, welche in der durch ITerrn Sicber veranstalteten Pflanzensammlung von Martinique enthalten sind.

Bot. Zeit. 1823. I. p. 360.

Note sur le Pteris cornuta de Palisot-Beauvois éspèce du genre Ceratopteris; par M. Le Prieur.

Annales des Sc. nat. Janv. 1830. p. 99.

\section{Lycopodiaceen. \\ Lycopodiaceac.}

Einc erst in neueren Zeiten von Richard aufgestellte kleine Familie, aus den Gattungen Lycopodium, Psilotum und T'mesipteris bestehend, wozu noch Decandolle das Genus Isoëtes brachte. Jussieuvereinigte die Lycopodien früher mit den Farrenkräutern, und die meisten Schriftsteller folgten dieser Ansicht, so zwar, dafs wenn man die neueren irbeiten über die Lycopodien kennen lernen uill, vorzugsweise die über Filices geschriebenen Abhandlungen zu vergleichen sind. So, um nur Einiges anzuführen, findet man Lycopodien in den Sie berschen Sammlungen von Farrenkräutern, Raddi in seiner Synopsis filicum brasiliens. beschreibt mehrere neue Lyc.podion $u$. s. $w$. Hier verdient aber nuchstehends Abhandlung besonders angeführt zu werden:

On the Gormination of Lycopodium denticulatum, in a Letter to the Secretary from R. A. Salisbury.

Transactions of the Linnean Society of London Fol. XII. P. II. p. 365. - Man sehe hierüber auch Isis Bd. II. p. 451 und Bd. XV. p. 347 des literarischen Anzeigers.

\section{Characeen.}

Characeae.

Nur eine einzige Gattung (Chara, Armleuchter) gehört hierher; ihr Schicksal in der systematischen Botanik ist eines der seltsamsten. Linné brachte sie zuerst zu den Cryptogamen, später aber zu der Monoecie der Phanerogamen. Jussieu zählte sie zu den Najaden, Robert Brown brachte sie in die Nähe der Hydrocharideen; mancher anderer Ansichten nicht zu gedenken. Richard betrachtete sie zuerst als besondere Familie und $A$ ghard trennt sie in zurei Guttungen (Chara und Nitella). Die Chara-Arten sind aber insbesondere noch durch die zahlreichen neuern $\boldsymbol{U}$ tersuchungen ihres innern Baues und ihres Saftlaufes merkwürdig geworden, wie aus nuchstehenden Bemerkungen erhellt.

Osservazioni ed esperienze, intorno la circolazione della limfa in alcune spezie di Care, di Paolo Barbieri. Mantova 1828.

Beobachtungen und Versuche über die Circulation der Lymphe in einigen Arten der Chara, von Paolo Barbieri, Aufreher des botan. Gartens zu ilantua.

Biblioteca italiana Nro. CLVIII. Febrajo 1829. - Froriep

Notizen Bd. XXV. p. 50. Nro. 532.

Observation sur la Circulation des Chara; par M. Lebaillif.

Bullet. des Sc. natur. Nov. 1827. $p$. $321-326$.

Circulation of the $S$ ap in the Chara vulgaris by Brainville.

Brewster Edinb. Journal of Science. Octob. 1827. p. 383.

Gozxi Kreislauf in der Chara.

Isis Bd. IV. p. 251. 
Ueber die Anatomie und den Kreislauf der Charen, von C. A. Aghard.

Verhandl. der $K$. Leopold. Akad. der Naturforscher Bd. XIII. Abth. 1. p. 87.

Experiences chimiques et physiologiques sur les Charas, par M. Raspail.

Société d'histoire natur. et philomatique de Paris. Séance du 11 et 15 Aoüt 1827. - Bullet. des Sciences natur. Sept. 1827. p. 74-77.

Beobachtungen und Bemerkungen über die Gattung Chara von Dr. Meyen.

Linnaea Bd. II. p. 55 u. d. $f$.

Erfahrungen über das Keimen der Charen, nebst andern Beiträgen zur Kenntnifs dieser Pflanzen-Gattung, mitgetheilt von Dr. G. F. Kaulfufs, aufserordentlichem Professor zu Halle. Mit einer Kupfertafel. Leipzig 1825.

Observations sur le genre Chara, par M. Aghard. Annales des Sc. naturelles. Janvier 1825.

Mémoires sur les Charagnes, par M. Vaucher.

Mém. de la Société de physique et d'hist. nat. de Genève. Tom.I. prem. part. 1821. p. 168.

Observationes in genus Chara. Praeside A. Bruzelio, respondente Eberhard Liljeval ch, Scano. Londoni Gothorum MDCCCXXIV. ex officina Berlingiana. 8. $24 \mathrm{~S}$.

Eine freie Bearbeitung dieser Schrift lieferte Herr Fürnrohr in der botanischen Zeitung 1826. II. p. 481 u. d. $f$.

Bertoloni. Sopra una nuove Specie di Cara.

Giorn. di fisica e chimica Tom. IX. p. 206. 1826. Pavia. Bim. tert. - Ist Chara ulvoides, im See von Mantua entdeckt. - Siehe Linnaea 18\%8. p. 93 der Literatur. - Man sehe auch Annales de la Soc. Linn. de Paris V. p. 325. Jul. 1826. und botanische Literaturblätter II. p. 12t. - Die Art gehört zu Aghard's Nitellen und zeichnet sich durch ihre ungewöhnliche Gröfse aus, daher sie auch die gigantische grenannt wird.

Chara tenuissima der Flora Monaster. ist nach Drees - Ectosperma caespitosa' Vaucher.

Linnaea 1827. p. 210.

Chara as pera; ein neuer Beitrag zur Flora Grofs-Brittanniens. $179-182$.

Edinburgh philosophical Journal Nro. XXVII. Jamuary 1826. p.

\section{Equisetaceen.}

Equ is et a c a e.

Nur die einzige Gattung Equisetum, von Decandolle zuerst als Familie betrachtet, gehört hierher. Die literarischen Arbeiten, welche zur näheren Kenntnifs derselben führen, dürften besonders folgende sein:

Smelov sky. Descriptio botanico - chemica Equiseti aruensis.

Mémoires de l'Academie impériale des Sciences de St. Petersbourg 1805. p. 316. Taf. $V$.

Reichenbach und Schubert über Entwickelung des Equisetum. Bot. Zeit. 1822. I. p. 335 u. d. f.; ferner 1827. II. p. 673. 
J. F. Wickstroem Trenne Arter of Växtläg tet Equisetum. Beskrifne. Stockholm 1821. 8.

Monographie des Prêles, par M. Vaucher.

Mém. de la Soc. de Physique et d’hist. nat. de Genève 1822. T. I. P. 2. p. 330.

\section{D r it e Section. \\ (Phanerogamen). \\ En do ge n e n. \\ Monocotyledonische Gefäfspflanzen.}

§. 14 .

Classis IX. Glumaceac. 0. 23. Gramineac. 24. Cyperaceac.

Cl. X. Juncinac. O. 25. Restiaceae. 26. Junceaceae. 27. Xyrideac. 28. Commclinaceat.

Cl. XI. Ensatae. O. 29. Burmanniaceae. 30. Hypoxideae. 31. Haemodoracene. 32. Irideac. B3. Amaryllideae. 31. Bromeliaceae.

Cl. XII. Liliaceae. O. 35. Asphodeleae. 36. Colchicacpae. 37. Smilaceae. 38. Dioscorcae.

C1. XIII. Orchideae. O. 39. Orchidene.

Cl. XIV. Scitaminea e. O. 40. Amomeae. 41. Cannaceae. 42. Musaceae.

Cl. XV. Palmac. 0. 43. Palmae.

Cl. XVI. Aroideac. O. 44. Callaceae. 45. Orontiaceac. 46. Pandlaneac. 47. Typhaceae.

C1. XVII. Helobia e. O. 48. Najadeac. 49. Podostemeae. 50. Alismaceae. 51. Butomeac.

C1. XVIII. Hydrocharideae. 0. 52. Hydrocharideae.

\section{Gramineen.}

Diese sehr grofse Familie, deren Glieder vorzugsweise clic grmäfsigten Zonen der Erde bewohnen, ist vielfältig untersucht, und freilich nach oft bedeutend von einander abweichenden Grundsätzen bearbeitet worden. B artling theilt sie in 10 Stümme, die er folgendermaafsen benennt: Stipacea, Agrostidea, Bromen, Hordeacea, Chloridea, Panicea, Saccharina, Phalaridea, Oryzea, Bambusacea, wozu noch mehrere Gattungen kommen, die nicht untergebracht werden konnten.

a. Schriften und einzelne Aufsätze, die Structur und systematische Eintheilung der Gräser betreffend.

C. B. Trinius. De Graminibus unifloris et sesquifloris. Dissertatio botanica, sistens theoriae constructionis floris graminei epicrisin, terminologiae novas rationes, de methodo disquisitiones, adjecta generum et specierum e tribu uni - et sesquiflororum plurium synopsi. Petropoli 1824. (Halae apud Schwetschke). 8.

G. Sinclair. Hortus gramineus Waburnensis, illustr. with numerous col. figures. 2de edit. 8 . Lond. 1825 .

Auf diese Schrift und ihre deutschen Bearbeitungen werde ich in dem dritten Theile, der der ölonomisch-technischen Botanik gewidmet ist, nüher zurückkommen. 
Nees v. Es enbeck (senior). Etwas übcr die Anlage zu einer dreizähligen Frucht bei den Gräsern, mit einer Abbild.

Linnaea 1830. IV. $p$. 679 .

Es s a i d'une classification générale des Graminées fondée sur l'étude physiologique des caractères de cette famille; par M. R aspail.

Annales des Sc. natur. Avril 1825. p. 423 Seconde partie; suite et fin. Aoüt 1825 p. 433-460. - Isis Bd. XX. Heft 11. p. 922.

Premier Mémoire sur l'anatomie comparée des Graminées par' M. Raspail.

Bullet. des Sc. natur. Mars 1827. p. 356-374. Mai p. 57-62. Botan. Literaturbl. II. p. 131.

Quelques idées sur les Graminées; par M. J. de la Harpe.

Annales des Sc. natur. Juillet 1825. p. อ̊35. - Bemerkungen hierüber von Raspail 1b. Mai 1826. p. 76-89.

Notice sur la plus interne des enveloppes florales des Graminées par M. Th. Lestiboudois. Prof. de Botanique à Lille. Paris 1824.

Ueber die Structur der Articulationen oder Lebensknoten in den grasartigen Gewächsen und Cyperaceen, von Herrn d e la Harpe.

Annales des Sc. naturelles Sept. 1825. p, $21-28$.

Observations sur la structure et la germination du blé; par M. Liec $0 \mathrm{q}$.

Mém. de la Soc. d’Emulation de Cambrai Année 182t. Janvier 1825. p. 330 .

Ueber den Blüthenbau der Gramineen von Dr. W. Cruse in Königsberg.

Linnaea 1830. II. p. 299.

U eber den Ba u und das Keimen verschiedener Saamen-Arten aus der. Familie der Gräser von Dr. Kittel.

Bot. Zeit. 1830. II. p. 623.

Sur la formation de l'Embryo dans les Graminées par M. Ras pail. Annales des Sc. natur. 'T. IV. p. 271. - Froriep's Notizen Nro. 24 :ै.

Ras pail's A blıandlung über die Bildung des Embryo in den Gräsern und Versuch einer Classification dieser Familie, mit Anmerkungen, übersetzt ron C. B. 'Trinius. Petersburg 1826.

Die Herren Trinius und Raspail entzweiten sich über einige Gegenstände, die Gramineen betreffend; beide führten ihre Streitsache zwar zum Theil herb genug, aber höchst scharfsinnig aus, man findet das Nähere in dem Bulletin des Sciences natur. Fevr. 1828. p. $213-231$.

Erster Aufsatz über die Graminologie, enthaltend die Analyse voin Embryo der Gräser.

Cassini im zweiten Bande der Opuscules phytologiques.

Ueber die Entwickelung der Fecula in den Fructifications-Organen der Cerealien, und mikroskopische Analyse der Fecula, nebst geeigneten Untersuchungen zur Erklărung des Uebergangs derselben in Gummi, von Ras pail. et suiv.

Annales des Sc. natur. Octobre 1825. p. 221-240. Dec. p. 38.

Additions au Mémoire sur l'analyse microscopique de la Fécula, par M. Ra s pail.

Annales des Sc. natur. Mars 1826. p. 325-335. 
C. B. Trinius. Fundamenta Agrostographiae, sive thenria constrnetionis floris graminci; adjecta synopsi generum graminum hucusque cognitorum. Vienn. 1820. 8.

\section{b. Topographie der Gräser.}

Dieser Gegenstand gehört zunc̈chst in die Abtheilung der Floren und der verwandten Abschnitte, hier mögen nur folgende Notizen einen Platz finden.

Agrostographia Brasiliensis, sive enumeratio plantarum ad familias Graminum et Cyperoidearum spectantium, quas in Brasilia collegit et deseripsit Jos. Raddius. Lucea, 188. 8 .

Ueber brasil. Gräser von Martius und Nees bearbeitet selie man dic Floren.

A. Monography of the British Grases, by G. Graves. London 1823. No. III.

Observations sur les Graminces de la Flore Belgique, par B. C. Dumorticer. Tournai 1823.

Bemerkungen über cinige Cyperaceen und Gramineen vom Vorgebirge der guten Hoffnung von Herrn Dr. Steudel in Efslingen.

Botan. Zeit. 1829. No. 9. 10. 20.

Revision des Graminées publiécs dans les Nova Genera et species plantarum de MM. Humboldt et Bonpland, précédée d'un travail général sur la famille des Graminées par C. S. K u nth. Ouvrage accompagnée de 100 planches coloriées d'après les dessins de Mme. Lïlalie Delille. Paris 1829.

c. Bearbeitung einzelner Gattungen, Arten u. s. w.

Essai d'expexiences et d'observation sur l'éspèce régétale en générale, et en particulier sur la valeur des caractères specifiques de Griaminées par Ra spail.

Annales des Sciences d'Observation. Mars 1829. p. 406.

Herr R. geht unbarmherzig mit sehr vielen, selbst alten Linneischen Arten um, deren eine nicht kleine Zahl eingezogen worden, man sche was er über Bromus mollis, nanus, grossus, secalinus, pratensis, über Festuca Myurus, nniglumis, ciliata, bromoides, stipoides sagt, ferner äber Festuca ovina, tenuifolia, duriuscula, glabra, cinerea, rubra, glauca, heterophylla, Lamarkii u. s. w. u. s. w. in schreiendem Contrast mit manchen andern Ansichten. - Medium tenuere beati!

Sprengel. Graminum minus cognitorum Decades duae.

Mémoires de l'Acad. de Petersbourg. II. $p$. 287.

C. B. Trinius. Species Graminum iconibus et descriptionibus illustrat. Petersb. 1825.

Man sehe auch Linnaea 1828. III. p. 153. Lit.

Koch. Bemerkungen über mehrere Gräser.

Bot. Zeit. 1828. I. p. 225.

Description of a Species of Aira found on Lach-na-gar in Aberdeenshire. By M. W. Macgilliway.

Jameson New. Edinb. Phil. Journ. Jul. - Octobrc 1830. p. 363. Aira montana?

Bemerkung über Anthoxanthum odoratum von Kunth. Annales des Sc. nat. Fevr. 1828. p. 221. Bot. Literaturbl. II. p. 117. 
Arundo Wilhelmsii Ledebour.

Mém. de l'Acad. de Petersbourg. VI. p. 593. f. 19.

Ca lam a grostis nutans und pulchella, zwei neue Arten, von Herrn Dr. Sauter in Bregenz.

Bot. Zeit. 1830. I. p. 289.

Ueber Cynodon Phragmites (Arundo Phragmites L.) und 2 neue Spielarten desselben, von $R$ as pail.

Bulletin des Sc. nat. Juni 1828. p. 230. - Bot. Literaturbl. II.

p. 114. Linnaea 1829. II. $p$. 22. der Literatur:

Note sur le Festuca Myurus de Linné et sur quelques éspèces voisines, par M. Soyez Willemet.

Annales des Sc. natur. Avril 1826. p. 440 - 447.

De Festuca loliacea Auctorum ad V. celebr. Dr. de Schlechtendal scripsit H. F. Link.

Linnaea 1827. $p$. 234 .

De Festuca heterophylla.

Botan. Zeit. 1828. II. p. 656.

Sur le Genre Hierochloa et ses analogies, et sur les analogics du Festuca flabellata Lamark, par M. Raspail.

Annales des Sc. d'Obscrvation Avril 1829. p. 70. - Botan. Literaturbl. II. p. 118.

De Libertia, novo graminum gencre commentatio, anctore $\mathbf{A}$. L. S. Liejeune.

Nova acta Acad. Caesar. Leopold. Nat. Cur. T. XII. P. 2.

p. 751. cum tab. aen.

Ueber diese Gattung sehe man Raspail's Kritik in dem Bulletin des Sc. natur. Juin 1826. p. 231 - 2038; er nennt die Pflanze Bromus auriculatus.

Lettre sur une varieté remarquable du Mais de Brésil, addressée à l'Academie des Sciences, par M. Aug. St. Hilaire.

Annales des Sc. d'Observations. Mars 1829. p. 458.

Note sur une nouvelle éspèce de Mais (Zea hirta) par M. Mathieu Bona a fons.

Annales des Sc. natur. Juin 1829. p. 156.

Observations on the genus Oryzapsis. By T. Nuttall. Read Juli. I. 1823. Part. I. p. 125.

Siehe Linnaea 1829. I. p. 42. Lit.

Monographie de deux éspèces de Panicum, qu'une erreur d'observation aroit érigées en genre sous le nom de Monach ne, accompagnéc de considerations relatives à quelques autres genres fondés sur des autres caractères tout aussi illusoires, par M. Raspail.

Annales des Sc. d'Observation, Mars 1829. p. 438.

Notice sur une Graminée de l'Amerique septentrionale, naturalisée depuis 1820 dans les environs de Bordeaux, par M. Ch. Des Moulins. (Paspalum Digitariae Poiret, Panicum Digitariae Raspail.

Bulletin de la Soc. Linn. de Bordeaux 11. livr. Juillet 1826.

Bemerkungen über die Gattung Phalaris von H. F. Link.

Linnaea I. Heft 1. p. 96.

Thysanachne, novum plantarum genus; descripsit C. B. Presl. Pragae 30 . Maji 1829. 4. c. tab. aen.

Siehe Linnaea 1830. II. p. 50. Lit. 
Die Sammlungen der Gräser der Herrn Weihe, Sieber u. s. u. sind bereits oben angezeigt; man sehe über erstere botan. Zeit. 1828. I. p. 115. und über Siebers Agrostothek. Das. p. 289.

Ueber die Cerealien werden die geeigneten Nachrichten in der Abtheilung für öconomische Botanik mitgetheilt werden.

\section{Cyperaceen.}

Sie zerfallen nach Bartling in zwei Stümme, die Caricinae und $\mathbf{C y}-$ perinae. Da sie mit den Gräsern grofse Aehnlichkeit haben, so wurden sie öfters mit ihnen zugleich bearbeitet, weshalb auch die dort angegebene Literatur zu vergleichen ist. Hierher gehören aber noch folgende:

Th. Lestiboudois. Essai sur la famille des Cyperacées. Paris 1819.

Caricography by Professor C. Dewey, Williams College. Benjam. Silliman American Journal' of Science and Arts. Yol.

IX. X. Durch mehrere Hefte. Man sehe bot. Zeit. 1827. I. p. 23.

Band XII. No. 2. Jun. 1827. p. 296. des amerikanischen Journals enthült einen Anhang, wo folgende Arten vorkommen: Carex filifolia Nuttall, C. Washingtoniana Devey, C. Barrattii Schw. et Torrey, C. caespitosa.

D. H. Hoppe, Caricologia germanica: oder Aufzählung der in Deutschland wild wachsenden Riedgräser, zum bequemen Gebrauch auf botanischen Excursionen u. s. w. Regensburg 1826. 8. Auch als Beilage zur botanischen Zeitung.

Note sur le genre Uncinia Personn, par M. Raspail.

Nouv. Bulletin des Sciences de la Soc. philomatique. Aout 1826. p. 121. - Linnaea 1828. p. 59. Lit.

Caricologische Bemerkungen von Herrn J. C. Schmidt, Lehrer der Naturgeschichte zu Lenzburg in der Schweiz.

Bot. Zeit. 1830. II. p. 633 .

Zuccarini, über den Utriculus der Carices. Bot. Żeit. 1827. I. p. 119.

Beschreibung ron zwei neuen Arten Cyperus des nördliçhen Amerika, und von vier Arten Kyllingia von der Küste Brasiliens und den Ufern des Platastroms.

Transact. of the Americ. philos. Society $I .2$. new series $p .167$.

Es sind: Cyperus sparsiflorus und ovatus Kyllingia tenuis, rigida, leucocephala, вquarrosa.

Cyperaceac der Reliquiae Haenkeana.

Siehe Linnaea 18\%8. IV. p. 176.

Einige Benerkungen über Kapische Restiaceen, Cyperaceen und Gramineen, von Herrn Dr. Ste thel in Elslingen.

Bot. Zeit. 18\%9. I. p. 129. II. p. 465.

Didymonema nov. plant. gen. descripsit C. B. Presl. Pragae 30. Maji 18\%9. 4. c. tab. aen.

Lepisia nor. pl. gen. descripsit. C. B. Presl. Pragac 30. Maji 1829. 4. c. tal). aen.

Siehe Linnaea 1830. II. p. 51.

Einige Wortc über Scirpus Holoschocnus von Herrn Hofrath Reichenbach in Dresden.

Bot. Zeit. 1830. II. p. 489. 


\section{-71
Restiaceen.}

Beitrag zur Kenntnifs der Familie der Restiaceen in Rücksicht auf Gattungen und Arten, von Dr. C. G. Nees von Esenbeck.

Linnaea 1830. 4. p. 627.

\section{Junceen.}

F. G. Rostkovius. Monographia generis Junci. Berol. 1801. 8.

E. Meyer, Junci generis monographiac specimen. Goettingae 1819.

I dem Synopsin Juncorum rite cognitorum. Ad inaugurandam ejusdem plantarum generis monographiam. Goetting. 1822.

Idem. Synopsis Luzularum rite cognitarum cum additamentis quibusdam ad Juncorum synopsin prius editam. Goett. 1823.

Ess a i d'une Monographie des vraies Joncées, comprenant les genres Juncus, Luzula et Abama, par II. Je an de la Ha r pe.

Mém. de la Soc. d'hist. natur. de Paris. T. III. p. 89. u. d. f.

Junceac collectae a Chamissone in itinere speculatorio Romanzoff. descript. a E. M cyer Prof. Region.

Linnaea. 1828. 4. p. 367.

Jun c e e der Reliquiae-Haenkeanae.

Bot. Zeit. 1828. II. p. 442.

Geographische Verbreitung der Juncus tenuis.

Messager des Sciences et arts 1. et 2. livrais. 1825.

Ueber die Zahl der Stanbgefäfse des Juncus conglomeratus und J. effusus von Hoppe.

Bot. Zeit. 1826. 1. p. 65.

Beschreibung der Kingia von Robert Brown.

Isis 1828. p. 175. Bd. XXI. Heft 2.

\section{Commelinaceen.}

Commelinea der Reliquiae Haenkeanae.

Siehe botan. Zeit. 1828. II. p. 440.

Bemerkung über die Tradescantia Zanonia, welche in den Gärten cultivirt wird, von Carl Bouché.

Verhandl. der Gesellsch. naturf. Freunde in Berlin. I. Stück 6. p. 392.

Die fragliche Pflanze ist eine von der wahren T. Zanonia verschiedene Art, und heijst jetzt T. marginata.

\section{Haemadoraceen.}

Pedilonia, novum plantarum genus. Descripsit C. B. Presl, in museo boh. custos, botan. Prof. extraord. Pragae 15. Maji I829. 4. c. Icone.

Siehe bot. Zeit. 1829. II. p. 568 . Linnaea 1830. II. p. 50 Lit. (Ist nach Tausch Wachendorfia hirsuta L. Bot. Zeit. 1830. II. p. 556.

Conostylis aculeata; schr genau beschrieben von Dr. Graham in Jameson New Edinb. philos. Journal Jan. - April 1830. p. 877.

\section{Irideen.}

Re, le Iridi coltivate. Padova 1823. 8. 
Bemerkungen üher Iris florentina: vom Herrn Prof. 'T'ausch.

Bot. Zeit. 1828. I. p. 234. siche auch 1830. II. p. 557.

Bemerkungen über Iris florentina und pallida vom Herrn Dr. Fresenius.

Daselbst 1830. II. p. 425.

Einige Beobachtungen über dic Gattung Crocus von Carl Bouché.

Linnaea 1826. p. 227.

Memoria sulle specic e varieta di Crochi della flora Napolitana. Del Dottore Mich. Tenore. Neapel 1526. 4. m. K.

Linnaca 1828. Lit. p. 96.

Bertoloni: Descrizione delle spezie italiane del genere Crocus. Bologna 1826. 4.

Siehe botan. Zeit. 1826. II. p. 577. Nuov. Collez. di Opusculi scientif. 1S26. p. $145-151$.

Ueber Safran-Arten von Gay.

Bulletin des Sciences naturelles Juillet 1827. p. 316 - 373. siehe cuch botan. Zeitung 1622. II. p. 551.

Ueber die Knollen oder Zwiebeln des Safrans ron Bory.

Isis Bd. VI. p. 592.

Harworth über die Cultur und die Arten von Crocus.

Siehe bot. Zeit. 1821. II. p. 656.

Hoppe über einige Arten von Crocus.

Daselbst 182t. I. p. 76.

Traclisel, Bemerkungen über Crocus vernus.

Daselbst 1825. II. $p$. 700 .

Bartling, Bemerkungen über einige Arten von Crocus.

Daselbst 1826. I. Beil. 71.

C. L. Goldbach, Dissertatio inauguralis Croci historiam botanico medicam sistens. Moscov. 1816. $54 \mathrm{~S}$. 8.

\section{Amaryllideen.}

A. 'Treatise on bulbous Roots. ete. Ahhandlung von den Zwiebelpflanzen, enthaltend eine botanische Beschreibung der Pflanzen aus den Gattungen Amaryllis, Cyrtanthus, Crinum et Pancratium, von William Herbert. London 18\%4.

Amaryllideae der Reliquiae Haenkeanae.

Siehe bot. Zeit. 1828. II. p. 433.

Ueber Amaryllis gravina rom Baron Tommasio Melazzo.

Giorn. di Scienz. Lett. ed Arti di Siciliu No. 2t. et Revista generale di Scienze, Lettere ed Arti per regne di due Sicilie An. 1825. fascic. $I$.

\section{Bromeliaceen.}

Bromeliaceen der Reliquiae Haenkeanae.

Siehe bot. Zeit. 1828. II. p. 435 .

Conte.

Neue Arten von Tillandsia in Nord-Amerika von John le Annals of the Lyceum of Nat. history of New York. 1826. Vol. II. p. 129. 
Es sind: Tillandsia utriculata, juncea, pinifolia, Bartrami, cespitosa, recurvata, usneoides.

Den americanska $A$ gaves eller den så kallade hundradeariga Aloe'ns Natural - Historia af J. E. Wikström. Stockholm 182S. 8. Siehe Linnaea 1830. III. p. 89. Lit.

Ueber mehrerc Arten von Pitcairnia sehe man Schrader's Blumenibachia. p. 24.

\section{Asphodeleen.}

Die zahlreichen Gattungen dieser Familie werden von Bartling in fünf Stämme abgetheilt, nämlich die Yuccea, Hemaracallidea, Anthericea, Scillea, Tulipea, wozu noch andere Gattungen kommen, die theils den Colchicaceen, theils den Allien, theils den Commelinaceen verwandt sind, wozu endlich noch mehrere Genera kommen, deren Affinität zweifelhaft ist.

$\mathrm{Z} u$ bemerken sind lier nachstehende Aufsätze:

Sur la nonvelle famille des Gillesiées, par M. John Lindley. Annales des Sc. natur. Nov. 1826. p. 26i; - 273.

Bemerkungen über mehrere Arten von Tulipa aus der Flor von Florenz, von Eugene de Rebaul. Antologia No. 82, 84. Nov, u. Dec. 1828. Vol. XXVIII. p. 290.

Bull. des. Sc. nat. Février 1829. p. 254.

Appendix ad nonnullarum specierum Tuliparum in agro Florentino sponte nascentium proprias natas; auctore Eugenio de Reboul. Florentiae 18\%3. 8.

Notice sur le Genre Pontederia, par M. Raspail.

Mém. du Muséum d'hist. natur. Sept. Année. Huit. Cahier $p$. 156. mit Abbildung.

Pontedereae der Reliquiae Haenkeanae.

Bot. Zeit. 1828. I. p. 352. ferner II. p. 436, wo die neue Gattung. Monochoria erörtert ist.

Ueber eine Art Ornithogalum. Vom Herrn Graf Leo Henkel v. Donnersmarck.

Bot. Zeit. 1819. I. p. อิ3.

Ornithogala der Flora von Dresden.

Das. 1822. II. p. 537 .

Beiträge zur nähern Kenntnifs der gelb blühenden Ornithogalen; vom Herrn Apotheker Hornung.

Das. 1824. I. $p$. 3 อె.

Dergleichen von Schulz in Zweibrücken.

Das. 1828. II. p. 662. mit Abbild.

Reichenbach, über Ornithogalum pusillum.

Das. 1829. 11. p. 617.

Schultes fil. über dieselbe Pflanze.

Das. 1830. I. p. 76.

Th h un berg, examen Liliorum japanicoruni institutum.

Mém. de l'Academ. de Petersbourg. III. p. 200. - Es ist da die Rede von Lilium pomponium, lancifolium, elegans, longiflorum, maculatum, japanicum, speciosum, cordifolium. 
Anthericum comosum, cine neue Pflanzen-Specics, aufgestellt von dem Grafen Kaspar von Sternberg.

Bot. Zeit. 1828. II. p. 609.

Ueber mehrere Arten von Drimia sehe man Schrader's Blumenbachia p. 28.

Bessera elegans. Genus novum IIexandriae Monagyniae, auctore J. H. Sehultes.

Linnaea 1829. 1. p. 121.

Ueber das Kícimen verschiedener Samen-Arten der Liliaceen und rerwandter Familien, von Dr. Kittel.

Bot. Zeit. 1830. II. p. 640.

\section{Colchicaceen.}

v. Voith, Beobachtung über Colchicum autumnale.

Botan. Zeit. 1826. I. p. 44.

Celchicum patens, eine neue Pflanzen-Species, beschricben von Herrn Dr. C. F. Schultz.

Das. p. 131.

Ueber die Melanthiaceen an Vorgebirge der guten Hoffnung, von Dr. v. Schlechtendal.

Linnaea 1826. p. 78.

Botanische Geschichte der Sippe Tofieldia, von J. S. Smith. Aus den Linn. Transactions in der 1sis 1825. Heft IX. p. 962.

Nachricht von mehreren amerikanischen Arten der Gattung Calochortus, von David Douglas.

Lond. hortic. Trans. T. VII. part. 2. p. 275 . Linnaca. $V$. p. 113. Lit.

A anteckening over het geslacht Calochortus. Door Jul. Herm. Schultes jun. etc.

Aus einer holl. Zeitschrift in der Linnaea. V.p. 185. Lit.

\section{Smilaceen.}

Sie zerfallen in zwei Abtheilungen, die Asparagea und Paridea, diese mit freien, jene mit verwachsenen Griffeln. (Bartling).

Generis Asparagi historia naturalis atque medica. Dissert. auet. Maurit. Bresler. Berolini 1826.

De Asparagi officinalis L. germinatione. Disseruit et pro venia legendi defendet Guilelmus Cruse, Dr. Regiomonti 1828. 8 .

Observations sur le Dracaena Draco L. pap Sabin Berthelot, Prof. Direct. du Collége de l'Orotava et du Jardin botanique etc., mitgetheilt und mit einer Einleitung versehen von Dr. F. C. Merten s, Prof. zu Bremén.

Nova acta physico medica Academ. Caesar. etc. Tom. X. p. 2. pag. 773 mit tab. $35-39 .-$ Annales des Sc. natur. Juin 1828. p. 137. - Brandes Archiv Bd. XXX. Heft. 2. p. 183.

Monographia generis Paridum, qua ad Scholas audiendas invitat D. C. F. Ledebour. Cum tabul, aenea." Dorpat, 1828. Fol. Bot. Literaturbl. II. p. 112.

Smilaceae der Reliquiae Haenkennae. Siehe bot. Zeit. 1828. II. p. 438. 


\section{Dioscoreen.}

Dioscoreae der Reliquiac Haenkeanae.

Siehe bot. Zeit. 1828. II. p. 439.

\section{Orchideen.}

Diese schöne Familie ist in neuerer Zeit mchrfach bearbeitet worden, besonders hat man ihre Blumentheile auf das genaueste untersucht und abgebildet, sowohl der systematischen Anordnung, als physiologischer Ansichten wegen, besonders haben sich die Herren Swartz, Richard, Trevira$n u s$ und viele andere um dieselbe verdient gemacht.

Claude Louis Richard, de Orchideis europaeis adnotationes. Paris 1817. 4.

Aubert du Petit Thouars, histoire des plantes Orchidées, recueillies sur les trois isles australes d'Afrique. Paris 1822. 8. arec fig.

Orchidearum sceletos, commisit Johannes Lindley, Londini 1826. IV. cum tab. trib. ligno inessis.

Wieder abgedruckt in der Linnaea 1827. p. 527. u. d. $f$.

J. Lindley. The genera and species of Orchideous Plants. Part. I. Malaxideae. gr. 8. London 1830. Treuttel et Würtz.

Remarks upon the Orchideous plants of Chile. By John Lindley. Esq.

The Quarterly Journal, January to March 1827. pag. 43 - 54.

Botan. Literaturbl. I. p. 54. Linnaea 1828. p. (68. Lit.

Monographie des Orchidées de l'ile Saint Maurice, par M. A. Richard.

Bulletin des Sc. natur. Juillet 1827. p. 345. Bot. Literaturbl. I. p. 60 .

Monographie des Orchidées des iles de France et de Bourbon, par M. A chille Richard.

Mémoires de la soc. d'hist. nat. de Paris. Tom. IV.p. 1 - $\mathbf{z 4}$. Bot. Literaturbl. II. p. 61.

Genera et Species Orchidearum et Asclepiadearum, quas in itinere per insulam Java collegerunt $D$. G. Kuhl et D. C. van Hasselt. Editionem et descriptiones curavit J. G. S. ran Breda, in universitate Prof. ordin., horti Gandavensis praefectus. Vol. I. Fascic. 1 et 2.

Messag. des Sc. et des arts 1827 - 1828. p. 393.

Orehideae arcticae descript. ab Adalb. de Chamisso.

Linnaea. 1828. 1. p. 25.

Orchideae der Reliquiae Haenkeanae.

Siehe botan. Zeit. 1828 I. p. 342.

Verhandeling over de Standelkruiden (Orchideac) van het Groothertogdom Luxemburg: medegedeeld door den Heer L. March and.

Bydragen tot de Natuurkundige Wetenschappen T. II. p. 423.

Linnaea 1829. I. p. 34. Lit.

Descriptions succinctes des Orchidées, qui croissent naturellement dans les environs de Falaise, par M. Alpbonse de Brébisson.

Mémoires de la Soc. Linn. de Calvadis. 1825. p. 367.

Remarks on the species of Corallorhiza indigenous to the United States. By Thom. Nuttall. 
Aus dem Journal of the Acalemy of nat. se of Philadelphia. Vol. III. in der Linnaca 1829. I. p. 43. Lit.

Colla Observations sur le Limodorum purpureum de M. de $I_{\text {a }}$ mark et création d'un nouvean genre dans la famille des Orchidées. Paris 18\%1. 8. avec une planche.

Iornschuch, über Ophrys atrata. Lindley.

Bot. Zeit. 1829. II. p. 607.

Stanhopea insignis Frost; cine südamerikanische Orchidec mit ausgezeichnet sichöner und grofser Blume, eine eigene Gattung bildend.

Aus dem Botanicul Magazin in dem Bull. des Sc. nat. Janv. 1830. p. 101.

\section{Scitamineen.}

Ilerr Dr. Bartling theilt diese Familie in zwei Ordnungen, die Amomeac und Canneae, besonders aus dem, mir eben so wichtig als richtig scheinenden Girundsatze, weil neben einigen Verschiedenheiten der Structur, erstere reich an aromatischen Bestandtheilen sind, welche letzteren mangeln.

Beschreibung verschiedener indischen Pflanzen aus der Klasse Monandria, welche zur Ordnung Scitamineae gehören, von W. Roxburgh.

p. 64.

Asiat. Research. T. XI. p. 518. Jahrb. der Gewächshunde. IIeft I.

Bemerkungen über Dr. Roxburgh's Beschreibungen der indischen P'llanzen ius der Monandria, von W. Roscoe.

Aus den Transuct. of the Linn. Soc. Sec. V. 7. Jahrbuch der Gewächsk. Heft 2. p. 59 .

Scitamincae der Reliquiac Haenkeanae.

Bot. 'Zeit. 1828. I. p. 319.

Mémoires sur le Canna indica et sur les familles des Balisiers et des Bananiers, par M. Them. Lestiboudois.

Recueil des traveaux de la Soc. d'amat. de Lille 1823 et 1824.

Linnaea 1829. Lit. p. 23.

Mémoire sur le Canna indica et sur les familles des Balisicrs et des Bananiers, par M. Th. Lestiboudois. Lille 1829.

Man sehe Bibliothèque universelle Aoút 1829. p. 345.

Eine Ucbersicht der Gattung Calathea gibt Lindley im Januarheft 1829 des Botanical - Register;

siche Bull. des Sc. natur. Aoút 1829. p. 235.

Notice sur le genre Hedychium de la famille des Musacées par Th. Lestiboudois.

Annales des Sciences naturelles Juin 1829. p. 113. u. d. f.

Nota. Bartling bringt die Gattung Hedychium zu den Amoineen.

Monandrian Plants of the Order Scitamineac. By W. Roscoe. Liverpool 1828 - 1829. I. Vol. Fol.

Siche Bibl. universelle Sept. 1830. p. 72.

\section{Palmen.}

Palmarum familia ejusque genera, denuo illustrata. Programma quo praelectiones academicas indicit. Dr. C. F. P. de Martius. Monachii 1824. 4. 
C. F. P. de Martius. Genera et species Palmarum, quas in Brasilia collegit. Monachii 1824. seq. gr. fol. cum 108 tabulis.

Siehe Linnaea 1826. $p$. 11 .

Drei neue mexicanische Palmen, bestimmt rom Hofrath Dr. v. Martius in München. (Chamaedorea Schiedeana, elegans, elatior.) Linnáa 1830. p. 204.

Ueber die geographische Verbreitung der Palmen ron Schouw Edinb. philosophical Journal 1825 u. 1826 durch melivere Hefte..

Ueber die Dattel-Palme in Italien von Dr. Brizner. Annalen der allgemeinen Schweizer Gesellschaft für die gesammten Naturwissenschaften. Band II. Heft 2. p. 192.

Herr Dr. B. fand in Neapel ein Exemplar, fast 200 Jahre alt; zu Terracina einen männlichen und einen weiblichen Baum, zu Rom fünfe; in Pisa gedeiht die Dattel noch, in Floren z ertrügt Chamaerops humilis kaum die Kälte.

Nachrichten von einem Chamacrops humilis des bot. Gartens zu Berlin von Otto.

Verhandl. des Vereins zur Beförderung des Gartenbaues in Preufsen. Erste Liefer. S. 34.

Germination du Cocos nucifera par Poiteau.

Annales de l'institut hortical de Fromont Aout 1829. p. 173.

Doppelte Cocosinul's.

Froriep's Notizen Bd. XVIII. p. 74.

Dissertatio botanica de Palmis, praeside Thunberg. Pars $I$. resp. O. U. Marin. Pars II. resp. C. S. Hultström. Upsaliae 1825. 4.

\section{Aroideen.}

Sic werden von Herrn Dr. Isartling in zwei Ordnungen getheilt, nemlich die Callaceac und Orontiaccac; erstere zerfallen wieder in die Cyclanthea, Callea und Pathoina, wozu noch als veruandte Gattungen Ambrosinia und Pistia kommen. Die Orontiaceen werden in die ächten (genuina), wozu Acorus, Orontium und Rohdea gehören, sodann in jene getheilt, die den Callaceen näher stehen, wie Dracontium us. $s, w$.

Aroideac der Reliquiae Hacnkeanae.

Siehe bot. Zeit. 182S. II. p. 445.

Cryptocoryna Fischer, näher beschriehen von Wydler.

Linnaea 1830. p. 428.

Bemerkungen über das Vaterland des Acorus Calamus $L$. von Dierbach.

Bot. Zeit. 1828. II. p. 545.

Ueber das Vaterland des Calmus (Aeorus Calamus L.) von Herrn Dr. A. K. Goep pert, Privat-Docenten an der Universität zn Breslau. Daselbst 1830. II. p. 473.

\section{Najadeen.}

Species generis Potamogeton.

Linnaea 1827. II. $p$. 149.

Potamogeton inontanum Presl.

Bot. Zeit. 1829. I. p. 341. 
Observatio de Zanichellia, auctore F. G. Drees.

Linnaca T. II. p. 237.

Altheniae novi plantarum generis descriptio, anetore F. Petit.

Annales des Sciences d'Observation. Mars 182!). p. 451.

Die Pflanze (Althenia filiformis), welche diese neue Giattung bildet, vächst in einem See der Insel Camargue im Departement der Rhone-Mündungen; sie ist die Zanichellia stipularis einiger Herbarien. Ihren Namen erhielt sie zu Ehren eines Herrn Althen, der den Krappbau in den sülllichen Provinzen Frankreichs einführte. -

Hierher gehört allem Ansehen nach die Belvalia a ustral is Delille oder Zanichellia vaginalis.

Siehe bot. Zeit. 1S30. II. p. 455.

Ueber die Gattung Najas sehe man Linnaea 1829. p. 498.

\section{Podostemeen.}

Sie zerfallen in die Juneaginea und Alismaceen. - Ueber mehrere hierher gehörige Pflanzen vergleiche man Linnaea 182\%. p. 149.

Tristichia be faria der Hänkischen Sammlung.

Bot. Zeit. 1828. I. p. 311.

Triglochis striatúm. Daselhst p. 541.

Botanisehe Bemerkungen über Stratiotes und Sagittaria ron E. F. Nolte. Koppenhagen 1S25.

Sur une variété gigantesque de la Sagittaire commune; par M. Ch. Des Moulins.

Bull. de la Soc. Linn. de Bordeaux. XI. livrais. Juill. 1826.

\section{Butomeen.}

Nur die Gattungen Limnocharis, Hydrocleis und Butomus begreifend.

Ueber Limnocharis Haenkei

sehe man botan. Zeit. 1828. I. p. 342.

\section{Hydrocharideen.}

Sie bilden nach Bartling eine eigene Klasse, die jedoch nur aus ciner einzigen Familie besteht, enthultend die Gattungen: Hydrocharis, Limnobium, Damasonium, Stratiotes, Vallisneria $u$. s. $w$.

Botanische Bemerkungen über Stratiotes und Sagittaria, von C. F. Nolte. Koppenhagen 1825. 4. mit 2 Kupfertafeln.

Siehe Jahresbericht der schwed. Akademie. Bjonn 1828. p. 150.

Monocotyledonische Gattungen, deren Stellung im System noch ungewifs ist, sind nach Herrn Dr. B. Ananthopus, Apidistra, Bulbospermum, Floseopa, Hollböllia, Hydrostachys, Ixiolirion, Lemna, Lewisia, Oneus, Phytelephas, Roxburghia, Vellozia, Wolfia.

Ueber Lemna wird Herr Prof. Horkel in Eerlin cine cigne Abhandlung herausgeben.

Isis 1829. $p .385$.

Ueber die Affinität der Vellozia schrieb kürzlich Herr Don eine Abhandlung, er hält sie nebst der Barbacenia und Xerophyta für Glieder der Hipoxydeae (Jameson New philos. Journ. Jan. 1830. p. 16t). Herr Dr. Bartling rechnet Barbacenia zu den Haemadoraceen und Xerophyta zu den Bromeliaceen. - 
Ueber die zu den Endogenen gehörende, von Schulte 8 aufgestellte Gattung Weldenia sehe man bot. Zeit. 1829. I. p. 1.

\title{
Vierte Section.
}

\section{Exogenen oder Dicotyledonen.}

\section{Chlamydoblasta.}

\author{
Exogenen mit verhülltem Embryo.
}

§. 15.

Classis XIX. Aristolochieae. O. 53. Balanophoreae. 54. Cytineae. 55. Asarineae. 56. Tacceae.

C1. XX. Piperinae. 0. 57. Saurureac. 58. Piperaceac. 59. Chlorantheae.

C1. XXI. Hydropeltideae. 0. 60. Cabombeac. 61. Nymphaeaceae. 62. Nelumboneae.

\section{Cytineen.}

Cytineae. Ad. Brongniart in Annales des Sc. naturelles 1524. I. p. 29.

Herr Dr. B. rechnet dahin: Apodanthes, Cytinus, Sarcophytum, (Ichthyosma Schi.) und als verwandte nüher $z u$ untersuchende Gattungen: Gonyanthes, Rafflesia und Aphyteja. Die hierher gewöhnlich gerechnete Nepenthes silheint ihm, und zwar üuhl mit Recht, der Typus einer eigenen Familie zu scin.

Nachricht von einer neuen capischen Pflanze, Ichthyosma Wehdemanni, von Herrn v. Schlechtendal.

Linnaea 1827. p. 671.

Nachtrag zu diesem Aufsatze.

Daselbst 1828. p. 194.

Nachricht ron einer nenen Pflanzengattung, genannt Rafflesia.

Robert Broun's vermischte Schriften. II. p. $605-674$.

Etwas über die Rhizantheae, einc neue Pflanzenfamilie und die Gattung Rafflesia insbesondere, durch C. L. Blume; mitgetheilt von $\mathrm{Nees} v$. Esenbeck.

Bot. Zeit. 1825. II. p. 609.

Kurze Beschreibung des Patma der Javaner von C. L. Blume. Batavia 1825. (Raffl. Arnoldi.)

Bulletin des Sc. natur. Janv. 1826. p. 75. - Geiger's Magazin Juni 1826. p. 264.

Ueber ein neues Pflanzengeschlecht: Brugmansia, aus der natürlichen Fanilie der Rhizantheen, von Herrn C. L. Blume.

Bydragen tot de Naturk. Wetenschappen T. II. p. 419. Linnaea 1S29. p. 34. Lit. Man vergleiche besonders die Flora Javae von Blumè.

Nouvelles observations sur le genre Nepenthes extraites d'unc lettre addressée ì M. Ad. Brongniart, par M. Nees d'Esenbeck. Annales des Sc. natur. T. III. p. 365.

Account of Nepenthes destillatoria mas etc., which have lately 


\section{$-\quad 80$}

flowered in the Royal Botanic Garden, Edinburgh. Communicated by Dr. Grahim.

Jumeson New Philosophical Journal. Jul. - Sept. 1827. p. 371.

Herr Professor Reinwardt will die Arten von Nepenthes auf drei nack der Form der Kapseln beschäult wissen; nemlich N. destillatoria, phyllamphora und maxima.

Bull, des Sc. natur. Juillet 1829. p. 85.

Nepenthes destillatoria foem. ist sehr genau beschrieben und alugebildet von Dr. Graham: er zeigt besonders, dafs die P'llanzc zu den Dicotyledonen gehört.

Jameson New Edinb. philos. Journal. Jan. - April 1880. p. 379.

\section{Tacceen.}

Tacceac Presl in reliquiis IIaenkeanis. III. p. 489.

Fon Tacea integrifolia wird ein eigenes Genus, Ataecia, gebildet.

\section{Saurureen.}

De Ionttuynia atque Saurureis disseruit E. H. F. Meyer. Regiomonti 1827.

Botan. Zeit. 1828. II. Ergeünzungsbl. p. 39.

\section{Piperaceen.}

Observations sur la structure des Poivres par C. L. Blume.

Annales des Sc. naturelles. T. XII. Octobie 1827. p. 216 - 224.

Piperaceae der Reliquiae Hacnkeanae.

Linnaea 1828. 4. p. 175. Lit.

\section{Nymphaeaceen und Nelumboneen.}

Sprengel über den Ban des Nelumbium speciosum.

Neue Entdeck. II. p. 176.

Ueber den Cyamus und Latus der Aegyptier und der Griechen, von IIerrn Custos Leopold Trattinnick.

Bot. Zeit. 1822. II. p. 577.

Observationes in Semina et fructus Nymphaeacearum, auctore II. Mertens et C. L. Goldbach.

Mémoires de la Soc. impér. des Naturalistes de Moscou. 1823.

T. VI. p. 187. Bulletin des sc. natur. Mai 1826. p. 56.

Sur les affinités naturelles de la famille des Nymphaeacées; par M. Decandolle.

Mémoires de la Soc. de Physique et d'hist. natur. de Genève.

T. I. p. 1. 1821. p. 209.

Spenner. Ueber Nuphar minima Smith.

Bot. Zeit. 1827. I. p. 113.

Description of a new genus of plants belonging to the order Nymphaeaceae: in a letter to $\mathbf{H}$. T. Colebrocke etc., by Nathanacl Wallich. (Barclaya)

Transactions of the Linncan Society Vol. XV. p. 2. p. 442 et tab. 18. Linnaca 1828. III. p. 144. Lit. Isis 1830. p. 167. 


\section{$-81$ \\ Fünfte Section.}

Gymnoblasta apetala.

Exogenen mit nacktem Embryo und ohne Corolle.

§. 16.

Classis XXII. Coniferae. O. 63. Cycadeae. 61. Abietinae. 65. Cupressinae. 66. Taxinae.

Classis XXIII. Amentaceac. 0. 67. Casuarineae. 68. Myriceae. 69. Betulaceae. 70. Cupuliferae. 71. Ulmaceae.

Classis XXIV. Urticinae. 0. 72. Monimicae. 73. Artocarpeae. 74. Urticeae.

Clas sis XXV. Fagopyrinae. 0. ร5. Polygoneae. 76. Nyctagineae.

Clas sis XXVI. Proteinae. 0. 77. Laurineae. 78. Santalaceae., 79. Elaengneae. 80. Thymelaeae. 81. Proteaceac.

Classis XXVII. Salicinac. 0. 82. Salicinae.

\section{Cycadeen.}

I. C. Richard, Botanices Professoris ete. Commentatio botanica de Coniferis et Cycaideis, characteres genericos singulorum utriusque familiae et figuris analyticis eximie ab antore ipso ad naturam delineatis ornatos complectens. Opus posthumum ab Achille Richard perfectum et in lucem editum. Stuttgardtiae 1826. Fol.

Siehe Linnaea 1827. pag. 496. Botan. Literaturbl. I. p. 405.

Recherches sur la structure des tiges des Cycadécs, par M. Adolph Brongniart.

Annales des Sciences naturelles. Avril 1829. p. $389-401$.

Ueber den Bau der weiblichen Blüthen der Cycadeen von Robert Brown.

p. 211.

Isis Bd. XXI. Heft 2. p. 176. Annales des Sc. natur. T. VIII.

Ueber die Vermehrung der Cycadeen aus den Schuppen ihrer bercits abgestorbenen Stämme, vom rufs. Kais. Obergärtner F aldermann zu Petersburg.

Verhandl. des I'ereins zur Beförderung des Gartenbaues Bd. III. p. $312-316$.

Nachricht über die im Gräf. Harrachischen Garten zu Bruck an der Leitha blühende weibliche Sago-Palme (Cycas revoluta) von Ch. Lü beck, Garten - Director.

Daselbst. Neunte Liefer. Berlin I828. p. 227.

Beschreibung einer blühenden Zamia horrida von Herrn Schelhas in Cassel.

Das. Zehnte Liefer. p. 186.

Ueber die Stellung der Familien der Cycadeen und Zapfenbäume im natürlichen Pflanzensysteme, von Dr. Kittel.

Bot. Zeit. 1830. II. p. 617.

\section{Abietineen.}

L. C. Richard. Commentatio botanica de Coniferis et Cycadeis. Stuttgardtiac 1826. p. 145 . 
E s s ai sur la distribution géographique des Coniféres, par M. Mirbel. Mémoires du Museum d'hist. natur. Sept. Année, premier Cahier T. XIII. Paris 1825. p. $28-76$.

Ueber die Tannenwälder Würtembergs und ihre Behandlung, so wie über die geographische Verbreitung der Nadelhölzer von Rogg. Correspondenzblatt des würtemb. Landwirthschaftl. I ercins. März 1826. p. 139 u. d. f.

Einige Bemerkungen zu Merrn von Rogg's Aufatz über die Tannenwailder Würtembergs, vom Forstinspector Emil André. Daselbst April 1826. p. 211. $u$. d. f.

Aylmer Bourke Lambert. Description of the genus piuns T. I. London 18U3. T. II. Lond. 1825. Fol. with plates.

Ueber den Bau der weiblichen Blüthe der Coniferen und Cyeadeen von $\mathrm{Rob}$. Brown.

Annales des Sc. natur. Juin 1826. p. 211 - 214. 1sis Bd. XXI. Heft 2. p. 176.

Note sur un effet en apparence très singulier, qui présente la coupe transversale du Pin maritime aux endroits des verticilles; par M. Ménard de la Graye; lue à l'Acad. roy. des Sc. le 18. Dec. 1826 . p. 169.

Nouv. Bull. des Sc. par la Sociélé philomatique Nov. 1826.

Ueber die indische Ceder, Pinus Deodora Roxburgh. Annales des Sc. naturelles Octobre 1825. p. $134-133$.

Neue Art Pinus in den vereinigten Staaten von Nordamerika. Revue Britannique. Mars 1827. Froriep's Notizen Bd. XVIII. p. 6.

Es ist dieses Pinus Lambertiana, wovon das Nähere in folgendem Aufsatze sich befindet. (Zucker-Fichte)

An account of a new species of Pinus, native of California: in a letter to Joseph Sabine ete., by M. Darid Douglas etc.

Aus den Linn. Transactions in der Linnaca 1828. III. p. 145. Lit. Isis 1830. p. 167.

\section{Amentaceen.}

Lange liefs man die Familie der Amentaceen unverändert stehen, wie sie Jussieu einst geordnet hatte; in neueren Zeiten aber glaubte man die Nothwendigkeit bemerkt zu haben; dafs sie in mehrere getrennt werden müsse, indessen sind diese Abänderungen in verschiedenem Sinne vorgenommen worden. Richard unterscheidet Salicineae, Myriceae, Bctulineae, und Cupuliferae; Lestiboudois hat nebst diesen noch aufgenommen: Corylaceae Mirbel, Ulmaceae und Plataneae. Fuhlrott liefs die Familie selbst unverändert, nimmt aber fünf Subordines an; Reichenbach brachte die Myriceae zu den Taxeen $u$. s. w. u. s. w. Die Ansicht des Dr. Bartling geht aus der oben angeführten Synopsis schon hervor; das IVesentlichste dabei aber ist, dafs die Salices weit von den übrigen Amentaccen entfernt werden, was allerdings nicht ohne Grund geschah. -

Ueber die Gattung Salix und die natürliche Familie der Amentaceen, von B. C. Dumortier.

Bydragen tot de natuurkundige Wetenschappen. I. p. 44.

Mirbel hat gelegentlich in seiner Abhandlung über die geographische Verbreitung der phanerogamischen Gewächse in der alten Welt, mehrere seltene oder neue Amentaceen beschrieben und abgebildet, wic Salix colu- 
teoides, Alnus castaneaefolia, A. acuminat. Fumb. et Bonpl. Fagus obliqua, F. Dombeyi, F. betuloides, F. dubia, Myrica macrophylla. M. spathulata.

Man sehe Mémoires du Museum. T. XIV.

\section{Artocarpeen.}

Observations sur un nouveau genre (Sychinium) de la famille des Urticées, et description de trois nouvelles éspèces du genre Dorstenia, par M. N. A. Desveaux.

$$
\text { Annales de la Soc. Linn. de Paris. Juillet } 1825 .
$$

Ueber eine neue Maulbeer-Art (Morus Morettiana) von Francesco Gera.

Aus dem Giornale di Fisica in der Linnaea 1828. p. 95. Lit siehe auch das. III. $p .128$.

Ueber den Kuhbaum (Galactodendron utile) von Humboldt.

Isis Band II. $p$. 469.

Observations on the Cow - tree of the Caracas. In a letter from M. David Lockhart, Curator of the botanical Garden in Trinidad to Aylmer Bourke Lambert.

Jamesun New Edinb. Philosophical Journal. Jul. - Sept. 1827.

p. 385. Froriep's Notizen Bd. XIX. p. 202.

Dafs der Kuhbaum eigentlich Brosimum Galactodendron heifsen müfse, wird bemerkt in Jameson New philos. Journal Jan. 1830. p. 171; er gehört also in die Familie der Urticeen, die aber sonst immer mit der der Artocarpeen vereinigt gelassen wurde. - Auch M. de Rivero und T.B. Bous singault haben über den Kuhbaum berichtet. Man sehe Edinb. Philos. Journ. 1825. No. 13. p. 97 - 102. und Schwedischer Jahresbericht, Bonn 1828. p. 162, wo er in die Familie der Sapoteen gerechnet und Galactodendron americanum Humb. genannt wird.

\section{Polygoneen.}

Monographiac generis Polygoni prodromus, auctore Car. Fricd. Meisner', M. D. accedunt tabulae aeneae VII. Genevae sumpt. auctor. 1825. 4 .

Siehe Linnaea 1827. p. 453.

Polygonea collectae in itinere speculatorio Romanz.

Linnaea 1828. I. $p$. 36.

Ueber Varietäten und Bastarde der Arten von Polygonum, von Lasch in Driesen.

Daselbst 1829. III. p. 424.

Ueb er Polygonum viviparum.

Bot. Zeit. 1830. II. p. 599.

Examen de deux mémoires de physiologie végétale, suivi de l'examen d'un passage d'un troisième mémoire, publié a Genève, comme les deux autres, sous le titre de Monographie generis Polygoni prodromus. Paris 1828. 8.

Von Dupetit-Thouars über die Zahl der Filamente bei Po-

Iygonum. Siehe Bull. des Sc. nat. Janv. 1828. p. 66. Linnaea 1828.

III. $p$. 126. Lit.

O bservations on the genus Eriogonum and the natural order Polygoneae of Jussieu. By Thomas Nuttall.

Journal of the Academy of natural sciences of Philadelphia.

Vol. I. p. 24. Linnaea 1828. p. 161. Lit. 


\section{$-84$}

Sur le Rumex nemorosus Sehr. et sur le Rumex Nemolapathum L. fil. par M. Desmazières.

Bulletin des Sc. natur. Juin 1828. p. 2234.

Die ächte Rhabarber nach Don. p. 109.

Edlinb, new. philos. Journal March. 1827. Bot. Literaturbl. II.

Note. Die nüheren Nachrichten über die Minulayu-Rhaburber und andere, die medicinische Botunik nüher angehende Gegenstünde, kommen im zueiten Bande vor. -

\section{Laurineen.}

De Cinnamomo. Disputatio, qua hortum medicum Bonnensem felicitcr instructum, rite inanguraturi res ejus viris rei herbariate studiosis commendant C. G. et Th. F. L. Nees ab Esenbeck, fratres. hurto medice Bonnensi praepositi. Cum tabulis septem iconographicis. Bonnae 1823. 4 .

Ueber den gemeinen Lorbecrbaum rom Abbé Salvadore Portal.

Giornale di Scicnza lettere ed arti di Palermo. Nov. 1821.

Ueber Laurus glandulifera Wallich.

Aus Transactions of the med. and physic. Journal of Calcutter. in Brandes Archiv Bal. XXV. Heft 2. p. 170.

Ueber den Nelkenzinmtbaum, von Herm Hofrath v. Martius in München.

Bot. Zeit. 1830. I. p. 17.

Dr. Blume stellt in den Beiträgen zur Flora des holländischen Indiens eine neue, zunächst mit den Laurineen verwandte Familie auf - II ernandieae, aus den Gattungen IIernandia und Inocarpus bestehend. Isis Bd. XXVIII. p. 2(i3.

\section{'Thymelaeen.}

Enumeratio specierum generis Daphnes; anctore Joh. Em. Wikstroem, Med. Doct. Stokholmiae 1820. 8. Typis E. A. Ortmann.

Dissertatio botanica de Daphne, anct. Joh. Em. Wikstroem, M. Dr. Editio altera emendata et aucta. Stolholmiae 1820. 4. 'Typis A. M. Strickholm et $\mathbf{C}$.

Beschreibung einer neuen Art Daphne ron Herrn Grateloup. (Daphne multiflora.)

Bullet. d'hist. nat. de la soc. Linn. de Borleuux II. p. 51. Mars 1828. Bot. Literaturbl. II. p. 108.

Darwinia fascicularis, als Gattung und Art beschriehen von Rudge.

Aus den Linn. Transactions in der Isis 1829. p. 146. (Man vergleiche die Myrtaceen.)

Ueber die Thymelacen und eine neue, ihnen verwandte Pflanzenfamilie die Penaeacen von C. S. Kunth.

Linnaiea 1830. IV. p. 667.

\section{Proteaceen.}

Ueper Jussieu's Proteacen won Robert Brown.

I'rmischte botanische Schriften II. p. 58. $u$. d. f. 
Thunberg. Descriptiones quatuor Proteae novarum specierum. (P. plumigera, coarctata, laevis, ovata.) Mémoires de l'Acad. de Petersbourg. VI. p. 516. t. 14 - 17.

\section{Salicineen}

De Salicibus europaeis commentatio; auctore G. D. J. Kock. Erlangae sumptibus Caroli Heyderi 1828. 8.

Abhandlung über das Geschlecht der Weiden und die natürliche Familie der Amentaceen ron B. C. Du mortier in Dornick.

Bydragen tot de Natuurkundige W'etenschappen. Amsterdam 1825. p. 41. Tom. I. No. 1. - Botan. Literaturbl. II. p. 110.

Classification der schwedischen Weiden, von Fries.

Physiograph. Saelskap Arsberaettel. Lund. 1825. p. 30. - Siehe Schwed. Jahresbericht Bonn 1828. p. 170.

Entdeckung des männlichen Stranches der Salix Forbyana Smith in Deutschland ron Weihe, mitretheilt ron Koch.

Bot. Zeit. 1830. I. p. 319.

Description of a Species of Salix found in Braemar. By W. Macgillivray.

S. Macnaliana, rerwandt mit S. prunifolia, carinata und Myrsinites. Jameson New Eillinb. Phil. Journal. July - Oct. 18æ0. p. 336.

$$
\begin{gathered}
\text { S e c h s t e S e c ti o n. } \\
\text { Gymnoblasta Monopetala. }
\end{gathered}
$$

Exogenen mit nacktem Embryo und einblättriger Corolle.

$$
\text { 5. } 17 .
$$

Classis XXVIII. Aggregata. O. 83. Plantagineac. 84. Plumbagineac. 85. Globularieae. 86. Dipsaceae. 87. Valerianeae.

Classis XXIX. Compositac. 0. 88. Calycereae. 89. Synanthereac.

Classis XXX. Campanulinae. 0. 90. Goodenovicac. 91. Stylideac.

92. Lobeliaceae. 93. Campanulaccae.

Class is XXXI. Ericincae. 0. 94. Vaccinceac. 95. Ericeae. 96. Epacrideae.

Clasis XXXII. Styracinae. 0. 97. Styraceae. 98. Ebenaccae. 99. Sápoteae.

Class sis XXXIII. Myrsineac. O. 100. Ardisiaceae. 101. Primulaceae. Classis XXXIV. Labiatiflorac. O. 102. Lentibulariae. 103. Scrophularinae. 104. Orobancheae. 105. Gessnerieae. 106. Sesameae. 107. Myoporinae. 108. Selagineae. 109. Verbenaceae. 110. Labiatae. 111. Acanthaceae. 112. Bignoniaceae.

Classis XXXV. Tubiflorac. O. 113. Polemoniaceae. 114. Hydroleaceae. 115. Convolvulaceae. 116. Cuscuteae. 117. Solaneae. 118. Hydrophilleae. 119, Borogineae.

Classi XXXVI. Contorta e. O. 120. Gentianeae. 121. Asclepiadeae. 122. Apocyneae. 123. Loganieae.

Classis XXXVII. Rubiacinae. O. 121. Lygodysadeaceae. 125. Rubiaceae. 126. Caprifoliaceac. 127. Viburneae.

Class is XXXVIII. Ligustrinae. 0. 128. Jasmineac. 129. Oleincac. 


\section{Plantagineen.}

Plantagineac collectae in expeditione speculatoria Romanzoffiana. Linnaea 1826. II. $p$. 165.

Esquisse de 1'histoire naturelle des Plantaginées, par M. Rapin. Annales de la Soc. Linn. de Paris. Nov. 1827. p. 437.

Don bestimmt die Stelle der Gattung Glaux im Systeme an das Ende der Familie der Plantagincen; er theilt ihre Charakteristik seler ausführlich mit, und spricht von ihrer I erwandtschaft mit den Primulaceen. Jameson New Philos. Journal. Jan. 1830. p. 166.

Ueber mehrere Arten von Plantago sehe man Schrader's Blumenbachia. $p$. 32.

\section{Globularieen.}

Monographie des Globulaires, par M. J. Cambessédes. Annules des Sc. naturelles. Tom. IX. Sept. 1826. p. $15-30$.

\section{Dipsaceen.}

Mémoire sur les Dipsacées, par Th. Coulter. Genève 1823.

Ueber den Pollen der Dipsaceen, von Dr. F. G. Bartling in Göttingen.

Linnaea 1828. p. 171.

Botanische Beobachtungen über einige Arten von Scabiosa, vom Herrn Prof. Tau s ch.

Bot. Zeit. 1829. II. p. 648.

\section{Valerianeen.}

Animadversiones botanicae in Valerianellas. Dissertatio inauguralis auct. E. F. Betcke. Rostochii 1826. c. tab. aen.

Siehe Geiger's Magazin Oct. 1826. p. 79.

Valerianeae collectae in expeditione speculatoria Romanzoffiana.

Linnaea 1828. II. $p$. 129.

\section{Synanthereen.}

Cassini und Bartling theilen diese grofse Familie in folgende Gruppen: Vernoniea, Eapatorina, Adenostylea, Tussilaginea, Mutisiea, Nassauviea, Senecionea, Asterea, Inulea, Anthemidea, Ambrosica, Helianthea, Tagetinea, Calendulacea, Arctotidea, Echinopea, Carduacea, Centaureacea, Carlinea, Lactucea.

Tableau synoptique des Synanthérées par M. II enri Cassini. Annales des Sc. natur. T. XVII. Aout 1829. p. $387-423$.

Frühere Untersuchungen über die Compositen theilte Cassini im ersten Bande seiner Opuscules phytologiques Paris 1826 mit. Die Arbeiten Robert Brown's über diese Familie sind im zweiten Bande der, vermischten botanischen Schriften enthalten.

Lessing de Synantheriis Herbarii regii Berolinensis dissertationes. Linnaea 1829. II. p. 240. III. p. 289. 1830. I. p. 1. II. p. 237. III. $p$. 837 .

Eв ваі monographique sur le Hieracium, et quelques genres voi-sins; par Auguste Mo nn ier. Nancy 1829. inprim. de Hissette. 92 page .

Mémoire sur le genre Absinthium par M. Besвer. 
p. $219-263$.

Artemisia glomerata Sieber. Die Mutterpflanze des barbarischen Wurmsaamens (Sem. Cin. barb.), beschrieben und abgebildet von J. B. Batka, mitgetheilt von F. Nees v. Esenbeck.

Nova Acta physico med. T. X. p. 2. p. 823. tab. 43. - Das

Nähere hierüber folgt im medicinischen Theile. husenia.

Ueber Barkhausia, Barckhausia, Borkhausia, Barkhausenia, BarkBot. Zeit. 18\%9. II. p. 510.

Bemerkungen über das Chrysanthemum indicum L. von J. Sabine. Linn. Transact. XIII. 2. Isis 1829. X. p. 1030.

Chrysanthemum indicum ist Matricaria sinensis Rumph. Amb. V. p. 259. tal 91. fig. 1. Chrysanthemum sinense ist Matricaria Kaempfer. Anthemis artemisiaefolia Willd. C. indicum Thunberg u. s. w.

An attempt at a new Classification of the Cichoraceae with some Observations on the geographical Distribution of this Family. By David Don. Jameson New Eidinb. Phil. Journal. Jan. - April 1829. p. 305. u. d.f. - Botan. Literaturbl. II. p. 71. - Linnaea V. p. 138. Lit.

Ueber Arnica und Doronicum von Herrn Prof. Tausch.

Bot. Zeit: 1828. I. p. 178.

Elephantopus Martii, sehr genau beschrieben vom Dr. Graham in

Jameson New Edinb. philos. Journal. Jan. - April 1830. p. 378.

Bemerkungen über Hieracium und einige verwandte Gattungen vom Herrn Prof. Ta usch in Prag.

Bot. Zeit. 1828. I. Ergäzungsblätter. p. 49.

$D a$ die Gattung Hieracium $\approx u$ den schwierigsten gehört, und die Synonymie, selbst der deutschen Arten, äufserst verwickelt ist, so verdient das Anerbieten des Herrn Heinhold in Dresden, die in der dortigen Gegend wild wachsenden Hicracien zu sammeln und tauschweise an andere Botaniker zu überlassen, besondere Beachtung.

Bot. Zeit. 1830.' I. p. 172.

Logurostemon Cassini, ausführlich erörtert von $H$. Wydler. Linnaea 1830. III. p. 425.

Ueber Leontodon palustre, ron Herrn v. Voith. Botan. Literaturbl. III. p. 534 .

Description d'une nouvelle éspèce de Seneçon (Senecio difficilis) par M. Leon Dufour.

Annales des Sc. natur. Aoút 1825. p. $428-430$.

Lessingia novum genus e familia Compositarum, auctore Adalb. de Chamisso.

Linnaea 1829. II. p. 203.

Diagnoses nonnullarum Solidaginis ot Helianthi species; a Prof. Tausch.

Bot: Zeit. 1828. II. p. 497.

Erigeron serotinus Weihe, eine neue Art.

Bot. Zeit. 1830. I. p: 258.

Erythrolaena conspicua Swett., eine mexicanische Pflanze und neue Gattung, in die Gruppe der Carduaceen gehörend. 
Aus dem Botanical Magazine in dem Bullet. des Sc. nat. Sept. 1829. p. 381.

Geigeria., genus e Compositarum fanilia, auctore Ludovico Griesseliạ, M. D.

Geiger's Maguzin Mürz 1831. p. 201. - Ist Dizonium longifolium Ilerbar. Willd.; siehe Linnucu 18iol. IIL p. 411.

A b hand l ung äber die Classification und Fintheilumg der Gattungen Guaphalium und Xeranthemum Linnate ven ILerrn D. Don.

Memoirs of the II ern. Socicty Iol. I. p. 503. - Lis kommen hier mehrere neue Gattungen vor, wie Lencostemma (Xeranthemum vestitum et lingulatum), Aphelexis (Xeranthemum sesamoides, Elichrysum fasciculatum u. 8. w.); Euchloris (Gnaphalium nudifolium).

Monographie dn genre Xeranthemum par M. Gay. Lue a la Soc. d'hist. nat. de Paris. Séance du !). Mars 1827.

Mémoires de la Soc. d’hist. nat. de Paris. T. III. Livr. 8. p. 325. Bulletin des Sc. natur. Avril 1827. p. 445.

Lasch über Sonchus arvensis und palustris.

Linnaéa 1829, III. p. 408.

Stylolepis norum Compositarum genus, auctore Lchmann.

Ibidem 1830. III. p. 385 .

Sauter über Picris crepoides und Carduus platylepis, zwei neue Tyroler Pflanzen.

Bot. Zcit. 1830. II. p. 409.

Lasch. Ueber verschiedene Formen von Scorzonera.

Linnaea 1827. p. 418.

Ueber den Werth der Unterschiede für Genera in der Familic der Compositae, von Dr. Griesselich.

Geiger's Magazin April 1830. p. 22.

8 chrader's Bemerkungen über mehrere Arten ron Senecio, Eupatorium und Echinops findet man in dessen Llumenbachia pag. 39 u. $d$. $f$.

Ueber den Ursprung und die Natur der zungenförmigen Strahlenblumen der Zinnia und über eine merkwürdige Vermehrung der Fructifications - Theile bei dieser Grattung, von D a v id Don.

Aus The Transact. of the Linnean Socicty of London Vol. XVI. in der Linnaea $V$. p. 168 Lit.

\section{Lobeliaceen.}

M émoire sur les Lobeliacées et les Stylidées nourclles familles des plantes, par M. A. Is de Jus sieu.

Uebersicht in der Isis 18\%6. Heft 8. p. 782 u. d. f.

Lobelia leptocarpa, beschrieben vou Griesselich.

Linnaea 1880. III $p .419$.

Cyphia volubilis $B$, racemosa.

Daselbst p.' 420.

Lobelia mollis et rugulosa; sehr genau beschricben vom Prof. Graham in Edinburg. New. Philos. Journal. Jan. 1830. p. 185.

Lobelia Kraussii; selur genau beschrieben von Dr. Graham in Jameson New philos. Journal. Jan. - April 1830. p. 378. 
Clintonia, eine neue Gattung, bestimmt nach einer in der Elene an Columbiaflusse wachsenden Pflanze von Dougla s (Clintonia elegans). p. 350.

Bottanical Register Nro. XV. - Bullet. des Sc.natur. Sept.1829.

\section{Campanulaceae.}

Monographie des Campanulées par A! ph. de Candolle. Paris 1830. 4. avec $20 \mathrm{pl}$. Chez M. V. Desray. 25 franes.

Thunberg. Campanulae capenses descriptae et depictac.

Mémoires de l'Acad. de Petersbourg $I V$. p. 361.

De Campanulis Suecon is. Dissertatio, quam venia exp. med. facult. U ps. P. P. Car. Pet. Forsberg Med. Dr. Ch. M. Botan. demenstrator E. O. et Joh. Phil. Arenander etc. Upsaliae 1829.4.

$Z_{w}$ eifel über die stigmatische Natur der Haare am Stylus der Campanulae ron Dr. Griesselich.

Geiger's Mag: April 1830. p. 3.

Wahlenbergia Schrader; siehe dessen Blumenbachia pag. 37.

\section{Ericineen:}

Diese Klasse begreift die beiden sehr nahe verwandten Familien der Vaccineae und Ericeae, deren Literatur hier zusammengefafst werden kann.

Epistola de Symphysia, novo genere plantarum ad ill. Lib. Bar. Joseph de Jacquin ete. data a C. B. Presl. 4to sine loco et anno. c. tab.

Siehe Linnaea 1829. I. p. 3. Lit.

Eric eae collectae in expedit. speculat. Romanz.

Linnaea 1826. IV. p. 511.

Obserrations sur deux noureaux genres de plantes par M. Degvea ux, Directeur du Jardin de Botanique à. Angers.

Hierher gehört nur das Genus Calodryum. - Annales des Sc. natur. Dec. 1826. p. $401-404$.

Ad a ms descriptio novae speciei Azaleae (A. fragrans).

Mém. de l'Acad. dé Petersbourg. II: p. 332. t. 14.

Geographische Verbreitung der Erica Tetralix von Ho ffmann, in seiner Schrift: De rallium in Germania boreali principalium directione memorabili congrua. Halae 1823. Drees.

Observationes circa Monotropam Hypopitys L. scripsit F. G.

Linnaea 1827. p. 237.

Mo notrope ae, ordo ut videtur proprius, descriptus a Dr. Bartling, in opere ordines naturales plantarum p. 156.

\section{Epacrideen.}

Epacrideac collectae in expeditione speculatoria Romanzoffiana. Linnaea 1826. IV. p. 539.

\section{Ebenaceae.}

Gaetzea Wydler, novun plantarum genus, e familia Ebenacearum. Linnaea 1830. III. p. 423 . c. tabul. 


\section{$-\quad 90$ \\ Ardisiaceen.}

Note sur l'Onestemum, nouveau genre de la famille des Ardisiacées, par M. Adrien de Jussieu.

Mém. du Mus. d'hist. nat. Dixième année deuxième Cahier p. 133.

Die neue Guttung ist folgendermafsen charakterisirt:

Calix 5 fidus partitusve. Corolla alte 5 loba. Stamina connata in masian ovoidcam cylindricamve, basi cum tubo corollae coalitam, apice 5 dentatam; antherae (e quibus eonferruminatis, pars major massae stamineae) crassae introrsac biloculares. Stylus simplex. Stigma subinfundibuliforme subintegrum denticulatumve. Ovarium 4-2 ovulatum.

Die Gattung begreift 2 Arten, Strüucher aus Madaguskar: O. Commersonianum und 0 . Capellieranum.

\section{Primulaceen.}

Primulacea e collectae in expeditione speculatoria Romanzoffiana.

Linnaea 1826. II. p. 212.

Beschreihung einer neuen Art Primula, bezeichnet mit dem Namen Primula sertulosa.

Annales de la Soc. Linn. de Paris. Mars 1825.

Neue Untersuchungen über cinige Arten der Gattung Primula. Daselbst. Sept. 1825.

Ueber mehrere Primulaceen von Ta $\mathbf{T}$ ch.

Bot. Zeit. 1829. II. p. 644.

Notice of a new Genus of Plants, discovered in the Rocky Mountains of North America by M. David Douglas. By John Lindley. Esq. (Douglasia nivalis).

The Quarterly Journal of Science. New Series Nro. IV. Oct. to

January 1828. p. 383-385. - Bot. Literaturbl. II. p. 322.

Ueber zwei unbeliannte Arten von Cyclamen, von Herrn Professor Tauch in Prag.

Bot. Zeit. 1829. II. p. 667.

Ueber Androsace imbricata D. C. von Walkex-Arnott.

Aus James on's Phil. Journal in den botan. Literaturbl. IV. p. 41.

\section{Lentibularieen.}

Observations on the North American species of the genus Utricularia. By Capt. John Le Conte.

Aus den Annals of the Lyceum of Natural History of New York übersetzt in der bot. Zeit. 1827. I. p. 8.

Utricularia neglecta Lehmann.

Siehe Linnaea 1830. III. 'p. 386.

\section{Scrophularineen.}

Dr. Bartling theilt diese ausgedehnte Familie in fünf Gruppen : Verhascea, Antirrhinea, Veronicea, Budileja und. Rhinanthea, wozu noch mehrere andere Gattungen kommen, deren Stellung ungewifs ist. Verbascum wurde bisher zu den Solaneen gerechnet, von denen sic allerdings in mehrfacher Hinsicht abweicht, was aber eben so gut von den Scrophularien gilt, sie dirfte mit einigen andern Gattungen passend ẹine familia intermedia zwischen den Solaneen und Scrophularien ausmachen. 
Esaa monographique sur le genre Scrofularia, par Henry Wydler.

Mémoires de la Soc. de Physique et d'hist. nat. de Genève. Tom. IV. Livr. 2. p. 121 - 170. - Abgebildet sind Scrophularia Urvillei Wydl. auf zuei Tafeln, S. cretacea Fischer, S. hypericifolia Wydler. Diese Abhandlung ist auch unter derselben Aufschrift besonders abgedruckt worden. Gienève 1828. in 4.50 pages avec $5 \mathrm{pl}$. Recensirt ist sie in dem Bulletin des Sc. natur. Mai 1829. p. 2ł3 von Aug. Duvau; - auch sehe man Bot. Literaturblätter III. p. 523. lectae.

Scrofularineae in expeditione speculatoria Romanzoffiana colLinnaea 1827. p. 555. 1828. $p .1$ et sequent.

Monographia generis Verbasci, auctore H. A. Schrader. Sect. 1 . Gottingae 1813. Sect. 2. 1823. 4. cum tabulis aeneis.

Observationes in varias Verbasci species et varietates auctore Lasch.

Linnaea 1829. III. p. 420.

Ba Isam o - Crivelli Jos. Gabriel. De Solanacearum familia in genere, addita Verbascorum Italiae indigenorum Monographia. Ticini 1824. 8.

Colla Osservazioni sopra il Verbascum cisalpinum del Sign. Beroli.

Memorie della R. Acad. di Torino. Tomo XXVI. p. 507. - Die Pflanze wird für eine leichte Abart des Verbascum phoeniceun L. erklärt.

Ueber eine neuc Art Verbascum, von Prof. Aghard; nemlich Verbascum bracteatum: stellato - tomentosum, foliis lingulatis decurrentibus, fasciculis florum bractea acuminata brevioribus, staminum tribus et pistillo medio pallide pilosis.

In sterilibus prope Holmiam.

Phisiograph. selskapets Aarsberïttelse. Lund. 1825. p. 73. in Prag.

Ueber Verbascum thapoides L. von Hern Prof. Tausch Bot. Zeit. 1828. I. $p$. 46.

John Lindley Digitalium Monographia London 1821. royal folio with 28 cop. plates.

Description of a new Genus belonging to the natural family of plantes, called Scrophularinae. By M. David Don (Lophospermum).

Aus den Transactions of the Linnean Society of London Vol. $\mathbf{X V}$.

P. 2. p. 349 in der Linnaea 1828. p. 1 ët Lit. - Bot. Literaturbl. I. p. 452. - 1sis 1830. p. 164.

Observation s on the Characters and Affinities of Darwinia, Brunfelsia, Browallia, Argylia, Eccremocarpus, and of plants improperly referred to the latter genus. By David Don.

Jameson New Edlinb. Phil. Journal. April-July 1829. p. 83. -

Brunfelsia ist nach $D$ on synonym mit Franciscea Pöhl und gehört zu den Solaneen eben so wie Browallia (alle drei gehören nach Bartling zu den Scrophularien). - Aus dem Botanioal Magazine in dem Bullet. des Sc. natur. Oct. 1829. p.' 79.

Observations on the North American Species of the Genus Gratiola. By Capt. John le Conte.

Aus den Annals of the Lyceum of Natural History of New York in der botan. Zeit. 1827. I. p.12. 
Aceount of a uew Caleenlaria ete. (C. purpurea) which have lately flowered in the Royal Botamie Garden Eilinburgh. Communicated by Dr. Graham.

Jameson New Philosophical Joumal. Jul. - Sept. 1827. p. 371.

Ueber Celsia Areturus von Carl Bouché.

Die bisher unter diesem Namen bekannte Pflanze ist nicht die wahre Linneische Art, sic heifst jetzt Celsia glandulosa. Sieber brachte die wahre species ans. Kreta. - I crhandl. der Giesellsch. naturf. Freunde in Berlin I. Stück (6. p. 39).

Nuttall.

Deseription of Collinsia, a new genus of plants. By Thomas

Aus dem Journal of the Acadcmy of natural seiences of Philadelphia, in der Linnaea 1828. III. p. 163 Lit.

Nachricht ron der Limosella ternifolia von Nuttall.

Daselbst p. 168.

Lindenbergia, novum genus familiae Scrophular. auctore Lchma an (L. urticaefolia, planta Nepalensis).

Bot. Zeit. 1830. I. p. 1-t.

Considerations générales sur le genre Veronica, et sur quelques genres de familles ou sections voisines, par M. A ug. Duvau.

Annales des Sc. natur. Juin 18:65. p. 163-186). - (Ueber Veronica sehe man auch Linnaea 1827. p. 445).

Monographia Pedicularis, auctore C. Steven.

Mémoires de la Soc. imp. des Naturalistes de Moscou. Tom. IV. p. 1. Moscou 1823.

Notice sur un genre nouveau (Duricua spicata) de la famille des Pediculariées par F. V. II ér at. avec un fig. lith.

Extr. des Mémoires de la société acudemique de Lille. 1829.

Mémoire sur le genre Tozzia, par M. Aug. de St. Milaire.

Mém. du Museum d’hist. nuturelle. Sept. Année, huit. Cahier. p. 94 et suiv.

Note sur le Melampyrum pratense et silvaticum par Soyer Willemet.

Bulletin des Sciences natur. Février 1826. p. 229.

Ueber mehrere Arten und Varietäten der Euphrasia, von Lasch in Driessen.

Linnaca 1829. III. p. 405.

Descriptions of Colmellia, Tovaria and Francoa, with remarks on their Affinities. By M. David Jon.

Jameson New Eclinb. Phil. Journal Oct. - Dec. 1828. p. 46.

Die Gruttung Columellia uvurde sonst zu don Jasmineen gerechnet, sie soll aber nach Don cine eigrene kleine Familie bilden (Columellieae) grenzend einerseits an die Jasmincen, anderseits an die Ilalesiaceen, wicher letzteren ihre Stelle zwischen alcn Oleincis und Ebenaceis anzuweisen wäre.

Bullctin des Sc. nat. Sept. 18\%9. p. 416.

\section{Orobancheen.}

Mémoire sur la Germination des Orobanches, par M. Vauchex à Genève.

Mém. du Mus. d'hist. nat. de Paris. T. X. 
M. G. P. Va u cher Monographie des Orobanches, histoire générale et physiologique de ce genre de plantes; description de 29 éspèces, distribuées en deux tribus. Genève 1827. 4. avec fig.

Siehe Linnaea 1828. p. 56 Lit.

Orobanches generis Sureçevi apud Bremanos celeberrimun Epistola. Scripsit F. G. Wa llroth. Francofurti ad Moenum apud Fredericum Wilmans 1825. 8.

Orobancheae in expeditione speculatoria Romanzoffiana collectae. Linnaea 1828. II. p. 132.

Einige Worte über Orobanche; rom Herm Itofrath Reichenbach in Dresden.

Bot. Zeit. 1529. II. p. 396.

Beitrag zur Keuntnifs der deutschen Orobanchen von F. W. Schultz. Mit einer lithographirten 'Tafel. München 1829. Fol.

\section{Gesnerieen.}

Gesnera latifolia Mart., eine nene Schmückpflanze warmer Hïuser, mitgetheilt durch F. Otto, mit Ablild.

Verhandl. des Vereins zur Beförder. des Gartenbaues. Zchnte

Liefer. Berlin 1828. p. 218. - Linnaea 1829. p. 17 Lit.

Ueber e ine neue Gattung aus der Familie der Gefsnerieen, von Herrn C. G. Nees von Esenbeck.

Es ist eine neue brasilische Pfanze, die eine eigene Gattung ausmacht, und zu Fhren des Universitüts - Gürtners Sinning in Bonn Sinningia Helleri genannt wurde. - Annales des Sc. natur. Nov. 1825. p. 290.

\section{Verbenaceen.}

Caractères généraux de la famille des Verbenacées tirés de l'ovaire par M. Auguste de Saint-Hilaire.

Nouveau Bulletin des Sciences, par la Société philomatique de Paris. Dec. 1825. p. 187.

\section{Labiaten.}

Caractères généraux de la famille des Labiées, tirés de l'ovaire, par. M. Augustede Saint-Hilaire.

Nouveau Bulletin des Sciences par la Société philomatique de $\mathrm{Pa-}$ ris. Dec. 1825. p. 187.

A ufz ähl $\mathrm{n}$ g und methodische Disposition der Gattungen und Arten ane der Familie der Labiaten, von Bentham.

Aus dem Botunical Register Nro.IX. Vol.2. Nov. 1829, in dem Bullet. des Sc. natur. Janvier 1830. p. 112. - Bot. Literaturbl. IV. p. 149.

Observationes botanicae in Ajugam genevensen auctore Fr. Guilielmo Drees.

Linnaea III. p. 78.

Chatekia; èine neue Pflanzeugattung, aufgestellt von den HH. Opiz und. Corda in Prag.

Bot. Zeit. 1830. T. p. 33. sis 


\section{$-94$}

Arten von Dracocephalum, dic in Rufsland wachsen, ron Doigoubsky.

Nouveau Magasin d'Ifist. nat. Nro. III. 1825. p. 149.

Histoire naturelle des Lavandes, par le baron Fréderic de Gingings Lassaraz. Genève 1826. 8. arec $11 \mathrm{pl}$. 4. chez Cherbuliez libr.

Observations sur les genres Leucas et Phlomis. Description de plusicurs éspèces, par M. Desfontaines.

Mémoires du Museum d'hist. nat. de Paris. Vol. XI. p. 1-10. Mierzu gehören die vortrefflichen Abhandlungen folgender P'fanzen: Lencas helianthemifolia, L. ternifolia, I. lamiifolia, L. lanceacfolia, L. marrubioides, L. procumbens, L. capitata, Phlomis Bruguieri.

Beiträ ge zur Aufklärung des Genus Mentha; vom Merrn Stud. Med. Fresenius in IIeidelberg.

Bot. Zeit. 1527. I. p. 225.

Enumeratio Mentharum. Prodromus disqnisitionis hujus generis accuratioris; accedunt Preslia et Pulegium; anctore Georgio Fresenius.

Sylloge plantarum novarum II. p. 225.

Bemerkungen über Mentha sativa L. von Herrn Prof. Tausch.

Bot. Zeit. 1828. p. 234.

Beitrag zur Kienntnifs der Varietäten und Bastarde der Mentha von Lasch in Driefsen.

Linnaea 1829. III. p. 417.

Ein Beitrag zur Kenntnifs des Genus Mentha, vom Regimentsarzt Dr. L. Griesselich in Karlsruhe.

Geiger's Magazin Nov. 1828. p. 97 . $d . f$.

Bemerkungen über die Gewächse, welehe man mit dem Namen Mentha crispa bezeichnet, von Dierbach.

Daselbst Februar 1830. p. 154.

$Z_{w}$ ei neue Arten von Satureja, entdeckt durch v. Ehrenberg; e sind:

Satureja Campanella: suffruticosa, ramis pubescentibus, foliis ovata lanceolatis acutis integerrimis hispidulis subsessilibus; florum cymis folio parum longioribus bracteatis, calycibus brevins campanulatis profunde quinquefidis. - Habitu proxime accedit ad S. rupestrem Wulf. Willd.

Satureja Olla: sufruticosa, ramis seticulosis, foliis anguste linearibus et lanceolato-linearibus acutis setoso-hispidis, florum cymis folio vix longioribus bracteatis; calycibus brevius campanulatis profunde quinquefidis.

Habitu propius accedit ad S. Thymbram, cujus flores et folia majora sunt.

Habitant prope Castel nuova.

Naturges. Reise durch Nord-Afrika.p. 13.

Cassini, Ueber den Kelch der Scutellaria galericulata.

Im zweiten Bande der Opuscules phytologiques.

Sphacele Lindleyi Benth (Stachys Salviac Lindley) genau beschrieben vom Prof. Graham im

New Edinb. Philos. Journal Jan. 1830. p. 184. - Die Gattung ist von Bentham benannt, wegren Aehnlichleit der Pflanze mit dem Salbey; es gehören dahin drei in Chili einheimische Arten. 
De la necessité de rejeter le genre Stachistarpheta, par M. A uguste de Saint Hilaire.

Nouveau Bulletin des Sciences de la Soc. philomat. de Paris. Nov.

1825. p. 170. - Man sehe auch Schrader's Blumenbachia p. 19.

Rudol ph, Commentatio in genus Ziziphora.

Mémoires de l'Academie de Petersbourg. I. p. 423-437. II. p. 307.

Bemerkungen über die sogenannten Drüsen auf den Blättern der Labiaten, und die in denselhen vorkommenden riechenden Bestandtheile, von Dr. Griesselich in Karlsruhe.

Geiger's Magazin August 1829. p. 105.

\section{Acanthaceen.}

Observations on the North American species of the genus Ruellia. By Capt. John le Conte.

Aus den Annals of the Lyceum of Natural History of New York in der bot. Zeitung 1827. I. p. 17.

Ueber die Cyrtandraceae, eine neue Pflanzen-Osdnung von W. Jack.

Aus den Linn. Transactions in der Isis 1825. Heft XI. p. 1176.

\section{Bignoniaceen.}

Fridericia novum plantarum genus Friderico Guilelmo III Borussorum regi potentissimo sacrum, auctore de Martius.

Nova acta physico-medica T. X. p. 2. c. tab. - Linnaea 1828.

II. p. 14 Lit. - Bot. Zeit. 1829. II. p. 413.

Observations on the Argylia, Eccremocarpus and of plants improperly referred to the latter genus. By David Don.

Jameson New Edinb. Philos. Journal April-July 1829. p. 83.

Ueber die Gattung Calampelis sehe man Daselbst Nro. 13. p. 89 und den verbesserten Gattungs - Charakter in der nemlichen Zeitschrift Jan. 1830. p. 171.

Calampelis gehört, wie Eccremocarpus, in die Familie der Bignoniaceen, und zwar in die Gruppe der Tourretien, sie verbinden diese Familie mit der der Cobeaceen.

Bulletin des Sc. natur. Oct. 1829. p. 83.

Bemerkungen üher eine neue natürliche Pflanzen-Familie, Cobaeaceac; von David Don.

Aus dem Edinb. philos. Journ. 1821, mitgetheilt von Beilschmied in der bot. Zeit. 1825. II. p. 525.

\section{Convolvulaceen.}

Note sur le genre Prevostea, par M. Choisy.

Annales des Sc. naturelles Avril 1825. p. 496.

L e de b our, Ueber Ipomoea Krusensternii.

Mém. de l'Acad. de Petersbourg. IV. p. 401. $t .8$.

Convolvulus arvensis ejusque varietates, von $L$ asch in Driefsen. Linnaea 1829. p. 407.

\section{Cuscuteen.}

Description de trois éspèces de Cuscute, qui croissent naturellement aux environs de Nancy; par M. Soy er-Willemet. Annales de Soc. Linn. de Paris. Sept. 1825. 


\section{Solanaceen.}

Mlistoire naturelle et médicale de la famille des Solanées, par A. F. P'auchet, Prof. de Botanique it Rouen. Ronen 1829) 8.

Balsamo Crivelli, Jos. Galbriel. De Solanacearum familia in genere, addita Verbascorum Italiae indigenorum Monographia. 'Ticini 1824. 8 .

De la Belladonne; Thèse sontenue ì la faculté de Médecine de Paris, par M. Pa uquy d'Amien 8. Paris 182!.

Note sur le Cestreau tinctorial par A. Chereau.

Journal de Chimic medicale Juillet 1826. p. 346.

Note on the native Country of the Potato (Solinum tuberosum). By Aylmer Burke Lambert.

Jumeson New Edinb. philos. Journal April Jun. 1827. p. 192. -

Man sehe auch Schued. Jahresberieht. Bonn 1826. p. 126, - ferner

Geiger's Magazin Bd. 17. p. 125.

Ueber das Vaterland der Kartoffeln und ihre Verpflanzung nach Europa, vom Herrn Grafen von Sternberg.

Monatsschrift der Gesellschaft des vaterlündischen Museums in Böhimen Febr. 18ะ2. p. 20.

Cestrum bracteat um, sehr genau beschrieben von Dr. Graham Jameson New Edinb. philos. Journal Jan. - April 1830. p. 378.

\section{Borragineen.}

De Asperifoliis Limnaei Commentatio, auctore II. A. Schrader, cum tabula aenea Gottingae 1820. 4.

Bemerkungen üher Arten von Cerinthe, Echium, Lithospermum, Anchusa, von Herm Prof. 'Tausch.

Bot. Zeit. 1829. II. p. 641.

Boragineac colloctae in expeditione speculatoria Romanzoffiana.

Linnaea 1829: IV. p. 435 .

Rudolph. Deseriptio novae speciei Mrosotidis (eiliata).

Mém. de l'Acad. de St. Petersb. 'T.1. tab.9.

Lobostemon Asperifoliarum genus novum, auctore Lehmann.

Linnaea 1830. III. p. 378.

Symphytum bulbosum, eine neue Pflanzenspecies; entdeckt und aufgestellt ron Herrn Carl Schimper in Heidelberg.

Bot. Zeit. 1825. I. p. 17.

Die Synonymie von Symphytum tuberosum und bulbosum betreffend, als Nachtrag zur Flora 1825. Nro. 2. von Herrn Alex. Braun in Heidelberg.

Daselbst p. 383.

Ueber dieselbe Pflanze ron Gay und Hoppe. Das. p. 297.

F. Mayer, Bemerkung ülıer Symphytum bullosum, bestätigend, dafs es S. wacrolepis sei.

Daselbst 1825. II. p. 720.

Ueber Sym ph y um bulbosum Schimper, von IIerrn Dr. Bisch of f inlleidelberg. Mit einer Abbildung. (Jetzt soll sic S. filipendulum heifsen). Dascilbst 1826. II. p. 561. 
Beschreibung des Symphytum Zeiheri und seiner zwei deutschen Verwandten des S. bulbosum Schimp. und S. tuberosum Jacq., nebst Erliuterungen über die Asperifolien überhaupt, namentlich über deren Blattstellung und Inflorescenz und das Pflanzen-Ei, von K. F. Schimper, der Philosophie Doctor.

Geiger's Magazin October 1829. p. 3.

\section{Gentianeen.}

Conspectus Generis Gentianae inprimis specierum Rossicarum. Auctore A. de Bunge.

Mémoires de la Société d'hist. nat. de Moscou. Vol. VII. avee figures. - Von dieser Abhandlung ist auch ein besonderer Abdruck erschienen: siehe Linnaea 1828. 111. p. 155. Lit.

Gentianeae collectae in expeditione speculatoria Romanzoffiana.

Linnaea 1826. II. p. 173.

Ueber Gentiana Amarella von Prof. Aghard.

Physiograph. selskapets Aarsberättelse Lund. 1825. p. 27.

Es schien dem Herrn I erf., dafs man in Schueden unter dem Namen G. Amarella 2 Arten verwechsle, nemlich 1. G. Amarella L. corollae limbo harbato, calice tereti, foliis ovato-cunentis acutis discoloribus trinerviis.

a. Linnaei calicis laciniis lanceolatis tubum corollae cylindricao subaequantibus; in Schweden, von Scamien bis nach Lappland.

B. thyrsoidea $\gamma$ germanica; calicis laciniis lancenlatis, corolla clarata dimidio brevioribus V. G. amarella Sturm. G. germanica Willd. S. uliginosa.

2. G. lingulata : corollae limbo barbato, calice tereti, foliis lingulatis subspathulatis oltusis utrinque concoloribus brevibus.

Ueber eine neue Art der Gattung Gentiana, von K. B. Presl. M. D. (G. excisa).

Bot. Zeit. 1828. p. 267.

Lomatogonium, ein neues Genus für Gentiana corinthiaca Froehl, von Dr. Alex. Braun.

Das. 1830. I. p. 221.

Chironia uliginos a Laperrouse ist - Hypericum elodes.

Bullet. des Sc. nat. Mai 1829. p. 250.

De Erythraea. Diss. inaug. botanico-medica, quam etc. palam defensurus est auctor G. L. Ewald Schmidt. Acced. tabul. II. aeneae. Berolini 1828. 4 .

Siehe Linnaea III. p. 165. Lit.

Curtia, novum plantarum genus, auctoribus Chamis s o et Schlechtendal.

Linnaea 1826. p: 209.

Die Verf. rechnen ihre Pflanze (C. gentianoides) $\approx u$ den Scrophularien, nach Dr. Bartling aber gehört sie hierher.

\section{Asclepiadeen.}

Ueber die Asclepiadeen, von Robert Brown.

Vermischte botan. Schriften II. p. $347-414$.

Genera et Species Orchidearum et Asclepiadearum, quas in itinere per insulam Javam collegerunt $K u h l$ et van Hasselt.

Siehe oben bei den Orchideen. 


\section{$-\quad 98$}

De antheris et polline Asclepiadearum. Sehlechtendalio sno Ehrenberg.

\section{Linnaca 1829. I. p. 94.}

A e counts and descriptions of the several plants belonging to the genus Hoya, which are cultivated in the garden of the horticultural society at Chiswick. By M. James Traill, Inder-gardener.

Transactions of the horticultural Socicty of London I"ol. III. part. 1. p. 16. - Linnaca 1828. II. p. 70. Lit. Coimbra.

Arauja, eine Pflanzen-Gattung aus Peru, von Prof. Brotero zu Isis 1829. Heft 2. p. 149.

\section{Apocyneen.}

Ueber den IIya-Iya oder Milchbaum ron Demarara (Tabernaemontana utilis) von James Smith. Esq.

Brandes Archiv Bd. XXXIII. Heft 2. p. 170.

\section{Rubiaceen.}

In dem kürzlich erschienenen vierten Bande des Prodromus theilt $D_{e-}$ candolle diese grofse Familie in folgende Stämme oder Tribus, deren mehrere wieder Subtribus haben, $a b$ : Cinchonaceac, Gardeniaceae, Hedyatideae, Iserticae, Ifamelieae, Cordierceae, Guettardaccae, P'acdericae, Caffeaceae, Spermacoceae, Anthospermeae, Stellatae, Opercularieae.

M émoire sur les Rubiacées par M. A. Richard, lue à la Séance de 1'Acad. des Seiences du 6. Juillet 1829. Le Globe 11. Juillet 1829. 438. - Bulletin des Sc. nat. Août 1829. p. 262. - Mémoires de la Soc. d'hist. nat. de Paris. T. V. p. $\mathbf{8 1 .}$

Rubiaceac collectae in expeditione speculatoria Romanzoffiana (Stellatae, Spermacoceae etc.). Linnaea 1828. p. 220. 309. 1829. p. 1 et 129.

Guilielm. Cruse: De Rubiaceis capensibus, praesertim de genere Anthospermo. Berolini 1825.

Die Fieberrinden in botanischer, chemischer und pharmacentischer Beziehung dargestellt, von Dr. S. Graaf. Wien 1824.

Versuch einer Monographie der China, von II ein rich von Bergen, Droguerie-Makler. Mit acht Kupfertafeln in Folio und zehn Tabellen. Ifamburg, auf Kosten des Verfassers, gedruckt bei Hartwig und Müller 1826. 348 S. 4.

Vergleichung der Synonyme des Geschlechts Cinchona und der verwandten, von C. A. F ée.

May 1825. p, 157.

Notiz über die verschicdenen Gattungen und Arten, deren Rinden unter dem Namen China verwechselt worden siud, von Herrn Professor Decandolle.

Bibliothèque universelle Juin 1829. p. 144-162.

Uebersicht der neuesten Arbeiten über die Abstammung der Chinasorten, von Dierbach.

Geiger's Magazin April 1830. p. 42.

Mémoirc sur une Rubiacée nommée par les jardinicrs Melanopoi- 
dium nigram et formation d'un genre nonveau sous lo nom de Viviana, par M. Louis Colla de Turin.

Annales de la Soc. Linn. de Paris. Mars 1825.

Cephalanthus Sarandi Chamisso et Schlecht.

Linnaea 1927. p. 610.

De Meyna Roxburghii (Vaugueria spinosa) Candollio summo Botanico Schlechtendalius.

Linnaea 1829. IV. p. 516.

Observatio in Plectroniae genus, auctore D. F. L. de Schlechtendal.

Linnaea 1830. III. p: 380.

Bartling bringt die Giattung fragweise zu den Rhamneen.

Don beschreibt ausführlich Lipostoma capitatum (Aeginetia cap. Graham, Hedyatis campanulaeflora Hooker) und L. sericeum; er zeigt dafs sie zu den Rubiaceen gehören.

Jameson New Philos. Journal. Jan. 1830. p. 168.

Ueber mehrere Arten Galium u. s. w. vom Herrn Prof. Tausch. Botan. Zeit. 1829. II. p. 645. Driesen.

Ucber Varietäten und Bastarde von Galium, von Lasch in Linnaea 1829. III. p. 415.

Thunberg Galii species capenses illustratac (G. rotundifolium, capense, mucronatum, expansum, asperum, glabrum, horridum). p. 326.

Mémoires de l'Academie imp. des Sciences de St. Petersbourg 1 .

\section{Caprifoliaceen.}

Ca prifoliac ea e collectae in expeditione speculatoria Romanzoffiana. Linnaea 1828. II. p, $13 \%$.

\section{Jasmineae.}

Observations sur les familles des Jasminées et des Oleinées, par M. A. Richard.

Annales des Sc. natur. Mars 1826. p. 350.

Palivaria Chamisso et Schlechtendal.

Linnaea 1826. p. 207.

Herr Dr. Bartling bringt diese Gattung zu jenen, deren Stello im System noch zweifelhaft ist.

\section{Siebente Section. \\ Gymnoblasta Polypetala.}

Exogenen mit nacktem Embryound vielblättriger Corolla.

§. 18.

Cl. XXXIX. Lorantheac. O. 130. Lorantheae.

Cl. XL. U m bellifl orae. 0. 1ŝ1. Umbelliflorae. 132. Araliaceae. 133. Hederaceae. I34. Hamamelideae.

Cl. XLI. Cocculin a e. O. 135. Berberidene. 136. Menispermeae. 
Cl. XILII. Trisepalae. 0. 137. Myristiceae. 138. Annnaceae.

Cl. XIIII. Polycarpicae. O. 13!). Magnoliaceac. 140. Dilleniacene. 141. Paeoniaceae. 142. Ranunculaceac.

Cl. XI.IV. Rhocadeac. O. 143. Tremantreac. 14. Polygaleae. 145. Resedaceae. 146. Fumariaceric. 147. Papaveraceae. 145. Cruciferae. 149. Capparideae.

Cl. XLY. Peponiferae. O. 150. Samydeae. 151. Inmalineac. 152. Passifloreae. 150. Turneraceae. 154. Loaseae. 155. Cucurbitaceae. 156. Grossularieae. 157. Nopaleae.

Cl. XI.VI. Cestiflorae. O. 158. Flacourtianeae. 159. Marcgrarieae. 160. Bixineae. 161. Eistineae. 162. Violarieae. 163. Droseraceae. 164. Tamariscineae.

Cl. XLVII. Guttiferae. 0. 165. Sauvagesieae. 166. Frankeniaceac. 167. Hypericineae. 168. Garcinieac.

Cl. XLVIII. Caryophyllinae. O. 169. Chenopodieae. 170. Amaranthacene. 171. P'hytolacceac. 172. Sclerantheae. 173. Paronychieae. 174. Portulaceae. 175. Alsineae. 176. Sileneae.

Cl. XLIX. Succulentae. O. 178. Ficoideac. 178. Crassulaceae. 179. Saxifrageae. 180. Cunoniaceac.

Cl. L. Calyciflorae. O. 181. Halorageac. 182. Lythrarieae. 183. Onagrarieae. 181. Rhizophoreac. 185. Vochysicac. 186. Combretaceac.

Cl. LI. Calycanth ina e. O. 187. Granateae. 188. Calycantheac.

Cl. LII. Myrtinae. O. 189. Memecyleac. 190. Melastomaceae. 191. Myrtaceae.

Cl. LIII. Lamprophyllae. O. 192. Camelliaceae. 193. Ternstroemiaceac. 194. Chlenaceae.

C1. LIV. Col umn iferae. O. 195. Tiliaceac. 196. Sterculiaceae. 198. Bütneriaceae. 198. Hermanniaceae. 199. Dombeyaceae. 200. Malvaceae.

Cl. LV. Gruinales. O. 201. Geraniaceac. 202. Lineae. 203. Oxalideae.

Cl. LVI. Ampelideac. O. 204. Sarmentacere. 205. Leeaceae. 206. Meliaceae. 207. Cedreleae.

Cl. LVII. Mal pighina e. O. 208. Malpighiaceae. 209. Accrineae. 210. Corsarieae. 211. Erythroxyleae. 212. Sapindaceac. 213. Hippocastaneae. 214. Rhizoboleae. 215. Tropaeoleae.

Cl. LVIII. 'Tricoccae. 0. 216. Staclihouseac. 217. Euphorhiacene. 218. Empetreae. 219. Bruniaceae. 220. Rhamneae. 221. Aquifoliaceac. 222. Pittosporeac. 223. Celastrineac. 224. Hippocratenceae. 225. Staphyleaceae.

cl. LIX. Terebinthinae. 0. 226. Ochnaceae. 227. Simarubeae. 228. Zanthoxyleae. 229. Diosmeae. 230. Rutaccae. 231. Zygophylleae. 232. Aurantiaceae. 233. Amyrideae. 234. Connaraceae. 235. Cassuvieae. 236. Juglandeae.

C. LX. Calophytae. O. 237. Pomaceae. 238. Rosaceae. 239. Dryadeae. 249. Spiracaceae. 241. Amygdaleae. 242. Chrysobalancae. 243. Papilionaceae. 2.4. Swartzieae. 245. Cacsalpineae. 216. Mimoseae.

\section{Lorantheen.}

Notice sur la végétation des plantes parasites et en particulier des Loranthacées, par M. De-Candolle, Prof. Bibl. univ. Mars 1830. p. 303 u. d. f. 
II émoirc sur la famille des Loranthacées, par M. A ug. Pyram. Decandolle, avec douze planches. Paris 1830. 4. chez Treuttel et Würtz. $5 \mathrm{fl} .24 \mathrm{kr}$.

Ist das 6. Heft der Collection de Mémoires pour servir à l'histoire du règne végétal. Abgebildet sind: $\mathbf{L}$ or a $\mathrm{n} t h u$ s pendulus, congener, farinosus, carinatulus, leptanthus, cinnamomeus, chinensis, Pentagonia, dodonacaefolins, calycatulus, Mi s o d e n d r um punctulatum, brachystachyum, quadriflorum.

Lorantheac collectae in expeditione speculatoria Romanzoffiana. Linnaea 18\%8. III. p. 200.

Einige Bemerkungen über Loranthus, von Herrn Hofrath Dr. von Martius in München.

Bot. Zeit. 1830. 1. p. 97.

Ueber eine neue Gattung aus der Familie der Lorantheen; von J. Preis sler, $k$. $k$. Fiscal-Adjuncten in Prag. (Tauschia hederaefolia). Daselbst 1828. I. p. 43.

Mémoire physiologique sur le gui (Viscum album L.), par B. Gaspard, M. D.

Magendie Journal de Physiologie experimentale et pathologique.

T. VII. p. 227-333. - Eine mit vielem Fleijs ausgearbeitete, selır schätzenswerthe Abhandlung!

Moquinia rubra Ant. Sprengel.

Linnaea 1830. III. p. 421.

Die Charateristik der Gattung A ueuba wird ron Don sehr genau mitgetheilt, er zeigt (was Andere schon früher wahrnahmen), dafs sie in die Familie der Loranthaceen gehöre, und die Structur der weiblichen Blume ganz so wie bei Viscum sei.

Jameson New Philos. Journal. Jan. 1830. p. 167.

\section{Umbelliferén.}

Neuvième Mémoire sur les caractères généraux des familles tirés des graines, et confirmés ou rectifiés par les observations de Gärtner. Araliacées, Ombelliféres. Par M. A. J. de J ussieu.

Wieder abgedruckt in der Isis 1826. Erstes Heft p. 23-29.

Generum Tribu umque plantarum Umbelliferarum nova dispositio, auctore Guilielmo Daniele Josepho Koch, M. Dr. Cum tabulis tribus lithograptis.

Nova Acta Acal. Caesar. Nat. Cur. Fol. XII. P. 1. p. 55-156.

(Ist auch besonders abgedrucht worden).

Beobachtungen über die Familien der Doldenpflanzen ron M. Mariana Lagaca.

Ocios de Espannoles emigrados. London T. Ir. Sept., Oct., Nov. 1825. - Linnaea 1828. III. p. 156. Lit.

Collection de Mémoires pour servir à l'histoire du règne végétal Nro. 5. Mémoire sur la famille des Ombelliféres, par M. A. P. Decandolle, avec 19 planches. Paris 1829. $4.8 \mathrm{fl} .6 \mathrm{kr}$.

Ein Beitrag zur Kenntnifs der Blätter, Scheiden und Hüllen der Schirmpflanzen, von Dr. Griesselich in Karlsruhe.

Geiger's Magazin Februar 1829. p. 17.

Ueber den Habitus in den einzelnen Dolden-Gattungen, von Demselben. 
U mbelliferae collectac in expeditione speculatoria Romanzoffiana. Linnaea 1826. III. p. 333 .

Note sur un nouveau genre dOmbelliféres (Gaya) par le Prof. Gaudin.

Fcuille du Canton de Vaud 1826. Nro. 157. p. 28. - Bulletin des Sc. natur. Oct. 182(6. p. 197. (Gaya simplex et pyrenaica).

Ueber Arracacia, eine neue Gattung aus der Familie der Dolden von Bincroft, sehe man André ükonom. Neuigkeiten 1826. Bd. II. p. 497. Nro. 63. aus englischen Zeitschriften. - Geiger's Magazin April 1827. p.9. - Verhandlungen des Vereins zur Beforderung des Gurtenbues 9. Liefor. Berlin 1828. 10982. - Linnaca 1829. I. p. 18. Lit. - De candolle in der Bibl. universelle 1829. Jan. p. 84 . - Brandes Archiv Bal. XXXI: Heft 3. p. 351. (Iferacleum tubcrosum Molina ist wohl dieselbe Pflanze).

Actinanthus Ehrenberg, norum Umbellifer. genus.

Linnaea 1829. III. P. 398.

Lejat u lus Ehrenberg, novum Umbellifer. genus.

Ibidem p. 400.

Pentacrypta Lehmann, novum Umbellifer. genus Arracaciae proximum.

Linnaea 1830. III. p. 380. Sauter.

Ueber Bupleurum junceum und B. Gerardi von IIerrn Dr. Bot. Zeit. 1826. I. p. 178.

\section{Araliaceen.}

Neuvième Mémoire sur les caractères généraux des familles tirés des graines et confirmés ou rectifiés par les observations de Ga ertner. Araliacées, par M. A. J. de Jussieu.

Wieder abgedruckt in der Isis 1826. Heft 1. p. 23-29.

Araliace a collectac in expeditione speculatoria Romanzofiana.

Linnaea 18\%6. II. p. 402.

Note sur le Gastonia palmata.

Messager des sciences et arts 1825. Livr. 1 et 2.

\section{Menispermeen.}

Ueber die indischen Gattungen von Menispermum.

Aus den Linn. Transactions übersetzt in der Isis 1825. Heft 9. p. 965.

\section{Anonaceen.}

Note sur le noureau genre Anaxagorea et sur le genre Xylopia, par. M. Aug. de Saint Hilaire.

Bull. de la Soc. philom. de Paris. Juin 1825. p.91. selben.

Bemerkungen über das Vaterland der Anona squamosa von DemIn derselben Zeitschifif Januar 1825.

\section{Magnoliaceen.}

Notice aur une nouvelle éspèce de Magnolia, par M. Soulange Bodin. Paris 1826. (M. Yulan $=$ discolor). 


\section{$-103$}

Bulletin des Sc. natur. Janv. 1828. p. 98. - Linnaea 1828. III. p. 127. Lit. - Bot. Literaturbl. II. p. 147.

\section{Paeoniaceen.}

Monographie der Gattung Paeonia von G. Anderson.

Aus den Linn. Transactions XII. P. 1. wieder abgedruckt in der Isis

1829. Heft 10. p. 102I. u. d.f. - Enthält auch die Synonyme der Alten!

Botanische und pharmakologische Bemerkungen über mehrere Arten von Paeonia; von Uierbach.

$$
\text { Geiger's Magazin August 1825. p. 105. u. d. f. }
$$

Bemerkungen über einige Arten der Gattung Paeonia, vom Herrn Prof. Tausch.

Bot. Zeit. 1828. I. p. 81.

Bemerkungen über Paeonia officinalis.

Daselbst 1829. II. p. 525.

\section{Rạnunculaceen.}

Dixième Mémoire sur les caractères généraux des familles, tirés des graines et confirmés ou rectifiés par les observations de Gärtner Renoneulacées, par M. A. L. de Jussieu.

Wieder abgedruckt in der Isis 1826. Heft 2. p. 167-176.

Verhandeling over de Ranunculaceae der nederlandsche Flora; medegedeeld door A. L. S. Lejeune, M. D. et R. Courtois.

Bydragen tot de Natuukindige W'etenschappen T. II. p. 69.

Esquisse d'une Monographie du genre Aconitum par Nicolas Charles Séring e. Genève 1823.

Scharfe Kritik dieser Schrift von Reichenbach in der bot. Zeitung 1821. I. Beilage $p .113$.

C. L. Reichenbach. Uebersicht der Gattung Aconitum, Grundzüge einer Monographie derselben. Regensburg 1819. 8.

C. L. Reichenbach. Monographia generis Aconiti iconibus omnium specierum coloratis illustrata latine et germanice elaborata. Lips. 1820. 1 Vol. fol. cum tab. VII.

C. L. Reichenbach. Mlustratio generis Aconiti atque Delphinii, oder neue Bearbeitung der Gattung Aconitum und Delphinium. Leipzig 1823. Fol. 12 Hefte. - Siehe botan. Zeit. 1826. p. 225, dann 1829. p. 61. Beilage.

Ueber die Schärfe der verschiedenen Arten ron Aconitum und über den vorzüglichsten Sitz derselben, von Dr. Griesselich in Karlsruhe.

$$
\text { Geiger's Magazin Januar 1829. Bd. } X X Y \text {. p. } 45 .
$$

L. C. Trevirani, Prof. Vratislav. in Observationes de Delphinii et Aquilegiae genere additamenta quaedam.

Jahrb. der Gewächskunde Heft 2. p. 43. $\approx u$ vergleichen mit dem früheren Aufsatze in dem zweiten Bande der Isis.

Bemerkungen über Aquilegia bicolor Ehrh. und Persoon, rom Herrn Fiscal-Adjuncten Preissler.

Bot. Zeit. 1828. I. p. 186.

Bemerkungen über einige deutsche Aquilegien von Koch.

Dáselbst 1830. I. p. 118. 
Bemerkungen über Anemone Nuttalli Decand. ron Thouna Nuttall.

Journ. of the Acarl. of nat. Sc. of Philadelphia Vol V.p.158. Linnaea 1830. II. p. 61. Lit.

Driesen.

Ueber dic Pulsatillen der Mark Brandenburg von Lasch in Linnaea 1828. p. 163, und 1829. p. 429.

Ueber Ranunculus anemonoides Zahlb. vom Herrn v. Welden. Bot. Zeit. 1828. I. p. 270.

Monographia generis Nigellac. Dissert. auet. F. Spenner. Friburgi 1829. 4 .

Ueber Myosurus minimus ron Cassini.

Im zweiten Bande der Opuscules phytologiques.

F. Hamilton. Nachricht von einigen Arten Caltha des Himalayagebirges.

Edinburgh Journal of Science Oct. 1824. p. 219. - Bulletin des Sc. natur. Février 1825. p. 221. - Geiğer's Maguzin Juli 1825. p.4.

\section{Polygaleen.}

A uguste de St. IIilaire et A. Moquin-Tandon premier Mémoire sur les Polygalées, contenant les recherches sur la symetrie de leurs organes.

Mémoires du Museum d'hist. natur. Neuvième année, Cahier $\boldsymbol{V}$. p. 313 et suiv.

Polygala multicaulis Tausch.

Bot. Zeit. 1821. II. p. 563.

Polygala Moriana Brittinger.

Daselbst 1826. II. p. 727.

Polygal a serpillacea Weihe.

Déselbst p. 745.

Botan is che und pharmakologische Bemerkungen über mehrere Pflanzen aus der Familie der Polygaleen.

Geiger's Magazin April 1829. p. 19.

Ueber Deutschlands Violae und Polygalae, ron Ifern Director Rossmāsler in Weida.

Bot. Zcit. 189. II. p. 708.

Conspectus Polygalearum Brasiliae meridionalis auctore A. de St. Hilaire.

Annales de la Soc. royale des Sciences, belles lettres et arts d'Orleans. T. IX. Nro. 1. p. 44.

\section{Resedaceen.}

Jules de Tristan. Mémoires sur les affinités du genre Reseda. Annales du Museum T. XVIII. p. 392.

Observations sur les Resedacées, par M. Rob. Brown. Annales des Sc. naturclles. Oct. 1826. p. 213-218.

Von der Yerwandtschaft der Resedaceen mit den Ranunculaceen tedet 10 on gelegentlich hei der Beschreibung der Gattung Tovaria, im Decemberhefte 1828. des Edinburgh new philos. Journal.

Man sehe noch Bull. des Sc. natur. Sept. 1829. p. 419. 


\section{$-105 \ldots$}

\section{Fumariaceae.}

Fumariaceac collectae in expeditione speculatoria Romanzoffiana. Linnaea 1826. IV. p. 556.

Rudolph. Descriptio novae speciei Fumariae (peregrina).

Mém. de l'Acadlemie de Petersbourg I. t. 18.

\section{Papaveraceen.}

Papareraceae collectac in expeditione speculatoria Romanzoffiana. Linnaca 1826 IV. p. 551.

Remarque sur l'affinité des Papaveracécs avec les Crucifères, par M. Mirbel. Annales des Sc. naturelles. Nov. 1825. p. 266.

\section{Cruciferen.}

Mémoire sur l'insertion des étamines des Crucifères, par M. Themistocle: Lestiboudois.

Recueil des travaux de la Soc. d'amateurs de Lille 1823 et 1824. p. 243. - Bulletin des Sc. natur. Mars 1828. p. 310. - Linnaea 1829. 1. p. 23. Lit.

Observations sur quelques Crucifères décrites par. M. D e candolle, dans le second volume de son Systema naturale regni vegetabilis, par J. P. Monnard, Principal du Collége de Nyon en Suisse, avec des notes de M. Gay.

Annales des Sciences naturelles. Avril 1826. $p$. 389-419.

Mémoire sur les fruits siliqueux, par M. Themistocle Lestiboudois.

Recueil des travaux de la Soc. de Lille. 1823. p. 194.

Beobachtungen über das Keimen der Saamen von Raphanus und anderer Cruciferen.

Cassini im zweiten Bande der Opuscules phytologiques.

Cruciferae collectae in expeditione speculatoria Romanzoffiana.

Linnaea 1826. I. p. 14.

Bemerkungen über die Cruciferas, rom Herrn Prof. Ign. Friedr. Tausch in Prag.

Botan. Zeit, 1930. I. p. 65.

Description de denx nouveaux genres (Selenia, Streptanthus) do l'ordre naturel des Crucifères, par Thomas Nuttall.

Journal of the academ. of Sciences of Philadelphia Vol. V. p.132.

Dec. 1825. - Bulletin des Sc. nat. Aouit 1827. p. 405. - Linnaea 1830. II. p. 62. Lit.

M émoire sur les differentes éspèces et varietés de Choux et Raiforts, cultivés en Europe, par M. De Candolle. Paris. 1822. 8. zig 1824.

Davon existirt auch eine deutsche Uebersetzung von Berg. Leip-

Ueber eine neue Art Brassica, die auf den Felsen am Meere im westlichen Ligurien wächst, von G. B. B a da ro.

Giorn. di fisica chem. etc. di Pavia. T. IX. 1826. p.411. - (Ein Mittelding von Brassica oleracea und balearica).

Beobachtungen über Barbarea arcuata Reichenb. von Carl Bouché.

Linnaea 1828. I. p. 68. 
Geschichte der Braya alpina von Sternb. et IIoppe nus dem Französischen nach J. Gay übersetzt von Ierrn Legationarath Felix, mit einem Nachtrage von il oppe.

Bot. Zeit. 1927. I. p. 241.

Barharea hirsuta Weihe.

Daselbst 1830. I. p. 257 .

C. Steven Alyssi rostrati descriptio.

Ním. de l'Academic de Petersbourg III. p. 295.

Ueber Alyssum von Walker-Arnott.

Aus engl. Zeitsehrift. in den botan. Literalurbl. IY. p. 42.

A h handlung über die zur Flora Deutschlands gehörigen Arten der Gattung Draba ; vom Herrn Dr. Koch in Kaiscrslatern, mit einem Vorund Nachberichte von Dr. Hoppe.

Bot. Zcit. 1823. II. p. 417.

Ueber Draba ciliata Scopoli, rom Herrn Prof. Tausch in Prag. Das. 1828. I. p. 269.

Draba aizoides und Aizoon, vom Herrn Apotheker IIornung in Aschersleben.

Das. 1829. II. p. 443 und 473.

Draba oblongrata, seltene Grönländische Art, beschrielsen von C. S. Sominerfelt.

Maguz. for Naturvidenskab. A.1824. Cah. I. p. 151.

Bemerkungen über Hutchinsia rotundifolia und cepeaefolia, von Hornung.

Bot. Zeit. 1829. II. p. 431.

Bemerkungen über Arabis pendula und bellidifolia von Demselben. Daselbst p. 668.

\section{Capparideen.}

Hoppe, üher Capparis spinosa und ovata.

Bot. Zeit. 1827. I. p. 186.

Tovaria, eine neue Gattung der Capparideen, beschrieben von Don in New Edinb. philos. Journal. Dec: 1828. -

Toyaria pendula Ruiz et Pavon hat den Habilus von Cleome, den Früchten nach aber gehört sie in die Nähe von Morisonia und Cratacva; die Narbe gleicht der der Papaveraceen und der Saume ganz jenem der Resela.

Man sehe Bullet. des Sc. natur. Sept. 1829. p. 416.

\section{Passifloreen.}

Beschreibung und Abbildung einer nenen Passionsblume (Passiflora kermesina), von Link und Otto.

Ierhandl. des Vereins zur Beförderung des Gartenbaues in den

K. Preufs. Stuaten. Berlin 1826. Bd. II. IIeft 4. p. 403.

Nachricht von einer neuen Passionsblume aus Brasilien (Passiflora racemosa) vom Prof. Brotero in Coimbra.

Isis 1829. Heft 2, p. 149.

A Description of the Genus Malesherbia of the Flora Peruviana; with remarks of its Affinities. By M. David Don.

Jameson the Edinb. New Philosophical Journal. Jan.-March

1827. p. $320-323$. 
Dr. Blume stellt in den Beitrâgen zur Flora des holländischen Indiens eine cigene Familie - Cariceae - auf, die ihre Stelle unmittelbar nach den Passifloreen einzunehmen hat. Isis 1828. $p$. 263.

\section{Loaseen.}

Blumenbachia, norum e Loasearum familia genus; adjectis observationibus super nonnullis aliis raxioribus ant minus cognitis plantis; anctore II enrico Adolpho Schrader; cum tabulis quatuor aeneis. Gottingae 1827. 4.

\section{Cucurbitaceen.}

Mémoire sur la famille des Cucurbitacées par Nicolas Charles Séringe, accompagnée de 5 tables et suivie d'une note de M. De Candolle sur la place de la famille des Cucurbitacées dans la série des familles naturelles. Genève 1815. 4 .

Extrait du 3. vol. des Mémoires de la Soc. de Physique et d'hist. nat. de Genève. florées.

Aug. de St. Hilaire. Mémoire sur les Cucurbitacées et les PassiMém. du Mus. T. IX. 1922.

Description du Joliffia africana, type d'un nouveau genre de la famille des Cucurbitacées, par M. Raffeneau Delille.

Mém. de la Soc. d'hist. nat. de P'aris III. p. 314.

Note supplémentaire à la description du Joliffia africana, par $\mathbf{M}$. Guillemin.

Ibid. p. 220. lille.

Nourelle description du Benineasa cerifera Savi, par M. De-

Mémoires de l'acad. royale des Sciences de l'institut de France. T. VII. p. 395. 1827.

Beschreibung ciner Winter-Melone, Cucumis serotinus, nebst deren Behandlung, vom bot. Gärtner S eitz in München.

Verhandl. des Vereins zur Beförd. des Gartenb. in den K. Pr.

Staaten. Bd. III. p. $376-378$.

Beschreibung einer neten Melonen-Art, Cucumis Melo persico odorus, von Demselben.

Daselbst. p. 379. - Linnaea 1S27. p. 489.

Sageret über Bastarde der Cucurbitaceen.

Annales des Sc. naturelles. Juillet 1826. p. 294 - $\mathbf{3 1 4}$.

\section{Grossularieen.}

Monographie ou histoire naturelle du genre Groseiller, contenant la description, l'histoire, la culture et les usages de toutes les groseilles connues, par C. A. Th ory. Paris 1829. avec 24 pl. (Opus posthumum +1827 .)

Ueber eine neue Art von Stachelbeeren, von dem Prof. Aghin rd, mit einer Abbildung.

Kongl. Suenska Landtbruks academiens annaler. IX. Jahrgang.

I. Heft. Stockholm 1823. p. 140.

Es ist hier die Rede von Ribes spinosissimum Mühlenberg und

R. aureum Pursh oder R. jasminiflorum Aghard. 


\section{Nopaleen.}

Rerue de la famille des Cactées, par M. A. P. Decandolle.

Mémoires du Museum d'hist. nut. IX. connće, Cah. I. p. 1-a0. - Botan. Literaturbl. III. p. 6iefi.

Teber die Gattungen Melocactus und Echinocactus, nebst Besehreihune und Abhildung der im Kinnigl. hot. Garten bei Berlin befindlichen Arten, von II. F. Linle und F. Otto.

Linnaca 1827. p. 490.

Nach IIooker ist Cactus coccinellifer Dec. in den plantes grasses nichts weiter als Cactus 'Tuna I. II. gibt Nachricht von den verschiccienen Arten, auf denen die Cochenille lebt, im Juniheft 1827 des Botanical Magazine.

\section{Cistineen.}

Cistineac, the natural ordre of Cistus, or Rook Rose No. 1. By Robert Sweet. London 1525.

ron disem Kupferwerke, ganz den Geraniaceis desselben Herausgebers ähnlich, erscheint alle Mönat ein Heft.

- Cistinea collecta in expeditione speculatoria Romanzoffiana.

Linnaea 1827. p. 148.

\section{Violaceen.}

Vinlaceae collectae in expeditione speculatoria Romanzoffiana.

Linnaea 1827. II. p. 145.

Description de quelques ésèces nourclles de Violacées reçues de M. Adalbert de Chamisso, eximinés en 1825. par M. de Gingins. Ibidem 1826. Heft III. p. 406.

Ueber nordamerikanische Violen ron John le Conte. p. 135 .

Annals of the Lyceum of nut. hist. of New York 1826. Vol. II.

Beschreibung einer neucn Art ron Viola, ron IIerrn Berthelot mit Abbild. (Viola Teydea vom Pic auf 'Teneriffia.)

Transuct. of the Americ. phil. societ. $V$. II. new series p. 418. Bot. Literaturbl. I. p. 249.

Ueber Viola canina und montana L. Vom Herrn Hofrath und Professor Dr. Koch in Erlangen.

Bot. Zeit. 1828. I. p. 1.

Viola occulta Lehmann.

Das. 1830. I. p. 15.

Rennie. Bemerkung über die Ausstrenung des Saanens bei Veilchen. Froriep's Notizen Bd. XXIII. p. 120. No. 492.

Nachricht von einer eigenthümlichen Samenbildung an gewöhnlichen Veilchen, von Dr. Steinheim, prakt. Arzte in Altuna.

Hecker's literar. Annulen. April 1828. p. 397.

Bemerkung über die Frucht von Viola odorata, von Dr. Griegselich in Karlsruhe.

Geiger's Mag. April 1830. p.-33.

Monographie des genres Sauvagesia et Lavradia, par M. Auguste de Saint-Hilaire. 
97 - 116.

Mémoires du Museum d’hist. nat. Fol. XI. p. 11 - 68 und

Abgebildet sind: Sauvagesia erecta, S. tenella, S. linearifo-

lia; Lavradia ericoides, elegantissima, glandulosa, capillaris.

Nota. Herr Dr. Bartling rechnet nur die Gattung Lavradia $\approx u$ den Violaceen, das Genus Sauvagesia bildet nach ihm unter dem Namen Sauvagesieae eine eigene Familie.

Viola umbrosa, eine neuc Veilchen Art, entdeckt und beschrieben von Dr. Hoppe.

Bot. Zeit. 1880. II. p. 521.

\section{Droseraceen.}

Droscraceae collectae in expeditione speculatoria Romanzoffiana.

Linnaea 1826. IV. $p$. 54 .

Ueber V arietäten und Bastarde der Droseea von $\mathrm{Las} \mathrm{ch}$ in Driesen. Daselbst 1829. III. p. 426.

\section{Tamariscineen.}

Sur In nouvelle famille de plantes, fondée sur le genre 'Tamarix, par M. Desveaux.

Annales des Sc. natur. Mars 1825. p. 344.

Ueber die Manna-Tamariske ncbst allgemeinen Bemerkungen über die Tamariscineen, von Dr. C. G. Ehrenberg.

Linnaea 1827. p. 241. u. d. f.

\section{Frankeniaceen.}

Observations nourelles sur les rapports des Frankeniées et des Caryophyllées, par M. Aug. de St. Hilaire.

Bullet de la Soc. philom. de Paris 1825.

Frankeniaceae collectac in expeditione speculatoria Romanzoffiana. Linnaea 1826. I. p. 35.

\section{Hypericineen.}

Onzième Mémoire sur les caractères généraux des familles tirées des graines Hypericées - Guttifères, par M. A. I. de Jussieu.

Wieder abgedruckt in der Isis 1826. Heft 7. p. 696 u. d. f.

Hypericineae collectae in expeditione speculatoria Romanzoffiana. Linnaea 1829. II. $p$. 115.

Ueber Varietäten und Bastarde ron Hypericum, von Lasch in Driesen.

Das. 1529. III. p. 413.

Scyphaea, norum plantarum genus. Descripsit C. B. Presl. Pragae, 30. Maji 1829. 4. c. tab. aen.

Siehe Linnaea 1830. II. p. 52.

Description d'un genre nouveau de la famille des Hypericées, par M. Cambassèdes.

Annales des Sc. natur. Aoút 1831\%. p. 399.

Eliea articulata (Hypericum articulatum Lamark). Die Gattung ist benannt zu Ehren des durch seine geognostischen Arbeiten bekannten Herrn Elie de Beaumont. 


\section{Garcinieen.}

Mémoire sur les familles des Ternstroemiacées et des Guttifércs, par J. Cambessèdes.

Mémoires du Museum 8. année 11. Cahier p. 369 - 408 und 12 Cah. p. 409 - 424. - Bot. Literaturbl. I. p. 386.

Description of a Plant of the Order of Guttiferae, which Dr. Roxburgh called Garcinia pedunculatia. By Francis Hamilton.

Breu'ster Edlinburgh Journal of Science No. XIII. July 1827. p. 45.

Die Pflanze ist auf nachstehende Wreise laruliterisirt: Foliis ohlongis parallelis nervilus natatis floribus diofeis longe pedunculatis, masculis numerosis, femineis subsolitariis in 5 phalangibus connexis, baccis decemspermis.

Siche auch Bullet. des Sc. nat. Juin 1829. p. 397.

\section{Chenopodeen.}

Essai sur la distribution géographique de la famille des Chenopodées, par M. Mirbel.

Mémoires du Museum Tom XIII. Cah. III. p. 192 - 203.

Chenopodieae. Bartling, in dessen Beitrïgen zur Botanik. Ifeft II. Göttingen 1825. p. 141.

Agriophyllum pungens M. v. B. ist synonym mit Granen orientale spicatum fruticosum, spicis echinatis in capitulum congestis Tournefort, ferner mit Cenchrus frutescens L. mit Eryngium lateriflorum Lamark und Corispermum pungens Autorum.

Bibl. universelle. Aouit 1829. p. 344.

Ueber die Gattung Blitum von Vignal. p. 108.

Bulletin du Sc. nat. Nov. 1528. p. 382. - Bot. Literaturbl. II.

Description des Thelygonum Cynocrambe, par M. le Prof. Delille. Annales des Sc. nat. Avril 1830. p. 370.

\section{Amaranthaceen.} p. 151.

Amaranthaceae, Bartling in den Beiträgen zur Botanik. Heft II.

Monographie der Amaranthaceen von C. F. P. Martius.

Nova Acta Acad. Caes. Leopold. Nat. Cur. Vol. XIII. P. I. p. 210. 1826. - Siche auch bot. Zeit. 1827. I. p. 97.

'Trommsd orffia, eine neue Pflanzengattung, aufgestellt rom IIcrrn Dr. r. M a rtius, aus der Pentandria Monogynia und der natürl. Familie der Amaranthaceen.

Froriep's Notizen Bd. XII. p. 343.

Brandesia, eine neue brasilianische Pflanzengattung, rom Hofrath und Ritter Dr. v. Martius in München.

Brandes Archiv Bd. XVI. Heft 3. p. 211 mit Abbildungen. In dieser Familie findet man noch die Namen melrerer meistens jetzt lehenden berühmten Chemiker, eine Berzelia M. Rosea Mart. Sertïrnera M. Pfaffia M. Bucholzia M.

\section{Phytolacceen.}

Phytolacceae. Bartling, Beitrăge zar Botanik. Heft II. p. 112. 
Gay.

Note sur les nouveaux genres Semonvillea et Gaudinia, par ì. J.

Bulletin des Sc. natur: Sept. 1829. p. 412.

Es ist hier die Rede von den am Senegal wachsenden Arten: Semonvillea pterocarpa, Gaudinia diffusa und viscosa. Die letztere Gattung wird aber einen andern Namen erhalten müssen, da schon eine Gaudinia, zu den Gramineen gehörend, von P. Brown besteht.

\section{Sclerantheen.}

Sclerantheae. Bartling, Beiträge zur Botanik. Heft II. p. 153.

Varietäten und natürliche Bastarde von Scleranthus, von $L$ a seh in Driesen.

Linnaea 1829. p. 410.

v. Voith, über Seleranthus annuus und perennis.

Bot. Zeit. 1826. I. p. 381.

Scleranthus neglectus Rochel.

Das. 1829. II. p. 614

\section{Paronychieen.}

Paronychieac. Bartling, Beitrïge zur Botanik. Heft II. p. 155.

Mémoire sur la famille des Onagraires et sur celle des Paronychiées, par M. A. Pyr. De Candolle. Paris 1829. 4. cn une seule livraison ornée de 9 grav. Prix y francs.

Steudelia, novum plantarum genus. Descripsit C. B. Presl. Pragac 1. Julii 1829. 4. cum tab. acn.

Linnaea 1830. II. p. 5\%. Lit.

Viviania Caranilles (oder Macraca Lindley) gehört nach Don zu den Caryophylleis neben Mollugo.

Jameson New. Philos. Journal. Jan. 1830. p. 180.

Nota. Bartlings Anordnung nach, ist diese Guttung hierher zu rechnen, er theilt die Familie in folgende Gruppen: Illecebrea, Spergulea, Molluginea, 'I'elephiea.

An account of a new Genus of Plants, named Macraea. By John Lindley Esq.

The Quarterly Journal of Science No. V. January to April 1828. p. $104-106$.

Beschrieben sind: M. grandifolia, parvifolia und M. rosea, sümmtlich in Chili einheimische Straucher. Lindley ist zweifelhaft, ob die Gattung zu den Lineen oder Caryophylleen gehöre.

\section{Portulaceen.}

Portulaceac. Bartling, Beitrïge zur Botanik. Heft II. p. 167.

Revue de la famille des Portulacées, par M. A. P. De Candolle. Lu à la Société d'hist. nat. de Paris le 23. Août 1828.

Mém. de la Soc. d’hist. nat. de Paris. T. IV. p. $17 t$ - 194. Bot. Literaturbl. II. p. 81.

Polpoda, novum plantarum genus. Descripsit C. B. Presl. Pragae, 30. Maji 1829. 4. c. tab. aen.

Linnaea 1830. p. 51. Lit.

Die Gattungen Cypselea, Aylmeria und Ginginsia, die Decandolle in der oben angezeigten 1828 erschienenen Abhandlung hierher recknet, stc- 
Juen in Bartlings Abtheilung bei den Paronychien, so wie Trianthema bei den Ficoilleen.

\section{Alsineen.}

Alsineae. Bartling, Beiträge zur Botanik. Heft II. p. 159.

Observations nouvelles sur les rapports des Frankeniacées et des Caryophyllées, par M. Ang. de St. Hilaire.

Bulletin de la Soc. philom. de Paris 1825.

Extrait d'une lettre de M. Gay, sur l'Arenaria triquetra.

Annales des Sc. natur. Janv. 1825.

Ueber Arenaria havarica und Moehringia muscosa, von F. Ma y er. Bot. Zeit. 1828. I. p. 205.

Bemerkungen üher Arenaria setacea Thureller ron Reichenbash Bot. Literaturbl. IV. $p .45$.

Alsineace collectae in expeditione speculatoria Romanzoffinna.

Linnaea 1826. $p$. 44.

Einige Beobachtungen über Cerastium rulgatum, viscosum und scmidecandrum L., von Carl B ouché.

Linnaea III. p. 64.

Ueber Cerastium repens und tomentosum L., vom IIern Prof. Tausch in Prag.

Bot. Zeit. 1828. I. p. 28.

\section{Sileneen.}

Sileneac. Bartling, Beiträge zur Botanik. Heft II. p. 160.

Caryophylleae (Tribus Sileneae) collectae in expeditione speculatoria Romanzoffiana.

Linnaea 1826. $p .37$.

System der Garten - Nelke. Berlin 1827.

Siehe Allgem. Monats-Bericht. VIII. August 1827.

Lychnis triflora, seltene Grönländische Pflanze, beschricben von C. S. Sommerfeldt.

Magaz. for Naturvidenskab. A. 1824. Cah. I. p. 151.

Lychnis inclusa Lehmann.

Bot. Zeit. 1830. I. p. 15.

Beobachtungen über mehrere Arten von Lychnis, Gypsophila, Dianthus, Silene u. s. w., vom Herrn Prof. Tausch.

Daselbst p. 242.

Ueber Silene polyphylla der deutschen Autoren, von Dr. Koch. Daselbst 1830. İ. p. 609.

Ueber einige Dianthus - Arten der deutschen Flora von Demselben. Daselbst p. 665.

\section{Crassulaceen.}

Mémoire sur la famille des Crassulacées, par M. A. P. Decanpolle. Paris 1828. 4. avec 13 'planches, $5 \mathrm{fr}$. p. 432.

Siche Linnaca 1829. 1. p. 20. Lit. - Botan. Literaturbl. I. 
Decades octo plantarum novarum succulentarum. Auct. A. H. Haworth.

Im Philosophical Magazine und Journal an mehreren Orten.

Dichotomische Tafel und Beschreibung der Arten der Gattung Sedum, von A. W. Haworth.

Daselbst Sept. 1825. p. 178.

Tillaea simplex, beschrieben ron Nuttall.

Aus dem Journal of natural sciences of. Philadelphia, in der Linnaea 1828. III. p. 162. Lit.

Description of the genus Francoa ete. with remarks on their Affinities. By M. David Don.

Jameson New Edinb. Philos. Journal. Oct. - Dec. 1828. p. 46. Adrian Jussieu rechnet die Gattung Francoa zu den Crassulaceen; nach Don ist sie mit Galax veruandt, und bildet eine eigene Familie (Galacineac), die in die Nachbarschaft der Philadelpheae und Saxifrageae zu bringen ist. Bartling setzt sie unter die Genera, deren Stelle zweifelhaft ist.

Man sehe Bullet. des Sc. nat. Sept. 1829. p. 420.

\section{Saxifrageen.}

Versuch einer Monographie der in Italien einheimischen Arten von Saxifraga, von M. Moretti.

Giornale di fisica, chimica etc. T. VII. bim. 2. et 3.

Moretti. Tentatiro diretto ad illustrare la Sinonimia delle specie del genere Saxifraga indigene del suolo italiano.

Giornale di fisica chimica etc. T. VIII. IX. Durch mehrere Abtheilungen.

Saxifraga pseudo - caesia Rochel.

Bot. Zeit. 1829. II. p. 614.

Saxifragae norae species.

Sylloge plant. nov. Ratisbon. 1824. p. 114. and 206. T. II. Ratisb. 1828. p. 240.

Escalloniae collectae in expeditione speculatoria Romanzoffiana.

Linnaea 1826. IV. p. 541.

Nota. Robert Brown bringt die Gattung Escallonia in die Nikhe der Grossularien, Bartling weist den Fiscallonien als eigene Familie cinen unbestimmten Platz an das Finde des Systems an; Decandolle betrachtet sie als eine Gruppe der Saxifrageen.

\section{Halorageen.}

Nach Bartling begreift diese Ordnung die Hippuridene Link, die Callitrichineae Lavieille, die Hygrobiae Richard, die Cereodianae Jussicu.

Remarques sur le Callitriche verna de Linné, par le Dr. F. U. Lavieille.

Annales de la Soc. Linn. de Paris Juillet 1824.

Das Wichtigste in diesem Aufsatze ist die Nachweisung, dafs die Gattung Callitriche nicht in die Familie der Najaden gehört, sondern in eine andere Familie der Dicotyledonen gebracht werden mufs. Herr L. beobachtete genau die zuei einander gegenüber stehenden Cotyledonen. Die Saamen keimen innerhalb 10 - 15 Tagen. 


\section{Lythrarieae.}

Revue de la fimille des Lythrariées, par M. Decandolle. Genève 1826. 4. avec trois planches.

Siche Linnaea 1827. p. 503.

Observations sur deux nouveaux genres de plantes, par M. Desvaux, Directenr dn Jardin de Botanique à Angers. - Physopedium. Annales des Sc. natur. Dec. 18\%6. p. $401-404$.

Salicarieac collectae in expeditione speculatoria Romanzoffiana. Linnaea 1827. III. p. 315.

Mémoire sur le Fatioa, genre nouveau de la famille des Lythraires, par M. Decandolle, Prof. ì l'academie de Genève.

Denkschriften der allgemeinen Schweizer Gesellschaft für die gesammten Naturwissenschuften. Bd. I. Abthcil. 1. p. 97 - 100, mit Abbildung der Fatioa nepaulensis.

Nicolas Faloa (dem die Gattung gewidmet ist) de Duillers aus Genf, schrieb über die Wirkung der Krümmung der Baumzweige, als Mittel zur Befürderung der Fruchtbarkeit.

Note sur les Elatinées, nouvelle famille des plantes, par J. Cambessèdes (Elatine, Bergia, Merimea).

Múmoires du Muséum d’hist. nat. Neuv. année 9. Cahier p. 225 - 231.

\section{Onagrarien.}

II émoire sur la famille des Onagraires et sur celle des Paronychićes, par M. A. Py r. Decandolle. Paris 1829. 4.

Onagreac arcticac.

Linnaea 1827. IV. p. 552.

Ledebour, über Oenothera Romanzoffii und 0 . stricta.

Mém. de l'Acad. de Petersbourg. VIII. p. 11. 12.

Beobachtungen über Philadelpheae und Granateae, zwei nene Pflanzenfamilien von David Don.

$132-135$.

Edlinburgh New Plilosophical Journal. April - July 1826. p.

Herr Dr. Bartling zählt die Philadelpheen $\approx u$ den Onagrarien,

die Granateen aber behält er als eigene Familie bei.

Deutzia scabra Thunberg beschreiht Don sehr ausführlich und zcigt dabei, daf's die Pflanze zu den Philatelpheen grehört.

Edinb. New Philos. Journal. Jan. 1830. p. 170.

\section{Combretaceen.}

Mémoire sur la famille des Combretacées, par M. Decandolle.

Mém. de la Société de physique et d’hist. nat. de Genève. Tóm. IV. p. $1-42$.

A review of the Genus Combretum. By M. George Don.

Transactions of the Linnean Society. Fol. XV. P. II. p. 412. Linnaea 1828. p. 139. Lit. - 1sis 1830. p. 166. - Botan. Literaturbl. I. p. 453.

\section{Granateen.}

Beobachtungen üher Philadelpheac und Granateac, zwei neue Pflanzen - Familien, von David Don.

Edinburgh New Philos. Journal. April - July 1826. p. 138 - 135 


\section{-115
Calycantheen.}

Note sur l'organisation d'un très vieux Calycanthus floridus du Potager royal de Versailles, par M. Mirbel. Annales des Sc. natur. Aoút 1828. p. $341-362$.

\section{Melastomaceen.}

Monographie des Melastomes et autres genres du même ordre, par A. Bon pland. II. Vol. Fol. Paris 1823.

Mémoire sur la famille des Melastomacées, par M. Aug. Pyr. Decandoll e. Paris 1828. 4. avec dix planches.

Siehe Linnaea 182. I. p. 19. Lit. - Botanische Literaturbl. II. $p .315$.

Melastomaceae, Don im

Edinbuirgh philos. Journal 1823. p. 180.

Ueber die Malayischen Arten von Melastoma, von Dr. W. Jack. Aus den Linn. Transact. XIV. P. I. p. 1. in der Isis 1829. Heft X. $p$. 1036.

Hier sind beschrieben: Melastoma obvoluta, malabathrica (Rumph Amb. 4. 137. t. 72.), erecta, decemfida, stellulata, nemorosa, bracteata, exigua, rotuudifolia, pallida, fallax, gracilis, glauca, viminalis, eximia, rubicunda, alpestris. -

An account of a new Genus of plants, called Diplogenea. By John Lindley Esq. F. R. S. Prof. of Botany in the University of London.

p. 121.

The Quarterly Journal of Science Nr. VII. July to Octob. 1828.

Die neue Gattung steht der Conostegia am nächsten. Bescliricben ist D. viscoildes, die einsige Art, voin Johannes Forbes auf Madagascar entdeckt, es ist ein, dem Ansehen nach parasitischer Strauch, der fast das Ansehen eines Viscum hat.

\section{Myrtaceen.}

Mémoire sur les Lecythidées, par M. A. Poiteau.

Mémoires du Muséum d’hist. nat. Tom. XIII. p. 141 - 165.

Abgebildet sind: tab. 1. Lecythis longipes. tab. 2. Lecythis corrugata. tab. 3. Bertholletia excelsa. tab. 4. Gustaria urceolata. tab. 5. Gustavia pteroarpa. tal,. 6. Détails du Gustavia. tal. 7. Détails des fruits du Bertholletia et du Couratari.

Observations sur le genre Couratari d'Aublet, par M. Achille Richard.

Siehe Isis 1826. Heft. II. p. 176 - 182.

Note sur les Myrtacées, par M. De Candolle. Article extrait du onzième volume du Dict. classique d'hist. natur. Paris 1826. 8.

Siehe Linnaea 1827. p. 504.

Observations on the Characters and Affinities of Darwinia etc. loy David Don.

Jameson new. Edinb. phil. Journal. April - July 1829. p. 83.

Darwinia gehört nach Don in die Familie der Myrteen zunëchst an Calythrix; Cunningham brachte sie sonst zu den Rhamneen, Bartling und Andere zu den Thymelëen. (Siehe die letztere Familie.) Bullet. des Sc. nat Oct. 1829. p. 78. 


\section{Camelliaceen.}

Collection de Camellias élevés à Bollwyller, dédice à Mr. Dr. Candolle, par M.M. Ch. et Nap. Bam ann. 1. livaison in 4. Bollwyller 1829. avee 12 planches color.

Siche Bibliothique universelle. Mai 1830. p. 109.

Liste des noms des Thés les plus éfibres de la Chine, traduite d'ua manuscrit chinois appartenant it II. Le baron de Sehilling; par M. Klaproth.

Journal asiutique 20. Cahier. p. 129. uddition pur .11. Abel Rémusat. Ib. 21. Cahicr. p. 187. 1824.

\section{T'ernstroemiaccen.}

Mémoire sur les Familles des Ternstroemiacées et des Guttifires, par M. J. Ca mbessèdes.

Mémoires du Muscum VIII. année Cah. 11. p. 369- 4118. Cah. 12. p. $409-424$.

\section{Sterculiaceen.}

Tilesius. Cheirnstemum platanoides Humboldti ob mirabilem interioris corollae structuram denuo pictum.

Mém. de l'Acall. de Petersbourg. V. p. 321. t. 9.

\section{Büttneriaceen.}

Comparaison des genres Buttneria et Commersonia, par M. A uguste de Saint Hilaire.

Annales des Sc. natur. Octobre 1825. p. $131-138$.

Ueber eine nete Pflanzen-Gattung Recresia aus der Familic der Büttneriaceen, von $\mathbf{J}$. Lindl ley.

Quarterly Journal of Science. New series No. III. Juty to Sept. 1827. p. 109. - Bot. Literaturbl. I. p. 248.

Die Guttung soll zuischen Pterospermm und Stercalia ihe Stelle erhalten; erstere gehört nach Turtlings Anorilnung $z$ den

Dombeyaceen, letztere in die eigene Fumilie der Sterculiacen.

Monographie du Cacąo, par M. Gallois. Paris 1827.

\section{Hermanniaceen.}

Observations sur les caractères du genre Melochia, par M. Ang. Saint Hilaire.

Bull. de la Soc. philomat. de Paris Oct. 1825. p. 154.

\section{Malvaceen.}

Bemerkungen über die Existenz des Eywcisses in den Malvaccen, von Herrn August von Saint-Hilaire.

Nouveau Bulletin des Sciences par la Socićté plitomatique de P'aris. Nov. 18\%5. p. 187.

Note sur le genre Malachra, par M.Auguste de Saint-Milaire. Ibiclem Aoút 1826. p. 120. - Bullctin des Sc. nat. 1827. p. 105. - Bot. Literaturbl. I. p. 219.

Bemerkungen über Lavatera, vom IIcrun Prof. J. F. Tausch Bot. Zeit. 1829. I. p. 177. 
Nachricht über Hibiscus fugax Martius und dessen Cultur, vom Hofgärtner Seitz in München.

I'erhandl. des I Ereins zur Beförderung des Gartenb. in den $\boldsymbol{K}$. Pr. Staaten. Bd, III. $p .102$.

Note sur les principales éspèces de Sida de la Flore de Brésil méridional, par M. Aug. de St. Hilaire.

Bulletin des Sc. natur. Février 1827. p. 273. - Linnaer: 1828.

p. 59. Lit.

D r. Biume stellt in den Bydragen tot de Flora van Nederlandsch Indie eine kleine Familie, dio Dipterocarpeae, auf, den Malracen und Bombaceen verwandt.

Isis 1828. p. 262.

\section{Geraniaceen.}

Geraniaceac, oder dic natürliche Familie der Geranien, von Robert Sweet. London 1824.

Ein Kupferwcrk, das heftweise erscheint, jede Lieferung enthält vier colorirte Figuren, nach der Natur gezeichnet und gemalt, mit Anzeige der systematischen und englischen Namen, so wie der Culturart. (Jede Nummer kostet drei Schilling.)

Neue Arten ron Pelargonien deutschen Ursprungs. Als Beitrag zu Rob. Sweet's Geraniaceen. Nelsst wissenschaftlichen Frläuterungen und rollständiger Anleitung zur Cultur derselben, mit Abbildungen, die sämmtlich nach lebenden Originalien gefertigt worden. Heransgegeben von einigen deutschen Gartenfreunden, der'Text von Leopold 'Trattinnik. Wien, auf Kosten der Herausgeber und zu finden bei Tendler und v. Manstein am Graben. 1825.

Beschreihung aller bekannten Pelargonien und Anleitung zur Erkennung und Cultur derselben, in Verbindung mit mehrern Freunden dieser Blumen herausgegeben von $\mathbf{J}$ acob Ernst v. Reider, K. $B$. erster Landgerichts-Assessor. Nürnberg 1829. $3 \mathrm{fl}$.

Rectification des caractères généraux des genres Erodium et Geranium, par M. A ug. St. Hilaire.

Bullet. de la Soc. philomatique de Paris. Férr. 1825.

Sur la necessité de comprendre dans le seul groupe des Geraniées cenx qui ont été formés sous les noms des Tropaeolées, Geranécs proprement dites, Oxalidées et Linées, par M. Aug. de St. Hilaire.

Bull. de la Soc. philomát. de Paris. Juillet 1825.

C. Steven, Erodii serotini descriptio.

Mém. de l'Acad de Petersb. III. p. 295.

Description d'un gence noureau de la fanille des Geraniacées, par J. Cambessèdes.

Mémoires du Museum. Ncuv. Année. 11. Cahier. p. 369.

Die Gattung ist benamnt zu Ehren des Herrn César de $\mathbf{S}$. IIlaire, Schiffskapitain; er brachte zuerst den Mokka-Kaffee nach Isle de France und Bourbon. Es sind zwei brasilische Arten beschrieben:- Caesarea albiflora und C. rubriflora. -

Die bereits oben bei den Paronychien berührte Gattung Macraea könnte vielleicht auch bei den Geraniaceen ihre Stelle finden.

\section{Lineen.}

Linea collectae in expeditione speculatoria Romanzoffiana.

Linnaea 1526. I. p. 65. 
Beschreibung einer neuen Art Linum, vom Prof. A. Bertoloni. Giorn. arcad. di Scienze 1828. T. XXXVII. p. 58.

Die neue Art ist L. serrulatum: foliis inferioribus obverse lanceolatis oltusis, eaulinis basi biglandulosis: calicibus eapsula brevioribus serrulatis. Nascitur in Umbria, agro Practutiano etc.

\section{Oxalideen.}

Monographie der amerikanischen Oxalis-Arten, von Dr. J. G. $\mathrm{Z}$ uccarini. Mit 6 Steinabdrücken. München 18:5. 4 .

Siche bot. Zeit. 1826. I. p. 337.

Ueber Oxalis corniculata und stricta von Demselben.

Daselbst p. 257.

Dr. Blume, in den Beiträgen zur Flora des holländischen Indiens, stellt eine neue Familie unter dem Nimen Hydrocereac auf; sie soll ihre Stelle zwischen den Oxalideen und Balsamineen erhalten und begrcift die Gattung Hydroccra. (Impatiens angustifolia.)

Isis 1828. p. 263.

\section{Balsamineen.}

Notice sur la Balsamine des jardins (Impatiens Balsamina L.), par M. Charles Kunth.

Mlémoires de la Soc. d’hist. nat. de Paris. Vol. III. Livr. 3. p. 384.

\section{Ampelideen.}

Giuseppe Acerbi di Castelgaffredo. Delle vite italiane, o sia materiali per servire alla classificazione, monografia e sinonimia, precéduti dal Tentativo di una classificazione geoponica delle viti. Milano. 1821. 8.

Siehe auch Biblioteca italiana. Juni 1823. André ökonom. Jieuigkeiten. Bd. XXXIII. p. 169.

Untersuchungen über Most - und Weintrauben-Arten Würtembergs. Inaugural - Dissertation (praeside Schübler), von Friedrich Köhler. Tübingen 1826.

Untersuchung über Obst - und Weintrauben - Arten Würtembergs, und die richtige Leitung der Gährung ihres Miostes. Inaugural-Dissertation (praeside Schübler) von $J_{0} \mathrm{~h} n \mathrm{Berg}$. Tübingen 1827.

Siche Geiger's Magazin. Februar 1828. p. 181. u. d. $f$.

Der Rheinische Weinbau, in theoretischer und praktischer Bezichung bearbeitet, von Joh. M etzerer, Universitätsgärtner zu Heidelberg. Heidelberg 182\%. Mit 17 Steintafeln.

Versuch einer systcmatischen Anordnung der vorzüglichsten, in den Rheingegenden cultivirten Varietäten des Weinstocks, von Dierbach.

Linnaea 1828. p. 142.

Ueber die Blüthe des Weinstocks, von Herrn Maximilian Kellex zu Freiburg im Breisgan.

$\boldsymbol{F}$ crhandl. des Ve eins zur Beförderung des Gartenbaues. Bd. II. p. 465.

Viele andere Schriften und Aufsätze, die Weinrebe, zumul deren Cultur betreffend, erhalten ihre Stelle in dem okonomisch-technischen Theile. 


\section{$-\quad 119$ \\ Malpighiaceen.}

Dixième Mémoire sur les caractères généraux des familles tirés des graines, et confirmés ou rectifiés par les observations de Gärtner. Par M. A. L. de Jussieu.

Wieder abgedruckt in der Isis 1826. Heft II. p. 167 - 176.

\section{Acerineen.} Prag.

Bemerkungen über Acer, vom Herrn Prof. J. F. Tausch in Bot. Zeit. 1829. II. p. 545.

\section{Sapindaceen.}

Cambess èdes. Mémoire sur la famille des Sapindacées.

Mém. du Muséum d’hist. nat. Année 9. Cah. 7. p. 1 - 50.

Sur les fleurs de quelques genres de Sapindacées, par Aug. St. Hilairc.

Annales des Sc. natur. Juillet 1828. p. 292.

Dodonaea cuneata, beschrieben von Rudge in den Linnean Transactions. Siehe Isis 1829. p. 144.

\section{Euphorbiaceen.}

En umeratio Euphorbiarum, quae in Germania et Pannonia gignuntur; auctore Joanne Raeper, M. D. Goettingae, typis C. E. Rosenbach. 1824. 4.

De Euphorbiacearum Generibus medicisque earundem viribus Tentamen, tabulis aeneis 18 illustratum, auctore Adriano de Jussieu. Parisiis ex typis Didot junioris. 1824.

Adriana, nouveau genre de plantes, par M. Gaudichand. Annales des Sc. natur. Juin. 1825. p. 221.

Description d'une nouvelle éspèce d'Euphorbe, Euphorbia Milii, par M. Charles:Desmoulins.

27. 1826.

Bullet. d'hist. nat. de la Soc. Linn. de Bordeaux. Tom. I. p.

Die Frucht der Aleurites brasiliensis, von $\mathrm{Agh}$ ard.

Suenska Laekare-Saelskaps Handlingar. X. 414. - Bull. des

Sc. med. XIII. 278. - Brandes Archiv 1829. Heft I. p. 26.

Mercurialis triandra Meyer.

Linnaea 1829. II. $p$. $23 \%$.

\section{Empetreen.}

On the Affinities of the Empetreae, a natural Group of Plants. By M. David Don.

Jameson New Edinburgh Philosophical Journal. Oct. - Dec. 1826. $p .59-63$.

\section{Bruniaceen.}

Mémoire sur la famille des Bruniacées, par M. Ad. Brongniart 
Annales des Sc. naturclles. Tom. VIII. Aug. 1826. p. 357 - 389. - Botun. Literaturbl. II. p. 86.

\section{Rhamneen.}

Mémoire sur la famille des Rhamnées, par Mr. Adolph Broneniart. Annales des Sciences nalur. Tom. X. Mars 18:7. p. 3201. Avril p. 337 - 386. - Bot. Literaturbl. II. p. 86.

\section{Ochnaceen.}

Diporidium Wendland, in dessen und Bartlings Beitriagen zur Botanik. Heft II. p. 24 .

\section{Zanthoxyleen.}

Note sur le Zanthoxylum des Caraihes, ou Clavalier dés Antilles, par M.M. Cherallier et Gabriel Pelletan. Journal de Chimie medicale. Juillet 1826. p. 314 - 321.

Zanthoxyleae collectae in expeditione speculat. Romanzoffiana. Linnaea 1830. p. 58.

\section{Diosmeen.}

Bartling und Wendland. Beitrïge zur Botanik. Erstes II ft. Auch unter dem Titel: Diosmeac descriptae et illustratac. Gottingac 18\%4.

Diosmeae collectae in exped. specul. Romanz.

Linnaea 18ü. p. 51.

Monographie du genre Phebalium, par M. Adrian de Jussieu.

Mém. de la Soc. d'hist. nat. de Paris. T. II. et Annales des sic. natur. Avril 18\%5. p. 481.

Philotheca australis, als Gattung und Art, beschrieben von Rudge in den Linncan Transàctions.

Siche Isis 18\%9. p. 145.

Eriostemon salicifolius, beschrichen von $R u d g e$.

Daselbst p. 148.

\section{Rutaceen.}

Mémoire sur la Groupe des Rutacées, par M. Adrien de Jussicu.

Mén. du Mus. d’hist. nat. Vol. XII. p. ̈84. et suiv.

Suite du Mémoire sur la groupe des Rutacées, par M. A. de Jussieu.

Mém. du Mus. Sixiòme Année, Cah. XII. p. 4.19. jusqu'au fin.

Rapport sur un Mémoire de M. Adricn de Jussieu, ayant pour oljet la famille des Rutilcées, par M. Desfontaines.

Annales des Sr. naturelles. Avril 1826. p: $419-428$.

Rutaceae collectae in expeditione specul. Romanz.

Linnaed 1830. I. p. 43.

\section{Zygophylleen.}

Zygopliylleae collect. in expedit. specul. Rom. Linnaca 1830. p. 4 .

Trichanthera et Anatropa, genera nova familiae Zygaphyllearum, auctore Ehrenberg.

Linnuea 1829. III. p. 401.402. 


\section{Aurantiaceen.}

Traité du Citrus, par Georges Galesio, précédé d'un extrait d'une lettre de M. Oscar Leclerc Thouin à M. C. 2e édition. Paris 18?9.

\section{Terebinthaceen.}

Mémoire sur les genres Connarus et Omphalobium, ou sur les Connaracées et Sarcolobées, par M. De candolle.

Mémoires de la Soc. d’hist. nat. de Paris. T. II. P. II. $p$. $379-396$.

Abgebildet sind: Connarus pubescens, Omphalobium Patrisii,

0.- Parrotetti.

On Boswellia and certain Indian Terebinthaceae. By Henry Thonas Colebrook.

Transactions of the Linnean Society Fol. XV. P. II. p. 355. Linnaea 1828. p. 13อ. Lit. - Isis 1830. p. 164.

Hemprichia Ehrenberg, genus novum ordinis Terebinthacearum et familiae Burseracearum.

Linnaea 1829. III. p. 396.

Aceount of the new genus Melannorrhea, or the Burmese Varnish Tree, with remarlis on each of the Genera to which it approaches. By N. Wallich, Superintendant of the Botanic Garden Calcutta.

Edinb. Journal of Science. Jan. 18:0. p. 66. - Brandes Archiv. Bd. XXIV. Heft 2. p. 129. - Annales des Sc. natur. Avril 1830. p.' 438 .

\section{Rosaceen.}

Rosarum Monographia, auctore J. Lindley. London 1824.

Observations sur la nomenclature et le classement des Roses, suivies d'un catalogue de celles, cultivées par J. P. Vibert. Paris 1824. $5 \mathrm{f}$.

Les Roses. Par J. J. Rédonté arec le teste de C. A. Thory. Paris 1825.

Erscheint in Lieferungen; die 17. ₹. B. enthält die Abbildungen von Rosa indica, R. gallica agatha, R. indica centifolia, R. Orbasseana. - Edition angmentée. Paris 1826. 8. 1 Rthl. 8 gr.

Saits Tobias. Die Rosen nach ihren Früchten. Ein unentbehrlicher Leitfaden zı ihrer richtigen Bestimmung, für Botaniker, Gärtner etc. oder alle bisher bekannte Rosenarten, nach Trattinniks Synodus karpologisch dargestellt: Prag 1825.

Die Rosen des Orients.

Geiger's Magazin. Bd. X. p. 146.

Examen critique du Rosa Candolleana elegans, injustement cité comme synonyme du Rosa rubella, par M. A. Ant. Thory.

Annales de la Soc. Linn. de Paris. Sept. 1825.

Rosea collectae in expeditione speculatoria Romanz.

Linnaea 182\%. p. 35.

Rosetum gallicum, ou enumeration méthodique des éspèces et varietés du genre Rosier, par W. Desportes. Paris 1828. 8. 124 S.

Desportes Roses cultivés en France, ou nombre de 2562 éspèces 
ou varietés, avec la synonymie françoise et latine. Paris 1830. 8. 1 Rthl. 8 gr.

Rosae plantarum generis Historia succincta accomodat a F. G. Wallroth. Nordhusae 1828. 8.

Ueber die brittischen Arten von Rosa, von Jos. Woods.

Aus den Transuct. of the Linn. Soc. Fol. XII. in den Jahrb. der Gewächsk. Heft II. p. 77.

Synopsis Rosarum Britanniae; auctore J. Woods. u. $d$. $f$.

Aus den Linn. Transact. XII. 1. in der Isis 1829. I. p. 602.

Catalogue des Roses cultivés, chez M. de Tocqueville. Paris 1822. 8 .

Cataloguc des Rosicrs, cultivés chez M. Godefroy ì Serres. Paris 1830. 12. $6 \mathrm{fr}$.

On the anomalous Structure of the Leaf of Rosa berberifolia. By M. David Don.

New. Philos. Journal. Jan. 1830. p. 175. - Don glaubt, diese

Art müsse von der Gattung Rosa getrennt werden.

Auguste Pronville. Nomenclature raisonnée des éspèces varietés et sous-varietés du genre Rosier, observés au jardin royal de plantex, dans ceux de Trianon, de Malmaison et dans les pepinières aux environs de Paris. Paris 1818.

\section{Pomaceen.}

Bemerkungen über die natürliche Gruppe der Pflanzen, welche Pomaceae heifsen, von J. Lindley.

Aus den Linn. Transactions in der Isis 1825. Heft IX. p. 972.

Lindley beschreibt zwölf Arten Catoneaster in 15. Bande des Botanical Register.

Siehe Bull. des Sc. nat. Sept. 1829. p. 378.

Crataegus kyrtostyla u. s. w., vom Dr. C. A. Fingerhuth.

Linnaea 1829. III. p. 372.

Ueber Crata egi von Lasch in Driesen.

Linnaea 1830. III. p. 439.

\section{Amygdaleen.}

Characteres novi Cerasorum germanicorum. Auctore G. Licgel. (Apotheker in Braunau.)

B uchners Repertorium. Bd. XXII. Heft 2. p. 215 - 220. und Bd. XXY. Heft 2. p. 169.

Memoria sul Pruno Coeumiglia di Calabria, del Cav. Michel Tenore. Napoli 1828. 4.

Jibl. universelle 1829. Juillet p. 214. - Linnaca 1829. III. p. 49. Lit.

$\Lambda \mathrm{mygdal}$ e a e collectae in exped. speculat. Romanz.

Linnaed 1827. IV. p. 542.

\section{Dryadeen.}

Nees v. Esenbeck und Weihe. Die deutschen Brombeersträucher, beschrieben und dargestellt. Bonn 1821. Fol. 10 Fascikel. 
Versuch über dic helvetischen Arten von Rubus, nebst Bemerkungen über Species-Bildung im Allgemeinen, von Dr. Johannes Hegetschweiler in Stäfa.

Denkschriften der allgem. Schweizer Gesellschaft für die gesammten Naturwissenschaften. Bd. I. Abtheil. 1. p. 1 - 47.

Ueber Fragaria vesca $L$. und semperflorens Hayne, von $L$ a s $h$ in Driesen.

Linnaea 1289. III. p. 409.

Ueber Potentillen von Demselben.

Das. p. 427. und 1830. III. p. 431.

Ueber einige Potentillen von Lehmann.

Bot. Zeit. 1830. I. p. 190.

Beschreibung der Cavania, einer neuen Pflanzen-Gattung und einer neuen Art Sieversia, von Herrn David Don.

Transact of the Linn. Soc. V. 14. p. 573.

(Cowania mexicana, Sieversia paradoxa oder Geum plumosum Sesse et Moeinno aus Mexiko.

Siehe Isis 1829. XI. p. 1287.

Note sur le feuillage des Clifforties, par M. Decandolle.

Annales des Sc. natur. T. 1. p. 447.

Dryadea e collectae in exped. speculatoria Romanz.

Linnaea 1827. $p .3$.

\section{Spiraeaceen.}

Kerria und Purshia, zwei neue Gattungen aus der Familie der Rsaccen, von Herrn De Candolle.

Linn. Transact. XII. p. 1. - Isis 1829. p. 499. u. d. f.

\section{Leguminosen.}

Mémoires sur la famille des Legumineuses, par M. Aug. Pyr. Decandolle. Paris 1825 et 1826. 4. Sept livraisons.

Notices sur quelques genres et éspèces nouvelles de Legumineuses, par. M. Decandolle.

Annales des Sc. naturelles. Janv. 1825.

Observations sur la famille des Légumineuses, par M. Desveanx. Annales des Sc. naturelles Dec. 1826. p. $40 t-438$.

Observations sur la famille des Legumineuses et sur quelques éspèces de l'Afrique centrale, par M. R. B rown. (Parkia africana etc.) Annales des Sc. naturclles Févr. 1827. p. 206 - 215.

Ueber Cassia rostrata Martius, vom Hofgärtner Seitz in München.

Verhandl. des Vereins zur Beförder. des Gartenbaues 1826. Heft V. p. $130 u$. 290.

Ueber die Gattung Copaifera, von F. G. Hayne. Linnaea 1826. p. $418-432$.

Mémoire sur la tribu des Coronillées, huitième Section des Legumineuses, par M. N. A. Desveaux.

Annales de la Soc. Linn. de Paris. Sept. 1825. p. 196.

Einige Bemerkung en über die Gattung Coronilla von T'reviranus. Linnaea 1827. p. 380. 
Note sur le Coronilla vaginalis, par M. Sendel.

Annules de's Sc. naturelles. Avril 1826. $p .455-45 \%$.

B emerkungen üher einige Arten Cytisus, vom Itern Prof. Tauseh. Bot. Zeit. 1829. II. p. 649. und 1830. I. p. 241.

Ueber die Gattungen Dolichos und Phascolus, vom Dr. Gaetano Savi.

Nuovo Giornali di Letterati. Jan. Febr. 1825.

Veher eine neue Edwarsia der Gürten, rom Prof. Wenderoth in Marburer.

Linnaca 1830. II. p. 2011.

Beschreibuner zwei neuer Arten Errthrina, von F. de Brotero. Lim. Transactions. XIV. 2. t. 10 - 12. p. 312. - Isis 1829. Heft X. p. 1040.

E. pajanthes und secundiflora.

Observations on the Genus Glycine and some of its lindred grencra. By Stephan Elliot of Charlestón.

Aus dem Journal of Philadelphia in der Linnaca 1828. p. 163. Lit.

II as aclia, neue Gattung ron Bentheim aufgestellt, dem Lotus verwandt. p. 72.

Aus dem Botanical Register im Bull. des Sc. natur. Oct. 1889.

Observations sur les caractires du genre Malochia, par M. A uguste de Saint-Hilaire.

Nouveau Bulletin des Sciences par la Société philomatique de Paris. Octobre 18\%5. p. 154.

Medicago corymbifera. Eine nene deutsche Art, aufgefunden und beschrieben rom Dr. W. L. E. Schmidt.

Linnaea 1829. $p$. 74.

Mimoses et autres plantes legumineuses du nouveau Continent, par C. S. Kunth, Paris 1820. I. Vol. Fol.

Repetition of M. Dutrochet's Experiments on the Mimosa pudicaBy Robert Spittal. Esq.

Jameson new Philos. Joarnal. Jan. 1830. p. 60 - 64.

Betrifft das angebliche Nervansystem dieser Pflanze.

Mémoire sur les Nauchées, grenre noureau dans la famille des Legumineuses, par M. J. Theod. Descourtilz.

Annales de la Soc. Linn. de Paris. Mars 1825.

Ueber. Orobus sylvaticus ron Koch.

Bot. Zeit. 18\%9. II. p. 733 .

Pultenaea ferruginea, elliptica und polygalacfolia, beschricben von $R$ udge in den Linn. Transactions.

Isis 1830. p. 146.

Soemueringia, novum plantarum genus constituit Dr. C. F. P'. de Martius.

Siehe Linnaca 18\%9. I. p. 3. Lit.

Note sur le Trifolium magellanicum, par M. Decandolle.

Anvalos des Se. naturelles. Janv. 1825.

Obscrvations sur la section des Trifles nommés Lupulina par Limú et sur une nouvelle éspèce de cette section, par M. Desveaux, directeur du jardin botanique d'Angers. (T. prionanthum.) 


\section{$-125-$}

Annales des Sc. natur. Mars 1828. p. 323 - 332. - Bot. Literaturbl. II. $p$. IU6.

Sur une variété nouvelle du 'Trigonella monspeliaca, trouvée aux environs de Paris, par M. Vignal.

Bullet des Sc. natur. Aoüt 1827. p. 436.

Zollernia novum plantarum genus Friderico Guilelmo III. Borussovum regi sacrum, auctor. Ne es ab Esenbeck.

Nova acta physico-medica. T. X. p. 2. cum tab. C.D. - Linnuea 1828. p. 45. Lit. - Bot. Zeit. 1829. II. p. 414.

Familien deren Stelle im System noch ungewifs ist.

$$
\text { §. 19. }
$$

\section{Ceratophylleen.}

Aquaticac quaedam recessit, ab Adalberto de Cham is so.

Linnaea 1829. p. 503.

Mantis'sa in genus Ceratophyllum.

Ibidem 1830. p. 336.

\section{Begoniaceen.}

Begonia diversifolia, eine mexicanische Pflanze, sehr genan beschrieben, ron Dr. Graham, Prof. der Botanik in Folinburg.

Jameson new Phil. Journal. Jan. 1830. p. 183.

Die Gattung Sarracenia sckeint den Typus einer eigenen Familie zu bilden. Man sehe:

Du genre Sarracenia en famille et description de la variété $\mathrm{S}$. purpurea, croissant à l'ile de Terre Neure, par M. B. de la Pylaie.

Annales de la Soc. Linneenne de Paris. Scpt 1827. p. 379. u. d. f. - Linnaea 188. III. p. 130. Lit.

\section{Dritter Abschnitt.}

\section{Greologie in Bezug a u $B$ otanik.}

\section{§. 20.}

Die Lehre von der Verbreitung der Pflanzen, odcr, wie man zu sagen pflegt, die Geographie des Gewächsreiches, ist erst in neueren Zeiten gehörig gewürdigt worden; wenn man gleich längst schon anfing, die Pflanzen einzelner Gegenden besonders zusammen $\approx u$ stellen. Die Betrachtung des Geuächsrciches je nach den besondern Slandorten, welche einzelne Arten, oder ganze Gattungen und Fumilien vorzuziehen pflegen; das klimatische Verhältnifs und der nicht zu verkennende Einflufs desselben, auf die Vegetation, veranlafste die Aufsuchung der Gesetze, die dic Natur bei der 
Vertheilung der Gewächse beobachtete und führte zu eben so lehrrcichen als interessanten Folgerungen, so zurar, dafs heut zu Tage gerade dieser Zuvig der Botanik besonders viele Freunde und höchst geistreiche Bcarbciter gefunden hat.

Sellust im Schoofse der Frde suchte man die Ueberbleibsel einer Vegetation auf, die lange vor den gegenuärtigen Zeitrechnungen der Schmuck unseres Pluneten ưaren, und sic uurden zu einem bestimmten Berührungspunkte zueier verschuisterter Naturwissenschaften, der Mineralogie und Botunik, die auf so manche W'eise gegenseitig sich beleuchten und erhollen. - Es gehört hierher also zuvörderst die

\section{Flora der Vorwelt.}

\section{§. 21.}

Die Untersuchung fossiler Vegetabilien hat man bis jetzt fast einzig den Mineralogen überlussen, so zuar, dafs in den meisten Lehrbüchern der Pflanzenkunde davon entweder gar nicht, oder doch nur beilüufig und mit wenigen $\boldsymbol{W}$ orten geredet wird ; dennoch diüften diese Reste einer längst vergangenen Zeit selbst für die systematische Dearbeitung der Pflanzenkunde höchst interessante Winke geben und zu folgenreichen Schlüssen die I'eranlassung werden, wenn man aufmerksam vergleicht, was einst war, was und wie es dagegen jetzt ist, wo ein allmähliges Fortschreiten und gröfsere Ausbildung der Vegetation, eine nach und nach vollkomminer gewordene Structur nicht verkunnt werden kann. Es licgt jedloch anfser dem Plane dieser Schrift, darüber tiefer einzugehen, aber es dürfte den Lesern angenehm sein, auch mehvere ältere Werke über fossile Vegetabilien hier benannt zu sehen, zumal da sich voraus sehen läfst, dafs dieser Gegenstand von den Botanikern bald näher und allgemeiner geuürdigt werden wirl.

J. Gesner. Dissertat. 1 et 2 . de Petrificatorum variis differentiis et de petrificat. variis origin. praecip. telluris mutationum testibus. Zurici 175\%2 et 1756. Zusammengedruckt unter dem Titel: Tract. phys. de Petref. Leyilen 1758. 8.

E. F. v. Schlotheim. Die Petrefactenkunde auf ihrem jetzigen Standpunkte, durch die Beschreibung sciner Sammlung u. s. w. erläutert. Gotha 1820.

Eb. Nachträge zur Petrefactenkunde. Gotha 1822. 2. Altheil. 1823.

Eb. Beschreibung merkwürdiger Kräuterabdrücke und Pflanzenversteinerungen; ein Beitrag zur Flora der Vorwelt. 1. Abtheil. Gotha 1804.

G. Ritter v. Cuvier's Ansichten ron der Urwelt, nach der zweiten Original-Ausgabe verdeutscht und mit Anmerkungen begleitet von J. J. Nöggerath. Bonn 1822.

Graf Casp. v. Sternberg. Versuch einer geognostisch - botanischen Darstellung der Flora der Vorwelt. Prag 1820. gr. Fol. mit Kiupfern. Viertes Heft. Regensburg 1825.

Essai d'un exposé géognostique-botanique de la Flore du monde primitif. Par le Comte de Bray. Pragae 1820. Ratisbonne 1826. Fol. avec 64 planches.

Siehe botan. Zeit. 1827. I. p. $321 u$. d. f.

J. G. Rhode: Beitrïge zur Pflanzenkunde der Vorwelt nach Abdrücken in Kohlensehiefer und Sandstein aus schlesischen Steinkohlenwerken. Leipzig 1821. mit Steindrücken. Jede Lieferung, gr. Fol., liostet 20 ggr. Die vierte Licferung Breslau 1824. 
H. F. Link. Die Urwelt und das Alterthum, erläutert durch Naturkunde. 2 Theile. Berlin 1821 und $1822.4 \mathrm{fl}$. $40 \mathrm{kr}$.

J. F. Krüg er. Geschichte der Urwelt. 2 Theile. Quedlinburg 1822 und 1823. 8. $11 \mathrm{fl} .24 \mathrm{kr}$.

J. G. J. Ballenstedt. Die Urwelt, oder Beweis von dem Dasein und dem Untergange von mehr als einer Vorwelt. 3 Theile. Quedlinburg 1819. $6 \mathrm{fl}$.

Derselbe. Die neue Welt, oder die jetzige Welt, ein Gegenstück zur Urwelt. 2 Theile. Hannover 1821. $6 \mathrm{fl} .18 \mathrm{kr}$.

Derselbe. Die Vorwelt und die Mitwelt, wie anch Nachträge zur alten und neueren Welt. 2 Theile. Braunschweig 1823. $3 \mathrm{fl}$. $54 \mathrm{kr}$.

Ueber Ballenstedt's Urwelt; ein Wort freimüthiger Prüfung und versuchte Ehrenrettung der ältesten bibl. Urkunden, von einem preufs. sächsischen Landprediger. Nordhausen 1825.

Geschichte der Erdoberfläche, von J. G. Sommer. Mit 5 Kupfertafeln. Prag 185.

J. G. S. van Breda. Oratio de Florae Mundi primigenii reliquiis in lithantracum fodinis praesertim conservatis. Gand 1523.

Antediluvian Phytology illustrated by a collection of the fossil remains of plants peculiar to the coalformation of great Britain. By Edmund Tyrell Artis. London 1825.

Siehe bot. Zeit. 1827. I. p. 129.

Prodrome d'une histoire des Végétanx fossiles ou Recherches botaniques et géologiques sur les végétaux renfermés dans les diverses couches du globe; par M. A. Brongniart. 4. Paris 1828. 1. et 2. livn avec 18 planches. 13 franes.

Siehe bot. Litera'urbl. I. p. 293. IV. p. 5. und Geiger's Magaz. Dec. 1829. p. 181.

Observations sur quelques végétaux fossiles du terrain houillier, et sur leur rapports arec les végétaux vivans, par M. Ad. Brongniart. Annales des Sc. natur. Janvier $18 \%$.

Cónsiderations générales sur la nature de la végétation, qui courrait la surface de la terre aux diverses periodes de la formation de son écorce; par M. Adolphe Brongniart.

Annales des Sc, natur. Nov. 1828. p. 225 u. d. f.

Essai d'une Flore du gré bigarré, par M. Adolphe Brongniart.

Annales des Sciences naturelles Dec. 1828. p. 435. - Siehe bot. Literaturbl. II. p. 245.

Ueber die von Brongniart aufgestellte Erklärungsart der Bildung der Flova der Vorwelt sehe man die Kritik des Herrn Prof. Hoffmann in Halle. (Poggendorf's Annalen der Physik und Chemie XV. Heft 3. p. 415. und botan. Literaturbl. III. p. 443).

Remarks on the Ancient Flora of the Earth.

Jameson new philos. Journal. Jan. 1830. p. 112. - Annalen der Physik v. Poggendorf 18थ9. Nro. 3. - Journal de Géologie. Juin 1830. p. 172.

Les Continens actuels ont ils été a plusieurs reprises submergés par la mer? Dissertation géologique par M. Constant Prevost. Iue 
à l'Acadenie royale des Sciences, dans les séances des 18. Juin et 2. Juillet 1827.

Vímoires de la Soc. d'hist. naturelle de Paris II. p. 219 u.d.f. -

Siehe bot. Literaturbl. II. p. 254.

A new System of Geology, in which the great Revolutions of the Farth and Animated Nature are reconciled at once to Modern Science and to Saced History. By Andrew Ure. London 1829. 8.

C. F. Sehulze. Iüre Betrachtung der versteinerten IIölzcr. Dresden 1754. 4.

J. J. Nöggerath, über anfrecht im Gelsiresstein eingeschlosscne fossile Baumstimme und andere Vegetahilien. IIistorisches und Beobachtung. Bonn 1819. 8. mit 2 Stcindruchtafeln. $12 \mathrm{gr}$.

besselben fortgretzte Remerlungen ïber fossile Baumstämme und andere Vegretabilien. Bonn 1830. $8.8 \mathrm{gr}$.

Siehe auch 1sis Bd.VII. p. 901.

Commentatio de Psarolithis, ligni fossilis genere. Auctore Antonio Sprengel, Phil. Dr. c. tal. aeri incisa. Halae 1828. 8. $42 \mathrm{~S}$.

Fossile Cycadoideae; nach Dr. Buckland.

Philos. Magaz. and Annals of Phi osophy. New Series. Nro. 12.

Sept. 1828. p. 225. - Botan. Literaturbl. II. p. 251.

Ueber die Gegenwart der Pecopteris reticulata in den Schichten der gleichzeitigen Formation in England und Frankreich; ron Ad. Brongniart.

Annales des Sc. nat. 1828. Mars p. 385 . - Botan. Literaturbl. II. p. 219).

Fossile Bäume in Ostindien, nach Dr. Buckland.

Edinb. philos. Journal. Oct. - Dec. 1827. p. (iề. - Journ. of science Jan. 1828. p. 10. - Jotan. Literaturbl. II. p. 250.

Nachrichten von fossilen Baumstämen bei Gallipolis in Ohio-Staate (in Nordamerilia) ron Dr. Sp. Hildreth.

silliman. American. Journ. of Sc. and arts XII. 2. Juni 1827. -

Froriep's Notizen Lid. XVII. Nro. 37t. - Lotan. Literaturbl. I. p. 850 .

Pflanzen-Fossilien am Ohio.

Revue brittannique. Mars 1827. - Botan. Literaturbl. II. p.253.

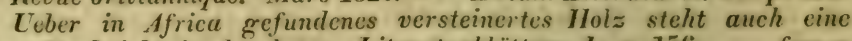
kurze Nachricht in den botan. Literaturblättern 1. p. 156, - ferner eine andere über versteinertes Holz in oler Isis J:d. VIII. p. 528. (Kaunitz).

Note über fossile Nüsse.

Annals of Philosophy. Dec. 1825. Nro. LX. p. 431. - Dulletin des Sc. natur. Mars 1826. p. 313. - Edinb. Journ. of Science. Juli 1825. $p .185$.

Untersuchung versteinerter Nüssc, rom Professor Marx, mit Abbildungen. p. 233.

Schweigger's Jahrb. der Chemie und Physik 1827. IIcft 2.

J. G. Lehmann. Untersuchung der sogenannten versteinerten Kornahren und Stangengraupen von Frankenberg in Ilessen. Berlin 1760. 4.

J. G. Waldin. Die Frankenberger Verstcinerungen nelset ihrem Ursprunge. Marburg 1778. 4. mit Kupfern. 
Q. F. Ho ffmann. Vegetalilia in Hercyniae subterraneis collecta, iconibus descripia et observationibus illustrata. Norimb. 1797-1823. 2 Vol. gr. Fol. 21 Rthl. $9 \mathrm{gr}$.

Untersuchungen über die Pflanzenreste des Kohlengebirges ron Ibbenbühren und rom Piesberge bei Osnabrück, von Herrn Dr. und Prof. Hof $\mathrm{fmann}$ in Halle.

Karsten's Archiv für Bergbau und Hüttenwesen İd. IIII. Heft 2. p. $266-282$.

Uebersicht über die Versteinerungen Würtembergs, nach dem gegenwärtigen Standpunkte der Peträfactenkunde. Stuttgardi 1825. 8.

Aus dem Correspondenzbl. des würtemb. landwirthschafll. Fereins abgedruckt.

Ueber dic Pflanzen-Versteincrungen, welche in dem Bauund Sandstein von Stuttgardt vorkommen, von Dr. G. F. Jäger. Stuttgardt 1827.

Im Auszug in den Annales des Sc. naturelles Sept. 1828. p. 92.

Ueber die rerschiedene Pflanzenabdrücke führenden Formationen und die Unterschiede der Vegetationen in denselben, rom Herrn Grafen Kas par v. Sternberg.

Bot. Zeit. 192t. II. p. 689.

Handbuch der physikalischen Erdbeschreibung, von H. F. Link.

In der ersten Abtheilung des zueiten Bandes (Berlin 1830, bei

Dümmler) sind die Nachrichten von den fossiten Gewächsen auf sehr lehrreiche Weise mitgetheilt.

Bemerkungen üher die Lagen von Süfswasserboden, welche in den Umgebungren von Sćte, nahe an dem mittelländischen Meere und unterhalb der Oberffäche des letzteren entdeckt wurden, vom Prof. Marcel de Serres.

Annales des Śc.natur. Tom. XI. Août 1827. p. 393-424. - Bot.

Literaturbl. 1. p. 153.

Note sur les plantes fossiles d'Armisson, par M. Adolple Brongniart.

p. 248.

Annales des Sc. nat. Sept. 1838. p. 43. - Bot. Literaturbl. II.

Sur un Gisement de Végétaux fossiles, situé au col du Chardonet, dep. des hautes Alpes, par M. Elie de Beaumont.

Annales des Sc. natur. Dec. 1828. p. 353. - Bot. Literaturbl.

II. $p .24$.

Ueber ein Lager von fossilen Pflanzen und Belemniten zu Petit-Coeur bei Montiers in Tarentaise, ron Elie de Beaumont.

Annales des Sc. nat. 18\%8. Juin p. 113-12\%. - Botan. Literaturbl. II. p. 242.

Ueber die fossilen Pflanzen des Fiohlendistrictes in den Alpen, von Ad. Brongniart.

lbid. p. 127-136. - Bot. Literaturbl. II. p. 212.

Ueber die-Brankohle von Chamberry.

Il propagatore 1828. p. 150. - Bot. Literaturbl. II. p. 264.

Osservazioni sulle Gessaje del territorio Sinigagliese, su i filliti, glictioliti ed altri oggetli contenuti nelle medesime fatte da Vito Procaccini Ricei, socio ord. dell' Acad. ital, etc. Roma 1828. 102 S. mit 5 Steintaf.

Botan. Literaturbl. I. p. 330. 
Ueber efnon rom Mecro bedeckten Wald an der Kïste ron Tirce (England), eincr der westlichsten Inscln. Von Herrn Colin Smith zul Inverary.

Jameson New Erlinb. Philos. Journal. April - June 1829.

Froriep's Notizen Bd. XXY. p. 290. Nro.5.17. - Lut. Literaturbl. III. p. 453.

Teber Torf, ron Mac Culloch. Aus dem Edinb. phil. Journ. III. IV. 1820. Jun. - Apr. Im Auszuge von Beilschmied.

Bot. Literaturbl. Das. p. 459.

Gedeon Mantell. Illustrations of the Geology of Sussex. London 1827. 4. Nit 20 Kupfern.

Daselbst I. p. 150.

IIerr Lindley, Prof. der Botanik in London, beschrieb und lief's abbilden einige fossile Pflanzenreste; namentlich Bruchsticke: von Podocarpus macrophylla, Laurus dulcis, Buxus balearica und Thuja articulata.

Jameson New Edinb. philos. Journal July - Octob. 1829. p. 2!IS.

In der Bai Gristhorp, bei Scarborough fand man eine grolige Masse von fossilen Vegetabilien, die mehrere moch nicht beschrichene Arten darstellen. Diese Ueberreste lagern in einem Thonschiefer, der mit reiner Thonerde (roche ferruginense) und einer dünen Lage Stcinliohlen abwcchselt, sie bestehen hauptsïchlich in Stengeln und BlätterAldrücken von Aequinoctial-Farrenkrä̈tern; mehrere Exemplare sind beträchtlich grofs und vollkommen gut erhalten.

Annales des Voyages. Février 1830. p. 251.

Observations sur les régétaux fossiles renfermés dans les grès de Haer en Seanie; par M. Ad. Brongniart.

Annales des Sc. natur. Févr. 1825. p. 200. - Rapport fait ì

la Société philomatique sur ce Mémoire, par M. Constant Prévost. - Bulletin de la Soc. philom. de Paris. Nov. 1825. p. 166.

Notice sur quelques végétaux terrestres fossiles, qu' on trouve mélés à des dépots de coquilles marines, dans la craie chloritie de Seanie, par S. Nilson.

Bulletin des Sc. natur. Juin 1826. p. 212.

\section{Statistik des Gewächsreiches.}

§. 22.

Fis ist bekannt, dafs sowohl die Höhe der Lünder, als zumal ilırer Giebirge, grofsen Einflufs anf die I'ertheilung der Pflunzen hat; es ist forner unbezueifelt, dafs auch dic verschiedenen ficlsurten und die übrige lieschaffenheit iles Borlens überhaupt für die Pflanzen-Geographie ein wichtiges Moment abgeben; und nicht minder ist es allgemein anerkannt, dufs das Klima und überhaupt die Tentaperatur der einzelnen Frdstriche mit ihrer Tegetation in dem genauesten Terhältnisse steht. Durum wird es nothwendig, die gengrnostische Bescheffenheit der Lünder und ihre physikalischen Eigenschaften überhaupt wüher kemnen zu lernen, wenn mun den statistischen Theil der Pflansenliunde gehörig beurbeiten will. Diesen Gesichtspunkten gemä̈s, glaube ioh den jungen Freunden der Pflansenliunde cinen kleinen Dienst zu leisten, wenn ich sie unf cinige literarische Ilülfsmittel aufmerksum mache. Zugleich kann ich den IT unsch nicht unterdrü̈lien, dafs auf Universitälen die mineralogischen und bolemischen Extursionen allezeit vereint vorgenommen werden möchten, da dus lnteresse beider Naturwissenschaften dadurch uur gewinnen könnte. 


\section{a. Geognostische Schriften:}

J. C. W. Cromo. Der Boden und sein Verhältnifs zu den Gewảchsen; oder Anweisung, den Boden, vorzüglich vermöge der darauf wild wachsenden Pflanzen kennen zu lernen, und seinen Werth zu beurtheilen, nebsto einer Beschreibung der Mergelarten, Moderarten und der Torflager. Hannover 1812. $1 \mathrm{fi} .40 \mathrm{kr}$.

G. Schübler. Uebersicht der für die Vegetation wichtigsten physischen Eigenschaften der Erdarten. Eine Tabelle in Folio. Tübingen 1821.

Boden, Klima und Lage, in wechsclseitiger Beziehung auf Vegetation, von Hundeshagen.

Forstliche Berichte und Miscellen Heft 1. p. 1-25.

v. Leonhard. Charakteristik der Felsarten. Heidelberg 1823. 1824. 8.

Agenda geognostica. Hülfsbuch für reisende Gebirgsforscher und Leitfaden zu Vorträgen über angewandte Geognosie. Von C. C. v. Le o nh a r d etc. Heidelberg 1829, bei Molix, mit 4 Steindruchtafeln. $4 \mathrm{fl}$.

Essai géognostique sur le gisement des roches dans les deux hémisphères, par A. de $\mathrm{Humboldt}$. 2. édition, conforme à la première. 1 Vol. Paris 1826. Deutsch ron Leon hard. Strasburg 182t. 8. 2 Rthl.

Tableau des Terrains, qui composent l'écorce du globe. Par Al. Brongniart. Paris 1829. 1 Vol. 8. Deutsch von Kleinschrod. Strasburg 1830. $5 \mathrm{fl}$.

Traité de Geognosie, ou exposé des connaissances actuelles sur la constitution physique et minérale dı globe terrestre, par J. F. D'A ubuisson de Voisins. Nouvelle édition revue et corrigée. Tom.I. Paris 1828.

Specimen Geographiae physicae comparativae, auctore Dr. J. F. Schouw, in Universitate Havn. Botanices Prof. Havniae 1828. 65 S. 4. Mit 3 lithographirten Tafeln.

Siehe bot. Literaturbl. IV. p. 10.

C. Keferstein. Tabellen über die vergleichende Geognosic. Ein Versuch. Halle 1825. gr. 4. $14 \mathrm{gr}$.

De statu Geologiae. Dissertatio inauguralis Auctore Aug. Alex. Ka mmerer. Heidelbergae 1829. 8.

Für Geognosie vergleiche man auch nebst den bekannten Zeitschriften der Herren v. Le onhard und Karsten, das neulich angefangene

Journal de Géologie, publié par MII. A. Boué, Jobert et Rozet. Paris 1830. 8. chez Levrault. Monatlich erscheint ein Heft.

\section{b. Höhenmessungen.}

C. v. Mechel's Tafel der höchsten Berge unserer Erde nach den gemachten Messungen, mit Beschreibungen. Basel und Leipzig 1808. gr: 4.2 Rthl. 16 gr.

W. A. Miltenberg. Die Höhen der Erde, oder systematisches Verzcichnif's der gemessenen Berghöhen und Beschreibung der bekanntesten Höhen der Erde u. s. w. Frankfurt 1815. gr.4. 3 Rthl.

J. Wfg. v. Göthe's Höhen der alten und neuen Welt verglichen. Weimar, Industrie-Comptoir, 1813. Fol. mit 1 K. ill. 2 Rthl. $12 \mathrm{gr}$. braun 1 Rthl. 12 gr. 
F. Schulz viber den allgemeinen Zusammenhang der IIöhen auf der Oberflähe der Erde, nebst Gebirgskarte von Liuropa. Weimar 1803. gr.4. 1 Rthl. $20 \mathrm{gr}$. Die Karte allein 1 Rthl.

II o f fman n. Höhenkarte. Stuttgarit 1825. $12 \mathrm{gr}$.

Oro-hydrographische Karte von Europa. Leipzig hei Ílcin.

Tableau comparatif des hautenrs des principales monlagnes et des lieux remarqual,les du globe au dessus du nivean de la mor par Perrat 1828. grand Fol. Nebst Beschreibung in Octav. P'reis schwar\% 10 francs, colorirt 15 francs.

\section{c. Temperatur - Verhältnisse.}

R. Kirwan. Angabe der Temperatur von den versehicdenen Breiten rerschiedener Länder und Städte. Aus dem Englischen von Crell. Berlin 1788.

G. F. Wucherer. Ueber die mittlere Temperatur Freiburgs. Freiburg 1818. 4 .

Beiträge zur vergleichenden Klimatologie; von Dr. J. Fr. Schouw, Prof. u. s. w. 1. Heft. Koppenhagen 182\%. 8.

Siehe bot. Literaturbl. 1. p.67.

Ueber dic Verbreitung der Pflanzen während des historischen Alterthums, nebst Schlufsfolgerungen über die klimatische Beschafienheit jener Zeit, von Schouw.

p. 265.

Fidinb. Journ. of Science XVI. p.311 - Botan. Literaturbl. II.

Vertheilung der mittleren Temperatur der Erde oder lignes isothermes von Herrn $K n p f$ fer.

Annalen der Physik und Chemie 1829. Nro. 2.

Skale der Schneelinie und der mittlern Temperaturhöhe.

Mém. du Mus. 8. XV. p. 298. tab. 9. 1828. 4. Cahier. - Bot. Literaturbl. IV. p. 133.

Ich kann nicht umhin, hier $\approx u$ bemerken, dafs Deutschlands Klima, was auch ein berühmter Naturforscher dagegen gesagt haben mag, einst milder und wärmer war, als es gegenwärtig ist; es wurden Gewaichse an Orten gezogen und mit Nutzen cultivirt, wo sie jetzt nicht mehr forlkommen würden, wovon ich anderwürts einige 13eispiele mittheilte, hicr möge nur noch eins eine Stelle finden. Im siebenzehnten Jahtrhunderle noch zog. man um Frankfurt Artischokken in Menge im Freien, was jetzt kuum mörrlich ist; in älteren Schriften wird öfters des Weinzehniden zu Jiechenheim, Niederrad und andern Orten gedacht, der an Stellen wuchs, wo es jetzt Mühe kosten würrle, nur ein Maafs zu erndten. In früheren Zeilen war die Weinlese (selbst den Unterschical der Zeitrechnung des damuls noch nicht gebräuchlichen Gregorianischen Kalenders in Anschlug gebracht, der 10 Tage betrïgt) immer um zwei bis drei Wochen früher, als in unscrn Zeiten $u$. s. w.

Man sehe Kirchner Geschichte der Stadt Franlifurt am Muin Bd. II. p. 491. 492. 


\title{
Schriften und einzelne Aufsätze über Pflan- zen-Geographie.
}

\author{
§. 23.
}

a. Allgemeine.

Die Literatur der Pflanzen-Geographie hat bereits Sprengel in seinen Grundzügen einer wissenschaftlichen Pflanzenkunde Seite 332 angegeben, worauf ich hier zuvörderst verweise, und nur wenige bereits dort angegebene Schriften hier anführe. 1783. 8.

Girand Soulavie Géographie physique du règne végétal. Paris

F. Stromeyer. Mistoriae vegetabilium geographieae Specimen. Dissertatio. Gottingae 1800. 4.

Ideen zu einer Geographie der Pflanzen, nebst einem Naturgemälde der Tropenländer von $A l$. von $H u m b o l d t$ und A. Bonpland bearbeitet yabd herausgegeben von dem erstercn. Mit einem Kiupfer. Tübingen. :-Auch unter dem besondern Titel: Voyage d'Al exandre de $\mathbf{H}$ umboldt et $\mathrm{A}$. Bonpland. Première partie. Physique générale et relation historique du voyage. Premier volume. Essai sur la Géograplie des plantes etc. A Paris 1807. XII und 155, S. in gr. 4. 10 Rthl. 12 gr.

A. de Humboldt. Prolegomena ad nora genera plantarum. Parisiis 1815. Fol.

A. de Humboldt. Ideen zu einer Gengraphie der Pflanzen mit erläuternden Zusätzen und Anmerkungen. Wien, Gerold, 1811. 8. $9 \mathrm{gr}$.

A. de Humboldt. De distributione gengraphica plantarum secundum coeli temperiem et altitudinem montium prolegomena. Paris 1818. 8. 2 Rthl. 8 gr.

Alexander's von Inmboldt nene Untersuchungen über die Gesetze der Vertheilung der Gewächse auf dem Erdhoden.

Annales de Chimie et de Physique Tom. XrI. p. 267. - Siehe

Sprengels Neue Entdeckungen III. p. 383.

Sur les lois, qu'on observe dans la distribution des formes régétales, par A. de II umboldt. Paris 1821. 8.

Dictionn. des Sciences natur. T, XVIII. p. 422.

Ansichten der Pflanzen-Geographie des Herrn Alexander von $1 I$ umboldt. Im Auszuge herausgegeben von Dr. Stirbes. Berlin 1827. 12.

A usichten der Natur mit wissenschaftlichen Erläuterungen, von Alexinder von If umboldt. Zwei Bände. Zweite verbesserte und vermelnte Ausgabe. Stuttgardt und Tübingen 1826.

$\mathrm{Im}=u$ eitch Tiande befinden sich die Ideen zu einer Physiognomik der Gieuriohse. - Min sehe auch A. v. II umboldt: Ueber Vertheilung der Pflenzen: Isis von $0 \mathrm{k}$ en Bd.I. 9. 11.

Géographie des Plantes, rédigée d'après la comparaison des phénnmines que présente la végélation dans les deux continens, par $\mathbf{A}$. de Humbold t et Ch. Kinnth. I Vol. in Fol. Ourrage précédé d'un Tahleau physique des rénions equinoxiales, par $\mathbf{A}$. de $\mathbf{H}$ umboldt et Aimé Bonpland. (Prospectus).

Lis werden duvon nur ill Erenpiure foctruckt und erscheint in

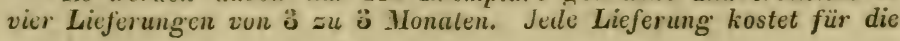


Subscribenten entweder 180 francs oder 201) franes, nach dem verschicdenen Papiere.

Bulletin des Sciences natur. Mars 1826. p. $314 u$. d. $f$.

Schouw. De sedibus plantarum originariis. Havniac 1316. 8.

Idem. Schema til en systematisk fremstilling of Plante-Geographien eller Planternes Stadlacre.

Sprengel neue Entdeck. II. p. 343.

F. F. Schouw. Grundzüge einer allgemcinen Pflanzen-Gengraphic. Berlin 182:. Von dem Verf. ans dem Dïnischen übersetzt. gr. 8. mit 2 Tabellen und Karten. 6 Rthl. $16 \mathrm{gr}$.

Ueber die Bezichung der Pflanzen zur geologischen Beschaffenheit des Bodens; von Will. Thomson.

Aus Loudon the Magaz. of natural History Nro. XI. Sept.1830. in den bot. Literaturbl. IV. p. 218.

C. W. Ritter. Die Erdlumle, im Verhältnifs zur Natur und zur Geschichte des. Menschen, oder allgemeine vergleichende Geographic u. s. w. 1. Theil. Berlin 1821. 8. 7 fl. $40 \mathrm{kr}$.

Derselbe. Sechs Karten von Europa, über Producte, physik. Erdbeschreibung und Bewohner dieses Erdtheils, mit erklärendem Text. Fol. Schnepfenthal 1820. $4 \mathrm{fl} .30 \mathrm{kr}$.

'itford's Sketches towards a hortus botanicus americanus; tatle of climates and habitats of plants. London 1811. 4.

K. P. Thunberg. Dissertatio geographiam plantarum adumbrans. Upsal. 1813. 8. dolle.

Essai élémentaire de Géographie botanique par A. P. de Can-

Dictionnaire des Sciences nat.. Tom. XVIII. p. 394 und Mímoires de la Soc. d'Arcueil. Tom. III. p. 295. dolle.

Grundrifs ciner allgemeinen Pflanzen-Geographie von Decan-

$$
\text { Froriep's Notizen Nro.8. Sept.1821. p.117u. d. f. }
$$

Gemälde der organischen Natur in ihrer Verbreitung anf der Erde - in grofsem Landkartenformate - herausgegeben vou Wilb rand und Ritgen, Prof. in Giessen; in Stcin gravirt von Jos. Päringer, $18^{\circ 22}$.

M. Mirbel Recherches sur la distrihntion géographiques des Véuétanx phanerogames dans l'Ancien monde, depuis l'équateur jusqu'au pole arctique.

Mémoires du Muséum d'histoire naturelle de Paris. Septième Année. Onzième et douzième Cahier p. 34!-45t. - Siehe bot. Literaturbl. I. p. 1 u. d. f. - Geiger's Magazin Februar 1828. p. 93 u. $d . f$.

Beilschmied. Ueher dic Methode, das mindere oder häufigere Vorkommen von Pflanzen gewisser Gegenden durch Zahlen anzudeutęn. Bot. Zeil. 1827. II. p. 427.

Derselbe. Ueher einige bei pflanzen - geographischen Vergleichungen zu beriicksichtigende Punkte, in Anvendung auf die Flora Schlesiens. Breslau 18:9.

Siche Linnaea 1830. III. p. 83. Lit.

IInrnschuch. Vergleichung der Pflanzen der Mellville-Insel mit denen der Gamserube.

Bot. Zcit. 1825. I. p. 249. 
Beobachtungen über das in der Natur olowaltende Gesetz des Vegetationswechsels.

Froriep's Notizen Bd. XII. p. 113 in Nro. 250 der Nro. 8 des zwölften Bandes.

Das Akklimatisiren von Forstgewächsen, als Gegenstand der Forstbotanik *).

II undeshagen forstl. Berichte und Miscellen Heft 1. p. 74-86.

Ueber Wiesen, ihre Wichtigkeit und ihre Ausdehnung in verschiedenen Ländern Europens, von Moreau de Jonnes.

Edinb. New Philos. Journal Oct. 18\%9. p. 272. - Dingler's polytechn. Journal Bd.-XXXV.p.58.

Picture of Vegetation on the Surface of the Globe.

Eidinburgh New Philosophical Journal. April - July 1826. $p$. 117 - 124. July-Oct. 1826. p. 255-262. Aus Poir et's Leģons de Flore. Paris 1825.

Etablishment of Vegetation of the Surface of the Globe.

Jameson New Edinb. Philos. Journal. Oct.-Dec. 1826. p. 61-73.

On the geographical distribution of Plants. By C. Pickering.

Aus den Transactions der amerikan. philos. Societät in dem Bulletin des Sc, natur. Mars 1829. p. 419.

\section{b. Zur Pflanzen - Geographie einzelner Länder. \\ $D$ e $u t$ s c $\boldsymbol{h} l \boldsymbol{l}$ a $n \boldsymbol{d}$.}

Für Deutschland glaubte ich einige literarische Nachweisungen, die geognostischen I erhültnisse und Höhen der Berge betreffend, angeben zu müssen, allein in Hinsicht der übrigen Lünder kann ich blos auf die allgemeinen Werke verweisen.

Observati ones geologico - geographicae de naturalibus soli in Germania formis, scriptae a G. B. Mende lssohn. Kíliae 1828.

Deuts chland geognostisch - geologisch dargestellt und mit Charten und Durchschnittszeichnungen erläutert. Eine Zeitschrift in freien Heften, herausgegreben von heferstein. Weimar 1821. Bereits der sechste Band 1829.

Auch unter dem Titel:

Mineralogisch - statistisch - geographische Beschreibung von Deutschland, ausgearbeitet von Ch. Keferstein.

Man findet darin unter andern im ersten Hefte eine Darstellung der geognostischen Ferhältnisse des Odenwaldes und Schwarzwaldes, und zuar im ersten Hcfte des ersten Bandes; in den späteren die Beschreibung der norddeutschen Ebene u. s. w.

Keferstcin geht bei seinen Beschreibungen auch die einzelnen Stïdte und selbst geringere Orte durch, wobei er neben vielen andern schützensuerthen Nachrichten angiebt, wo Mineralogen sich aufhalten und welche Sammlungen anzutreffen sind.

Hätten wir doch auch einen solchen Wegweiser durch Deutschland für Botanik!

*) Ueber den Einflufs des Klima auf die Pflanzen und ähnliche Gegenstände vergleiche man den Abschnitt über Anatomie und Physiologie der Pflanzen. 
C. Keferstein. Versuch einer vergleichenden Darstellung der gengnostischen Verhältnisse in Wirtemberg und Norddentschland. Mit Anmerliungen rom Prof. und Bergrathaassessor Sehübler.

Würtemb. Korrespondenzbl. 1824. V. p. 331 - 373.

Geognostisehes Gemïlde ron Dentschland. Mit Rücksicht auf dic Gebirgsbeschaffenheit nachbarlicher Staten. Von $\mathbf{A}$ m i Bon ausgeguhen ron C. C. ron Leonhard 1829. 1. Bd. 8. Mit acht Steindrucktafeln.

Geognostische Umrisse der Rheinländer zwischen Basel und Mainz von Oeynhasen, Dechen und Laroehe, Éssen 1825. "2 Bände.

\section{2. $\mathrm{gr}$.}

J. Steininger. Geognostische Studien am Mittelrhein. Mainz 1819.

Mémoire géologique sur le terrain diluvien de la vallée du Rhin, par M. Rozet.

Journal de Géologie Nro. 1. Mai 1830. p. 25. 1821. 8 .

M. v. Engelhard. Wanderungen durch die Vogesen. Strasburg

Behlen. Der Spessart, Versuch einer Topographie dieser Waldgegend. Leipzig 1823. 3 Bände.

K. F. V. Jïgersehmid. Das Murgthal, besonders in Hinsicht auf Naturgeschichte und Statistik. Nürnberg 1800. 8. mit Kupfern.

Selb. Geognostische Beschreibung des Kinziger Thales. p. 349.

Denkschriften der Aerzte und Naturforscher Schwabens Bd. I.

II undeshagen. Beitrïge zur Kenntnifs der Gehirge Schwabens. $808-844$.

v. Leonhard Taschenbuch für Mineralogie Jahrg. 1821. $p$.

J. Nöggerath. Das Gebirge in Rheinland-Westphalen. Bonn 18221826. 4 Bände.

Im ersten Bande befindet sich eine geognostische Reiscbeschreibung z̈ber die Ciebirge der Bergstrafse, die Ilard, den Donnersberg und den Hundsrück.

O. Lisenlohr. Geognostische Beschrcibung des Kaiscrstuhls bei Freilurg im Breisgau. Karlsruhe 1829. 8.

A. Lilipstein. Gedrängte Uchersicht der Ergehnisse einer gengnostischen Frforschung des Odenwaldes. Darmstadt 1829. 4. Mit einer Karte. $3 \mathrm{fl} .80 \mathrm{kr}$. olhne die Karte $26 \mathrm{kr}$.

Gaea Meidelbergensis, oder mineralogische Beschreihung der Gegend ron IIeidelberg. Mit einer petrographsichen Fiarte. Von Dr. II. G. Bronn, Prof. etc. Heidelberg und Leipzig 1830.

A. v. Iroff, Gemälde der physischen Btschaffenheit und inslocson-

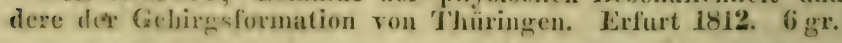

J. F. Gloeker. Beitrïge zur mineralogischen líenntnifs der Sudetenländer, inshesondere Schlesiens. 1. IIeft. Mit einer Charte und einer Steindrucktafel. Breslan 1827.

G. F. W. Cinappentiex. Beitrïge zur grengnostischen Kenntnifs des Itiescngehirges, sehlesischen Antheils. Leipzig $180 t$.

K. v. Itavimer. Die Gehirge Niederschlesiens, der Grafschaft Glatz u. 6. w. Berlin 1819. 
C. v. Oeynhausen, Versuch einer geognostischen Beschreibning ron Oberschlesien und der zunächst angrenzenden Gegenden. Essen 1822. 8. m. 1 Karte und 3 Specialabrissen.

De vallium in Germania boreali principalium directione memorabili congrua," auct. D. $\mathrm{H}$ of f man n. Halae 1823.

F. Uoffmann. Beiträge zur genauern Kenntnifs der geognostischen Verhältnisse Nord-Deutschlands. 1. Theil. Auch unter dem Titel: geognost. Beschreibung des Herzogthums Magdeburg, des Fü:stenth. Halberstadt und ihrer Nachbarländer. Berlin 1823. mit 2 illumin. Karten.

J. C. Stübner, geographisch - naturhistorische und rorzüglich mincralogische Beschreibung des Marzgebirges. Leipzig 1800. 2 Bände. 8.

Das Harz- und Thüringer Waldgebirge im Jahre 182!. Magdeburg 1824. 2 lithogr. Bl. Fol.

J. C. L. Zinken, der östliche Harz, mineralogisch und bergmännisch betrachtet. Leipzig 1825. 8. mit 1 Karte.

J. F. Weiss, Südbaicrns Obcrfläche, geognostisch-topographisch entworfen. München 18\%1. 8. mit Kiupfern und liarten.

W. Edler v. Sänger, Versuch einer Orylitographie der gefürsteten Grafschaft Tyrol. Inspruck 1520.

Leopold de Buch lettre à M. Alex. de II umboldt, renfermant le tableau géologique du Tyrol meridional 1823. 4.

Geognostische Kiarte der Rheinländer von Basel bis Mainz, von Oeynhausen, Dechen und Laroche. Zwei Blätter. Berlin 1826. Bei Simon Schopp u. Comp.

Charte von dem Königreich Würtemberg, dem Grofsherzogthum Baden und den Ländern der Fürsten von Hohenzollern, entworfen von $C . F$. W e iland. Geognostische Illumination ron $C h$. Leferstein. Weimar in Verlage des Landes - Industric - Comptoir 1828.

Charte vom Königreich Baiern, nach den vorzüglichsten Hülfsmitteln, und der neuesten Kreiscintheilung entworfen ron C. T. Weiland. Geognost. Illumination von lieferstein. Weimar 1826.

Bronn, Geognostische Karte des Neckarkreises. Im zwciten Bande des Badischen Archives von Mone. "Karlsruhe 1827.

In der oben angeführten Gaea Heidelbergensis ist diese Karte jedoch gröfser und mit manchen I erbesserungen ebcnfalls enthalten.

C. F. Wieland, Höhenkarte, oder bildlich vergleichende Uchersicht der bedeutendsten Berge in Deutschland und der Schweiz, nelsst Andentungen der Hiihen vieler Städte, Dörfer, Seen etc. nach den besten Barometer-Messungen entworfen. Weimar 1825.

Barometrisches Nirellement des Schwarzwaldes und der benachbarten Gegenden von E. II Mi cha el is, Kön. Prenfs. Hauptmann. u. d. $f$.

Hertha. Dritter Jahrgang. Dd. X.' Heft 3. Sept. 182\%. p. 196

Höhenmessungen im bairischen Kreise, von dem geh. Rath v. Rau.

v. Leonhard Zcitschrift für Minerulogie. Jahrg. 1826. Bd. I.p. 501-507. - Hertha May 1827. p. 141. - In dieser letzten ZeIt schrift, namentlich in dem ersten Bande derselben findet man noich manche andere Höhenmessungen verzeichnet. 
Auch in C. W. Muncke: Anfangrgruinde der Naturlehre. 2. Abtheilung. Heidellerg 150. sind derselben viele sorgfüllig zusammengetrugen.

Dic Berghähen Würtembergs sind in nachstehendem Tjurhe verscichnet.

F. v. Alberti. Die Gebirge des liönigreichs Würtemberg, mit Anmerkinngen und Beilagen, vom P'rof. Schübler. Stuttg. nnd Tübingen 1826. 8 .

J. 13. Steiner, gengraphische Karte des ol der Ensischen Salzkanmerguts und Gehireshibhen des Salzkammerguts in Oestreich ob der Ens nach der 'Traunstrimung, nelost den vorzïglich sichnbaren Gebirgen Steiermarlis und Salzhuress. Salzhurg 1825. 2 BI. in Fol. 2 Rthl.

Toussaint v. Charpentier. Darstelhur der Höhen verschiedener Berge, Flüsse und Orte Schlesiens. Breslau 1813. 4. Mit 1 ill. Karte 2 Rthl.

Geognostisehe Specialkarte rom nordwestlichen Deutschland, von II of $\mathrm{fmann}$. Berlin 18:!).

Geognostischer Atlas ron Nord-Deutschland. Stuttgardt 1830.

Beschreibung der orographischen und geognostischen Verhältnisse rom nordwestlichen Dentschland. Leipzig 1830. Ifl. $12 \mathrm{kr}$.

Geognostische Charte von Dentschland und den umliegonden Staaten, in 42 Blättern; nach den vorzüglichsten mitgetheilten Materialien herausgegeben von Sim o n Sehropp in Berlin, 1826 his 1829.

Uebersicht der Vegetation bentsehlands nach ihren natürichen Familien, von D. J. IB. Wilbrand, Profefsor zu Gicfsen.

Isis Band XV.

Untersuchungen üher die pflanzengengraphischen Verhältnisse Deutschlands und der Schweiz, rom Prof. Schübler und Dr. A. Wiest.

Bot. Literaturbl. III. $p$. 46i8. u. d. f. - Dic Abhandlung wurde früher als Dissertation in Tübingen 1827 herausgegeben, kam aber nicht in den Jiuchhandel. Man sehe Linnaca Band II. p. 680. und Hertha Juli 1827. Bd. X. Ileft 1. p. 39 - 666 , wo sie ganz abgedruckt ist.

Joannes II euffel, Mungarus Madro-Posoniensis. De distributione plantarum geographica per Comitatum Ifungariae Pestiensem. Pest 1827. 39 Seiten. 8.

A. E. Santer. Salishurgensis. Dissertatio inauguralis geographicobotanica de territorio Vinstobonensi. Vindobon. 1826.

Bemerkungen über das Klima und die Vegetations-Verhältnisse der Ikheinlïnder, vom Ilerrn Prof. J. Ste ininger in Trier.

Herthe Brl. X. Heft 2. Lugust 1827. p. 155 - 194. - Gicistreic/ und vortrefflich!

Ueber einigre Vigrenthümlichkeiten der böhmischen Flora, und die klimatisehe Verbreitung der Pflanzen der Vorwelt und der Jetztwelt. Yon dem Grafen Caspar von Sternberg.

Botan. Zeit. 1829. II. p. 65. u. d. f. der Beilage.

Bemerkungen über Terbreitung der Pilinzen in den Salzburger und 'I'yroler Alpen, von Link.

Bot. Zeit. 1827. I. p. 217.

Das Miltizer Kastanienwäldchen, ron C. M. Beyer.

Sickler, der deutsche Obstgürtncr. "W'eimar 1804. Bd. XXII. p. $28-32$. 
Ueber die Pflanzen-Geographie Schlesiens vergleiche man, die oben angezeigte Schrift von Beilschmied.

Die Bergpflanzen der Rheinpfalz, von Dierbach in Heidelberg. Bot. Zeit. 1826. II. p. 657 .

Pflanzen - Geographie der bayerischen Alpen, nach Forstmeister Ferchel.

Behlen's Zeitschr. für das Forst - und Jagdwesen. II. 2. Bot. Literaturbl. IV. p. 16. - Hertha Ed. XIIII. - Geogruph. Zeit. p. 6 .

\section{$S \subset h$ we $i$ z.}

C. S. Gruner. Die Eisberge des Schweizerlandes beschrieben. 8 Theile. Bern 1760 .

C. Bernoulli. Geognostische Uebersicht der Schweiz. Auch unter dem Titel: Taschenbuch für die Schweizerische Mineralogie. Basel und Halle 1811.

P. Merian. Uebersicht der Beschaffenheit der Gebirgsbildung der Gegend ron Basel, mit besonderer Rücksicht auf das Juragebirge im Allgemeinen. Basel 1821. Mit 1 Steintafel.

K. F. Hoffmann. Das Berner Oberland, oder der höchste Theil der deutschen Alpen, nach den rorzïglichsten IIülfsmitteln hearbeitet. Stuttgardt 1824. Queer Folio. 48 kr.

Stercorama oder Relief des Montblanc-Gebirges und dessen nächster Ungebungen, von K. M. K u m m er; nebst erläuternder Beschreibung von $R$ itter. Berlin 1824. 8.

A. Rengger. Beiträge zur Geognosie, besonders zu derjenigen der Schweiz und ihrer Umgebungen. Stuttgardt. I. 1824. 8.

Esquisse des directions, qui dominent dans quelques systémes de Montagnes (Chaine principale des Alpes) cine Karte im Januarheft 1830 der Annales des -Sc. naturelles.

A. Sticler, die Berge der Schweiz, hydrographisch geordnet, mit Angabe bekannter Höhen. Gotha 1820. Mit einer Karte. It gr.

Ueber die Vegetations-Grenze im Berner Oberlande, von Kasthofer.

Hertha. Bd. IX. Heft 3. p. 236.

Die Bildung der Alpenpflanzen, und die Schneelinie in der Schweiz. Morgenblatt vom 30. und 31. Dec. 1829.

Dissertatio inauguralis botanica de distributione geographica plantarum Helvetiae, auctore Ringier, praeside Schübler. Tübingae 1823.

Projet d'une Flore physico-géographiquē de la vallée du Leman, par M. De Cand olle. Grenève 18\%1.

Bois Duval, Versuch über dic geographische Verbreitung der Cryptogamen in den Alpen.

Bot, Literaturbl I. p. 173.

\section{Frankreich und spanien.}

Etat de la Vegetation an sommet du Pic de Midi de Bagnères. 
Mémoiro lue à l'Acalemic des scicnces, en sa séance du 16. Janvier 1826, par M. le Baron $R$ a mond.

Mémoires du Musíum d'hist. nat. T. XIII. Cah. 4. p. 217-281.

Daron ein Auszug in den Annales des Siciences naturelles. Mai 1826. p. 96 - 100. - Man sehe anch Froriep's Jotizen. Bd. XIV. p. 27:3. in No. 394 und :315. - Ferner Bot. Literalurbl. I. p. 48. u. d. f. - Endlich moch David Brewster Edinburgh Journal of Scienee No. VIII. Apiil 1826. p. 380.

Herr Ramond untersuche besonders den Pic du mili; er sammetle 130 phanerogumische und cryplogamisshe Pflan=en. Mehrere davon waren Arten aus denselben Giattungen, aus denen der C'apitain Parry ouf der Melville-Insel Speries gesammell halle; von 113 . Irten, die auf dieser Insch gefunden wurden, wachson is auch auf dem l'ie du midi.

Sur les proportions relatives des éspèces de plantes, de la Flore Bordelaise.

Bullet. d'hist. nat. de la Soc. Linn. de Bordecunx 1826. T. I. p. 12. - Bulletin des Sciences natur. Avril 1826. p. 4t. - Bol. Lilcraturbl. I. $p$. 66 .

Description statistique du Departement de la hante Loire, par M. Deribier de Choissae. Paris 1828. 8. I. Vol. 522 p.

Coup d'o eil sur la végétation de la Basse - Normandie, par A. de Brébisson.

Mémoires de la Soc. Lim. de Calvados. 1528. T. IV. - Siche Bull. des Sc. natur. Nov. 1829. p. 281.

Végétation et sol de la hasse Normandic. Sćance de la Soc. Linn. de Normandie du 1. Dec. 1828.

Journal de Caen et de la Normandie. IV. Dec. 1828. - Bull. des. Sc. nat. Janv. 1829. p. 533.

Iïniges hierher Gehörige kommt auch in den sogenunnten medicinischen Topographien vor; z. L. der von Strasburg von Rennes, (Mím. de Med. Chir. et Pharm. mil. Yol. XXIV. p. 1.), der von Metz, bearbeitet durch J. C. B rault (Daselbst Bd. XXII.) u. s. w. Siehe II e us ingers Zcilschrift. Bd. III. Meft 4, wo noch mehrere angegeben sind. -

\section{I $t$ a $l i$ is $u$.}

J. F. L. I a usmann. Commentatio de Apcuninorum constitutione geognostica. Goettingae 18\%3. 4. 9 gr.

G. F. Schonw. Prospetto di una descrizione erengrafica delle piante d'Italie e di Sicilia, con un Sitgrio di monografia delle specie del genere Campanula indigene dell' Italia ete.

Giumn. di fisica etc. Parin 1'201. Bim. 1. T. VII. - Siche anch die Isis, im achten Bande. 1821. Ileft 4.

Ueberst a t. ciner P'flanzen-Geographic von Italien und Sicilien.

Ftoriep's Nolizen. No. X. p. 151.

Bossi hugi. Applicarione del sistema di geografia botanica ded Signore de Candolle al Regno d'Italia; in

Givinale d. S. d'Incoragr. de Miluno. VII. S. B2:3.

Veber die Vegetation des Festlandes in Italien, ron II. Samuel brunner in Bern.

Filura 1826. H. p. 625. 
Sulla naturalizazione delle piante. Osserrazioni del D. Gaetano Savi, Professore di Botanice, e Direttore del giardino dell I. e R. universita di Pisa. Pisa 1822. 8.

Bot. Literaturbl. II. p. 159.

Versuch einer botanischen Gengraphie des Beckens des mittclländischen Meeres. Auszug aus der Flora Lybica des Prof. Dom. V iviani.

Giorn. di fisica. et chem. Sept. et Oct. 1824. p. 419.

IIistoire naturelle des principales productions de l'Europe meridionale et particulièrement de celles des environs de Nice et des Alpes maritimes. Par M. A. Ris s o. Paris 1828. V.' Vol. 8.

Tenore. Essai sur la géographie physiq̨ue et botanique du royaume de Naples, arec deux plans. gr. 8. $8 \frac{1}{2}$ Bogen. Br. 4 fl. 12 kr. Wien 1828. Naples 1827.

\section{E $n$ g $l$ a $\quad \boldsymbol{n}$ d.}

R. Bakewell. Einleitung gin die Geologie, nebst eincr Geologic und Mineral - Geographie ron Éngland. Frei aus dem Engl. übersetzt von K. H. Müller. Freiberg 1819. 8.

Skizze der geographischen Verbreitung der Gewächse in der Grafschaft York, von J. Atkins on.

Mémoirs of the W'rnerian Society $1823-1821$. Iol. V. part. 1. p. 278.

Herr 1. spricht von dem Einflusse des Bodens auf die Tegetation und scheint überhaupt annehmen zu wollen, dajs man schon an letzteren den ersten erkennen könne. Er fragt: fand man je Heiden auf Kalkboden? Findet man auf diesem nicht immer folgende Pflanzen: Chlora perfoliata, Astragalus glyciphyllos und Hypoglottis, Neottia spiralis, Orchis pyramidalis, Cistus lielianthemum? - Pinguicula vulgaris, Vaccinium Oxycoccos. Fimpetrum nigrum, zeigen sie nicht ein aus Sandstein bestehendes Erdreich an?

Pflanzen des südlichen Finglands, die nicht in Yorkshire wachsen, sind: Clematis Vitalba, Orohanche minor, Tamarix gallica, Campanula patula und C. Trachelium. Die des Nordens von England, die nicht in der genannten Provinz vorkommen, sind: Ligusticum scoticum, Cerastium tetrandrum, Aira laevigata und Sagina maritima. In Yorkshire scheinen folgende Pflanzen ihre nördlichste Grenze erreicht zu haben: Butomus umbellatus und Viscum album; dagegen die südlichste Grenze: Trientalis europaea. -

Auf bedeulenden Höhen nur, : sagt er, kommen vor: Arbutus Uva Ursi, Saxifraga oppositifolia, Rubus Chamaemorus. Auf gewisse partielle Localitäten eingeschrünkt: Dryas octopetala, Gentiana verna, Cypripedium Calceolus.

J. N. Winch Esq. An Essay on the Gengraphical Distribution of Plants, through the Coninties of Northumberland, Cumberland and Durham. 2. edit. Neiwcastle 1828. 8.

Bemerkungen über die Naturalisation der Pflanzen und den Ǧartenbau: von Guernsey, von Ma c Culloch.

Quaterly Journal of Science. N. XIII. p. 200. - Lotan. Jicraturbl. I. p. 356 .

Bemerkungen über eine Anzahl Bäume und Stauden, velchs in Irland naturalisirt wurden, mebst einigen Nachrichten von sehr ijrofisen Bäumen, von 'T ow nsend-Mackay. 
Dublin philos. Journ. Nov. 1825. p. 437. - Botan. Literaturbl.

II. p. 16 .5.

Dendrolocia Britannica or Trees or Shrubs that will live in the open Air of Britain thromonth the year, loy P. W: Watsow. London 1825. II. Vol. 8. Mit 172 colorirten Abbililungen.

In der Nithe des Dorfes Newton bei Bromfield in Wales, befindet sich cine Allee trefflich grünender ächter liastanienbüume, die man kaum im Süden üppigrer geschn zu haben rlauben möchte.

Spieker Reisen in Fingland, "W'ales und Schottland. Bd. II. p. 80.

$$
S \in h w \in d \in n \text {. }
$$

G. G. Hermelin. Minerographie von I appland und Westbothnien, nelost einem Auszur aus Wahlenbergs Toporraphie von Kemi Lappmark. Aus dem Schwed. von Blumhof. Freiberg 1813.

II isinger. Versuch riner mineralog. Gengraphie von Schweden. Vebursetzt ron Bläde. Freiberg 1819. Neuc umgearbeitete Auflage von Wöhler. Leipzig 18\%6.

Anteckningar i Physik och Geognosi under Resor uti Sverige och Norrige, af W. IX isinger. Upsala första häftet 1819, andra 1820, tredje häftet $18 \geq 3$.

G. Wahlenlerg. Bericht über Messungen und Beobachtungen zur Bestimmung der IIöhe und Temperatur der Lappländ. Alpen unter dem 67. Breitegrade, angestellt im Jahre 1817. Aus dem Schwed. mit Anmerkungen von $\mathrm{H}$ a usm a nn. Göttingen 1812. 20 gr.

G. Wahlenberg. Conspectus Regni et Vegetationis.

Im zweiten Bande der Flora Sueciae desselben.

Ueber die Region des ewigen Schnces in Norwegen und Schweden. Vom Obristlicutenant Hagelstam.

jus Jameson Edinb. New. Philos. Journal. 1828. Jul. - Sept.

p. 315. in den botan. Literaturbl. II. p. 38. - Hertha Band XIII. p. ยี12.

Vegetation am Nordcap und in Gröland, von Capell Brocke. Botan. Literaturbl.

Daselbst $p_{.} 45$.

Jeschreibung einiger seltneren Pflanzen der nördlichen Gegenden vun Schweden; mit Bemerkungen über die Geographic der Gewïchse, von L. L. Lestadius.

Kongl. Vetenskaps Academiens Ilandlingar. Slockholm 1821. p. 160.

Uebersicht der Veretation in Schonnen, vom Herm Prof Fries.

Man selie hierüber Schwed. Jahresbericht. Bonn 1826. p. 182.

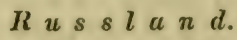

Gr. de Razoumovski. Coup d'ocil geornostique sur le nord de l'Europe én général et partieulièrement de la Russic. Berlin 1820.

M. v. Engelhard. Darstellungen aus dem Felsgehäude Russlands. 1. Licf. Geognost. Unril's von Finnland. Herlin 1820.

A perçu de la Géographie botanique de la Follynie et de la Podolie, par le Dr. Besser, Prof. d'hist. nat. au lycie de Volhynic.

Mém. de la Soc. impériale des Naturalistes de Moscou. T. VI.

1823. p. 185. 


\section{Der Urwald ron Bialowicza in Lithanen.}

Mémoire descriptif sur la forèt impériale de Bialowicza, par le Baron de Brincken, conscrvateur en chef des forèts etc. Orué de 4 grav. et d'une carte. Varsovie 1826. - 4.

Bot. Literaturbl. III. p. 599. - Hertha. Bd. XIII. p. 305.

$$
\text { A s } i \text { e } n \text {. }
$$

Bemerkungen zur physikalischen Geographic und zur Ethnographie des Gebirgs-Districtes des Himalaja, zwischen den Flüssen Siamuna und Satadru, rom Dr. Med. George Govan.

Hertha. Zweiter Jahrg. Bd. V. Heft 3. Erste Abtheil. p. 331 - 359.

Topographie médicale et végétale de quelques parties de l'Ilimelaja, par le Dr. Govan.

Asiatic Journal. Aug. 1828. - Nouvelles Annales des voyagres.

Nov. 1828. p. 249.

Herr $\boldsymbol{G}$. fund in den Himelaja-Gebirgen unter andern Himbeeren, Erdbeeren, Birnen und Acpfel; cultivirt suh er eine neue Art ITeizen (Ladakh) und eine besondere Art Gerste (oua), die, seiner Mcinung nach, auch in Schottland eingeführt werden könnten.

Ueber den Charakter der Vegetation auf den Inseln des Indischen Archipels. Vortrag, gehalten in der Versammlung dentscher Naturforscher und Acrzte in Berlin, am 20. Sept. 1822, ron Dr. C. G. C. Reinwardt, Prof. der Chemie, Botanik, u. s. w. in Leyden.

Brandes Archiv. Bd. XXVIII. Heft 1. p. 8. u. d. f.

Lettres on the Climate, Inhahitants, Productions ete., of the Neilgherries or Blue Mountains of Coimbatur, South India, by J. Ilough. London 1829. 8.

Bot. Literaturbl. II. p. 281.

II. P. Gerard. Nachrichten über das Kilima und den Ackerbau in Soobathoo und Kotgurh.

Aus dem Fidinburgh Journal of Science by Breuster. No. XVIII

p. 233 , übersetzt in II eusinger's Zeitschrift. Bund III. Ileft p. 52t. $u$. $d . f$.

Sketches of the Meteorology, Geology, Agricultury, Botany and Zoology of the Southern Mahratta Country, by Aleximiter Turnbull Christie.

Jameson Ncw Edinb. Phil. Journal. April - July 1829. p. 52. Geiger's Magaz. Dec. 1829. p. 226. - Bot. Literuturbl. IY. p. 25.

Einige Bemerkungen üher die Pflanzen verschieldener Theile Ostindiens, mit Rücksicht auf die Benennung der Gegenden im Sanskrit, von Hamilton.

Aus dem Engl. von Beilschmied. - Lotan. Zeit. 1826.

II. p. 689 .

$$
A f r i k a \text {. }
$$

Leep. v. Buch. Allgemeine Uebersicht der Flor auf den canarischen Inseln.

Abhandl. der Akad. der Wissenschaften in Berlin. 1816 und 1817.

p. 337 - 381. - Sprengel neue Eintdcokungen. III. p. 386.

Botanische Geographie von Madcira, ron Kuhl und Hasselt. Annales générales des Sciences physiques. Fevr. 1821. - Froriep's Notizen. October 1821. No. XII. p. 182. 
Excursions to Madeira and Porto Santo during the Antumn. 1823. By the late Eilward Bouduh, with an Appendix containing zoological and botanical descriptions. Lendon 1825.

Observations made dnringr a Visit to Madcira, and a Residence in the Canary Islands. By Baron Leopold von Buch.

The Jaw. Edinb. Philosophical Journal by Jameson. April - Oct. 1826. p. $380-384$.

T. r. Bueh. Physilialische Beschreibung der lianarischen Inseln. Berlin 1825. 4.

Besehreihung der Insel St. IIclena. nach ihrer geognostischen Beschaffenheit und Bildung, nebst Nachrichten von dem filima, der Naturgeschichte ete, von 'T'. F. Eh rmann. Weimar 1807.

$$
\Lambda m e r i k \text { a. }
$$

Vegetation in Grönland und am Nordcap, ron Capell Brocke. Bot. Literaturbl. II. p. 45.

Bemerkungen üher die Tloren der Karagies-Inseln und cines Theils des Landes an der Behrings-Strafse, aus den Briefen des Dr. Mertens an die IIerren Fischer und Trinius.

Linnaea 1830. I. p. 60.

Berieht über eine Excursion auf den Gipfel des Werstevoi bei Neu-Archangel in Norfolksund, von Dr. Heinrich Mertens.

Linnaea 1829. I. p. 58.

Notice sur l'ile de Terre Neuve et quelques iles voisines; par M. B. de la Pilaye.

Annales de la Soc. Linn. de Paris. 5 Livrais. Nov. 1825. p. 417 - 547.

Enthält einen Vergleich der Flor des nördlichen Ameriku, mit der in Europa und Asien unter gleicher Breite.

Beobachtungen über das Klima und die Producte der Washington-Country im Ohiostaate, von Dr. S. P. II ildreth.

Sïlim. Amer. Journ. XII. 2. p. 206. - Botan. Literaturbl. I. p. 361 .

Die Pflanzen - Geographic einiger amerikanischen Landstriche berühren auch die Mittheilungen des Dr. Richardson von der Expedition des Capitaine Franklin.

Bulletin de la Société de Geographic. Tom VII. No. 45 et 46.

Paris 1827. - Siehe Linnaea 1827. p. 519.

Memorias para servir de introduccion à la Ilorticnltura Cubana. Par D. R a mon de la Sagra etc. Nueva York 182\%. 8.

Siche Linnaea 1828. III. p.169. - Botan. Literaturbl. II. p. 283.

Botanische Berichte aus Mexico ron Sehicde. p. 4 6ỉ.

Linnace 1529). Heft II. p. 205. Heft IV. p. 55t. 1830. IIeft III.

Extrait d'une lettre de M. Bertero, D. M. et voyageur naturaliste, addressée ì M. Guillemin et datée de Ramcagua, le 12. Juillet 1828.

Isull. des Sc. nut. Junvier 1829. p. 112. - Enthült Nachrichten über die Vegetation in Chili.

IIistoire physique des Antilles françoises etc, par A. M. de Jonne 8. Paris 1823. 8. 
W. จ. Eschwege. Geognostisches Gemälde von Brasilien. Wei$\operatorname{mar} 1822.8$.

v. Martius. Die Physiognomie des Pflanzenreichs in Brasilien. Eine Rede, gelesen in der am 14. Febr. $182 t$ gellaltenen Sitzung der Akademie der Wissenschaften. München 1824.

Höhenbestimmungen in der peruanischen Andeskette; ron Pentland.

Geiger's Magazin Juni 1829. p. 132.

A $11 \mathrm{gemeine} \mathrm{naturhistorische} \mathrm{Beobachtungen} \mathrm{über} \mathrm{die} \mathrm{Insel} \mathrm{Soledad}$ (cine der Malouinen) von R.P. Lesson.

Botan. Literaturbl. I. p. 160.

Blüthenzeit der Wallnufs in Amorika.

Silliman. Americ. Journ. XIII. 2. p. 228. - Bot. Literaturbl.

II. $p$. 284 .

$$
N \text { e } u-H \text { o } l l a n d .
$$

Geographical Memoirs on New South Wales, edited by Baron Fields. London 1825.

Siehe Linnaea II. p. 120.

Einige allgemeine Bemerkungen über die Vegetation vorzüglich der Nordwestküste von Australien, von A. Cunningham.

Isis 1828. p. 172. Bd. XXI.

Auszug cines Briefs des Dr. Zippelius an den Prof. Blume, enthaltend Notizen über eine botanische Rcise auf Neu-Guinea.

Algemene Konst en Letterbode. 8. Mai 1829. No. XIX. p. 294. -

Bulletin des Sc. naturelles. Juillet 1829. p. 90.

W. Ellis, Polynesia. Researches during a Residence of nearly six years in the South-sea Islands, including descriptions of the Natural History and Scenery of the Islands etc. Lond. 1829. II. Vol. with plates.

Re is ebeschreibungen,

Notizen enthaltend, die für die Geographie der Botanik benutzt werden können.

\section{§. 21.}

Die erste Kenntnifs mancher Gewïchse und so vieler anderer Naturprodukte verdanken wir einzig den rühmlichen Bemühungen gelehrter Reisenden, auch haben sie in ihren Berichten häufig die interessantesten Nachrichten über das Beobachtete niederge'egt; deshalb ist die Literatur der Reiscbeschreibungen ein gar nicht unwichtiger Zweig für die Pflanzenkunde; leider mufs man sich aber durch eine Masse von Schriften winden, bis man cine, oder die andere trifft, in der sich Bemerkungen finden, die den aufmerksumen Kenner und Freund der Natur bezeugen. Möchte doch jeder Gebildete wenigstens einen Zweig der Naturkunde cultiviren; wie viel hätte dadurch die Wissenschaft gewonnen, und welchen Genufs mehr hätten sich die Reisenden selbst verschaffen können!

Die literarischen Nachrichten, die ich hier gebe, dürften allerdings ziemlich unvollständig gefunden werden, allein ich konnte nicht mehr Zeit auf die Lectüre der Reisebeschreibungen verwenden, als ich wohl gerne gemocht hätte. Auch hier hat indessen der fleissige und umsichtige Schultes in seinem oben angeführten Werke vorgearbeitet, worauf ich also, so wie auf das Handbuch der deutschen Literatur von Ersch verweisen muss. 
I'ichtige newere, den Potaniker vorzugswcise interessircnde W'erke glaube ich nicht übergungen zu haben, und entschuldige mich daher mit Columella, der einst sagte:

In magna silva boni venatoris est, indacranter feras complures capere, nee cuiquam culpae est, non omnes cepissc. -

\section{Einige Zeitschriften.}

Journal für die neuesten Land- und Seereisen und das Interessanteste aus der Völker - und Länderkunde, zur angenchmen Unterhaltung für gelildete Leser aus allen Ständen. Redigirt ron Dr. Gr. G. Friedenberg. Berlin. 8. mit gemalten Kupfern. Jotat 65 Bäinde.

Tasehenbuch zur Verhreitung gengraphischer Kenntnisse von Joh. Gottfried So numer. Achter Jahrgang. Prag 1830.

Bibliothek der neuesten und wichtigsten Reisebeschreibungen und gcographische Nachrichten zur Erweiterung der Erdliunde ete., von M. 6. Sprengel und T. F. Ehrmann. Mit Kupfern und Karten. 50 Bände. Weimar 1800 - 1814.

Nene Bibliothek der wichtigsten Reiscbeschreibungen, zur Frwciterung der Erd- und Völkcrkunde; nach Bertuchs Tode bearbeitet und herausgegeben von mehreren Gelehrten. Weimar seit 1814. Jetzt 55 Bände. Der 55. enthält Clapperton's zweite Reise in das innere Afrika.

Magazin ron merkwürdigen neuen Reisebeschreibungen, mit Kupfern und Iiarten. Berlin. 8. seit 1790. Der letzte mir zu Gesicht gelommene Band ist der 37. vom Jahre 1828, enthaltend Bukingham's Reise naich Mesopotamien.

Nouvelles Annales des Voyages et des Sciences géngraphiques, publiées par M.M. Eyries et Klaproth. Paris. 8. chez Gide fils. Jetzt sind 17 Bände der zweiten Serie vorhanden. -

Bibliothek naturhistorischer Reisen für die reifere Jugend. $1-4$. Bïndehen, cnthält Alex. v. II umboldt und $\Lambda$ imé Jonpland Reisen in die $\Lambda$ equinoctialgegenden des neuen Continents, bearbeitet von $\mathbf{G}$. $A$. Wimmer. Wien 1830. 12. Mit Kupfern und Karten.

Journal des Voyages, découvertes et navigations molernes, ou Archires géographigues du 19. siècle; par une société de géographes, de voyagcurs et de litterateur français et étrangers. Publić par $\boldsymbol{\Lambda}$. R. de Le uven. Paris. 8. Au burean rue Saint-Lazarc. No. 42.

\section{Feisen um die Welt.}

W. G. Tileøius. Naturhistorische Früchte der ersten K. Russ., unter Kirusenstern vollbrachten Frdumsegelung. Petershurg und Lcipzig. 1813. 4.

G. II. v. Langsdorf. Bemerkungen anf einer Reise un die Welt in den Jahren $180 \S$ - 7. Frankfurt a. M. 1513. 2 IBde. 4.

Voyace autour du Monde, cxécutée sur les corvettes de lvianic et la P'hysicienne. sous les ordres du Cap. Lonis de Freycinct, en 1818 - 1820. Partic hotanique; par M. Ch. Gaudichaud, pharmacien de la marine. In 4. avec atlas in fol. de $120 \mathrm{pl}$. Paris $1826-27$. Livr. I - IV. Pillet aine.

Siche bolan. Lileraturbl. I. p. 62.

Entdecliungareise in die Südsee und nach der Berings - Stragse 
zin Erforschung einer nordostlichen Durchfahrt. Unternommen in den Jahren 1815 - 181S, auf Kosten Sr. Erlaucht des Herrn Reichskanzlers Grafen Romanz off auf dem Schiffe Rurick, unter dem Befehl des Lieutenants der Russ. Kaiserl. Marine O tto v. Kotze bu e. Weimar 1821. 8 Vol. 4.

Im dritten Theile kommen botanische Nachrichten vor von $A \mathrm{del}$ bert v. Chamisso über Teneriffa, Brasilien, Californien, Chili, die Philippinen, Marianen, die Oster-Insel, Sandwich-Inseln, Kamtschatka u. s. w.

Voyage autour du Monde etc., par Duperrey. Partie botanique livr. I. Paris 1827.

Diesen botanischen Antheil besorgen die Herren d'Urville, Bory de St. Vincent und Ad. Brongniart. Den Anfang macher Cryptogamen, namentlich Algen. -

Voyage medicale antour du monde, executée sur la corvette la Coquille, pendant les années 1822 - 1825, par R. P. Lesson. Paris 1929. 8 .

Note sur les collections et les observations recueillis par $J$. M. d'Urville, durant de la campagne de la Coquille autour du monde, en $1822,1823,1821$ et 1825 .

Annales des Sc, natur. Mai 1825. p. 62.

D. Vollmer. Natur- und Sittengemälde der Tropenlãnder, Skizze einer Reise durch Süd-Amerika und um die Welt in 14 Vorlesungen. Zweite Auflage. Leipzig 1829 bei Brockhaus. 2 fl. 42 kr.

\section{Reisen in Deutschland, Ungarn und Holland.}

Voyage dans le Tyrol aux salines de Salzbourg et de Reichenhall, et dans une partie de la Bavière, par M. le Chevallier de Bray, Conseiller intime d'Etat de $\mathbf{S}$. M. le Roi de Bavière etc. Paris chez F. Schoell, rue de Fossés - Saint Germain l'Auxerrois No. 29. 1808.

Nach der Vorrede ist diese cben so instructive als unterhaltende Reisebeschreibung, die den Botaniker besonders ansprechen wird, zuerst in deutscher Sprache in Berlin herausgekommen; ich habe aber nur die französische Edition zu lesen Gelegenheit gehabt.

C. F. Germar. Reise durch Oesterreich, Tyrol nach Dalmation, in dem Gebiete von Ragusa 1 . s. w. Leipzig 1814. 2r Theil 1817.

H. Hoppe und C. F. Hornschuch. Tagebuch einer Reise nach den Küsten des adriatischen Meeres und den Gebirgen von Krain, Kärnthen, Baiern und Böhmen, vorzüglich in botanischer und entomologischer Hinsicht. Regensburg 1818. 8. Der zweite Theil dieser interessanten Schrift ist nicht heraus-
gekommen.-

M. S. v. Uechtritz, kleine Reisen eines Naturforschers. Breslau 1820. 8.

Sehr lehrreich und brauchbar!

J. F. A. Dehne. Spaziergang von Leipzig nach dem Harze und den umliegenden Gegenden, vorzüglich in botanischer und mineralogischer Hinsicht. Quedlinburg 1819. 8. 54 kr.

F. Meyer. Bemerkungen auf einer Reise durch Thüringen, Franken, die Schweiz, Italien, Tyrol und Baiern in Jahre 1816. Berlin 1818.

Tousв v. Charpenticr. Bcmerkungen auf einer Reise von Bres- 
lau üher Salzhurg und Tyrol, der südlichen Schwelz, nach Rom, Neapel etc., im Jahre 1818. Leipzig 1820.

In geognostischer. Hinsicht wichtig.

J. A. Schulte s Reisen auf den Glockner. Wien 1822. 4 Theile. 8.

K. Graf v. Sternberg. Reise durch Tyrol nach Italien im Jahro 1804. Regensburg 1806.

J. A. Schultes. Ausflïge nach dem Schnecherg in Unter-Oestreich, mit beigefügter Fauna und Flora de? südwestlichen Gegend um Wien bis auf den Gipfel des Schneeberges; ein Taschenbuch auf Reisen nach demselben. Wien 1822. 2 Theile. 8.

Berättelse om en botanisk Resa till Österrike och Nordöstra Italien ar 1827 af C. A. Agardh s. titulo 8. 15 S. Stockholm 1828.

Verslag van een plant - end landbauwkundig reisje, gedaan in July 1826, langs de oevers der Maas, van Luik naar Dinant, in de Ardennes en het Groothertogdom Luxemburg; door M. Bronn en R. Court ois ete. Bydragen tot de Natuurk. Wetens. Vol. II. p. 450. - Siehe Linnaea 1829. I. p. 35 der Lit.

A. Rochel. Naturhistorische Miscellen über die nordwestlichen Karpathen in Ober-Ungarn. Pesth 1820.

Bruchstücke aus dem Tagebuch einer naturhistorischen Reise von Prag nach Istrien, ron dem Herrn Grafen Caspar v. Sternberg. Bot. Zeit. 1826. I. Beilage p. 1. u. d. f.

Reisen in der Schweiz und den benachbarten Alpen.

Alpina. Eine Schrift der genauen Kenntnifs der Alpen gewidmet; herausgegeben ron K. V. Sali s und J. R. Steinmüller. Winterthur seit 1806.

K. Kasth of er. Bemerkungen auf einer Alpenreise über den Sïsten, Gotthard, Bernhardin und über die Oberalp, Furka und Grimsel. Aarau 1822. $5 \mathrm{gr}$.

K. Kasth ofer. Bemerkungen auf einer Alpenrcise üher den Brüning, Bragel, Kirenzenberg und über den Flüelen, den Maloyen und Splügen. Bern 1825. 8. 1 Rthl. 12 gr.

Der Monte Rosa, eine topographische und naturhistorische Skizze; nebst einem Anhang der von Herrn $Z$ umstein gemachten Reisen zur Ersteigung sciner Gipfel; herausgegeben von L. v. Welden. Wien 1821. 8.

Hö́chst schätzbar und belehrend!

H. G. Bronn. Ergebnisse meiner naturhistorisch-öconomischen Reisen. Erster 'Theil. Briefe aus der Schweiz, Italien und Sädfrankreich im Sommer 1821. Heidelberg und Leipzig 1826.

J. G. Ebel. Anleitung auf die nützlichste und genufsvollste Art die Schweiz zu bercisen. 4 Theile mit Kupf. u. Karte u. neugest. Bergketten. Zürich 1810. 8.

Gibt auch die Pflanzen einzelner Gegenden an.

W. Gerhard. Spaziergang über die Alpen. Gotha 1824. 8. mit 6 Kupfern.

$V$ on den besuchtesten und interessantesten Stellen der Schweiz gibt der Herr Verf. die Vegetation an. Das Buch verdient den Botanikern bekannter zu werden, als es zu sein scheint. 
Villars précis d'un voyage botanique fait en Suisse etc. en 1811. Strashourg 1812. 8. chez Levrault. 64 Seiten, mit Abbildungen mehrerer Hieracien.

Flug von der Nordsee zum Montblane; durch Westphalen, Niederrhein etc., von W. L. M üll er. Altona 1821. 2 Theile.

Im 2. Bande p. 498. befindet sich eine Aufzählung der Pflanzen des Rigi; sie dürfte jedoch eben nicht zuverlässig sein.

J. Hegetschweiler. Reisen in den Gebirgsstock zwischen Glarus und Graubündten in den Jahren 1819, 1820 und 1822. Nebst einem botanischen Anhang und mehreren lithographirten Zeichuungen. Zürich 1825. 8. $2 \mathrm{fl}, 12 \mathrm{kr}$.

Wanderungen in weniger besuchte Alprngegenden der Schweiz und ihrer nächsten Umgebungen, von Hirzel-Esch er. Zürich 1829. 8. $168 \mathrm{~S} .1 \mathrm{fl}$.

Mehr für den Geognosten als Botaniker brauchbar.

Naturhistorische Alpenreise von J. J. Hugi. Solothurn und Leipzig 1830. 378 S. 8.

Reisen in Frankreich, Italien und Griechenland.

J. A. Schultes. Briefe über Frankreich auf einer Fufsreise im Jahre 1811. Leipzig 1815. 2 Theile. 8.

1818. 2 Teihle. 8.

A. Thieb. v. Berneaud. Schilderung der Insel Elba, aus dem Franz. mit Anmerkuugen von Eh rmann. Weimar 1809. 8.

Brunner. Strcifzug durch das östliche Ligurien, Elba, die Ostkïste Siciliens und Malia; zunächst in Bezug auf Pflanzenkunde, in Sommer 1826. Winterthur 1828.

G. v. Martens. Reise nach Venedig. 1r Thl. von Stuttgart über Ulm, Wien und Triest nach Venedig. 2r Thl. Venedig, Eugoneen, Alpen von Belluno, Tyrol, Baiern. Naturgeschichtl. Anhang. Mit 2 Kpf. und 7 lithograph. Abbilit. gr. 8. Stuttgardt 1824.

F. W. Sieber. Reise nach der Insel Kreta im griechischen Archipelagus im Jahre 1817. 2 Bd. mit Kupfern und Karten. Leipzig 1822. 8. 9 fl. $54 \mathrm{kr}$.

Viaggio in alcuni luoghi della Basilicata e della Calabria citeriore, effetuato nel 1826 da L. Petegna, G. Ferrone e M. Tenore. Napoli 1827. 8.

Siehe botan. Literaturbl. II. p. 283.

Travels in various parts of Europe, Asia and Africa, by E. D. Clarke. London, 1810 - 16. 4 .

Siche Sprengel's neue Entdeckungen. Bd. III. p. 157.

Reisen in England und den übrigen Ländern im nördlichen Europa.

Reise eines Gallo-Amerikaners (M. Simonds) durch Grofsbrittannien in den Jahren 1810 und 1811. Deutsch von Schlosser. Leipzig 1817 - 1818. 2 Theile mit Kupf.

F. Garnett. Reise durch die schottischen Hochlande und einen Theil der Hebriden. Aus dem Engl. von Kosegarten. Leipzig 1802. 
Loopold v. Buch. Reise durch Norwegen und Lappland. Berlln 1810... 2 Bïnde.

Für Planzen-Geographic vorzugsweise wichtig!

J. F. L. Hausmann. Reise durch Skandinavien in den Jahren 1806 - 7. Güttingen 1811 - 18. 5 Bände.

Der Geognosie gewidmet.

Graf Vargas Bedemar's Reise nach dem hohen Norlen durch Seliweden, Norwegen und Lappland, in den Jahren 1810 - 14. Frankfurt 1819.' 2 Theile.

F. W. v. Sehubert. Reise durch Schweden, Norwegen, Iappland, Finnland und Ingermannland, in den Jahren 1817 - 20. Leipzig 1823. 3 Bïnde.

Entheilt einige, doch im Ganzen nur wenige die Botanik betreffende Notizen.

Reisen im Norden Europa's, vorzïglich in Island, in den Jahren 1820 und 1821, von Th ienemann und $G$ ïnther. Leipzig 1827.

Unter vielen interessanten Notizen mögre folgende hier cine Stelle finden: Am 8. Juni waren in Leipzig die Erdbeeren reif und die Stuchelbeeren fingen an sich zu fürben, am 15. Juni waren erstere in Hamburg so selten, dafs $601 \mathrm{~K}$ Rthl. $8 \mathrm{gr}$. kosteten. In Koppenhagen waren die Erdbeeren erst am 3. Juli reif und das Korn fing an $\approx u$ blïhen; in Arendal erreichten die Erdbeeren erst am Ende des Juli ihre Reife, und das Korn blühte im Anfang des August.

Accrbi. Reisen durch Schweden, Finnland und Lappland; aus dem Engl. von Weyland. Berlin 1803. - Magazin merkw. Reiscbeschrcibungen. Bd. XXVI.

Enthült einige allgemeine Kennzeichen vom Frühling und Sommer in Ulaborg, nach 2tjährigen Beobacht. von Julin (p. 201.), dann besonders andere botanische Nachrichten von Herrn Castrein, Prediger zu Kemi, über die dortige Flor u. s. w. (p. 205.)

\section{Reisen in Asien und Afrika.}

Burkhard. Reise in Syrien und dem gelobten Lande. Jena 1823.

Sieber. Reise von Cairo nach Jerusalem. Prag 1823. 1 Rthl. 12 gr.

J. D a vy's Reisen im Innern der Insel Ceylon. Jena 1822.

Voyage d'Orembourg à Boukhara, fait en 1820, redigé par M. le Baron Georges de Meyend orf. Paris 186. Partie botanique.

Siehe Bulletin des Sc, natur. Sept. 1826. p. 48.

Ein Verzeichnifs von Pflanzen, die auf einer Reise von Orenburg nach den Lande der Kirgisen gefunden wurden, von Herrn IN aréline, steht im

Lulletin de la Soc. des natural. de Moscau 1829. p. 150.

E. Eversmann's Reise ron Orenburer nach Buchara; nehst cinem Wortverzeichnif's der Afghan Sprache, begleitet von naturhistor. Anhang und Vorrede von II. Lichtenstein. Berlin 1823. 4. mit Kupf. 3 Rthl.

Relation abregée d'un voyage, fait an mois de Mai 1827 dans les stepues situées entre les parties meridionales du fleuve Oural ct du Volga, par M. E. Eversmann, M. Dr.

Nouvelles Annales des Voyages Juin 1828. p. 281. $u$. d. $f$. 1829.

Reisc durch das Altaigebirge, von C. T. v. Ledebour. Berlin 
Reise zu den altaischen Kalmucken im Herbste 1806, von dem Bergmeister Gregorii Iwanowitsch Spaski.

Hertha März 1827. p. 237 - 255.

Colebrooke's Bericht über die neueste Reise der Gebrüder Gorard in das Setlej (Satadru) Thal im Himclaja - Gebirge, während des Jahres 1821.

Hertha. Zweiter Jahrgang. Bd. V. Heft 8. erste Abtheilung. p. $286-330$.

J. Berggren. Reisen in Europa und dem Morgenlande. Aus dem Schwedischen ron Ungewitter. Darmstadt bei Leske 1825. Erster 'Theil 2 Rthl.

Höchst interessant!

Von Herrn Bè ra n g e r haben wir eine besonders für Botanik reiche Ausbeuts enthaltende Reisebeschreibung nach Ostindien durch den Norden von Europa, die Provinzen des Caucasus, Georgien, Armenien und Persien nächstens zu erwarten. Man sehe

Bulletin des Sc. natur. Févr. 1829. p. 267. Nov. 1829. p. 813.

Burkhard. Reisen in Nubien und Arabien. Aus dem Englischen. Jena 1820. 8.

Eduard Rüppell. Reisen in Nubien, Kordofan und dem petrâischen Arabien, vorzüglich in geographisch - statistischer Hinsicht. Mit 8 Kupfern und 4 Karten. Frankfurt am Main 1829.

Enthält nur höchst dürftige Nachrichten über dic Vegetation der bereisten Länder.

Menu v. Minutoli. Reise zum Tempel des Jupiter Ammon in der lybischen Wüste und nach Ober-Aegypten in den Jahren 1820 und 1821. Berlin 182.

Naturhistorische Reisen durch Nord-Afrika und West-Asicn in den Jahren 1820 bis 18\%, von Dr. W. E. Hemprich und Dr. C. G. Ehrenberg. Historischer 'Theil, mit Charten und Ansichten. 1. Bd. 1. Abtheil. Mit einer Landkarte und einer Ansicht des Lybischen Wüstenabfalls. gr. 4 . Berlin 188. $6 \mathrm{ff}$. $45 \mathrm{kr}$.

Eines der vor züglichsten Werke in diesem Zweige der Literatur.

Della Celle. Reise von Tripolis an die Grenzen von Aegypten im Jahre 1817. Aus dem Italien. W cimar 1821. $1 \mathrm{fl}$. $35 \mathrm{kr}$.

Ist auch im. 29. Bande von Bertuch's neuer Biblitithek, auch vollständig in Malte Brun Annales des voyages 1823. Febr. - Mai.

'Travels in the interior of southern Africa by W. J. Burchell, with unew map and coloured and black Engravings. London. Vul. I. 18\%t. Vol. II. 1825. 4.

Voyage de M. Leprieur dans la Senegambie.

Bulletin des Sc.natur. Aout 1829. 1.261.

Notice sur le voyage de M. Perrotet au Scnegal, en Gambie of en Casmanic. Extrait d'une lettre de ce voyageur it M. De Candolle.

Bibl. universelle. Nov. 1829. p. 265. et suiv.

A Voyage to Abyssinia ete. by Henry Salt. Esw. F. R. S. Lond. 1814. 4. Mit Kupf. und Kart.

Sielse bot. Zeit. 1821. I. Beil. p. 61. 


\section{Reisen in Amerika und Australien.}

W. E. Parry. Tagebuch einer Entdeckungkreise nach den nördlichen Polargegenden im Jahre 1818. a. d. Engl. Hamburg 1820. 8.

W. E. Parry. Zweite Reise zur Entdeckung einer nordwestlichen Durchfahrt aus dem atlantischen in diss stille Meer, in den Jahren 1819 und 1820. Aus dem Engl. Hamburg 1822.

v. Chateanbriand. Reise in Amerika; übersetzt von Dr. Per$1 \mathrm{cb}$. Freiburg 18:8. 1827.

Hamilton, Reisen in den innern Provinzen von Columbien. Jena

Alex. v. Humboldt und Aimé Bonpland, Reise in die Aequinoctialgegenden des neuen Continents, in den Jahren $1799-1804$. Aus dem Franzôs. Stuttgardt 1815 - 1829. 6 Bände.

Alex. v. Humboldt. Reise in das Innere von Südamerika und um die Welt. Hamburg 1815. 8. 6 Bände.

Alex. v. Humboldt und Aimé Bonpland. Reise u. 6. w. im Auszuge aus ihren Memoiren von J. C. Delametherie. Aus dem Französ. Erfurt 1806. $8 \mathrm{gr}$.

Reisen in Süd-Amerika, während den Jahren 1819, 1820, 1821, enthaltend eine Schilderung des gegenwärtigen Zustandes von Brasilien, Buenos Ayres und Chile von Alexander Caldcleugh Esq. Weimar 1826. Ist auch der 41. Band der neuen Bibliothek der wichtigsten Reisebeschreibungen.

Enthält manche interessante die Vegretation der bereisten Gegenden betreffende Notizen, z. B. über den. Theebaum von Paraguay, die cultivirten Arten der Musa, Citrus u. s. w. Der Verf. kennt auch, was bei Englündern selten ist, mehrere Schriften der deutschen Botaniker, namentlich führt er die Herren Hornschuch und Hoppe, doch bei einer seltsamen Gelegenheit, an.

Mx. Prinzen von Neuwied's Reisen nach Brasilien in den Jahren 1815 - 17. Frankf. a. M. 1S15 - 20. Zwei Bände. 4. Mit 22 Kupfern. 25 Rthlr. Wohlfeile Ausgabe 2 Bände. 8. 2 Rthl. $16 \mathrm{gr}$.

Desselben Beiträge zur Naturgeschichte Brasiliens 2 Bände. Weimar 1825 , und 1826.

N a chri chten von den K. K. Oestreichischen Naturforschern in Brasilicn und den Resultaten ihrer Betricbsamkeit. Aus der Amtsrelat. der K. K. Gesantten und aus den Berichten und Briefen an IIerrn v. Schreibers. Brünn 1820. Zweites Heft. Leipzig 1823.

W. L. v. Eschwege. Journal von Brasilien, oder verm. Nachrichten aus Brasilien, auf wissenschaftlichen Reisen gesammelt. Weimar seit 1818.

Desselben. Brasilien, die neue Welt, in topogr., geognost., hergmänn., natırhistorischer แ. s. w. Hinsicht, während der Jahre 1810 1821. Braunschweig 1824. Zwei Bände. 8.

J. P. v. Spix und K. F. P. v. Martius Reise in Brasilien, anf Befehl Sr. Majest. Maxim. Jos. I. gemacht in den Jahren 1817 - 1820. Minchen 1823. gr. 4. Mit Karten und Abbildungen. Zweiter Theil, herausgegeben von v. Martius. 1828.

A. de Saint-IIilaire. Voyage an Bresil, dans les provinces de Rio de Janeiro et des Mlinas geracs. Paris 1830. 8. II. Vol. 
Schilderung der Insel Van Diemens Land u. 8. w., nach den von F. L. v. Bibra gesammelten Materialien, bearbeitet von $\mathbf{C}$. N. Röding. Hamburg 1823.

Voyages of Discovery undertaken to complete the Survey of the western Coast of New Holland, between the years 1817 - 1822 by Philip Parker King etc. London 1826. 8. 2 Vol.

Siehe Linnaea 1827. p. 690.

Neueste Forschungreisen im Innern und an der Küste von Neu-Süd-Wallis auf Befehl der Brittischen Regierung in den Jahren 1817, 1818 und 1819 unternommen von John Oxley, Oberaufseher der Colonial - Ländereien und Marine - Lieutenant. Im Auszuge bearbeitet nach dem Englischen. Jena 18\%1: 8.

Enthält botanische Notizen von den Herren Fraser und Cunning ham.

Reisebericht einiger holländischen Naturforscher über ihre Entdeckungen in Neu-Guinea.

p. 142.

Bydragen tot de natuurkundige Wetenschappen. Vyfde Deel. 1830.

\section{Literatur der Floren.}

§. 25.

Einen der interessantesten Zuveige der Botanik machen die Floren einzelner Länder, Provinzen und Städte aus; ilire Bearbeitung ist nicht selten die Lieblingsbeschäftigung der Freunde der Gewächskunde. Seitdem Thalius im 16. Jahrhunderte die Pflanzen des Harzes aufsuchte und beschrieb, sind dergleichen Monographien in grofser Zahl gefolgt, doch wie man schon voraus sehen konnte, von sehr ungleichem inneren Gehalte und Werthe.

Heut zu Tage ist es ein sehr bedenkliches Unternehmen geworden, die Flor selbst nur eines kleinen Districts beschreiben $z u$ wollen, denn die Forderungen, welche man mit Recht jetzt an eine solche Arbeit macht, sind so grofs, dafs nur ein langjühriges und vielseitiges Studium zureichen kann, sie alle vollkommen oder auch nur einigermafsen genüg-end zu erfüllen.

Gewöhnlich richten sich die Floristen bei Abjassung ihrer W erke nach politischen Grenzen, oder dehnen diese willkührlich und unbestimmt, ja olne alle Rücksicht nach jeder Himmelsgegend aus, allein sollen überhaupt Floven zugleach dazu dienen, die Pflanzen-Geographie zu erhellen und die Verbreitung der Gewächse oder die Gesetze ihrer Vertheilung darzuthun, so wird man diesem Zicle nur dann sich nühern können, wenn man die Untersuchungen streng und einzig nach natürlichen Grenzen vornimmt. Diese Nuturgrenzen sind wohl nirgends als in der Richtung der Gebirge und in dem Laufe der Ströme zu suchen, der von jenem nothwendig abhängt. In diesem Sinne habe ich auch die Literatur der Floren einiger europäischen Lünder geordnet, hoffend, es werde eine Zeit kommen, wo man das Belehrende und Nützliche jener Bearbeitung der Floren nicht blos anerkennen, sondern auch befolgen wird. Nicht die I Igetation sler Umgebungen ciner Stadt, oder die einer politisch begrenzten Provinz sollte man künftig aufzeichnen, sondern die Verbreitung der Pflanzenwelt nach den Gebirgszügen und Stromgebieten, von ihrer Quelle bis zur. Mündung ausnitteln, um ein richtiges Bild von den Gesetzen, die die Natur bei der Anordnung der Vegetation befolgt, sich entwerfen zu können.

Es war keineswegs möglich, scharfe Grenzlinien zu ziehen zwischen 'den Aufsätzen, die als Beiträge' zu den Floren anzusehen sind, oder $z u$ dem Abschnitte mit der Aufschrift: Statistik des Gewächsreiches, gehören, 
ich glaubo deshalb den Leser darauf aufmerksam machen zu můssen, dafs beide Abschnitte zu vergleichen sind, indem sie wechselseitig sich ergünzen.

\section{A. Nord - Alpen - Länder.}

Hierher gehört vorzugsweise Deutschlund, daher die allgemeinen Schriften über die Vegetation unsers V Iaterland's zuvörderst cine Stelle finden mögren.

Deutschlands Flora in Abbildungen nach der Natur mit Beschrcilumen von J. Sturm u. s. w. 1. Abtheil. Ileft 54. Nürnberg 1828. 16 ill. Kupfer und ehen so viel 'Texthlitter in 12.

Ueber dieses neueste Heft der beliebten Schrift sehe man Bot. Zeit. 1830. I. p. 143 der Ijeilage. - I on der sueiten Ablheilung, Kryptogumen enthultend, sind jetzt 17 Hefte vorhanden. Die drille Abtheilung ist für die pilze bestimmt and wird von den Ilerren Rostkovius und Corda bearbeitet. Ueber das neueste (6te) Heft derselben sehe man Bot. Zeit. 1829. II. p. 127 der Ergünzungrsblütter.

Enumeratio plantarum Germaniae IIelvetiaeque indigenarum, sen P'rodromus, quem Synopsin plantarum Germaniae IIelvetiaeque adituri Botanophilisque adjuvandam commendantes scripserunt E. S te ud el, M. D., et Ch. F. Ilochstetter, Prof. Stuttgardtiae et Tubingae, sumptibus J. G. Cottae, MDCCCXXVI. 8. 2 fl. 45 kr.

Hierüber vergleiche man IIoppe in der bot. Zcit. 1827. II. p. 497.

Nachträge und Bemerkungen zur Enumeratio plantarum, auctore Steudel et Hochstetter; von Herrn Dolliner, Chirurgiae Magister in Wien.

Bot. Zeit. 1829. II. p. $5+1$.

Diese Nachträge gehören sämmtlich zum Donaugebiet, besonders sind es mehrere Pflanzen der Gegend um Wien.

Gemeinnütziges II andbuch der Gewächskunde, welches mit Ansnahue der 24. Klasse des Limneischen Systems, die wilden Gewächse Deutschlands enthält, und von den ausländischen diejenigen, welche dem Arzte und Apotheker, dem Färber, Gärtner und Landwirth Nutzen bringen, von Dr. Johann Christoph Mössler. Zweite Auflage, nach den neuesten $A$ nsichten dor Wissenschaft umgearbeitet, vermehrt und mit einer Anordnung der bekannteren in - und auslïndischen Pflanzengattungen nach den natïrlichen Verwandtschaften versehen, von Dr. II. G. Isudwigr Reichenbiech, Königrl. Sächs. Hojiathe. Altona, bei Joh. Friedr. Hammerich, 1827. 3 'Theile.

Anleitung die im mittleren und nördlichen Dentschland wachsenden Pflanzen auf eine leichte und sichere Weise durch eigne Untersuchung zu bestimmen. Von F. P. ('urie. Zweite sehr verumehrte Aufląge. Görlitz, bei C. G. Zobel, 18². 2 fl. $6 \mathrm{kr}$.

Ilierzu gehört cine Tabellarische Uebersicht der Flora des mittleren und nördlichen Deutschlands von J. F. Arendt. Osnabrück, bei Rachhorst, 1831. 1 Bogen Folio. $18 \mathrm{kr}$.

II andbueh der Botanik, oler systematische Beschreibung allev dentsehen P'lanzen, so wie anch derjenigen ansländischen, wclehe für den Arzt, Apotheker, Landwirth, Forstmann, Gïrtner, Schuluanu u. s. w. wichtig sind, von Friedrieh David Dietrieh, mekerer geJehrlen Giesellschaften Mitgliede. Eister Theil, crste Abtheilung. Jenie, bei August Schmid, 18\%8. 211. $15 \mathrm{kr}$.

J. C. R̈̈hling's Dentschlands Flora. Nach vinem verïnderten und erweiterten P'lane hearheitet von Franz Carl Mertens, Dr. und Prof., Vorsteher der Ilandelsschule in Biremen elc. und Wilhelm $\mathbf{D}$ aniel Jo- 
seph Koch, Dr. der Arsneiwissensch. etc. Erster Band in y Abtheilungen. Frankfurt am Main 1823. Zweiter Band 1826. 8.

Reicht bis zur siebenten Linneischen Klasse.

Compendium Florae Germaniae. Sect. I. Plantac phanerogamicae seu vasculosae. Scripserunt Math. Jos. Bluff et Carol. Ant. Fingerhuth. Tomus I. Norimbergae sumptibus J. L. Schrag 1825. Tomus . II. 1825.

Albert Guil. Roth, M. D. etc. Enumeratio Plantarum phaenogamarum in Germania sponte nascentium. Pars prima. Sectio prior (Classis I-V). Lipsiae sumptibus J. Gleditsch, MDCCCXXVII. Sectio posterior (Classis VI - XIII) MDCCCXXVII.

Manuale botanicum peregrinationibus botanicis accommodatum, sive Prodromus enumerationis plant. phaenogam. in Germania sponte nascentium ab Alberto Guilielmo Roth, Med. Doct. etc. Fasc. I. Classis I - VIII. Lipsiae 1830 in bibliopolio Hahniano. Fascic. II. Class. IX - XVI. Lipsiae 1830.

Flora Germanica excursoria ex affinitate regni vegetabilis naturali disposita, sive Principia Synopseos Plantarum in Germania terrisque in Europa media adjacentibus sponte nascentium cultarumque frequentius, Auctore Ludorico Reichenbach, Consil. aul. Reg. Saxon. etc. Insunt plantae Acroblastae et Phylloblastac. Lipsiae apud Carolum Cnobloch. 1830.

Beiträge zu Deutschlands Flora, gesammelt aus den Werken der ältesten deutschen Pflanzenforscher, von Dr. J. H. Di erbach, Prof. der Medicin zu Heidelberg. Erster Theil mit dem Bildnisse des Hieronymus Tragus Heidelberg 1825. Zweiter Theil mit dem Bildnisse des Leonhard Fuehs 1928. Dritter Theil mit dem Bildnisse des Carl Glusius 1830.

Deutsch lands kryptogamische Gewächse, nach ihren natürlichen Standorten geordnet, von $P$ h. M. O p i z. Ein Anliang zur Flora Deutschlands von Röhling. Prag 1817. 1 Rthl.

Bciträge zur Flora Deutschlands.

Bot. Zeit. 1830. II. p. 742.

\section{a. Gebiet der Donau mit ihren Nebenflüssen.}

Die Donau durchströmt ein sehr grofses Gebiet und überschreitet weit die politischen Grenzen Deutschlands, indem sie in das schwarze Hcer mündet. Ein Theil des Grofsherzogthums Baden und Würtembergs hat scine sbdachung gegen die Donau, so wie fust das ganze südliche Deutschland. Lüngst huben einige Geographen Zweifel erhoben, ob nicht richtiger gesagt werde, die Donau falle in den Inn, als umgekehrt, und wenn man die hohen Gebirge Tyrols vergleicht, aus denen der Inn herab kommt, mit den bei weitem niedrigeren des Schwarzwaldes, wo die Donau ihre Quelle hat, und dabei noch manche andere Umstände erwägt, so können jene Zweifel nicht als ganz grundlos angesehen werden.

Zur botanischen Kenntnifs des Danubial-Gebietes tragen aber nachstehende Schriften und einzelne Aufsätze bei:

\section{$A u g s b u r, g$.}

Joh. Bapt. Schwarz. Uebersicht der Augsburger Flora. Bot. Zeit. 1820. 'I. p. 34. 
Verzeichnifs der seltnern um Augsburg wild wachsenden Pflanzen, vom Herrn Dr. Dobler.

Daselbst 1822. I. p. 314.

A ugsburgische Blumenlese, oder systematisches Verzeichnil's der in der Gegend um Augsburg wild wachsenden Pflanzen, als Finleitung zu einer Flora von Augsburg, von Johann Wilhelm von Alten, I). der Philos., Magister der freien Künste, Apotheker zum goldenen Fingel in Augsburg u. s. $w$. Angsburg 1822, in der Wolfischen Buchhandlung. 214 S. in 8.

Man vergluiche über diese Schrift bot. Zeit. 1823. I. p. 49. der Beiluge. - Merküüdig sind die vielen Alpenpflanzen, welche unstreitig der Lech herabführt.

Schrank: neue Beitrïge zur Flora von Baiern.

Aus den Denkschriften der $k$. Akademie der Wissenschaften $\approx u$

München in der bot. Zeitung 1821. II. p. 599.

Flora der Gegend um München, von Dr. J. G. Zucearini, anfserordentl. Professor der Botanik an der Ludwig-Maximilians-Universität $u$. s. $w$. Erster Theil, Phanerogamen I. bis XI. Klasse. München 1829. 8. Jos. Lindauersche Buchhandlung.

Siehe Brandes Archiv Bd. XXX. Heft 1. p. 92.

Auch die Gegend um München besitzt mehrere Alpenpflanzen, offenbar durch die Isar herabgeführt, sie hat daher offenbar mit der Flor von Augsburg grofse Aehnlichkeit.

Botanische Bemerkungen auf einer Reise nach dem Wildbad lireuth; von Herrn Hofrath und Prof. Dr. Ko ch in Erlangen.

Bot. Zeit. 1830. I. p. $113 u$. d. f.

Auch Herr Prof. Zucearini schricb schätzenswerthe Nachrichten über die Vegetation des hinter Tegernsee hoch im Gebirge liegenden Bades Kreuth; ferner sehe man die Schrift des Dr. Krämer betitelt: Die Molken und Bade-Anstalt lireuth in Baierschen Hochgebirge bei Tegernsee. München 1829.

Tyrol. Inn-Gebiet.

Botanische Bemerkungen anf einer Reise durch Tyrol und Oberitalien gesammelt von L. C. Treviranus, Prof. zu Bresluu Jahrbücher der Gewä̈hskunde Ileft II. p. 1-42.

C. T. Hilsenberg: über die Flora von Tyrol. Bot. Zeit. 18:0. II. p. (j3̈0.

Hargasser's botanische Wanderung in Tyrol. Daselbst 1825. II. p. 435.

Fleis cher's botan. Reise in Tyrol. Daselbst 1826. I. p. 81 und 273.

Eschweiler botan. Reise in Tyrol. Das. p. 204.

Link. Wanderung in den 'Tyroler und Salzhurger Alpen. Das. 1827. I. p. 217.

Ueher die Vegetation der tyrolischen Gebirgsgegend um litzbühl, rom Herrn Dr. Sauter in Bregen\%.

Das. 1830. II. p. 457. 


\section{$-157$}

Botanische Notizen über cine im Juli 1823 nach Tyrol und Oberitalien gemachte Reise, vom Herrn Dr. Zuccarini.

Das. 1824. I. p. 257.

W and erung nach dem Wormser Joch, vom Herrn Apotheker Funk in Gefrees.

Das. 1828. II. p. 488.

$$
S a l=b . u r g .
$$

Verzeichnifs einiger merkwürdigen Pflanzen, welche bei Elixhausen wild wachsen. Vom Herrn Vicarius Michl.

Bot. Zeit. 1820. I. p. 312 .

Vegetation um Salzburg.

Das. 1822. I. p. 343 .

Botanis che Al penreis e durch Salzburg und einen Theil von Kärnthen und 'Tyrol im Juni und Juli 1822, von Herrn G. W. Bisch off.

Das. 1823. I. p. 209.

Sa ter, Reise von Wien nach Salzburg.

Das. 1825. II. p. 699.

C. Gruner, botan. Reise in den deutschen Alpen.

Das. 1822. II. p. 699.

Botan ische Topographie des Goldberges im Thale Rauris. Von dem k. k. Secretär an der landärztl. Schule zu Salzburg, Herr F. A. v. Braune.

Das. 1819. I. p. 305.

v. Sauter, Pflanzen auf dem Watzmam.

Das. 1824. I. p. 141 .

Botanisches 'T'aschenbuch, oder eine Flora von Salzburg und Berchtesgaden. Salzburg 1818. 2 Bde. 2 fl. 24 kr.

Siehe auch über eine ähnliche Schrift desselben Verfassers botan.

Zeit. 1821. II. p. 681 .

Kärnthen, Steiermark, Krain." (Gebiet der Drave und Save).

Hoppe: Vegetation um Heiligenblut im obern Mälthale, Oberkärnthens.

Bot. Zeit. 1819. I. p. 227. und 273.

Bartenstein, Excursion auf die Pasterze.

Das. 1823. II. - p. 477.

Vegetation der Villacher Alpen in Kärnthen; vom Herrn Apotheker Hauser in Villach.

Das. 1829: II. p. 561.

Vegetation der Kuhwegcr-Alpe im Gailthale in Kärnthen; rom Herrn Apotheker Haus er in Villach.

Das. 1830. I. p. 161.

Bericht ühor einige botanische Excursionen durch Oestreich, Steiermark und Salzburg, vom Herrn Dr. v. Sa uter.

Das. 1824. I. p. 209.

J. Zahlbruckner, botan. Excursion in Steiermark.

Das. 1823. I. P. 220.

Bruchstücke einer botanischen Reise in Krain, von Dolliner.

Das. 1827. I. p. 248. 
Berieht über eine botan. Reise, unternommen im Sommer 1825, vom Baron v. Welden. Botan. Zeit. 1826. II. p. 497.

Verzeiehnifs der ron dem Jahre 1801-1819 auf meinen lontaniselien Reisen dureh und in Steiermark selbst beobit:hteten, gésanumelten und bis auf wenige bereits in meinen Centurien getrocknet gelieferten P'llanzen ete., von J. N. Gebhard. Grätz 18\%̈. 12.

\section{Mähren. Gebiet der Morava oder. March.}

Tebersieht des Merkwürdigsten aus Mährens Flora, vor. G. F. Inochisteter.

Botan. Zcit. 1825. II. p. 513, 529.

\section{Wien und Floren Oestreichs überhaupt.}

v. Sternberg. Die Frühlingspflanzen um Wien.

Bot. Zeit. 18ะ8. I. p. 114. Ergänzungsbl.

I. Trattinnik. Flora des österreichischen Kaiscrthums. Wien 1815. 4. 24 IIefte.

Nicolai Thomac Host, Caes. Reg. Archiatri. Flora Austriaca. Volumen I. Viennac, sumptihus Car. Ferd. Beck, MDCCCXXVII. 8.

Reicht bis zur Dodecandria inclusive. Host rechnet $\approx u$ Flor. austriaca auch die italischen Besitzungen Oestreichs, die zu einer ganz andern Abtheilung der Vegetation gehören.

Gemeinfafsliche Anleitung, die Bämme und Sträncher Oestreichs ans den Blïttern zu erkennen. Zum Sellsstuntericht entworfen von Franz Hoss, öfentl. ordentl. Prof. der Forst-Naturliunde. Mit 10 Kupfertitfeln. Wien 1830. $3 \mathrm{fl} .50 \mathrm{kr}$.

\section{Gebiet der untern Donau.}

Gottl. Baumgarten. Enumeratio stirpium magno Transilvanae principatim pracprimis indigenarum, in usum nostratum botanophilorum conseripta, inque ordinem sexuali naturalem concinnata. Vindobon. 1816. 3 Vol. 8.

Jos. Sadler, Verzcichnifs der um Pesth und Ofen wild wachsenden phanerogam. Gewächse mit Angabe ihrer Standorte und Blïhezeit. Pesth 1818.

Idem. Dissertat. inaugural - med. sistens descriptionem plantarum epiphyllospermarum Inngariae et provinciarum adnexarum atque Transylvaniae indigenarum. Pestini 8. (Sine anno).

Finra Comitatus Pestiensis, anctore Jos. Salleretc. Pars I, MTonandria-Decandria. Pestini 1825. 335 P. Pars II, Dodecandria-Polygamia. Pestini 1826. 398 P. 8.

Siehe, Linnaea 18\%8. p. 39 der Literatur.

Plantac Banatus rariores, iconibus et descriptionibus illustratae; praemisso tractatu phytographico et subnexis additumentis in terminologiam botanioum; Anctore Antonio Rochel, Chirurgiae et artis olsstetriciae nee non culturae horti botanici regii Scient. univ. Hungar. Magistro ete. Accedunt tabulae botanicae $\mathrm{XI}_{1}$ et mappae duae tithograptac. Pestini typis Ludovici Landerer de Füskiit MDCCCXXVIII. Folio. 
 \\ b. Gebiet des Rheins und seiner Nebenflïsse.}

Die Flora des Rheinthals und seiner zahlreichen Nebenzweige ist Deutschlands vorzüglichste Zierde und ohne Zweifel eine der reichsten in Europa. $\boldsymbol{I}$ on seiner Quelle auf dem Gotthard an bis zu seiner Mündung in die Nordsee durchlëuft der schöne Strom ein fruchtbares und gesegnetes Land, dessen Vegetation schon vielfültig untersucht worden ist, daher vielleicht eine Chloris rhenana vor allen andern bearbeitet werden könnte, um als Muster zu dienen, wie cigentlich Floren dargestellt werden müssen, um durch sie die Gesetze auszumitteln, dic die Natur bei der Vertheilung der Iegetation befolgt. Welches Interesse muifste es haben, wenn wir $\approx$. B. die Rhein - Flora genau mit der der Donau zu vergleichen im Stande wären, wie sie auf der einen Seite von Süden nach Norden, von der andern in entgegengesetzter Richtung laufend, sich verhält und verändert! Möchte diese Bearbeitungs - Art nicht lange ein frommer Wunsch bleiben!

Gebiet des Ober-Rheins, dengrössten Theil der Se hweiz $u m f$ ass end.

J. R. Suter. Helretiens Flora. Vermehrt herausgegeben ron Joh. Hegetschweiler. 2 Theile, enthaltend die phanerogamischen Gewächse. Zürich 1822. 3 Rthl.

C. F. Zollikofer. Tentamen florac alpinae Helvetiae, iconibus lapide incisis et descriptionibus illustratae. St. Gall. 4. 1826. Auch unter dem Titel: Versuch einer Alpenfora der Srhweiz in Abbildungen auf Stein nach der Natur gezeichnet und beschrieben. Jedes Heft mit 10 Bl. gr. 4. Schwarz $1 \mathrm{f}$. $30 \mathrm{kr}$. illum. $3 \mathrm{fl}$. $30 \mathrm{kr}$.

Flora Helvetica sive historia stirpium hucusque comnitarum in Helvetia et in tractibus conterminis aut sponte nascentium aut in hominum animaliunque usus vulgo cultarum continuata. Auctore J. Gaudin V. D. M. Ecclesiae hevidunensis Pastore etc. Vol. I. cum IV. tab aeneis. Turici sumptibus Orellii, Fuesselini et sociorum MDCCCXXVIII. Vol.II. cum XV tab. aeneis 1828. Vol. III. 1828. Vol. IV. eum quinque tab. aeneis 1829. Vol, V. cum tab. aenea 1829. Vol. VI. cum tribus tab. acneis. 1830.

Diese sechs Bände enthalten blos die Phanerogamie, übrigens darf kaum erinnert werden, dafs einige Districte der Schweiz zu den West-andere zu den Süd-Alpenländern gehören.

Sammlung ron Schweizer-Pflanzen, nach der Natux und auf Stein gezeichnet von J. D. Labram. Text von Dr. Joh. Hegets chweiler. Zürich, bei Esslinger Praeceptor. 36 Hefte 8.

Siehe bot. Literaturbl. IV. p. 23.

J. G. Krauer Prodromus Florae Lucernensis, seu stirpium phanacgamarum in agro Lucernensi et proximis ejus confiniis sponte nascentium catalogus. Lucernae 1824. 12.

Berichte über cine Reise nach Mailand und zurück nach Carlsruhe, von F. Mayer

Botan. Zeit. 1823. I. p. 49.

Enthält interessante Beitrïge zur nëheren Kenninifs der Fegetation in der Nähe des Ursprungs iles Rheins.

In der newen Alpina Bd. I. p. 72 sind die Pflanzen des Rheinthales über Constanz anfgezcichnet von Dr. Cu s tor.

Reise durch einen Theil der Schweizer Gebirge, vom Herrn Apotheker Carl Stein in. St. Gallen.

Botan. Zeit. 1826. 1. p. 310 u. d. f. 
Ich darf diesen Reiscbericht den Totaniliern zur Berüclsichtigung auf dus Lestimmteste empfehlen, da der Herr Ferf. mir viele Alpenpflanzen aus der Giegend von St. Gallen zu übersenden die Güte hatte, die eben so schön erhalten, als richtig bestimmt waren.

Zur Kenntnifs der Flora der Kantone Schaffhausen und Thurgau, so wie eines Theils des angrenzenden Alt-Schwabens, ven C. E. Dieffen ba eh. Pronisor der Apotheke zu Nidun im Kinton Lern.

Bot. Zeit.. 1826. II. p. 4(i5 u. d. f.

Nachrieht von einer naturhistorischen Reise durch die Schweiz und durch Ober-Italien. Yom IIerrn Prof. I). J. B. Wilbrand in Gielsen.

Das. 1529. I. Beiluge p. 1 u. d. f. - Höchst interessant und belehrend!

Botanisehe Bemerkungen vom Herrn Dr. Trachsel in Rüggisberg bei Bern.

Bot. Zeit. 1827. II. p. 481 und 1828. p. 145.

Ergebnifs zweier botanischer Excursionen in die Alpen des schweizerschen Cantons Freyburg, im Sommer 1827, mit einigen beigefügten Bemerkungen mitgetleilt vom Ifern Dr. Trachsel in Rüggisberg bei Bern.

Das. 1828. II. p. 476.

Finige Bemerkungen zu Steudel's und Hochstetter's Enumeratio Plantarum - seu Prodromus ctc. von Demselben.

Das. 1829. 1. p. 17 . . d. $f$.

A uszug aus dem Bericht über eine Besteigung des olnweit Bern sich erhebenden Stockhorn, im Juli 1827, vom fIerrn M. J. Lühr, Candidaten der Pharmacie zu Coblenz.

Das. 1830 I. p. 53. - Auch vergleiche man über die Pflanzen der Stockhornkette die Annalen der Schweizer Giesellschaft für Naturkunde. Bd. II. Heft 1. p. 72.

Vegetation um Basel, von Frnst Grolh in Dresden. Bot. Zeit.' 1819. I.' p. 265.

Tentamen Florae Basileensis, exhibens plantas phanerogamas sponte nascentes secundum systema sexuale digestas, adjectis Caspari Bauhini Synonymis ope horti cjus sicci comprobatis. Cum effigie Casp. Bauhini et duabus iconibus coloratis Auctore C. F. Hagenbach, Med. Doct. Vol. I. Basileae typis J. G. Neukirch 1821. 8.

Reicht bis zur Dodecandria inclusive. - Warum wird uns die Fortsetzung dieses vorzüglichen W'erkes so lange vorenthalten?

Flora des Rotthals in der Schweiz, nach II ugi. Bot. Literaturbl. IV. p. 260.

\section{Gebiet des Mittel-Rheins.}

Flora Badensis alsatica et confinium regionum eis et transrhenana, plantas phanerogamas a lacu bodamico usque ad confluentem Mosellate et Rheni sponte nascentes exhibens secundum systema sexuale cum iconihus ad naturam delineatis, auctore Carolo Christiano Gmelin, Med. D. ete. 'Tom. IV. Supplemento cum Indicibus. Carlsruhae in officina aul. Mülleriana. 1826.

Bei- und Nachträge zur Flora Badensis et confinium regionum. Vom Regimentsarzt Dr. Griesselich in Karlsruhe. 
Geiger's Magazin für Pharmacie Sept. 1828. p. $157 u . d . f$. nebst Polemik hierüber März 1829. p. 1 u. d. f. Aprilheft p. 81. Neue Beiträge von Griesselich. Februarheft 1830. p. $106 u$. d. f.

In der Gegend ron Immendingen im Bad. Seckreise wachsen nach Angabe des Pfarrers A m tsbühler: Lonicera alpigena, Aconitum Napellus, A. Lycoetonum, Anemone narcissiflora, Dentaria pentaphylla, Cineraria alpina, Satyrium Epipogium, Ophrys Corallorhiza, Cypripedium Calceolus etc. p. 177.

I'erhandl. des G. Bad. landwirthschaftl. Vereins 5. Jahrg. I828.

Flora Friburgensis et regionum proxime adjacentium, auctoro F. C. L. Spenner, societatis naturae curiosorum Friburgensis sodali. Tomus I. cum tabulis duabus aeneis. Friburgi Brisgoviae typis Friderici Wagner 1825. Tomus II. 1826. Tom. III. cum tabula lithograpta 1829. 8.

Rastadis Flora ron Jos. C. Frank. Heidelberg in Commission bei C. F. Winter 1830. 8 .

Zentner, das Renchthal und seine Bäder. Freiburg $182 \%$.

Die Iegetation der Gegend ist in dieser Schrift von Spenner angegeben, die meisten Beiträge rühren aber eigentlich von dem Apotheker Hartmann in Kork.

W. A. Rehmann, Rippoldsau und seine Heilquellen, in historischtopographischer, natur- und heilkundiger Beziehung. Freiburg 1830. 8. Mit 2 Abbildungen.

Description historique et topographique des deux departemens du Rhin, par J. F. A ufschlager. Strasbourg 1828. 8.

Enthält in dem Supplemente eine vom Herrn Kirschleger abgefafste Liste seltnerer Pflanzen des Elsafses und der Vogesen.

Herr Doctor Petif in Muschbach bearbeitete eine Synopsis plantarum Palatinatus, nach dem natürlichen Srsteme geordnet, mit specicller Angabe der Standorte; ich sah die sehr schätzbare Schrift im Manuscripte, das mir der Herr Verf. zur Durchsicht zuschickte; es soll seitdem gedruckt worden sein, ich habe aber noch kein Exemplar davon gesehen und kann daher den Titel nicht angeben.

Neckargebiet.

Flora Mannhemiensis et vicinarum regionum cis et transrhenanarum, auctore F. G. L. Succow, Med. Doct. etc. Pars I. continens plantarum phaenogamarum Class. I - XIII. cum charta topographica. Mannhemii 1812. Pars II. Class. XIV - XXII. 1822.

Systematis che Uebersicht der um Heidelberg wild wachsenden, und häufig zum ökonomischen Gebrauche cultivirten Gewächse. Von J. H. Di erbach. Erstes Heft. Karlsruhe 1827. 8.

Flora von Stuttgardt. Herausgegeben rom Prof. Zenneck in Hohenheim. Sichtbar blühende Pflanzen. Stuttgardt bei Zuckschwerdt 1822.' 4. Mit einer 'Tafel in Steindruck. $55 \mathrm{~S}$.

Die ä chten und unächten Farren Stuttgardts, als Bcitrag zur cryptogamischen Flora dieser Gegend.

Bot. Zeit. 1825. I. p. 254 .

Systematisches Verzeichnifs der bei Tübingen und in den umliegenden Gegenden wild wachsenden phanerogamischen Gewächse, mit Angabe ihrer Standorte und Blüthezeit, vom Prof. Schübler. 
Beilage zu und abgedruckt aus Dr. Fisenbach's Geschichte und Beschreibung der Stadt und Universität Tübingen. 1822. 60 S. klein 8.

Siche bot. Zeit. 1823. I. p. 198.

Ueber Würtembergs Flora.

Correspondenzblatt des würtombergischen landwirthschaftlichen Fercins. Siebenter Band. Stuttg. und Tübingen 1825. p. 333 u.d.f.

Veber die würtembergische $A l p$, ron Georg von Martens.

Hertha Band FI. Ileft 1. erste Abtheilung p. 5!) u. d.f. - Linthält auch die Angabe der merkwürdigsten Pflanzen jener Gegend, die, wie bereits früher erinnert, zum Theil in das Donaugebiet gehört.

Beiträge zu Würtembergs Flora.

Buchhalter $\boldsymbol{R}$ ösler zu Christophsthal lieferte Flechten; Köstlin, Oberamtsarzt in Sulz, Moose; Merl. Stud. 13. Feit z aus Gimünd, Schwämme; Apotheker $L a n g$ in Heilbronn, officinelle Pftanzen.

Correspondenzblatt des Würtemb. landwirthschaftl. Fereins $B d$. XIII. p. 301.

Das Sirona-Bad bei Nierstein und seine Mineralquellen. Mit lithographirter Abbildung. Mainz 1827. 79 S. 8.

Im vierten Abschnitte befindet sich eine Aufzählung der seltnern Gewrichse um Nierstein mit mancher interessanten Bemerkung, vom Medicinalrathe $\mathbf{Z} i \approx$ in Mainz.

$$
\text { Main-Gebiet. }
$$

Flora der Gegend um Frankfurt am Main, ron Johannea Becker u. s. w. Erste Altheilung, Phanerogamie. Frankf. a. M. 1828, bei Ludwig Reinherz. 558 S. in 8. Zweite Abtheilung, Cryptogamic. 112 S. 8. 1828.

Becker und Fresenius: Anzeige ciniger um Frankfurt gefundenen Pflanzen.

Bot. Zeit. 1830. II. p. 430.

Supplementum Florae Wirceburgensis, continens plantarum genera quaedam atque species in magno ducatu Wirceburgensi recenter detecta, nec non observationes nonnullas cirea plantas indigenas earumque loca natalia. Quod patriac Florae curatoribus affert D. F. X. Heller, Botan. in univ. reg. Wircelo. Prof. etc. Wirceburgi apud Josephum Stahel 1815. 8.

Hepp, D. Ph. Lichenen - Flora von Würzburg oder Aufzählung und Beschreibung der um Würzburg wachsenden Flechten. Mit Abbildungen. Mainz 1824. 8.

Verzeichnifs der Wasser - Algen, welche sich in der Gegend um Würzhurg vorfinden; vom Ilerm Dr. Leiblein, Prosector bei der zootomischen Anstalt der Universität in Würzburg. Bot. Zeit. 1827. I. p. 257 u. d. f.

Flora cryptogamica Frlangensis, sistens vegetabilia e classe ultima Linn. in agro Erlangensi hucusque detecta, auctore C. F. P. Martio, Med. et Chir. Dr. ete. Norimbergae Sumpt. J. L. Schrag 1817. cum tah. duab. aeneis muscos nonnullos et quatuor lapidi incisis Jungermannias germanicas foliosas illustrantes. 
Excursion in die Gegend von Muggendorf, vom Herrn Prof. Nees v. Esenbeck und seinen Zuhörern aus Erlangen; erzählt vom Herrn Provisor Ra ab. Bot. Zeit. 1819. I. p. 289.

Ad Floram Lrlangensem auct. Schweigger et Koerte adnotationes nonnullae. Scripsit Dr. G. G. Zuccarini. Das. 1821. II. p. 605.

Koch, über einige bei Erlangen gefundene Pflanzen. Das, 1825. I. p. 190.

J. S. Winterschmid: Nürnbergische Flora oder 1. Abtheil. der bayrischen Flora. Nürnberg 1818. 8. 6 Hefte, jedes mit 8 Kupfern nebst Text.

Frankens und Thüringens Flora in naturgetreuen Abbildungen von F. P. Ekart. Erstes Heft, mit einem Theile der Klee-Arten. Bamberg und Aschaffenburg bei J. C. Dresch. 1828. 4. $3 \mathrm{fl}$.

Siehe Geiger's Magaz. Sept. 1828. p. 231.

$$
\text { Gebiet der } L a h n \text {. }
$$

Beiträge zu der Flora von Hessen, rom Herrn Prof. Wenderoth.

Schriften der Gesellschaft zur Beförderung der gesammten Naturuissenschaften zu Marburg 1823. p. 118 - 152. - Bot. Zeit. 1823. II. p. 716.

Botanische Reise in den Gebirgen Hessens. Von Demselben. Daselbst 1826. I. p. 252.

Dafs ein Theil der hessischen Lande in das Weser-Gebiet gehört, braucht wohl kaum erinnert zu werden.

S. L. Hergt. Versuch einer Flora ron Hadamar, mit einer Anleitung zur Pflanzenkenntnifs für Schulen. Hadamar 1822. 8.

$$
\text { Ge biet der Mosel. }
$$

Trierische Flora von M. Schäfer, Lehrer der Mathematik und Naturgeschichte. Zwei Theile. Trier 1826. bei J. J. Linz.

II. Schäfer, 'Trierische Flora, oder kurze Beschreibung der im Regierungsbezirke Trier wild wachsenden Pflanzen. 3. Theil. 23. Klasse. $30 \frac{1}{4}$ Bogen 8. Trier 1830. 1 Rthl. 12 gr.

Bemerkungen über Herrn Schäfers Trierische Flora; von Herrn Fr. Wilh. Schultz in Zweibrücken.

Bot. Zeit. 1827. II. p. 65\%.

Nachtrag zu den Bemerkungen über Schäfers Trierische Flora, vion Demselben.

Das. 1828. II. p. 587.

C. A. Fingerhuth. Tentamen florulae Lichenum Eiffliaeae, sive Enumeratio Lichenum in Eifflia provenientium. Norimb. 1828. 8. $54 \mathrm{kr}$.

Verzeichnifs der Laubmoose, welche in der Nähe ron Zweibrïcken und einigen benachbarten Gegenden vorkommen; vom Herrn Apotheker Bruch in Zweibrücken.

Bot. Zeit. 1824. II. p. 760.

Observations sur quelques plantes de France, suivies du Catalogue des plantes rasculaires des environs de Nancy, par H. F. Soy erWillemet. Nancy 1828. Bontaux et Grimblat. 2 Fr. 50 C. 
Flore de la Moselle, on Manuel d'herhorisation dans le departement de la Moselie, contenant la description des plantes qui y croissent avec l'indication de l'époque ou elles fleurissent, des lieux ou elles se trouvent, et des usages yn'en font les arts ct l'économie domestique. Par J. II ol andre. Metz 18:9. chez la veuve Thiel, Paris chez Treuttel et Würtz. 1 Vol. 18. 4 francs.

\section{Grebiet des Unter-Rheins.}

Beiträge zur Charakteristik der Flera von Bonn und seiner Ungegend, mitgetheilt rom Herrn Dr. Friedr. Nees von Ésenbech.

Bot. Zeit. 1822. I. p. 17\%.

Verzeiehnifs der seltueren Pflanzen der Gegrend ron Bonn. Von Demselben.

Das. p. 231.

Oligsehläger, Arzncipflanzen im nstrheinisehen Theile des Regierungsbezirkes Düsseldorf.

Brandes Archiv Bd. XXVI. Heft 3. p. 327.

Beschreibung einer Reise in das Bisthum Paderborn im August 1819. Von Herrn Dr. A ugust Weihe in Mennighöffen.

Bot. Zeit. 1820. I. p. 246.

Excursionen in der Umgegend von Padcrborn; von Herrn Dr. Weihe in Herford.

Das. 1825. II. p. 753.

Physikalisch - chemische Untersuchung der Mineralquelle zu Ründeroth, von A. Marder. Köln bei Bachem 1827. $27 \mathrm{kr}$.

Fnthïlt im dritten Kupitel eine Flora von Ründeroth, jedloch uur die in der nüchsten L'mgebung der Ǫuelle vorkommenden PJlanzen.

Versuch einer Flora der Ufer des Nieder-Rheins, der Roer, Maas, Ourte und der angrenzenden Gegenden. Nach Anleitung ron $\mathbf{J}$. W. Meigens und L. Weniger systematischen Verzeichnissen. Cälln 1823. ifl. $48 \mathrm{kr}$.

Revue de la Flore des environs de Spaa; contenant l'éunmeration de toutes les plantes y décrites, arec les observations, les descriptions, les additions et les corrections nécessaires pour la mettre le plus possible à la hauteur de la science; par A. L. L. Lejeune, Dr. en Medecine etc. liège 18"4. chez Juvivier. 26i3 S. 8.

Siehe bot. Literaturbl. I. p. 369.

Compendium Florae Belgicae; Anetore A. L. S. Lejeune et R. Courtois. Liège 1828. 1 Vol. 12. 26it S. $2 \mathrm{fr}$.

Geht bis zur Polyandria Pentagynia.

Flora Belgii septentrionalis sive index plantarum indigenarum, quae hucusque in septem provinciis focderatis repertie sunt, auctore II. C. van Hall. 1 Vol. 1 et 2 part. Amsterdam 1825. 8. plantas phanerogamas continens.

Herr van II all lieferte auch mehreve Nachlesen $\approx u$ seiner Flor des nördlichen Belgiens, die in den Bydragen tot de Nutuurlundige Wetenschappen enthalten sind. Man sehe Linnaca 1828. I. p. 31 und 182!. I. p. 32 der Literatur, und Isis 18\%9. Heft 9. p. 922.

A. L. S. Lejeune. Choix des plantes de la Belgique. Liège 1826. 
Catalogue des plantes omises dans la Botanographie Belgique et dans les Flores du nord de la Francé etc.; par J. B. H. J. Desmazière в. Lille 1823.

Botanographie Belgique on Flore du nord de la France et de la Belgique proprement dite; ouvrage disposé selon la methode naturelle, contenant les tableaux analytiques de Fr. Jos. Is estiboudois, publiée par Themistocle Lestiboudois, D. M. Professeur de Botanique à Lille etc. 2 Vol. in 8 . de 854 pages arec 22 tableaux. Prix 16 francs à Paris chez Roret, libraire rue Hautefeuille, à Lille chez Vanackere, père et fils.

Es ist nicht unpassend hier zu erinnern, dafs ein Theil von Belgien in das Schelde - Gebiet gehört, das sich in seiner Vegetation von der des. Rheines allerdings unterscheidet.

Bemerkungen über den Boden und die Gewächse der Provinz Luxemburg, mitgetheilt yon Herrn Tirant junior in Luxemburg.

Bydragen tot de Natuurkundige W etenschappen p. 61.

Abhandlungen über einige cryptogamische Pflanzen des Grofsherzogthums Luxemburg, von Marchand.

Ibid. Tom. I. Nro. 4. 1826. p. 406. - Siehe bot. Literaturbl. II. p. 69. - Linnaea 18\%8. Heft 1. p. 36 der Literatur. Ferner Linnaea V. p. 183. Lit.

Initia florae Groningianae etc. Groningae 1825. fi6 S. S.

Sie werden von der naturforschenden Gesellschaft in Gröningen herausgegeben.

Beiträge zur Kenntnifs der Floren an der Maas zwischen Dinant und Haslie, von J. J. de Cloet.

Siehe Linnaea $V . p .182$. Lit.

\section{c. Gebiet der Weser und Ems.}

Ion weit niedrigeren Gebirgen als der Rhein, nimmt die Weser ihren Ursprung, sie durchläuft eine weit kürzere Strecke, und die Vegetation ilhres Gebietes ist daher auch bei weitem nicht so mannichfaltig und reich, wenn gleich dem Thüringer Walde, dem Harze, dem Rhürgebirge, dem V'ogelsberge und Teutoburger Walde u. s. w., die der $\mathbf{W}$ eser und ihren $\mathrm{Ne}_{c}$ benflüssen, so wie dem Küstenflusse Ems, ihre Wasser liefern, eine interessante und schöne Flor nicht abgesprochen werden kann. Fs scheint übrigens, als ob das We esergebiet, besonders einige Strecken desselben, noch nicht so sorgfältig untersucht wären, als sie es zu sein verdienen. Das Neneste, uas wir aus dieser Gegrend Deutschlands in botanischer Hinsicht erfuhren, dürfte vorzugsweise in nachstehenden Schriften $\approx u$ suchen sein:

Die Mineralquellen zu Liebenstein; ein historiseher, topographischer und heilkundiger Versuch, von Dr. H. G. Schlegel, Brunnenarst $\approx u$ Liebenstein u. $s$. w. Meiningen 182\%. 8.

Siehe Bot. Zeit. 1828. I. p. 109 der Beilagen.

Flora Brunsvicensis, oder Aufzählung und Beschreibung der in der Ümgegend ron Braunschweig wild wachsenden Pflanzen,

$$
\text { od er } \mathbf{r}
$$

Flora der Ungegend von Braunschweig, bearbeitet von Dr. H. W. L. Lach mann jun. 1. 'Th.: Chorographie, Geognosie, Meteorologic. Allgemeine Vegetation. Nebst einer geognostisch - bocanischen colorirten Kiarte, einer lithographirten Tafel und 4 gedruckten Tabellen. Braunschweig bei G. C. E. Meyer 182\%. 8. $3 \mathrm{fl}$. $36 \mathrm{kr}$. - Zweitcr Theil, Phanerogamen. 1. Abtheilung: Conspectus generum, Specierun. Class. I-XI. Braunschweig 1828. 496 s. 8 . $4 \mathrm{fl}$. $30 \mathrm{kr}$. 
Neuo physikalisch-chemische Beschreibung der Mineralquellen zu Pyrmont, nebst naturgeschichtlicher Darstellung ihrer Umgebungen, von Dr. R. Brandes, Fürstl. Wuldeck. Hufruth und Apotheker au Salsuften u. s. w. und F. K' rüger, Fürstl. Waldeck. Medicinaliathe und Hofapotheker zu Pyrmont u. s. w. Pyrmont 1826. 8.

Die zuvite Abtheilung dieser Schrift enthült ein systematisches rerzeichnifs der in der ímgerend von Pyrmont wachsenden phanerogumischen Pflanzen, deren Zuhl sich gegren 700 beläuft. Als _Anhung ist noch eine Aufzählung mehrever dort einheimischen Crypto-

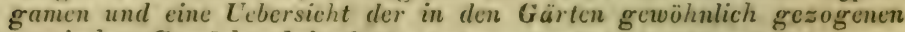
exotischen Gewächse beigefügt.

Index systematicus plantarum florae Ravensbergicae pracsertim circum Bielefeldiam sponte nascentium, collectarum ab Dr. Ernesto Friderieo. Aselioff.

Brandes Archiv Bd. XXVI. Heft 3. p. 313.

G. F. W. Meyer. Erste Anlage zur Flora des Königreichs Iannorer, oder Beiträge zur chorographischen Kenntnifs des Flufsgebietes der Innerste in den Fürstenthümern Grubenhagren und Hildesheim, mit besonderer Rücksicht auf die Veränderungen, die durch diesen Strom in der Beschaffenheit des Bodens und in der Vegetation lewirkt worden sind. Göttingen 1820. 8. 2 Theile, mit einer illum, und einer schwarzen Tafel in Steindruck.

Möchten uir doch auch bald die lëngst versprochene Flora von IIannover selbst, wozu ganz vorzüglich schöne Abbildungen bereits vorhanden sein sollen, erhalten!

Prodromus Florae Monasteriensis. Westphalorum. Auctore C. M. F. a Boenninghausen, J. U. D. etc. Phanerogamia. Monasterii sumptibus Frid. Regensberg 1824. 332 S. 8.

\section{d. Gebiet der Elbe.}

Aus zwölf Quellen des Riesengebirges entspringt dieser schöne Strom, der auf seinem Laufe in die Nordsee eine grofse Zahl grïfserer und kleinerer Flüsse aufnimmt, die schönsten oft mit subulpiner Flor geschmückten Thüler bildet, und ohne Zuvifel nach dem Rhein und der Donau der vorzïglichste deutsche Strom genannt werden kann, dessen Iegetation auch sehr genau untersucht und beschrieben wurde. Man kann das Elb-Giebiet gleich dem des Rheines eintheilen:

\section{oberes Elbe-Gebiet. Böhmen.}

M. P. Opitz. Böhcims phanerogamische und cryptogamische Gewächse, nebst Angabe ihrer gebränchlichsten Provinzial-Namen, ihres Vorkommens, ihrer Verbreitung, Anführung der Fundorte der seltneren nebst ihren Findern und Andeutung des landesiublichen Gebrauchs, ihres Nutzens und ihrer Schädliehkeit. Prag 1823. 1 fl. $12 \mathrm{kr}$.

Kostelezli Clavis analitica in floram Bohemiae. Pragae 1824.

Opiz. Nachtrïge zn seinen Gewächsen Böheims.

Botan. Zeit. 1829. II. p. 114. Beilage.

Es sind deren mehrere in der botanischen Zcitung mitgetheilt worden, wovon die angeführten die ncuesten sind, z. B. Jahrgang 1822. I. p. 266, die aber eigentlich die Flora cechica des Horrn Dr. Presl angehen.

Ueber die zweifelhaften Planzen der Flora Bohemica Schmidt's von Herrn Prof. Tansch.

Bot. Zeit. 1528. II. p. 417. 
Lichenum in Bohemia observatorum dispositio succinctaque de6criptio. Auctore Wenzeslao Mann. Dissertat. inauguralis. Pragae 1825.

Beobachtung einiger neuen Pflanzen um Prag. Vom Herrn Prof. Tausch. Bot. Zeit. 1819. II. p. 461.

Mittleres Elbe-Gebiet.

Sachsen, Theile von Preufsen, Weimaru. s. w.

Verzeichnifs der merkwürdigsten Phanerogamen von Sachsen. Botan. Zeit. 1819. II. p. 483 .

H. Ficinus Botanisches Taschenbuch oder Flora der Gegend um Dresden. 'Theil I. Dresden 1821. 'Theil II. 1823.

E. Schmalz Dispositio synoptica generum plantarum circa Dresdam et sponte crescentium et in agris frequentius cultarum, adjectis familiis naturalibus. Dresdae 1822.

Groh, Pflanzen im Plauenschen Grunde, der Loschwitzer Gegend, um Pirna und andern Orten; um Dresden.

Bot. Zeit. 1820. I. p. 123.

Beiträge zur Flora Dresdensis, von Herrn Prof. Reichenbach. Daselbst 1822. I. P. 289. 305.

Zweiter Beitrag zur Flora ron Dresden. Von Demselben. Das. 1822. II. p. 529.

Kryptogamische Beiträge zur Flora der Gegend um Dresden. von M. Eduard Schmalz.

Das. 1823. II. p. 566.

A. G. L. Reichenbach. Flora Lipsiensis pharmaceutica sistens plantarum agri Lipsiensis nunc et olim officinalium venenatarumque diagnoses, descriptiones, synonyma, locos natales, qualitates, vires et usum. Lipsiae 1817. 8.

C. G. L. Pappe. Synopsis plantarum phaenagamarum agri Lipsiensis indigenarum. Lipsiae 1828. 8. Voss. $12 \mathrm{gr}$.

Flora der phanerogamischen Gewächse der Umgegend von Leipzig, von Gustav Theodor Klett und Herrmann Eberhard Fricdrich Richter, Baccal. der Medicin. Mit einem Worte von H. L. Reichenbach. Leipzig 1830. $4 \mathrm{fl}$. $48 \mathrm{kr}$.

Beitrag zur Kenntnifs der Flora Weida's im Grofsherzoglich Sãchsischen Neustädter Kreise, von Herrn Prof. Rofs mässler in Weida. Bot. Zeit. 1830. I. p. 225.

Winke für Botaniker, welche auf ihren Forschungsreisen die Schwarzburg - Rudolstädtischen Lande vorderer Herrschaft berühren; von Herrn Pfarrer S chönheit in Feichröda.

Das. 1824. II. p. 449.

F. W. Wallroth Schedulae criticae de plantis Florae Halens. select. Corollar: novum ad C. Sprengelii Floram Halensem etc. Halae 1822. 8.

J. C. F. Graumüller. Flora Jenensis oder Beschreibung der in der Nähe von Jena und einem grofsen Theiie des Grofsherzogthums Sachsen-Weimar, Eisenach und des Herzogthums Altenburg wild wachsenden Pflanzen, nebst genauer Angabe ihrer Wohnorte, Blüthezeit, Nutzen u. s. w. 1. Bd. Fisenbers 1824. 
Flora Jenensis, oder Beschreibung der Pflanzen, welehe in der Ungegend ron Jena wachsen, herausgegeben von Friedrich David Dietrich. Ersten Bandes erster Theil. Jena 1826. 8. $400 \mathrm{~S}$.

Eine Flora ron Magdeburg hat der Pharmacent IIerr Fricdrich Kützing learbeitet; zufïllig sáh ich disvon das Manuscript und cinige dazu gehörige Abbildungen, wortunter Gymnadenia bifida und Stachys Hornungiana Kuitzing.

\section{Berlin. Gebiet der Spree u. s. w.}

Schlechtendal, D. F. L. de. Flora Berolinensis. Pars prima, Phanerogamia. Berolini 18:3. Sumptibus Ferdinandi Dümuler. P’ars secunda, Cryptogamia. Berol. 1824. 8.

Im zuciten Theile befinilet sich ein sehr schïtzbarer Aufsatz ziber Boden, Klima und $I$ ertheilung der Pflanzen um Berlin, wobei besonders treffend auf den Lnterschied der Vegetation an der Elbe und Oder aufmerlesam gemacht wird.

A. Dietrich. Flora der Gerend um Berlin, oder Aufzählung und Beschreibung der in der Mittelmark wild wachsenden und angebauten Pflanzen. Mit einer Vorrede begleitet von Dr. H. F. Link. 1. Theil, Phanerogamen. Berlin 1824.

Brandt. Flora Berolinensis, sive descriptio plantar. phanerogamarum circa Berolinum sponte crescentium vel in agris cultartum additis Filicibus et Charis 16 maj. Berolini 1824.

C. T. Strempel. Filicum Berolinensium Synopsis. Berolin. 1823.

Flora der Mark Brandenburg und Nicherlausitz, von Joh. Fridr. Ruthe, Oberlehrer am Cölnischen Real-Giymnasium and Lehrer an der Berlinischen Gieurerbschule. Erste Albtheilung, Phanerogamen. Auch unter dem Titel: Versuch einer Naturgeschichte der Mirk Brandenburg und der Niederlausitz. - Pflanzen. - Berlin, bei lleinrich Adolph Wilhelm Logier, 1827.' 2 fl. $42 \mathrm{kr}$.

Unteres Elbe-Gebiet mit dem der nahe liegenden Küstenflüsse.

G. Eimbcke Flora Iamburgensis pharmacentica; oder Verzeichnifs und Beschreibung der um Hamburg und in den angrenzenden Ländern wild wachsenden Arzneypflanzen. Mamburg 1822. 8.

II amburg in naturhistorischer und medicinischer Beziehung. Zum Andenken an die im September is30 statt gefundene Versammlung der deutschen Naturforscher und Aerzte. Hamburg 18と0). 8.

Detharding Comspectus plantarum ducat. Megapol. Phanerogam. Rostochii 18\%8. Fs. 8.

Siehe bot. Zeit. 18:9. I. p. 140.

Novitiae Florae Malsaticae sive supplementum alterum primitiarum Florae Halsaticate (t. II. Weleri, auctore E. F. Nolte, M. D., Prof. Botan. etc. Kilonii prostat in libraria acadenica 1826. 8.

\section{e. Gebiet der. Oder.}

Die Iegetation der norddeutschen Fbene, won der Weser, Flbe und Oder durchströmt, hat olne 'areifel die grïjste Gleichartigkeit, wenn man besondere Loculitäten und I erhältnisse ausnimmt; verfolgt man sie aber nach den Quellen dicser Ströme, so vird der Unterschied schon detitlieher hervor- 
treten, wie dies auch geuifs bei der Flora des Riesengebirges und der der Sudeten, woher die Oder ihren Ursprung nimmt, nicht zu verkennen ist. 'Die Lünder, welche die Oder mit ihren Nebenflüssen bewïssert, sind neucrdings vielfältig in botanischer Hinsicht untersucht worden, wie die nachstehende Literatur zeigt.

\section{Südliches Odergebiet. Schlesien.}

A. Krocker. Supplenentum seu Vol. quartum Florae Silesiae, contin. 800 plant. phanerogam. non solum in Silesia, sed et aliis vicinis provinciis rarissime detegendas etc. Vratislaviae 182 1 . 8.

F. W. Neygefind: Enchiridium botanicum continens plantas Silesine indigcnas, cui adjungitur Calendarim botanicum, et tab. aenea montium Sudetic. Schmiedel. et Misen. 18:1. 8.

Enumeratio stirpium Phancrogamarum, quae in Silesia sponte proveniunt. Vratislaviae apud Guilielmum Theophilum Korn MDCCCXXIV. Die Torrede haben die Herren C. Günther, H. Grabowski

und F. Wim iner unterzeichnet.

Flora Silesiae. Scripserunt Fr. Wimmer et II. Grabowski. Pars prima. Cl. I. - X. Cum tahnla lithographica. Vratislaviase 1827. Pars secunda -Vol. I. Cl. XI. - XV. cum imagine Güntheri. Vratislayiae $18 \% 9$.

Vegetation der Wiesenbaude auf dem Riesengebirge, ron Funk. Botan. Zeit. 1819. II. p. 609. 1820. I. p. 65 ,

Beschreibungen neuer Pflanzen aus dem Riesengebirge, von Herrn Prof. Tauseh.

Das. 1821. II. p $55 \%$.

Prodrom us Fiorae Lusatiac von $B$ urk ha rdt.

Bis zur 14. Klasse in den zuei ersten Heften des ersten Bandes der Abhandlungen der naturforschenden Gesellschaft in Gürlitz. -

Nördliches Odergebiet mit den Küstenflüssen.

Hortus Francofortanus, oder Verzeichnifs der in meinem Garton cultivirten Gewächse, mit Ilinzufügung der in der Nähe Frankfurts wild wachsenden Pflanzen, von J. N. Bach, Apotheker. Frankf. 182-..

Flora Sedinens is, exhibens plantas phanerogamas sipntaneas nec non plantas praccipuas agri Swincmundii, anctoribus F. G. T. Rostkovi o, Dr. Med. Sed. Consil. et E. L. G. Schmidt, Med. et Chir. Stud. Cum tabulis duabus lapicic incisis Sedini Formis Struckianis. 152!. 8.

Prodromi Florae Stargardiensis supplenentum primum, adjectis observationibus; auctore C. F. Schultz. Neubrandenburgi sumptibus Auctoris 1819. : 8.

Botanische Bemerkungen, die Flora Stargardiensis lvetreffend, von Herrn Dr. C. F. Schultz.

Bot. Zeit. 1824. I. p. 113.

St. Endlicher, Flora Posoniensis, exhibens plantas circa Posonium sponte crescentes aut frequentias cultas, methodo naturali dispositas. 8. Lips. 1830. Andreae. Weifs Dapier mit illum. Kupfern j̃ fl. $24 \mathrm{kr}$. ordin. Papier mit schwarzen Kupfern $3 \mathrm{fl}$. $36 \mathrm{kr}$.

Flora von Pommern oder Beschreibung der in Vor- und Finter-Poumern sowohl einheimischen als auch unter frciem Himuel leicht 
fortkommenden Gewåchse; nehst Bezcichnung ihres Gebrauches für die Arznei -, Forst - und Landwirthselhaft, Gärtnerci, Färberei u. s. w., ihres etwaigen Nutzens oder Schadens. Herausgegreben von G. G. J. Homann, Prediger zu Büdow bei Stolpe in Pommern. Erster Band enthaltend die 10 crsten Klassen des Linn. Pllanzensystems. Cöslin 1 ro28. Druck und Verlag von C. G. IIendess. 2 fl. 42 kr. Zweiter Band 1830. 8. 2 fl. $42 \mathrm{kr}$.

Beitrãge zu der Flora von Pommern, von den Pastoren Neuschild zu Grofs-Mellen bei Piritz und Prochnow za Marienthal bei Bahn.

Bot. Zeit. 1830. I. p. 38.

Botanische Bemerkungen über die Insel Rügen, von den Herren Hanshagen und Hornschuch.

Bot. Zeit. 1819. II. p. 477.

Beiträge zur liryptogamischen Flor der Insel Rügen, vom Herrn Prosector La urer in Greifswalde.

Flora der Insel Usedom nach Kastner.

Archiv für die ges. Naturlehre XVIII. 2. p. 211. 1829. - Bot. Literaturbl. IV. p. 20.

f. Unteres Gebiet der IVeichsel und der nahen Küstenflüsse.

$W_{\text {enn }}$ gleich die hier in Betracht kommenden Lü̈nderstreclen geographisch richtiger nach ihrer Lage rücksichtlich der Karpathen geordnet worden w'ären, so glaubte ich sie doch gerade hier anreihen zu müssen, da einerseits das Odergebiet unmiltelbar an das der I'eichsel stöfst, und dann die Gegenden an dem oberen Theile der W $\boldsymbol{W}$ eichsel in botanischer Hinsicht wenig bekannt sind, somit auch die gedachte Fintheilung für den vorliegenden Zueck wenig Nutzen stiften kann. Es gehören hierher aber vorzugsweise die Floren Preufsens.

C. G. Hagen. Preufaens l’flanzen. Königgsberg 1818. 2 Bände. 8.

C. G. Hagen. Chloris Borussica. Regiomont. 1819. 12. $446 \mathrm{~S}$.

Die um Danzig wild wachsenden Pflanzen nach ihren Geschlechtstheilen geordnet und beschrielien von G. Rey ger. Neue ganz umgearbeitete und vermehrte Auflage yon J. G. Weiss. Erster Theil. Danzig 1825. 8. Zweiter Theil. Danzig 182ii. 8. mit 3 hiupfertafeln.

Leo Henkel Victor a Donnersmark. Enumeratio plantarum circa Regiomontanum Borussorum sponte crescentium. Regiom. 1817. 8.

Botanische Notizen über einige Pflanzen Ost-Preufsens, von Gr. Henkel v. Donnersmark.

Bot. Zeit. 1819. II. p. 552.

C. G. Lorek. Flora Prussica. Abbildungen sämmtlicher bis jetzt aufgefundenen Pflanzen Preufsens. Königsberg 1826. Lexiconformat nit 18 Kupfern. (Ohne Seitenzahl).

E. Meyer. Nachrichten von den Pflanzen um Königsberg. B̈ot. Zeit. 1826. II. p. 562 u. d. $f$.

Botanische W anderung an der Küste von Samland, von Herrn Prof. Baer in Königsberg.

Daselbst 1S21. II. p. 897 u. d. $f$. 
g. Halbinsel Jütland, dänische Inseln und Helgoland.

Bemerkungen über die Vegetation der dänischen Provinzen, vom Professor Jons Wilken Hornemann, Ritter vom Dannebrog ctc. Koppenhagen 1821.

$$
\text { (In dünischer Sprache.) }
$$

Bemerkungen über die Vegetation der dänischen Provinzen; von Prof. Hornemann. Aus dessen Abhandl. in den Verhandl. d. dïn. philosoph. Gesellsch. 1821 ins Engl. übertragen in dem Edinb. philos. Journ. 1824. Jan. und aus diesem ins Deutsehe übersetzt ron Beils chmied. Botan. Zeit. 1825. II. p. 537. u. d. f.

F. Holl. Vegetation der Gegend um Eickenförde. Daselbst 18ㄹ․ I. p. 73.

Botanische Bemerkungen anf einer Reise durch Schoonen und Seeland im September und October 1819. Von Herrn Dr. Hornschuch. Daselbst. 1820. II. p. 413.

Beobachtungen über die Vegetation und die Fauna von Helgoland, von M. Hoffmann.

Schriften der naturforschenden Gesellsch. zu Berlin. Bd. I. Heft

4. 1824. p. $228-260$.

\section{B. West - Alpen - Länder.}

Dahin gehört nebst kleinen Stücken der Schweiz und der sardinischen Länder hauptsächlich Frankreich, dessen allgemeine Floren also zuvörderst hier, anzuführen sind.

La Flore et la Pomone françaises, ou description, histoire et culture des fleurs et des fruits de Françe, par M. Jaume St. Hilaire. Livraison 1. à 12. Paris 1928. chez l'auteur rue de Fürstemberg No. 3.

Flore générale de France on Iconographic descriptive et histoire de toutes les plantes phanerogames, cryptoganes et agames, qui croissent dans ce royaume, disposées suivant les familles naturelles; par M.M. Loiseleur-Deslongchamps, Persoon, Gaillon, de Brébisson et Bois-Duval. Paris 1828. grand in 8 .

Die drei crsten Hefte, jedes mit 12 Tafeln, enthalten die Ranunculaceen, jedes Heft kostet $\mathbf{6}$ francs, in 4 . auf Felin-Papier 12 francs.

Manuel complet de Botanique, deuxième partie. Flore française par M. F. A. Boisdural. 3 Vol. in 18. de 317,370 et 396 pages. Paris 1828.

\section{Auch unter folgendem Titel:}

J. A. Bois-Duval. Flore française ou description synoptique de toutes les plantes phanerogames et cryptogames qui croissent naturellement sur le sol français avec les caractères des genres et l'indication des principales éspèces. Paris chez Roret 1828. 3 Vol. de 1120 pages. 10 francs.

Methode analytique comparative de Botanique, appliquée aux plantes phanerogames, qui composent la Flore française, par B. L. Payre. Paris 1828. 4.

Flora gallica seu enumeratio plantarum in Gallia sponte nascentium, secundum Linnaeanum systema digestarum, addita familiarum naturalium synopsi, auctore J. A. L. Loiseleur-Des longchamps. Edit. secunda, aucta et emendata cum tab. XXXI. Deux Vol. 8. Pris 
16 francs. Paris 1828. chez Boillère rue et vis à vis l'école de Médecine No. 13.

Nourelle Notice sur les plantes is ajouter a la Flore de Françe, par M. Loiseleur Deslongehamps.

Annales de la Soc. Linn. de Paris. Sept. 1827. p. 396. - Siche bot. Literaturbl. I. p. 202.

Nouvelles observations sur la denxieme édition du Flora gallica de M. Leiseleur, pour servir de replique a la reponse quil a faite aux premières observations de M. Felix Petit.

- Innales des Sciences dobsercation Mars 182!) p. 46\%.

Aug. Pyram. De Candolle. Botanicon gallicum seu synopsis plantarum in flora gallica descriptarmm. Editio sccumdit. Fix Herbariis et Schedis Candullianis propriisque digestum a J. F. Duliy V. T). M. pars prima plantas vasculares continens. Paris Ve Desray, rue IIantefeuille No. 4. 1828.

Dufour.

Observations sur quelques plantes de la Françe, par M. Leon Annales des Sc. naturelles. Mui 1825. p. 82.

Ol,serrations sur quelques plantes rares ou nouvelles de la Flore française, par M. Requien.

Annales des Sc. natur. Aont 1825. p. $381-386$.

Plantes nouvelles pour la Flore française, par feu L. F. E. Ramiond.

Annales de la Soc. Linn. de Paris Sept. 182'T. p. 4333.

Album floral des plantes indigènes de France, ou Botanique élémentaire, à l'usage des jeunes personnes, par L. Lefebure et ch. Leforestier. Paris 18\%8. 8. avec 5 pl.

\section{a. Gebiet des Adour.}

Tief in den Pyrenc̈en bei Barcges entspringt dieser eben nicht sehr. grofse Flufs, aber das von ihm durchzogene Gebiel hat eine ganz vor züglich reiche und ausgezeichnele Fegetution. Die frunsösische Seite der Pyreneien ist weit hüufiger von IJlanzenliennern besucht worden, als die spanische, es diüfte daher nichl ungerne gesehen werden, wenn hier die literarischen Notizen über die Flor dieser Gebirge vercinigh würden.

J. Bergeret. Flore des Basses Pyrenées. Pall 1803. 2 Vol. 8.

N. Dralet. Description des Pyrences. Paris 1813. 2 Fol. 8.

Philippe Pieat de la P'eyrouse. Figures de la Flore des Pyrenécs. Paris 1795-1811. Fol. Flore des Pyrenées. Toulouse 18 til. Histoire abrégée des plantes des Pyrences et Itineraire des hotanistes dans ces montagnes. 'Toulouse 1813.

Kurze Uebersicht der Monographic üher die in den Pyrenien wachsenden Steinlsecharten in sehrader's Journal für die Botanili. 1stil. p. 199. Man sehe auch ïber Pyrenien-Pflanzen Isis Lid. III. p. 1505 .

L. F. F. R amond de Carhonnieres. Voyage au mont Perdu et dans la partie adjacente des Ilautes P'yrenées. Pairis 1811. 8.

Catalogues des plantes indigènes des Pyrenées et du Bas Languedoc, par George Bentham. Paris 182ii. 8.

Siche bot. Litcraturbl. I. p. 65. 
A tour to the South of France and the Prrenees in the year 1825.

Reise durch das suidliche Frankreich und nach den Pyreneen im Jahre 1825 ron G. A. Walker-Arnott. Aus einem Briefe an den Prof. Janeson im

New Edinburgh Philosophical Journal. April - July 1826. $p$. 66 - 78. July - Oct. 1826. p. 268 - 275. April - Juni 1827. p. 157 164. July - Sept. $350-356$.

J. Thore. Essai d'une Chloris du Departement des Landes Dax 1803. 1 Vol. 8.

Florula littoralis Aquitanica, a Bayona usque ad Caput Batos (vulgo Tête de Buch) auct. J. P. S. Grateloup.

Bulletin d'hist. natur. de la Soc. Linn. de Bordeaux 1826. (Juillet Aoút - Nov.)

b. Gebiet der Garonne mit ihren Neben- und nahen Kïstenflüssen.

N. Baron. Flore des départemens méridionaux de la Françe et principalement de celui de Tarn et Garonne, on description des plantes, qui croissent naturellement dans ce departement etc. Montauban 18³. 8.

D. J. Tournon. Flore de Toulouse ou description des plantes, qui croissent aux environs de cette ville, par le docteur T. a Toulouse. 1 Vol. 8. 1827.

N. Cazeau. Catalogue des plantes qui croissent dans le Departement du Gers, in Annuaire du departement de l'an XII.

C. Saint Amans. Flore Agénoise, ou description methodique des plantes qui croissent dans le departement de Lot et Garonne, et dans quelques parties des departemens voisins. Agen 1818. 8.

Flore Bordelaise et de la Gironde, description characteristique des plantes, qui eroissent naturellement aux environs de Bordeaux et dans les autres parties du Département avec l'étymologie, le site, l'époque de la floraison ete., leurs propriétés et leurs principaux usages, précédée de notions élémentaires de Botanique, par J. F. Laterade. 3. édition. Bordeaux 1829.

F lora Burdigalensis praefecturaeque Garunnae, Addenda prima, auct. J. F. Laterrade.

L'Ami des champs Juillet 182\%. p. 270. Man sehe auch diese Zeitschrift.,Aug. u. Sept. 1825.

Rapport sur une excursion faite à la Bastide et à Bouillac près Bordeaux, par M. Ch. Desmoulins.

L'Ami des champs Juin 1827. p. 228.

Notice sur le Lychnis corsica et autres plantes méridionales de la Gironde.

Bulletin d'hist. nat. de la Soc. Linn. de Bordeaux 1826. T. I. p. 81.

J. L. M. Guilla ume. Calendrier de Flore, des environs de Niort, précédé d'un abrégé élément. de Botanique. Niort 1801.

Lou is Perrat, botanische Reise in Languedoc. etc.

Bot. Zeit. 1819. I. p. 216.

Hier kommen auch Nachrichten von den Pyrenüen vor, wie denn auch das Garonne-Gebict seinem Ursprunge nach den Pyrenäen angehört.

Liste des Mousses, Hepatiques et Lichens, observés dans le departement de la Lozére, par M. F. C. Prost. 
Extr. des Mémoires de la Société d'agriculture, commerce, sciences et arts de Mende Bull. des Sc. natur. Louit 1829. p. 258.

Notice sur la Flore du Departement de la Lozére, par M. Prost. Ibidem annéc 1827.

\section{c. Gebiet der Loire mit ihren Nebenflüssen.}

J. A. M. $A$ rnaud. Flore du Département de la IIaute Loire, ou tableau des plantes, qui y croissent, disposée suivant la methode naturelle. Paris 1825. S. Au Puy, chez Pasquet.

Antoine de l'Arbre. Flore d'Aurergne. 2. édit. Riom et Clermont 1800. 2 Vol. 3.

Navière-Laboissière. Botanographie du Département de la haute Vienne. Paris. 4

Im Auszug in der Statistique du Depart. de la Vienne.

N. Dubois. Méthode déprouvée aves låquelle on peut parvenir facilement à comnoitre les plantes des environs d'Orleans. Orleans 1803. 8.

A. de Saint Hilaire. Notice sur $\mathbf{7 0}$ plantes à ajouter à la Flore d'Orleans. Orleans 1812. 8.

T. Bastard. Essai sur la Flore du departenient de Maine et Loire. Angers 1809.

G. E. Merlet de la Bonlaye. Herborisations dans le departement de Maine et Loire et aux environs de Thouars, publiées par M. Davy de la Roche. Angers 1209.

Flore d'A njou, ou exposition methodique des plantes du Departement de Maine et Loire et de l'ancien Anjou, par M. Des reaux. Paris 1827. Chez Fourier-Mame. 6 fr.

Flore de Maine et Loire. Par Guepin. Angers 1830. in 12. Tom. I.

d. Gebiet der Seine und ihrer Nebenflüsse.

Lorey et Duret. Catalogue des plantes, qui croissent naturellement dans le departem. de la Cote d'or, et des éspèces le plus généralement cultivées, observées jusqu'aujourd'hui. Dijon 1825.

Fin Theil dieses Departement gehört noch in das Gebiet der Rhone, namentlich die Gegend um Dijon u. s. $w$.

$$
P \text { a } r i s .
$$

Flore Parisienne, on description des plantes des environs de $\mathbf{P a}-$ ris, suivant la methode du jardin des plantes. Paris 1801. in 16.

Flore économique des plantes qui croissent aux environs de $\mathbf{P a -}$ ris, au nombre de plus de 400 genres et 1400 éspèces. Paris 1802. 8.

Vademecum du hotaniste vorageur aux environs de Paris, à l'usage des persomnes qui ont la flore de M. Thuillier, contenant la carte botanique portative de E. P'. Ventenant. Paris 1803 . Eine andere Ausgaluc. Paris 1807. 12.

J. D. Dupont. Double flore Parisicnne, on description des plantes, qui croissent naturellement aux environs de Paris. Paris 1805. 12.

Turpin et Poiteau. Flore Parisienne, contenant la description des plantes qui croissent naturellement aux environs de Paris; ouvrage 
orné de figures et disposé selon le système sexuel. Paris 1808. Fol. 9 'Lieferungen mit 68 Tafeln.

A. F. Plée. Herborisations artificielles des environs de Paris, on Recueil des plantes qui y croissent naturellement. Paris 1810. 8. I - XVII. herbor. avec les détails anatomiques en couleurs.

Bennoit. (Madame). Herbier élémentaire ou récueil de gravures au trait ombré, contenant la collection compléte des plantes utiles, agréables et usuelles, qui croissent sons ancune culture aux environs de Paris dans le rayon de 8 - 12. myriamètres, soigneuscment dessinées d'après nature, classées d'après le systéme de Linné. Paris 1811. Fol.

L. B. Francoeur. Flore Parisienne. Paris 1811. 12.

G. V. Mérat. Nouvelle flore des environs de Paris suivant'le système sexuel de Linné avec l'indication des vertus des plantes usitées en medecine, des détails' sur leur emploi pharmaceutique etc. Paris 1812. 2 Vol. 8. Deux Edit. Paris 1821.

A. Saint-Hilaire. Obserrations sur la nourelle flore des environs de Paris. Paris 1812. 4.

A. Vigneux. Flore pittoresque des environs de Paris, contênant la description de toutes les plantes, qui croissent naturellement dans un rayon de 18. - 20 lieues de cette capitale etc. ourrage orné de plus de 250 figures presque toutes de grandeur naturelle etc. Paris 1812. 4. avec 68 pl. et une carte. Supplément Paris 1814.

F. F. Chevallier. Flore générale des environs de Paris, selon la methode naturelle etc. Tom. I. 1. Vul. 8. fig. noires. (12 francs.) Paris 1826. shez Ferra jeune, rue des Grands Augustins. No. 23.

Tableau annalytique de la Flore Parisienne, d'après la methode adoptée dans la flore française de M.M. Lamaŕck et De Candolle, contenant toutes les éspèces de phanerogames des environs et de la description des familles naturelles et des genres par E. Bautier. Paris 1827. 18. Chez Béchet jeune.

N. Dumarchais. Flore du Departement de l'Aine: Im Auszuge in der Statistique du departement du l'Ain. Paris 1806. 4.

N. Brouard. Catalogue des plantes du departement de l'Eure. Paris 1820.12.

P. A. Renault. Flore du Departement de l'Orme. Alençon 1804. 8.

J. A. G. Boucher. Extrait de la flore d'Abbeville et du Departement de la Somme. Paris 1803. 8.

F. Rouçe1. Flore du Calvados. Caẹn 1806. 8. en 1816.

Turquier de Longchamps. Flore des environs de Rouen. Rou-

\section{e. Gebiet der Schelde und ihrer Nebenflüsse.}

Schon oben ist bemerkt worden, dafs ein Theil von Belgien in das Schelde-Gebiet gehört; das rechte Ufer dieses Stroms aber kommt hier noch besonders in Betracht.

F. Rouçel. Flore du Nord de la France, ou description des plantes indigènes et de celles cultivécs dans les departemens de la Lys, de l'Escaut, de la Dyle, et des deux Nythes y compris les plan- 
tes qui croissent dans les pays limitrophes de ces departements. Paris 1803. 8. 2 'T'om.

J. B. II. J. Desmazières. Agrostographie des departemens du Nord de la France, ou Analyse et description de toutes les Graminées qui croissent naturellement, ou que l'on cultive généralement dans ces departemens. Paris 1812. 8.

Plantes eryptogames du Norl de la Françe, par Desmazières. Fascic. I. Lille 185. Leleux. Paris. Trentel et Würz.

\section{f. Gebiet der Thone und ihrer Nebenfliisse.}

Nebst dem Adlour und der Guronne, denen die Nähe der Pyrenäen ihre schöne Flor grewaihst, dürfle die Rhone in botanischer Hinsicht das interessanteste Stromgebiet der "W'est-Alpenlünder sein; es ist zugleich am sorgfültigsten und f:ühesten untersucht worden, ja die Lfer der Rhone kann man die Wiege für Frankreichs Pflanzenkunde nennen, wie jene des Rheins es für Deutschlands Botanik sind.

Chantrans-Girod. Essai sne la Géographic physique du departement du Doubs. Paris 1810. 2 Vol. 8.

N. Guyetaut. Catalogue des plantes à fleurs visibles qui croissent dans les montagnes du Jura. Besançon 19u8. 8.

N. Guyetaut. Catalogue des plantes à fleurs doubles qui naissent dans le Canton du Jura et dans les pleines qui s'étendent dépuis ces montagnes jusqu'a la Saone. Paris 1809.

Flore Lyonnaise, ou description des plantes qui croissent dans les environs de Lyon et sur le Mont-Pilat, par le Docteur J. B. Balbis, Prof. de Botanique, Direet. du jardin des plantes, Président de la Soc. Linnéenne. Tom. I. Première et seconde partie. 8. Lyon 1827. Inprimerie de C. Coque. 890 S. Tom. II. Lyon 1828.

Siehe botan. Literaturbl. I. p. 210. III. p. 513.

J. Guerin. Fragmens d'une topographie physique et médicale du departement de Vaucluse.- Montpellier. 4.

J. Guerin. Description de la fontaine de Vaucluse. 2. édition. Avignon 1813.

P. C. Ambreux. Etat de la végétation sous le climat de Montpellier. Montp. 1809. 8.

Sa $\mathrm{z}$ mann. Enumeratio plantarum rariorum in Gallia australi crescentiun Montispelii 1818.

Flore du Departemens meridionaux de la France, par M. Baron. Montauban 182:

Diese Sclurift umfafst auch einen Theil des Stromgebietes der Garonne, das an vielen Stellen an das der Rhone grenst, wie denn überhaupt die jetzt vorhandenen botanischen Schriften über die Floren einzelner Gegenden sich nie streng nach natürlichen Grenzen ordneñ lassien.

Ueber die Vegetation um Nizza hat Herr Risso in seinem bcreits oben angeführten Werke Bericht erstattet. -

Beautés meridionales de la Flore de Montpellier. Par un ancien herboriste de cette ville. Montpellier 1827. 8.

Vegetation der Umgebung von Nizza, nach $R$ isso. Bot. Literaturbl. IV. p. 255. 
 \\ C. Süd-Alpen - Länder.}

Es ist zweckmäfsig, zuvörderst hier die Schriften und Aufsätze anzuzeigen, welche von der Flora Italiens z̈berhaupt handeln.

Giuseppe Moretti. De quibusdam Italiae plantis. -

Unter dieser Aufschrift hat Herr M. in dem Giornale di fisica Chim. etc., welches zu Pavia herauskommt, namentlich in den Heften des Jahres 1824 mehrere Aufsätze, die Nachrichten über italische Pflanzen enthalten, bekannt gemacht.

I dem. Il Botanico italiano ossia discussioni sulla flora Italica. Giornale di fisica etc. Pavia 1826. Bim. I. II. III.

Flora Italica, seu descriptio plantarum omnium, quae in Italia sponte nascuntur e systemate sexuali distributarum, auctore G. M oretti. 1825.

M. Savi. Flora Italiana. 2 Vol. Pisa 1825. cum figar.

Anton. Bertolonii, Prof. Bonon. Amoenitates italicae, sistentes opuscula ad rem herbariam et zoologiam Italiac spectantia. Bonon. 1819. 472 S. in Quart und 6 Kupfertafeln.

Siehe Sprengel neue Entdeck. II. p. 271.

Antonii Bertoloni, M. D., praelectiones rei herbariae et prolegomena ad Floram Italicam. Bononiae 1827.

Fragmente über die Vegetation in Italien im Allgemeinen und den Anbau des Oelbaums insbesondere.

Carus Analekten zur Naturwissenschaft und Heilkunde. Dresden 1829. $p$. $26-45$.

Ueber die Vegetation des Festlandes von Italien, von Herrn Samuel Brunnex, Doctor der Medicin in Bern.

Botan. Zeit. 1526. II. p. 625. 641.

a. Gebiet der kleineren Alpenflüsse, Etsch, Brenta, Piave, Tagliamento, Isongo u. s. w.

De plantis quibusdam Italiae borealis et Germaniae australis rarioribus. Dissert. inaugural. botan. auctore J. E. L. A vé-Lall ema n t L ube censi. Berol. 1829. 4.

Der Verf. besuchte mehrere Gegenden, daher seiner Schrift nach der angegebenen Ordnung auch hier nur ein partieller Platz angewiesen werden kann.

Angabe der Standörter über die in der Umgebung von Botzen im Sommer 1824 gesammelten seltenen Pflanzen, ron F erdinand Elsmann.

Bot. Zeit. 1825. II. p. 503.

Beschreibung einiger botanischen Ausflüge auf etliche in der Nähe von Botzen g'elegene Berge, von Demselben.

Daselbst p. 705., ferner 1826. II. p. 405.

Vegetation des Schleerngebirges von Demselben.

Daselbst 1826. I. p. 366. und 283.

D. C. Pollini. Flora Veronensis, quam in prodromum florae Italiae septentrionalis exhibet. Veronac. Tom. I. II. 1823. III. 1825. 8. mit Kupfern. 
Pflanzen um Verona, vom ILern Grafen Henkel von Donner $\mathbf{m} \mathbf{m}$ ark.

Bot. Zeit. 1820. I. p. 49. rona.

Brief des Dr. Cir. Pollini über die Flor und den Boden von Ve-

Bibl. ital. Mars 1827. No. 135. p. 411.

1823. 8.

Romano D. Girolamo. Le piante fanerogame euganee. Padova

Ignatz Edler v. Hildenbrand. Pflanzen in den Euganeen.

Botan. Zeit. 1820. II. p. 736. $u$. d. $f$.

Ciro Pollini. Elencho delle piante del territorio di Vicenza. Milano 1802. 8.

G. Marzari Pencati. Eleneho dell piante spontanee de Vincentino. Milano 18u2. 8.

Giuseppe Moretti. Notizia sopra diverse piante da aggunsiarai alla flora Vicentina. Pavia 1815. 4.

Giuseppe Ruchinger. Flora dei Lidi Veneti. Venezia 1818. 8.

Steph. Moricand. Flora Veneta, seu enumeratio plantarum circa Venetiam, secundum method. Linn. disposita. Genova 1820. 8.

Botanische Bemerkungen über Venedigs Umgebungen.

Botan. Zeit. 1819. II. p. 321.

G. v. Marten s. Bemerkungen über einige Pflanzen um Venedig. Daselbst 1828. II. p. 507.

Noecari. Flora Veneta Vol. I. Venezia 1826. 4. Die vicr ersten Klassen.

$$
\text { Triest, Istrien u. s. w. }
$$

Vegetation um Triest von Dr. Hoppe.

Botan. Zeit. 1821. II. p. 461.

Excursionen um Triest, von Demselben.

Daselbst 1827. I. p. 346. Triest.

Dr. Biasoletto. Blüthezeit mehrerer Pflanzen in Istrien und um

Daselbst p. 188.

Vegetation in der Valle Rutte bei Triest, von Demselben.

Daselbst 1829. I. p. 154.

Bericht über eine Reise durch Istrien, vom Herrn Dr. Biasoletto in Triest.

Dasclist 1829. II. p. 513.

Uebersicht der Vegetation in den Ungebungen von Triest, von Herrn Gustav Heinhold zu Dresden.

Daselbst p. 657.

Vegetation um Fiume, im Thale der Isongo u. 8. w., von Dr. Bartling.

Botan. Zeit. 1819. I. p. 53. u. d. f.

Bruchstücke aus dem Tagebuch einer naturhistorischen Reise von Prag nach Istrien, von dem Herrn Grafen Caspar v. Sternberg.

Botan. Zeit. 1826. I. Erste Beilage. 


\section{b. Gebiet der Küstenflüsse der Gebirgskette Poglicza.}

\section{D a $l m a t i$ e $n, C r o a t i e n$.}

Enumeratio plantarum in Dalmatia collectarum a Francisco de Portensehlag-Ledermayer U. J. D. Vindobon. apud Gaertner 1824. c. tab. 12 aeneis.

Plantae Dalmaticae nunc primum editae a Roberto de Visiani, M. D. pluriumque academiarum sodali.

Botan. Zeit. 1830. I. p. 49.

U eber die Vegetation Dalmatiens, vom Herrn General Baron v. Welden zu Zara in Dalmatien.

Daselbst p. 193.

Frühlingsflor in Dalmatien; von Demselben.

Daselbst $p .251$.

Plantac rariores in Dalmatia recens detectae a Roberto de Visiani, M. D.

Daselbst 1829. I. Ergänzungsbl. p. 1. $u$. d. f.

Stirpium Dalmaticarum specimen, auctore Roberto de Visiani. Patavii typis Crescinianis MDCCCXXVI.

v. Welden. Ueber den Charakter der Vegetation in Dalmatien und über einige Dalmatiner Pflanzen.

Bot. Zeit. 1829. I. p. 105. und 300.

Pflanzen - Producte der Insel Meleda.

Aus P. Partsch Bericht über das Detonations - Phänomen auf.

der Insel Meleda bei Ragusa. Wien 1826. Bot. Literaturbl. I. p. 360.

\section{c. Gebiet des Po und seiner Nebenflïsse.}

Die Landschaften, welche der Po und die in ihn sich ergiefsenden Flüsse bewässern, gehören in botanischer Hinsicht zu den interessuntesten, nicht nur in Italien, sondern in Europa überhaupt. Entspringend aus dem höchsten Theile der Alpenkette, deren Klima und Vegetation an den hohen Norden erinnert, durchlüuft er dann ein reiches, fruclutbares, durch die Milde seiner Luft und durch die Schönheit seiner Flor berühmtes Land, auf seinem Wege zu dem adriatischen Meere. Wenn ein geübter, mit allen nöthigen Kenntnissen versehener, und mit scharfer Beobachtungs-Gabe ausgerüsteter Botaniker das Pothal mit seinen zahlreichen Nebenzweigen von dem Ufer der Adria an, bis hinauf $\approx u$ den beeisten Höhen der kottischen und rhätischen Alpen wiederholt und mehrere Jahre lang durchsuchte, die eben so reiche als mannichfaltige Flor dieser Gegend in jeder Rücksicht beobachtete und aufzeichnete; welche Ausbeute würde davon für alle Theile der Botanik, zumal für die Pflanzen-Geographie zu erwarten sein!

Ein ige Bem erkungen über die Temperatur, Entstehung und das Verschwinden der Vegetation auf dem grofsen St. Bernhard, 7668 Pariser Fufs über dem mittelländischen Meere, im Jahre 1822, von Herrn Baronv. Welden. Bot. Zeit. 1823. II. p. 561.

Prodromus einer Flora des Monte Rosa, gesammelt am 24. und 25. August 1821, von Demselben.

Daselbst 1822. I. p. 265.

Plantarum a Josepho Comellio, M. D. in Lariensi provincia lectarum enumeratio, quam ipse in botanophilorum usu atque commodo exhibet, uti Prodromun Florae Comensis. Novo Comi 182t. 8. 
Flora Comensis, plantas sponte in provincia crescentes atque illas quae indigenatum nactae coluntur exhibens earumque proprietates et usus enumerans a Josepho Comellio, olim Botanices atque Arrariae in patris Lycen Professore, nunc ejusdem Reetore atque I. R. Medico provinciali ete. secundum systema sexuale digesta Novo Comi. Ex typis C. Petri Ostinelli 1826.

Alpenflor von Bresciana und Bergamasco, nach Dortor Zantedeschis.

Commentarii del Ateneo di Brescia per il 1825. - Giorn. di Fisica e stor. natur. Dec. II. T. X. p. 329. 1827. - Botan. Literaturbl. I. p. 355.

Joannes Franciscus Re. Appendix ad Floram pedemontanam. Taurini 1824. 8.

Reliquiae Bellardianae, auctore Prof. Re.

Mem. Acad. Sc. Torin. T. XXXIII. - Ist auch ein Nachtrag zur Flora von Piemont, wie Cyperus oryzetorum B., Anemone latifolia Bell.

J. F. $\mathbf{R}$ é ad Floram Pedemontanam appendix altera.

Mem. del real Acad. di Torino 1827. p. 189. - Bot. Literaturbl. II. $p$. 60.

Flora torinese del Dottore in Medicina Gioanni Francesco Re, Professore di Botanica, di Chimica e di Materia medica etc. Tom. I. et II. 8. Torino della Tipografia Bianco. 1825 - 1827.

Botan. Literaturbl. IIÎ. p. 616.

Nocca, Dominicus et Balbis Jo. Baptista. Flora Ticinensis, seu enumeratio plantarum, quas in peregrinationibus multiplicibus plures per annos solertissime in papiensi agro peractis observarunt et collegerunt etc. Pavia 1821. 2 Vol.

Siehe botan. Zeit. 1824. I. p. 143.

Nocca. Clavis rem herbariam addiscendi absque praceptore, sen Enchiridion ad excursiones botanices in agro T'icinensi. Ticini 18:3. 2 Vol. 8.

G. Jahn. Flora Italiae superioria. Parma 1820.

Moretti. Versuch eincr Naturgeschichte der Umgegend von Mantua. Auszug eines Briefes des Dr. Lanfossi an Moret ti.

Giornale di fisica etc. T. X. p. 279. Nov. Dec. 1825. Jan. Febr. 1826. Mars. Jun. Sept. Oct. 1827. p. 235. - Bot. Literaturbl. I. p.68.

\section{d. Gebiet der Apenninen - Flüsse.}

Bergamaschi. Lettera sopra rarie piante degli Apennini, colli altrepodani e della campagna pavese da aggiungersi alla Flora ticinese. Giornale di Fisica etc. Pavia 1821. Bim. III. et IV.

Prodromus der Mai-Flora um Genua.

Botan. Zeit. 1822. I. p. 263.

A usflug nach dem Vorgebirge Porto fino, im Monat April 1823, von dem K. K. Obersten Herrn Baron v. Welden. Daselbst. 1823. II. p. 577.

D. C. B. Badaro. Osservazioni sopra diverse piante della Ligurie occidentale e della Sardegna.

Giornale di fisica etc. Pavia 1824. Bim. V. p. 363. 
Beobachtungen über einige Pflanzen-Arten des westlichen Lignriens, aufgenommen in dem Botanico italiano des Prof. Moretti.

Giornal. Ligustico di Scienze etc. Mars 182\%. p. 115.

Anonym und gegen Moretti gerichtet.

Botanicon Etruscum, sistens plantas in Etruria sponte crescentes; auctore Cajetano Savi D. M. Pisa 18\%5. Der erste Band erschien 1808, der zweite 1815 , der dritte 1818, der vierte 1825.

A usflug von Rom nach Ostia im Jahre 1823, von Herrn Dr. Brunner in Bern.

Bot. Zeit. 1825. II. p. 465.481.

Marat ti D. Joann Frana abbatis Vallumbrosani Flora Romana, opus posthumum. Roma 1822. Vol. II: 8.

Sickler. Pflanzen um Rom.

Isis. Bd. IV. p: 578.

Notizen über eine kleine Zahl Pflanzen, gehörend zum Prodromus der Flora von Rom, ron Mad. Elisabetta Fiorini.

Giornal. arcad. di Ścienze Mai 1823. p. 161.

Plantae rariores quas in itinere per oras Jonii ac adriatici maris et per regiones Samnii ac Aprntii collegit Jo annes Gus sone, Med. Doct. et praefectus h. r. botanici in Boccadifalco. Neapoli 1826. 4.

Siehe Linnaea V. Lit. p. 99.

Botanischer Ausflug nach dem Agnano-See, der Solfatara, Pozzunli und dem Monte nuovo, unternommen am 29. März 1823, vom Dr. Herbich.

Bot. Zeit. 1823. II. p. 614.

Botanischer Ausflug nach dem Monte St. Angelo bei Castellamare, von Demselben.

Daselbst. 1821. I. p. 71.

Botanischer A usflug nach der Insel Capri, von Demselben.

Daselbst. 182t. II. p. 481.

Botanische Reise nach dem Vesuv, von Demselben.

Daselbst. 1823. I. p. 98.

Synopsis novarum plantarum, quae in Prodromo Florae Neapolitanae describuntur, auctore Mich. Tenore. Neapol. 1819.

Flora Neapolitana, auctore Tenore. In Folio. Fasciculi XXIV. cum figuris coloratis. Neapoli 1824.

Siehe Bulletin des Sc. natur. Juin 1826. p. 209.

Ad Florae Neapolitanae Prodromum Appendix quinta, exhibens Centurias duas plantarum nuperrime detectarum, nec non specierum novarum vel minus rite cognitarum characteres et illustrationes. Neapoli 1826.

Siehe Linnaea 1828. Heft II. p. 97. der Lit.

Italische Inseln.

Corsika.

Ph. Salzmann. Kurze Bemerkungen auf einer botanischen Exoursion nach Corsica, im Jahre 1820.

Bot. Zeit. 1821. I. p. 101.

Catalogue des plantes, qui croissent spontanément en Corse, 
nouvelles pour la Flore Française, et recueillies par M. P. C. Marie de Pouzolz.

$$
\text { Annales de la Soc. Linn. de Paris. Nov. 1825. p. 5601. }
$$

F l orae Corsica epecierum novarum vel minus cognitarum diagnosis, quam in Florae Italicae fragmenti alterius prodromum exhibet $\mathbf{D}$ o minicus Viviani. Genuae 1824.

Ejusdem Appendix ad Florae Corsicae prodromum. Genuae 1825.

$$
\text { S } a r d i n \text { i } e \text { n. }
$$

Stirpium Sardoarum Elenchus. Auctore Josepho Hyacyntho Moris, in regio Caralitano Athenaeo clinices Professore, collegii medicorum Taurinensis et r. societ. agrar. et oecon. Caralitanae socii. Fasc. I. Carali ex typis regiis 18\%7. 4 .

Siehe Linnaea 1829. III. p. 59. Lit. - Botan. Literaturbl. I. p. 26 - 45., wo auch zugleich Beiträge zur Flora von Sardinien von Herrn Müller beigefügt sind.

Stirpium Sardoarum Elenchus. Fascicul. II. Dec. 18\%7. p. 197.

Siehe Linnaca 188̊0. III. p. 91. Lit. - Botan. Literaturbl. I.

Stirpium Sardoarum Elenchus, auctore J. H. Moris. Fasciculus III. Augustae Taurinor. 1829. 4 . Botan. Literaturbl. II. p. 303.

$$
S \text { i c } i \text { l } i \text { e } n \text {. }
$$

Catalogus plantarum, quae asservantur in regio horto Ser. Franc. Borbonii in Boccadifalco prope Panormam, auctore Gussone. Neapol 1821. 8.

In dieser Schrift sind zugleich 15 neue sicilianische Pflanzen beschrieben; nemlich: Chrysanthemum hybridum, Cineraria gibbosa, C. ncbradensis, Colchicum bivonae, Crepis spathulata, Erica sicula, Euphorbia stenocarpa, Euphrasia Bocconii, Genista Cupanis, Hyacinthus dubius, Ononis sicula, Seseli Bocconi, Trifolium congestum, T. intermedium, Trigonella littoralis, Urtica rupestris. Bullet. des Sc. nat. Juin. 1826. p. 212.

Florae Siculae Prodromus, sive plantarum in Sicilia ulteriori nascentium enumeratio secundum systema Linneanum, auctore Joanne Gussone. Vol. I. Neapoli 1527. 8. (Die ersten 12 Klassen.)

Flora Sicula, exhibens plantas vasculosas in Sicilia aut sponte crescentes aut frequentissime cultas, sccundum systema naturale digestas, auctore C. B. Presl, Med. Doct. etc. Tomus prinus. Pragae suimptibus A. Borrosch. 1826. 8.

Einige seltene Pflanzen Siciliens, von dem Herrn Director Ritter von Schrank.

Bot. Zeit. 1819. II. p. 381.

Coceo, Anastasio. Per lo stabilimento della Flora Messincse di piante in rilievo orazione. Messina 1824. 8.

D. Pyrenäische Halbinsel nebst den balearischen Inseln.

Berieht über eine hotanische Reise nach eincm Theile von Spanien, nach Gilhraltar und Tanger, ron Herrn Salzmann in Montpellier. Bot. Zeit. 1825. I1. p. 737. 


\section{- $183 .-1$}

Verzeichnifs und Characteristik neuer Pflanzen aus der Flora Zusitano - Maderensis.

Daselbst 1830. 1. p. 129.

Excursion dans les iles Baleares par M. C. Cambessédes, correspondant de la Société d'hist. natur. de Paris.

p. 5 - 37 .

Enumeratio plantarum, quas in insulis Balearibus collegit $\mathbf{J}$. Cambessédes, earumque circa Mare Mediterraneum distributio geographica.

Mémoires du Muséum d’hist. natur. Sept. Année neuvième, Cahier p. 173. bis zu Ende des Heftes.

Hier sind abgebildet: Brassica balearica Pers., Helianthemum Serrae Cambessédes, Silene disticha Willd, Sillene villosa var. nana, Genista lucida Camb. Der Rest der Abhandlung steht in dem zehnten Hefte p. 253 - 335. der genannten Mémoires. Dort findet man die Abbildungen von Lotus tetraphyllus, Helichrysum Lamarckii Camb., H. Fontanesii. Camb., Disandra africana Camb.

\section{E. Brittische Inseln.}

$$
\text { E } n \text { g } l \text { l a } n \boldsymbol{d} \text {. }
$$

W. Withering. Systematic arrangement of british plants, corrected and enlarged by his son. Lond, 1812. 8. 4 Tom. with plates.

R. Thornton. The british flora; or the genera and species of british plants arranged after the reformed sexual system. Lond. 1812. 8. 5 Vol.

S. F. Gra y. A natural arrangement of british plants etc. London 1822. 8.2 Vol.

Jam. Edward Smith. The english-Flora. London 1823, 1824, 1825, 1828. 4 ' Vol. 8. London.

A natural arrangement of british plants by Samuel Fred. Erey.

P. W. Watson. Dendrologia Britannica or Trees and Shrubs that will live in the open air of Britain throughout the year. London 1825. 8. 2 Vol. with plates.

W. Johns. Practical botany; and improved arrangement of the generic characters of british plants, with a familiar introduction to the Linnean System. London 1826. 8.

A n introduction to english Botany; by J. M. Butt. Londen 1824. 8.

Zusaatze zu Flora Britannica. (Convallaria verticillata, Reseda alba, Myosotis collina.)

Aus dem Edinb. Journal of nat. and geograph. Sciences in dem Bull. des Sc. nat. Juillet 1880. p. 75.

J. C. Loudon. Hortus Britannicus, a Catalogue of all the Plants indigeneous, cultivated in, or introduced into Britain. London, Longmann et Comp. 1827.

A Synopsis of the British Flora, arranged according to the natuwal Orders; containing vasculares or flowering Plants. By John Lindley. London 1829. 8.

Siehe Bibliothègwe univerelle. Mai 1830. n. 107 
Plantae Varvicenses sclectae, by W. G Perry. Warwick 1820.

Flora Devoniensis, or a descriptive Catalogue of Plants growing in the county of Devon, arranged both according to the Linnean and matural System, with an account of their geographieal distribution ete. By the Rew. J. P. Jones and J. F. Kingston. London 1830.8.

The British Flora; comprising the phenogamous or flowering plants, and the ferns by William Jackson Hooker. London 1830.

\section{Sc. $h$ o $t \operatorname{t} l a n d$.}

Will. Jackson Hooker. Flora Scotica. London 1821. 4.

Liste seltener schottischer Pflanzen.

Liste seltener in Pertshire gefundener Gewächse.

Edinburgh philosophical Journal. No. XXVII. January 1826. p. 179 - 182.

In Pertshire cinheimische und seltene Pflanzen, von Herrn David Bishop.

Edinburgh philosoplical Journal. Jan. 1826. p. 280.

A Flora of Berwick upon Tweed. By George Johnston, M. D. Vol. I. Phaenogamous Plants. Berwick 1829. 8.

Graham. Botanische Excursion in Sätherlandshire in Schottland. Jameson New Edinb. Phil. Journ. Oct. - Dec. 1827. p. 193. - Botan. Literaturbl. II. p. 283.

A Memoir regarding the natural History of the District of Alford in Aberdeenshire. By Alex. Murray.

Jameson New Edlinb. phil. Journal Oct. - Dec. 1828. p. 133. u.d.f. Physionomie der Gegend, geognostische Bemerkungen, Verhültnifs des Borlens zu den Gewä́chsen, Aufzählung der vilduachsenden Pflanzen, von der Monandria bis zu einem Theile der Pentandria.

Flora Edinensis, or a description of plants growing near Edinburgh, by Kaye Greville. London 1824.8.

Waadford. Catalogue of Phaeno:amic Plants growing spontaneously in the envirens of Edinburgh. London 1821.

Ei-Notice of a new Scottish Localithy for the Linnaea borealis, by John S. Bushnan.

Jameson New Edinb. Philos. Journal Jan. - April 1829. p. 300.

Dieser neue Standort der Linnaca, der nördlichste in Grofs Brittannien ist ein Hügel swischen Elgin und Forres.

Scottish Cryptogamic Flora, or coloured figures and descriptions of cryptogamic plants, belonging chiefly to the order Fungi; and intended to serve as a continuation of English Botany. By Robert K. Greville. Edinburgh $1821-1825.4$ Vol. 8.

Notice of Plants observed in an Excursion made by Dr. Graham with part of his botanical Pupils ete.

Jameson New Edinb. Pliil. Journal July - Oct. 1830. p. $360 \rightarrow 363$.

$$
\text { I } r \text { rla } n d \text {. }
$$

James Towrsind Mackay. A Catalogue of the plants, found in Ireland with deseriptions of some the rarer sorts. Dublin 1825. P. I. 498 S. Grevisberry.

Siehe Allgem. Literatur-Zeitung 1825. Sept. No. 210. p. 14. 


\section{$-\quad 185$ \\ F. Scandinavien.}

Svensk Botanik, Flora von Schweden, herausgegeben durch die königl. Akademie der Wissenschaften zu Stockholm. Heft 103. 104 und 105. 17 S. 8. Mit Abbildungen. Upsala 1825.

Siehe Linnaea 1828. Lit. p. 106.

Flora Suecica, enmuerans plantąs Sueciac indigenas, cum synopsi classium ordinumque etc. post Linnaeum edita : a Georgio Wahlenberg, Botanices Demonstratore Upsaliensi. Pars prior. Upsaliae impensis Palmblad et C. 182t. Pars posterior 1826. 8.

Elia e Fries, Prof. reg. Acad. Sc. Holm. Membr. etc. Noitiae Florae Succicae Edit. altera, auctior et in formam commentarii in C el. Wahlenbergii Floram Suecicam redacta. Lundini Gothorum ex officinia Berlingiana 1828. 8.

Hornschueh. - Nachricht von mehreren in Schweden gefundenen, für dessen Flora neuen Pflanzen.

Bot. Zeit. 1830. II. p. 439.

H. Fri es. Botanische Excursionen in einigen Provinzen von Schweden. Physiograph. Saelskap Aarsberättelse. Lund. 1825. p. 54. (Myosotides, Polygalae etci.).

Botanische Bemerkungen auf einer Reise durch Schoonen in September und October 1819, von Herrn Dr. Hornschuch.

Bot. Zeit. 1820. II. p. 413.

Försök till en Blekingsk Flora. Versuch einer Flora von Bleckingen, von G. C. A spegren. Carlscrona $18 \% 3$.

Supplementum Florae Lapponicae, quam edidit D. G. Wahlenberg, auctore S. Sommerfeld. Christiania 1826. 8. cum tabul. colorat. tribus. Borgia et Grosdahl. $5 \mathrm{fl} .24 \mathrm{kr}$.

Flora Upsaliensis, enumerans plantas circa Upsaliam sponte crescentes. Fnchiridion excursionibus studiosorum Upsaliensium accommodatum a Georg. Wahlenberg. Bot. Demonstrat. Upsal. 18². $496 \mathrm{~S} .8$.

Flora Gothoburgensis, Pars I. anctore Petr. Frid. Wahlenberg. Upsal. 1820. (Stockholm bei Wickhond 1825).

Stirpium agri Femsionensis index; auctore Elia Fries. Lundac 1825. Fascicul. III. 1. resp. Winding. 2. Hemmes. 3. Loyen Dissertationes inaugurales.

Stirpes agri Ratnoviensis, praeside $\mathrm{Agh}$ ard, offert Auctor A. E. Lind $\mathrm{b}$ lom pars 1 , Lundae 1826.

Flora Paroeciae Braenkyrka; praeside prof. Sillen, dissertat. 1 part. resp. Lundequist. 2. part. resp. Kjelleborg. 3. part. resp. Gellaerstedt. Upsal. 1827.

\section{G. Rufsland.}

Prytz et Hartwall: Florae Fennicae breviarium. Abo 1821.

Skizze der Pflanzenwelt in Liefland; Rede, gehalten von dem Grafen Bray.

Abhandl. der literar. Gescllschaft von Curland II. 1822. p.9t-100.

V. S. Besser, M. D., Prof. Zoologiae et Botanices etc. Enumeratio plantarum hucusque in Volhynia, Podolia, Gub. Kioviensi, Bessara- 


\section{$-186$}

bia cis-tyraica et circa Odessam collectarum, simul cum olservationilus in Primitias Florae Galiciae austraciae. Vilnae typis Josephi Ziawadski $18 \% 2.8$.

Pflanzen um Wilna.

Bot. Zeit. 1821. II. p. 683.

Plantae ueranieac descriptae a D. de Schrank.

Das. 1822 II. p: 641.

Ledebour: Decades sex plantarum novarum in imperio rossico indigenarum.

Mémoires de $\boldsymbol{V}$ Acad. de Petersb. V. p. 514. vitch.

Liste der Pflanzen der Flor von Moskau, von Herrn Maximop. 203.

Neues Magaz. der Naturgeschichte Nro. XI. Moskau Nov. 1826.

Nomenclatur der Pflanzen, die im Distriet von Vologda wachsen, vom Herrn Prof. Fortounatof.

Das. $p$. 207. ning.

Observationes de plantis Tanaicensibus, auctore Joanne Henp. 63.

Mémoires de la Soc. imper. des Naturalistes de Moscou Tom. VI.

Ueber die Flora von Petersburg, von Nikolaus Sehtscheglow. St. Petersburger Zeitschrift 1824. Juli p. 111.

Verzeichnifs cier um Petersburg vorkommenden Pflanzen, von J. B. Longmite.

Annals of philosophy 1823. p. $191-197$.

Catalogue des plantes qui croissent spontanément dans le district de Dimitrieff sur la Svapa, dans le gouvernement de Koursk, par F. M. S. V. Ha efft, Doct. en Med. ete. Moscau 18\%(\%. 8. XXIII und 66 Seiten.

Moskofiks Telegraph Nro. 6. Mars 1826. p. 156. - Linnaea 1828. Lit. p. 146.

Liste der in Curland, Liefland und Esthland gefundenen Pflanzen, von Herm Fleis cher.

Bulletin de la Soc. imperiale des naturalistes de Moscou. Ann. 1829. p. 74 .

\section{H. Griechenland.}

J. Dumont D'Urville. Enumeratio plantarum, quas in insulia Archipelagi aut littoribus Ponti Euxini anni 1819 et 1820 collegit atque detexit: Paris 1822. 8.

M. T. Pieri: Flora Corcirensis. Centuria prima et secunda, sive cnumeratio ducentarum plantarum, quas in insula Corcira invenit. Corcirae 1824. 8. c. tal). aen.

Beobachtungen über die Pflanzen, welche in der Gegend von Constantinopel wachsen, deren Saamen eingesammelt und an die Londner Gartenbau - Gesellschaft von Herrn R. W al s ch eingeschickt wurden. P. 1. $p$. 32.

Transactions of the horticultural Society of London. Vol. VI.

Some account of a Collection of Cryptoganic plante from the Joians islands. By Rob. Kaye Greville. 
Transactions of the Linnean Society of London. Vol. XV. P. 2. London 1827. p. 335. - Siehe Linnaea 1828. III. p. 131. Lit.

\section{Asien.}

Flora altaica. Scripsit D. Carol. Fridericus a Ledebour, adjutoribus C. A. Meyer et D. Al. a Bunge T. 1. Classis I-V. Berolini 1829. $3 \mathrm{fl} .36 \mathrm{kr}$.

J. H Rudolph. Dissertatio exhibens novissimas plantas Sibiriae orientalis : Campanula expansa, Sedum cyaneunt, Pedicularis longiflora. Mémoires de l'Acad. de Petersbourg IV. p. 339.

Diagn oses plantarum minus cognitarum e Flora Sibirica Gmelini, a Prof. Tauseh.

Bot. Zeit. 1828. II. p. 481.

Herr Szowits untersucht, beauftragt von der russischen Regierung, in botanischer Hinsieht den Landstrich zwischen dem See Ormiah' und den Gebirgen von Kurdistan, hauptsächlich die Umgegend der Stadt Khoi in der Provinz Aberbeidjan. Er hat eine beträchtliche Pflanzen-Sammlung mitgebracht, die Herr Fisch er in Petersburg ordnet, und worunter 200 neue Arten sein sollen. Die Gegend ist besonders reich an Dolden.

Bibl. universelle Juillet 1829. p. 248.

Vegetation von Georgien und Armenien, nach Belanger.

Bulletin des Sc. natur. Fevr. 1829. p. 267. - Botan. Literaturbl. II. p. 282.

Berggren: Gewächse in Morgenlande, mit einem Vorworte von Göran Wahlenberg.

Isis. Bd. XXI. Heft 10. 1828. p. 971 u. d. f.

Will. Roxburgh: Flora Indica; or descriptions of Indian plants. edited by $W$. Carey. To which are added descriptions of plants more recently diseovered by N. Wallich. Vol. I. Calcutta 1820. Vol. II. Serampore 1824.

Siehe Isis Bd. XXI. (1828), p. 835.

Einige Nachrichten über die Pflanzen versehiedener Theile Indiens und über die Sanscritnamen dieser Gegend von Francis Hamilton.

Froriep's Notizen Nro. 228. August 1825.

Benj. Heyne: Novae plantarum species, praesertim Indiae orientalis. Cum deseriptionibus et observationibus, auctore All. Guil. Roth. Halberst. 1821. 8.

Col ebrooke über verschiedene indische Pflanzen, Sabia, Strychnos u. 8. w.

Isis Bd. XVII. p. $\mathbf{8 1 5}$.

Plantae asiaticae rariores; or Descriptions and Figures of a Select Number of unpublished East Indians Plants. By N. Wallie h, M. D. 3 Vol. Fol. London 1829 . $300 \mathrm{pl}$. col.

Nachrichten von den Früchten und Blumen Indiens ans der Geschichte und Statistik Hindostans, von Mir-Cher-Ali-Afsos entlehnt.

Blätter für literar. Unterhaltung 1830. Nro. 155. p. 538. 


\section{$-18 S$}

Conmentarien über das IIerbarium amboinense des Rumphius; von F. Hamilton.

Memoirs of the $W^{\prime}$ en. nat. hist. Society $V^{\text {ol. }} \boldsymbol{V}$. p. 307.

Commentarien über den Hortus malabaricus des Rheede, von $\mathbf{F}$. II amiltan.

Aus den Linn. Transactions übersetzt in der Isis in verschiedenen

Bänden. Siehe auch Linnaea Jahrg. 1828. $p$ 76 der Literatur.

Flor von Ava. Mechanies Register 1827. Nro. 34. p. 306. - Bot. Literaturbl. I. p. 72.

Tentamen Florae Nepalensis illustratae, consisting of botanical descriptions and lithographic firures of select Nipal-Plants. By $\mathbf{N}$. Wallich, M. et Ph. Dr. 1 fascicle. Calcutta, and Serampore 182t. Fol. Siche Linnaea Jahrg. 1827. p. 532.

Prodromus Florae Nepalensis, seu enumeratio vegetabilium, quae in itinere per Nepaliam proprie dictam et regiones conterminas Inno 1892 - 3 detexit atque legit Fr. Hamilton (olim Buchanan). Accedunt plantae a D. Wa llich nuperius missae. Secundum methodi naturalis normam disposuit atque descripsit $\mathbf{D a v i d} \mathrm{Don}$. Londini 1825.8. Siehe Linnaea Jahrg: 1826. p. 1503.

Biidragen tot de Flora von Nederlandsch Indic. Beiträge zur Flora des holländischen Antheils von Ostindien; herausgegeben von C. L. Blume, M. D., Director des bolanischen Gartens zu Liuitenzorg. Erstes Heft.' Bátavia 1825.

Siehe Geiger's Magaz. Juni 1826. p. 260. - Linnaea 1826. p. 490. - Ueber das zueite Heft Linnaea das. p. 6t3. - Diese Hefte tragen auch den Titel:

Kruidkundige Waarnemingen uitgegeven door C. L. Blume. Batavia 1825. 8.

Beschreibung einiger auf einer Excursion nach dem Berge Salak in Java beobachteten Pflanzen von C. L. B I u in e.

Verhandl. van het Bataviasch Genootschap etc. $t$. IX.

Schetsen uit myne Javansche Portefeuille, dóor G. H. Nagel. Amsterdam 18\%8. 8.

Diese Slizzen enthalten unter andern eine Beschreibung der Flora des ausgebranten Fulkans Tjerimai von Dr. Blume. (Lot. Literatu, bl. II. (p. 282).

Flora Java nec non insularum adjacentium, auctore C. L. Blume, adjutore J. B. Fis che r, M. D., cum tabulis lapide acrique incisis. Fascicul. 1 et 2. Bruxell. 1828. Fol.

Siehe Bot. Literaturbl. III. p. 606.

Enumeratio plantarum Javae et insularum adjacentium minus cognitarum vel novarum ex herbariis Reinwardtii, Kuhlii, Hasseltii et Blumii; auctore C. I. BI um e. Leydae 1827.

Enumeratio plantarum Javae et insularum adjacentiun minus eognitarum vel novarum ex herbariis Reinwardtii, Kuhlii, Iasseltii et Blumii. Curavit Ca rol. Lud. BI ume, M. D., Naturae nuper investigator in Coloniis belgicis Indiarum Orientalium ibique rebus medicis pracpositus, horti botanici Bogoriensis Directore etc. Fasciculus 1. Edition. novae. Hagac apud fratres F. et H. Hartmannum Bibliopolas 1830. Fascicul. II. 1830. 3 fl. $36 \mathrm{kr}$.

Florula Javanica; praeside Thunberg. P. 1. resp. L. Winberg. P. 2. resp. F. 0. Widmark. Upsal 1825. 4. 
Enumeratio plantarum cryptogamicarum Javae et insularum adjacentium quas a Blumio et Reinwardtio collectas describit edique curavit C. Nees ab Esenbeck, Prof. Wratislav. Fasciculus prior, Hepaticas complectens, ab editore illustratas. Vratisl. 1830. 4.

Icones plantarum sponte in China nascentium e bibliotheca Braamiana excerptae. London 1821. gr. Fol. (30 col. Tafeln in Steindruck).

Plantarum japonicarum norae species. Dissert. praeside Thunberg. Upsaliae 1824.

\section{K. Afrika.}

A da m s afrikanische Pflanzen.

Isis : Band 1.

Alire Rafeneau Delille; Mémoires botaniques extraits de la Description de l'Fgypte. 1 Vol. Fol. Paris 1813.

De lille, wilde Pflanzen in Aegypten; siehe Isis III. p.1161. Angebaute Das. 1315 und 1512.

Centurie des plantes d'Afrique du voyage à Mérae, recueillies par M. Fr. Cailliaud, décrites par M. Raffeneau-Delille, Prof. de botanique à Montpellicr. Paris 1826. 8 , avec $3 \mathrm{pl}$. lithogr.

Siehe Linnaea 1828. Lit. p. 125.

Ehrenberg. Vegetation in Aegypten.

Bot. Zeit. 1s22. II. p. 692.

Die Oase von Siwa, nach Caillaud.

Bot. Literaturbl. I. p. 35 !.

Vegetation von Sennar, von Broechi. Biblioteca italiana April 1823. - Bot. Literaturbl. I. p. 352.

Dominicus Viviani; Florae lybicae specimen, sive plantarum enumeratio, Cyrenaicam, Pentapolim, Magnae Syrteas desertum et regionem 'Tripolitanam incolentium. Genuae 1824. cum 27 tabulis.

Siehe bot. Zeit 1827. II. p. 93. Beilage.

Narrative of Travels and Discoveries in northern and central Africa 1822 - 1824. By Major Denham, Capitain Clapperton and the late Dr. Oudney. London 1826.

Mit einem botanischen Anhang von Robert Brown; siehe Isis 1829. Heft 12. p. 1254. Dieser ist betitelt:

Observations on the Structure etc. Beohachtungen über die Structur und Verwandtschaft der wichtigsten Pflanzen, welche von dem Dr. Ondney, Major Denham und Capitain Clapperton in den Jahren 1822, 1823 und 1824 während der Expedition zur Kenntnifs des innern Afrika gesammelt wurden; von Robert Brown. London 1826.

Siehe Linnaea Bd. II. p. 283 u. d. $f$.

O b servations sur quelques Legumineuses de l'Afrique centrale, par M. Robert Brown. (Parkia africana etc.).

Annales des Sc. naturelles. Févr. 1827. p. 206.

Beschreibung der von dänischen Naturforschern, und namentlich vom Staatsrath Thonning an der Küste von Guinea gefundenen Pfianzen, von Prof. F. G. Schumacher. Koppenhagen 1827. (In dänischer Sprache).

Systematische und geographische Bemerkungen über die von Herrn Prof: Christian Smith in der Nachbarschaft des Congo-Stroms gesammelten Pflanzen.

Rob. Brown, vermischte botan. Schriften Bd.I. p. 167 u. d. f. 


\section{K a p.}

C. P. Thunberg. Flora Capensis, sistens plantas promontorii bonae spei Africes secundum systema sexuale emendatum etc. edidit et pracfatus est J. A. Schulte s. Vol. II. Stuttgardtiae 1823: 8.

\section{E. F. Jarosz Plantae capenses. Berolini 1823.}

Plantarum capensium descriptiones ex schedis derelictis Berginnis. Commentatur D. F. L. de Schlechtendal.

Linnaea 1. Heft 2. p. 250.

Adumbratio plantarum (Capensium) edidit D. F. L. de Schlechtend a l. Berolini 18²5. Fascicul. IV. c. tab. aen. 4.

Topographisches Verzeichnifs der Pflanzensammlung von C. F. Ecklon. Erste Lieferung, oder Standorte und Blüthezeit derjenigen Arten, welche bis jetzt auf dem Vorgebirge der guten Hoffnung beobachtet und gesammelt worden sind, von C. F. Écklon. Efslingen 1827. 8. nebst Grundrifs.

Siehe Isis 1828. p. 265. - Linnaea III. p. 6. Lit.

Plantarum capensium Species novae Dissertat. praeside Thunb erg. Upsaliae 1824 .

\section{Afrikan. Inseln.}

Excursions dans les iles de Madera et de Porto Santo; faites dans l'automne de 18ะ3 pendant son troisième royage en Afrique, par T. E. Bowdich ete. ouvrage traduit de l'anglois et accompagnés de notes par M. le baron de Cuvier et de Humboldt. Paris 1826. 8. avec un atlas en 4.

Excursion nach dem Pico Ruivo auf der Insel Madeira; vom Herrn Friedr. Holl in Dresden.

Bot. Zeit. 1829. II. p. 689.

Verzeichnifs der auf der Insel Madeira beobachteten Pflanzen, nebst Beschreibung einiger neuen Arten; von Demselben. p. 130.

Daselbst 1830. I. p. 369; auch sehe man in demselben Bande

Ueber die Flora von Teneriffa, von Berthelot, Director der gelehrten Schulen $z u$ Oratova.

Hertha Bd. XI. Heft 4. p. 109; der geograph. Zeitung. April 1828. - Bot. Literaturbl. I. p. 345.

Derniers momens de Napoleon, ou complément du mémorial de St. Heléne, par le docteur F. A u toma rché. Bruxelles chez H. Tarlier. 18\%5. 2 Vol. 8 .

Im zueiten Bande ist von p. 255-425 eine Esquisse de la Flore de St. Heléne enthalten.

Vegetation von Madagaskar; aus einem Schreiben von L. T. Hilsenberg an Sieber.

Nouvelles Annales des voyages. Fívr. 1829. - Bot. Literaturbl. I. $p .348$.

Some account of the island of Tristan da Cunha and of its national productions, by Dug. Carmichacl.

Transact. of the Linn. Society. T. XII. P. 2. - Enthäl auch eine Flor der Insel. 


\section{1 \\ L. Amerika. \\ Polar. Länder.}

Vorläufige Nachricht von den Pflanzen der Melville-Insel, einer vom Capit. Parry bei der Nord-Pol - Expedition entdechten Insel. Von Herrn F. S. Voigt, Prof. der Botanik zu Jena.

Bot. Zeit. 1821. I. p. 201.

Flora der Melville-Insel von R. B rown, nach dem Englischen bearbeitet und mitgetheilt von Herrn Prof. Kunze in Leipzig.

Daselbst 1824. II. zweite Beilage.

Chloris Melvilliana. London 1823. 52 pag. 4.

Verzeichnifs der Pflanzen, welche von den Officieren der Englischen Expedition zur Entdeckung einer nordwestlichen Durehfahrt auf der Melleville's - Insel gesammelt wurden; nebst den Characteren und Beschreibungen der neuen Arten.

Rob. Brown's vermischte botan. Schriften Bd.I. p. 357 u. d. f.

Verzeichnifs von Pflanzen, welche an den Küsten der Baffins-Bay zwischen $70^{\circ} 30^{\prime}$ und $76^{\circ}$ nördlicher Breite an der Ostseite, und der Possessions - Bay bei $73^{\circ}$ nördl. Breite an der Westseite gesammelt worden sind.

Daselbst p. 337.

Verzeichnifs in Spitzbergen gefundener Pflanzen.

Daselbst p. 864.

Verzeichnifs von Pflanzen von der östlichen Küste Grönlands, mit einigen Bemerkungen von Dr. Hooker, Prof. der Botanik zu Glasgow.

\section{Daselbst $p$. 351.}

Auszug aus den Briefen des Dr. Richardson Drummond und Douglas, über die Expedit. des Kapit. Franklin und über die Vegetation im Norden von Amerika, von Hooker.

Edinb. Journ. of Science Nro. XI. Jan. 1827. p. 107.

Vegetation der nordamerikanischen Polarküste.

Narrative of a second expedition to the shores of the Polar Sea,

by J. Franklin. London 1828. - Bot. Literaturbl. II. p. 43.

Botanischer Anhang zu Capit. Franklin's Bericht von einer Reise nach den Küsten des arktischen Meeres von John Richardson mit Nachträgen von Rob. Brown; auch betitelt: Richardson's Flora der Polar - Länder.

Vermischte botan. Schrift. von R. Brown. Bd.I. p. 465 u.d.f.

Aufzählung einiger Pflanzen aus Labrador, von Herrn geh. Rath v. Schrank.

1- 30.

Denkschriften der Kön. bot. Ges. zu Regensburg 1818. Seite

Ernest. Meyer. De plantis labradoricis Libri tres. Lips. 1830. 8.

Uebrige nordamerikanische Länder.

A Flora of North America, by W. P. C. Barton. 3 Vol. 4. 1825.

Flora boreali-americana, or the Botany etc.; by William Jackson Hooker. London 1829. with plates.

Siche bot. Literaturbl. IV. p. 146. - Linnaca V. p. 101. Lit. 
Specimen Florae Americae septentrionalis cryptogamicae, sistens musces hepaticos ete. Anctore Lewis, D. de Schweinitz. Raleigh 1821. 8. $27 \mathrm{~S}$.

Siche Linnaea 1829. I. p. 41. Lit.

Verzeichnifs der Farrenkräuter und Moose der vereinigten Staaten Nord-Amerika's, ven L. W. Beck.

Silliman's Americen. Journal XY.p. 287. 1829. - Bot. Literaturbl. II. p. 309.

A. Eaton. Manuel of botany for the northern and middle states of America 3 ed. 12. Albany 1822.

A Compendium of the Flora of the northern and middle states: containing generic and specific descriptions of all the plants, exclusive of the Cryptogania, hitherto found in the United States, north of the Patomac. By John Torrey, M. D., Professor of Chemistry in the West Point Military Academy.' New York 18\%6. 12 kr.

Flore de Terre neuve et des iles Saint Pierre et Miclou, avec figures dessinées sur la plante vivante. Lirr. 1. Paris 1529. 4.

Florula Bostoniensis, auctore Jacobo Bigelow, M. D. 2 edit. 1 Vol. 8. 1821.

W. P. C. Bart on Compendium Florac Philadelphiae: 2 Vol. Philadelphia 1824.

Ueber eine frühere Bearbeitung der Flora von Philadelphia siehe Jahrb. der Gewüchsk. Heft 3. p. 181.

Liste seltener in der Gegend von Easton in Pensylvanien gefundenen Pflanzen, von M. L. de Schweinitz.

Amer. Journ. of Science and arts Aug. 1821. p. 267.

Flora Cestrica, an essay towards a Catalogue of the phaenogamous plants, native and naturalized, growing in the vicinity of the borough of West Chester, in Chester County, Pensylvania : with brief notices of their properties and uses in medicina, rural economy and the arts etc. By William Darlington, M. D. West Chestere Penn 1826. 8. Paris Arthur, Bertrand.

Steph. Elliot, A sketch of the botany of South - Carolina and Georgia in two Volumes. Char Astown 18\%1. 8.

Lis te seltener in Alabama gefundener Pflanzen, von Melines C. Leavenworth. Mitgetheilt in einem Briefe ven Dr. Karl II ooker.

P. Silliman American. Journal Fol. IX. Nro. 1. February 1825.

p. 74. - Auch sehe man daselbst Vol. VII. Nro. 1. Nov. 1823. 1817.

Flora of Louisiana by Robin and Rafinesque. New York

Siehe hierüber und über Robin's Reise nach Louisiana, deren dritter Theil auch eine Flora von Louisiana enthäl, Jahrb. der Gewächskunde Heft 2. $p$. 165 .

Contributions towards the Botany of the States of Illinois and Missouri; ly Lew is C. Beck, M. D. (Prof. der Botanik zu Albany).

silliman American. Journal. Vol. IX. Juni 18\%5. p. 167. Vol. X. Nro. 2. Febr. 1826. p. 257-264. Vol. AIV. Nro. 1. April 1828. p. $112 u$. d. f. - Die Pflanzen sind nach Linne's System geordnet, und reichen bis zur Monodelphia.

A description of some new species of plants, recently introduced 
into the gardene of Philadelphia, from the Arkansa territory. By Thomas Nuttall.

1829. p. 39. Lit.

Catalogue of Plants etc. Catalog von Gewächsen, die auf einer Reise nach den Felsenbergen (Rocky Mountains) im Sommer 1820 gesammelt wurden, von L. P. James, Begleiter der Expedition des, Major Long, Ingenieur der vereinigten Staaten von _Amerika, mit Beschreibungen vou. John Torrey.

Transactions of the Americ. philosoph. Society Vol. II. new Series

p. 172. - Annals of the Lyc. of Nat. Hist. of New York Dec. 1826.

März et Juni 1827. - Siehe auch Bot. Zeitung 1827. I. p. 5 und 18.

P. de la Liave et J. Lexarga, nororum regetabilium descriptiones fascicul. I 'et II. Mexici 1824 et 1825. (Lipsiae 1 Rthl. $16 \mathrm{gr}$.).

Siehe Bot. Literaturbl. I. p. 245.

Plantarum mexicanarum a cel. viris Schiede et Deppe collectarum recensio brevis auctoribus $D$. de Schlechtendal et Ad. de Chami sso.

Linnaea 1830. I. p. 72 . II. p. 206. IV. p. 554.

Descriptiones plantarum Norae Californiae, adjectis florum exoticorum analysibus anct. J.' Fr. Escholtz.

Mím. de l'Acad. imp. des Sc. de Petersbourg. X. p. 281. 1826. -

Bot. Literaturbl. I. p. 385. - Linnaea 1828. III. p. 149.

\section{Westindien, Antillen.}

Prodromus plantarum Indiae oecidentalis hucusque cognitarum, tam in oris Americae meridionalis, quam in insulis Antillicis sponte nascentium, aut ibi diuturne hospitantium; Nova Genera et species hactenus ignotas complectens. Digessit Guil. Hamilton, M. B. Londini 1825. 8. c. tab. aen. pict. (Lipsiae 1 Rthl. $16 \mathrm{gr}$.).

Siehe Linnaea III. p. 23. Lit.

Flore des Antilles ou Histoire générale botanique rurale et économique des végétaux indigènes de ces iles et des exotiques, qu'on est parvenu a y naturaliser, décrits d'après nature et classés selon le système sexuel de Linné et la methode naturelle de Jussieu, enrichée de planches color. par le cheralier F. R. de Tus sac. Tom. III. Paris 1826. Erscheint in Lieferungen, seit 1848.

Sketches towards a Hortus botanicus americanus: or coloured Plates with a catalogue and concise and familiar. Deseriptions of many Species of new and valuable Plants of the West-Indies and of North and South-America etc. by W. J. Tittford, M. D. London 1826. in 4 .

Flore pittoresque et medicale des Antilles, on Histoire naturelle des plantes usuelles des Colonies françaises, anglaises, espagnoles et portugaises, par M. E. Descourtilz. Paris. 1828. 8.

Bereits sind 110 Lieforungen davon erschienen; bis zur 5tsten trug

das Werk auch den Titel: Flore médicale des Intilles.

Florula Insulae Sti Thomae Indiae occidentalis, concinnata a D. F. L. de Schlechtendal.

Linnaea 1828. p. 251.1829 . I. p. 78. II. p. 177. 1830. IV. p. 682.

Öfrersigt af ön Sanct Barthelemis Flora af Joh. Em. Wickströ m.

Kongl. Vetenskaps-Academiens Handlingar för är 1825. Stockholm 182(j. p. 411. - Linnaea 1828. p. 106. Lit. 
Öfversigt af in Goundeloupe's Flora af Joh. Em. Wikström.

K. Tetenskaps Academ. Handlingar 1827. St. 1. p. $51-79$. Linnaea 1830. III. p. 89. Lit. - Isis 1830. Ileft 1. p. 35 u. d. f.

\section{Süd - Amerika.}

Synopsis plantarum, quas in itinere ad plagam aequinoctialen orbis nevi collegerunt Alex. de II umboldt et A. Bonpland; auctore C. S. K u nth. Tom. I - IV. Paris 1822 - 1825. chez Levrault.

Synonyma ad plantas Humboldtianas e Mantissa tertia Roemeri et Sehultesii relata a Caról. Sigism. Kunth.

Linnaea 1830. Heft 3. $p$. 366.

Voyage de MII. IIumboldt et Bonpland. Partie botanique in fol. Chez Gide fils. Livr. XXXIV. 100 franes; planches en couleurs 180 francs; grand colombier $200 \mathrm{fr}$. in $4 .-36 \mathrm{fr}$.

Reliquiae Iaenkeanae seu Descriptiones et Icones plantarun, quas in America meridionali et boreali, in Insulis Philippinis et Marianis collegit Thaddaeus Haenke. Pragae 1825.

G. F. G. M eyer Primitiae Florac Essequeboensis aljectis descriptionibus centum circiter stirpium novarum observationibusque criticis. Gotting. 1818. 4 .

Plantarum Surinamensium corollarium primum edidit Ernestus Meyer.

Nova Acta Acad. Caesar. Leopold. Tom. XII. P. 2. p. 759.

\section{Brasilien.}

His toire des plantes les plus remarqualles du Brésil et du Paraguay, comprenant leur description et des dissertations sur leurs rapports, leurs usages etc. avec des planches noires ou color., par M. A. de Saint Hila ire. Paris 1824. 4. 3 Vol.

Frscheint in Lieferungen zu 5-6 Blättern Text und eben so viel Kupfertafeln.

Flora Brasiliae meridionalis, auct. Aug. de St. Hilaire. Accedunt tabulae delineatae a Turpinio aerique incisae. Paris 18\%. 4. chez Belin.

Erscheint gleich dem vorigen in einzelnen Heften.

Tableau monographique des plantes de la Flore du Brésil meridional, appartenant au groupe, qui comprend les Droseracées, les Violacées, les Cistinées et les Frankeniées; par M. A ug. de Saint Hilaire.

Mémoires du Muséum d'Hist. nut. Vol. XI. p. $335-853$ et 445 -498; ferner. Vol. XII. p. 75 - 91.

Rapport verbal sur la Flore du Brésil meridional de M. $\mathbf{A}$ ug uste de Saint Hilaire par M. Alexandre de II umboldt. Annales des Sc. naturelles. Oct. 1825. p. 222-224.

Flora Brasiliae meridionalis; auctoribus Angusto de Saint Hilaire, Adriano de Jussieu, Jacobo Cambessèdes; accedunt tabulae delineatae a Turpinio aeriqne incis. Parisiis 1827. Auch davon sind mehrere Hefte erschienen.

Note sur les principales éspèces de Sida de la Flore du Brésil meridional, par M. A ug. de St. Hilaire.

Bulletin des Sciences natur. Févr. 1827. p. 278. 
Mémoire sur la Série lineaire des plantes polypetales, et en particulier de celles qui font partie de la Flore Brasilienne, par M. Aug. de Saint Hilaire.

Mémoire du Muséum d'Hist. nat. Sept. Année, Huit. Cahier. p. 120 et suiv.

Crucifera rum, Elatinearum, Caryophyllearum Paronichiearumque Brasiliae meridionalis Synopsis. Auct. J. C a m b es sè de 8. Paris 1829.

Portulacearum, Crassulacearum, Ficnidearum, Cunoniacearumque Brasiliac meridionalis Synopsis, auct. J. Cambessèdes. Paris 1829. 8. $4 \mathrm{~S}$.

Fnthält die neue Gattung Belangera aus der Familie der Cunonaceen.

Plantarum brasiliensium Decas tertia. Dissertat. praeside Thunberg, respond. C. O. Holm. Upsaliae 1821. in 4. c. tab.

D. C. F. P. de Martius Genera et Species plantarum, quas in itinerc per Brasiliam annis $1817-1820$. depingi curavit etc. Fasc. I et II. cum 49 tab. Imp. Fol. Mouachii 1824.

Ejusdem Nova genera et species plantarum, quas in itinere per Brasiliam annis 1817 - 1820. collegit et descripsit. Pingendas curavit et secund. auctoris schedulas dig. D. J. G. Z u c carini. Vol. I. Monachii 1S24. Vol. II. 1826. Vol. III. 1829. Fol.

Siehe bot. Zeit. 1826. I. p. 358. 1827. I. p. 97 und 369. - Linnaea 1830. p. 34. Lit.

C. F. Ph. Martius. Plantae novae, quas in itinerc jussu et auspiciis Max. Jos. Bav. regis per Brasiliam suscepto observarit et depingit. Monach. 1817.

Fiora Brasiliensis seu enumeratio plantarum in Brasilia tam sua sponte quam accedente cultura provenientium, quas in itinere auspiciis Maximiliani Josephi I Bavariae regis annis 1817 - 1820: peracto collegit partim descripsit; alias a Maximiliano seren. principe Widensi, Sellovio aliisque advectas addidit, communibus amicormu propriisque studiis secundum methodum naturalem dispositas et illustratas edidit C. F. Ph. de Martius. Vol. II. Pars prior, Gramineae a Necsio ab Esenbeck expositac. Stuttg. et Tüb. 1829, 8 .

$$
\text { Auch unter dem Titel: }
$$

Agrostologia Brasiliensis seu descriptio Graminum in imperio Brasiliensi huc usque detectorum auctore G. G. Neesio ab Esenb e ck etc. etc.

Icones selectae plantarum cryptogamicarum quas in itinere per Brasiliam annis 1817 - 1820 jussu et auspiciis Maximil. Josephi I Barariae regis aug. suscepto collegit et pingendas curavit Dr. C. F. P. de Martius etc. Fasc. I. c. tab. I - XIV. Algae et Lichenes. Monachii 15 April 1828. 4to maj. $30 \mathrm{~s}$.

Siehe Linnaea 1830. III. p. 71. Lit.

Crittogame Brasiliane raccolte e descritte da Giuseppe Raddi. Atti della Societa Italiana delle Scienze, residente in Modena Tom. XIX. 1926.

Raddi Descrizione di 40 piante nuove provenienti dal Brasile. Memoria della Societa italiana. Vol. XVIII.

Plantarum brasiliensium nova genera et species novas rel minus cognitas, collegit et descripsit Joseph Raddius. Pars I. Florentiae 1825 . 
Ueber mehrere von Raddi in Brasilien gesammelte Pfanzen sehe man auch die aus ital. Zeitschriften entlehuten Nachrichten in der Isis Bd. XX. Heft 11. p. 920. Wien.

Neue Brasilianische Gewächse, von Herrn Dr. Pohl in Bot. Zeit. 1827. I. p. 145.

Plantarum Brasiliae Ieones et descriptiones hactenus ineditae. Auct. J. E. Pohl. Fascic. I. Vindobonac 1826. Fol. II. 1827.

Man sehe Linnata 1828. p. 51. Lit. - Bot. Zeit. 1829. I. p. 118.

A ccount of the Poison Plants of the Southern Parts of Brazil.

Fdinburgh Philosophical Journal Vol. XIV.p. 100 und in Nro. XXVIII. p. $264-270$.

Notice sur la Botanique de Brésil.

Bibl. univers. T. XXXII. Nov. 1827. p. 204.

Delectus Florae et Faunae Brasiliensis. Fascicul. III. Vindobonae 1823. Fol.

\section{Peru, Chili u. s. w.}

Zoologische und botanische Beschreibung der Gegend ron Payta und Colon in Peru, ron Lesson. p. 275.

Froriep's Notizen Bd. XY. p. 129. - Botan. Literaturbl. II.

Pöppig. Vegetation von Chili im Mürz.

Botan. Literaturbl. II. p. 278.

Flore des Malouines, par M. Gaudichaud.

Nouveau Bulletin dés Sciences par la Société philomatique de $\mathrm{Pa}$ -

ris. Octobre 1825. p. 156. - Annales des Sc. natur. Mai 1825.

Rapport de M. Mirbel et Desfontaines sur la flore des iles Malouines par M. Ga u di chaud.

et suiv.

Annales des Sc. natur. Mai 1821. p. 89. Decemb. 1825. p. 469

Flore des Malnuines, par M. J. Dumont d'Urrille.

Annales de la Soc. Linn. de Paris. Janv. 1826. p. 578-621.

Account of the natural Productions of Staten Islands, and Cape Horn. By Capitain W. II. B. We bster. In a Letter to John Barrow.

Edinb. Journ. of Science. Jan. 1830. p. 26 u. f. - Brandes Archiv Bd. XXIV. Heft 2. p. 218.

Liste des plantes observés au Chili dans l'année 1828, par le Dr. Bertero.

Aus dem Mercurio Chileno Nro. 12, 13 et 14 Mars, April, Mai 1829 in dem Bull. des Sciences natur. Janv. 1830. p. 105.

Descriptions of the new genera and species of the Class. Compositae belonging to the Floras of Peru, Mexico and Chile. By/David Don.

Aus dem 16. Bande der Transactions of the Linn. Societ. in der Linnaea V. p. 169. Lit.

\section{Australien.}

Allgemeine geographische und systematisclte Bemerkungen über dic Flora Australiens. 
Robert Brown vermischte botan. Schriften Bd. I. p. 3-166. Der dritte Theil dieser Schriften enthält einen abermaligen Abdruck des ersten Theils des bekannten Prodromus Florae Novae Hollandiae.

La Billardière Sertum Austro-Caledonicum Pars prior 1824, pars posterior 18\%5. Paris, Strasbourg, London. 4 maj.

Flora australasica, or the plants of New Holland and the SouthSea Islands. Each number will contain four faithfoully coloured figures with magnified dissections of their most essential parts, their names, descriptions and a full account of the best method of cultivation and propagation. By Robert Sweet etc. London 8. Nro. I. June 1827. XII. Mai 1828.

Linnaea 1829. I. p. 25. Lit.

Icones lithographica e plantarum Australasiae rariorum Decades duae, quas Botanicis affert J. B. A. Guillemin. Paris 1828. 4. m. K.

Beschreibung mehrerer neuer Pflanzen-Gattungen aus NeuHolland, yon $\mathbf{E}$. R udge.

Limn. Transactions XI. P. 2. - Isis 1829. Heft 2. p. 1 H.

Mertens. Bemerkungen über die Vegetation der Südsee-Inseln.

Froriep's Notizen Bd. XXIV. Nro. 507.

Vegetation von Neu - Süd - Wallis.

Aus Cunningham's Two years in New South Wales. London 1827. - Botan. Literaturbl. I. p. 343.

On two kinds of Timber Trees peculiar to New Zeeland.

Fidinburgh Philosophical Journal Nro. XXII. Octob. 1825. p. 378.

Es ist hier die Rede von Dammara australis und Dacrydium taxifolium Solander.

\section{Vierter Abschnitt.}

\section{Anatomie und Physiologie des Gewächsreiches.}

\section{§. 26.}

Auch dieser Zweig der Pflanzenkunde ist mit grofsem Eifer und Vorliebe bearbeitet worden, wie schon die nachstehenden kurzen literarischen Nachrichten zur Genüge zeigen, dennoch ist gerade die Anatomie und Physiologie der Pflanzen noch am wenigsten aufgeklärt, kein anderer Zweig der Botanik ist noch mit so vielen Hypothesen und gewagten Schlüssen überladen, in keinem andern bleiben noch so viele Zweifel zu lösen, so viele Fragen zu beantworten, so viele Desideranda übrig, die auch den eifrigsten und gewandtesten Naturforschern noch Jahrhunderte lang Beschäftigung gewähren dürften. 


\section{Schriften und einzelne Aufsätze über die Structur} und die Function der Pflanzentheile.

\section{s. 27.}

Commentatio de fontibus vegetationis plantarum. Auctore Eug. Dạvid s. Lug d. Batáv. 1822.

Colloquia de rehus praecipuis physiologiae Vegetabilium atque Botanices, quaestionibus et responsis ad isum studiosae juventutis accommodata, auctore Archibaldo Robertson. Lovanii 1822. cum tabul. 4.

Link Elementa Philosophiae botanicae cum tahulis aeneis quatuor. Berolini 1821.

Essai sur la metamorphose des plantes; par J. W. de Goethe. Trad. de l'allemand sur l'édition originale de Gotha (1890), par M. Fréd. de Gingins Lassaraz. Genève 1829.

J. W. Gothe. Zur Naturwissenschaft überhaupt, hesonders zur Morphologie, Erfahrung, Betrachtung, Folgerumg, durch Lebenscreignisse verbunden. I. Bd. Tübingen 1820. Bd. II. Heft 1. 1823. p. 991.

Ueber Göthe's botanische Arbeiten sehe man auch Isis Zd. II.

G. D. Ki e ser Mémoire sur l'organisation des plantes. Harlem 1822. 4. avec 12 pl. et $345 \mathrm{p}$.

Mlan sehe auch Isis Bd. XII. p. 371.

Die Pflanzen und das Pflanzenreich. Nach einer neuen natürlichen Methode dargestellt, von Dr. Carl Heinrich Schulz. Auch unter dem Titel: Die Natur der lebendigen Pflanze. Erweiterung und Bereieherung der kintdeckungen des Kreislaufs im Zusammenhange mit dem ganzen Pflanzenleben u. s. w. Frster Theil, das Leben des Individuums. Mit 4 Kupfertafeln. Berlin 1823. Zweiter Theil, die Fortpflanzung und Ernährung. Mit 3 Kupfertafeln. Stuttgart und Tübingen 1828.

Lettre de M. C. H. Sehulz, Prof. is Berlin à M. Decandolle, contenant un resumé de ses obserrations sur la Physiologie végétale.

Biblioth. universelle Tom. XXXVI. Nov. 1827. p. 18t-201. Dec. p. $297-310$.

Betrachtung des Pflanzenreichs, oder Erklärung des Wachsthums und der Ausbildung der Pflanzen. Nelsst einem Anlange über die ursprüngliche Entstehung der Gewächse; ron C. M. Klinkhardt. Berlin 1828. Auf Kosten des Verf. 2 fl. 24 kr.

Erfahrungstheorie der Pflanzen- und Thier-Production, nebst Anwendung derselhen zu Feststellung sicherer Grundregeln für den Feldbau und die landwirthschaftliche Thierzucht.

Aus den Erscheinungen der Aatur, so wie sich solche den Beobachtungen in der Chemie in angestellten Versuchen und in den Firfolgen der Lundlwirthschaft darbieten, begleitet und entwickelt von W. A. Kreyssig, einem Ostpreufsischen Landwirthe. Zwei Theile. Königsberg 1828. Im Verlage der Gebrüder Bornträgerer. 6 f $f .36 \mathrm{kr}$.

De Organis Plantarum. Seripsit Joannes Raeper, Med. D., Botan. Prof. Basil. Basilaae 1828. 4. typis Augusti Wielandi.' tafeln.

F. J. Ferd. Meyen. Phytotomie. Berlin 1830, 8. Mit 14 Kupfer-

Bemerkungen zur Pflanzen-Physiologie, aus einem Briefe des Dr. Westrumb, ausübendem prakt. Arzte in Hameln, an Prof. Kastner. Dessen Arehiv Bll. VII. Heft 3. p. 351. 
Einige Bemerkungen über den Bau der Pfanzen besonders im Vergleich mit dem der Thiere; vom Herrn Prof. Ritgen in Giefsen. Bot. Zeit. 1828. I. p. 241.

Beiträge zur Anatomie, Physiologie und Organographie der Gowåchse; von Herrn Dr. Med. Bald. Kittel in München.

Bot. Zeit. 1828. II. p. 657. Jahrg. 1830. I. p. 81.

Fragmente aus der Pflanzen-Physiologie des sechzehnten Jahrhunderts; von Dierbach.

Geiger's Magazin Bd.VIII. p. 121.

Versuch über die Trennung oder Vervielfältigung der Organe der Gewächse. Von Herrn Moquin-Tandon.

Bibliothèque universelle Mars 1827. - Froriep's Notizen Bd.

XVII. Nro. 372.

Recherches anatomiques et physiologiques sur la structure interne des animaux et des régétaux, par M. D u troch et. Paris 1821.

L'agent immediat du mouvement vital, dévoilé dans sa nature et son mode d'action chez les végétaux et les animaux, par M. H. Dutroch et. Paris 18\%4. chez Baillière.

Siehe botan. Literaturbl. I. p. 461.

M. H. Dutrochet: nourelles recherches sur l'Endesmose et l'Exosmose, suivies de l'application experimental de ces actions physiques à la solution du problème de l'irritation végétale et à la determination de la cause de l'ascension des tiges et de la descente des racines. Paris 1828. 8. chez Baillère. 106 pages.

Cours de Phytologie, on de Botanique générale par lo Chev Aubert du Petit-Thouars. Paris 1824.

Siehe Bull. des Sc. natur. Mars 1824. p. 38.

Précis d'Anatomie végétale, par M. Romain Feburier. Versailles et Paris 1824. 8. 71 pages.

Physiologie végétale par C. L. Leschvin. Paris 1825.

Opuscules phytologiques par M. Henri Cassini. Premier Recueil. II. Vol. Paris 1826. 8. avec planches.

Im zweiten Bande befindet sich eine Abhandlung über die Phytonomie, enthaltend allgemeine Betrachtungen über den Bau der $V^{\prime}-$ getabilien, besonders anatomische Beobachtungen über Borago officinalis.

Organographie végétale, ou description raisonnée des organs des plantes, pour scrvir de suite et de développement à la Théorie élémentaire de la Botanique, et l'introduction à la Physiologie végétale et à la description des familles; avec 60 planches en taille douce, par A ug. Pyr. De Candolle. Paris chez Deterville 1828.

Davon ist eine deutsche Uebersetzung vorhanden, von Dr. C. F. Meisner besorgt, unter dem Titel: Aug. Pyramus De Candolle's Organographie der Gewächse oder kritische Beschreibung der Pflanzen - Organe etc. Stuttgardt und Tübingen 1828. 2 Bände 8.

Nouveau Manuel de Botanique, ou Principes élémentaires de Physique végétale; par M.M. J. Girardin et J. Juillet. Paris 1827. 8. avec pl.

Siele bot. Literaturbl. II. p. 148. 
Extrait de l'Analyse des travaux de l'Academic royale des Sciences pendant l'année 1827, par la minéralogie, la géologie, la physiologie végétale et la botanique; par M. le baron Cuvier.

Annales des Sc. natur. Juillet 1828. Tom. XIV. p. 300. - Botanique p. 314.

Aghard: Essai de reduire la plyssiologie végétale à des principes fondamentaux. Lund. 1828. $8 \mathrm{gr}$.

Les Végétanx curieux, ou Recucil du particularités les plus remarquables, qu' offrent les plantes considerées sous leurs rapports naturels etc., par B. Allent. Paris $182 t$.

Résumé complet de Botanique, par Lamouroux. T. II. Physique végétale. Paris $\mathbf{1 8 2 6 .}$

Lettres d'un frère à sa soeur, sur la botanique et-la physiolngie des plantes, par Edouard Rastoin. Paris 1828. in 18. rue des grands Augustins Nro. 18. '3 Fr. 50 C.

\section{Schriften.}

Man vergleiche noch die oben angezeigten propädeutischen

Principes de physiologie comparée on histoire des phènoménes de la vie dans tous les étres, qui en sont doués, depuis les plantes jusqu? aux animaux les plus complexes, par M. J. Bourdon. Paris 1830. 8.

Manuel de Plyysiologie végétale, de Physique de Chimie et de Minéralogie, appliquées à la culture, par M. Poitard. Paris 18:y. In 18 de 356 pag. avec planches.

Popular lectures on the study of natural history and the sciences, regetable Physiology, Zoology etc. by W. Lemprière. London 1827. 8.

Conversations on Vegetable ete. London 1829.

Ueber diese der Pflanzen-Physiologie gewidmete Schrift sehe man Bibliothèque universelle. Sept. 1829. p. $47 u$. d $f$.

On the Functions and Structure of Plants. B B G. F. Burnett Esq.

The Quarlerly Journal of Science Oct. to Dec. 1829. Niro. XII. p. $289-2 y 2$.

Primitive Entwicklung der niedersten regetabilischen Organismen und Ansichten über die Metamorphosen derselben.

๑. 28.

Beobachtungen über die Urformen der niedern Organismen. Yon Georg Friedrich Märklin, Apotheker in Wiesloch $u$. s. w. Heidelberg 1823. 8.

Beiträge zur Kenntnifs der Metamorphose der Gewächse, vornemlich in Hinsicht ihrer innern und äufsern Bedingungen. Von F. G. Gmelin.

Würtemb. Naturwissenschaftl. Abhandl. Bd. I. Heft 1.p. 73-132.

Entwicklungs-Combinationen organischer Wesen, von Dr. Mathias Joseph Bluff. Kölln 1827. $36 \mathrm{kr}$.

Bischoff, über Fintwicklung der Pflanzen-Substanz. Isis Bd. VII. p. 731. 
Entdeckungen über die Entstehung des organischen Lebens, von Dr. A. Koell e, K. Preufs: Finanzrath.

Kastner's Archiv. Bd. XII. Heft 3. p. $318-361$.

Ueber die Anfeinanderfolge des ersten Auftretens der rerschiedenen organischen Gestaliten, rom Prof. Rit gen in Giefsen.

Isis 1828. p. 488. - Man sehe auch das dritle Heft der Mar-

burger Schriften zur Beförderung der Naturwissenschaften.

Wiederbelebung urweltlicher Algen oder See-Tange, beobachtet vom Prof. Dr. Henschel in Breslau.

Kastner's Archiv Bd. XIII. Heft 2. p. 229.

Untersuchungen über die Individualität im Pflanzenreiche, ron M. Ursin.

Lycée Armoricain 6อ̃. Livraison. 11 Vol. p. 178.

Observations sur la formation de la matière verte de Priestley; par M. Maximovitch.

Neues Magaz. der Naturges. Moscau. Januar 1826. No. I. p. 63.

Die Priestley'sche Materie ist nach den Ansichten des Herrn M.

die primitive und unvollständigste Alge.

De la liaison du règne végétal et du règne animal, par le Dr. W. Edward s.

Extrait d'un Mémoire lue à l'Academie des. Sciences le 8. Mai 1826. - Bulletin des Sciences naturelles. Juin 1826. p. 188. Froriep's. Notizen. Bd. XIV.p. 232. in No. 301.

Lettre de M. Desmazières sur l'Animalité de quelques Hydrophytes et des Mycodermes en particulier.

Annales des Sciences naturelles Juin 1828. $p$. 206.

Ueber den unmittelbaren Uebergang des sprossenden regetativen Lebens in das bewegte infusorielle und umgekehrt, und zunächst über die Metamorphose der Ectosperma clavata Vaucher, rom Herrn Dr. F. Unger in Stockerau bei Wien.

Bot. Zeit. 1830 . II. p. 569.

\section{Forschungen über die Elementartheile des Gewächsreiches.}

\section{ङ. 29.}

P. J. F. Turpin. Organographic végétale. Observations sur quelques végétaux microscopiques, et sur le ròle important, que leurs analogues jouent dans la formation et l'accroissement du tissu cellulaire.

Mémoires du Muséum T. XIV. $p .15-67$.

Aperçu organographique sur le nombre deux, consideré comme multiplicateur de $4,8,12,16,32,64$, dans la structure des végétaux d'un ordre inférieur, et dans les parties résiculaires ou élémentaires, dont se composent les masses du tissu cellulaire des régétaux d'ordres plus élevés, suiri de la description de plusieurs genres et éspèces nouvelles très remarquables, découverts parmi des productions végétales et microscopiques, par P. J. F. Turp in.

Mémoires du Musćum 8. année. Cahier X. p. $295-344$.

Organographie microscopique élémentaire et comparée des Végétaux, par P. J. F. Turpin.

Mémoires du Muséum. 9. année. Cahier IX. p. 161. et suiv.

Es ist sehr schwer, wenn nicht unmöglich, den wesentlichen Inhalt dieser drei Abhandlungen mit wenigen W'orten anzudeuten; immer- 
hin gehören sie aber $\approx u$ den interessantesten dieser 50 schwierigen Untersuchungen über den Bau zumal des Zellengewebes im Pflanzenreiche.

Recherches chimiques et physiologiques, destinées a expliquer non seulement la structure et le développement de la feuille, du tronc, ainsi que des organes, qui n'en sont qu'une transformation, mais encoro la structure et le développenent des tissus animaux, par M. Raspail.

Mémoirs de la Société dhistoire naturelle de Paris. Tom. III.

p. 17. et suiv. ferner p. $209-309$.

Ueber den ersten Theil dieser sehr ausgedehnten Abhandlung habe ich bereits in dem Januarheft 1828 von Geiger's Magazin p. 84. u. d. f. kurzen Bericht erstattet. Aus dem allgemcinen Resumé physiologique, welches Herr $R$. selbst aus seiner Arbeit über dic orgunischen Gewebe zicht, hebe ich nur einige Sätze aus:

W'asserstoff und Sauerstoff verbinden sich mit dem Kohlenstoffe, in ciner solchen Proportion, dafs daraus die organischen Moleculen cntstehen. Diese legen sich spiralförmig zusammen zur Bildung der W ände eines 13läschens. Jedes solche Bläschen wird eine Werkstätte, in der sich durch Hülfe der Luft andere Bliëschen billden, die ihrerseits durch verschiedenen Combinationen in den Zustand des Gummi, Schleim, Gluten und der Holsfaser übergehen. Ist das Blëschen bis zur Holzsubstanz gelangt, so scheint sie die Elemente der Luft nur zur Zersetzung zu gebrauchen, und indem sie ihre Principie mit dem Stickstoffe der Luft verbinslet, kann sie alle Producte des Thieres erzeugen $u$. s. $w$.

Ueber dic Poren des Pflanzenzellengewebes, ron Hugo Mohl, Dr. Med. Mit 4 Kupfertafeln. Tübingen bei Laupp. 18थ8. $4.1 \mathrm{fl} .12 \mathrm{kr}$. Sielı bot. Zeit. 1830. 1. p. 137 Ergänzungsbl.

Anatomisch-physiologische Untersuchungen uiber den Inhalt der Pflanzen - Zellen, von F. J. F. M eyen, Med. et Chir. Dr. Berlin bei Hirschwald 1829. $54 \mathrm{kr}$.

Siehe auch Isis. Bd. XXI. p. 436.

Hayne. Ueber Zellgewebe der Pflanzen.

Isis. Bd. XXI. p. 499.

Ueber dic Structur der Pflanzenzellen von Eduard Heis, Mitglied des naturhistorischen Seminars in Bonn.

Isis. Bd. XXI. Heft 11. p. 109.

Der Verf. untersucht besonders das Zahlenverhältinifs der Zellen, worauf auch Turpin aufmerksam gemacht hatte.

On the general presence of Spiral Vessels in the Vegetable Strueture; and on the peculiar Motion observable in detached pieces of the living Bark of Urtica nivea. By M. David Don.

Jumeson New Eidinb. Philos. Journal. Oct. - Dec. 1828. p. 21. u. d. f. - Froriep's Notizen. Bd. XXIV. p. 55. - I3otanische Literaturblütter. I. p. 496.

Betrachtung der Spiralgefäfse der Pflanzen, von J. L a st drager. Aus einer holländ. Zeitschrift in den Literaturblättern für Botanik. 1. p. 492.

Bisch off de vera vasorum plantarum spiralium structura et functione commentatio. Bonmae 1830. 8. maj. Weber. $1 \mathrm{fl}$.

Die Spiralgefäfse sollen im Pflanzenreiche die Lungen der Thiere vertreten. - Man sehe hierüber Bibliothèque universelle. Mai 1830. p. 69. 
Note sur les Raphides ou poils microscopiques interieurs, observés dans plusieurs éspèces de végétaux, par Alph. De Candolle.

Mém. de la Soc. de Physique et d'hist. nat. de Genève. T. III.

P. 2. p. 115. - Siehe Linnaca 1828. Heft III. $p$. 126. der Literatur.

U e ber die Structur der Zellen der Epidermis, von F. A. Morren. Aus holländ. Zeitschr, in der Linnaea. V., p. 192. Lit.

Analyse microscopique du tissu cellulaire de la moelle et de l'écorce du Cereus peruvianus, ou Cierge du Perou, et de l'immense quantité d'agglomerats de cristanx prismatiques d'oxalte de chaux, qui se forment dans l'interieur de chacune des vesicules de ce tissu; par P. J. F. Turpin.

Annales des Sc. natur. Mai 1830. p. $26-42$.

\section{Untersuchungen über die Organe der Nutrition, Wurzel,} Stengel, Blätter und ihre accessorischen Theile.

§. 30.

Note sur l'existence du canal medullaire dans l'interieur des raçines par M. Achill Richard.

Bulletin des Sciences natur. Fćvr. 1824. p. 135.

Bemerkung über die unterirdischen Stengel der Monocotyledonen, vom Herrn Raspail.

Nouveu Bulletin des Sciences par la Société philomatique de Paris. Aoüt 1825.

Nach Turpin haben wahre Wurzeln in ihrem ganzen Verlanfe nirgends Lebensknoten (noeuds vitaux) es können folglich aus ihnen auch niemals sich blattähnliche Organe entwickeln. Die Vermehrung ihrer Aeste ist nur eine rein zufällige (adventice).

Mémoires'du Muséum. T. XIX, p. 16.

Ueber die Wurzelausleerung der Gewächse, von G. L. Hartig.

Erfahrungen und Bemerkungen bei dem prakt. Forst-und Jagdwesen. p. 269 - 277.

Premier Mémoire sur les Lenticelles des arbres, et le developpement des racines qui en sortent, par M. De Candolle.

Annales des Sc. naturelles. Janv. 186 p. 1 - 26. - Froriep's Notizen. Bd. XIV. p. 129. No. 296. und 298. - Eine ziemlich scharfe Kritik dieser Abhandlung lieferte Raspail in dem Bulletin des Sc. natur. Mai 1826. p. 45. u. d. $f$.

Revision der bei dem Vereine vorgekommenen Verhandlungen über das Beschneiden der Pfahlwurzeln, vom Herm Dr. v. Schlechtendal. Verhandl. des Vereins zur Beförder. des Gartenbaues. Bd. $1 I I$. p. 231 - 239 .

Dissertatio botanico-medica inauguralis de radicum plantarum physiologia earumque virtutibus medicis plantarum physiologia illustrandis, auctore Gerard Backer. Amstelodam. ap. Münster 1830. 4.

Abhandlung über das Wachsthum des Stammes der Dicotyledoneen, vom Herrn Alexand er Fischer.

Bullet. des la Soc. impér. des natural. de Moscou 1829. p. 333.

- Bibl. univ. Aoút 1830. p. 418.

Nach Turpin sind die Stengel an ihrer Oberfläche allezeit mit symetrisch gestellten Lebensknoten versehen, auch besitzen sie immer blattähnliche Anhängsel, Organe, die biswcilen zu einem rudimentōsen 
Zustande herabkommen oder selbst rerkümmern (avorté). Knospen und Zwieheln nehmen ihren Ursprung aus diesen Lebensknoten, welehe ihnen zu Behältern (ćonccptacles) dienen.

Mémoires du Muséum. T:IXX. p. 16.

Mirbel. Mémoire sur l'Origine, la developpement et l'organisation du liber et du bois.

Mémoire du Muséum. T. XII. p. 9-36. avec planches.

Remarques lues à la société philomatique le 17. Août 1816 sur une note relative au Cambinm et an Liber, lue par M. Mirbel is la mème société le 6. Juillet 1826. par $A$ ubert du P'etit Thouars. Paris 1828. 8. $20 \mathrm{~s}$.

Papi us. Der Holzwuchs in der Natur. Mainz 1826. 8. 68 Seiten.

Ueber die Stärke oder Cohäsionskraft verschiedener Hölzer. Aus dem New London Mechanic's Register No. X. p. 237. - In

Dingler's polytechnischem Journal. Bd. XXIV. p. 2f. $u$, d. f.

Da in diesem Aufsutze die bäume nicht mit ihren systematischen Namen bezeichnet sind, so bleiben dabei noch manche Zuveifel zu lösen übrig; es kommen vor: Courbaril, Jujuba, Populus, Ccdrus $u$. s. $w$.

Versue h zur verhältnifsmäfsigen Bestimmung des Grades von Hitze, welche sich bei Verbrennung der vorzïglichsten Arten von Holz und Kohlen, die man in den vereinigten Staaten als Brenn-Material verwendet, entwickelt, nebst Angabe der verhältnil'smälsisen Menge ron Wärme, welche bei den gewöhnlichen Apparaten zur Verbrennung derselben verloren geht, von Mareus Bull.

Aus den Transactions of the americun philos. Society. New Scries 18\%6. In Gill's, technical Repository. Dec. 18\%6. p. 312. Jan. 182\%. p. 3. Febr. p. (18. und cius diesem in Dingler's polytechnischem Journal. Bd. XXIV. p. 251 - 261. ferner p. 3ü6 - 3t8.

Note sur la conductibilité relative, pour le colorique des differens bois, dans le sens de leurs fibres, et dans le sens contraire, par M. Auguste de la Rive et Alphonse Deeandolle.

Mém. de la Soc. de Physique et d'hist. natur. de Genève. T. IV. P. 1. p. 70-75. - Biblioth. univ. T. XXXIX. Nov. 1828. p. 206-211. Nach der Leitungslivaft der W'ärme folgen die Hölzer in nachstehender Ordnung: Cratacgus Aria, Juglans, Quercus, Abies, Populus $\boldsymbol{u}$. s. w.

Marcet, über die Farbenveränderung in dem Holze gewisser Bäume. Bibl. univer's. F'́vr. 1830. p. 228.

Er sucht sie in einer Art von Oxygenisation.

Ueber hohle Bäume; vom Prof. Amos Eaton. Aus Silliman Amer. Journ. XIII. 1. p. 194. Sept. 1827. in den botan. Literaturblättern. I. p. 495.

Ueber Inschriften in lebenden Bäumen, vom Prof. A ghard in Lund. Aus dem Schwedischen von Johnst on im

Edinburgh Journal of Science by Brewster. July 1830. p. 98. u. $d$ : $f$.

Considerations sur les fonetions physiologiques des Epines et sur les rapports, qu'elles paroissent avoir avec les météores electriques, par M. Astier.

Annales de la Soc. Linn. de Paris. Nov. 1525. p. 566.

Die amerikanische Buche, kein Leiter für den Blitz. Edinb. philos. Journal. March 1827. p. 392. - Dingler polytechn. Journal. Bd. XXIV. p. 469. 
Nach den Erfahrungen des Herzogs Heinrich von Würtemberg ersetzt sehr trocknes. Weils - Buchenholz die Glasstangen bei elektrischer Isolation. Auch werden Buchen selten rom Blitze getroffen.

Wedekind und Behlen allgemeine Jahrbücher der Forst-und Jagdkunde. Bd. I. p. 113.

Ueber die Structur der Blätter und die Beziehungen derselben zur Respiration der Pflanzen in der Luft und dem Wasser, hat Herr Brongniart in der Sitzung der Academie des Sciences am 1. Februar (1830) cine Abhandlung vorgelesen.

Nach den darin vorgetragenen Ansichten hat das Parenchym unregelmäfsige Höhlen, durch die mittelst der Poren die Respiration geschieht. In Wasser lebende Blätter haben keine Oberhaut und keine Höhlen im Parenchym.

Froriep's Notizen. Bd. XXYI. p. 295. No. 569.

Schimper. Ueher die Stellung der Blätter.

Geiger's 'Magazin. Jan. 1830. p. 1. u. đ. f. Sucht die Gesetze der Blattstellung darzuthun. Man vergleiche auch:

Raumer. Die Zahlenverhältnisse der Pflanzen.

Isis: Bd. XIII. p. 836.

Ueber die Bewegung der Blätter der Mimosa pudica; ron Herbert Majo.

I. $p$. Fror 124.

Froriep's Notizen. Bd. XVIII. No. 396. - Botan. Literaturbl.

Burnet und Majo wollen bei sensitiven Pflanzen nebst der Bewegung zugleich Farbenveränderung bemerkt haben; auch soll, wenn der sich bewegende Theil geschwärzt wird und Sonnenlicht anfsaugt, die Wiederaufrichtung der Pflanze zum ursprünglichen Zustande nach längerer Zeit erfolgen.

Brewster. Journal of Edinburgh, Jan. 1829. p. 186.

Sur les feuilles du Maloxis paludosa, par M. J. S. Henslow, Prof. de Botanique à l'Université de Cambridge.

Annales des Sc. naturelles. Janv. 1830. p. 103.

An den Elättern dieser Orchidee beobachtete Herr H. kleine Zwie-

belchen, was durch eine Abbildunglerläutert wird.

Pleurothallis prolifera Lindley; eine neue in der Gegend von Rio Janeiro einheimische Orchidee, die sich besonders durch ihre folia prolifera auszeichnet. In den Blattwinkeln bilden sich neue Würzelehen, und was noch besonders eigen ist, es entsteht das erste Blatt eines jeden neuen Individuum auf der nemlichen Seite des Blattwinkels der Mutterpflanze, wie das alte Blatt ans. dem es sich entwickelt. Diese scheinbare Ausnahme von dem allgemeinen Gesetze der Blattbildung ist das Resultat des Abortus eines frihheren Blattes das in Gestalt einer röthlichen Schuppe sich zeigt, während das nachfolgende zwcite Blatt sich vollständig ausbildet.

Bullet. des Sc. natur. Févr. 1S3̈0. p. 288.

Obserrations sur les feuilles de l'Ornithogalum thyrsoides, par M. Poiteau.

Bull. des Sciences natur. Août 1826. p. 432.

Die Blätter wurden für; ein Herbarium eingelegt, es entwickelten sich dabei kleine Zwiebelchen an ihnen. -

Rapport fait à l'Academie royale des Sciences sur un Mémoire de M. Turpin, ayant pour objet la reproduction d'un végétal phaneroǵame (Ornithogalum thyrsoides) au moyen de bourgeons dereloppés à la surfaçe des feuilles, par M. Cassini.

Annales des Sc. natur. Janv. 1829. p. 44. 
Grippert. Ueber das Kíeimen der Blätter, (Citronen, Pomeranzen) ans dem Vortrag der naturgeschichtl. und botanischen Section der schles. Gesellschaft fïr vaterländisehe Cultur im Jahre 1828.

Kastner's Archiv. Bd. XV. Ileft 2. p. 161. - Man sehe auch Bertiner Gesellschafter 1829. No. 92. S. 468.

Mémoire sur la coloration des feuilles par Macaire Princep.

Mémoires de la Soc. de physique et llhist. natur. de Genève. T. IF. p. 43 - 53. - Annales des Sc. natur. Nov. 1828. p. $851 .-$ Geiger's Magazin. Lugust 182!9. p. 115. - Bibliothique universelle. T. XXXIX. Oct. 18\%8. $p: 125-135$.

Beobachtungen über die verschiedenen Färbungen der Blätter, von Robinet und $G$ uibourt.

Journal de Chimie medicale III. p. 161. - Froricp's Notizen.

No. 377. p. 37. - Brandes Archiv. Bd. XXY. Heft 2. p. 183.

Ueber das Phänomen des Blattfalls, von John Murray.

Edinb. philos. Journal. Oct. 1823. p. 237.

Sur la chute des fenilles, par le Prof. Vaucher.

Mémoires de la Société de physique et d'hist: natur. de Genève.

Tom. I. Part. I. 1521. p. 12!. - Bulletin des Sc. natur. Mai 1826. p. 5.. Linnaea 1828. III. p. 126. Lit.

Ueber das Winden der Pflanzen. Eina botanisch-physiolegische Alhandlung, von Ludwig II einrich Palm, Dr. der Med. und Chir. Mit 3 Steindrucktafeln. Stuttgardt bei Löfflund und Solnn 1827, $1 \mathrm{fl}$.

Ueber den Bau und das Winden der Ranken und Schlingpflanzen. Fine gekrönte Preisschrift ron H ugo Mohl. Mlit 13 Steintafeln. Tübingen bei Heinrich Laupp. 1827. 3 fl.

Siche bot. Zeit. 1830. 1. p. 46. Ergünzungsbl.

\section{Ernährung und Wachsthum der Pflanzen.}

S. 31.

J. R. Göplert, nonnulla de plantarum nutritione, Dissertatio. Berolin. 1825 . 1829.

Aghard. Essai sur le déreloppement intericur des plantes. Lund

Observations sur l'Aecroissement des Vérétaux, par M. A mici. . Aninales des Sc. natur. Sept. 1830. p. 92.

Joannis Devyver responsio ad quaestionem propositam: Definiatur nutritio corporum organicorum in genere, comparaturque mutritionis processus vegetahilium cum processu animalium. Lovanii 1823. 134 S. 4.

Nichts Neues oder Eigenes.

Ueber das Nahrungsverhältnifs der Gewächse ron A. Desberger.

Wedekind und Behle $n$, allgemeine Jahrbücher der Forst-und Jagdkunde. Heft I. p. 132.

Merkwürdige Ernährung und Verzweigung einiger Lindenwurzeln von Schmalz.

Dresdner Zeitschrift für Natur und Hcilkunde. Bd. II. p. 107.

Ueber die Aussaugung der Bodenkraft durch die Vegetation der Pflanzen, von W. A. Kreyssig.

Möglinsche Annalen der Landwirthschaft. Bd. XVIII. Stück I. p. $195-247$. 
Die Erdarten in Gebiete der Pflanzen-Vegetation im Culturund Nichtcultur - Zustand, nebst den Folgen einer Vernachlässigung des Ersteren. Nach Grundsätzen der Naturlehre, Chemie und Landwirthschaft bearbeitet von G. F. H. Schoder, Kameralverwalter $\approx u$ Ludwigsburg. Ludwigsburg 1825. 98. S. 8. $54 \mathrm{kr}$.

Welche Bestandtheile des Bodens begünstigen oder hindern das Wachsthum der Wucherblume, und welches wären also die Mittel zu ihrer Vertilgung? Weshalb entkräften manche unsrer Gewächse so sehr den Boden? von Dr. C. Sprengel.

Die Wucherblume (Chrysanthemun segetum) wird getödtet durch Mangan, Anwesenheit von Eisen dagegen begünstigt das Wachsthum derselben, man soll daher zu ihrer Vertreibung die Aecker mit manganhaltigen Fossilien bestreuen. - Quecken verschuinden nach Düngung mit Mergel gänzlich, dagegen dann Leguminosen in Menge oft erscheinen.

Zur Lösung der zweiten Frage wird bemerkt. (im Widerspruche mit andern Ansichten) Reps, der in Saamen gelassen werde, nütze dem Acker noch, weil die Wurzeln darin blieben, viele Blätter abfielen, und also dem Borlen wieder gegeben werde, was ihm entzogen sein sollte: dagegen durch gänzliches und frühzeitiges Herausnehmen der Pflanze der Boden nothwendig verliere. -

Erdmann. Journal für ökonomische und technische Chemie Bd. IV. Heft 3. - André, ökon. Neuigkeiten. Bd. XXXVIII. p. 513. u. $d . f$.

In wie ferne hängt von dem Zersetzungs- und Verwesungsprocesse der Gewächse überhaupt die Bildung der tragbaren Erde von dem Einflusse der Wälder hierauf, sowohl jine als die Fruchtbarkeit des Bodens alb, von $R$ euter.

Allgem. Forst- und Jagdzeitung 1829. - Siehe Wedekind und Behlen allgemeine Jahrbücher der Forst und Jagdkunde. Heft I. p. 126.

U e b e r d en $E$ in 1 l fs der verwesenden Laub- und Nadelabfälle u. s. f. in chemischer und mechanischer Beziehung auf Waldvegetation, mit einem Hinblick auf den Gesichtspunkt der Zulässigkeit der Waldstreunutzung u. s. f.'

Daselbst. p. 129.

Versuche und Beobachtungen über den Einflufs der Düngungsmittel auf die Erzeugung der nâhheren Bestandtheile der Getreidearten von Sig. F. Hermstädt.

Je reicher an animalischen, stickstoffhaltigen Bestandtheilen der Dünger, desto gröfser der Klebergehalt des damit gerlüngten Weizens, je ärmer an diesen Bestandtheilen, desto gröfser der Amylumgehalt.

Schweigger. Jahrb. der Chemie und Physik. Bd. XVI. Heft 3. p. 278 - 285 .

Effects of certains Manures on the qualities of plants.

Edinb. new philos. Journal. Jan. - March 1827. p. 397.

Neueste Abänderung in der Theorie über den Humus.

Hundeshagen forstl. Berichte und Miscellen. Heft 1. p. 51.

D ie Humussäure und ihre Verbindangen, vorläufige Bemerkungen von Dr. Spreng el in Göttingen.

Kastner's Archiv. Bd. VII. Heft 2. p. 163. 
Das humnsa a re Kali als Düngungsmittel für dio Weinberge, von Demselben.

Duselbst. p. 168, u. $d: f$.

Tieber Pflanzenhumus, Humussiure und humussaure Salze, von Dr. Sprengel, Privatdocent der. Chemie und Oeconomie in Göttingen.

Daselbst. Bd. VIII. Heft 2. p. 145 - 220.

Abhandlung über die Anwendung des Kochsalzes auf den Feldund Gartenbau. Von Luthbert Wilhelm Johnson. Aus dem Engl. der zwciten Ausgabe übersetzt von C. J. I. Leipzig bei Cnobloch 1826. 218 S. 1 Rthl. $4 \mathrm{gr}$.

Bemerkungen über die Anwendung des Kónchsalzes auf Feld - und Gartenbau, vom geh. Ober-Medicinalrath und Prof. Dr. Hermbstädt in Berlin.

Terhandl. des rereins zur Beförderung des Gartenbaues. Bd. III. p. $111-116$.

Bemerkungen üher die Anwendung des salzsauren Kalkes als Düngungsmittel, von dem Ober-Medicinal-Assessor Horrn Schrader in Berlin.

Daselbst. Bd:II. p. $425-431$.

Fortsetzung der Versuche üher Düngung mit salzsaurem Kalk und Poudrette, vom Hofgärtner Vos s in Potsdain.

Daselbst. Bd. III. p. $390-398$.

E. Leuchs Vollstindige Düngerlehre. Nürnherg 1825. 8.

Beobachtungen üher die Activität der Vegefation im Vergleiche mit den verschiedenen Tageszeiten, vom Herrn. Prof. Meyer in Kïnigsberg.

Lnnalen der Berliner Garten-Cultur-Gesellschaft. Bd.V. Heft 1. 1828. p. 110.

Herr Prof. M. beobachtete Amaryllis Belladonna im September; sie wüchst dloppelt so viel am Tage als bei der Nacht.

Ueber das periodische tägliche Wachsthum der Getreide-Arten ron Demselben.

Linnaea. 1829. Heft I. p. 98.

Ueber das Wachsthum eines Blattes der Urania speciosa, von Claas Mulder.

-Lus holl. Zeitschr. Das. 183n. p. 188. Lit.

Beobachtungen üher das Wachsthum einer Blumenknospe des Cactus grandiflorus, von Clas Mulder.

Das. p. 191. Lit.

Saftbewegung in den Pflanzen.

5. 32.

Knight, üher Bewegung des Pflanzensaftes.

Isis. Ed. 1. p. 1399.

Ueber den eigenen Saft der Gewiähse, seine Behälter, seine Bewegungen und seine Bestimmung, von L. C. Treviranus.

Zcitschrift für Physiologie. In Ferbindung mit mehreren Gelehr-

ten herausgegebon von F. Tiedemann, G.R. Treviranus und

L. C. Treviranus. Ed.II. No. VI. p. 147.

C. H. Schultze. Ueber den Kreislauf des Saftes im Schöilkraut 
und in mehreren anderh Pflanzen, und über die Assimilation des rohen Nahrungsstoffes in den Pflanzèn überhaupt. Berlin 1822.

Ueber den Kreislauf des Saftes in den Pflanzen. Erläuternde Bemerkungen von K. H. Schultz, Arzt und Privat-Docent in Berlin. Berlin 1824 .

Ueber die Bewegung der Pflanzensäfte. Briefe des Herrn Prof. Schultz in Berlin an den Herrn. Prof. De Candolle in Genf. Bot. Zeit. 1828. I. p. 17. u. d. f.

Sur le mouvement, que l'on apperçoit au microscope dans les feuilles de la grande Chelidoine, Chelidonium majus, par M. Dutrochet.

Journal complem. du Dictionnaire des Sciences medicales. Tom. XIX. p. 290. et Bulletin des Sc. naturelles et de Géologie. Decembre 1824. p. 343.

Ueber Dutrochet's Ansichten der. Saftbewegung sehe man auch Isis. Bd. XVI. p. 572. - Geiger's Magazin. Juniheft 1826. p. 232. Ueber eine pliysiologische Erscheinung, welche die Circulation zu erklären scheint, die man an dem Stengel mehrerer Pflanzen beobachtet, las Dutrochet eine Abhandlung am 18. Januar 1830. vor der Academie des Sciences zu Paris (Froriep's Notizen. Bd. XXVI. p. 279. No. 568.). Dic Causa efficiens jener Beuegung wird in der,verschiedenen Temperatur gesucht, die zwischen den beiden Flächen der Röhrè Statt finde. -

Zenker. Ueber die Bewegung des Saftes im Schöllkraute. Isis. Bd. XIV. p. 332 .

A mici. Ueber den Kreislauf des Pflanzensaftes.

Isis. Bd. X. p. 665.

Ue Ue r die Eirculation des Lebenssaftes in den Pflanzen, von F. J. Meyen.

Linnaea. If. 1827. p. 632.

Ueber die eigenthümliche Säfte-Bewegung in den Zellen der Pflanzen, von F. J. F. Meyen.

Nova acta physico-medica. T. X. P.2. p. 839. cum tab. - Linnaea 1828. $p$. 47. Lit.

Ueber die Organe der Saftbewegung in den Pflanzen, von Dr. M e yen "aus Bonn.

Isis 1S28. $p .436$.

Supplement zur Lehre von dem Kreislaufe; von Dr. Meyer. Heft I. Supplemente zur Biologie des Blutes und Pflanzensaftes. Bonn 1827. 4.

Zw eifel und Gewissensfragen an Physiologen und Botaniker, die Sichtharkeit der Bewegung der Säfte in den Pflanzen betreffend, von J. P. Wilbrand.

$$
\text { Isis 1828. p. } 281 \text {. }
$$

Ha yne, über Bewegung des. Saftes in den vollkommenen Gewächsen. (1) Isis 1830. p. 502 .

U.eber das Aufsteigen des Saftes in den Weinstöcken, vom Hofrath Muncké.

Mittheilungen der. $k$. $k$. Mährisch-Schlesischen Gesellschaft zur Beförderung des Ackerbaues, der Natur-und Landeskunde in Brünn. Bd. VIII. p. 60 . 
Memolre anr la sève d'Anùt, par le Prof. Vaucher.

Mém. de la Soc. de physique et d'hist. nat. de Genèvo. T. I. P. 2. p. 289. - Siche auch Linnaea 1828. III. p. 126. Lit.

Ueber das Ringeln der Obstbäume, vom Herm Rudolph Workmoister zu Charlottenburg.

Verhandl. des Vereins zur Beförderung des Gartenbaues in den

K. Preufs. Staaten. Bd. I. Berlin 15\%4. p. 47.

Der Narben-oder Astring; nicht Zauberring. Ausfïhrliche, anf Erfahrung gegründete Anweisung zur naturgemäfsen Obstbaumzucht. Mit einem Kupfer. Leipzig 1821.

Considerations sur l'incision annnlaire ses canses, ses effects, et particulièrement anon emploi dans la culture de la vigne, par Bailly de Merlieux. Paris 18\%5.

Ueber, den Ringelschnitt nach Bailiy.

André ökonomische Neuigkeiten. 1826. No.21. p. 167.

Der Ringelschnitt der Reben.

Verhandlungen des Grofsherzogl. Bad. landwirthsehaftichen Vereins. Jahrgang III. p. 139.

Untersuchungen über die Vermehrung der Gewächse durch Theilung, oder die individuelle Reproduction.

\section{§. 33.}

v. Berg. Ueber den Unterschied zwisehen dem Veredlungstricbe und dem Vermehrungstriebe bei den $\mathrm{Z}$ wiebelgewächsen.

Bot. Zeit. 1829.' II. p. 705.

Ueber das Vermögen der Zwiebeln und Zwiebelknollen sich zu jedem Vegetationsacté zu reproduciren, von Treviranus.

p. 193.

Vermischte Schriften anatom. und physiolog. Inhalts. Bd. IV.

Bemerkungen über die Vermehrungsarten der Tulpo; vom Oberconsistorialrath Dr. J. J. Bell ermann.

$V$ Vrhandl. der Gesellsch. naturforschender Freunde in Berlin.

Bd. I. St. 5. p. 318. u. d. f.

Vrolik. Ueber dic Verẩnderungen, welche die Blumenzwiebel, wăhrend ihres Wachsthums bei der Tulpe erleidet.

Bot. Zeit. 1829. II. p. 721.

II isto ire des développemens de quolques genres bulbiféres, et principalement de celles du Colchique, par M. Tristan.

Mémoires du Muséum 1823. T. X. p. 36.

Ueber das Vorkommen von Zwiebelchen an den Blättein ciniger

Endogenen ist oben Nuchricht gegeben worden. -

Observations sur lorigine commune et la formation de tous lés corps propagateurs végétaux, et particulièrement sur un nouveau mode de cea corps propagateurs, par P. J. F. Turpin.

Mémoires du Muséum. T. XVI. 8. année. Cahier 9. p. $157-200$.

Mémoire sur l'organiation interioure et exterieure des tuhereules de Solanum tnberosum et de l'Helianthus tuberosus, considérés commo une véritable tige souterraine, et sur un cas particulier de l'une de ces tiges, par M. Turpin.

Mémoires du Musćum. T. XIX. p. 1 - 56. mit Abbildung. 


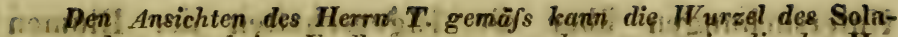
num tuberosum keine Knollen erzeugen, eben so wenig die des Helianthus tuberosus. Die Topinambours; wahre unterirdische Stengel nur erzeugen diese, wie die Kartoffeln. Die Knollen des Convolvulus Batatas und der Dahlia sind blos aufgeschwollene, verdickte Wurzeln. Die Kartoffel kann ihren unterirdischen Stengel sucvessive ser äbteln, und so bis vier neue Generationen von Knollen hervorbringen, gerade wie die Baumtriebe von verschiedenen Jahren, z. B. von 1826, 27, 28, 29. (erläutert durch. Abbildungen). ${ }^{*}$ )

Bei mikroskopischer Betrachtung des Zellgewebes der Kantoffeln unterscheiden sich drei Organisationen: 1) die zahlreichon distincten fast sphärischen, weifseh durchsichtigen Mutterbläschen, die.unregelmäfsig liegen und Zuischenräume (meatus) zwischen sich lassen.

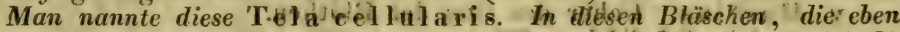
so viele Oraria sind, entwickeln sich ardere Bläsehen, nemlich 2) die Globuline orler die facula der, Karteffel. Sie sind gerade oder gerollt, voll oder hohl, mit Klappen versehen oder nicht, und bilden das tissu tigellulaire (vasculaire). 3) Die Epidermis, wel-

1s: che das Ganze umhült.' schweig.

Ueber den innewn Bau der Kartoffeln, vom Prof. Marx in Braun-

Schweigger-Seidel. Jahrb. der Chemie und Physik, 1829. Heft VIII. p. 478.

Ueber Entwickelung ter Knollen in den Orchideen, von F. A. Morpen.

h. Aus: Bydragen tot de natuturkundige Wetenschappen in der Linnaéa. K. p. 189. Lit.

Sur la formation des arbres naturelle ou artificielle etc., par le Prof. Aubert du Petit-Thoút - Paris 1824.

Mémoire sur la sève d'Aont et sur le divers. mobles de reproduction des arbres, par le Prof. Vaucher.

Mém. de la Société de physique et d'hist. nat. de Genkve. "T.'T. P. 2. p. 289:-

Mémo ir e sur une plantatión à travers des arbres, dite Grefféded

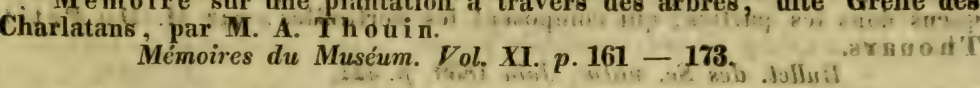

Ueber das Pfropfen hinter der Rinde und über die Art desselben mit dem geschlossenen Auge, von Herrn Benade Pastor priman, und Reator zu Hoyensverda.

Verhandl. des Vereins zw. Beförder des Gartepbaues in Preussen. Bd. I. p. 169.

his Ueber das Pfropfen der Pfinsche auf den Mandelbaum, it Isis. Bd. VIII. p. 533.

Ii: Robherches snu la roprodubtion des vegettux y par M. Leic oq.

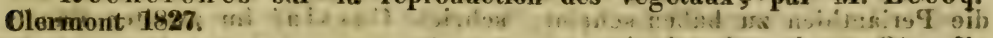
Besonders von der Fortpflanzungsart der Ghara ist Tievolio Rede.

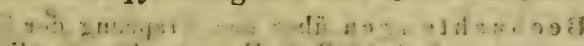
1) $\cos$

*) Was hier Herr Turpin als neu vorträgt, findet, man gnalsentheils schon in einem Aufsatze des Herrn Mauz, überschrieben: Ueber Entwickelung und Ansetzung der Knollen von Solanum tuberosum. Bot.

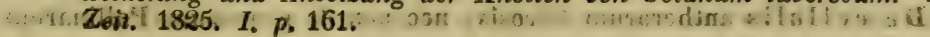


ร. 31.

- Observationes aliquot in florum inforescentiarumque naturam. Auctore Joanne Roeper.:

Linnaea I. Heft 3. p. 433 - $466 \%$

Bemerkung über die Extraaxillar - Infloréscenz von Aug. St. Filaire.

Nouv. Bull. de la Soc. philomatique. Mai 1826. p. 75. - Bot. Literaturbl. I. p. 250.

v. Martius. Ueber den Bau der Blüthen.

(2. asius...Isis. Bd, XXI, p. 499.

Werselbe. Ueber die Architectonik der Bithen.

Isis 1828. p. 522. 18\%9. p. 333. u. $d . f_{\text {. }}$

Versuch einer wissenschaftlichen Blüthenlehre. In einem Briefe von $R$. Wackernagel an W. Bucholtz in Berlin.

Kast ner's Archiv für die gesammite Nuturlehre. Bd. VI. Hëft 3. p. $257-296$.

Considerations sur la nature et les rapports de quelques uns des organes de la fleur, par M. T. Dunal, Prof. de Botanique à la faculté des.Sciences à Montpellier. Montpellier 1829. 4.

Die Blume besteht nach ihm aus mehreren Reilhen quirlförmig ge- wis stellter Organe, nemlich: 1) Verticilletehlicrnal. 2) Androcée exterieure, gebildet von der Corolle und den Filamenten. 3) Androa) cée interieure, begreifend den unmittelbar um den Fruchthnoten stehenden Quirl. 4) Verticille carpellati, die Frucht odter das Gynaecium.

Considerations sur les fonctions des organes floraux colorés et glanduleux, par Michel Felix Dunal. Paris et Montpellier chez

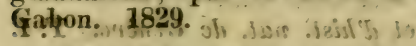

Considerations sur la position mutuelle des étamines sur les vertị̣illes de la fleur en général, et sur les:nombres qui sont les plus fréquens dans les parties, qui composent les verticiles, par M. D u Petit Thouars.

Bullet. des Sc. natur. Juin 1830. p. 422.

Observations sur les enveloppes florales de tégétaux monocotylellons, par M. Alex Boreau.

Annales de la Soc. Linn. de Paris. Mai 1827, p. 139-171.

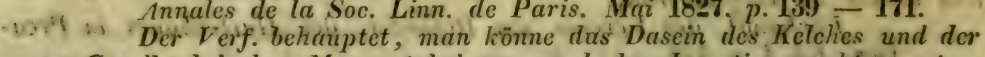
Corolle bei den Monocotyledonen nach den Insertionspunkten unterscheiden:- Bei Convallaria seien aben die, 3 petala und ebew so viel Sepala verwachsen.

yo Von dem E in flus.8 e, welehen vas. Fehlschlagen der Stanbfäden auf die Perianthien zu haben scheint, schrieb Cassin im gweiten Bande seiner Opusicules phytologiques.

Beobachtungen über den Ursprung der Stanbfäden in den einblättrigen Blumen lieferte Derselbe, an dem nemlichen Orte.

Ueber Reizbarkeit der Staubfiden in den Blumen der Berberis, ion Dr. Gôppert.

\section{Linnaea 18\%8. p. 234.}

De cellulis antherarum fibrosis, nec non de granorum Pollinarum 
formis. Commentatio phytotomica; auctore Joanne Ev. Purkinie; Med. Dr. et Prof. publ. ordin. in universitate literaria Vratisl. ete., aceedunt tabulae lithographicae XVIII. Vratislaviael sumptibus J. d. Gruessonie. MDCCCXXX. 4. $6 \mathrm{fl}$. $15 \mathrm{kr}$.

Zahlreiche Untersuchingen der Pollenfơrnien u. s. w. nach natürlichen Fanilien geordnet, dem Systeme Reichenbach's gemäfs. "N

Mehrere andere Untersuchungen übër dèn Polleñ oder Antherenstaub kommen unten bei Gelegenheit der Sexualität der Gewächse vor.

Ueber die fibrosen Zellen der Antheren, von Dr. Hugo Mohl.

Flora 1830, II. $p .697 ., u, d . f$.

Ueber die Organisation des Stempels in den Blumen, vom Herm Prof. Dr. Schultz in Berlin.

Bot. Zeit. 1828. I. 353. nifolium.

Nachricht von der Irritabilität des Griffels bei Stylidium grami-

Nouvelles Annales des Voyages. Mai 1829. p. 222.

Note sur l'Irritabilité du Stigmate de Mimulus, par M. Henri Braconnot.

Annales de Chimie et de Physique. Juillet 1825. T. XXIX. p. 333.

Sur l'Irritabilité du Stigmate dans le Pinus Larix, par M. David. Dan.

Aus den Annals of Philosophy, in den Amales des Sciences natur. Janvier 1828. p. S3. - Botan. Literaturbl. I. p. 518.

Mémoire sur le Nectaire; par M. Soyer-Will emet de Nancy. Annales de la Soc. Linn. de Paris. Mars 1826. T.V. p.1.

Recherches sur les appareils secretaires de Nectar, ou de nectair dans les fleurs, par M. Desveaux.

Daselbst. $p .53$.

Ueber die Ursache der Blumenfarben, von John Murray.

Edinb. philosophical Journal. Oct. 1823. p. 287. - Man sehe auch Isis. Bd. II. p. 468.

Untersuchungen über die Farben der Blüthen, von C. A. Franck. Inaugural -Abhandlung unter dem Präsidium von G. Schübler. Tübingen 1825 . 8.

Untersuchungen über die Farben der Blüthen und einige damit in Beziehung stehende Gegenstände, voin Prof. Schï̈bler in Tübingen. Schweigger. Jahrb. der. Chemie und Physik. Bd. XVI. Heft 3. p. 285 - 321 .

Ueber die Veränderung der Farbe der Blüthen des Hibiscus mutabilis, von Ramon de la Sagra zu Havana.

Aus der Bibliothèque universelle in den bot. Literaturbl. II. p. 397.

Umgekehrte Reaction der Säuren auf Pflanzenfarben im chemischen und im Lebensprozess, von Dr. Hollunder in Bendzin in Polen.

Kastner's Archiv. Bd. XII. Heft 4. p. 397.

Hortensien, deren Corolle gewöhnlich roth sind, werden blau, wenn die Erde, worin sie vegetirt, mit alaunhaitigem W'asser begossen, , oder mit Caput mortuum Vitrioli, so wie mit ockerhaltiger Erde vermengt wird.

Hebenstreitia dentata ist nach Scanagara in Pavia in den Mor- 
genstunden gänzlich geruchlos, um die Mittagøzeit riecht sic) ckelhaft, und am Abend duftet sie mach Hyacinthen.

n>t is Morgenblatt, 1812, p. 982.

Ueber das electrisohe Lieuchten ciniger Blumen; von Dr. Alexander Zawadski in Lemberm.

Baumgartuer und Kitinghausen Zcitschrift für Physik und Mathematite. Bd. 6. Heft 4. p. $459-462$.

Man beobachtete es nach ihm an mehreren gelbrothen Blumen,

i uve Calendtala officinalis, Tropacolum viajus et minus, Liliuni loul-

hiferum, Tagetes patula et erecta, und Arten von. Helimthus, und Gorteria rigens; und zuar im Juli und August während der Be1.79llfruchtung wach Untergaing der Sonne an wurmen heitren Tagen; nie bei feuchter Lnft.

Rich. Morris. The botanist's Manual: or a Catalogue of hardy exntic and indigenous plants, arranged according to their respective months of flowering. London 18:5.

Observations, on the flowering of plants, ripenings of fruits etc. in the past year, by $\mathrm{A}$. W. Bowen. u. $d . f$.

Silliman-American Journal. Fol. XVI. No. 1. Aprit 1829. p. 45.

E. Enthält auch einen Vegetations-Kalender der Districte Germanflatte, Herkimer country. Ucber den Blüthe-Kalender von Deerfield siehe bot. Zeit. 1822. I. p. 209.

Untersuchungen über die Zeit der Blüthenentwickelung mehrerer Pflanzen der Flora. Deutschlands und benachbarter Länder; vom Herrn Prof. Schübler in Tülingen.

Bot. Zeit. 1830. I. p. 353.

E. H. Blumenhain. Die Pflanzen-Uhr in Garten und Zimmer, oder Beschreibung und Behandlung derjenigen Blumen und I’flanzen, an welchen zu bestimmten Stunden des 'Tages auffallende Veränderungen zu bemerken sind... Brünn 1827. 8.

Untersuchungen der Früchte und Saamen.

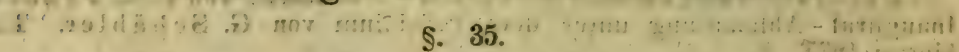

Mémoire sur l'organisation du péricarpe, par M. Mirbel. Annales des'Sc. nutur. Dec. 1825. p. 486.

De l'influence des fruits verts sur l'air avant leur maturité, par M. Th. de Saussure.

Mém. de la Soc. de Physique de Genève. T. I. P. 1. p. 2 t.

C'ouverchel, üher das Reifen der Früchte.

E's soll in zwei Zciträumen gesshehen, so zwar, dirss in dem ersten die Entwicklung und Bildung der Grundstoffe vorgeht, wobei dèr Einflufs der Pflanze auf die Fruclet unläugbur sei. Im zuciten Zeitraume erfolge dus Reifen im eigentlichen Sinne, wo die Süuren von der Wärme unterstïtzt, sich in Pflanzengallerte und Zuckersteff umbildeten; dieser Erfolg sei rein chemisch und unabhängig vom pflanzlichen Leben.

Schweigger-Seidel Jahrb. 1830. Heft VI. p. 262.

Die früheren Untersuchungen der allmähligen Stoffbildung in den Früchten der Adansonia digitata von V a u uelin stehen im achten Bande der Mémvires du Musćum, und jene von Bérard, verglcichende Analy- 
sen reifer und unreifer Obstsorten enthaltend, findet man in dem 16. Bande der Annales de Chimie p. 152. und 225.

Colonel Thaekery gibt Nachricht von dem Gebrauche der Caprification auf Malta und an andern Orten am mittelländischen Meere. Jameson New Edinb. philos. Journal. Jul. - Oct. 1829. p.378.

Ueber das Insekt, welches die wilden Feigen in Oberitalien bewohnt, von L. C. Treviranus, Prof. zu Breslau. (mit Abbild.)

Linnaea. Bd. III. p. 70.

Du Poirier et de la grosscur de ses fruits, par M. Jaume Sainte-Hilaire.

Annales de lagriculture franç. T. III. 1829. - Bull. des Sc. nat. Févr. 18810. p. 264:

Die Birnen sollen gröfser werden, wonn sic zufällig von einem Gegenstande am Baume getragen odcr zwischen swei Zweigen gehalten werden.

Drei physiologisch-botanische Abhandlungen. 1) Befruchtungsprocefs und Reifung des Saamens. 2) Der Bau des Saamens, besonders die Lage, Richtung und Bestimmung des Funiculi umbilicalis und des Ductus spermatici. 3) Die Entwickelung des Saamens, die Ursache der heruntersteigenden Richtung des Rostelli, Corculi und der aufsteigenden der Plumella, von J. F. von $\mathbf{H o f f m a n n}$. Warschau 1828. 8.

Siehe bot. Zeit. 1829. I. p. 103. Ergänzbl. - Brandes Archiv. Bd. XXVIII. Heft 2. p. 293.

Einige Vermuthungen über den Zweck der Flügel, besonders des .Nadelholzsaamens.

Sturm. Beiträge zur deutschen Landwirthschaft. Bd. III. Bonn 1823. p. $90-95$.

J. Macvicar, ron der Erhaltung der Arten (Species) in der Natur, erläutert durch den Bau des Samens der Stipa pennata.

Edinburgh new philosophical Journal by Jameson. March 1827. Heusinger Journal für die organische Physik. Bd. I. Heft 1. Juli 1827. $p$. 145 .

Ueber die Eintheilung der Pflanzen nach den Cotyledonen, und besonders über den Saamen der Monocotyledonen, von C. A. A ghard.

Verhandl. der k. Leopold. Acad. der Naturforscher. Bd. XIII. Abtheil. 1. p. 87.

Observations sur les prétendus bulbilles, qui se développent dans l'interieur des eapsules de quelques éspèces de Crinum, par M. Ách. Richard.

Annales des Sciences natur. T. 11. p. 12. - Bulletin des Se. nat. Juin 1825. p. 218.

Bei den Gattungen Crinum, Amaryllis, Agave u. s. w. fand man bisweilen in den Kapseln fleischige Körper, die von mehreren Botanikern für Zwiebelchen angesehen wurden. Nuch den Beobachtungen des Herrn $R$. aber sind es wahre Saamen, welche aus unbekannten Ursachen so grofs geworden sind, dafs sie an Dicke die gewöhnlichen Saamen ungefähr $50 \mathrm{mal}$ übertreffen. In einer Abbildung ist der Embryo und das Eyweifs deutlich nachgewiesen, so dafs über die Identität dieser Körper mit dem Saamen kein Zweifel mehr sein kann.

Ueber eine besondere Saamenbildung bei Viola odorata sind die literar. Nachweisungen oben bei der Familie der Violaceen angegeben worden. Man sehe auch bot. Literaturbl. I. p. 251. 
Mémoiro concernant l'onverture que Grew à décrite sur le Test des graines, par M. Raspail.

Mímoires du Musćum d’hist. natur. Sept. Annéc. Cah. 8. p. 130. etc. avec planches.

M émoire sur l'hordeine, le gluten et sur la difficulté d'isoler par les procétés en grand les differens principes, dont se compose une farine, par M. Raspail.

Mémoires du Muséum d'hist. nut. Huit annće, Cah. 10. p. 253 - 294.

Dieser Aufsatz dient insbesondere zur nüheren Kenntnifs des inneren Baues des. Saamens der Cerealien.

Untersuchungen über das Eirrengewicht der Samen und nähere Bestandtheile des Pilanzenreichs, Dissertation von C. F. Renz zur Erlangung der med. Doetorwïrde, unter Prïsidium des Prof. Schübler. Tübingen 1826.

Auch in Kastner's Archiv. Bd. X. Heft 4. p. 401. u. d. f.

Nähere Darstellung der Verhältnisse, welche günstig oder ungünstig auf die Keimungsprocesse einwirken, nebst Betrachtung der Nahrungsstoffe keimender Pflanzen auf sämmtliche Boden - und LuftartenVerhāltnisse, von A. F. R euter, Prof. zu Aschaffenburg.

Kastner's Archiv. Bd. XVI. Heft 4. p. 385 - 474. Bd. XVII. Heft 4. p. 492.

Ueber die äufscren und inneren Bedingungen des Keimens und der Ernährung der keimenden Pflanzen, von D e s b e r g er.

$W$ edekind und Behlen allgemeine Jahrbücher der Forst- und Jagdkunde. I. p. 132.

Ueber die Bedingungen des Keimens der Saamen und über einige Besonderheiten, die dabei vorkommen, von Dr. Pönitz aus Dresden. Isis 1829. $p$. 385.

Ueber das Keimen der Saamen in verschiedenen Medien von unorganischen Kï̈rpern, namentlich ron Erden, Metalloxyden und Salzen, voin Hofrath und Prof. Vogel in München. Isis 1830 . p. 499.

De l'influence du Desséchement sur la germination de plusieurs graines alimentaires, par M. Theodor de Sa ussure.

Anuales des Sc. natur. Janv. 182\%. p. 68 - 93. - Froriep's

Notizen. Bd. XIX. p. 295. - Bot. Literaturbl I. p. 539.

U eber die Ke imzeit verschiedener P'flanzen. der Lit.

Aus einer spanischen Zeitschrift in der Linnaea 1829. p. 159.

Ueber die aufserordentlich lange daucrnde Keimkraft des türkischen Korns.

Annales de Chimie et de Physique und daraus in Froriep's Notizen. Bd. V. p. 157. - Mittheilungen der k. k. Mährisch-Schlesischen Gesellschaft. Bd. VIII. $p .144$.

Man hat die Saamen von Zea Mais in Gräbern der Peruaner gefunden, die lange vor Ankunft der Europäer in Amerika golebt haben. Sie waren so frisch, dafs sie gesëet sehr gut aufgingen und Saamen trugen.

Ueber die Dauer der Keimfähigkeit der Saamen einiger PflanzenArten, insbesonlere der Cucurbitaceen, und über die aus ihrer Schale genommenen fierne der Obstsorten.

Verhandl. des Vereins zur Beförder. des Gartenbaues in den $K$. Preufs. Staten. Bd. IV. Heft 2. p. 275, 308 und 379. 
Ueber die Fähigkeit verschiedener Salzlösungen den Keimùngs und ferneren Entwickelungsprocefs mehrerer Saamen zu befördern. Auszug aus dem Tarebuche des Dr. Hopff.

Kastner's Archiv Bd. VII. Heft 2. p. 170.

Ueber die Anwendung des mit Wasser verdünnten Chlor zur Beförderung des Keimens der Saamen. p. 146.

Annales de la Soc. Linn. de Paris. Nov. 1827. - Bull. Linn.

Ueber die Richtung des Würzelehens im Embryo zur Zeit des Keimens, von Keith.

Isis $B d$. VII. p. 379.

Herr Pinat stellte Keimversuche an, und bemerkte, dafs die radicula auf Quecksilber sich entwickele, ja selbst in dieses Metall eindringe.

Jameson New Philos. Journal. Jul. - Oct. 1829. p. 376.

Nouvelle explication des directions, que prennent la racine et la tige d'une jeune plante mue circulairement dans un plan, soit vertical, soit horizontal etc., par M. Poit ea u.

Annales de la soc. d'horticult. de Paris. T. IV. p. 297. - Bullet. des Sc. natur. Aoút 1829. p. 223.

Herr P. macht besonders aufmerksam auf die gröfsere Schwere der radicula und spricht von einem den Gewächsen eigenen polarischen Verhalten.

Mulder.

Ueber das Keimen der Saamen auf Quecksilber, ron Claas Aus holl. Zeitschr. in der Linnaea V. p. 191. Lit.

Ueber die Ursache, welche die radicula embryonis in ihrer Rich. tung bestimint, von Dutrochet.

Nouv. Bull. de la Soc. philom. Mars 1826. - Froriep's Notizen Bd. XIV. p. 40. - Bot. Literaturbl. I. p. 499. macht.

Es wird besonders auf den Einflufs des Lichts aufmerksam ge-

The unsatisfactory Nature of the Theories, proposed to account for the Descent of the Radicles in the Germination of Seeds, shewn by Experiments. By HenriJohnson.

Jameson New Edinb. Philos. Journal. Jan. - April 1829. p. $312-$ 317. - Botan. Literaturbl. II. p. 152. - Linnaea V. p. 145. Lit.

\section{Sexualität der Pflanzen.}

ฐ. 36.

Caroli Linna ei Exercitatio botanico-physica de nuptiis et sexu plantarum. Edidit et latine vertit M, J. A. Afzelius. Upsalae 1828. (Lipsiae Brockhaus 89).

Von der Sexualitât der Pflanzen. Studien von Dr. August Henschel. Nebst einem historischen Anhange von Dr. F. J. Schelver. Breslau 1820. 8.

Die Lehre vom Geschlechte der Pflanzen, in Bezug auf die neuesten Angriffe erwogen, von Ludolf Christian Treviranus. Bremen 1822. 8.

F. G. Schelver's zweite Fortsetzung seiner Kritik der Lehre von den Geschlechtern der Pflanzen. Karlsruhe 1823. 8. 
H. F. Antenrieth, Disquisitio quaestionis academicae de discrimino sexuali jam in seminibus plantarum dioicarum apparente, praemio regio ornata. Additis quibusdam de sexu plantarum argumentis generalibus. Tübing. 1821. 4. c. tabal,

J.e. F: Mauz. Versuche und Beobachtungen über das Gesehlecht der Pflanzen und die Veründerungen derselben durch Einwirkung äufsener Einflüsse.

Sprengel, Neue Entdeck. Bil. III. p. 311.

ats Gieht es in der Pflanzenwelt eine wirkliche Geschlechtsverschiedenheit und eine hierauf gegründete wirkliche Befruchtung? Vom Herm Prof. J. B. Wilb rand in Giefsen.

$$
\text { Bot. Zeit. 1830, II. p. } 585 \text { u. d. f. }
$$

Ueber die Erzeugung durch zwei Geschlechter im Pfanzenreiche, nebst einem Nachtrige zu dieser Abhandlung ïber das Geschlecht der Pflanzen, vom Prof. L. C. Treviraus.

Vermischte Schrift. anatom. und physiolog. Inhalts Bd. IV. p. 95 und 172.

Bemerkungen über den Bau der Befruchtungstheile und das Befruchtungsereschäft der Gewächse, ron Demselben.

Tiedemunn, Zeitschrift für Physiologie Bd.II. Heft 2. Darmstadt 1827. p. $185-250$.

Recherches microscopiques sur le Pollen et Considerations sur la génération des plantes, par M. Guill em in.

Mém. de la Soc. d'hist. nat. de Paris. Tom. Il. - Annales des Sc. natur. T. $I V \cdot$ p. 271.

Mémoire sur la Génération et Developpement de l'Embryon dans les végétaux phanerogames, par M. Adolphe Brongniart, D. M. Lu à l'Academie des Sciences le 26. Decembre 1826.

Annales des Sciences naturelles Tom. XII. Scpt. 1827. p. 14-53. Oct. p. 145 - 172. Nov. p. $225-288$.

Man findet hier zuhlreiche Bemerlcungen über den Pollen, über die Sexualität der Pflanzen, zumal über die Einführung der befruchtenden Substanz in das Ovulum, über die Bildung des Fmbryo und seiner Fintuickelung, über die Formation der verschiedenen Gewebc des Saamens u. s. w. durch Abbildungen versinnlicht. Herr B. behauptet, der Pollen enthalte dem männlichen Saamen ähnliche Kürperchen (granules spermatiques), sie sind nach ihm den in dem Suctmen der Thiere enthaltenen Thierchen ähnlich; er sah sie deutlich durch das achromatische Mikroskop des Prof. Amici; sie variiren schr nach der Gröfse und Gieslalt in verschicdenen Vegetabilien; er glaubte zu bemerken, dafs sie ein eigenes Leben haben, das sich durch selbststündige Bewegung äufsere, was jedoch wohl nock nüher untersucht werden dürfte.

Man sêhe auch Journal de Pharmacie Dec. 1827. p. 621. - Botan. Lileruturbl. 1. p. 276 und 540 . - Nouvcau Bulletin des Sciences de la soc. philomat. Auait 1826. p. 170. - Froriep's Notizen B3d. XII, p. 310. - Brandes Archiv Bd. XXVIII. Hejt 2. p. 197.

Nouvelles recherches sur le Pollen et les granules spermatiques des végétaux, par M. Idolphe Brongniart.

Le Gilobe 201. Juillet 1828. - Bemerkungen z̈ber diesen Gegenstand von Arago, Mirbel und Bory de St. Vincent Ibid. 30. Juillet 1828. - Man sehe auch Jjulletin des Sc. natur. Sept. 1828. p. 90 und Annales des Sc. natur. Dec. 1828. p. 381 u. d. f. 
Ra pport fait à l'Academie royale des Sciences sur un Mémoire de M. Adolph Brongniart intitulé Observations sur les Granules spermatiques des Végétaux, par M. H. Cassini.

Annales des Sc. natur. Février 18ะ8. p.146-158.

Die Abhaidlung von Hoffmanu über den Befruchtungsprocefs der Pflanzen ist. bereits im vorigen $\$$. angezeigt worden.

Recherches sur la reproduction des végétaux, par M. Lecoq. Clermont 1827. 4.

Siehe Linnaea 1828. III. p. 127. Lit.

Experiences sur la génération des plantes par M. C. Girou de Buzaringues.

Annales'des.Sc. natur. Févr. 1829. p. 140. Mars 1830. p. 297.

Sur la fecondation des plantes, par Maximoritch.

Neues Magaz. der Naturgeschichte, herausgegeben in Moscau.

Jan. 1826. Nro.1. p. 13.

"Sur la structure de l'Ovule anterieurement à l'imprégnation dans les plantes phanerogames, par M. Robert Bxown.

Annales des Sc. naturelles Juin 1826. p. $211-244 .-$ Isis Bd.

XXI. Heft 2. p. 176. - Linnaea 1827. Heft 4. p. 691 u. d. f.

L. C. Treviranus; de Ovo regetabili ejusque mutatione. Vratislav. 1828. 4.

Nouvelles recherehes sur la structure et les developpemens de l'ovule végétale, par M. Mirbel.

Annales des Sc. naturelles. Juillet 1829. p. 302.

A. W. Henschel, Nachricht von einigen die Bestäubung der Pflanzen betreffenden Versuchen. Berlin 1828. 4.

Verzeichnifs eines Systems von Versuchen über die Bestäubung der Pflanzen, aufgestellt in den Jahren $1821-1829$, von Dr. A. W. Henschel. (Ohne Druckort und Jahrzahl).

Siehe Isis 1829. p. $262 u$. d. f. - Botan. Zeit. 1830. I. Ergänzungsbl. p. 1335 .

Ueber die Befruchtung der Blüthen der Birnbäume, ron Herrn G. Suaine, correspondirendem Mitgliede der Lond. Horticultural Society.

Dingler, polytechn. Journal Bd. XIX. Heft 3. p. 306 u. d. f.

Beobachtungen und Erfahrungen hinsichtlich der künstlichen Befruchtung bei Blunen und Olstbäunen. Vom königl. Hofgärtner Herrn Tintelmann auf der Pfauteninsel.

Verhandl. des I ereins zur Beförder. des Gartenbaues etc. Dritte Lieferung $p$. 136.

Ueber cine merkwürdige Anomalie der Befruchtungsart bei der rundblättrigen Campanula.

Cassini im zweiten Bande der Opuscules phytologiques.

Curious Phenomenon in Vegetable Physiology communicated by. M. Alix:

Quarterly Journal of Science Oct. to Dec. 1829. Nro. XII. p. 425.

Herr A. machte 'Versuche zur' Befruchtung eines Apfelbaums mit verkümmerten männlichen Genitulien und Blumenblättern, was auch gelaing.

Einige Bemerkungen über künstliche Rosenbefruchtung, derenErgebnisse und die Erziehung der daraus zu gewinnenden Sämlinge, von Herrn Dr. Stiehlex in Dresden. 
$207-210$.

Ferlandl. des Vereins zur Beförder. des Gartenb. Bd. III. p.

Ch. J. G. Sehiede. De plantis hybridis sponte natis. Cassell. 1825.

Ueber die Bastard-Erzeugnifs im Phanzenreich, von Dr. A. F. Wiegmann. Braunschweig 1828. 1 il. $39 \mathrm{kr}$.

Man sehe auch Brandes Archiv Jid. XXVIII. Heft 2. p. 203.

Nachrichten von Versuchen, dic Befruchtung einiger Gewächso, betreffend, von C. F. Gärtner.

Natururissenschaftl. Abhandlungen. Merausgegeben von einer Ge-

sellsch. in W'ürtemberg. Hid. I. Heft 1. T'übingen 186. p. $35-66$.

Nach richten über einige Bastard-Pflanzen, von Demselben.

Botan. Zeit. 1827. I. p. 7t. 1528. II. p. 553. 1829. II. p. 681.

Ueber Bastarde in Pflanzenreiche.

Bot. Zeit. 1826." II." p. 593.

Ueber Varictäten und natürliche Bastarle, von Lasch in Driesen.

Linnaea 1829. III. p. 410 . 1830. III. p. 431. Erslingen.

Beobachtungen über die Bastard-Erzeugung, von Dr. Ma uz in Correspondenzblatt des würtemb. landwirthschaftl. Vereins, Bd. VIII. Sept. 18\%5. p. 201.

Herr Ma z bemerkt: allgemein zeige sich die Frscheinung, dafs"diejenigen Blüthen, welche castrirt und mit fremden Pollen befruchtet wurden, eine bedeutend längere Zeit blühen, als wenn sie ihrer nutürlichen Befruchtung überlassen werden. Ferner zeigen viele Jilüthen, welehe künstlich befruchtet werden, namentlich mit Pollen von einem andern Genus, einen wirklich krankhaften Zustand, der öfters mehrere Tage dauert. Sodann uerden diese Gewächse, wie z. B. Brassica-Arten, wenn sie castrirt worden sind, nie wieder von Bienen, oder überhaupt von Honig suchenden Insekten besucht, vielmehr von denselben verabscheuet. Endlich zeigen diejenigen Gewächse, welche künstlich befruchtet wurden, eine viel gröjsere Vegetationskraft; gewöhnlich setzen sie viele kleine Aestchen unter den befruchteten Zueigen an, so dafs sich diese Gewächse gerade verhalten, wie diejenigen, velche beschnitten wurden.

Versuche und Beobachtungen über das Geschlecht der Pflanzen, von Demselben stehen auch im Correspondenzblatt Jahrgang 1822. pag. 71. und bei Gelegenheit einer Abhandlung über den IIanfbau Daselbst p. 214 u. d. f.

Considerations sur la Production des Hybrides, des Variantes et Varietés en général, et sur celles de la famille des Cucurbitacées en particulier, par M. Sageret, Membre de la Soc. royale et centrale d'Agriculture de Paris:.

Annales des Sc. natur. Juillet 1826. p. 291-314.

Beobachtungen über Bastardpflanzen der Passifloren, von Joseph. Sabine.

Transact. Horticult. T. V. p. 70.

Hybrids produced by Species of Rhododendron, Azalea and Rhodora, fertilized each other.

Jumeson New Edinb. Phil. Journal Oct - Dec. 1828. p. 193.

Ueber Zwitter - Kastanien von Prince.

Isis Bd. XII. p. 646.

Bastard - Magnolic. Siche Literaturblätter für Botanik II. p. 147. 
Ueber den Einflufs des Beisammenstehens beider Geschlechter der Dattelpalme auf den Stamm und die Blätter, von Berthollet.

Daselbst $p$. 148.

-tí Observations sur la Stérilité des Hybrides, par M. Aug. de Saint Hilaire.

Mém. de la Soc. d'hist. nat. de Paris. Vol. I. P. 2. p. 373.

Sur une monstruosité de Pommier, par M. Tillette de Clermont.

Revue Encyclop. Sept. 1829. - Bull. des Sc. natur. Juin 1830. p. 425.

Die Staubfäden fehlten in den Blumen dieses Apfelbaums, man befruchtete-ihn daher künstlich, was auch gelang.

Bei dem auf so überzeugende W' $W^{\prime}$ ise eruiesenen Dasein einer Sexualität im Pflanzenreiche ist es gleichwohl auffallend, dafs einige Phänomene darauf hinzudeuten scheinen, wenigstens von einigen Naturforschern so angesehen werden, als ob sie vernuthen liefsen, es könnten dennoch selbst höhere Pflanzen, unter gevissen Umständen ohne Saamen sich erzeugen. Es gehören hierher folgende Aufsätze:

F. M a yer. Ueber das durch zufällig örtliche Veränderungen bewirkte Erscheinen der Pflanzen.

Botan. Zeit. 1826. I. p. 65.

Entstehen alle Pflanzen aus dem Keime eines Saamens?

Hermes. Juli 1326. Nro. 176. p. 702.

Die Erscheinung, dafs wenn ein Wald ausgerottet ist, junge Bäume von ganz verschiedener Art auf der Stelle der alten wachsen, hat man auch in Neu-Süd-Wales wahrgenommen, wo sehr häufig Acacien auf den Ländereien herrorwachsen, welche, nachdem sie eine Zeit lang cultivirt worden sind, wieder dem Naturzustande überlassen werden.

Cunningham's Two Years in New South Wales. - Froriep's Notizen Bd. XVIII. p. 231.

Das Entstelien der Unkräter betreffend, von Prof. FIörke in Rostock.

Neue Annalen der Mecklenburg. Landwirthschafts-Gesellschaft. 12. Jahrg: 3. Quartal 1825. - André ökonom. Neuigkeiten Bd. XXXIII. p. 81. - Der Herr Verf. stimmt für das von. selbst Entstehen dieser Giewächse.

Ueber das oft plötzliche Erscheinen ron Pflanzen, an Orten, wo man sie nicht vermuthen sollte, vom Apotheker Burkhardt in Niesky.

Abhandl. der naturforschend. Gésellsch. zu Görlitz. Bd. I. Heft $\mathbf{1}$.

p. 153. - Erklärt die Sache durch die lange Dauer der Keimkraft der Saamen.

U eber die natürliche Umwandlung der Wälder, oder die sogenannte Wanderung der Pflanzen.

Hundeshagen forstl. Berichte und Miscellen Heft 1. p. 36. Ueb er das Herauswachsen parasitischer Gewächse aus den Wurzeln anderer Pflanzen; von Herrn Dr. J. Meyen in Berlin.

Bot. Zeit. 1829. I. p. 49.

\section{Secretionen vegetabilischer Theile.}

§. 37.

Ueber Regen bẫume (Arbres pluvieux).

Journal de Pharmacie...Mars 1826. p. 117. 


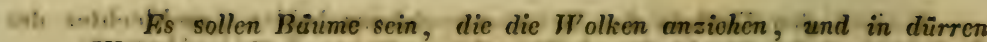
Wüsten zu Regren auflösen. Das. Wahre daran ist, dafs es in Brasilien eincn Baum gilbt, dessen junge Zweige Wassertropfen ausschwitzen, die wie Regen fallen; e's ist Cacsalpinia. pluviosa Decandolle.

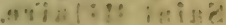

Be merkung über eine auffallende Erscheinung an den Blättern der Calla aethiopica. Vom Prof, Schôn in Würzburge.

Mittheil. der K. K. Mährisch-Schlesischen Gesellschaft Bd. VHI. 16. 1. p: 135.

Einige auf die Bildung des Thaues bezügliche Thatsachen. Von Gebrg Harvey.

$$
\text { Froriep's Notizen Bd. XII. p. } 196 \text { in Nro. } 255 .
$$

U eb e r eine Wasser absondernde Art Tillandsia, von Capitain Andrew's.

Dasılbst Nro. 380.' p. 88. - Bibliothèque universelle Tom. XXXVI. Nov.1827, p. 294. - Geiger's Magazin für Pharmacie. May 1828. p. 131 .

A new. Plant which supplies limpid and wholesome water.

Breuster Edinb. Journal of Science. Octob. 1827. p. 381. Froriep's Notizen Bd. XIIII. p. 250 :

Etwa 5 äbor die wäfsrigen:Absonderungen blättriger Pflanzentheile, von $L$. C. T'reviranus.

Zeitsihrift für Physiologie Bd. III. Heft 1. p, 72.

- vini. Mls Wassér absondertide Gewächse werden genanit: Nepenthes, Anrracenia, Cephalotus, Zingiber, Zerumbet, Maranta gibba, Lu24.7n dolfia glaucescens $u$." s. $w$.

Wine reichliche $W$ asserrabsonderung bemerkte ich selbst an Borago 1. Iaxiflora.

Phytocre ne gigantea: Wallich.

Diese Pflanze, zur Familie der Araliaccen gehörend, wird Mannshoch, und aus in den Stamm gemachten Einschnitten fliefst eine groai) "se' Itengi cines hellen schmackhaften Saftes, welcher in den brennenden W'üsten, wo diese Pflanze wächst, ein sehr gesundes Getränk Noil gibt.

A. Gibt. Journal de Pharmacie XIV, 258. - Brandes Archiv Bd. XXV. - that Hift 3. p. 285.

U Gber die sülsen Ausschwitzungen der, Blïtter, von L. C. Treviranti:s.

Germischte Schriften cuatom, und physiolog. Inhalts Bd.IV.p.'s1.

Ue be r das Vorkommen von künigen gemeinen Zucker in den Blumen des Rhoslodendron ponticum, vou Dr. Georg. Jäger.

Tiedeniann, Zeitschirift fur Physiologic. Ziveiter Bd. Erstes Heft. p $173-175$,

Bemerkungen über die sauren Ausschwitzungen der Kiehererbse,

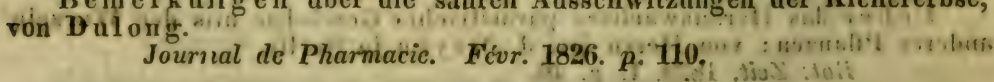

Existence dans les végétaux de cristaux d'oxalate de chaux. Academie des Sciences. Inistitut de France Séan du 25. Juin 1827. - Bulletin des Sc. natur. Juillet 1827. p. 376.

Göppert, über kohlensauren Kalk, als in Pltinzen organisch aus-

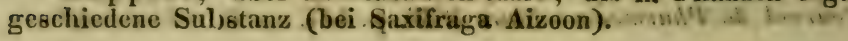


Aus den Verhandl: der schlesisehen Gesellschaft für vaterländische Cultur in Kastner's Archiv Bd. XVII. Heft 1. p. $139-142$.

Ueber die krystallinische Form mehrerer Unterharze, von Bonastre.

Annales de la Soc. Linn. $V$. p. 518. Nov. 1826. mit tab. XIV. Botan. Literaturbl. I. p. 113.

Salzpflanzen entdunsten Chlor, beobachtet von Dr. Sprengel, Privatlehrer der Chemie in Güttingen.

Kastner's Archiv Bd. VII. Heft 2 p. 161.

Das Leuchten der Rhizomorpha subterranea, eine Beobachthng'des Oberbergamts-Referendarivis Hr. v. La roche, mitgetheilt vom Oberbenghauptmann Gerhard.

Íthandl. der Gesellsch. naturforschender Freunde in Berlin.

Bd. I. Stück 4, p. 222 u. d. f.

Beobachtungen über die Temperatur der Vegetabilien; eine Inaugural - Abhandl. vôn F. A. Hal de r unter dem Präsiditum ron S c h übler. Tübingen 1826.

p. 581.

Auch in Pòggendorf's Annàlen dè Physik. Bd.X. Nro. 4.

Untersuchungen ïlier die Tempexatur-Veränderungen der. Vegetabilien und verschiedene damit in Beziehung stehende Gegenstände. Inangural - Dissertation ron WV. Neuffer ausiEfslingen, unter dem Präsid. von G. Sch übler. Tübingen 1829. 8. Druék von Hopfer de l'Orme.

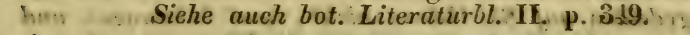

Ueb er die Wäme-Eritwickelun in den Planzen, deren Gefrieren und die Sehutzmittel gegen dasselbe, von H. R: Gồpert. Breslau 1830. 8.

\section{Wirkung der Elemente auf die Pflanzen und dieser, auf ihre

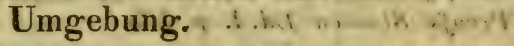

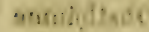

(4hi: in: in

5. 38.
lips
Uebex den Einfluls des Lichts auf die Pfanzen, von Henry Phil-
Quarterly Journal of Scicnce. Nro. 41. Wond. 1826. p. 190. -
Froriep's, Notizen. Bd. XIV. p. 42.
Bei Mimosen entfalten sich die Blätter nur. in dem Sonnenlichte, keineswegs in dem künstlichen.

Ueber die Wirkung des Lichts auf die Pflanzen.

Kastiner's Archiv. Bd $\mathrm{X} V$.'Heft 3. $p$.' 300 .

Wirkungen des Lichts auf die Bäume, vom-Prof. Amos Eaton.'

Aus Silliman. Americ. Journal. Vol. XIII. Nro. 1. p. 193. Sept.

1827 in den Literaturbl. für Botanik I. p. 522.

Ue ber das lebhafte Ergrünen der Pflanzen, abhängend vom Einflusse des Sonnenlichts.

Das. II. p. 396.

Einflufs des Mondes auf die Holzplanzen, rom Oberförster Saue $\mathbf{r}$ in Selan.

Pohl's Archiv fuir die deutsche Landwirthschaft 1827. März. -

Heusinger, Zeitschrift für die organische Physik. Bd. I. Heft 1.

Juli 1827. p. 79. 
Tom Neumond bis zum Follmond steigt der Saft bis in die Spitze;

- I bei abnchmendem Monde greht derselbe zurück, unid das Holz enthält daher im letzten riertel um wenigsten, weshalb dies die zweckmäJigrste Zcit ist, die Bäume zu fällen. Dagregen mufs das Ausschlageholz, wo mniglich vom, Neumonde bis zum Vollmonde gehauen werden, weil damn die Stöcke besser ausschlagen.

W'aldbüume mufs man pflanzen vom ersten Viertel bis zum Vollmond, und zurar itm besten im Frühjahre bis die Knospe aufplatzt und zeitigt; im Herbste gleich nach den Hundstagen.

Fon dem Einflusse des Mondes auf die thierische und vegetabilische Oeconemie; von N. Mill.

Annals of Philosophy. Nro. 57. Sept. 1825. p. 218.

Ueber das Klima, in Bezichung auf Gartenbau, ron John Frederic $D$ aniell.

4i $p: 31 \tau-322$.

Verhandl. des Vereins zur Beförder. des Gartenbaues. Bd. III.

CRemarks on the Naturalization of Plants in colder Climates by J. Mac Culloch.

Quarterly Journal of Soience April to Jun. 1829. p. $221 u . d . f$.

Disquisitions on the influence of Soil and Climats in improving the nourrishing Quality of Tegetables by William Macks. Edinbuirgli.

W.: Man vergleiche die im zueiten Abschnitte angegebenen Schriften . winüber Temperatur - Verhältnisse.

Kirschbäume gedeiken auf St. Helena nicht, und Johannes - und Stachelbeersträucher werden dort immergrünende Pflanzen, ohne je Fruchte $\neq 4$ tragen.

Froriep's Notizen Bd. XIV. p. 42.

Einige Bemerkungen über die Wirkung des Frostes auf die Gewåchse im Winter 1822-1823, von H. F. Link in Berlin.

Tdt In: Ierhandl. des Nereins zur Beforder. des: Gartenbaues: in don

Preufs. Stauten Bd.I. p.-165.

Wirkung des Frostes im Winter $18 \frac{2}{2} \frac{2}{3}$ anf verschiedene Obstbäume and andere Holzarten, vom Hofgärtner Bosse in Oldenburg.

4hi" s"Aus Iderselb. Zeitsehrift angezeigi in der Liinaca 1827. p. 489.

Ueber den Einflufs der Kälte, auf die Vegetation, ron Dr. Goepperf in Breslau.
Froriep's Notizen, Bd." XXV.p. 85. Nro. 531.

Man vergleiche auch dessen im vorigen \$. angegebene Schrift nebst den dort ebenfalls notirten Schübler'schen Dissertationen.

Ueber die Wirkungen des Frostes auf den Pflanzenkörper, und die Schutzmittel, namentlich der Weinberge gegen dieselben. Vom Prof. Plieningex in Stuttgardt.

dy Correspondenzblatt des Würtemb. landwirthschaftl. Vereins. Januar 1826. p. $3-37$.

Ueber Sicherung der W cinberge gegen Frost.

$\because 3$ Verhandl. des Grojsherz. Bad. landwirthschaftl. Vereins zu Carlsruhe. Siebenter Jahrgang p. 12 .

Ueber den Einflufs, den die tiefe Lage des Bodens, auf das Gefrieren der Verretabilien wïhrend der Nacht äufsern kann, von Prevost. Froriep's Notizen Bd. XIX. Nro. 399.

Erfrorne Bäume in Winter $18_{5}^{2}$.

Bot. Zeit. 1830. II. p. 405. 
Desgleichen; mitgetheilt vom Grafen v. Sternberg in Brzezina. Daselbst $p$. 562.

Von dem Einflusse der Kälte auf einige Individuen der Familie der Agaricoideen, vom Herrn Lieveillé.

Annales de la Soc. Linn. de Paris. Juillet 1825.

- Der Winter $18_{30}^{29}$ im botanischen Garten zu München, vom IIrn. Prof. Dr. Zuccarini.

Flora 1830. II. p. 715.

Ueber den Einflufs desselben Winters anf die Gewächse im botanischen Garten zil Marburg; von Merrn Prof. Wenderoth.

Das. p. 761 u. d. f.

Ueber den Einflufs der Walder auf die Temperatur, die Gesundheit, die Luft u. s. W., ron Moreau de Joannes.

Mémoires de l'Academie royale de Fruxelles. Tom. $\mathrm{Y}$. - Heusinger Zeitschrift für die organ. Physil. Eid. I. Heft 2. August 1827. p. 26!) u. d. f. Heft 3. p. 312. Heft 4. p. 467. Heft 5. p. 614 .

Es ist hier sehr ausführlich und mit einem Aufuande von grofser Belesenheit die Rede von dem Einflusse der Wülder auf die Temperatur der Lünder, auf die Hüufigkeit und Mengee des Regens, auf die Feuchtigkeit dler Atmosphüre, auf die Quellen und Flüsse, auf die Winde und die Gesundheit der Luft, auf die Fruchtbarkeit des Bodens und den gesellschaftlichen Zustand der Völker.

Untersuehungen über die Feränderungen, die durch die Ausrottung der Wälder in dem physischen Zustande der Länder entstehen, von A. Moreau de Jonnes. Aus dem Franzins. von W. Widenmann, Prof. der Forstuissenschaft zu Tübingen. 'Tübingen 1828. $1 \mathrm{fl} .30 \mathrm{kr}$.

U eber dic Abhängigkeit der Temperatur der Länder überhaupt, nit besonderer Berieksichtigung des Einflusses der Wälder auf dieselbe und der Frage: Ob durch Ausrottung der Waldungen in der Temperatur der Länder Aenderungen vorgregangen seien, von Reuter.

Ein grofser Aufsatz in der allgemeinen Forst-und Jagdzeitung des Jahres 1829.

Mémoire sur l'influence plyssique du deboisement des forêts, par A. Bos son. Paris 18\%5. chez MI. Hussard.

Etat général des végétaux originaires, ou moyen pour juger, mème de son calinet, de la salubrité. de l'atmosphère, de la fertilité du sol et de la propriété des habitans dans toutes les localités de l'univers, par J. Liary, D. M. Paris 1830. 8.

Beucker Andreae r. Lecuwarden. Beobachtungen über einiqe Gewächse, die bei Ueberschwemmungen dem Einflusse des Sce-Wassers ausgesetzt waren.

Messager des Sciences et arts $1826 \quad$ 27. $p$. 387.

Alle Läume, die ticfe Wurzeln schlaggen, litten weniger; Pfirschensorten, die sonst eine fade Frucht tragen, lieferten jetzt eine schmachhafte; die Beeren der Vitis und Rilies nahmen dagegen einen salzigen Geschmack an. Erdbeeren verdarben sehr bald. Zuiebeln, Cellerie, Spinat, Sauerumpfer u. s. w. wuchsen nachher vorzüglich schön, eben so einige Grasarten, wie Festuca ovina, maritima. 


\section{Untersuchungen über die Wirkung mehrerer Gasarten und Gifte auf die Pflanzen.}

§. 39.

Einige Beobachtnngen über lic Wirkungen rersehiedener Gasarten auf Pflanzen, von Dr. G. F. J ̈̈ ger, Prof. der Naturgesehichte in Stuttgardt.

Tuchner's Repertorium Iid. XXII. IIeft 2. p. 169-184. Geiger's Maguz. Juni 18201. p. 205t.

On the Effects of the Poisonous Gases on Vegetables. By Edward Turner, M. D. Lecturer on Chemistry, and Robert Christison, M. D. Professor of Medical Jurisprudence and Police in the University of Edinburgh.

Fdinburgh med. and surg. Journal. Oct. 1527. p. 856 - 363. Salzburerer Med. Zeit. 1820. II. p. 141. - Kastner's Archiv Bd. XII. Heft 3. p. 269-ê11. - Brandes Archiv Lid. XXV. Ileft 2. p. 201 .

Man sehe ferner Philosophical Magazine and Annals of Philosophy Oct. 1827. - Geiger's Magazin Februar 18:!). p. 89.

Ueber die Wirkung der Gifte auf das Pflanzenleben, ron F. Marcet.

Aus den Mémoires de la Société de Physique et d'hist. natur. de Genive abgedruckt in den Annules de Chimie et de Physique XXIX. 200; im Auszuge nitgetheilt in Buchner's Repertorium Isd. XXII. Heft 3. p. 330-399. - Ferner in Schweigger's Joumal Bd. XV. Heft 3. p. 310. - Froriep's Notizer Nro. 218 oder Nro. $\mathbf{6}$ des. zuilften Jundes. - Geiger's Magazin für' Pharmacie. Juni 1826. p. 241. - Enallich noch in dem Quarterly Journal Nro. XXXIX. unter der Aufschrift: Actions of Poisons upon the Fegetuble Kingdom. By M. Marcet.

Diese I ersuche von Marcet, die so vicles Aufsehen machten, schliefsen sich an die frïheren weit weniger bekannten von Carradori an, die doch nicht mindere Aufmerksamkeit verdienten. Man sehe Antologia. Octo3. 1\%25. p. 15. - Bulletin des Sciences natur. Janv. 1826. p. 16. - Gieiger's Magazin. Juni 1826. p. 238.

Ueber die Wirknng der Gifte auf Pflanzen, rom Herrn Macaire. Isibriothique universelle XXXI. 241. übersetzt in Quarterly Journal of Science 18\%6. Nro. XLII. p. 403.

Von der Wirkung de: Gifte auf solehe Pflanzen, an welchen sich Bewegungen auf äulsern Reiz wahmehmen lassen. Von Macaire Princep.

Froricp's Votizen 13d. XIV. p. 81. Nro. 292. - Brandes Archiv Ijd. XXVIII. Ileft 3. p. 3iż. unter der Aufschrift: Einflufs der Gifle auf irritable Pflanzen.

Versuche über den Einfluls der Gifte auf sogenannte Sinn- und andere: Pllanzen, von Cla a $\mathbf{M}$ ulder, Prof. zu Franceker.

Froriep's Notizen Ijd. XVIII. Nro. 392. aus Bydragen tot de Natuulaudige IV etenschappen Beed II. Nro. 1. 1827. - Bot. Litcraturbl. I. 1. 120. - Linnaea 182!). I. p. 3̊2. Lit.

Note sur l'empoisonnement des végétaux par les substances veneneuses, quils fournssent eux memes, par M. Ma eaire Princep.

Hémoires de la Sioc. de physique de Cience T. IV. p.91-93. -Annales de Chim. et de Physique XXXIV. p. 55-95. - Brandes Archiv Ijd. XXVIII. IIeft 3. p. 333. Ld. XXXIV. IIeft 1. p. 74. 
Meyer's Versuche über die. Wirkung der Gifte auf die Pflanzen stehen in

$$
\text { Froriep's Notizen Nro. } 141 .
$$

Ueber die Wirkung einiger Substanzen auf die Gewächse, von Herrn E. Leuchs. p. 153.

Poggendlorf, Annalen der Physik und Chemie 1829. Nro. 1.

Untersuchungen über die Einwirkung verschiedener Stoffe des organischen und unorganischen Reichs auf das Leben der Pflanzen. Inaugural-Dissertation von $\mathbf{E}$. Alb. Zeller, praeside Schübler. Tübingen 1826. 58 S. 8. Schönhardt.

Abgedruckt in Schweigger's Jahrbüch. der Chemie und Physik 1827. Bd. 2. Heft 1. p.51. - Siehe Froriep's Notizen Nro. 382.

Ueber das Einsaugungs - Vermögen der Wurzeln, von Dr. A. F. Wiegmann in Braunschweig. Marvurg, bei Krieger, 1828. 8.

Besonders abgedruckt aus den Schriften der Gesellschaft zur Beförder. der ge ammten Naturwissenschaften. Bd. II. Heft 1. Siehe uuch Isis 1826. HIeft 2. Bd. XVIII. p. 165. - Ferner Kastner's Archiv Bd. X. Heft 4. p. 502. unter der Aufschrift: Ueber Aufsaugen aufgelöster Metalle etc. durch die Wurzel lebender Pflanzen, vom. Apotheker Wiegmann in Braunschweig.

Versuche über Vergiftungen der Pflanzen. Vom Apotheker C. Moldenhauer in Frankfurt an der Oder.

Geiger's Magazin Aug. 1828. p. 83.

Versuche über die Resorbtion der Pflanzen, von H. F. Link.

Linnaea 1830. I. p. 12. Lit.

Ueber die Wirkung des Kupfers auf Pflanzen.

Isis $B d$. XI. p. 926.

Der Camphor: ein Mittel zur Wiederbelebung welker Pflanzen und Pflanzentheile, von IIerrn r. Droste zu Heilshof. p. 321.

Verhandl. des Vereins zur Bieförder. des Gartenbaues Bd. III.

Ueber dic Einwirkung des Camphors auf die Vegetation, von Herrn Dr: Göppert.

Daselbst Bd. VI. p. 65-80. Aus dem Bericht an die naturwissenschaftl. Section der schles. Gesellschaft für vaterländ. Cultur im Jahre 1828 in K a stner's Archiv Lid. XVII. Heft 3. p. 375. - Botan. Zeit. 1830. II. p. 66. der Ergänzungsbl.

Ueber die Einwirkung des Moschus auf die Vegetation, von Dr. Göp pert in Breslau.

Tiedemann Zeitsehrift Bd. III. Heft 2. p. 269.

Göppert: De acidi hydrocyanici vi in plantas Commentatio. Vratislav. 1827. 8.

U eber die Einwirkung der Blausätre und einiger anderer Substanzen auf die Pflanzen, von Dr. Göppert.

Isis 1828. p. 510.

Ueber die Einwirkung der Blausäure, des Camphors und der narkotischen Gifte auf die Vegetation, von Dr. Göppert.

Isis 1829. $p .285$.

Ueb er die Einwirkung des regulinischen Quecksilbers auf die Vegetation, von Herri Dr. Göpper 
Ferhandl. des I'ercins zur Beförder. des Gartenbaucs. I3d. IV. IIeft 1. Berl. 1829. p. 55. - Isis 1828. p. 507. - Bot. Zeit. 1830. I. p. 72. Ergünzungsbl.

\section{Untersuchungen über die Bewegungen niederer Organismen und Molecülen.}

\section{§. 40 .}

Beobaehtungen über die Bewegung dex girünen Materic in den Gewächsen, von L. Ch. Treviranus. Annales des Sc. natur. Janv. 1827. p. 22. T. X.

Ueber innere und äufsere Bewegung im Pflanzenreiche und Thicrreiche; und inshesondere über Ersatz der äuiseren durch innere oder chemische, mit Rüeksicht auf Gestaltungsverschiedenheit. Vom Prof. Dr. Bartels zu Marburg. Marlurg 1828. 8. 206 S.

Auch in den Schriften dev Marburger Gesellschaft fïr Naturwissenschaften Bd. II. Heft 2.

Kurze Nachricht ron mikroskopischen Beobachtungen, die in den Monaten Jun., Jul. und Aug. 182厅. gemacht wurden, über die Theilchen, welche im Pollen der Pflanzen enthalten sind und ïber die allgemeine Existenz selbstständig beweglicher (active) Molecüle in organischen und unorganischen Körpern. Von Robert Brown (Lond. 1828).

Aus dem Fnglischen von Beilschmied in den botan. Literaturblättern I. p. 253 u. d. f. - Man sehe auch Froriep's Notizen Bd. XXII. Nro. 473. - Annales des Sc. naturelles. Aont 1828. p. 341 - 31\%. - Bibliothéque universelle T. XXXIX. Oct. 1828. p. $136-157$.

Hofrath Muncke hält die Bewcgungen der Molecüle, die man als cine neue und sehr wichtige Fntdeckung anzusehen schien, für einr mechanisch, und zwar durch ungleic Temperatur des stark erleuchteten Wassers, durch Verdampfung desselben, durch Luftzug, Wärmeströmung $u$. s. $w$. p. 160.

Poggendorf's snnalen der Physik und Chemic 1829. Nro.9.

Ueber die Ursachen der Bewegung kleiner Körper unter dem zusammengesetzten Microscop, von $R$ udolphi.

Bot. Zeit. 18s0. I. p. 1 u. d. f.

Schulz, Dr. C. A. S. Mikroskopische Untersuchungen über des Herrn R. Brown Entrleckungen lebender, selbst im Feuer unzerstörbitrer 'Theilchen in allen Körpern, und über Erzengung der Monaden. Mit 1 liupfer. Freiburg 1830. 4. Herder. $54 \mathrm{kr}$.

Ueber Robert Brown's Molecïle und deren cigene Bewegung, sehe man auch dic abweichenden Erfahrungen von Marx in Braunschweig. u.d. $f$.

Schweiggger-Seidel Jahrb. für Physik 1830. Heft 10. p. 173

Additional remarks on active Molecüles by Robert Brown. London 18\%9.

Man sehe Froriep's Notizen Nro. 518. - Bulletin des Sc. natur. Aout 1829. p. 221. - Biblioth. univers. Oct. 1829. p. 114. Jameson Erlinburgh. new Philos. Journal. Jan. 1830. p. $41-46$. Annales des'Sc. natur. Janv. 1830. p. 104. 
Uebersicht der verschiedenen Verhandlungen ûber die Bewegung der Molecülen.

Aus Edinburgh. Journ. of nat. and geograph. Sciences. Nro. 3.

Dec. 1829. p. 193 in dem Bull. des Sc. natur. Juillet 1830. p. 48.

\section{Krankheiten und Mifsbildungen der Gewächse.}

ฐ. 41.

Die Pathologie der Pflanzen kann zwar als ein besonderer für sich bestehender Zweig der Botanik angesehen werden, allein da sie eben nicht mit besonderer Vorliebe bearbeitet wurde, und ihr Zusammenhang mit der Physiologie ohnedem nicht zu verkennen ist, so habe ich das dahin Gehörigc hier gleich beigefügt. Fleifsiger hat man die Mifsbildungen der Pflanzen gesammelt, und durch sie die wahre Structur der Organe zu erklären gesucht, eine Ansicht, die nichts veniger als neu ist; jene Deutung verlangt aber einen reifen Verstand und grofse Umsicht, wenn nicht dadurch die seltsamsten Irrthümer verbreitet werden sollen; möchten doch manche Botanophili recht oft erwägen, was einst ein scharfsinniger Römer sagte: Non omnes qui citharum habent, sunt citharoedi.

Philippo Ré. Ueber die Krankheiten der Pflanzen. Aus dem Ital. von Dr. Ströhlin. Stuttgardt 1821.

De corporum heterogeneorum in plantis animalibusque genesi. Dissert. inauguralis nosomorphologica; auctore Josepho Herrmann Schmidt. Berol. 1825. 4. S. 44.

Essai sur les dédoublemens ou multiplications d'organes dans les végétaux, par A. Moquin Tandon. Montpellier 1826. 4. avec figures.

U e ber den Unterschied von Monstrosität und Bildungsabweichung, vom Oberlehrer Wimmer in Breslau.

Kastner's Archiv Bd. XV. Heft 2. p. 162.

Die Erhaltung und Vermehrung der Pflanzen, ihre Physiologie, Krankheiten und Mittel dagegen, nebst Anweisung zum Verpacken und Versenden der Gewächse, von Lou is Noisette. Aus dem Französischen von Sigwart, Prof. und aufserordentl. Lehrer der Botanik und Chemie in Tübingen. Stuttgardt 1827. $1 \mathrm{fl} .48 \mathrm{kr}$.

U eber die Kräusel - Krankheit der Kartoffeln oder Erdäpfel, und über die Ursache und Cur derselben; von Herrn Wilh. Hollins.

Aus englischen Zeitschriften in Dingler's polytechnischem Journal Bd. XX. Heft 3. p. 295.

Beiträge zur speciellen Pathologie der Pflanzen, von Herrn Dr. F. $\mathbf{T}$. Unger in Wien.

Bot. Zeit. 1829. I. p. 289.

J. Th. Ch. Ratzeburg: animadversiones quaedam ad Peloriarum indolem definiendam spectantes. Berolini 18\%5.

Ueber das Füllen der Blumen theilten die Herren Lindley in London und Pastor Meinzel zu Tonndorf ihre Ansichten mit.

Man sehe Garten-Magazin. W'eimar 18\%. T II. Heft 4. p. 164-170. Heft 5. p. 197-200. - Bemerkungen hierüber schrieb

Duvau in dem Bulletin des. Sc. natur. Nov. 1829. p. 260-269.

U e be r die mit dem Namen Brand oder Krebs belegte Krankheit der Obstbäume, von Wiederhold zu Höxter, nebst einem Gutachten über diese Abhandlung und einem Zusatze zu derselben von Link.

Verhandlungen des Vereins zur Beförder. des Gartenbaues in don

K. P. Staaten. Bd. II. p. 5 u. d. $f$. 
Von dem Iropfen und dessen Kirankheit, Rus genannt; von Githe, Nees v. Esenbeck und Bermeister Lössl.

Göthe, zur Naturwissenschaft. Bd. II. Heft 2. p. 7.4-77. 100.

Note snr une torpenr trìs longue, particulière aux racines du Murier noir; par M. Dureau de la Molle.

Annales des Sc. natur. T. IX. p. 338. Nov. 1826.

Ueber den Rost im Weizen sehe man:

Mittheil. der KK. K. Mährisch-Síhlesischen Gesellsch. zur Beförder. des Ackerbaues. Bd. VIII. p. 244.

Eine neue Art Rost in dem Getreide (und an 'Tannen, Pinus Abies) beschrieb Prof. Fries. Fs ist Aecidium curruseans: pseudo-peridiis laxis membranaceis elongatis congestis albidis, sporidiis globosis aureis. - Es schadet sehr dadurch, dafs es dic jungen Halme angreift und das Wachsthum hindert.

Physiograph. Suelskap arsberacttelse. Lund. 1825. p. 92.

Observations sur le developpement du Charbon dans les Graminées et sur les modifieations qu’il détermine dans les parties de ces plantes qu'il attaque, par M. Adolph Brongniart.

Annales des Sc. natur. Juin 1830. p. 171-176. avec une planche.

Ueber die Ursache des Brandes im Getreile, ron R. Westenhoff. Aus holl. Zeitschr, in der Linnaea V. p. 190. Lit.

Ueber den Unterschied zwischen Schmier und Flugbrand, von II. C. van Hall:

Daselbst p. 191.

Beobachtungen und Bemerkungen über einen an Kiorn - und Trespe-Aehren befindlichen durch Insektenstich verursachten rothen Auswuchs, von J. C. Lauer.

Mittheil. der K. K. Mühr.-Schles. Gesellsch. Bd. VI1I. p. 128.

Notiz über die rotlıen Auswüchse an Korn - und Weizenähren, vom Prof. Müller in Breslan.

Kastner's Archiv Bd. X. Heft 4. p. 428.

Ueber den Ursprung oder die Entstehung des Mutterkorns (Ergot), von Martin Field.

Quarterly Journal Nro. XXXIX. p. 189. - Geiger's Magazin May 1826. p. 201. - Dingler's polytechnisches Journal Bd. XX. Heft 1. p. 73 u. $d . f$.

Mémoire sur l'ergot par M. Leveillé.

Annales de la Soc. Linn. de Paris. Jan. 1827. p. 565.

On the Ergot in Maize, and its Effects on Man and Animals. By M. Roulin.

The Edinb. new philosophical Journal. July - Oct. 1829. p. 217. - Geiger's Magaz. Dec. 1829. p. 232.

Ueber des Multerkorn ist noch besonders der Abschnill über medicinische Pflanzenkunde zu vergleichen.

Beobachtungen über Pflanzen - Mifsbildungen, vom verstorbenen Professor Eysenhardt in Königsberg. (Dictamus, Barleria cristata, Matricaria Parthenium).

Linnace 1826. p. 576.

Pflanzen-Mifsbildungen, gesammelt von Dr. F. L. v. Schlechtendal. (Cerasus ovium, Euphorbia degener, Fritillaria imperialis, Vitis, vinifera capsulifera).

Ibidem 1830. 1. p. 175. III. p. 492. 
Ueber eine Monstrosität der gemeinen Gatrten - Tulpe (Tulipa Gesneriana L.), von Demsellien.

Linnaea 1826. p. 595.

Ueber eine ästige Entwickelung weifser Lilienblumen, von G. Vrolick.

Aus einer holländ. Zeitschrift in den botan. Literaturbl. II. p. 336.

Beobachtungen und Beschreibungen über eine Monstrosität der Scabiosa Columbaria.

Cassini im sueiten Bande der Opuscules phytologiques.

De metamorphosi partium floris Trapaeoli majoris in folia, auctore G. Jaeger.

Nova acta physico-medica. T. X. p. 809. - Linnaca 1828. p. 46. Lit.

Mifsbildung der Cotyledonen ron Convolvulus arvensis, beobachtet von Simonet.

Bot. Literaturbl. II. p. 147.

Observation d'une monstruosité des fleurs de Lilus vulgaire, par M. Guillemin.

Mém. de la Soc. d’hist. nat. de Paris. T. IV. p. 363. - Bot.

Lit.- II. p. 344.

Ueber eine doppelte Cocos - Nufs sehe man

Jameson new philos. Journal. April - June 1827. p. 192.

Ueber eine besondere Prolification der Reseda lutea var. crispa, von Herrn Kops.

Aus einer holl. Zeitschrift in der Limaea III. p. 35. Lit.

De Digitali purpurea heptandra, Adalbertus de Chamisso. Linnaea 1826. p. 571. 1829. p. 78.

Obserrationes de quibusdam Pini silvestris monstris, auct. Jäger. Stuttg. 1828. 4. c. tab. lithogr.

Besch reibung einiger Pflanzen-Mifshildngen, von R. Courtois (Erysimum cheiranthoides, Veronica media) und von Koning (Lonicera Periclymanum).

Aus Bydragen tot de natuurk. Wetenschappen in der Linnaea 1829. p. 33. Lit.

Ueber Monstrositïten und Abarten, vom Herrn Dr. Schimper. Bot. Zeit. 1829. II. p. 417.

On the natural History and Properties of Tabasheer, the Siliceous concretion in the Bamboo. By David Brewster.

Edinb. Journal of Science. April 1828. p. 2\$5. - Froriep's Notizen Nro. 452. Bd. XXI. p. 179. - Man sehe auch Geiger's Magazin Juli 1829. p. 33 . bach.

Ueber einige Mannaartige Produkte des Pflanzenreichs, von Dier-

Geiger's Magaz. Februar 1826. p. 97 u. d. f.

Es werden hier mehrere Gründe angeführt, dafs der Honigthau und die Manna, die von sehr verschiedenen Gewächsen abgesondert wird, für ein krankhaftes Erzeugnifs anzusehen scin dürfte.

Einige Beobachtungen ron Mifsbildungen, rom Herrn OberstBergrath v. Voith in Regensburg.

Bot. Literaturbl. IV. p. 157. 


\section{- 232 \\ Fïnfter Abschnitt.}

\section{Gesehichte de r B ot a n i k.}

Nur einzelne und sehr zerstreute Beiträge sind es, welche die jüngste Zeit zu diesem interessuntesten und lehrveichsten aller Zueige der Pjlunzenkunde geliefert hat; ich glaubte sie unter folgende Abtheilungen bringen zu können.

\section{Untersuchungen über die Pflanzenkenntnifs früherer Zeiten.}

\section{§. 41.}

Flora Classica. Herausgegeben von Dr. Julius Billerbeck in Hildesheim. Leipzig 1524. J. C. Hinrichssche Buchhandlung. 8.

$W^{\top}$ äre Herr $\bar{B}$. eben so erfahren in der Pflunzenkunde, als in den ailteren Sprachen, so würde seine Schrift sehr brauchbar geworden sein.

Ohservationes hicrobotanicae (Biblische Flor). Auctore Chr. Lyngbye, Cand. Theol. Fascicul. 1.

Iidensk Ferhandl. ued Fyends stifto landmode Odense. II. Vol.

1. Heft. $p .27$.

Seripture natural History by W. Carpenter. Lond. 1828. Wightmann and Cramp. $608 \mathrm{~S} .8$.

Naturgeschichte der Bibel, oder Notizen über Zoologie, Botanik und Geologie der heiligen Schrift.

Remarks on the Moustard Tree, mentioned in the new Testament, with a couloured Plate, hy John Frost. London 1828.

Man sehe auch Quarterly Journal of Science Liter. and the arts.

Oct. 1825. p. 57. (Phytolacca).

Notic e sur quelques végétaux réprésentés sur les anciens monumens de l'Kgypte, par M. Bonastre.

Journal de Pharmacie Oct. 18.30. p. 642; es ist hier die Rede von Musa paradisiaca, Nymphaea Lotus, Arum Colocasia, Cyperus

Papyrus, Punica Granatum u. 8. w.

Recherches sur les Plantes trouvées dans les tombeaux égyptiens par M. Passalaequa, par M. K unth.

Annales des Sc. natur. Tom. VIIr. Aoút 1826. p. $418--423$. -

Man sehe auch die Nuchrichten von Jul. Fontenelle in dem Journal de Chimic médicale. Oct. 1826. p. 486. - Geiger's Magazin Febr. 1827. p. 16 i.

Fs kommen hier unter andern vor: Triticum vulgare, Cyperus esculentus, Phoenix dactylifera, Cucifera thebaica, Olea europaca, Mimusops Elengi, Citrus Aurantium, Balanites aegyptiaca, Mimosa farnesiana, Ficus Sycomorus u. s. w.

Notice sur la determination specifique des Cereales trourés par M. Passalacqua dans un tombeau égypticn, et sur le mode de préparation, qu'on leur à fait subir, par M. Raspail.

Mémoires du' Muséum d’hist. nat. Huit. année, deuxième Cahier.

T. XV. p. $145 u, d . f$. 
Herr R. sucht zu beweisen, dafs die gefundenen Getreidekörner kein Waizen, sondern vielmehr geröstete Gerste sei.

Recherches sur l'histoire ancienne, l'origine et la patrie des Ceréales et nommément du Blé et de l'Orge, par M. Dureau de la Malle. Annales des Sciences natur. T. IX. p. 61 - 82. Sept. 18\%6. Geiger's Magazin Februar 1827. p. 115.

Ueber eine Getreideart, deren Homer gedenkt, von Dierbach. Bot. Zeit. 1827. I. p. 281.

Sur le jardin des Hesperides. Fragment inédit de la Relation d'un voyage dans la Cyrenaique, par M. Pacho.

Nouvelles Annales des Voyages. Mai 1828. p. 25̄3. - Botan.

Literaturbl. II. p. 215.

Ueber den Cyamus und Lotus der Aegyptier und der Griechen. Vom Herrn Custos Leopold Trattinnick.

Bot. Zeit. 1822. II. p. 577. 593. - Man sehe auch über denselben Gegenstand die Ansichten des Herrn Fée im Journal de Pharmacie Januar 1823.

On the Substance called Fine Linen in the Sacred Writings. By the Rev. David Scott, M. D. Read before the Wernerian Society 10. Mar. 1827.

Jameson Edinburgh new Philosophical Journal April - June 1827. p. $71-81$.

On the Cotton, of the Ancients.

Ibidem Jul. - Sept. 1827. p. 387.

Theophrast's Naturgeschichte der Gewächse. Uebersetzt und erläutert von $K$. S prengel. Altona 1822. 2 Bände. 8.

Ueber Schneider's Bearbeitung der W'erke des Theophrast sehe man Sprengel's neue Entdeckungen Bd. I. Abschnitt - alte Literatur.

Ueber einige Pflanzen des Theophrast, von Dierbach.

Bot. Zeit. 1821. I. p. 177.

Ueber die breiten Nüsse des Xenophon (Castanea resca), ron Demselben.

Daselbst 1825. I. p. 49.

Ueber die Nachrichten der griechischen und römischen Autoren von der indischen Feige (Banyan Tree, Ficus indica), von G. H. Noehden. Part 1.

Transact. of the royal asiatic. Society of Great Britain T. I.

Die genannte asiatische Societät wählte sich die indlische Feige zu ihrem Emblem mit der Umschrift: Quot rami, tot arbores. Siehe auch bot. Literaturbl. II. p. 216.

Opera medicorum graecorum, quac extant. Edit. cur. Dr. C. G. Kuhn. Vol. XXIII. Lips. apud Cnobloch. 8.

Enthä!t auch eine neue, mit Commentarien von Sprengel begleitete Ausgabe der Werke des Dioscorides, wobei ich bemerke, dafs die näheren Angaben über die Untersuchungen der Arzneipflanzen der Alten dem zweiten Theile des Repertorii vorbehalten sind.

Diese Bearbeitung des Dioscorides macht den 25. Band der Sammlung aus, und hat den besondern Titel:

Pedanii Dioscoridis Anazarbei de Materia medica Libri quinque. Ad fidem codicum manuscriptorum, editionis aldinae principis usquequaque neglectae, et interpretum priscorum textum recensuit, varias 
addidit lectiones, interpretationem emendavit, commentario illustravit Curtius Sprengel, med. et phil. Doet. ete. Lipriae 1829.850 S. 8. 5 Rthl. Fs ist bis jetzt nur der erste Theil erschienen und der Commentar noch zu erawrten.

Catalogus plantarum ad septem varias editiones Commentariorum Mathioli in Dioscoridem. Ad Linnacani systematis regulas elaboravit Comes Caspar ab Sternberg. Pragac 1521. Sumptibus F. Tempsky, Firma J. G. Calve. Folio.

Examen des plantes, anx quelles les Anciens ont donné le nom de fleur de Soleil ou Heliotrope, par M. Thiehaud de Bernaud.

Annales de la Soc. Linnenne de Paris. Mai 18\%(j. p. 164.

Recherches sur les plantes connues des anciens sous les noms de Chara Cacsaris, de Lapsana et de Armoracia, par M. Thiebaud de Bernaud.

Annales de la Soc. Linn. de Paris. Nov. 1826. p. 516 - 518.

Werden erklürt für Crambe tartarica, Chou Colza var. floribus albis, Cochlearia Armoracia.

Iristoire naturelle de Pline. Traduction nonvelle, arec le texte en regard et des notes, par M. A jas son de Grandsagne, ennotée pour toute la Zoologie par M. Cuvier. Paris chez M. C. L. Panckouck 8. (Seit 1829.)

Jeden Monat soll ein Band von 400 - 450 Sciten erscheinen, der 7 francs kostet. Die Commenturien für Botanik liefern die Herren Decandolle, Desfontaines, Fée, de Jussieu. Kunth: für Materia medica die Herren Descuret, Daë, Guibourt, Robiquet, Thibaud; für Mineralogie die Herren Beudant, Brongniart, Larosse.-

Die Gesellschaft deutscher Naturforscher und Aerzte hat schon seit mehreren Jahren eine neue und sehr ausführliche Bearbeitung des Plinius versprochen; möchte die Suche doch auch wirklich auf eine des deutschen Namens würdige W'

Flore de Virgile, ou nomenclature methodique et critique des plantes, fruits et produits régétales mentiomées dans les ouvrages du prince des poetes latins, par A. L. A. Fée. Paris 18\%2.

Osserrazioni sulla Flora Virgiliama. Auetore Tenore. Napoli 1826. Flor.

Retz und Martini bearbeiteten bereits früher die Virgilische

B emerkungen über den Cytisus der Alten, von Herrn F éc. Bibliothèque universelle. Mars 1830. P. ̈̈16 - 320.

Sprengel de Patribus rei herbariae.

Isis. Bd. III. p. 1326.

Die botanische Terminologie älterer Zeiten in Auszuge, von Dierbach.

Geiger's Magazin: Bd. VII. p. 99.

Fragmente aus der Pflanzen-Physiologie des 16. Jahrhunderts, von Demselben.

Daselbst. Bd. VIII. Nov. 1821. p. 121. u. d. f.

Verdienste des Ruellius um die Entdeckung vaterländischer Pflanzen; von Demselben.

Bot. Zeit. 1826. I. p. 97. 


\section{$-235-$}

Clavis Agrostographiae antiquioris. Uebersicht des Zustandes der Agrostographie bis auf Linné und Versucli einer Reduction der alten Synonyme der Gräser auf die heutigen Triviaglnalımen. Tọn Dr. Carl Bernhard Trinius, Kais. Rufs. Hofrath etc. Mit einer Tafel in Steindruck. Coburg. 1822.

Zusätze und Berichtigungen zur Clavis Agrostographiae. Bot. Zeit. 1823. I. p. 229.

Ueber den Mays und dessen Verbreitnng in Europa, vom Grafen Kaspar Sternberg etc. Prag. 1830. 8.

Siehe bot. Zeit. 1S30. II. Frgänzungsbl. p. 75. - Enthält höchst schätzbare historische Untersuchungen.

Recherches sur les époques historiques de l'Orme en France, par M. 'Thiebaud de Bernaud.

Annales de la Soc. Linn. de Paris. Sept. 1825.

On the Ancient Forests of Scotland. By P. F. Fytler.

Jameson New philos. Journal. Jan. 1830. p. 105.

F. A. Mayer. Fin paar Worte über ein paar Druiden-Bäume in Königreiche Baiern. Mit zwei lithographirten Tafeln. Eichstält 1826. $3 \frac{1}{2}$ Bogen. 8 gr.

Allbekannt ist die Göttin der Blumen, welche die Griechen und Römer verehrten; aber auch unser Iaterland kannte ehedem eine solche, denn wie Kirchner (Geschichte der Stalt Frankfurt am Main. I. p. (6lt) sagt, ist Apollonia die Flora der Deutschen, die freundliche Patronin des Frühlings; noch sind Bruchstücke (einer Statüe?) vorhanden, mit den dorh kicum noch erkennbaren Worten Have Apollonia! Die Art, wie das Fest dieser deutschen Flora alljährlich (am 18. April) begangen wurde, beschreibt der gedachte Schriftsteller.

Die Deutung der Blumen, nach orientalischer Art, wovon schon $R u m p h$, Roxburgh und Andere Nachricht gaben, ist wohl sehr alt und gehört der Geschichte jener Gegenden an; man wird es nicht ungerne sehen, darüber einige literarische Nachweisungen hier, $z n$ finden.

Die Blumensprache oder Bedeutung der Blumen nach orientalischer Art. 10. Auflage. Berlin 1826. $36 \mathrm{kr}$.

Die Blumensprache oder Bedeutung der Pflanzen, Blumen und Kräuter nach Occidentalischer Art. Ein Hülf - und Nothbüchlein. Zweite verbesserte und vermehrte Auflage. Berlin, 182\%, bei Flittner. $1210 \mathrm{gr}$.

Die Sprache der Blumen; zum Theil nach dem Orientalischen des Selam. Würzburg 1826. $16 \mathrm{gr}$.

Neuhof, neueste Blumensprache, der Liebe und Freundschaft gewidmet. 1 Bogen. 12. Halle 1828. $4 \mathrm{gr}$.

Die Blumensprache in ihrem ganzen Umfange durch 210 nach der Natur gezeichnete Blumen, Pflanzen und Gewächse erläutert. Mit einem Anhange, die Blumen in gemessenen Dichtungen über Liebe und Freundschaft. $2 \frac{1}{2}$ Bogen. 16. Eisleben 1829, bei Reinhardt. geh. 16 gr.

\section{Beiträge zur Geschichte der Botanik, besonders der neueren Zeit.}

5. 42.

Zustand der Botanik am Ende des ersten Viertels des neunzehnten 
Jahrhunderts. Aus dem Berichte der Königl. botan. Gescllschaft an ilhren Präsidenten, IIcrrn Grafen von Bray. Bot. Zeit. 182. 11. p. 46.5.

Schouw. Berichtigungen und Zusätze zu Schultes Gesehichte der Botanik.

Bot. Zeit. 1825. I. Beil. p. 97. - Betrifft die dänische Literatur über Pflanzenkunde.

De l'état actuel de la Botanique générale, par. M. Decandolle. Revue françoise. Mars 1829. No. VIII. p..3อ.

Discours sur l'état ancien et moderne de l'arriculture et de la Batanique dans les pays bas, par M. Charles van II 1 them. Gand 1817.

Adamsliy. Prodromus historiae rei herbariac in Polonia a suis initiis usque ad nostra tempora. Vratislav. 18\%5. jon 18:8.

Histoire de la Botanique de la Bourgogne, par M. Vallat. Di-

Ueber den Zustand der Botanik in Amerika, von J. Honker. American Journal of Sciences and arts. Vol. IX. p. 2 (ti3.

Notice sur la Botanique de l'Inde orientale et les encouragemens que la compagnie anglaise lui a accordés, par M. De candolle.

Bibl. universelle. Dec. 1829. p. 312.

Nachrichten von Anstalten für Naturwissenschaften, besonders von botanischen Gärten.

§. 43.

a. In Deutschland.

Ueber botanische Gärten in Sachsen und Prenfsen. Vorgelesen in der an 10. Nor. 18:1 zu Regensburg gehaltenen Sitzung der $k$. botan. Gesellschafi von J. A. Schultes.

Bot. Zeit. 1822. I. p. 129. 145.

Nachlese zu Ferrn Hofrath Sehultes Nachrichten über die deutschen botanischen Gärten, vom Herrn grcheimen Rath Grafen Caspar v. Sternberg.

Dasclbst 1824. II. p. 737.

Verzeichnifs der botanischen Gürten in der österreichischen Monarchic.

Daselbst 1820. I. p. 111.

Ueber hotanische Anstalten in Altenburg, vom Herrn G. F. Winkler. Bot. Zeit. 1828. II. p. 768

Berlin. Botanische Anstalten in Berlin, vom Ilerrn Prof. Nees v. Esenbeck.

Das. 1819. II. p. 578.

Sehreiben aus Berlin. Das. 1821. I. p. 203.

Botaniker und botanische Anstalten' in Berlin, vom Kerrn Prof. Hornschuch mitgetheilt.

Daselbst $p .2+1$.

Bonn. Ueber botanische Anstalten in Bonn, vom Herrn Prof Nees v. Esenbeck.

Das. 1819. I. p. 8. ferner II. p. 405. 


\section{Von Herrn v. Martius über den Garten in Bonn.}

Daselbst 1822. II. p. 742.

Einen Plan von diesem jetzt sehr reichen Garten findet man in der Abhandlung des Herrn Nees v. Esen beck. „De Cinnamomo disputatio" Bonnae 1823. 4., die sie bei Gelegenheit der Eröflnung desselben herausgaben. -

Breslau. Beschreihung des botan. Gártens der königl. Universität, von H. R. Göppert. Breslau 1830.

Brzezina. Mittheilungen über Brzezina in Böhmen, dem Lustschlosse des Grafen von Sternberg, von Hornschuch.

Bot. Zeit. 1827. II. p. 746.

Hortus Carlsruhanus, oder Verzeichnifs sämmtlicher Gewïchse, welche in dem Grofsherzoglichen botanischen Garten zu Carlsruhe cultivirt werden, nebst dem Geschichtlichen der Botanischen - und Lustgärten von 1530 - 1825 und einem Situationsplan im Grund - und Aufrifs und im Durchschnitt von sämmtlichen Gewächshäusern. Herausgegeben vom Garteninspector Hartweg. Carlsruhe 1825. 8.

Darmsta dt. Botanische Anstalten daselbst.

Bot. Zeit. 1820. II. p. 619.

Nachrichten yom Herrn Prof. Nees v. Esenbeck.

Daselbst 1822. II. p. 697.

Dresden. Nachricht ron den dortigen Anstalten.

Bot. Zeit. 1821. I. p. 152. Dergleichen von Reichenbach. Daselbst 1822. II. p. 553.

C. G. v. Hoffma nnsegg. Verzeichnifs der Pflanzen in den Gräfl. Hoffmannseggischen Gärten zu Dresden und Rammenau. Dresden 1824. Ungarn.

Der Fürstlich Esterhazysche botanische Garten zu Eisenstadt in Bot. Zeit. 1820. II. p. 470.

Frankfuit a. M. Nachrichten über dic dortigen Gärten ron Wenderoth.

Bot. Zeit. 1819. II. p. 712. - Ferner 1821. II. p. 125. - Nachrichten von Martius. Daselbst 1822. II. p. 739. - Ferner von Schultes 1824. II. p. 677.

Freiburg. Nachricht ron den dortigen Anstalten.

Bot. Zeit. 1820. II. p. 647. - Dergleichen von Perleb. Daselbst 1826. II. p. 616.

Natalitia augustissimi principis Ludovici Guilielmi Augusti indicit D. C. J. Perleb. Disseritur simul de horto botanico Friburgensi. Friburgi 1829. 4. $36 \mathrm{kr}$.

Giessen. Nachricht von den dortigen Anstalten von Wilbrand.

Bot. Zeit. 1823. II. p. 757. - Ferner 1825. I. p. 163. und 1826. II. p.513, wo das Geschichtliche des Gartens mitgetheilt wird. Man sehe auch Hesperus den 17. Dec. 1829. No. 301. - Ferner eine kurze Nachricht von Schultes in der bot. Zeit. 1824. II. p. 678.

Göttingen. Nachricht von den dortigen Anstalten von Wenderoth. Bot. Zeit. 1819. II. p. 705. - Eine andere von Schultes 1824. II. p. 681 .

Hamburg. Nachricht von den dortigen Anstalten ron Wenderoth. Bot. Zeit. 1819. II. p. 709. - Ferner 1821. I. p. 124.

Verzcichnifs der Topfpflanzen und der im Lande ausdauernden Stau- 
dengewächse, wclche im IIamburger botanischen Garten abgegeben werden kïnnen. (Nebst Preisen). Hamburg 1826.

Semina in horto Iamburgensi botanieo 1826 collecta, quae pro mutua commutatione afferuntur. Ilamburg 1826. Alia collectio 1827. 'Tertia 1529.

\section{Man sehe Linnaen 1830. II. p. 45 . Lit.}

Index scholarum in Mamburgensimu Gymmasio acalemico a Pascha 1828 usigue ad Pascham 1\%29 hahendarum, editus a J. G. C. Lehmanno. Continetur his plagnlis pugillus novarum quarumdam plantarum in botanico Hamburgensium horto occurrentim. Hamburgi 1828. 4.

Ileidelberg. Botanische Anstalten daselbst, Mittheilung von Dierbach. u. $d . f$.

Bot. Zeit. 1820. I. p. 216. - Ferner 1821. I. Beilage. p. 65.

II errenhausen. Nachricht ron dem dortigen Garten von Wenderoth.

$$
\text { Bot. Zeit. 1819. II. p. } 708 .
$$

Kiel. Hortus Kiliensis, oder Verzeichnifs der im botanischen Garten der Universitït zu fiiel cultivirten Gewächse, von F. Weber. Kiel 1822.

Kö̈nigsherg. Nachricht von den dortigen Anstalten für Botanik, von Ernst Meyer.

I. p. 165.

Bot. Zeit. 1826. II. p. 562. Ferner 1828. I. p. 320. Endlich 1830.

Laybach. Nachricht ron dem dortigen botanischen Garten, vom Apotheker Heinrich Freyer jun.

Bot. Zeit. 1829. I. p. 173.

Marburg. Index seminum horti academici Marburgensis anni 1829. 4.

Siche Linnaca 1830. II. p. 53. Lit.

Eine hurze Nachricht von den Garten galo Sehultes.

Bot. Zeit. 1824. II. p. 679.

München. Nachricht von den dortigen Anstalten, von A. E. F ürnrohr.

Bot. Zeit. 1823. I. p. 35 3ิ.

Martius. Hortus botanicus academiac Monacensis. Monach. 1825. 4. cum tabul. lithogr.

Hortus regius monacensis. Verzeichnifs der in königl. botan. Garten zu München wachsenden Pflanzen. München 1829. im königl. Central - Büeher - Verlag und Leipzig bei Fleischer. 210 S. 8.

Siehe bot. Zeit. 1829. II. p. 569.

Mïnster. Der Garten für die deutsche Flora daselbst, vom Prof. Wernekink.

Zot. Zeit. 1821. II. p. 645.

Nymphenburg. A. Sterler Hortus Nymphenburgensis seu enumeratio plantarum in horto regio $\mathrm{N}$. cultarum. Nonachii 1821. 8.

Pesth. Botaniker und botanische Anstalten daselbst. Bot. Zeit. 1822. I. p. 235 .

Prag. Nachricht von dem Museum dasclbst.

Bot. Zeit. 1820. I. p. 27. ferner dergleichen mitgetheilt von Hornschuch 1828. I. p. 257 . 
Regensburg. Nachricht von dem dortigen botanischen Garten.

Bot. Zeit. 1821. II. p. 739.

Salm-Dyk. Nachricht ron dem Garten des Fürsten.

Siehe bot. Literaturbl. III. p. 689.

Salzburg. Nachricht von den dortigen Gärten und Botanikern.

Bot. Zeit. 1822. II. p. 625. ferner 1823. I. p. 310., endlich 1824.

I. p. 187. und 1828. II. p. 646. (von Hop pe.)

Stuttgardt. Nachricht ron den dortigen Anstalten von Martius. Bot. Zeit. 1822. II. p. 737.

Hortus Belvederanus, odcr Verzeichnifs der bestimmten Pflanzen, welche in dem Grof'sh. Garten zu Belredere bei Weimar zu finden sind. Weimar 1820.

Wien. Nachricht ron den dortigen Anstalten.

Bot. Zeit. 1822. II. p. 465. ferner 1825. I. p. 182.

Ueber den Zustand der Botanik in Wien, vom Obristen v. Welden. p. 446.

Daselbst 1826. I. p. 2.11. ferner 1827. II. p. $4 \hat{3}$. und 1828. II.

Würzburg. Zustand der Botanik daselbst.

Bot. Zeit. 1819. II. p. 520, ferner 1824. II. p. 675.

Ueber die holländischen Gärten, namentlich jene zu Utrecht, Amsterdam, Harlem und Leyden theilte Herw Hofrath Schultes lesenswerthe Nachrichten mit.

Bot. Zeit. 182t. II. p. 405 - 721.

T. G. Bruckmann. Elenchus plantarum, quae in horto Lugd. Batav. coluntur 1818.

\section{b. In Frankreich.}

Notice historique sur le jardin botanique de Caën, par M. Lange.

Mémoires de la Soc. Lim. de Calvados. Année 18\%5. p. 34. Bulletin des Sc. natur. Octobre 1926. p. 210.

Catalogue des végétaux de plcine terre, d'orangeric et de serre du jardin de Fromont près Ris (Seine et Oise) pour 1826. Paris chez M. Húzard.

Catalogus plantarum horti regii Parisiensis cum annotationibus de plantis novis ant minus connitis. Auct. R. Desfontaines. Edit. 3. Paris 1829. Chaudé 7 francs.

Man sehe auch über dicsen Garten bot. Literaturbl. III. p. 681.

Nachrichten über die butanischen Gürten in Paris findet man auch in der bot. Zeit. 1830. I. p. 2è2. dann II. p. 671.

Nachrichten von dem Garten in Strasburg theilte Herr Hofrath v. Martius mit.

Bot. Zeit. 1822. II. p. 738.

Catalogue des plantes cultivées au jardin botanique de la marine royale du port de Toulon, à l'usage des élèves de l'école de médecine. Avignon 1821. 8.

Alfroy. Catalogue des arbres fruitiers, arbres d'alignement et forestiers, arbustes et plantes etc., qui se trouvent dans la pepinière de Lieursaint. Paris 1821. 
Jacques. Catalogue alphahetique des plantes, arbres ou arbustes croissant naturellement, on enltivés en plein air, orangerics ou serres chaudes dans les jardins de Nenilly, du Rainey et de Moussoaux, appartenant ì S. A. R. Mgr. le Due dOrleans. Paris $\mathbf{1 8 2 5 .}$

$\mathrm{Agl}$ a e $\mathrm{Ad}$ anson. Catalogue des arbres, arbrisseaux, arbustes et plantes vivaces cultivées en pleine terre, à Baleine près Moulin, Depart. de l'Allier. Paris 18'5.

Catalogue général des arbres, arbustes et plantes tant de 6erre que de pleine terre, cultivées dans les jardins et pépinières de L. Noisette. Paris 1826.

Pépinière de M. Cels. Paris 1826. 8.

\section{c. In Italien und Spanien.}

Nachrichten von Acerbi über Botaniker und botanische Anstalten in Italien.

$$
\text { Bot. Zeit. 1821. I. p. } 93 .
$$
Garten.

Bologna. Man sehe Brunners Nachrichten über den dortigen p. 280.

Bot. Zeit. 1828. I. p. 161. - Geiger's Magazin. Juny 1826.

Viridarii Bononiensis Vegetabilia, cum aliis regetalilibus commutanda ad annum MDCCCXXIV. Anctore Dr. Bertoloni.

Giornale Arcad. Feb. 1824. p. 189. In derselben Zeitschrift März 1826. $p$. $3+1$ theilt Herr Prof. Bertoloni Nachrichten von einigen im Garten zu Bologna cultivirten Pflanzen mit.

Borromäische Inseln. Ueber die Gärten derselben lese man Brunner's Nachrichten in der

bot. Zeit. 1828. I. p. 170.

Naupoil, Carlo. Catalogo degli alheri e delle piante coltivati nei 6uoi vivai e giardini al Dolo. Venezia 182.t. 8.

Ferrara. Ueber den dortigen Garten sehe man Brunners Nachrichten in der

bot. Zeit. 1828. I. p. 166.

Florenz. Ueber den dortigen Garten sehe man Brunners Nachrichten in der

$$
\text { bot, Zeit. 1827. II. p. } 577 .
$$

Genua. Ueber den dortigen Garten sehe man Brunner s Nachrichten in der

$$
\text { bot. Zeit. 1827. II. p. } 385 .
$$

Mailand. Nachrichten über die dortigen Anstalten gab Graf IIenckel von. Donnersmark.

Bot. Zeit. 1820. I. p. 38. und Dr. Brunner daselbst 1828.

I. p. 169 .

Mon v. Donnersmark.

Bot. Zeit. 1820. 1. p. 44. Catalogus plantaru
tabul. (auct. Ros osi.)

Fs sind hier sogenannte Luflpflanzen beschrieben: Tillandsia diantoidea, Pourretia aerantha, Tillandsia stricta Curtis.

Neapel. Nachrichten von den dortigen Gärten gab IIerr Dr. Brunner. Bot. Zeit. 1825 II. p. 658, und 721.

Semina 1825. collecta, quae hortus botanicus Neapolitanus pro mutua commutatione offert. Auct. Michacl Tenore. 
Index Seminum et Plantarum riventium, quae in horto regio Neapolitano pro mutaa commutatione offeruntur, vel venalia prostant. Anno 1829. Accedunt de re herbaria adnetationes nonnullạe.

Palermo. Ueber den botanischen Garten daselbst von Dr. E. Güntz. Bot. Zeit. 1830. II. p. 441.

Index seminum anni 1925, quae ab horto regio in Boccadifalco pro mutua commutatione exhibentur. 4.

Catalogus plantarum, quae asservantur in regio horto Ser. Franc. Borbonii in Boccadifalco prope Panormum. Auctore Gussone. Neapol. 1821. 8.

In dieser Schrift sind sugleich 15 , neue sicilianische Pflanzen beschrieben: nemlich Chrysanthemum hybridum, Cineraria gibbosa, C. nebrodensis, Colchicum bivona, Crepis spathulata, Erica sicula, Euphorbia eccremocarpa, Duphrasia Bocconii, Genista Cupeni, Hyacinthus dubius, Ononis sicula, Sescli Bocconi, Trifolium congestum, T. intermedium, Trigonella littoralis, Urtica :"upestris. Bulletin des Sc. natur. Juin 1826. p. 212.

Paria. Nachricht von dem dortigen botanischen Garten ron Dr. Brunier.

Bot. Zeit. 1821. I. p. 168.

D. Nocea. Historia atque iconographia horti botanici Ticinensis. Ticini 1824.4.

Pisa. Nachrichten von dem dortigen Garten von Dr. Brunner. Bot. Zeit. 1827. II. p. 616

Notizie per servire alla storia del Giardino e Musco della I. e. R. Universita di Pisa di Gaetano Savi, Professor di Botanica. Pisa 1828. 8. 1830. p. 92.

Bot. Literaturbl. II. p. 208. - Bulletin des Sc. natur. Suillet

Hortus Ripulensis sen enumcratio plantarum, quae Ripulis ab Aloys Colla coluntur, additis stirpium rariorum vel nondum satis cognitarum aut forte novarum, natis, descriptionibus et iconibus. Turin 1824.

Man sehe über diese und einige andere hierher gehörige Schrif-

ten Linnaea 1829. III. p. 50. Lit.

Rom. Ueber die dortigen Gärten sehe man Dr. Brunners Nachricliten in der

bot. Zeit. 182\%. Ii. p, 392.

Supplement au catalogue des plantes du jardin de St. Sebassian, par le marquis de $\mathbf{S} p$ in. Turin 1823.

Ueber die Gärten zu Verona, Treriso und Venedir sehe man dic Nachrichten des Grafen Henckel von Donnersuark in der

bot. Zeit. 1820. I. p. $53-56$.

Ueber Botaniker und botan. Anstalten in Spanien theilte Herr ron Martius einige Nachrichten mit.

Bot. Zeit. 1821. I. p. 76.

d. In England, Dänemark, Schweden und Russland.

Flora Historica, or the thrce seasons of the british parterre. Ilistorische Flora, oder dic drei Jahrszeiten der brittischen Gärten, von W. Philips. London und Edinburg 182t. 2 Vol. 8.

Hortus suburbanus Londinensis; oder Catalog von Pflanzen, 
die in der Gegend von Inndon cultivirt werien, nnch dem Linnelschen System geordnet, mit Angabe der natürlichen Familien zu denen sie gehören, der Werke, in denen man sie heschrieben findet, Vaterland und Blüthezeit, von Robert $\mathbf{S}$ weet. London 1824.

Nachricht von mehreren betanischen Gïrten in England, mitgctheilt von $\mathrm{Sehulte}$.

Bot. Zeit. 1825. Bd. Y. Reil. I. Dergleichen von Ilerrn Otto. Bot. Literaturbl. III. p. 683.

Einen medicinisch-botanischen Garten, worin die in den brittischen Pharmakopöen aufgenommenen I'flanzen cultivirt werden, hat flerr A iton in einer besondern Abtheilung des küniglichen botanischen Girtens zu Kew eingerichtet.

Froriep's Notizen. Bd. XXVI. p. 106.

Page's Prodromus or a general Nomenclator of all the plants, indigenous and exotic coultivated in the Southampton botanic gardens. London 1818.

Nachrichten von der Finrichtung des hotanischen Gartens in Lirerpol, findet man bei Spieker, Reise durch England, Wales und Schottland. Bd. I. p. 379.

Conr. Loddiges. Catalogue of plants which are sold at Hackney. London 1826.

Nachricht ron dem botanischen Garten in Koppenhagen, von Hornsehuch. p. 492.

Bot. Zeit. 1820. II. p.430. ferner sche man daselbst 1823. II.

Botaniker und botanische Anstalten in Lund.

Flora 1820. II. p. 415.

Enumeratio plantarum horti botanici unirersitatis regiac Fredericianae Christianiensis. Christianiae 1823. 8. uann.

Nachricht ron dem botanischen Garten in Paulorsk ron WeinBot. Zeit. 1820. II. p. 668.

Nachricht von dem botanischen Garten in Petersburg.

Das. 1823. I. p. 29. II. p. 655. - Bot. Literaturbl. III. p. 690.

Beschreibung des kaiscrlichen Gartens zu Nikita vom Dr. von Steplen zu Sympheropol.

Verhandl. des Vereins zur Beförderung des Gartenbanes. Zehnle Lieferung. Berlin 18ะ8. p. 103.

Index reminum in horto botanico Dorpatensi 1819 collectorum.

Siehe Flora 1820. I. p. 271.

F. E. L. Fischer. Index plantarum anno 1824 in horto Impcriali Petropolitano vigentium. Petropol. 1824.

In Jahre 1653 enthielten die vorzïglichsten botanischen Gürten in Europa die nachstehenden Zahlen an Bäumen, Sträuchern und krautarigen Gewächsen.

1. Der königliche Garten in Coppenhagen . . . . . 1518.

2. Der könirliche Garten zu Parif. . . . . 2121.

3. Der königliche Garten in der Vorstail zi Warschan. 780 .

4. Der königliche Garten in Schlosse zu Warschau . . 740.

5. Exotische Pflanzen, die in der Umgegend von Warschau cultivirt wurden 


\section{$-243$}

6) Medicinischer Garten zu Oxford

7) Der Garten zu Padua

8). Der Garten zu Leiden

9) Der Garten in Gröningen

Bullet. des Sc. natur. Janv. 1S330. p. 12.6.

\section{e. Ausserhalb Europa.}

Nachricht ron dem Garten im Thale Cauca in Columbien.

Bot. Literaturbl. III. p. 692.

Nachricht ron dem botan. Garten in Rio Janeiro.

Daselbst $p$. 6 i91.

Arruda. Die Gärten in Brasilien.

Isis III. p. 1502.

A n a c count of the botanic garden of St. Vincent by Guilding. Glasgow 1825. 4. with plates.

Catalogue des plantes cultivées au jardin royal de Pondichery, pour l'année 1827, par le jardinier pepinièriste Rich ard.

p. 409.

Annales maritimes et coloniales 13. Année Nro. 3. Mars 1828.

Catalogue des plantes cultivées au jardin botanique et de naturalisation de l'ile de Bourbon (Année 1825), par N. Bréon.

Ibid. Nro. 6. Juin 1828. p. 761 .

Catalog ue général des plantes cultivées anx Colonies; Supplement nu Catalogue des plantes cultirées aux jardins de Botanique et de naturalisation de l'ile Bourbon et de Richard - Tol au Senegal.

Annales marit. et coloniales. Dec. 1829. $p$. 884 .

Catalogus ran eenige der merkwaardigste zoo in als uit-heemsche Gewassen, te vinden in 'sLands Plantentuin te Buitenzorg. Opgemaakt door C. L. Bl ume, M. D. Batavia 1823.

Siehe botan. Zeit. 1825. I. p.97 u. d. f.

South African Institution.

Philosophical Magasine and Annals of Philosophy 1830. Sept.

p. 222. - Botan. Zeit. 1830. II. p. 676.

\section{Nachrichten von mehreren Botanikern und Freunden der Gewächskunde.}

\section{§. 44.}

Noch bis auf den heutigen Tag besitzen wir keine biographische Uebersicht der Botaniker, die von den ältesten Zeiten an bis auf unsere Tage die Pflanzenkunde betrieben, und durch ihren Fleijs und rühmliche Anstrengung diese gemeinnützige IVissenschaft, zu jenem Grade von Vollkommenheit brachten, den sie wirklich erreicht hat. Sollte liünftig ein Gelehrter sich mit Abfassung biographischer Nachrichten beschäftigen uollen, so werden die nachstehenden Notizen die Nachforschungen erleichtern.

Amoreux. Notice sur Pierre Joseph Amorenx, par M. Thiebaud de Berneaud.

Annales de la Soc. Linn. de Paris Janv. 1826. p. 688.

Aschoff: geboren am 25. Novemb. 1758 zu Weeze im Clevischen. † zu Bielefeld ain 13. Juny 1827.

Man sehe dessen Nekrolog von Weihe in der Flora 1829. II. p. 1u6. der Ergünzungsblätter. - B randes, dessen pharm. Zeitung 1828. 325. 
Bank ค. + 19. May 1820 .

Bloge historique de M. Banks, par M. le baron Cuvier. M!moires du Muséum d’hist. natur. Septième Annće. Cinquième Cahier $p$. 29) -326 .

Vine kurze Nachricht von dem Vermüchtnifs seiner botanischen Scheitze an das brittische Museum und Robert Broun steht in der Flora 1821. I. p.. 123.

Baue (Erasmus), Pfarror zu Tittmoning, vorzüglicher Kenner der Kryptogamen. †' 15. März 1825.

Siehe Flora 1825. I. p. 304.

Bauer: trefflicher Pflanzenmaler, Begleiter Robert Brown'a. $\dagger$ zu Wien 17. März 1826.

Siehe Flora 1826. I. p. 240.

Bellardi (Carl Ludwig). † 4. May 1826 in einem Alter von 86 Jahren zut Turin.

Siehe Flora 1826. II. p. 432.

Bergfeld (Arnold), chemal. Sekretair der botan. Gescllsch. † zu Regenshurg am 15. October 18:30, 81 Jahre alt. Flora 1830. II. p. 6966.

M. r. Bieberstein (Friedrich Angust), geho:ren zu Stuttgardt am 10. August 17fis. † zu M!aref, 8 Werste ron Charkow, an 28. Jini 1826.

Siehe dessen Nekrolog in der Flora 1827. II. p. 5366.

Bok, geboren zu Naumburg. † 4. Januar 1822.

Interessante Nachrichten von demselben stehen in der Flora 1822. II. p. 629 .

Bose: Notice hiographique sur M. Bosc.

Bulletin des Sc. natur. Oct. 1828. p. 281. - Biography of M. Bosc by Buron Cuvier. - Jameson New Edinb. philos. Journal. Jul. - Oet. 1829. p. $2 \pi 4$.

v. Bridel (Samuel Elias), geboren zu Cressig im Kanton Waadt. + 7. Januar $1828 \mathrm{zu}$ Gotha, 67 Jahre alt.

Siehe Flora 18:8. I. p. 335.

Brotero. t in Lissabon.

Siehe bot. Zeitung 1829. I. p. 210.

Campaigne. Notice sur Pierre Campaigne.

Ami des Champs de Bordeaux. Mai 1826. p. 173.

Cas sel, gestorben 1821 in Gent.

Siehe Flora 1821. II. p. 761.

Cerrantes (Vicente), Prof. der Botanik in Mexico. t am 28. Juli, 1829, 70 Jahre alt.

Annales des Sc. nat. Mars 1S30. - Revue bibl. p. 31.

Clemente de Roxas, ein spanischer Botaniker. † im Februar 1827. Flora 1827. II. p. 736.

C. Clusii Atrelatis et Conr. Gesneri Tigurini Epistolae ineditac. Fx arehetypis edidit adnotatiunculas adspersit, nec non praefatus est Lud. Christ. Treviranus. Lipsiae 1830.

v. Cobres (Paul). † am 26. Dec. 1823 zu Göggingen bei Augsburg,

76 Jahre alt. Flora 1821. I. p. 48. 
Correa de Serra. Nachrichten von dem Leben und den Schriften desselben lieferte Herr Dr. François d'Almeida in den

Mémoires du Muséum d'hist. natur. Vol. XI. p. 215 -229.

Er starb am 10. October 18ะ3 zu Lissabon; sein Geburtsort ist Serpa in Portugall.

Man sehe Flora 1823. II. p. 672. und 1825. II. Beilage p. 70.

Dickson (James). † im Frühjahr 1823.

Dittmarse h (Eduard) aus Dresden. + 23. Juni 1821 auf der Sachsenburg in Thüringen.

Flora 1821. II. p. 555.

Duval (Carl Jeunet), geboren zu Roie in der Picardie 1751. f zu Jolbach 10. Sept. 1828, 78 Jahre alt.

Flora 1828. II. p. 619. 1830. I. p. 185. auch 1829. I. p. 39.

Elliot (Stephen), Prof. der Botanik. + zu Charlestown in Süd-Carolina im April 1830.

Bot. Zeit. 1830. II. p. 693.

Fisch er. Garten-Inspector in Göttingen.

Flora 1820. II. p. 622.

Freyreis.
Flora 1tzo. Leopoldinia in Brasilien an 1. April 1825. 1825.

Gärtner (Philipp Gottfried), Apotheker in Hanau. † 27. December

Flora 1826. I. p. 31.

Gebhard (Joh. Nepomuck), gebürtig aus Freisingen. † zu Grätz 9. Juni 1827, 63̈ Jahre alt.

Flora 1828. II. p. 495. ferner 1830. II. p. 533.

Goldbach (Carl Ludwig), geboren den 12. April 1793 in Leipzig. † 13. März 1824 zu Moskau.

Man sehe die interessanten Nachrichten, mitgetheilt von $K u n z \epsilon$ in der Flora 1824. I. p. 380.

Graumüller in Jena. † im Jahre 1824.

Flora 1824. II. p. 752.

v. Guckenberger, Collegien-Assessor. † im Jahre 1821.

Flora 1821. II. p. 460.

Hagen (Carl Gottfried). † zu Königsberg am 8. März 1829, 80 Jahre alt.

Flora 1829. I. p. 187.

Hargasser (Johann Georg), geboren 1785 zu Mühldorf in Baiern. † auf dem hohen Göhl am 22. August 1824.

Flora 182t. II. p. 557. 1825. II. p. 445.

v. Harrach (Graf Carl). † zu Wien am 19. October 1829.

F'ora 1830. I. p. 256.

Heim (Ernst Ludwig), hiographische Nachricht von demselben Flora 1824. II. p. 506. 1825.

Heinrich (Joseph Placidus). † zu Regensburg an 18. Januar

Flora 1825. I. p. 95.

Heinze, früher Apotheker in Heidelberg. $t$ am 15. Juni 1823 in Weinheim.

Bot. Zeit. 1823. II. p. 752. 


\section{$-245$}

Iemprich. † zu Massana in Abyssinien, ain 60. Juni 1824, 80 Jahre alt.

Flore 1825. II. p. 768.

v. Hentschel. † zu Kastelec in Mühren im Jahre 1823.

Flore 1824. I. p. 191.

II eyne (Benjamin). † am 6. Februar 1819 zu Vappera bei Madras. Flora 18\%. 1. p. 192.

II ils e nhere aus Erfurt. † auf der Insel Madigasear. Bot. Zeit. 1825. II. p. 608.

II of fmann (Georg Franz), geboren 1760 im Bayreutisclien. $\div 5$. März 1826, nach einer andern Nachricht am 17. Mïrz.

Bot. Zeit. 1826. I. p. 336. - Biographische Nachrichten von demselben findet man in dem neuen Vugazin für Natargeschichte

Nro. 4. Moscau. April 1826. p. 238-256, mitgetheilt vom Herrn Maximovitch; auch sehe man Bulletin des Sciences natur. Janv. 1829. p. 107.

r. II ohenwarth, Fürstbischoff von Linz. t am 22. April 1825, 80 Jahre alt.

Bot: Zeit. 1825. I. p. 367 .

v Kerner (Juliann Simnn). t zu Stıtrgardt am 13. Juni 1830.

Flora 18ü. II. p. 472.

Kohaut (Franz) aus Prag. + in der französischen Kolonic am Scnegal im Jahre 18\%?.

Bot. Zeit. 1823. I. p. 111 und p. 190.

Iiroker, Verfasser einer Flora Silesine. † in Jahre 1823.

Bot. Zeit. 1824. I. p. 191.

Kuhl (Heinrich) aus Hanau. † auf der Insel Java am 16. September 1821.

Bot. Zeit. 1822. I. p. 238. ferner 1822. II. p. 633. - Eine biographische Skizze ron ilm findet man auch in dem Edinburgh New Philosophical Journal April-July 1826. p. 1-22.

Lamark. + zu Paris am 20. Dec. 1829.

Bot. Zeit. 1830. I. p. 64 .

Lamouroux. Eloge historique de M. Lamouronx, par un de ses élères. Caën 1828. † an cincm Schlagflussc zu Caën àn 26. März 1825, 46 Jahre alt.

Man sehe unch Bullet. des Sc. nutur. Juin 1830. p. 458.

Lee (James), in Hammersmith bei London. f am 10. Juni 1824. Bot. Zeit. 18\%5. II. p. 463 .

Lestiboudois. Fotice biographique sur J. B. Lestiboudois, par M. Bottin.

Recueil des amat. de Lille année $\mathbf{1 8 2 3}$ et 1824. p. 357.

Linné. Auch über den berïhmtesten und geschätztesten der Botanilier sind in den letzten Jihren mehrere Nachrichten bekant geworden.

Linnés eigenhändige Anzeichnungen über sich selbst, mit Ammerkungen und Zusätzen von Afzelius. Aus dem Schwedischen ühersetat ron Kiarl Lappe. Mit einer Vorrede von Dr. K. A. Rudolphi. Nebst Linnés Bildnifs und Handschrift. Berlin 186. Gedruckt und verlegt bei G. Reimer. 2 fl. $15 \mathrm{kr}$.

Siche Geiger's Magazin Nov. 1826. p. 170. - Bot. Zcit. 1827. I. p. $29 ! 9$. 
Antiquitates Iinnaeanac. Programma quo ad solemnem tnaugurationem Philosophiae Doctorum crastina luce celebrandam magnos hospites, literarum patronos, patres civesque academicos et urbicos reverenter et perofficiose invitat legitime constitutus Promotor Carolus Adolphus Aghard, Philos. Doctor, Botanices et Oecon. pract. Professor R. O. Lundae MDCCCXXVI. Litteris Berlingianis.

Ueher Linné's Standbild siehe bot. Zeit. 1823. I. p. 30. ferner 1830. II. p. 632.

Caroli Linnaci eq. literas XI. ad Alex. Gardenium, D. Med. Carolinensem datas necdum promulgatas edidit A. F. Lueder's, M. D. Prof. P. O. Kil. Regiis impensis. Kíliae Holsatorum 18æ9. 4.

Siehe Linnaea 1830. III. p. 81. Lit.

Féte Linnéenne.

- Siehe bot. Zeit. 1827. I. die erste Beilage.

Gedächtnifsfeier : Lin n é's in München.

Daselbst p. 99. der Beilagen.

Notice sur un opuscule relatif de Linné, par le baron Deggenettes.

Journal compl. Tom. XXX. Mars 1829. p. 91.

Maratti. + am 12. März 1829, 96 Jahre alt.

MarteIli. † zu Rom im 95sten Jahre seines Lebens.

Bot. Zeit. 1829. I. p. 240.

Märklin. † zu Wiesloch bei Heidelberg am 31. Juli 1823.

Bot. Zeit. 1823. II. p. 752.

Mayer (Friedrich). $\dagger$ in Italien am 21. December 1828.

Bot. Zeit. 1829. I. p. 46.

Mecse. Oratio de meritis Dav. Meese, tum botanicis, tum aliis. Groningae 1823.

Mertens (Heinrich). † zu St. Petersburg am 30. Sept. 1830, 34 Jahr alt.

Bot. Zeit. 1830. II. p. 694.

Moser (Herrmann). $\dagger$ in Paris am 28. Jun. 1826.

Bot. Zeit. 1826. II. p. 623.

v. Nenfville (Wilhelm Robert). † zu Bonn am 12-Dec. 1819.

Bot. Zeit. 1820. I. p. 63.

Palisot de Beauvois in Paris.

Biographical Memoir of Baron de Beauvois, By Baron Cuvier. Aus dem Französischen in The Edinb. New Philos. Journal by Jameson. Oct. -Dec. 1828. p. 1-21.

Panzer (Georg Wolfgang Franz). † am 28. Juni 1829 zu Her'bruck bei Nürnberg, 75 Jahre alt.

Bot. Zeit. 1829. II. p. 400.

v. Portens chlag (Franz Edler). † am 7. November 1822, 51 Jahre alt, in Wien.

Bot. Zeit. 1822. II. p. 81. Beilage.

Pursh (Friedrich), geboren am 4. Febr. $1784 \mathrm{zu}$ Grossenhayn in Sachsen. $t$ am 22. Sept. 18\%5. in Canada.

Bot. Zeit. 1827. I. p. 192. II. p. 491. 528. - Notice sur Pursh; Annales de la Société Linnéenne de Paris. Janv. 1826. p. 710. 
ran Raalten. $千$ auf der Insel Timor.

Bot. Zeit. 1830. I. p. 48.

Raddi (Joseph). † auf Rhodos. Eine kurze Nachricht ron ihm findet man in der

Bibl. universelle. Fúvr. 1830. p. 220); auch sehe man botan. Zeit. 1829. II. p. 752.

Ramond. Historical Eloge of Louis François Elisabeth Baron Ramond. By M. le Baron Cuvier.

Edinb. Journal of Scicnce. January 1830. p. 1-20.

Ra u (Ambrosius). + zu Würzburg am 26. Januar 1830.

Bot. Zeit. 1830. I. p. 256 .

Reynier. $\dagger$ in Lausanne im November 1821.

Bot. Zeit. 1825. II. p. 464.

Richard (Claude Louis). † in Paris am 7. Juni 1821, 67 Jahre alt. Man sehe Eloge historique de M. Richard, par M. le baron $\mathrm{Cu}$ vier. - Mímoires du Mus. d'hist. nat. 'ol. XI. p. 215-229.

Roscoe (William). Mehrere Nachrichten von den zum Theil widerwärtigen Schicksalen dieses auch in Deutschland bekannten und geschätzten Botanikers gibt Spieker (Reise nach England, Wales und Schottland Bd. I. p. 389 u. d. f.), eine Skizze seines Lebens ist auch enthalten in Dictionary of living Authors. London 1816. S.

$\mathbf{R}$ oth in Vegesack, Nestor der deutschen Floristen, feicrte am 17 Sept. 1828 вcin 50ijihriges Doctorjubilacum. Bot. Zeit. 1829. II. p. 704 .

Sehäffer (D. J. U.), feierte in Regensburg am 20. September 1825 6ein 50jähriges Jubilaeum als Arzt. Bot. Zeit. 1825. II. p. 638.

Schmidt (Joseph) zu Rosenhcim in Baiern; cine kurze Nachricht von ilım steht in der botan. Zeit. 1820. II. p. 606.

Sehott. Botan. Gärtner an der Unirersităt in Wien. † an 18. Juli 1819, 56 Jahre alt:

Bot. Zeit. 1819. II. p. 491.

Schweigger (A. F.), ermordet in Sicilien.

Siehe bot. Zeit. 1821. II. p. 747. und 1822. I. p. 30. - Bruchstücke aus dem Leben des als Opfer sciner Wissenschaft gefallenen Dr. A. F. Schueigger, vormals P'rof. der Medicin und Botanik in Königsberg. Halle 1830.

Sibthorp. In der Abteikirche zu Bath ruht dieser berïhnte Kenner der Gewächse Griechenlands; sein Denkmal ist von Flaxmann gearbeitet, es stellt ihn ais Pilgrim dar, in den elisäischen Feldern landend, mit einer Pflanze in der IJand.

Spieker's Reisen Bd. II. p. 158.

Smith (James Edward). † an Norwich im März 1828.

Bot. Zeit. 1828. II. p. 560. - Biographical Notice of the late

Sir J. F. Smith, l'resident of the Linnean Society etc. By the Rev.

E. B. Ramsay.

Edinburghth Journal of Science. July 182!). p. 1-16.

Smith (Christian). Biographical Memoir of the late Chr. Smith, M. B. Naturalist to the Congo Expedition. By Baron Leopold v. Bucis. 
The Edinburgh New Philos. Journal by Prof. Jameson. July Oct. 1826. p. 209-215. - Notice sur Chrctien Smith, communiquce à M. Decandolle, par. M. le baron Leopold de Buch.

Bibliothèque universelle. T. XXXIII. Janv. 1828. p. 5̇.

Sow erloy (James). + in October 182?.

Bot. Zeit. 1825. II. p. $4(63$.

Sprengel (Christian Courad). Erinnerungen an Denselben, nebst einigen Bemerkungen aus seinem Leben, stehen in der

bot. Zeit. 1819. II. p. 541. (höchst lesenswerther Aufsatz!).

Swartz (Olaf), geboren zu Noorköping am 21. Dec. 1760. † am 19. September 1818 in Stockholm.

Bot. Zeit. 182!. II. p. 550.

Adnotationes botanicae, quas reliquit Olavus Suartz. Post mortem Auctoris collectae, examinatae et in ordinem systematicum redactae atque natis et praefatione instructae a Johanne $\mathrm{Em}$. Wickström. Accedit Biographia Suartzii auctoribus C. Sprengel et C. A. Aghard. Adjectis effigie Swartzii delineatione monumenti ejus sepulcralis atque duabus tabulis botanicis. Holmiae 1829. 8.

Linnaea 1830. III. p. 87. Lit.

Targioni Tozetti (Ottav.). † zu Florenz am 6. May 18 29, $7 t$ Jahre alt.

Bot. Zeit. 1829. II. p. 480.

Thomas (Ludwig) aus Bex. $\dagger$ in Neapel den 9. Januar 1823, 39 Jahre alt.

\section{Bot. Zeit. 1824. II. p. 464.}

Thouin. Eloge histerique de M. A. Thouin, lu dans la séance publique annuelle de l'Academie du lundi 20. Juni 1825, par M. le baron Cuvier, Sécrćtaire perpetuel.

Mémoires du Muséum d’Hist. nat. Tom. XIII. Cahier 4. p. $205-$ 216. - Er wurde geboren am 10. Februar 1747. † 23. Sept. 1824 in Paris.

Es scheint, dafs zwci Herren Thou in in Paris lebten; im 12. Bande $p .1$ u.d. $f$. der angegebenen Mémoires, wo Nachrichten von dem Leben und den Schriften desselben mitgetheilt sind, ist der Todestag am 27. October 1824 angemerkt, womit auch die Nachricht in der bot. Zeitung 1821 11. p. 704. übereinstimmt.

Thunberg (Carl Peter), geboren zu Jönköping den 11. Nov. 1713. † 8. August 1828 zu Upsala.

Bot. Zeit. 1828. II. p. 89. der Ergänzungsbl.

Thyssen (H. F.). $\dagger$ in Amsterdam am 8. Januar 1S30, 42 Jahre alt. Bot. Zeit. 1830. I. p. 176.

Tournefort. Eloge historique de Tournefort, prononcé dans la séance publique du 24. Mai 1825, par M. Roberge.

Mémoires de la Soc. Linn. de Calvados. Année 1825. p. 318.

v. Waldstein (Graf Franz). † am 22. May 1823 zu Obcrleitensdorf in Böhmen.

Bot. Zeit. 1823. I. p. 383.

Wallich. Nachrichten von diesem noch lebenden und durch scine zahlreichen Entdeckungen so berühmt gewordenen Botaniker findet man in der

bot. Zeit. 1820. II. p. 440. 
Willdenow (Carl Lndwig), geboren zu Berlin an 22. August 1765, gesterben daselbst am 10. Jul. 1812 .

Bot. Zeit. 1821. I. p. 204.

$W_{0}$ If $(\ldots)+.\div$ am 2r. Juli 1825 zu Schwcinfurt, 82 Jahre alt. Bot. 'Leit. 18\%5. II. p. 7(i8.

Wredow (Joh. Christ. Lulwig). † am 11. Angust 1823 in Schwerin. bot. 'Zeit. 1823. II. p. (iuld.

Wyttenbach (Samnel). $\dagger$ in Bern am 22. Mai 1830, 82 Jahre alt. Dus. 1830. II. p. 694 .

Zippelius. t auf der Insel Timor. Bot. Zeit. 1830. I. p. 48.

$\mathrm{Ziz}$ (Johanu Baptist). $\dagger$ am 1. December 1829 in Mainz. Bot. Zeit. 1829. II. p. 131. der Ergănzungsbl.

Ein Yerzeichnifs der in Bölemen lebenden Botaniler ist milgetheilt in der bolanischen Zeitung 1824. II. p. 136 der Beil. und 1825. II. p. 49. der Beiluge.

\section{Literatur der Botanik.}

5. 45.

Sie ist jetst schon schr ausgedehnt, und eine vollständige Darstellung dersellen dü̈fte zu den schwierigsten Aufguben gehören. Alphabctische Terzcichnisse botanischer Schriften lieferten die Herren Decandolle und Wencieroth; ersterer in seinem alibekannten Regni vegetabilis Systema naturale, letzterer in seinem geschätzten Lehrbuche der botanik (Marburg 1821). Aufserdem sind aber noch besonders folgende Werke zu nennen:

Grundrifs einer Geschichte und Literatur der Botanik von Theophrastos Eresios bis auf die neuesten Zeiten; nebst einer Geschichte der hotanischen Gürten, von J. A. Schultes. Wien 1817.

Kurt Sprengel, Gesehichte der Botanik. Neu bearbeitet. In zwei Theilen. Altemburg und Leipaig 1817.

Biblintheca botanica secundum Botanices partes locos, chronologian, formam, auctores, volumen, titulos, pretium et recensiones concinnata ". 8. w. und zug'eich mit dem deutschen Titel: II a ndbuch der botanisehen Literatur für Botanilier, Bibliothekare, Buchhäudler und Anctionatoren, mit Angalye der Preise und Recensionen, von Friedrich von Miltit\%, Kön. Sächs. Kummerherrn etc. Mit einer Vorrede von Dr. Ludwig Reichenhach. Berlin, bei Angust Rëcher 16?!). 8.

Sehr brauchbar, wemn gleich, so gut wie bei dem vorliegenden Repertorium, noch Manches zu wünschen übrig blcibt.

Literatur der Mathematik, Natur- und Gewerbskunde, von J. S. Firseh. Nene Ausgale von F. W. Schweigger-Seidel. Leiprig, bei Brockhaus 1528. 4 Rthl.

Jahresherieht der Schwedischen Academie der Wirenschaften uiher die Fortschritte der Naturgeschichte, Anatomic und P'lysiolorie der Thiere und Pflanzen. Aus dem Schwedischen mit Zusïtzen von Dr. Joh annен M ̈̈ll ex, aufserordentl. Prof. der Medicin an der Universität zu Jonn, 1824. Der Uebersetzung erster Jahrgang. Bonn, bei Adolph Marcus 1816. 8. 'Zweiter Jahrgane 1825. Bonn 18\%8.

Es ist zu bedauern, dafs diese sehr sorgjällig abgefafsten Jahresberichte so spät zur Kenntnifs des deutschen Publikums kommen. Ge- 
vifs wîrde es höchst willkommen sein, wenn IIerr Proj. IVickst $r \dot{m}$, I'erfasser des botanischen Antheils, eine in lateinischer, franzosischer oder dcutscher Sprache geschriebene Ausgabe dem deutschen Buchhandel übergeben wollte.

Ueber die botanische Literatur des Königreichs beider Sicilien in der neuest:" Zeit. Ton Albrecht ron Schönberg in Neapel.

Linnaea 1827. IV. p. 612.

Uebersicht der neuesten italienischen Literatur.

Bot. Zeit. 1820. I. Erste Seilage vom Grafen Henkel v. Donnersmark. Nachtrag von Herrn v. Martens 1820. II. p. 80. Ferner 1820. II. Beilage p. 98 - 101; - sodann 182?. II. p. 8т-93.

Nachricht ron einem wenig bekannten Kupferwerke des $A .0$. Rivinus, von L. C. Treviranus in Breslau.

Linnaea bid. II. $p .47$ u. d. $f$.

- Notice sur un livre de Botanique des plus rares et sur son auteur, par M. le docteur A mo reux à Montpellier.

Annales de la Snc. Linn. de Paris. Mai 1825. p. 118 - 131.

Es ist hier die Rede von Rudbeck und seinem Buche, betitelt:

Campi Elysaei Liber primus et secundus. Upsaliae 1701 et 1702. 
E r s t e s

oder

\section{Autoren - Register.}

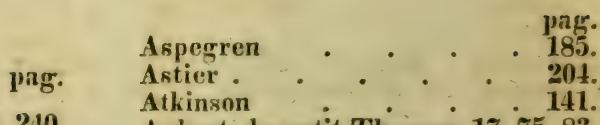

Accrbi . . 118. 159. 240.

Acharius . . . . . 56 .

Adams . . . . 8!. 18!).

Adamsky . . . . 236.

Adanson . . . 240.

Afzelius . . . 217.

Aghard 18. 41. 4i. 46. 47. 65. 91.

97. 107. 119. 148. 185. 2ilo.

204. 206i. 215.

Aiguebelle . . . . 21 .

Alberti . . . . . 138

Alfroy . . . . 239.

Alix . . . . 219.

Allent . . . 201\%.

Alten . . . . . 156 .

Ambreux . . . . 176.

Amici . . . . 206. 209.

Amoreux . . . . 251 .

Amtsbïhler . . . . 16i.

Anderson . . . . . 103.

André . . . . . 82.

Andreus . . . . 222.

Anguetin . . 16.

Antoine de l'Arbre . . 174.

Apel . . $\quad 30$.

Arienander . . . . 89.

Ariendt . . . . . 154.

Arnaud . . . . 174.

Arruda . . . . 213.

Artis . 127.

Aschoff . . . . . 166.
Aubert du petit Thounrs 17. 75.83 .

Aubuissen 131

Aufschläger . . . 161.

Autenrieth . . . . 218.

Antomarchi : . . 190.

Avé Lallemant . . . 177.

B.

Bach • • . . • 169.

Backer . . _. . 2113.

Batlaro . . . . 105. 180.

Bater. . . . 170.

Bailly . . . . . 210.

Bakewell . . . 141.

Balbis . • . 176. 180.

Ballenstedt . • • . 127.

Bancroft . . . . 102.

Bang

Barbieri . . . 6t

Baron . . 173. 176.

Bartels . . . . . 228.

Bartenstein . . 187.

Bartling 30. 40. 72. 86. 89. 110.

111. 112. 120. 178.

Barton . . . . 191. 192.

Bastard . . . - 174.

Batka . . . . 30.87.

Bauhard . . . 19.

Baumann - . 30.31. 116. 
pag.

Baumgarten $\quad \therefore \quad \cdots \quad 158$.

Bauticr $\quad 175$.

Beck 48.60. 192.

Becker $\quad$. 162.

Bedemar . . . . 150.

Behlen . . . . 136.

Beinert • • • • 19.

Belanger $\quad . \quad{ }^{\circ} \quad 187$.

Bellermann . . . . 210.

Benale - $\quad$ • 211.

Bennoit . . . . 175.

Bentham . . . 93. 124. 172.

Beranger $\bullet \quad 151$.

Bérard _ . . . 214.

Berg . . . 118. 210.

Bergamaschi . . . 50. 180.

Bergen - • • • . 98.

Bergeret ? . . 172.

Berggren . . . 151. 187.

Bernhardi . . . 13. 33.

Bernoulli • • • 139.

Bertero - . . 144. 196.

Berthelot - . 74. 108. 190.

Berthold . . . . 30.

Berthollet . . 221.

Bertoloni 18. 37. 65. 72. 118. 177.

Besser : . 86. 142. 185.

Betcke . . . . . 86.

Bencker . . . . . 205.

Beyer $\quad$.,$\quad . \quad 138$.

Biasoletto . . . . 178 .

v. Bibra . . * . 153.

Bigelow . . . . 192

Billardière . . . 197.

Billerbeck . 232.

Bischoff 15. 38, 43, 58. 62. 96. 157.

Bishop $\quad 184$

Bivona . . . . 45.

Bluff $\quad$ 155. 200.

Blume 57. 79. 80. 107. 117. 118.

188. 243.

Blumenhain : . . 214.

v. Boenninghausen $\quad 166$.

Boistuval . . . 139. 171.

Boitard . $\quad$ 16. 41. 200.

Bonafons . : : $\quad 69$.

Bonastre - . 223. 232.

Bonnemaison . . . 45.

Bonpland . . 115. 194. vergleiche auch Humboldt und Kunth.

Boreal . . . . 212.

Bory de Saint Vincent 14. 45. 63.

72. 147.

Bossange : . . . 17.

Bosse $\quad-224$

Bossi - . . . . 140.

Bosson - . . . 225.

Bouché - 71. 72. 92. 105. 112

Boucher - . . . 54. 175.

Boudier . . . ! 52.

Bouduh . . . . . 144.

Boué - . . . 131. 1336.

Botmann _. . . $\quad .50$.

Bourdon $\quad \because \quad \therefore \quad \quad 200$.

Bourwieg • $~ • \quad 51$.

Bowdich - . . 190.

Bowie . . . . 26. 214.

Braconnot $\therefore \quad \therefore \quad 213$.

Brainville . . . . (64.

Brimdes . . . . 13.

Brandt . . . . 168.

Braun :.. . . 96.97.

v. Branne : . $\quad 157$.

de Bray 126. 147: 185. 2อె6.

Brebisson . 28. 75. 140.

Breda . . 16. 75. 127.

Bréon . . . . . 243.

Bresler . . . . 74.

Brentel . . . 60. 61.

Brewster . . . _ 2031.

Bridel . 1 . . . . 59.

Briganti . . . . 50.

Briucken . . . . 143.

Brocchi _. . . 189.

Brocke . . . 142. 144.

Brondean . : :50.51.54.

Brongniart 49. 79. 81 . 119. 120.

127. 128. 129. 130. 131. 147. 205. 219. 219. 230 .

Bronn . . . 136. 137. 148.

Brotero : 98.166 .124$.

Brouard 175.

Brown (Robert) 41. 59. 60. (63. 71. 81. 82. 84. 97. 104. 123. 191. 197. 219. 228.

Bruch _ . ‥ 60. 163.

Bruckmann : : : . 239.

Brumer 77. 140. 149. 177. 181.

v. Buch - 137. 143. 144. 150.

Buckland . . . 128.

Bukinghain . . . . 146.

Bull . . $\quad 204$.

Bunge . . . . 97. 187.

Burchell . 151.

Burkhardt - 150. 15i. 169. 221.

Burnett : . $\quad \therefore$ 200. 205.

Bushnan : : : 184.

Butt : . . . 18. 183. 
+1. C.

Caillaud par.

Caldcleugh $\quad 152$

Cambessedes 86. 109. 110. 114. 116. 117. 119. 183: 194. 195.

Carey . . 187

Carmichael . . . 190.

Carpenter . . . . 202

Cassebeer . 44.

Cassini 46. 51. 53. 57. 67. 86. 94.

Castle . . . . 18.

Cazcau $\div \quad$. 173.

Celle (Della) . 151 .

Chamisso 75. 87. 125. 147. 231.

Chantrain - Girod : 176.

Charpentier : $\quad 136.138 .147$.

Chateaubriand - . 152

Chauvin . . . fiò

Chaznl

Cherean - . . . 96,

Chevallier .

Choisy - . . 95.

Christie : . . . 143.

Christison . . . 226.

Clapperton : : . 146. 189.

Clarke . . . . 14!.

Cloet : : . . 165

Cocco :

Colebrooke : 12i. 15i. 187.

Colla $\quad$ 86. 76.91.99. 241.

Comellio . . . 179. 180.

Conrad . . 63

Cente 72.90.91. 95. 1118

Cooke - . 20.

Corda $\because \quad$ 57. 61.93.

Cordier . 49 .

Conlter 86

Courtois. . . 118. 231

Couverchel . ... . 214 .

Crivelli . . . 91.965.

Crome - . . 131.

Cruse . . . 67. 84.98

Cumningham - 145. 153. 197. 221.

Curie . . . . 154

Curtis $\quad \because \quad \because 3$

Custor : . . . 159

Cuvier . 126. 200.

D.

Daniell . . . . . 221.

Dargassies . . . 17

Darlington . . . 192

Davids . . : 198.

Dary . . . . 150.
Decandolle 35, $41,47,51$ pag. 98. 100.101 105. 108.11 112. 114. 115. 121. 123. 124. 134. 139. 172. 199.

2ข3. 201. 236.

Deline 14 .

Delile . . 61. 107. 110. 189.

Delise . . . 28. 56.

Demerson . . . 16.18.

Denhan

Deppe • . . 193.

Deribier . . . 140.

Desherger : $\quad 206,216$.

Descourtils ! 49. 124. 193. 174.

Desfontaines 94.239.

Desmazieres 28. 51. 52. 54. 84 . 165. 176. 201 .

Desmoulins ․ 119. 173. 69.78.

Vespertes : : : 121 .

Despreaux : : 4 45.83.

Desvenux 60. 63. 89. 109. 114.

Detharding : . . 168.

Deville • . . . 17.

Deryver * . 2 (i6.

Dewey . . . . 70.

Dieffenbach $\because 160$.

Dierbach : 12. 77. 94. 98. i03. 118. 139. 155. 11. 199. 231. 233. 234. 238.

Dietrich 11. 12. 27. 28. 59. 154 .

Diostoride :

Dioscorides . . • 203 .

Doigroubsky : : 94.

Dolliner * i54. 157.

Don 78. 84. 87. 88, 91,92.95. 99. 101. 114. 106. 113. 114. 115 . 119. 122. 123. 188. 196. 2192.

213.

Douglas : 74.82.89.90.191.

13 ralet . . 172.

Drees . . 65. 78. 89.93.

v. Droste ‥ ․

Drumond : • 18.59.

Dubois

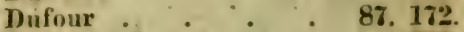

Dulong

Dumarchais _ . . 175

Dumortier : $\quad$ 68. 82. 85 .

Dunal * . . 212.

Dupont 174.

Dureau de la Malle : 230.233.

Duret do 174.

Durieu : $\quad \therefore \quad 52$.

Duvau . . . 30. (92.

Duvernay . . . 62. 
Dutrochet . . 199. 209 pag. van Dyk.
E.

Eaton • _ 192. 204. 222.

Ebel

Ecklon

Edwards

26. 190

Ehrenberg 94. 98. 109. 120. 151.

Ehrmann . • . 144. 146.

Eimbcke . . . . 168.

Eisentohr . . . . 136i.

Ekart . . . 22. 28. 1 (6i3.

Elie de Beaumont 、 . . . 129.

Elliot . . . . 121. 192.

Ellis . . 145.

Elsniann . … 28. 177.

Emmons … . (60)

Endlicher . . . 169.

Engelhard . . . . 136. 142.

Erey : . . . . 183.

Ersch * * . * . 250.

Escholtz . . . . . 193.

Eschwege _. . 145. 152.

Eschweiler : . . 2. 55.156.

Eversmann . . . . 150.

Eyries * . 116.

Eysenhardt . . 44. 46. 53. 230.

F.

Faldermann . . . . 81

Farini . . . . 41.

Feburier . . . . 199.

Fée ○ . 56.98. 234.

Ferchel . . 139.

Ficinus . . . 167.

Ficlds . . 145. 230 .

Fingerhuth : . 1 122. 163.

Fiorini . . . 181.

Fischer .. ․ . 188. 213. 212.

Fleischer . . . 156. 186.

Floerke . 56. 221.

Flotow . 28. 57.

Forlier . . . . 20.

Forsberg - . . _ 89.

Fortunatof $\quad \ldots \quad \ldots \quad 186$.

Franck (C. A) . 213.

Francoeur . . . 175.

Frank ... . . 161 .

Franklin . . 191

Fraser . _ 153.

Fresenius - . 72. 94. 162.

Freycinet . . 146.

Freyer * 238 pag.

Friedenber . 146.

Fries 28. 29. 42. 48. 49. 53. 56, 85 . 142. 185. 230.

Froriep

Frost .

18. 232.

Fürnrohr . . 61: 65. 238.

Funk . . . 27. 158.169.

Fytler . . . . 235 .

\section{G.}

Gachet : . . . . 52. 53 .

Gaertner . . . . 220.

Gaillon. . . . . 44. 45 .

Galesio _. . . 121.

Gallois . . . 116.

Garnett . . . . 149.

Gaspard : $\quad 111$.

Gaudichaud . : 119. 146. 196.

Gaudin . . 102 159.

Gay 72. 88. 96. 106. 111.:112.

Gebhard . . . . 158.

van Gecl . . . $\quad 35$.

Gera . . $\quad 83$.

Gerard - . 143.

Gerhard . . 22. 148. 223.

Germar . . . 147.

Gesner . . . 126.

Gingings . . . 94. 108. 198.

Girardin . . . . 17. 199.

Gireu • • + 219.

Glocker . . . . 136.

Gmelin . . 49. 160. 200.

Godefroy . $\quad 122$.

Göppert 77. 206. 212. 222. 2203.

224. $227.23 \pi$

G . 131. 198. 230.

Goldbach . . . 72. 80 .

Govan . . . 143

Gozzi ․ . 64.

Graaf : . . . 98.

Grabowsky … 169 .

Graham 36. 71. 80. 87. 88. 92. 94.

Grateloup 96. 125. 154.

Graumüller . . . 167.

Graves . . . . . 68

Gray - 183

Greville 46. 49. 54. 5่8. 59. 60. 6อ̃. 181. 186.

Griesselich 34. 38. 89. 94. 95. 101.

Groh 103. 18. 160

- 160. 168.

Guerin • • . 139.157.

Guibourt - • . • . 206

Guillaume . . . 173 . 
Guillemin 14. 107. 197. 218. 231. Guillery

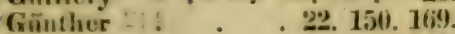

Giunt\%

(Bissome

Guyetant

181. 182. 211

II.

IIacefit

186.

Haenke - . . 63. 194.

Hagelstam . . . . 142.

Hiaren . . . . . . 170.

Hagrenbach , . . . . 160.

Halder .. . . . . $\quad 223$. vain Ilall . .. . 53. 16it. 2030.

Hálsey .. . . 5. 65.

Ilamilton 104. 110. 143. 152. 187.

188. 1 198.

Hankhigen . . . . . 170.

Hargasser : 。 _. . 156.

Harpe (de lia) _. . . 67. II.

Hartig. • . 203.

Hartmann. 28.

Hartwall . . . 185.

Hartweg . . . . 237.

Harvey. ․ . 222

Hasselt $\because$ 75. 97. 143.

Hauser

Пай́mann . .. . 140. 150.

Haworth • . . 72. 113.

Hayne , . . 123. 202. 209.

Hedren . . . . 38

Hedwig . . . . . . 59

Heer

Heretschweiler 61. 123. 149.15\%.

Ileinhold: 87.178.

Heis. 202

Heller 162

Ilenkel (a Donnersuark) 73. 180.

240. 241. 251.

IIennine : 186.

Hensthel 。 201.217.219.

Henslow 205 .

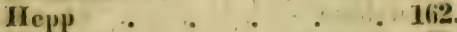

IIerliert . ․ 72

Iferbieh . . . 181 .

Hergt . . . 163.

If ermbatädt . • . 207. 2018.

Hermelin . . . 142.

Herr , , . 15.

IItenffel . . . 138.

IIoyne . . . 187.

Ilildenbrand : 178.

Hildreth. 128.144.

Milsenlierg ‥ 156.190.

Hinterhulier pag.

Hirzel-1scher • • $14 !$ !

llitcheock. 52

Hochistetter . 29. 154. 158.

v. IIoff

Uoftinann 89. 129. 132. 137. 138.

Hoflimannsegg _. . . 237.

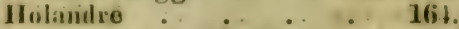

Hlolboen ...

Holl . . . . 171. 199.

Hollins - . * . 229.

Hollunder . . . . . . 218.

Holm . . . . . 195.

Homiain : . … 169\%.

Hooker 3. 58. 60. 62. 184. 191.

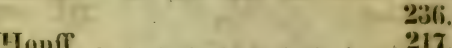

Hoppe 2. 23. 70. 71. 72. 96. 106.

109. 147. 157. 178. 239

IIorkel . . . . . 78

Hornemann - . . 17.

Hornung . . . 22. 73. 106 .

Hornschuch 60. 61. 76. 134. 147. 17v. 171. 185. 23̈6. 237. 240.

Hoss . . . . . 158.

Host . . . . . 158.

Hough - . . . 143.

Huri . . . 149. 160.

Hulthem $\cdots, 236$.

IInltström

Humboldt 68. 83. 13ิ1. 133. 146.

; 152. 194.

Hundeshagen ..... 131. 136.

I.

Jack _. . . . 95. 115.

Jacques . . . . 240.

Jacquiin - 18. 31.

Jäger . 129. 222. 226. 251.

Jïrersehmidt • • • 136.

Jahn : . . . . 181\%

Jamies • • • . 193.

Jan * * - - 24.

Jares\% . - . 190.

Johns • • • 183.

Johnsen - • 205. 217.

Johnston . . . . 184.

Jones • $\because \quad 184$

Jounes - . 144

Juillet • • 13.17.199.

Junghahn i . • • 50

Jürgens 29

Jussieu 41. 88. 90. 101. 102. 108. 109. 119. 120 . 
K.

\section{Kachler}

pag.

Kaemmerer

Kalm *

Kärèline

Kasthofer

11. 12.

Kastner

Kaulfus.

Keferstein

18.

150.

13\%. 148.

Keith

Keller

Kephalides

Ker

Kickx

Kicllenberg

Kieser

King.

Kingstom

Kirwan

Kittel

Klaproth

17. 19. 59. 67. 74. 81 . 199

Klett

116. 146

Klinkhardt

Klipstein

167.

Klug :

136.

Kluk

22.

Krieiff

11.

Knight . 208

Koch 14. 19. 68. 85. 191. 1113. 106.

108. 124. 154. 156. 163.

Koehler _ . . . . 118.

Koclle $\quad$. $\quad . \quad \ldots 201$.

Kops _ . . 2031.

Kosteletzky $\quad . \quad \ldots \quad 166$.

Krämer • • • • • 156.

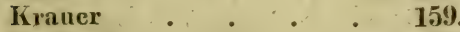

Kirebs . . . 26.

Kreyssig - _ . 198. 206.

Krocker . . . . . 169.

Kŕmbholz . • $\quad{ }^{\circ} 51$.

Krüger . . . . 127. 166

Kützing @ 2 22. 168.

Kulil . . . 75. 97. 143

Kummer a 139

Kunth 68. 84. 118. 121, 194. 232.

Kunze . . . • . 191.

Kupffer:

132.

L.

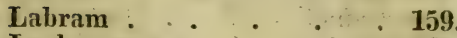

Lachmann ․ . $\quad 165$

Lagasca . . 2 25. 101.

Lambert

82. 96.

Lainouroux

Lang
16. 41.45 .200$.

24.
Lange. pag.

Langsdorff . . . 146.

Laroche $\because \quad 136.137 .223$.

Lary - . 2 225.

Lasch 20. 51. 83. 88. 91.92.91.95. 99. 104. 109. 111. 122. 123.

Lastdraeger . . $\quad \begin{aligned} & 20202 \\ & 202\end{aligned}$

Laterade _. . . i 173.

Lamer ... . . . . . $\quad 230$.

Laurer . . . . . 5\%.170.

Lavieille . . . . . 113.

Leavenworth _ _ _ _ 192.

Lebaillif . • . 64.

Lecoq 13. 17. 19. 67. 211. 219.

Ledebour 69.74. 95. 150. 186. 187.

Lefebüre ～. . . 17. 172.

Leforestier .. . 172.

Lehmann 33. 34. 38. 58. 88. 92.96.

123. 128.238.

Leiblein . . 44. 162

Lejeune ․ 24. 69. 103.164.

Lemprière . . . . 200.

Leo $\ldots$. . . 12

Leon - Dufour . . . . . 51 .

v. Leonhard . . . . 131.

Leprieur . . . . 151.

Leschvin $\quad . \quad \ldots \quad 199$.

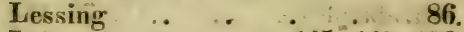

Lesson . . . 145. 147.196.

Lestadius . . . . 142.

Lestiboudois 16. 67.76. 105. 165.

Letellier . . . . .

Letourneur . - . 17.

Leuchs . . . . 296. 208.

Leuwen 146.

Leveillẻ . . 51. 52. 54. 225. 230 .

Lewis _ . 53. 57. 192.

Lexargå . . . . . 193.

Lhotsky _. . . . 27. 27.

Liave (P. de la). . . 193.

Libert ... ․ 45.52.58.

Liden . . . . 38

Liegel . - . . 122.

Lilievalch. . . $\quad . \quad 65$.

Lindenberg $\quad 57$.

Lindley 18. 73. 75. 76.91. 111. 115.

116. 121. 122. 130. 183.198.

229.

Link 14. 34. 39. 40. 62. 69. 106. 108. 12\%. 129. 138. 156.

224. 227 .

Linné : . . . . 37. 38. 60 .

Lloyd .. $\ldots \quad \ldots \quad \ldots \ldots 12$

Locke . $\quad . \quad 18$.

Lockhardt . . . . 83.

Loddiges _. . อ 242. 
Lag.

Löhr • . . . 23.160.

Löв81 - . . . . 230.

Loiseleur-Deslongchampe 35.171.

Longmite $\quad . . .6172$.

Lorek … …170.

Lorcy . . . . 174.

Louden - . . 38. 183.

Louis * - 20.

Lübeck . . . . . 81 .

Lüdersdorff . . . . . 19. 20.

Lyngbye . . . . . 232

\section{M.}

Macaire Princep 47. 51. 206. 226.

Mac Culloch - 130.141 2204.

Macgilliway _. . . . . . (88. 85.

Mackay _ . 141. 181.

Macks . . - : 224.

Macvicar . . . . 215.

Maerklin . . . 46. 2ul.

Majo . . . . 205 .

Mann . . . . 57. 167.

Mantell . . . . 130.

Maratti . . . . . 181.

Marcel _. . . . 12!.

Marcet . . 47. 50. 204. 226.

Marchand .. .. .. 75.165.

Marder . . . . . 164.

Marin . . . . . 77.

Marker .... . / . 28.

Marquis . . $\quad$.. $\quad . \quad \ldots 3$.

Martens - 46. 149. 162. 178.

Martinof .. $\quad . . \quad 12$.

v. Martins 21. 34. 55. 76. 77. 84. 95. 111. IT10. 124. 145. 15\%. 162. 195. 212. 237. 239.

Marx . . . 128. 211. 228.

Maund . . . . . 36 .

Mauz . . 218. 220.

Maximovitch 186. 201. 219.

Mayer . 96. 112. 159. 221. $2: 55$.

Mechel. . 131.

Meigen . . . . . 16 .

Meinzel o @ 229.

Meisner : _ . $\quad . \quad 83$.

Melazzo ‥ ‥ 72.

Menard de la Graye . . 82.

Mendelssohn _. . 1335

Merat . . . 92. 175.

Merian . . . . 139.

Merlet . . . 174.

Merrem . . . 38.

Mertens (pater et filius) 29. 46.

Metrger 。 80.144. 154. 251.
Meyen 44. 54. 65. 71. 198. 202 pag. 221.

Meyendorf 150.

Meyer (fratres) 32. 45. 55. 62. 81 . 147. 166. 170. 187. 191. 194. 208. 227. 238.

Michaelis . . . . 137.

Michel . . . . . 24 .

Michl . . . . . 156.

Mill _ . . . 224.

Miltenberg . . . . 131.

Miltitz • . . . 250.

Minutoli . . ... 151.

Mirbel 45. 82. 105. 110. 115. 134.

204. 214. 219.

Mir-Cher-Ali-Affos _ $\quad 187$.

Mitchill . . . . 55.

Moesler . . . . . 38

Mohl _ 202.206.213.

Moldenhauer . . . . 227.

Monnard . . . 105.

Monnier _ . . . . 86.

Mentagne . . . . 52.

Moquin-Tandon - 199. 229.

Moreau de Jonnes a 135. 225.

Moretti 20. 113. 178. 178. 180. 181.

Moricand . . . 178.

Morren . 32. 48. 211.214.

Morris .. . . . 36.182.

Moucheron - $\quad 17$.

Mïller . 29. 60. 149. 230. 250.

Muhl

Mulder

208. 217.226 .

Muncke . . . . . 138. 209.

Murray. . - 181.206. 213.

\section{N.}

Naceari . . . . . . 46.

Nagel _ $\quad . \quad \ldots \quad$. $\quad 188$.

Naupoil . . . . . 240.

Naviere Laboissière .. . . 174.

Nees (al) Viscubeck fratres) 4. 33. 50. 53. 54. 57. 58. 62. 67. 70. 79. 84. 93. 122. 125. 164.

189. 280.

Neuffer 203.

Neuhof . . . 235.

Neuschild . . . 170.

Neuwied (Prinz von) • - 152.

Neygenfind … 169.

Nilssen : . . . 130.

Nocea

180. 211.

Noeggerath. . 53.128.136.

Noehiden . . . 233.

Noisette . . 229.240. 
Nuttall 18. 69. 75. 83.92. 104. 105.

113. 195.

O.

Oeynhausen . . 136.137.

Oken . • . . . 4. 39.

Olagnier . • • • 35.

Oligschläger 164

Opiz - 15. 29. 93. 155. 166.

Otto - . 34. 78. 93. 106. 108.

Oudney — • • — — 189

Oxley — • — _ 153

P.

Pacho . . . . . 233.

Page . - . 242

Palm . . . . . 206

Papius . . . 204

Pappe . . . . . 167.

Parry _. . . 47. 152

Pauchet . . . . 96.

Pauqu'y d'Amiens . . 96.

Pauls $\quad 4.48$.

Payre . . . 171.

Pelletan .. , 120.

Pencati . . . 188.

Pentland . . 145.

Perleb . . . 15, 39, 237.

Perrat . . . 132 173.

Perrotet . 151.

Perry . . . . 184.

Persoon . . 20.45.

Petif,$\quad . \quad 161$.

Petit $\quad 78$

Petter + . 25.

Phillips . : 223. 241.

Pickering . . . . 135 .

Picot de la Peyrouse - . 172.

Pieri $\because \quad$. 186.

Pinat . . . . 217.

Pizacolli - . . 29. 49.

Plée . . . . 175.

Plieninger , . 234.

Plinius $. \quad . \quad 234$.

Plösl $\quad \ldots \quad \ldots \quad \ldots \quad 30.31$.

Pohl . . . 196.

Poiret . . 16.

Poiteau - 115. 174. 205. 217.

Pollini : . . . 177. 178.

Ponitz $\quad . \quad 216$.

Pöppig . .

Portal 84

Portenschlag. , . 179.

Pouzolz $\quad \ldots \quad$. . 182.
Presl (fratres) 25. 34. 69. 70 pag. 89. 97. 109. 111. 182.

Preissler . . . 101. 103.

Prevost: . . . . 127. 224.

Prieur \& ... . 14.

Prince . . . . . 220.

Pritchard ․ . . . 31.

Prochnow $\quad \ldots \quad \ldots \quad .170$

Pronville . . . . . 122.

Prytz . . . . . 185.

Purkinic . . . 213.

Pylaie (de la) . 44. 125. 144.

R.

Raab _ $\quad \ldots \quad 163$.

Raddi . . 63. 68. 195.

Rafinesque .. 192

Ramond . 140. 172

Ramon de la Sagra , 144. 213.

Rapin . . $\quad$ S6.

Raspail 29. 65. 67. 68. 69. 70.73.

202. 203. 215. 232.

Rastoin . . . . . . 200.

Ratzeburg . , , _ 229.

Rau - . • . . 137.

Raumer . . . 136. 205.

Razoumowsky - $\quad 142$.

Ré • 71. 180.229.

Rebaul .. • 73.

Redlouté .. . . 35. 21.

Rehmann 161.

Reichenbach 4. 15. 23. 28. 32. 33. 39. 65. 70. 73. 93. 103. 112.

154. 155. 167.

Reider 117.

Reinwardt _ . \& 143

Renault . . @ 175.

Rengger . . . 138.

Rennié . . . . 108.

Renz . . . 216.

Requien. . . . 172.

Reuter - . 207. 216. 225

Reyger . . . . 170.

Rhode . . . • 126.

Ricei - . 129.

Richard 14. 17. 75. 81.98. 99. 115.

203. 215.

Richter . . 15. 167.

Ricord-Madianna . . 55.

Ringier . . . . 139.

Risso . . 141. 176.

Ritgen . . 39. 199: 201.

Ritter . . 184. 138.

Rivière . . . 16.

Roberge.

99. 


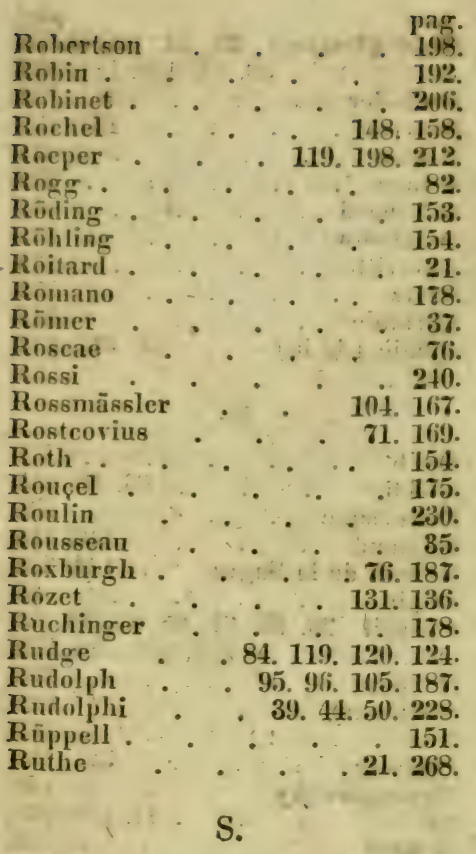

Sabine . $\quad \ldots \quad \ldots \quad \ldots 8$ 87. 220. Sadler . - 24.158. Sageret . : 107. 220. Saint-Amans $\therefore .173$ Saint-Hilaire (Jaume) 69. 92. 93. 95. 102. 104. 1117. 108. 109. 116. 117. 119. 121.152. 171 . 174. 175. 194. 195. 212. 215.

Saits 220 .

Sali $\quad . \quad \ldots \quad 121$.

Salis • • . . . 148.

Salisbury : 64.

Salt 20151 .

Salzmann 24. 26. 27. 176. 181. 182.

Sỉnger . . . 137.

Saller . . . . 223.

Saussure 216.

Sauter 22. 69. 88.102. 138. 156.

Savi 121.141.177, 181. 241.

Schaefer . 1fis.

Schaerer . … 28.

Schaub. . . . .

Sehelhas 。 . . . 81 :

Seliclver . . . 217.

Schiede . . . 27. 144. 219.

Schilling . . .

Schillinger .. . 54
Schimper 96. 97 205 pag. v. Schlechtendal 3. 50. 54. 63. 74. 79. 99. 168. 190. 199.

Schlegel

Schlotheim

1(15).

Schmals 50.167.

Schmidt 15. 70, 97. 124. 169. 229.

Schoider • • . 207.

Schomburgh $\ldots \quad \ldots \quad \ldots \quad 27$.

Schü̈ . . . . . 222.

Schünbergr . . . 251.

Schönheit ......167

Schouw 77. 131. 132. 134. 140, 236.

Schrader (in Berlin) . 2118.

Schrader (in Güttingen) 88. 96. $10 \%$.

Schrank - 156. 182. 186. 191.

Schiropp . . . . . 138.

Sehteheglow $\quad 186$.

Schubert 28. 65. 150.

Schübler 34. 39. 45. 1331. 138. 161. 213. 214

Schultes (pater et filins) 37. 73. 74. 148. 149, 190. 250.

Schulz (F.) … . . 132.

Schulz (in Berlin) 198. 208. 21!!).

S 213.

Schulz (in Frciburg) - . 228.

Schulz (in Neu-Brandenburg) 59.

61. 24. 16i9.

Schulz (ron Zweibrücken) Te้ 93.

- 163.

Schulze _.. . . 128

Schumachẹ $\quad . \quad \ldots \quad . \quad 18 \%$.

Sehummel
Sehiitz

Schwabe $\quad . \quad 53$.

Schwägrichen . 59.60 ,

Schwarz . . 155,

Scott . . . . 2.

Scitz . 62.107.117.123.

Selb . . . . 136.

Sendel . . . 12t.

Séringe .. 3. 23. 35. 103. 107.

Sette . . . . 48.

Sickler .

Sieber . 23, 25. 26. 149,150.

Sigwart . . . 2y.

Sillen . . . . 185.

Simonds . . . 14\%.

Simonet . . . 231.

Sinclair . . . . . (i6.

Sinning . . . , , 33.

Smelowsky.

Swith . 74.98. 150. 183, 189.

Sommer . 34, 127, 146

Sommerfelt 23. 47.53.106, 112 
Soulange - Bodin

Soulavie $\quad 133$

Spaskii * . 151

Spenner . . . 80.104:161.

Spin . . . . . . $\quad 211$.

Spittal $\ldots \quad \ldots \quad \ldots \quad .52124$.

Sprengel (pater et filius) 13. 37. 38. 68. 80. 128. 146. 207. 208. 2203. 233. 234. 2050.

Stein

22. 159.

Steinheiin . . . . . 108.

Steiner . . . 138.

Steininger $\quad \therefore \quad$ 136. 138.

Steinmüller $\because \ldots \quad 148$

Stekhoven . . . 12

Sterler $\quad 2 \quad 2 \quad 28$.

Sternberg (Graf Caspar von) Tt.

96. 126. 129. 138. 148. 158 . 178. 225. 2:4. 2ชือ.

Steudel - $\quad 12.29 .68 .70 .154$.

Steven a 02. 106.118. 242.

Stieler • . . . 139. 219.

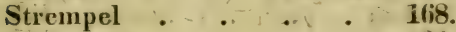

Stromeyer . . $\quad$. $\quad$. $\quad . \quad 1333$.

Stubner

Sturm _. . . . 60. 15.4.

Suaine $4 \quad 219$

Suckow . . 21. 161.

Suhr $\quad . \quad . \quad 09$

Suter . . . 159.

Swcet . 108.117. 212

Szovits . . . 25. 187.

T.

Talrich

29.

Targioni Tozzetti- . 12. 45.

'Tausch 34. 72. 86. 87. 90.91.94. 96. 99. 103. 105. 146. 112. 116. 119. 124. 166. 167. 169.

187.

Tènore 122. 141. 149. 181. 234.

Tettelbach _ . 15.

Thackery . . . 215.

Thede . . . . . 22

Theophrast $\quad \bullet_{233}$

Thiebaud de Berneaud 149. 234

Thienemann. .1 .

Thiele . . . 55.

Thomas . . . 24.

Thomson . . 18.134.

Thon : . . 19.

Thonning - . . 189.

Thore . . . 173

Thornton $\quad \ldots \quad 18.183$.

Thory . . 107. 121. 


\section{- $262 \quad-$}

Walker-Arnott . 59. 90.173

Wallich 80.81. 121. 187. 188 .

Wallroth : 56.93.122. 167.

Walsch . . . . 186i.

Watkon . . . . 183.

Weber . . . 186. 258.

Wehster . . . . . 196.

Weihe . . . 22.122 164.

Wéiland . . . . . 137.

Weinmann . . . 242.

Weiss .137

v. Welden 104. 148. 158. 179. 180.

23!).

Wendernth - 121. 163. 225. 237.

Wendland . . 33. 120.

Weniger . . . . 164.

Werkmeister * * 210.

Wernekink . . 238.

Westenhoff . . . 230 .

Westrumb . . . . . 198 .

Wickstrïm . 63. 73. 193. 194.

Widenius . . . . 18.

Widmark . . 188.

Wiederhold . . ‥299.

Wiegmann . 47. 220. 227.

Wiest.

29. 138 .

Wilbrand 134. 138.160. 209. 218 .

Willemet . 69.92.95.163.213.

Wimmer • • . 169. 229.

Winberg . . . . 188.

Winch :. . . . . 141.

Winkler . . , . 236.

Withering . . . . 183.

Woods . . . 122.

Wrangel . . . 54.

Wucherer.

Wydler ... . . 77. 87.91.

\section{Z.}

Zahlbruckner $\quad . \quad 157$

Zantedeschi . . 50.180.

Zawadski . . 214.

Zeller . . . 227.

Zenker . . . 15. 28. 209.

Zennek . . . 46.57.161.

Zeyher . . . . 26.

Zimuermann . . . 16.

Zinken . . . 137.

Zippelius : . . ., 145.

Liz . . . . . 162.

Zollikofer . . 159.

Zuccarini 70.118. 156.157. 163. 


\section{Z w e i t e s \\ oder}

\section{Pflanzen - Register.}

Familien nebst neueren Gattungen enthaltend.

A.

Alietinae $\cdot$

Acanthaceáe . . . . 95.

Actinanthus . . . . . 102.

Accrineare . . . . . . 119.

Actinomyce . . . 54.

Adriana . . . . 119.

Algae . . . . . . . 43.

Alismaceac . . . . 78.

Alsineae . . . . . . 112.

Althenia . . . . . . 78 .

Amaranthaceac : . . . 110.

Amaryllideae . . . . 72.

Amentaceae . . . 82

Amomeae . : . 76.

Ampelideae . . . . 11\%.

Ainggdaleae. . . . 122.

Anatropa : . . . . 120.

Anaxngorea . . . 1102.

Anonaceae .. . . . . 102.

Apocyneae .. . $\quad . \quad \ldots \quad .98$.

Arauja $\quad . \quad$.. $\quad \ldots \quad$. $\quad .97$.

Araliaceac . - . 102.

Ardisiaceae . . . 90.

Aroideae . $\because \quad . \quad 76$ 77.

Artocarpeac . . . . 83.

Asclepiadeae . . . . 97.

Asparageae . . . . . 74.

Asphodeleac .. . . 73.

Aurantiaceac . . . . 121.

B.

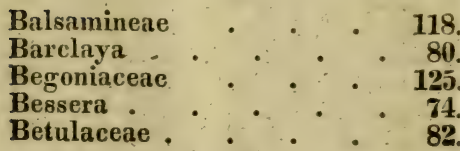

95.

$\underset{\text { Blimenbachia . . . } 107 .}{96 .}$

Brissocarpus . . . 58

Bromeliaceae . • . 72.

Brnniaceae . . • . 119.

Büttneriacease . . . 116.

Butomeae . . . . 78.

C.

Cactere .. . . . 108.

Calampelis . . . . 95.

Callaceace .77.

Callitrichinene . . . 113.

Calochortus . . . 74 .

Calodryum . . . . 89.

Calopliytae . . . 100.

Calycantheae . . . 115.

Camelliaceae. . . 116.

Campanulaceae . . . 89.

Cannacease . . . 76.

Capparideare . . . 106.

Caprifoliacene . . 99.

Caricene . . . 107.

Caryophylleae . . . 112.

Ceratophylleac . . . 125.

Cercodianae . . . . 113.

Characeac . . . . 64.

Chenopodiese . . 110.

Chotekia . . . 93.

Cistincae . . . 108.

Clintonia . . . . 89.

Colvacaceae . . . 95 ,

Colchicacease ${ }^{\circ}{ }^{\circ}$. 74.

80. Collinsia . . . 92

25. Columellieae

4. Combretaceac . . 114.

2. Commelinaceas . 71. 
pag.

Confervate . . . 44.

Convolvuleac . . . 9.

Cowania - . . 123.

Crassulacene . . . 112.

Cruciferae . . I05.

Crucigenia . . . 48.

Cryptocaryna * . . 77.

Cucurbitaceac $\quad . \quad 197$.

Curtia - . . . 97.

Cuscutene - . . 95.

Cycadeae . . . . 81.

Cyperaceac : : . . . 70.

Cyrtandraceac . . . 98.

Cytineâc . . . . 79.

D.

Desmaziarella . . … . 52.

Dietyophora . . . ....... 5̇t.

Didynomena . . . . . .

Dioscorene . . . . . . 75 .

Diosmeae . . . . . 120.

Diplogenea . . . 115.

Dipsaceae. . . . . 86.

Dipteroearpene ․ . 117.

Douglasia . . . 90.

Droseraceac . . . . 109.

Dryadeac . . . . 12\%.

Duricua . . . . .

E.

Ehenaceae . ¿ : . _ . 89.

Eccremocarpus . . $\quad 95$.

Eetosperma .. . . 44.

Elatineae .. . . . . 114.

Eliea . . . . . . 109.

Empetreae . . . 119.

Epacridene . . . $\quad 89$.

Equisetaceae . . . . 65.

Ericeae -. . . . 89.

Erponema * . _ 53.

Erythrolaena _ . . 83.

Escallonieae ., 113.

Euphorbiaceae * $\quad 119$.

F.

Fatioa . . . . 114.

Filices . 62

Franeoa . . - 113

Frankeniaceac 。 . . 109.

Fridericia 。 . 95.

Fucoideae _ . . 46.

Fumariaceae _ . . 105.

Fungi : . . . 48.

pag.

Galatineace $\bullet .113$.

Garcinieae . . . 110.

Gaya . . . . 102.

Geigeria . . . 88.

Gentianeae . . . 97.

Geraniaceac . . 117.

Gesnerieae . . . . 9:3.

Gilleriene . . . 7is.

Globularieae . . . . 86.

Goctzea :. . . . 8?.

Gramineae . . . fif.

Granateae . . . 14.

Grevilleana . . 60.

Grossularieae . • 1117.

Guentheria . . . . 58.

Guttiferae . . . 110.

Gyropodium . . . . 52.

\section{H.}

Haemadoraceae . . . 71 .

Halorigeas . . . . 113.

Hemprichia ... . 121.

Hepaticae ... 57.

Hermanniaceae. . . 116.

Hippurideae. • • . $118 ̈$.

Hosackia . . . . 124.

Hydrocereae. . • . 118.

Hydrocharideae . . . 88.

Hydrurus . . . . . 45.

Hygrobiac. . . . 113.

Hypericincae . . . . 109.

1.

Jasmineàe - . . . 9!9.

Inoconia . . . 45.

Joliffia • . . 108.

Irideae . . . . 11.

Juncagineae $\quad . \quad$. 78

Junceae . . . 81 .

I.

Labiatac $\quad \therefore \quad . \quad$. 93 .

Laurineae.. .98.

Lecythileac ‥ . 115.

Leguminosac . . . 123.

Lejatulus . . . 102.

Lejetinia . . . 58.

Lentibularieae : . . 90.

Lepisia . . . Tl.

Lessingia - . . 87.

Libertella * . . 52.

Libertia $\quad . \quad$. (i9.

Lichenes _. . • 55 .

Liliaceac . 73. 
pag.

Lineae . . . . . 11 .

Loaseae . . . 1117.

Lobeliaceae . . . . 88.

Lobostemon • • 96.

Lomatogonium . . . 97.

Lorantheae * . 100.

Lycopodineae.$\quad .64$.

Lythrarieae

114.

\section{M.}

Macraea

111.

Magnoliaceae

1112.

Malesherbia .

106.

Malpighiaceae

Malvaceae

119.

116.

Marsiliaceae :

Melanthiaceae

Melastomaceae

Melanorrhaea

Menispermeac

Monotropeae

Musci frondosi

Myrtaceae

61.

74.

115.

121.

102.

89.

58.

115.

N.

Najadeao

7ร.

Nelumboneae . . . 80.

Nepentheae . . . 79

Nopaleae

Nostochineae .

108.

Nymphaeaceae

46.

80.

\section{o.}

Ochnacene • • • 120.

Onagrarieae,$\quad 114$.

Oncostemúm . . . . 90.

Orchideae . . . 75.

Orobancheae . . . 92.

Orontiaceae . . 77

Oxalideae $\quad 118$

Oxymitra . . . 58.

Papaveraceae

Parideae

Paronychieae

Passifloreae

Pedilonia

Pentacrypta

Philadelpheae

Philotheca .

Physopodium

Phytolaccene

P.

Piperaceae

105

74.

- 111.

106.

71.

102.

114.

120.

114.

110.

80.
Plantagincae . . . 8 86.

Podostemeae . • . 78.

Polpoda. • • 111.

Polygaleae . . . 104.

Polygoneae . . . 83.

Pomacene . . . 122.

Pontedereae * . 73.

Portulaceae . . 111

Prinulaceae .990

Proteaceae . . . 84

Protococeus . . . 44.

R.

Rantunculaceae . . 103.

Reevesia • . . 116.

Resedaceae . . . 104.

Restiaceae . . . . 71 .

Rhamneae • . . 120.

Rhizantheae * . 79

Rhizocarpeae . • . 61 .

Rosaceae . . 121.

Rubiaceae * . 98.

Rutaceae 120

S.

Salicarieae • • 114

Salicineac . . . 85

Sapindaceae . . . 119.

Sarrocenia • . . 125

Saurureae . . 80.

Sauvagesieae . . . 109.

Saxifragene . . 113.

Seitamineae . . 76.

Sclerantheae . . 111.

Scrophularinae . . 90.

Scouleria . . 61 .

Scyphaea . .109.

Selenia .0105.

Semonvillea . . . 111.

Sileneae. .0112

Sinningia . . 93.

Smilaceae * . 74 .

Soemmeringia . . 121.

Solaneae . . . 96.

Sphacele . . . 94.

Sphaerella : . . 47.

Spiraeaceae . . 123.

Spirogyra . . . 44.

Sporendonema,$\quad .54$

Stanhopea . . . 76.

Sterenliaceae . . 116.

Steudelia . 111.

Stroptanthus - . . . 105

Stylideae 


\section{$-\quad 266$}

Stylolepia . . $\quad 88$.

Synantherene . . . 80.

'T.

Tacceao . . . . 80.

Tamariscineae - . . 109.

Tauschia . . . . 101.

Terebinthaceac . . . 121.

Ternstrocmiaceae . . 116.

Thymelaeae . . . 84.

'Thysonachne . . . . 90.

Tovaria . . . 106.

Trichanthera . . . 120.

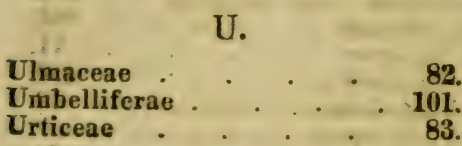

Unbelliferae . . . . 101.

83.
V.

Valerianeac $\mu$ pag.

Valonia . . . . 46.

Yerbenaceae. . . . 93.

Violariene . . . . 108.

Viviana . . . . . 99.

W.

Weldenia \& . . $\quad 79$.

Z.

Zanthoxyleae . . . 120.

Zygophylleae . . . 120. 


\section{D r u c k f e h l e r.}

Seite 16 Zeile 26 Statt Aristole lese man Aristote.

- 19 - 2 von unten Statt Therebint. lese man T'erebinth.

- 25 - 19 Statt abviarum lese man obriarum.

- $51 \quad-16$ von unten Statt Liveillé lese man Leveille.

- 54 - 13 - - - Eratiun lese man Erotium.

- 72 - 21 Statt Harworth lese man Haworth.

- 73 - 10 - Hemaracall. lese man Hemerocallid.

- $80-14$ - Ataeeia lese man Ataccia.

85 - 5 - Kock lese man Koch.

$89-10$ - Sueconis lese man Suecanis.

89 - 2 von unten Statt Gaetzca lese man Goetzen.

93 - 3 - _ - Chatekia lese man Chotekia.

99 - 14 Statt Hedyatis lese man Hedyotis.

$108-16$ - disem lese man diesem.

109 - 14 - Droseea lese man Drosera.

- 112 - 12 - Thureller lese man Thuiller.

- 113 - 9 von unten Statt Cercodianae lese man Cercudianae.

- 123 - 13 Statt Cavania lese man Cowania.

- 123 - 17 - Moeinno lese man Mocino.

- $124-18$ - Hasaekia lese man Hosackia.

- $124 \quad-3$ von unten Statt Trifles lese man Tréfles.

- 129 - $2-$ - Ricei lese man Ricci.

- 178 - 21 Statt Noecari lese man Naccari.

- 185 - 19 von unten Statt Wahlenberg lese man Wahlberg.

- $\quad 208$ - 22 Statt Poitard lese man Boitard.

- 228 - 1 von unten Statt Göpper lese Göppert.

Mehrere andere wird der geneigte Leser mit der Fiffernung des $V \mathrm{cr}$ fassers vom Druckorte entschuldigen und leicht verbessern. 


\section{Sีn unierm Berlage ift nod) cridtienen:}

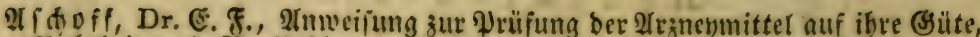

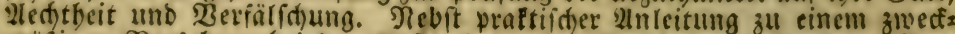

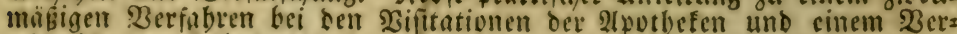
acid)niffe Der gebräud)(id)ften d)emiiden Reagentien, zum (Bebraud) für Whrjici, Aergte, Apothefer uno Droguiften. gr. 8.

18 ggr.

Brandes, Dr. R., Dev 2 potbefer= Betein in nivblidten Teutfdlano, ges (d)ilbert nad) ! feinen 2 Birfungen in bem erften Decennium feines Defte=

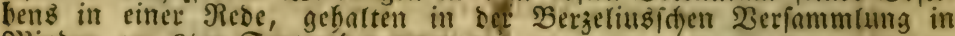
Dimben all 8ten September 1850. 4.

10 ggr.

- Befeututung ber Sombopatbie vom pharmacentifd)en Standpuncte. Rebft cinem श्ञnbange vom alten Eriticus. gi: 8.

8 ggr.

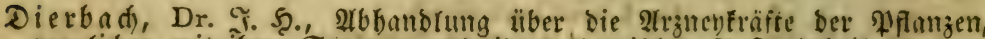
verglidjen mit iber Structur und ibren bemiften Seftandbeilen. gr. 8 .

1 Rtb. 8 ggr.

Inftruction bes Gefunbheitzconfeils zu भaris über bie 2unfertinung ber

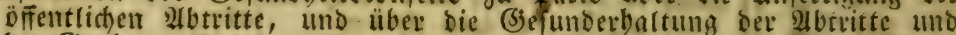

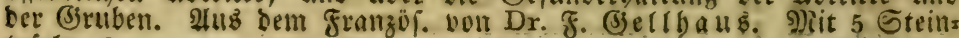
tafeln. 8.

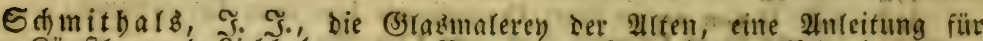

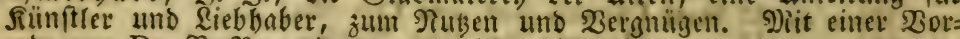
rede volt Dr. R. $\mathfrak{B}$ tande 8 . gr. 8.

$8 \mathrm{ggt}$.

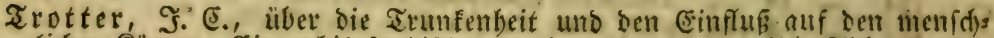
ridten Rörper. (eine phirofophifd)e, mebicinifde und d)emifd)e glbhandrung.

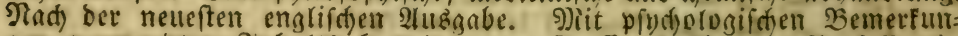

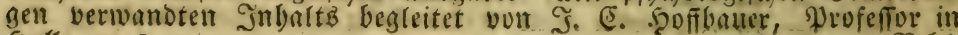
Şalle. gr. 8.

1 Riter.

Weftumb, Dr. 2 . S. L., Netre Toricorogie, ndet die Lebre von ben (3iften und Bergiftungen in demifdfer, whyfinologifder, pathologifder uno thetapeutifder 2 ezielung. AHB Dem Frangif. Sez Guerin de Mamers. 8.

20 ggt.

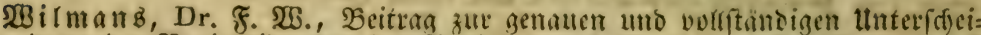
bung Der Zarioloid = von ber Bariora = und Bariceflen = Rranflecte, nebit befonberen Einmulten gegen die Soentitat ber Eontngien jener beiden erfteren Exantbeme etc. 8.

18 ggr.

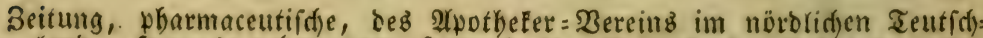
rano. Serauggegeben vom Sofratb Dr. Branses. Jabrgang 1831.

शr. $1-26$ incl. gr. 8. (Diefe Seitfdrift wirb audf p. 1852 fortgefe(3t).

1 Rtbr. 8 ggr. 


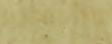

$y^{2}=6,-3$

$-$

- 3,, $2=$

$-\quad-x^{2}=x^{2}=$

$S$

8

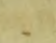

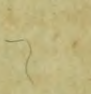

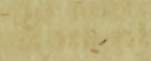

\section{-}

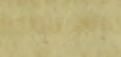

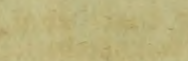

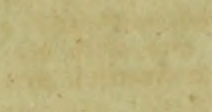

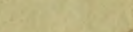





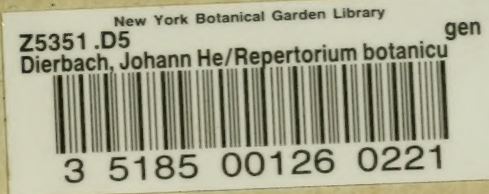




\section{1@29a}

$-0 s$

$200 \%$ - 02

10.20101

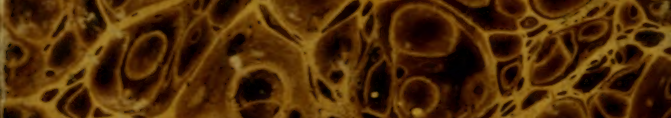

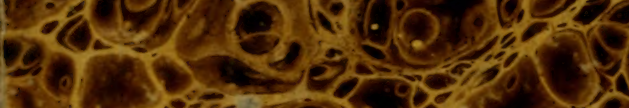

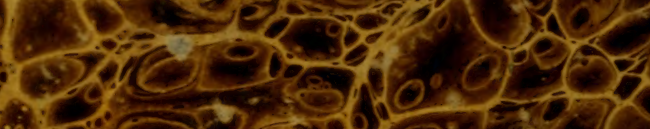
Q. 200 cos $\lim _{10}$

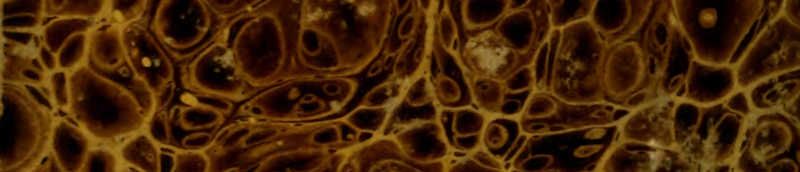
$100 \%$
H(-2)

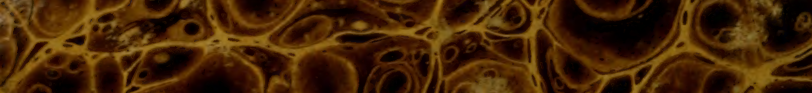

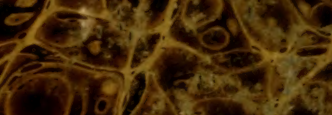

\section{$\operatorname{lig}_{2}$}

$(x, 3)$

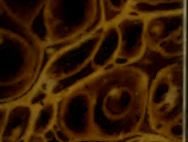

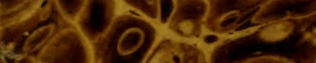

S.

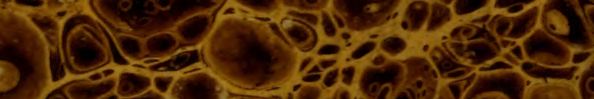

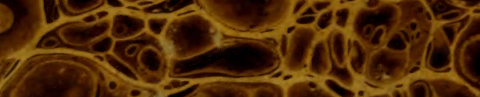

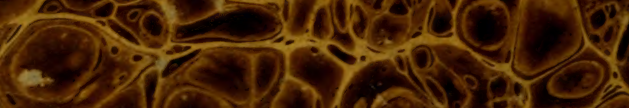<smiles>C1C2C3CC4C1C1C2C3C41</smiles>

10

tis

$\int_{0} 20$

ef

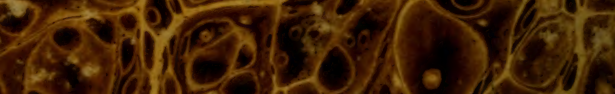

Q.

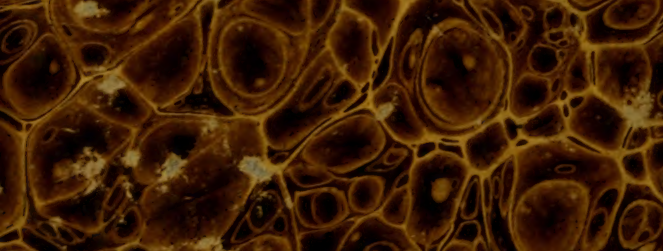

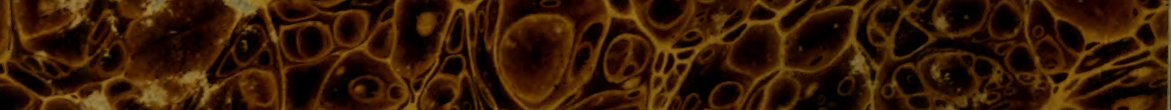

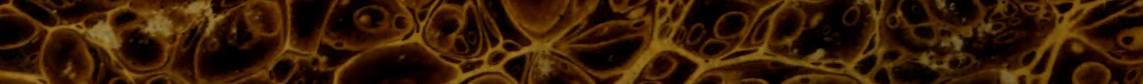
ST(3) 1.

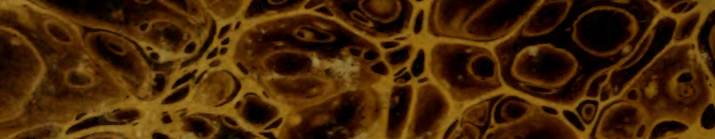

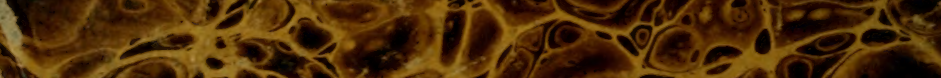

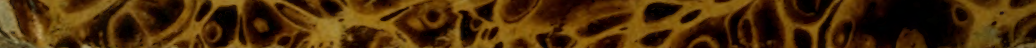

